\title{
Strike-slip tectonics during rift linkage
}

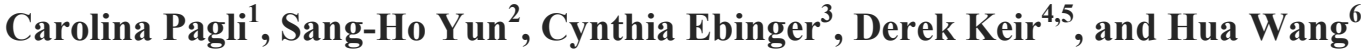

${ }^{I}$ Dipartimento di Scienze della Terra, Università di Pisa, Via S. Maria 53, 56126 Pisa, Italy

${ }^{2}$ Jet Propulsion Laboratory, California Institute of Technology, 4800 Oak Grove Drive

Pasadena, CA 91109, USA

${ }^{3}$ Department of Earth \& Environmental Sciences, Tulane University, 101 Blessey Hall

New Orleans, LA 70118, USA

${ }^{4}$ Ocean and Earth Science, University of Southampton, Waterfront Campus National

Oceanography Centre European Way SO14 3ZH, Southampton, UK

${ }^{5}$ Dipartimento di Scienze della Terra, Università degli Studi di Firenze, Via La Pira 4, 50121

Florence, Italy

${ }^{6}$ Department of Surveying Engineering, Guangdong University of Technology, 100 Waihuan

Xi Rd., Panyu District, Guangzhou, 510006, China

\section{Supplemental Material}

\section{Table of Contents}

Earthquake modelling ...........................................................................................................................2

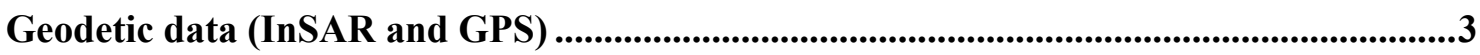

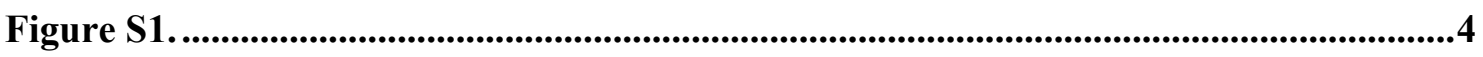

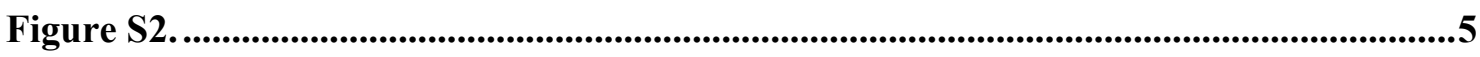

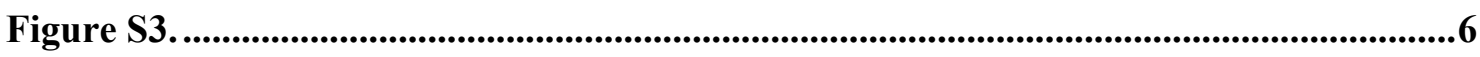

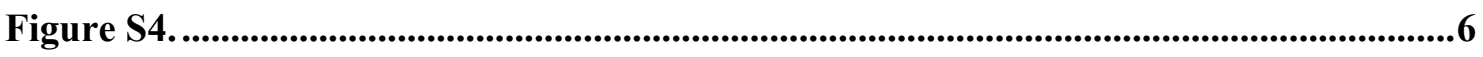

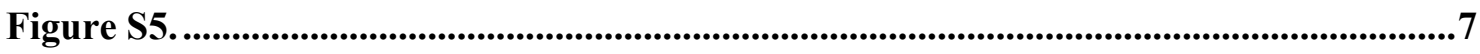

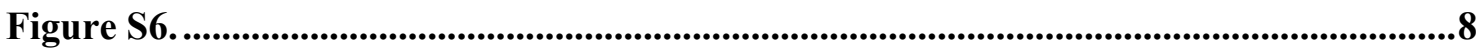

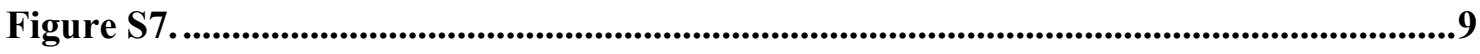

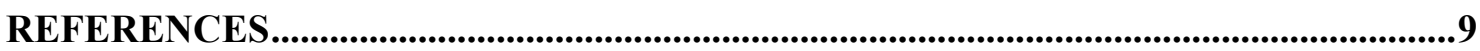




\section{Earthquake modelling}

The DMH intruded rift is modeled as rectangular crack experiencing uniform internal pressure in an isotropic, homogeneous, linear, elastic half-space. A Poisson's ratio of 0.25 and shear modulus of $30 \mathrm{GPa}$ were initially used. We used $4 \mathrm{~m}$ of opening along the entire DMH rift based on previous results (Wright et al., 2012). This value corresponds to the total opening of the rift including the first largest intrusion in Sept. 2005. Although our seismicity recordings start a month after that intrusion we still use the total opening so that our modelling results are an upper bound. We inverted the assumed dike opening for magma pressure on the dikes walls using a boundary element method (Yun et al., 2006) and find that a uniform excess pressure on the walls of the dike of $23 \mathrm{MPa}$ matches the $4 \mathrm{~m}$ opening. As intrusions induce faulting above the dike (Grandin et al., 2009), we assume faults in the uppermost $2 \mathrm{~km}$, with a dip angle of 60 degrees towards the rift axis. At deeper depths faults are assumed to be vertical as the dike opening prevents fault slip. The dike induced earthquakes are then simulated based on the seismicity-rate theory of Dieterich (Dieterich, 1994) as described in Segall et al. (2013), using a time duration of 10 days which is based on the total durations of the DMH intrusions. Specifically, we calculate the dike-induced seismicity rate, $R$, as follows

$$
\begin{aligned}
& R=\frac{r}{\gamma \dot{\tau}_{r}} \\
& \gamma=\left(\gamma_{0}-\frac{1}{\dot{\tau}}\right) \exp \left(\frac{-t \dot{\tau}}{A \sigma}\right)+\frac{1}{\dot{\tau}}
\end{aligned}
$$

where $r$ is the reference seismicity rate (once every 30 days, based on the seismicity in our study area during non co-intrusive periods) and $\dot{\tau}_{r}$ is the reference stressing rate $(0.1$

$\mathrm{MPa} /$ year), $\gamma$ is a state variable, $\gamma_{0}$ is the initial state variable equal to $\left(\frac{1}{\dot{\tau}_{r}}\right), \dot{\tau}$ is the shear stressing rate due to the dike, $t$ is time, $A$ is the fault frictional constitutive parameter, and $\sigma$ is the normal stress. Then we integrated the seismicity rate to calculate the number of events at hypothetical receiver faults in each voxel (volume elements) surrounding the intruded dike. We assumed faults to be parallel to the dike in $\mathrm{DMH}$, as observed in the field. We assume that all stresses imposed by diking were elastically accommodated, and the calculated number of events were plotted randomly within each voxel.

A fault frictional constitutive parameter of 0.01 is initially assumed (Fig. 2c) but we also test a friction of 0.004 as suggested from studies of dike intrusions in Kilawea (Segall et al., 2013). Decreasing this parameter generates a larger area of dike-induced seismicity (Fig. S4) 
but this still does not explain the seismicity lineament southeast of DMH. The mean effective normal stress is $66 \mathrm{MPa}$ in the intrusion zone and above it, from 0 to $9 \mathrm{~km}$ depth. We also tested a $15 \mathrm{GPa}$ shear modulus, as may be assumed in volcanic areas, and found that this change makes no difference in the induced seismicity pattern compared to assuming a 30 GPa modulus. Although halving the shear modulus doubles the dike opening, this also makes the material twice as compliant, negating the effect of the larger opening.

\section{Geodetic data (InSAR and GPS)}

InSAR from eight different tracks together with all the available GPS data 2007-2010 were combined to obtain a continuous high-resolution 3D velocity field and strain field of the Afar rift (Fig. S5) as described in (Pagli et al., 2014). First, the line-of-sight (LOS) average surface velocities 2007-2010 were obtained for each InSAR track, using a multi-interferogram method to mitigate common sources of errors in the interferograms, such as orbital and atmospheric delay errors (Wang et al., 2012). In all the analyses contributions from sudden events such as those caused by dike intrusions and eruptions were removed. The GPS data analysis included data from different sources. The GPS sites near Dabbahu-Manda Hararo and in central Afar were measured between 2007 and 2010 as a part of the Afar Rift Consortium and NSF projects (Nooner et al., 2009), while GPS velocities from the Red Sea coast, the Gulf of Aden and the main Ethiopian rift are from other sources (Kogan et al., 2012; McClusky et al., 2010; Saria et al., 2014; Vigny et al., 2007; Vigny et al., 2006) (Fig. S5). All GPS velocities were combined in the common International Terrestrial Reference Frame (ITRF 2008) and then converted the velocities with respect to (w.r.t.) Nubia (Fig. S6). Offset corrections were estimated and subtracted from the GPS time series in order to remove the contributions from dike intrusions and eruptions so that GPS velocities are directly comparable with the InSAR velocities. The InSAR velocities were combined with the GPS velocities, using a velocity-field inversion (Wang and Wright, 2012) to obtain surface velocities in the 3D components together with their associated uncertainties (Fig. S6), as described in(Pagli et al., 2014). Uncertainties are generally very small in central and southern Afar, less than $3 \mathrm{~mm} / \mathrm{yr}$, as many InSAR observations with different viewing geometries are available. Finally, based on the geodetically-derived velocity field we calculated the horizontal strain rates tensor and the associated uncertainties, using spherical approximations equations (Savage et al., 2001). The first invariant of the horizontal strain rate and the maximum horizontal shear strain rate are shown in Fig. 3 and their uncertainties are in Fig. 
S7. Uncertainties on the strain rates in central and southern Afar are generally less than $\sim 0.3 \times 10^{-7} / \mathrm{yr}$, about an order of magnitude smaller than the observed extensional strain rates (Fig. 3a).
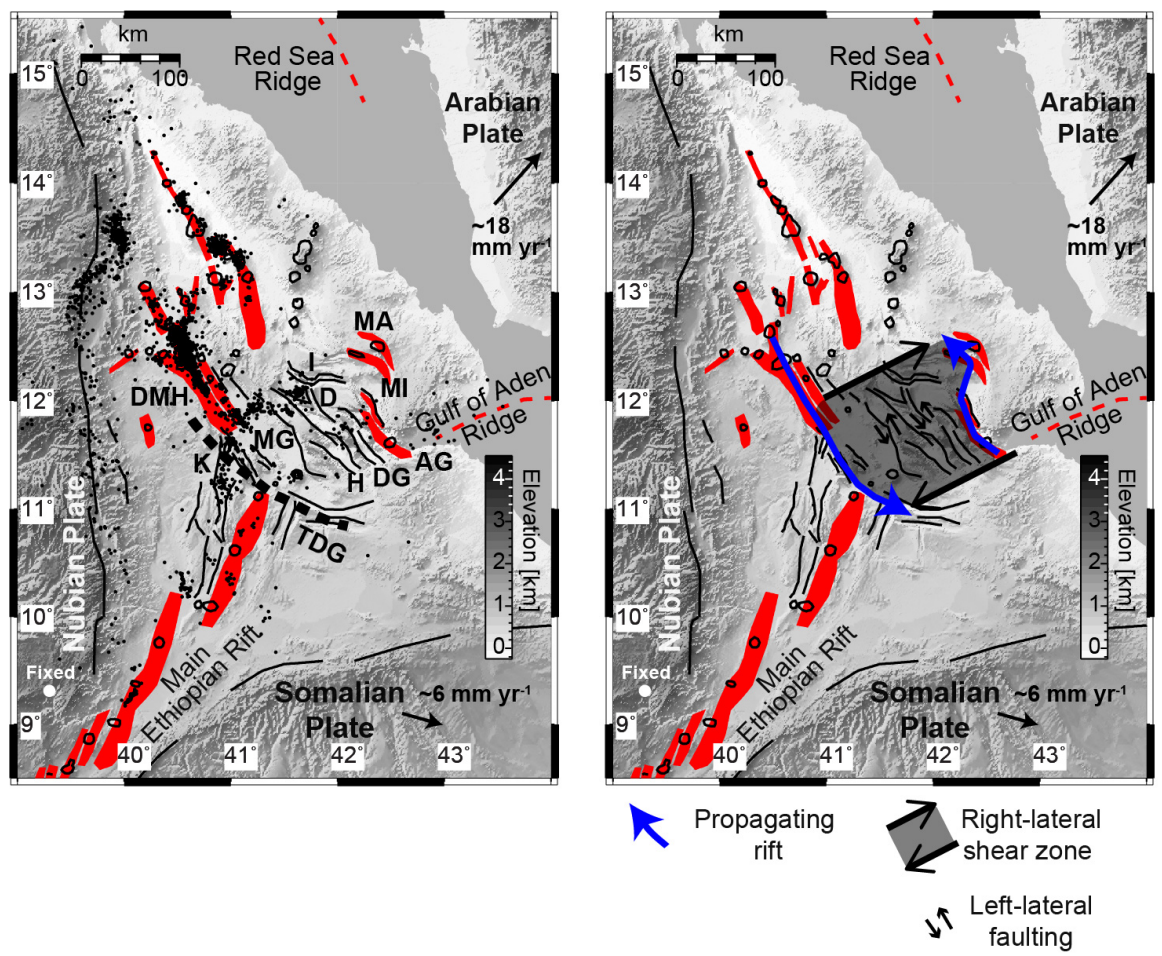

Figure S1.

a) Afar rift and local seismicity 2005-2009 (black dots). Solid red polygons are the Holocene magmatic rift segments: DMH Dabbahu-Manda Hararo, MA Moussa Alli, MI Manda Inakir and AG Asal-Ghoubbet segments. Volcanoes are marked by black outlines. Dashed line mark the Tendaho-Goba'ad discontinuity (TDG). Black lines are faults and the tectonic rift segments are: K Karrayu, MG Manda Gargori, D Dobi, I Immino, H Hanle and DG Derele Gaggade. Seismicity from Ebinger et al. (2008), Keir et al. (2009) and Belachew et al. (2011). Rift segments and faults modified from Wright et al. (2012) and Varet (1975). b) Afar rift and bookshelf model (modified from Tapponier et al., 1990). Blue arrows mark the two propagating rifts that create a broad zone of right-lateral shear in central Afar (dark grey area bounded by arrows), where a series of rift-parallel left-lateral strike-slip faults are assumed to accommodate deformation by bookshelf faulting. 

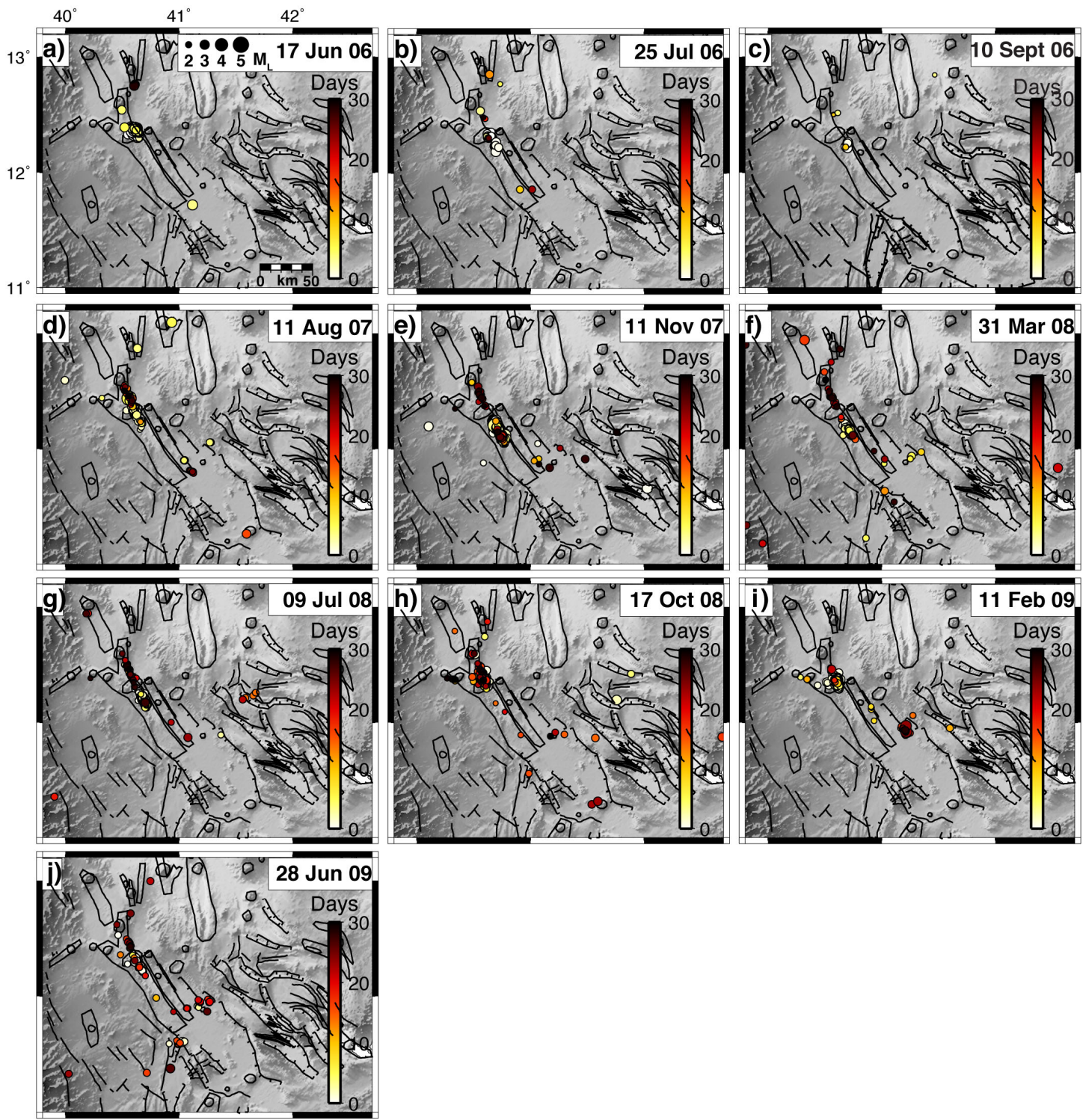

Figure S2.

Seismicity triggered by intrusions in 2006-2009. Coloured dots are earthquakes that occurred within 30 days after an intrusion. Earthquakes are colour coded by day of occurrence since onset of an intrusion. The date of each intrusion is given in the upper right corner. The $\mathrm{km}$ scale and the earthquakes magnitude scale shown in panel a) apply to all panels. 

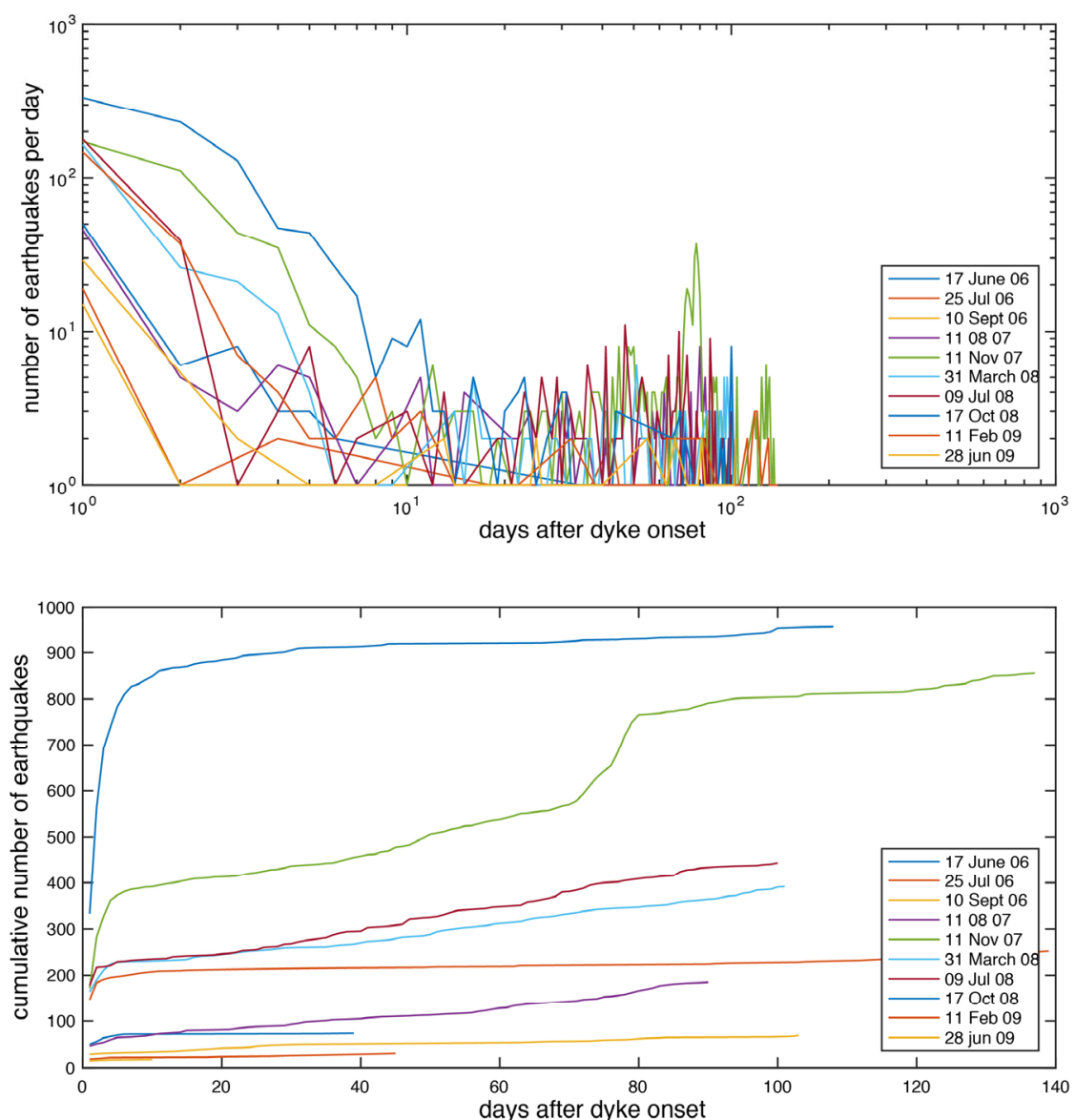

Figure S3.

Seismicity decay in Dabbahu-Manda-Hararo (DMH) after the intrusions in 2006-2009. Lines show the seismicity associated with each intrusion.

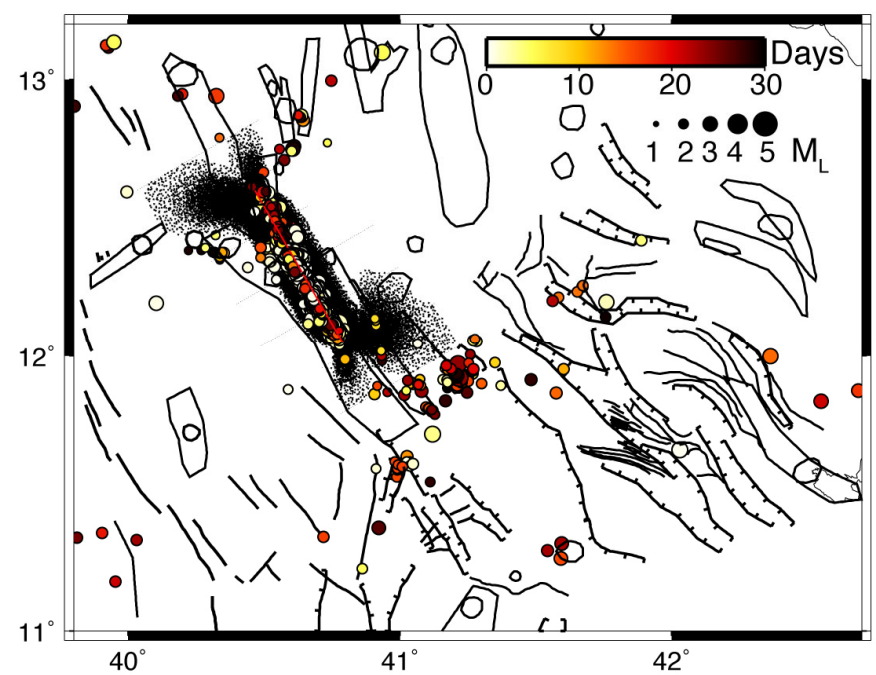

Figure S4.

Modelled dike-induced seismicity assuming a friction parameter of 0.004 . 


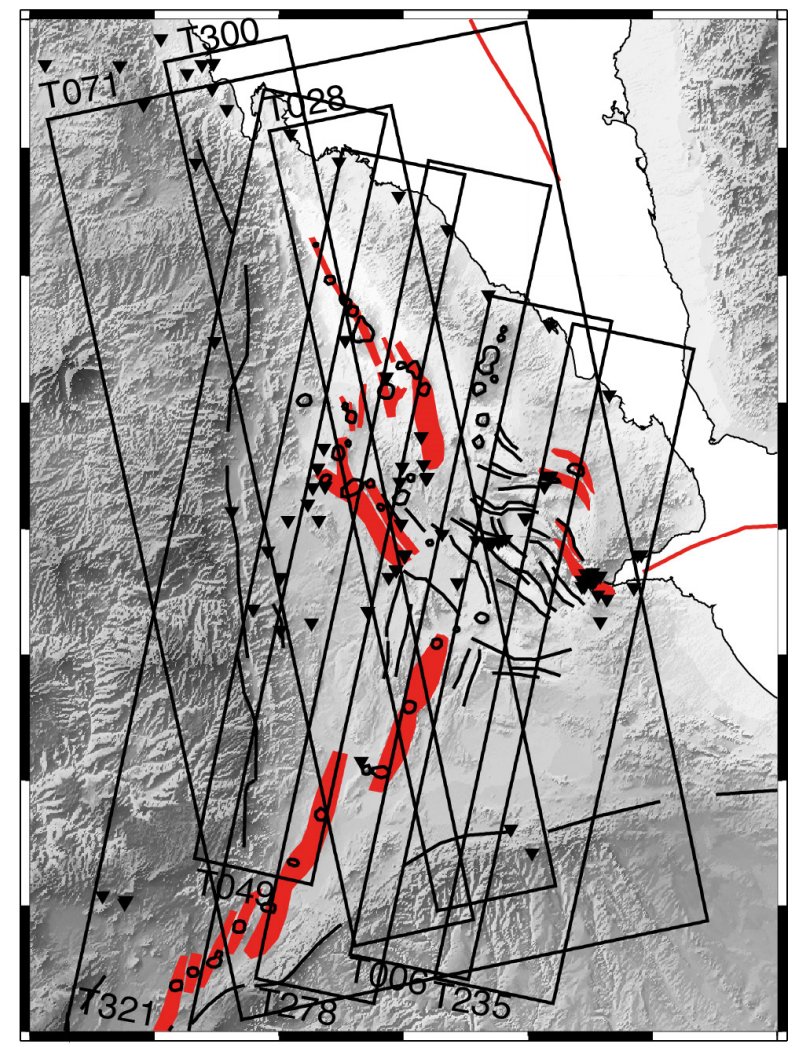

Figure S5.

Geodetic dataset used to obtain the 3D velocity field in Fig. S6. Black rectangles mark the InSAR tracks; the black triangles are the GPS sites (figure modified from Pagli et al., 2014). 

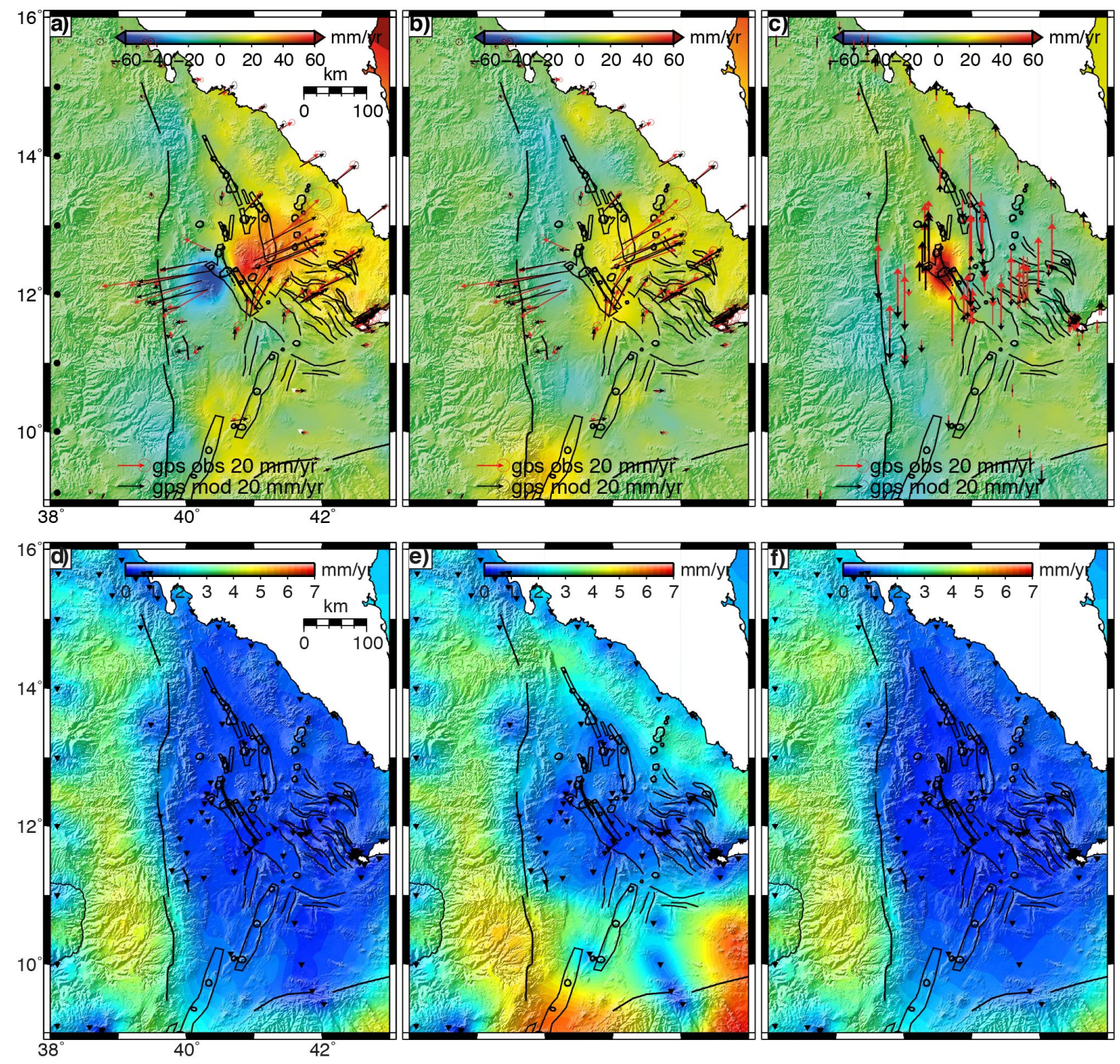

Figure S6.

Continuous 3D velocity field of Afar 2007-2010. a) East-West surface velocities (positive values are movements toward the East), observed horizontal GPS velocities (red arrows) and modelled horizontal GPS velocities (black arrows). b) North-South surface velocities (positive values are movements toward the North), observed horizontal GPS velocities (red arrows) and modelled horizontal GPS velocities (black arrows). c) Vertical surface velocities (positive values are uplift), observed horizontal GPS velocities (red arrows) and modelled horizontal GPS velocities (black arrows). GPS velocities are w.r.t. Nubia. d) Uncertainty on the East-West surface velocities. e) Uncertainty on the North-South surface velocities. f) Uncertainty on the Vertical surface velocities. Figures modified from Pagli et al., (2014). 

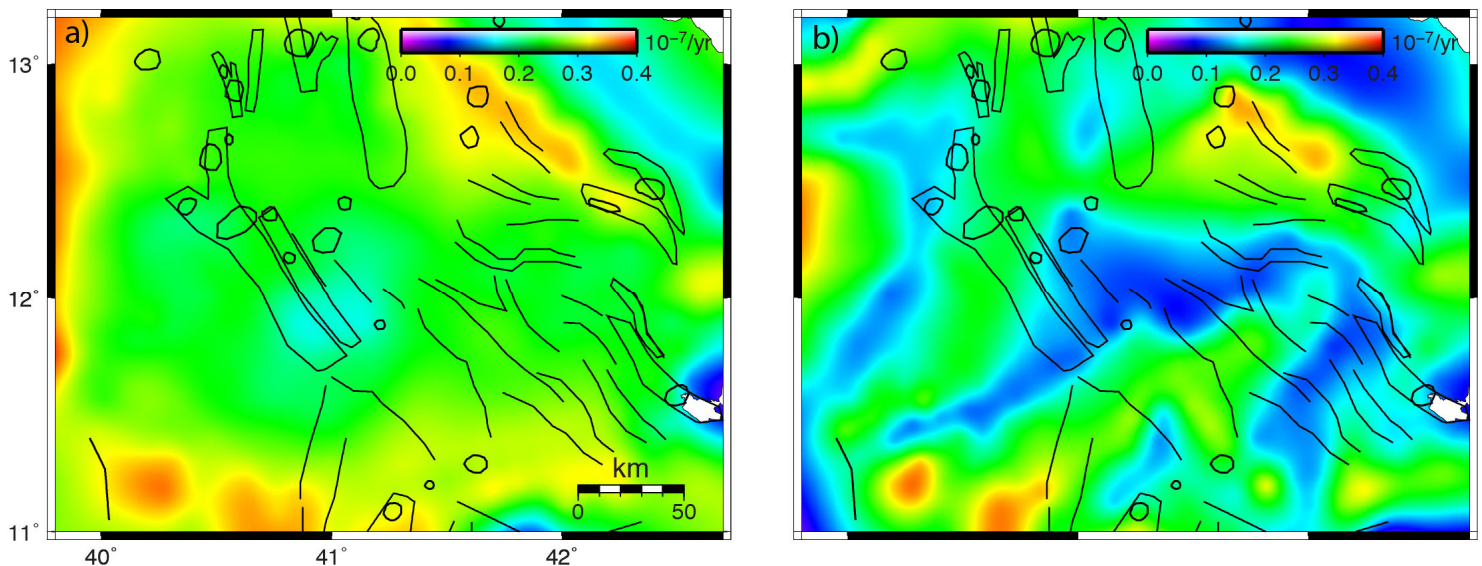

Figure S7.

Uncertaintis on the horizontal strain rates. a) Uncertainty on the first invariant of the horizontal strain rate. b) Uncertainty on the maximum horizontal shear strain rate. Figures modified from Pagli et al., (2014).

\section{REFERENCES}

Belachew, M., Ebinger, C., Cote, D., Keir, D., Rowland, J. V., Hammond, J. O. S., and Ayele, A., 2011, Comparison of dike intrusions in an incipient seafloor-spreading segment in Afar, Ethiopia: Seismicity perspectives: Journal of Geophysical Research, v. 116, B06405, doi:10.1029/2010JB007908.

Dieterich, J., 1994, A constitutive law for rate of earthquake production and its application to earthquake clustering: Journal of Geophysical Research, v. 99, p. 2601-2618, doi:10.1029/93JB02581.

Ebinger, C. J., Keir, D., Ayele, A., Calais, E., Wright, T. J., Belachew, M., Hammond, J. O. S., Campbell, E., and Buck, W. R., 2008, Capturing magma intrusion and faulting processes during continental rupture: seismicity of the Dabbahu (Afar) rift: Geophysical Journal International, v. 174, p. 1138-1152, doi:10.1111/j.1365246X.2008.03877.x.

Grandin, R., et al., 2009, September 2005 Manda Hararo-Dabbahu rifting event, Afar (Ethiopia): Constraints provided by geodetic data: Journal of Geophysical Research, v. 114, p. B08404, B08404, doi:10.1029/2008jb005843.

Keir, D., et al., 2009, Evidence for focused magmatic accretion at segment centers from lateral dike injections captured beneath the Red Sea rift in Afar: Geology, v. 37, p. 5962, doi:10.1130/G25147a.1.

Kogan, L., Fisseha, S., Bendick, R., Reilinger, R., McClusky, S., King, R., and Solomon, T., 2012, Lithospheric strength and strain localization in continental extension from observations of the East African Rift: Journal of Geophysical Research: Solid Earth, v. 117, 10.1029/2011JB008516.

McClusky, S., et al., 2010, Kinematics of the southern Red Sea-Afar Triple Junction and implications for plate dynamics: Geophysical Research Letters, v. 37, L05301, doi:10.1029/2009GL041127. 
Pagli, C., Wang, H., Wright, T. J., Calais, E., and Lewi, E., 2014, Current plate boundary deformation of the Afar rift from a 3-D velocity field inversion of InSAR and GPS: Journal of Geophysical Research, v. 119, p. 8562-8575, doi:10.1002/2014jb011391.

Saria, E., Calais, E., Stamps, D. S., Delvaux, D., and Hartnady, C. J. H., 2014, Present-day kinematics of the East African Rift: Journal of Geophysical Research: Solid Earth, v. 119, p. 3584-3600, doi:10.1002/2013JB010901.

Savage, J. C., Gan, W. J., and Svarc, J. L., 2001, Strain accumulation and rotation in the Eastern California Shear Zone: Journal of Geophysical Research-Solid Earth, v. 106, p. 21995-22007, Doi 10.1029/2000jb000127.

Segall, P., Llenos, A. L., Yun, S. H., Bradley, A. M., and Syracuse, E. M., 2013, Timedependent dike propagation from joint inversion of seismicity and deformation data: Journal of Geophysical Research, v. 118, p. 5785-5804, doi:10.1002/2013JB010251.

Varet, J., 1975, Geological map of Central and Southern Afar: CNRS-CNR, scale 1:500,000.

Vigny, C., de Chabalier, J. B., Ruegg, J. C., Huchon, P., Feigl, K. L., Cattin, R., Asfaw, L., and Kanbari, K., 2007, Twenty-five years of geodetic measurements along the Tadjoura-Asal rift system, Djibouti, East Africa: Journal of Geophysical Research, v. 112, B06410, doi:doi:10.1029/2004jb003230.

Vigny, C., Huchon, P., Ruegg, J. C., Khanbari, K., and Asfaw Laike, M., 2006, Confirmation of Arabia plate slow motion by new GPS data in Yemen: Journal of Geophysical Research: Solid Earth, v. 111, 10.1029/2004JB003229.

Wang, H., and Wright, T. J., 2012, Satellite geodetic imaging reveals internal deformation of western Tibet: Geophysical Research Letters, v. 39, L07303, doi:doi:10.1029/2012g1051222.

Wang, H., Wright, T. J., Yu, Y. P., Lin, H., Jiang, L. L., Li, C. H., and Qiu, G. X., 2012, InSAR reveals coastal subsidence in the Pearl River Delta, China: Geophysical Journal International, v. 191, p. 1119-1128,

Wright, T. J., et al., 2012, Geophysical constraints on the dynamics of spreading centres from rifting episodes on land: Nature Geoscience, v. 5, p. 242-250, doi:10.1038/Ngeo1428.

Yun, S., Segall, P., and Zebker, H., 2006, Constraints on magma chamber geometry at Sierra Negra Volcano, Galápagos Islands, based on InSAR observations: Journal of Volcanology and Geothermal Research, v. 150, p. 232-243, doi:10.1016/j.jvolgeores.2005.07.009. 
Supplemental table DR1

Seismicity in Afar from seismic surveys between 19 0ctober 2005 and 07 0ctober 2009 (Ebinger et al., 2008), Keir et al., 2009), and Belachew et al., 2011). Data are also available on the ISC (International Seismological Center) catalogue.

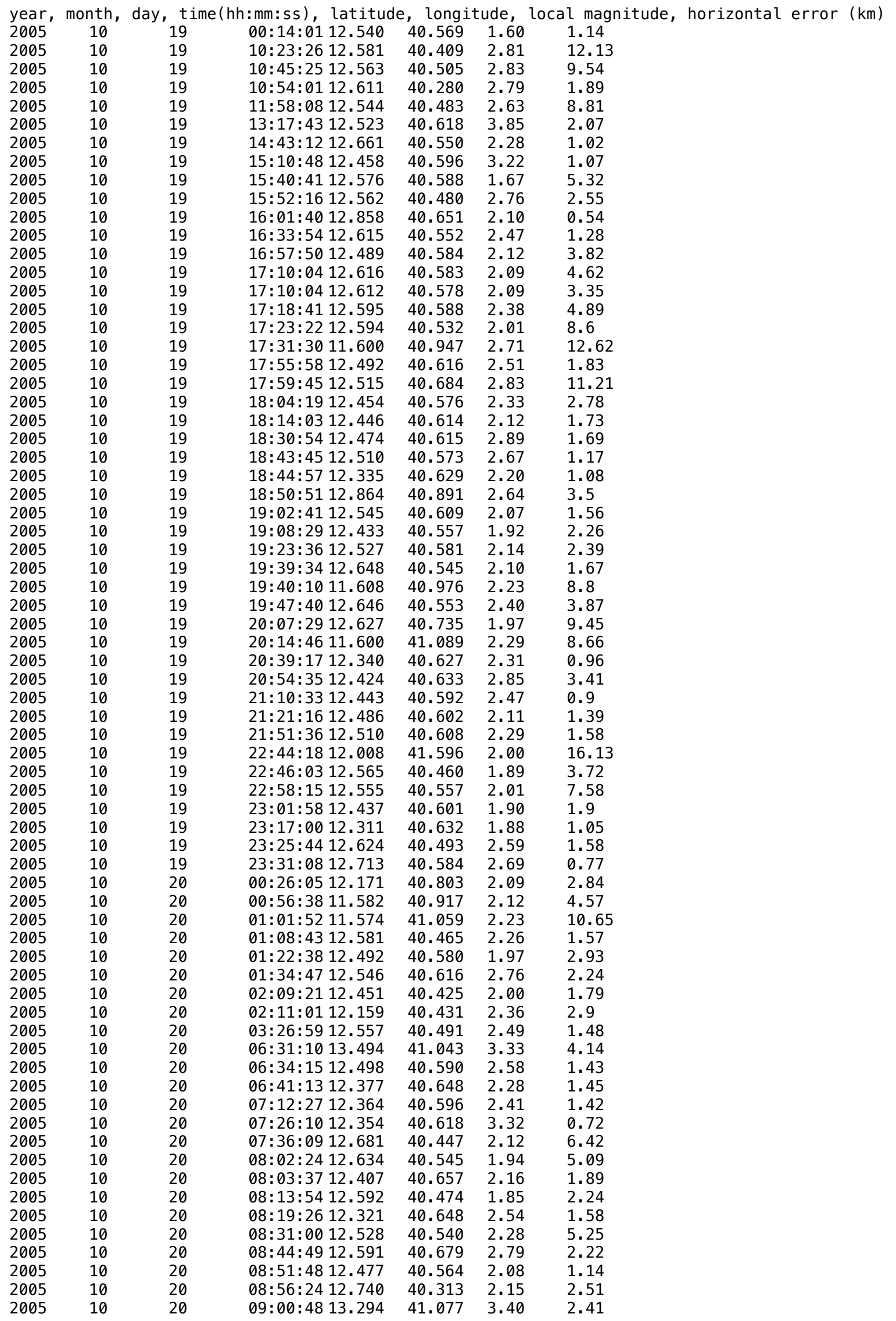




\begin{tabular}{|c|c|c|c|c|c|c|}
\hline 2005 & 10 & 20 & $09: 09: 0412.702$ & 40.321 & 1.92 & 3.77 \\
\hline 2005 & 10 & 20 & $09: 28: 2612.627$ & 40.480 & 2.45 & 1.72 \\
\hline 2005 & 10 & 20 & $09: 28: 2712.608$ & 40.522 & 2.47 & 2.1 \\
\hline 2005 & 10 & 20 & $09: 28: 4612.353$ & 40.592 & 2.44 & 1.31 \\
\hline 2005 & 10 & 20 & $09: 35: 5912.368$ & 40.627 & 2.43 & 13.02 \\
\hline 2005 & 10 & 20 & $09: 40: 3612.579$ & 40.548 & 2.78 & 1.84 \\
\hline 2005 & 10 & 20 & $09: 49: 0912.582$ & 40.475 & 2.33 & 1.31 \\
\hline 2005 & 10 & 20 & $09: 57: 0912.664$ & 40.376 & 1.54 & 3.89 \\
\hline 2005 & 10 & 20 & $10: 18: 2512.557$ & 40.525 & 2.52 & 3.08 \\
\hline 2005 & 10 & 20 & $10: 28: 1612.577$ & 40.510 & 2.46 & 3.2 \\
\hline 2005 & 10 & 20 & $10: 40: 3412.444$ & 40.584 & 2.20 & 0.8 \\
\hline 2005 & 10 & 20 & $10: 41: 4112.479$ & 40.560 & 2.18 & 0.66 \\
\hline 2005 & 10 & 20 & $10: 43: 4912.482$ & 40.562 & 2.17 & 2.41 \\
\hline 2005 & 10 & 20 & $10: 45: 3012.525$ & 40.578 & 2.46 & 10.13 \\
\hline 2005 & 10 & 20 & $10: 58: 1512.579$ & 40.475 & 2.15 & 2.24 \\
\hline 2005 & 10 & 20 & $11: 15: 2012.607$ & 40.458 & 2.06 & 8.48 \\
\hline 2005 & 10 & 20 & $11: 35: 1612.535$ & 40.564 & 2.66 & 2.45 \\
\hline 2005 & 10 & 20 & $11: 50: 1012.488$ & 40.557 & 2.41 & 0.74 \\
\hline 2005 & 10 & 20 & $12: 11: 3312.479$ & 40.556 & 2.83 & 0.62 \\
\hline 2005 & 10 & 20 & $12: 22: 2212.506$ & 40.539 & 2.80 & 0.79 \\
\hline 2005 & 10 & 20 & $12: 22: 2212.500$ & 40.555 & 2.81 & 0.63 \\
\hline 2005 & 10 & 20 & $13: 46: 2412.590$ & 40.473 & 2.44 & 2.97 \\
\hline 2005 & 10 & 20 & $13: 52: 4712.588$ & 40.481 & 2.11 & 3.9 \\
\hline 2005 & 10 & 20 & $14: 13: 2413.409$ & 40.856 & 2.83 & 3.16 \\
\hline 2005 & 10 & 20 & $14: 33: 1912.435$ & 40.572 & 2.23 & 1.13 \\
\hline 2005 & 10 & 20 & $15: 14: 0712.380$ & 40.590 & 1.95 & 2.45 \\
\hline 2005 & 10 & 20 & $15: 33: 5112.631$ & 40.479 & 2.80 & 1.57 \\
\hline 2005 & 10 & 20 & $15: 38: 1413.376$ & 40.913 & 2.63 & 1.44 \\
\hline 2005 & 10 & 20 & $15: 48: 5112.619$ & 40.452 & 2.07 & 4.3 \\
\hline 2005 & 10 & 20 & $15: 56: 4812.719$ & 40.585 & 2.84 & 0.89 \\
\hline 2005 & 10 & 20 & $15: 58: 1812.625$ & 40.475 & 2.28 & 6.8 \\
\hline 2005 & 10 & 20 & $16: 02: 0612.486$ & 40.550 & 2.76 & 0.73 \\
\hline 2005 & 10 & 20 & $16: 34: 1012.620$ & 40.520 & 1.84 & 2.18 \\
\hline 2005 & 10 & 20 & $16: 34: 1012.614$ & 40.544 & 1.98 & 1.96 \\
\hline 2005 & 10 & 20 & $16: 38: 2712.470$ & 40.564 & 3.65 & 0.61 \\
\hline 2005 & 10 & 20 & $16: 58: 4712.557$ & 40.520 & 2.19 & 4.65 \\
\hline 2005 & 10 & 20 & $17: 12: 4412.444$ & 40.585 & 2.18 & 0.56 \\
\hline 2005 & 10 & 20 & $17: 15: 5412.600$ & 40.490 & 2.08 & 3.61 \\
\hline 2005 & 10 & 20 & $17: 40: 5212.565$ & 40.472 & 2.57 & 1.74 \\
\hline 2005 & 10 & 20 & $17: 51: 3712.444$ & 40.580 & 2.40 & 0.67 \\
\hline 2005 & 10 & 20 & $18: 02: 0912.646$ & 40.547 & 1.94 & 1.98 \\
\hline 2005 & 10 & 20 & $18: 14: 4312.422$ & 40.569 & 2.00 & 0.89 \\
\hline 2005 & 10 & 20 & $18: 24: 5012.574$ & 40.496 & 2.48 & 1.35 \\
\hline 2005 & 10 & 20 & $18: 39: 5312.517$ & 40.530 & 1.93 & 2.02 \\
\hline 2005 & 10 & 20 & $19: 09: 1612.578$ & 40.442 & 1.78 & 2.75 \\
\hline 2005 & 10 & 20 & $19: 13: 5612.424$ & 40.593 & 2.41 & 0.79 \\
\hline 2005 & 10 & 20 & $19: 34: 2212.580$ & 40.518 & 1.91 & 0.58 \\
\hline 2005 & 10 & 20 & $19: 38: 0112.473$ & 40.641 & 2.27 & 13.88 \\
\hline 2005 & 10 & 20 & $19: 43: 0512.320$ & 40.631 & 2.78 & 0.68 \\
\hline 2005 & 10 & 20 & $19: 49: 2312.317$ & 40.631 & 3.28 & 0.52 \\
\hline 2005 & 10 & 20 & $19: 49: 4612.331$ & 40.623 & 3.27 & 0.98 \\
\hline 2005 & 10 & 20 & $19: 53: 0912.409$ & 40.589 & 2.71 & 0.43 \\
\hline 2005 & 10 & 20 & $20: 08: 0112.261$ & 40.652 & 2.14 & 0.94 \\
\hline 2005 & 10 & 20 & $20: 30: 2212.428$ & 40.576 & 2.10 & 0.99 \\
\hline 2005 & 10 & 20 & $20: 43: 3312.604$ & 40.504 & 2.42 & 0.87 \\
\hline 2005 & 10 & 20 & $21: 24: 5712.438$ & 40.611 & 1.90 & 1.25 \\
\hline 2005 & 10 & 20 & $21: 45: 5012.611$ & 40.466 & 2.13 & 2.84 \\
\hline 2005 & 10 & 20 & $22: 00: 1412.441$ & 40.586 & 2.20 & 4.86 \\
\hline 2005 & 10 & 20 & $22: 03: 3112.573$ & 40.526 & 2.59 & 1.09 \\
\hline 2005 & 10 & 20 & $22: 13: 1012.592$ & 40.484 & 2.70 & 0.58 \\
\hline 2005 & 10 & 20 & $22: 26: 4512.577$ & 40.513 & 1.71 & 2.61 \\
\hline 2005 & 10 & 20 & $22: 29: 4912.482$ & 40.553 & 2.23 & 0.77 \\
\hline 2005 & 10 & 20 & $22: 34: 4512.310$ & 40.627 & 2.06 & 0.66 \\
\hline 2005 & 10 & 20 & $22: 44: 4512.367$ & 40.599 & 2.45 & 0.57 \\
\hline 2005 & 10 & 20 & $23: 00: 4412.595$ & 40.509 & 2.91 & 0.53 \\
\hline 2005 & 10 & 20 & $23: 04: 3412.611$ & 40.484 & 1.77 & 2.9 \\
\hline 2005 & 10 & 20 & $23: 05: 3112.572$ & 40.527 & 1.99 & 0.8 \\
\hline 2005 & 10 & 20 & $23: 13: 2212.521$ & 40.534 & 2.43 & 1.44 \\
\hline 2005 & 10 & 20 & $23: 36: 2212.594$ & 40.510 & 2.11 & 1.16 \\
\hline 2005 & 10 & 20 & $23: 39: 5012.616$ & 40.525 & 1.53 & 6.91 \\
\hline 2005 & 10 & 20 & $23: 44: 1812.380$ & 40.610 & 2.43 & 0.59 \\
\hline 2005 & 10 & 20 & $23: 47: 2712.394$ & 40.609 & 1.91 & 0.5 \\
\hline 2005 & 10 & 20 & $23: 54: 0512.715$ & 40.550 & 1.97 & 3.26 \\
\hline 2005 & 10 & 21 & $00: 01: 4112.633$ & 40.538 & 1.93 & 1.17 \\
\hline 2005 & 10 & 21 & $00: 02: 4912.533$ & 40.544 & 2.20 & 1.36 \\
\hline 2005 & 10 & 21 & $00: 04: 2812.623$ & 40.482 & 2.72 & 0.98 \\
\hline
\end{tabular}




\begin{tabular}{|c|c|c|c|c|c|c|}
\hline 2005 & 10 & 21 & $00: 11: 1912.400$ & 40.594 & 2.15 & 0.58 \\
\hline 2005 & 10 & 21 & $00: 14: 3812.653$ & 40.534 & 1.76 & 0.69 \\
\hline 2005 & 10 & 21 & $00: 43: 0612.254$ & 40.657 & 1.75 & 1.25 \\
\hline 2005 & 10 & 21 & $00: 48: 1112.357$ & 40.656 & 1.85 & 2.17 \\
\hline 2005 & 10 & 21 & $01: 14: 5312.635$ & 40.552 & 1.67 & 2.44 \\
\hline 2005 & 10 & 21 & $01: 30: 1012.462$ & 40.567 & 2.93 & 0.5 \\
\hline 2005 & 10 & 21 & $01: 34: 1813.400$ & 40.904 & 2.17 & 1.8 \\
\hline 2005 & 10 & 21 & $01: 38: 1612.617$ & 40.513 & 1.87 & 1.97 \\
\hline 2005 & 10 & 21 & $01: 57: 5712.590$ & 40.514 & 1.84 & 3.68 \\
\hline 2005 & 10 & 21 & $02: 02: 5112.570$ & 40.526 & 2.52 & 0.74 \\
\hline 2005 & 10 & 21 & $02: 06: 2612.607$ & 40.439 & 2.17 & 6.47 \\
\hline 2005 & 10 & 21 & $02: 13: 5112.550$ & 40.496 & 2.41 & 0.68 \\
\hline 2005 & 10 & 21 & $02: 33: 5612.542$ & 40.570 & 2.05 & 3.29 \\
\hline 2005 & 10 & 21 & $02: 47: 2612.365$ & 40.609 & 2.40 & 2.04 \\
\hline 2005 & 10 & 21 & $02: 53: 1712.590$ & 40.455 & 2.58 & 2.32 \\
\hline 2005 & 10 & 21 & $03: 04: 1612.445$ & 40.586 & 2.52 & 2.61 \\
\hline 2005 & 10 & 21 & $03: 52: 1412.542$ & 40.527 & 2.13 & 3.2 \\
\hline 2005 & 10 & 21 & $03: 53: 0012.363$ & 40.610 & 2.46 & 1.24 \\
\hline 2005 & 10 & 21 & $04: 00: 4212.541$ & 40.534 & 2.41 & 1.41 \\
\hline 2005 & 10 & 21 & $04: 17: 5413.395$ & 40.936 & 3.23 & 1.7 \\
\hline 2005 & 10 & 21 & $04: 26: 4812.550$ & 40.507 & 2.26 & 3.42 \\
\hline 2005 & 10 & 21 & $04: 46: 1012.387$ & 40.597 & 2.78 & 0.67 \\
\hline 2005 & 10 & 21 & $04: 49: 5312.583$ & 40.518 & 2.03 & 0.62 \\
\hline 2005 & 10 & 21 & $04: 52: 3212.395$ & 40.598 & 2.68 & 0.55 \\
\hline 2005 & 10 & 21 & $05: 09: 3712.726$ & 40.315 & 2.46 & 2.2 \\
\hline 2005 & 10 & 21 & $06: 24: 2912.559$ & 40.476 & 2.06 & 1.86 \\
\hline 2005 & 10 & 21 & $07: 09: 4512.461$ & 40.492 & 2.43 & 3.13 \\
\hline 2005 & 10 & 21 & $07: 14: 4412.598$ & 40.502 & 2.18 & 1.14 \\
\hline 2005 & 10 & 21 & $07: 30: 0212.577$ & 40.487 & 2.83 & 1.17 \\
\hline 2005 & 10 & 21 & $07: 37: 4712.395$ & 40.602 & 3.09 & 0.7 \\
\hline 2005 & 10 & 21 & $08: 09: 2912.547$ & 40.609 & 2.29 & 2.52 \\
\hline 2005 & 10 & 21 & $08: 26: 2812.422$ & 40.609 & 2.95 & 0.86 \\
\hline 2005 & 10 & 21 & $08: 32: 0012.845$ & 40.633 & 2.58 & 0.65 \\
\hline 2005 & 10 & 21 & $08: 48: 0012.619$ & 40.609 & 2.18 & 5.43 \\
\hline 2005 & 10 & 21 & $09: 23: 3712.626$ & 40.479 & 3.08 & 1.25 \\
\hline 2005 & 10 & 21 & $10: 11: 1512.447$ & 40.574 & 2.69 & 0.52 \\
\hline 2005 & 10 & 21 & $10: 28: 3512.611$ & 40.476 & 1.83 & 5.73 \\
\hline 2005 & 10 & 21 & $10: 55: 4412.632$ & 40.434 & 1.75 & 9.19 \\
\hline 2005 & 10 & 21 & $11: 07: 2512.591$ & 40.517 & 2.46 & 1.04 \\
\hline 2005 & 10 & 21 & $11: 30: 5612.505$ & 40.549 & 2.16 & 1.86 \\
\hline 2005 & 10 & 21 & $11: 49: 5812.732$ & 40.290 & 2.26 & 2.61 \\
\hline 2005 & 10 & 21 & $12: 13: 5912.727$ & 40.345 & 2.47 & 3.6 \\
\hline 2005 & 10 & 21 & $12: 26: 2612.451$ & 40.591 & 2.64 & 0.65 \\
\hline 2005 & 10 & 21 & $12: 50: 5212.595$ & 40.507 & 2.40 & 1.06 \\
\hline 2005 & 10 & 21 & $12: 59: 2012.489$ & 40.557 & 2.49 & 0.94 \\
\hline 2005 & 10 & 21 & $14: 50: 0712.484$ & 40.546 & 2.40 & 1.29 \\
\hline 2005 & 10 & 21 & $14: 53: 0212.936$ & 40.292 & 2.58 & 1.7 \\
\hline 2005 & 10 & 21 & $15: 27: 2912.690$ & 40.391 & 2.39 & 10.38 \\
\hline 2005 & 10 & 21 & $15: 41: 4812.550$ & 40.497 & 2.93 & 0.61 \\
\hline 2005 & 10 & 21 & $15: 50: 4912.609$ & 40.511 & 2.17 & 2.65 \\
\hline 2005 & 10 & 21 & $16: 06: 2612.608$ & 40.462 & 3.10 & 1.16 \\
\hline 2005 & 10 & 21 & $16: 10: 5412.579$ & 40.467 & 2.65 & 1.23 \\
\hline 2005 & 10 & 21 & $16: 22: 1412.638$ & 40.547 & 1.95 & 4.11 \\
\hline 2005 & 10 & 21 & $16: 43: 3812.575$ & 40.526 & 2.89 & 0.84 \\
\hline 2005 & 10 & 21 & $16: 55: 0712.588$ & 40.525 & 2.94 & 0.63 \\
\hline 2005 & 10 & 21 & $17: 13: 5012.383$ & 40.605 & 2.71 & 0.43 \\
\hline 2005 & 10 & 21 & $17: 23: 4712.302$ & 40.635 & 2.26 & 1.04 \\
\hline 2005 & 10 & 21 & $17: 29: 3012.589$ & 40.457 & 3.12 & 0.99 \\
\hline 2005 & 10 & 21 & $17: 54: 2712.625$ & 40.531 & 2.17 & 0.93 \\
\hline 2005 & 10 & 21 & $18: 05: 2512.354$ & 40.599 & 2.14 & 0.87 \\
\hline 2005 & 10 & 21 & $18: 15: 3012.549$ & 40.477 & 1.80 & 1.31 \\
\hline 2005 & 10 & 21 & $18: 47: 4112.576$ & 40.547 & 2.08 & 0.79 \\
\hline 2005 & 10 & 21 & $18: 58: 2512.479$ & 40.528 & 2.05 & 1.1 \\
\hline 2005 & 10 & 21 & $19: 17: 1812.639$ & 40.551 & 1.95 & 1.64 \\
\hline 2005 & 10 & 21 & $19: 19: 1512.394$ & 40.602 & 2.37 & 1.26 \\
\hline 2005 & 10 & 21 & $19: 23: 2912.470$ & 40.572 & 2.36 & 0.56 \\
\hline 2005 & 10 & 21 & $19: 26: 1012.451$ & 40.584 & 2.00 & 1.15 \\
\hline 2005 & 10 & 21 & $19: 36: 5412.580$ & 40.475 & 2.32 & 1.13 \\
\hline 2005 & 10 & 21 & $20: 19: 4312.581$ & 40.513 & 2.00 & 2.32 \\
\hline 2005 & 10 & 21 & $20: 34: 5112.564$ & 40.525 & 2.25 & 1,00 \\
\hline 2005 & 10 & 21 & $21: 03: 4112.760$ & 40.446 & 2.34 & 3.58 \\
\hline 2005 & 10 & 21 & $21: 23: 3512.604$ & 40.504 & 2.59 & 0.74 \\
\hline 2005 & 10 & 21 & $21: 58: 1412.568$ & 40.536 & 2.47 & 1.3 \\
\hline 2005 & 10 & 21 & $22: 20: 0512.569$ & 40.524 & 1.89 & 0.98 \\
\hline 2005 & 10 & 21 & $22: 25: 2712.593$ & 40.481 & 2.28 & 1.74 \\
\hline 2005 & 10 & 21 & $22: 45: 0412.485$ & 40.538 & 2.15 & 1.07 \\
\hline
\end{tabular}




\begin{tabular}{|c|c|c|c|c|c|c|}
\hline 2005 & 10 & 21 & $22: 48: 2512.565$ & 40.538 & 2.16 & 1.14 \\
\hline 2005 & 10 & 21 & $23: 32: 5812.612$ & 40.477 & 2.89 & 0.77 \\
\hline 2005 & 10 & 21 & $23: 44: 2112.527$ & 40.536 & 2.35 & 0.56 \\
\hline 2005 & 10 & 22 & $00: 04: 2112.576$ & 40.487 & 2.37 & 1.41 \\
\hline 2005 & 10 & 22 & $00: 06: 3412.389$ & 40.592 & 2.40 & 0.92 \\
\hline 2005 & 10 & 22 & $00: 31: 3012.459$ & 40.560 & 2.12 & 0.74 \\
\hline 2005 & 10 & 22 & $00: 34: 3712.371$ & 40.599 & 2.66 & 0.56 \\
\hline 2005 & 10 & 22 & $00: 47: 0512.539$ & 40.543 & 1.84 & 0.88 \\
\hline 2005 & 10 & 22 & $00: 59: 5312.507$ & 40.541 & 2.21 & 0.83 \\
\hline 2005 & 10 & 22 & $01: 02: 4712.540$ & 40.519 & 2.09 & 4.3 \\
\hline 2005 & 10 & 22 & $01: 29: 0412.449$ & 40.561 & 2.40 & 0.88 \\
\hline 2005 & 10 & 22 & $02: 08: 4612.877$ & 40.401 & 2.45 & 4.69 \\
\hline 2005 & 10 & 22 & $02: 32: 0212.583$ & 40.519 & 2.96 & 0.86 \\
\hline 2005 & 10 & 22 & $03: 16: 5612.464$ & 40.557 & 2.08 & 1.42 \\
\hline 2005 & 10 & 22 & $03: 52: 0912.387$ & 40.605 & 2.32 & 2.31 \\
\hline 2005 & 10 & 22 & $04: 27: 2412.427$ & 40.592 & 2.99 & 0.99 \\
\hline 2005 & 10 & 22 & $04: 34: 1512.309$ & 40.658 & 2.67 & 1.08 \\
\hline 2005 & 10 & 22 & $06: 36: 2212.556$ & 40.542 & 2.71 & 0.67 \\
\hline 2005 & 10 & 22 & $06: 40: 5412.870$ & 40.318 & 2.67 & 0.73 \\
\hline 2005 & 10 & 22 & $06: 53: 5412.392$ & 40.606 & 3.25 & 1.07 \\
\hline 2005 & 10 & 22 & $07: 05: 3012.476$ & 40.559 & 2.68 & 0.52 \\
\hline 2005 & 10 & 22 & $07: 54: 4612.463$ & 40.570 & 3.12 & 0.79 \\
\hline 2005 & 10 & 22 & $09: 52: 5012.619$ & 40.469 & 3.00 & 0.84 \\
\hline 2005 & 10 & 22 & $10: 00: 1612.448$ & 40.578 & 2.33 & 0.62 \\
\hline 2005 & 10 & 22 & $10: 58: 2812.487$ & 40.557 & 2.90 & 0.49 \\
\hline 2005 & 10 & 22 & $11: 13: 2112.568$ & 40.484 & 2.47 & 0.69 \\
\hline 2005 & 10 & 22 & $12: 04: 3212.528$ & 40.533 & 2.55 & 1.23 \\
\hline 2005 & 10 & 22 & $12: 42: 4912.472$ & 40.558 & 2.27 & 0.77 \\
\hline 2005 & 10 & 22 & $13: 29: 3512.545$ & 40.551 & 2.70 & 3.06 \\
\hline 2005 & 10 & 22 & $14: 14: 0312.339$ & 40.638 & 2.99 & 1.25 \\
\hline 2005 & 10 & 22 & $15: 06: 3112.469$ & 40.569 & 2.33 & 1.1 \\
\hline 2005 & 10 & 22 & $15: 49: 1112.570$ & 40.455 & 2.77 & 0.66 \\
\hline 2005 & 10 & 22 & $16: 42: 1712.507$ & 40.551 & 2.00 & 1.82 \\
\hline 2005 & 10 & 22 & $17: 16: 5612.605$ & 40.473 & 3.24 & 1.06 \\
\hline 2005 & 10 & 22 & $17: 51: 2012.559$ & 40.479 & 2.27 & 1.45 \\
\hline 2005 & 10 & 22 & $17: 55: 0712.576$ & 40.462 & 2.48 & 1.09 \\
\hline 2005 & 10 & 22 & $17: 59: 2412.477$ & 40.561 & 2.53 & 0.7 \\
\hline 2005 & 10 & 22 & $18: 07: 1012.389$ & 40.596 & 2.47 & 0.44 \\
\hline 2005 & 10 & 22 & $19: 06: 3212.582$ & 40.532 & 1.97 & 1.42 \\
\hline 2005 & 10 & 22 & $19: 28: 3312.457$ & 40.586 & 2.02 & 0.87 \\
\hline 2005 & 10 & 22 & $19: 32: 2612.639$ & 40.542 & 2.02 & 3.02 \\
\hline 2005 & 10 & 22 & $19: 36: 3212.628$ & 40.531 & 1.92 & 1.86 \\
\hline 2005 & 10 & 22 & $19: 52: 3412.562$ & 40.522 & 2.29 & 2.22 \\
\hline 2005 & 10 & 22 & $19: 55: 3012.592$ & 40.519 & 2.91 & 0.56 \\
\hline 2005 & 10 & 22 & $22: 56: 5012.372$ & 40.610 & 3.45 & 0.85 \\
\hline 2005 & 10 & 22 & $23: 10: 0812.428$ & 40.566 & 2.20 & 1.43 \\
\hline 2005 & 10 & 23 & $00: 43: 3812.595$ & 40.514 & 3.13 & 0.71 \\
\hline 2005 & 10 & 23 & $01: 14: 0912.575$ & 40.518 & 2.38 & 0.72 \\
\hline 2005 & 10 & 23 & $01: 49: 0712.569$ & 40.522 & 2.33 & 0.77 \\
\hline 2005 & 10 & 23 & $02: 26: 0312.534$ & 40.502 & 2.13 & 1.22 \\
\hline 2005 & 10 & 23 & $02: 42: 3712.585$ & 40.501 & 2.54 & 1.25 \\
\hline 2005 & 10 & 23 & $04: 45: 5812.611$ & 40.480 & 2.99 & 0.97 \\
\hline 2005 & 10 & 23 & $04: 54: 0712.589$ & 40.518 & 3.76 & 0.65 \\
\hline 2005 & 10 & 23 & $08: 39: 5812.586$ & 40.454 & 3.12 & 1.12 \\
\hline 2005 & 10 & 23 & $09: 02: 3712.464$ & 40.566 & 2.69 & 3.34 \\
\hline 2005 & 10 & 23 & $09: 09: 4912.491$ & 40.556 & 2.43 & 0.72 \\
\hline 2005 & 10 & 23 & $09: 28: 1612.586$ & 40.514 & 2.88 & 1,00 \\
\hline 2005 & 10 & 23 & $09: 55: 1112.587$ & 40.516 & 3.31 & 1.47 \\
\hline 2005 & 10 & 23 & $10: 54: 4612.586$ & 40.488 & 3.16 & 0.98 \\
\hline 2005 & 10 & 23 & $11: 13: 4212.462$ & 40.572 & 2.99 & 0.7 \\
\hline 2005 & 10 & 23 & $11: 57: 0112.388$ & 40.611 & 2.63 & 0.53 \\
\hline 2005 & 10 & 23 & $12: 18: 0512.375$ & 40.614 & 3.30 & 0.56 \\
\hline 2005 & 10 & 23 & $13: 20: 0812.504$ & 40.757 & 3.23 & 1.37 \\
\hline 2005 & 10 & 23 & $14: 06: 5512.353$ & 40.618 & 3.26 & 1.24 \\
\hline 2005 & 10 & 23 & $14: 16: 3212.345$ & 40.625 & 2.96 & 0.54 \\
\hline 2005 & 10 & 23 & $14: 29: 4612.361$ & 40.618 & 2.91 & 0.57 \\
\hline 2005 & 10 & 23 & $15: 14: 5812.460$ & 40.572 & 2.82 & 0.54 \\
\hline 2005 & 10 & 23 & $15: 31: 2212.464$ & 40.570 & 2.65 & 1.81 \\
\hline 2005 & 10 & 23 & $15: 41: 4612.610$ & 40.486 & 3.07 & 1.19 \\
\hline 2005 & 10 & 23 & $16: 18: 0212.577$ & 40.485 & 2.43 & 1.56 \\
\hline 2005 & 10 & 23 & $16: 28: 1513.417$ & 40.848 & 3.20 & 3.7 \\
\hline 2005 & 10 & 23 & $18: 05: 3812.321$ & 40.620 & 2.33 & 0.8 \\
\hline 2005 & 10 & 23 & $18: 24: 2312.585$ & 40.470 & 2.27 & 1.47 \\
\hline 2005 & 10 & 23 & $18: 36: 0813.027$ & 41.022 & 2.61 & 9.79 \\
\hline 2005 & 10 & 23 & $19: 25: 4212.474$ & 40.573 & 2.64 & 0.51 \\
\hline 2005 & 10 & 23 & $19: 29: 0812.594$ & 40.515 & 3.10 & 0.7 \\
\hline
\end{tabular}




\begin{tabular}{|c|c|c|c|c|c|c|}
\hline 2005 & 10 & 23 & $19: 42: 1312.460$ & 40.565 & 2.39 & 0.58 \\
\hline 2005 & 10 & 23 & $20: 21: 3612.591$ & 40.488 & 2.32 & 0.97 \\
\hline 2005 & 10 & 23 & $20: 30: 1112.438$ & 40.584 & 2.84 & 0.44 \\
\hline 2005 & 10 & 23 & $20: 41: 2012.505$ & 40.648 & 2.52 & 12.01 \\
\hline 2005 & 10 & 23 & $21: 06: 3312.570$ & 40.487 & 2.39 & 0.82 \\
\hline 2005 & 10 & 23 & $21: 34: 5912.541$ & 40.525 & 1.72 & 0.82 \\
\hline 2005 & 10 & 23 & $22: 09: 5112.586$ & 40.464 & 2.16 & 1.31 \\
\hline 2005 & 10 & 23 & $23: 13: 5112.520$ & 40.543 & 2.77 & 0.63 \\
\hline 2005 & 10 & 23 & $23: 34: 4912.513$ & 40.530 & 2.46 & 1.17 \\
\hline 2005 & 10 & 23 & $23: 48: 4612.515$ & 40.655 & 2.35 & 1.94 \\
\hline 2005 & 10 & 23 & $23: 52: 3312.474$ & 40.558 & 1.78 & 1,00 \\
\hline 2005 & 10 & 24 & $00: 15: 1812.509$ & 40.516 & 1.90 & 3.1 \\
\hline 2005 & 10 & 24 & $00: 17: 4512.556$ & 40.507 & 1.90 & 2.01 \\
\hline 2005 & 10 & 24 & $00: 23: 3412.555$ & 40.492 & 2.84 & 0.61 \\
\hline 2005 & 10 & 24 & $01: 14: 3312.402$ & 40.596 & 2.71 & 0.42 \\
\hline 2005 & 10 & 24 & $01: 46: 5212.330$ & 40.648 & 2.45 & 1.01 \\
\hline 2005 & 10 & 24 & $01: 52: 5112.468$ & 40.674 & 2.38 & 10.16 \\
\hline 2005 & 10 & 24 & $02: 24: 3812.614$ & 40.519 & 2.32 & 1.24 \\
\hline 2005 & 10 & 24 & $03: 14: 1512.597$ & 40.457 & 3.42 & 1.22 \\
\hline 2005 & 10 & 24 & $04: 06: 5412.550$ & 40.502 & 2.98 & 0.6 \\
\hline 2005 & 10 & 24 & $05: 58: 4612.572$ & 40.475 & 2.54 & 2.21 \\
\hline 2005 & 10 & 24 & $07: 00: 0313.203$ & 40.315 & 3.19 & 0.85 \\
\hline 2005 & 10 & 24 & $09: 17: 0713.060$ & 40.931 & 3.60 & 0.82 \\
\hline 2005 & 10 & 24 & $09: 24: 3413.058$ & 40.924 & 2.96 & 1.36 \\
\hline 2005 & 10 & 24 & $09: 31: 0912.584$ & 40.468 & 2.62 & 0.81 \\
\hline 2005 & 10 & 24 & $09: 33: 4713.041$ & 40.935 & 2.71 & 1.97 \\
\hline 2005 & 10 & 24 & $09: 36: 2812.461$ & 40.585 & 2.79 & 1.07 \\
\hline 2005 & 10 & 24 & $10: 16: 3613.051$ & 40.922 & 3.31 & 0.64 \\
\hline 2005 & 10 & 24 & $10: 24: 1113.052$ & 40.949 & 2.31 & 3.53 \\
\hline 2005 & 10 & 24 & $11: 07: 0413.096$ & 40.976 & 2.67 & 1.19 \\
\hline 2005 & 10 & 24 & $11: 10: 3312.598$ & 40.465 & 2.32 & 1.07 \\
\hline 2005 & 10 & 24 & $11: 49: 5712.462$ & 40.566 & 2.49 & 0.5 \\
\hline 2005 & 10 & 24 & $12: 28: 5012.478$ & 40.556 & 2.67 & 1.17 \\
\hline 2005 & 10 & 24 & $13: 58: 1512.456$ & 40.563 & 2.72 & 1.31 \\
\hline 2005 & 10 & 24 & $16: 58: 1912.588$ & 40.484 & 2.47 & 1.59 \\
\hline 2005 & 10 & 24 & $17: 45: 5312.389$ & 40.596 & 2.54 & 0.77 \\
\hline 2005 & 10 & 24 & $17: 51: 2612.379$ & 40.603 & 2.66 & 0.43 \\
\hline 2005 & 10 & 24 & $18: 17: 5312.385$ & 40.597 & 2.66 & 0.52 \\
\hline 2005 & 10 & 24 & $18: 36: 3712.575$ & 40.525 & 2.31 & 0.71 \\
\hline 2005 & 10 & 24 & $20: 39: 1612.474$ & 40.572 & 2.38 & 1.08 \\
\hline 2005 & 10 & 24 & $20: 43: 4112.498$ & 40.559 & 2.32 & 0.69 \\
\hline 2005 & 10 & 24 & $21: 04: 4912.494$ & 40.550 & 1.95 & 1.14 \\
\hline 2005 & 10 & 24 & $21: 21: 2612.502$ & 40.541 & 2.24 & 0.77 \\
\hline 2005 & 10 & 24 & $21: 28: 0012.455$ & 40.564 & 2.09 & 0.97 \\
\hline 2005 & 10 & 24 & $21: 43: 1612.619$ & 40.533 & 2.24 & 0.56 \\
\hline 2005 & 10 & 24 & $22: 24: 3412.309$ & 40.613 & 2.67 & 0.79 \\
\hline 2005 & 10 & 24 & $22: 29: 5912.207$ & 40.675 & 2.66 & 0.78 \\
\hline 2005 & 10 & 24 & $23: 00: 1512.283$ & 40.648 & 2.42 & 0.64 \\
\hline 2005 & 10 & 24 & $23: 02: 1012.475$ & 40.556 & 2.05 & 1.06 \\
\hline 2005 & 10 & 24 & $23: 48: 2012.594$ & 40.486 & 3.83 & 1.02 \\
\hline 2005 & 10 & 24 & $23: 55: 1212.550$ & 40.553 & 2.54 & 0.91 \\
\hline 2005 & 10 & 24 & $23: 57: 1012.592$ & 40.479 & 2.32 & 0.96 \\
\hline 2005 & 10 & 24 & $23: 59: 2312.435$ & 40.584 & 2.54 & 0.6 \\
\hline 2005 & 10 & 25 & $00: 13: 3512.434$ & 40.582 & 2.04 & 0.62 \\
\hline 2005 & 10 & 25 & $00: 17: 2512.416$ & 40.581 & 2.30 & 0.79 \\
\hline 2005 & 10 & 25 & $00: 20: 3712.588$ & 40.500 & 2.25 & 0.84 \\
\hline 2005 & 10 & 25 & $00: 42: 4312.312$ & 40.628 & 2.54 & 0.55 \\
\hline 2005 & 10 & 25 & $00: 55: 1612.466$ & 40.578 & 2.47 & 0.49 \\
\hline 2005 & 10 & 25 & $00: 56: 4512.464$ & 40.579 & 2.74 & 0.48 \\
\hline 2005 & 10 & 25 & $01: 04: 1612.634$ & 40.532 & 1.99 & 0.58 \\
\hline 2005 & 10 & 25 & $02: 36: 3112.881$ & 40.251 & 2.77 & 0.77 \\
\hline 2005 & 10 & 25 & $02: 39: 5612.890$ & 40.248 & 3.19 & 0.76 \\
\hline 2005 & 10 & 25 & $02: 55: 1012.563$ & 40.485 & 2.44 & 0.78 \\
\hline 2005 & 10 & 25 & $07: 17: 1312.541$ & 40.529 & 3.06 & 8.36 \\
\hline 2005 & 10 & 25 & $08: 22: 3712.582$ & 40.511 & 2.55 & 1.81 \\
\hline 2005 & 10 & 25 & $09: 37: 4712.588$ & 40.508 & 2.54 & 0.66 \\
\hline 2005 & 10 & 25 & $09: 38: 4512.591$ & 40.507 & 2.93 & 1.05 \\
\hline 2005 & 10 & 25 & $09: 49: 5712.559$ & 40.468 & 2.15 & 0.97 \\
\hline 2005 & 10 & 25 & $10: 01: 4612.886$ & 40.276 & 2.88 & 1.4 \\
\hline 2005 & 10 & 25 & $11: 14: 5112.494$ & 40.538 & 2.39 & 0.62 \\
\hline 2005 & 10 & 25 & $11: 19: 5512.477$ & 40.559 & 2.36 & 0.57 \\
\hline 2005 & 10 & 25 & $12: 24: 4612.901$ & 40.279 & 2.77 & 1.59 \\
\hline 2005 & 10 & 25 & $12: 37: 2112.604$ & 40.533 & 3.33 & 1.44 \\
\hline 2005 & 10 & 25 & $12: 49: 3812.586$ & 40.466 & 2.93 & 0.93 \\
\hline 2005 & 10 & 25 & $12: 52: 2512.731$ & 40.541 & 2.72 & 0.69 \\
\hline 2005 & 10 & 25 & $13: 07: 0712.300$ & 40.643 & 2.83 & 1.22 \\
\hline
\end{tabular}




\begin{tabular}{|c|c|c|c|c|c|c|}
\hline 2005 & 10 & 25 & $13: 19: 2812.578$ & 40.601 & 2.89 & 10.62 \\
\hline 2005 & 10 & 25 & $15: 08: 0812.561$ & 40.507 & 2.74 & 1.25 \\
\hline 2005 & 10 & 25 & $16: 07: 0212.572$ & 40.531 & 2.62 & 0.64 \\
\hline 2005 & 10 & 25 & $16: 29: 4812.569$ & 40.529 & 3.08 & 0.82 \\
\hline 2005 & 10 & 25 & $17: 33: 1912.595$ & 40.474 & 2.67 & 1.2 \\
\hline 2005 & 10 & 25 & $18: 15: 2512.590$ & 40.491 & 2.47 & 1.3 \\
\hline 2005 & 10 & 25 & $18: 28: 0812.644$ & 40.553 & 2.29 & 4.81 \\
\hline 2005 & 10 & 25 & $18: 43: 1012.388$ & 40.596 & 1.41 & 0.48 \\
\hline 2005 & 10 & 25 & $19: 24: 2812.595$ & 40.533 & 2.68 & 1.07 \\
\hline 2005 & 10 & 25 & $19: 35: 4412.585$ & 40.474 & 2.90 & 1.18 \\
\hline 2005 & 10 & 25 & $19: 59: 0212.479$ & 40.570 & 2.42 & 0.56 \\
\hline 2005 & 10 & 25 & $20: 19: 5112.534$ & 40.538 & 2.77 & 0.72 \\
\hline 2005 & 10 & 25 & $20: 25: 0112.392$ & 40.599 & 1.71 & 0.68 \\
\hline 2005 & 10 & 25 & $21: 00: 4312.571$ & 40.482 & 2.45 & 0.64 \\
\hline 2005 & 10 & 25 & $21: 35: 2812.395$ & 40.596 & 2.84 & 0.5 \\
\hline 2005 & 10 & 25 & $21: 39: 4712.466$ & 40.563 & 2.35 & 0.97 \\
\hline 2005 & 10 & 25 & $21: 49: 0912.534$ & 40.542 & 2.21 & 0.95 \\
\hline 2005 & 10 & 25 & $23: 08: 2012.320$ & 40.624 & 2.53 & 0.68 \\
\hline 2005 & 10 & 25 & $23: 13: 2112.595$ & 40.458 & 2.81 & 0.76 \\
\hline 2005 & 10 & 25 & $23: 26: 0612.310$ & 40.623 & 2.78 & 0.93 \\
\hline 2005 & 10 & 25 & $23: 39: 2811.908$ & 42.219 & 4.38 & 16.05 \\
\hline 2005 & 10 & 26 & $01: 03: 4112.453$ & 40.572 & 2.37 & 1.73 \\
\hline 2005 & 10 & 26 & $01: 29: 4612.379$ & 40.610 & 2.36 & 0.84 \\
\hline 2005 & 10 & 26 & $02: 09: 2412.563$ & 40.511 & 2.44 & 2.37 \\
\hline 2005 & 10 & 26 & $02: 36: 5212.502$ & 40.561 & 2.38 & 3.6 \\
\hline 2005 & 10 & 26 & $02: 57: 3012.594$ & 40.472 & 2.85 & 1.33 \\
\hline 2005 & 10 & 26 & $03: 31: 4212.879$ & 40.266 & 3.00 & 5.77 \\
\hline 2005 & 10 & 26 & $05: 32: 2612.360$ & 40.644 & 3.15 & 1.75 \\
\hline 2005 & 10 & 26 & $06: 04: 4812.380$ & 40.594 & 3.13 & 0.61 \\
\hline 2005 & 10 & 26 & $08: 52: 1112.555$ & 40.488 & 2.34 & 0.87 \\
\hline 2005 & 10 & 26 & $09: 25: 1412.512$ & 40.576 & 2.80 & 4.27 \\
\hline 2005 & 10 & 26 & $11: 28: 0412.424$ & 40.601 & 2.75 & 1.9 \\
\hline 2005 & 10 & 26 & $12: 31: 0112.555$ & 40.588 & 3.12 & 10.81 \\
\hline 2005 & 10 & 26 & $13: 39: 2112.550$ & 40.532 & 2.80 & 1.1 \\
\hline 2005 & 10 & 26 & $14: 44: 3412.582$ & 40.478 & 2.98 & 2,00 \\
\hline 2005 & 10 & 26 & $15: 05: 0112.602$ & 40.511 & 2.79 & 1.41 \\
\hline 2005 & 10 & 26 & $16: 11: 1412.394$ & 40.617 & 2.70 & 0.5 \\
\hline 2005 & 10 & 26 & $16: 52: 0512.568$ & 40.539 & 2.87 & 1.85 \\
\hline 2005 & 10 & 26 & $17: 05: 5112.376$ & 40.620 & 2.55 & 1.15 \\
\hline 2005 & 10 & 26 & $17: 52: 3412.463$ & 40.592 & 2.50 & 0.73 \\
\hline 2005 & 10 & 26 & $19: 33: 3112.534$ & 40.545 & 2.73 & 0.93 \\
\hline 2005 & 10 & 26 & $20: 16: 0612.399$ & 40.598 & 2.46 & 0.64 \\
\hline 2005 & 10 & 26 & $20: 19: 0212.414$ & 40.589 & 2.31 & 0.67 \\
\hline 2005 & 10 & 26 & $20: 45: 1512.307$ & 40.625 & 1.64 & 1.26 \\
\hline 2005 & 10 & 26 & $20: 54: 0012.392$ & 40.596 & 2.73 & 0.59 \\
\hline 2005 & 10 & 26 & $21: 18: 2212.435$ & 40.587 & 2.56 & 0.46 \\
\hline 2005 & 10 & 26 & $22: 20: 4911.975$ & 40.445 & 3.45 & 11.2 \\
\hline 2005 & 10 & 26 & $22: 36: 0512.569$ & 40.481 & 2.78 & 1.27 \\
\hline 2005 & 10 & 26 & $23: 03: 3712.596$ & 40.509 & 2.38 & 0.8 \\
\hline 2005 & 10 & 26 & $23: 19: 5912.539$ & 40.530 & 1.74 & 1.78 \\
\hline 2005 & 10 & 26 & $23: 41: 3712.499$ & 40.618 & 2.65 & 1.91 \\
\hline 2005 & 10 & 26 & $23: 45: 2512.316$ & 40.607 & 2.12 & 0.84 \\
\hline 2005 & 10 & 27 & $00: 21: 1612.480$ & 40.554 & 2.23 & 1.1 \\
\hline 2005 & 10 & 27 & $01: 01: 2712.479$ & 40.557 & 2.50 & 1.12 \\
\hline 2005 & 10 & 27 & $04: 08: 5912.654$ & 40.522 & 2.45 & 1.31 \\
\hline 2005 & 10 & 27 & $04: 53: 3612.593$ & 40.449 & 3.27 & 2.09 \\
\hline 2005 & 10 & 27 & $04: 56: 3912.572$ & 40.474 & 2.96 & 0.77 \\
\hline 2005 & 10 & 27 & $06: 44: 0112.597$ & 40.481 & 2.82 & 1.4 \\
\hline 2005 & 10 & 27 & $08: 39: 0112.698$ & 40.235 & 2.93 & 1.84 \\
\hline 2005 & 10 & 27 & $08: 44: 5112.531$ & 40.523 & 2.98 & 1.37 \\
\hline 2005 & 10 & 27 & $09: 11: 2612.527$ & 40.540 & 2.53 & 2.24 \\
\hline 2005 & 10 & 27 & $09: 59: 0812.477$ & 40.557 & 2.53 & 0.58 \\
\hline 2005 & 10 & 27 & $10: 44: 1311.321$ & 40.948 & 3.18 & 2.49 \\
\hline 2005 & 10 & 27 & $11: 10: 1912.538$ & 40.536 & 2.66 & 0.48 \\
\hline 2005 & 10 & 27 & $13: 35: 0612.591$ & 40.475 & 2.95 & 2.92 \\
\hline 2005 & 10 & 27 & $13: 38: 4112.557$ & 40.519 & 3.59 & 1.76 \\
\hline 2005 & 10 & 27 & $14: 39: 0312.618$ & 40.472 & 3.46 & 1.84 \\
\hline 2005 & 10 & 27 & $16: 21: 5012.373$ & 40.606 & 3.49 & 0.56 \\
\hline 2005 & 10 & 27 & $17: 14: 2612.615$ & 40.504 & 3.04 & 1.11 \\
\hline 2005 & 10 & 27 & $18: 49: 0912.537$ & 40.550 & 2.61 & 1.66 \\
\hline 2005 & 10 & 27 & $19: 18: 2912.530$ & 40.529 & 2.36 & 2.88 \\
\hline 2005 & 10 & 27 & $20: 00: 1112.382$ & 40.616 & 2.35 & 0.68 \\
\hline 2005 & 10 & 27 & $20: 06: 5012.384$ & 40.609 & 3.01 & 0.62 \\
\hline 2005 & 10 & 27 & $20: 45: 3412.573$ & 40.538 & 2.54 & 0.99 \\
\hline 2005 & 10 & 27 & $21: 36: 4812.636$ & 40.538 & 1.60 & 0.77 \\
\hline 2005 & 10 & 27 & $22: 14: 0612.335$ & 40.638 & 2.30 & 0.58 \\
\hline
\end{tabular}




\begin{tabular}{|c|c|c|c|c|c|c|}
\hline 2005 & 10 & 27 & $22: 32: 2312.613$ & 40.537 & 1.90 & 1.1 \\
\hline 2005 & 10 & 27 & $23: 27: 2812.621$ & 40.554 & 2.12 & 1.34 \\
\hline 2005 & 10 & 27 & $23: 38: 2212.591$ & 40.498 & 2.43 & 1.12 \\
\hline 2005 & 10 & 27 & $23: 44: 3312.585$ & 40.457 & 2.28 & 1.02 \\
\hline 2005 & 10 & 28 & $00: 56: 3912.592$ & 40.490 & 3.66 & 1.14 \\
\hline 2005 & 10 & 28 & $01: 32: 0812.614$ & 40.493 & 3.35 & 1,00 \\
\hline 2005 & 10 & 28 & $02: 21: 3012.444$ & 40.577 & 2.22 & 0.93 \\
\hline 2005 & 10 & 28 & $03: 36: 2112.578$ & 40.507 & 3.04 & 1.65 \\
\hline 2005 & 10 & 28 & $07: 13: 1912.577$ & 40.482 & 2.91 & 2.36 \\
\hline 2005 & 10 & 28 & $09: 35: 0212.589$ & 40.466 & 3.54 & 1.2 \\
\hline 2005 & 10 & 28 & $12: 03: 2412.325$ & 40.618 & 2.81 & 0.63 \\
\hline 2005 & 10 & 28 & $12: 40: 2312.441$ & 40.581 & 2.93 & 0.54 \\
\hline 2005 & 10 & 28 & $13: 05: 3212.386$ & 40.614 & 2.63 & 0.99 \\
\hline 2005 & 10 & 28 & $13: 18: 3012.573$ & 40.535 & 3.17 & 2.69 \\
\hline 2005 & 10 & 28 & $14: 29: 0312.565$ & 40.484 & 2.62 & 1.99 \\
\hline 2005 & 10 & 28 & $16: 04: 3512.597$ & 40.468 & 2.75 & 1.3 \\
\hline 2005 & 10 & 28 & $16: 10: 1412.534$ & 40.539 & 2.47 & 2.45 \\
\hline 2005 & 10 & 28 & $16: 38: 2912.590$ & 40.522 & 2.83 & 1.02 \\
\hline 2005 & 10 & 28 & $16: 57: 0612.397$ & 40.597 & 3.03 & 0.46 \\
\hline 2005 & 10 & 28 & $17: 29: 3512.516$ & 40.560 & 2.70 & 2.97 \\
\hline 2005 & 10 & 28 & $17: 54: 1412.585$ & 40.487 & 2.55 & 1.51 \\
\hline 2005 & 10 & 28 & $18: 00: 1012.392$ & 40.596 & 2.57 & 0.6 \\
\hline 2005 & 10 & 28 & $18: 31: 4712.508$ & 40.553 & 2.49 & 1.47 \\
\hline 2005 & 10 & 28 & $19: 07: 3112.577$ & 40.474 & 2.40 & 1.29 \\
\hline 2005 & 10 & 28 & $19: 57: 0412.569$ & 40.524 & 2.31 & 1.88 \\
\hline 2005 & 10 & 28 & $20: 11: 2712.578$ & 40.480 & 2.15 & 1.31 \\
\hline 2005 & 10 & 28 & $20: 53: 2512.611$ & 40.511 & 2.16 & 1.12 \\
\hline 2005 & 10 & 28 & $21: 11: 4812.561$ & 40.508 & 2.51 & 1.3 \\
\hline 2005 & 10 & 28 & $21: 14: 4912.325$ & 40.620 & 2.22 & 0.82 \\
\hline 2005 & 10 & 28 & $21: 16: 1612.459$ & 40.553 & 2.26 & 0.86 \\
\hline 2005 & 10 & 28 & $21: 27: 0512.559$ & 40.554 & 2.83 & 1.01 \\
\hline 2005 & 10 & 28 & $21: 46: 1212.417$ & 40.585 & 2.67 & 0.48 \\
\hline 2005 & 10 & 28 & $21: 51: 1912.488$ & 40.545 & 1.96 & 1.83 \\
\hline 2005 & 10 & 28 & $22: 06: 1712.596$ & 40.492 & 3.22 & 0.81 \\
\hline 2005 & 10 & 28 & $22: 52: 5412.419$ & 40.586 & 3.58 & 0.52 \\
\hline 2005 & 10 & 28 & $23: 06: 3512.415$ & 40.590 & 2.82 & 0.56 \\
\hline 2005 & 10 & 28 & $23: 08: 1612.609$ & 40.476 & 2.77 & 1.61 \\
\hline 2005 & 10 & 28 & $23: 16: 4212.376$ & 40.602 & 2.64 & 0.53 \\
\hline 2005 & 10 & 28 & $23: 26: 4312.426$ & 40.584 & 2.71 & 0.8 \\
\hline 2005 & 10 & 29 & $00: 20: 1512.603$ & 40.485 & 2.10 & 1.03 \\
\hline 2005 & 10 & 29 & $00: 22: 3212.625$ & 40.525 & 1.88 & 1.45 \\
\hline 2005 & 10 & 29 & $00: 24: 2912.381$ & 40.579 & 1.84 & 1.04 \\
\hline 2005 & 10 & 29 & $00: 39: 5412.610$ & 40.496 & 2.03 & 1.81 \\
\hline 2005 & 10 & 29 & $00: 54: 4612.606$ & 40.531 & 2.15 & 0.79 \\
\hline 2005 & 10 & 29 & $00: 56: 3112.404$ & 40.600 & 2.46 & 0.61 \\
\hline 2005 & 10 & 29 & $00: 57: 0412.419$ & 40.604 & 2.69 & 1,00 \\
\hline 2005 & 10 & 29 & $01: 06: 5312.592$ & 40.510 & 3.13 & 0.89 \\
\hline 2005 & 10 & 29 & $01: 23: 5212.585$ & 40.462 & 3.02 & 0.95 \\
\hline 2005 & 10 & 29 & $01: 25: 0812.702$ & 40.386 & 2.78 & 3.29 \\
\hline 2005 & 10 & 29 & $04: 39: 1812.743$ & 40.623 & 2.68 & 0.83 \\
\hline 2005 & 10 & 29 & $06: 43: 0012.585$ & 40.477 & 2.71 & 1.76 \\
\hline 2005 & 10 & 29 & $06: 46: 5212.594$ & 40.468 & 3.26 & 1.34 \\
\hline 2005 & 10 & 29 & $08: 08: 5012.555$ & 40.497 & 2.85 & 0.56 \\
\hline 2005 & 10 & 29 & $09: 12: 4612.567$ & 40.489 & 2.52 & 0.86 \\
\hline 2005 & 10 & 29 & $10: 11: 2312.557$ & 40.516 & 3.14 & 2.18 \\
\hline 2005 & 10 & 29 & $10: 45: 5012.579$ & 40.489 & 3.31 & 1.85 \\
\hline 2005 & 10 & 29 & $11: 54: 2612.365$ & 40.635 & 3.07 & 0.69 \\
\hline 2005 & 10 & 29 & $11: 56: 2612.310$ & 40.632 & 3.12 & 0.77 \\
\hline 2005 & 10 & 29 & $12: 09: 2812.682$ & 40.355 & 2.46 & 11.51 \\
\hline 2005 & 10 & 29 & $12: 31: 1812.598$ & 40.429 & 2.00 & 1.12 \\
\hline 2005 & 10 & 29 & $13: 34: 1312.479$ & 40.565 & 2.44 & 0.99 \\
\hline 2005 & 10 & 29 & $14: 35: 2012.486$ & 40.565 & 2.39 & 1.48 \\
\hline 2005 & 10 & 29 & $15: 38: 3712.646$ & 40.204 & 2.38 & 1.38 \\
\hline 2005 & 10 & 29 & $16: 42: 3512.318$ & 40.641 & 2.56 & 0.92 \\
\hline 2005 & 10 & 29 & $17: 05: 2412.609$ & 40.492 & 3.02 & 1.24 \\
\hline 2005 & 10 & 29 & $17: 26: 1712.589$ & 40.503 & 2.28 & 1.91 \\
\hline 2005 & 10 & 29 & $18: 07: 5412.611$ & 40.515 & 2.09 & 1.3 \\
\hline 2005 & 10 & 29 & $18: 15: 2612.598$ & 40.480 & 1.97 & 1.66 \\
\hline 2005 & 10 & 29 & $18: 18: 5312.451$ & 40.590 & 2.48 & 0.67 \\
\hline 2005 & 10 & 29 & $18: 41: 4612.530$ & 40.552 & 2.29 & 2.71 \\
\hline 2005 & 10 & 29 & $19: 19: 2912.539$ & 40.532 & 2.39 & 0.49 \\
\hline 2005 & 10 & 29 & $19: 46: 0512.463$ & 40.562 & 2.28 & 1.14 \\
\hline 2005 & 10 & 29 & $20: 02: 0712.594$ & 40.491 & 2.20 & 1.84 \\
\hline 2005 & 10 & 29 & $20: 35: 2712.582$ & 40.468 & 3.24 & 1.07 \\
\hline 2005 & 10 & 29 & $21: 51: 0912.967$ & 40.645 & 3.02 & 0.47 \\
\hline 2005 & 10 & 29 & $22: 47: 3312.432$ & 40.586 & 2.08 & 0.6 \\
\hline
\end{tabular}




\begin{tabular}{|c|c|c|c|c|c|c|}
\hline 2005 & 10 & 29 & $23: 22: 3713.123$ & 40.961 & 2.51 & 0.93 \\
\hline 2005 & 10 & 30 & $01: 18: 3012.468$ & 40.566 & 3.42 & 0.67 \\
\hline 2005 & 10 & 30 & $02: 04: 2112.624$ & 40.457 & 2.42 & 1.9 \\
\hline 2005 & 10 & 30 & $04: 06: 2112.403$ & 40.591 & 2.98 & 0.52 \\
\hline 2005 & 10 & 30 & $09: 54: 2612.318$ & 40.623 & 2.76 & 0.98 \\
\hline 2005 & 10 & 30 & $10: 27: 2912.470$ & 40.559 & 2.56 & 1.21 \\
\hline 2005 & 10 & 30 & $12: 26: 2212.540$ & 40.532 & 2.25 & 1.39 \\
\hline 2005 & 10 & 30 & $13: 00: 0212.577$ & 40.503 & 2.96 & 1.58 \\
\hline 2005 & 10 & 30 & $13: 27: 3812.593$ & 40.476 & 2.58 & 1.2 \\
\hline 2005 & 10 & 30 & $13: 32: 0412.584$ & 40.485 & 2.85 & 1.84 \\
\hline 2005 & 10 & 30 & $14: 03: 4312.597$ & 40.482 & 3.72 & 1.89 \\
\hline 2005 & 10 & 30 & $16: 12: 0313.382$ & 40.915 & 3.01 & 1.9 \\
\hline 2005 & 10 & 30 & $16: 12: 5713.389$ & 40.922 & 3.02 & 4.38 \\
\hline 2005 & 10 & 30 & $16: 29: 4812.600$ & 40.461 & 3.60 & 1.3 \\
\hline 2005 & 10 & 30 & $16: 39: 5212.579$ & 40.569 & 2.62 & 8.95 \\
\hline 2005 & 10 & 30 & $17: 03: 3112.589$ & 40.513 & 2.68 & 1.1 \\
\hline 2005 & 10 & 30 & $17: 33: 1712.369$ & 40.604 & 2.37 & 1.19 \\
\hline 2005 & 10 & 30 & $17: 56: 4412.576$ & 40.518 & 2.34 & 1.51 \\
\hline 2005 & 10 & 30 & $17: 57: 2512.562$ & 40.526 & 2.36 & 2.09 \\
\hline 2005 & 10 & 30 & $18: 28: 1712.595$ & 40.472 & 2.22 & 1.45 \\
\hline 2005 & 10 & 30 & $18: 39: 2812.595$ & 40.481 & 2.15 & 2.26 \\
\hline 2005 & 10 & 30 & $19: 04: 0812.582$ & 40.480 & 2.52 & 1.63 \\
\hline 2005 & 10 & 30 & $19: 16: 3212.443$ & 40.582 & 2.55 & 0.51 \\
\hline 2005 & 10 & 30 & $19: 43: 3512.570$ & 40.513 & 2.07 & 1.08 \\
\hline 2005 & 10 & 30 & $19: 54: 1212.547$ & 40.485 & 2.07 & 1.34 \\
\hline 2005 & 10 & 30 & $20: 07: 1712.492$ & 40.564 & 2.51 & 0.57 \\
\hline 2005 & 10 & 30 & $20: 12: 2512.463$ & 40.574 & 2.16 & 1.59 \\
\hline 2005 & 10 & 30 & $20: 19: 0512.645$ & 40.561 & 2.27 & 0.86 \\
\hline 2005 & 10 & 30 & $20: 44: 0212.553$ & 40.547 & 2.59 & 0.93 \\
\hline 2005 & 10 & 30 & $21: 06: 5112.595$ & 40.475 & 3.27 & 2.03 \\
\hline 2005 & 10 & 30 & $21: 27: 0812.586$ & 40.514 & 2.70 & 1.32 \\
\hline 2005 & 10 & 30 & $21: 30: 1012.532$ & 40.537 & 2.23 & 0.81 \\
\hline 2005 & 10 & 30 & $21: 40: 2112.441$ & 40.581 & 2.81 & 0.42 \\
\hline 2005 & 10 & 30 & $21: 46: 4412.562$ & 40.544 & 2.48 & 1.27 \\
\hline 2005 & 10 & 30 & $21: 50: 3512.611$ & 40.449 & 2.22 & 5.95 \\
\hline 2005 & 10 & 30 & $22: 01: 2912.607$ & 40.459 & 2.39 & 3.47 \\
\hline 2005 & 10 & 30 & $22: 11: 0012.480$ & 40.551 & 2.38 & 1.24 \\
\hline 2005 & 10 & 30 & $23: 20: 1812.650$ & 40.549 & 2.02 & 0.64 \\
\hline 2005 & 10 & 30 & $23: 35: 0912.530$ & 40.495 & 2.42 & 1.38 \\
\hline 2005 & 10 & 30 & $23: 38: 1112.568$ & 40.543 & 2.41 & 1.83 \\
\hline 2005 & 10 & 30 & $23: 53: 4712.557$ & 40.490 & 2.47 & 0.6 \\
\hline 2005 & 10 & 31 & $00: 20: 4412.293$ & 40.633 & 2.10 & 1.03 \\
\hline 2005 & 10 & 31 & $00: 23: 1112.711$ & 40.535 & 2.63 & 0.61 \\
\hline 2005 & 10 & 31 & $01: 18: 0712.559$ & 40.471 & 2.40 & 0.97 \\
\hline 2005 & 10 & 31 & $01: 24: 2712.711$ & 40.212 & 2.93 & 1.65 \\
\hline 2005 & 10 & 31 & $02: 01: 0512.596$ & 40.488 & 2.82 & 0.9 \\
\hline 2005 & 10 & 31 & $02: 15: 3912.431$ & 40.611 & 2.13 & 1.33 \\
\hline 2005 & 10 & 31 & $02: 43: 3812.453$ & 40.592 & 2.20 & 1.25 \\
\hline 2005 & 10 & 31 & $03: 22: 3012.627$ & 40.531 & 1.97 & 1.21 \\
\hline 2005 & 10 & 31 & $04: 13: 3312.582$ & 40.476 & 3.06 & 1.39 \\
\hline 2005 & 10 & 31 & $04: 47: 0112.557$ & 40.486 & 2.57 & 1,00 \\
\hline 2005 & 10 & 31 & $06: 52: 3212.479$ & 40.549 & 2.55 & 1.62 \\
\hline 2005 & 10 & 31 & $07: 19: 5012.562$ & 40.487 & 2.51 & 2.7 \\
\hline 2005 & 10 & 31 & $10: 09: 2912.551$ & 40.529 & 2.88 & 2.16 \\
\hline 2005 & 10 & 31 & $10: 44: 2812.440$ & 40.590 & 2.76 & 0.56 \\
\hline 2005 & 10 & 31 & $10: 52: 3612.303$ & 40.638 & 3.15 & 0.84 \\
\hline 2005 & 10 & 31 & $14: 49: 1712.595$ & 40.514 & 2.93 & 1.53 \\
\hline 2005 & 10 & 31 & $15: 42: 1712.312$ & 40.638 & 2.76 & 1.09 \\
\hline 2005 & 10 & 31 & $16: 18: 1712.603$ & 40.465 & 2.56 & 1.92 \\
\hline 2005 & 10 & 31 & $16: 40: 0812.576$ & 40.506 & 2.27 & 2.83 \\
\hline 2005 & 10 & 31 & $16: 58: 1312.585$ & 40.465 & 2.19 & 2.22 \\
\hline 2005 & 10 & 31 & $17: 10: 0012.436$ & 40.587 & 2.92 & 0.41 \\
\hline 2005 & 10 & 31 & $18: 24: 5813.470$ & 39.942 & 3.42 & 1.64 \\
\hline 2005 & 10 & 31 & $18: 30: 5712.442$ & 40.581 & 2.34 & 0.55 \\
\hline 2005 & 10 & 31 & $18: 38: 3812.446$ & 40.583 & 2.86 & 0.61 \\
\hline 2005 & 10 & 31 & $18: 52: 0112.596$ & 40.527 & 2.81 & 0.9 \\
\hline 2005 & 10 & 31 & $19: 00: 5812.560$ & 40.477 & 2.28 & 0.89 \\
\hline 2005 & 10 & 31 & $20: 21: 1112.426$ & 40.590 & 2.62 & 0.49 \\
\hline 2005 & 10 & 31 & $21: 08: 2612.507$ & 40.545 & 2.38 & 0.71 \\
\hline 2005 & 10 & 31 & $21: 30: 0712.607$ & 40.486 & 2.12 & 0.86 \\
\hline 2005 & 10 & 31 & $21: 37: 5812.632$ & 40.552 & 2.21 & 1.19 \\
\hline 2005 & 10 & 31 & $22: 11: 1612.466$ & 40.561 & 2.23 & 0.54 \\
\hline 2005 & 10 & 31 & $22: 23: 3611.255$ & 41.089 & 3.34 & 7.14 \\
\hline 2005 & 10 & 31 & $22: 50: 4912.574$ & 40.494 & 2.31 & 2.11 \\
\hline 2005 & 10 & 31 & $23: 19: 0112.587$ & 40.498 & 3.02 & 1.58 \\
\hline 2005 & 10 & 31 & $23: 25: 1712.627$ & 40.458 & 2.48 & 1.87 \\
\hline
\end{tabular}




\begin{tabular}{|c|c|c|c|c|c|c|}
\hline 2005 & 10 & 31 & $23: 39: 2312.476$ & 40.574 & 1.88 & 1.35 \\
\hline 2005 & 10 & 31 & $23: 49: 5412.586$ & 40.513 & 2.54 & 1.15 \\
\hline 2005 & 11 & 1 & $00: 14: 1712.584$ & 40.475 & 2.10 & 2.56 \\
\hline 2005 & 11 & 1 & $03: 25: 0412.595$ & 40.465 & 2.82 & 2.29 \\
\hline 2005 & 11 & 1 & $03: 59: 0012.379$ & 40.588 & 2.66 & 0.48 \\
\hline 2005 & 11 & 1 & $04: 19: 0512.479$ & 40.554 & 2.78 & 0.56 \\
\hline 2005 & 11 & 1 & $04: 43: 4312.364$ & 40.597 & 2.54 & 0.54 \\
\hline 2005 & 11 & 1 & $04: 58: 1212.652$ & 40.452 & 3.64 & 2.54 \\
\hline 2005 & 11 & 1 & $11: 13: 5912.568$ & 40.525 & 2.27 & 0.69 \\
\hline 2005 & 11 & 1 & $11: 22: 5712.403$ & 40.608 & 3.47 & 0.68 \\
\hline 2005 & 11 & 1 & $13: 18: 1112.601$ & 40.502 & 2.78 & 0.79 \\
\hline 2005 & 11 & 1 & $13: 18: 4912.570$ & 40.487 & 2.86 & 0.99 \\
\hline 2005 & 11 & 1 & $13: 20: 5412.440$ & 40.578 & 2.85 & 0.54 \\
\hline 2005 & 11 & 1 & $13: 25: 0812.355$ & 40.615 & 2.92 & 0.71 \\
\hline 2005 & 11 & 1 & $16: 02: 5112.555$ & 40.493 & 3.04 & 0.44 \\
\hline 2005 & 11 & 1 & $17: 33: 5212.556$ & 40.493 & 2.59 & 0.65 \\
\hline 2005 & 11 & 1 & $18: 35: 3512.566$ & 40.525 & 2.26 & 0.83 \\
\hline 2005 & 11 & 1 & $18: 56: 3412.590$ & 40.485 & 2.21 & 3.05 \\
\hline 2005 & 11 & 1 & $19: 12: 5512.582$ & 40.510 & 2.29 & 0.78 \\
\hline 2005 & 11 & 1 & $20: 08: 0412.598$ & 40.505 & 2.27 & 1.57 \\
\hline 2005 & 11 & 1 & $20: 16: 0112.579$ & 40.483 & 2.41 & 1.29 \\
\hline 2005 & 11 & 1 & $20: 44: 3012.486$ & 40.715 & 2.24 & 2.47 \\
\hline 2005 & 11 & 1 & $21: 53: 4112.472$ & 40.774 & 2.44 & 12.94 \\
\hline 2005 & 11 & 1 & $22: 31: 2112.629$ & 40.541 & 2.72 & 0.91 \\
\hline 2005 & 11 & 1 & $22: 50: 0812.624$ & 40.528 & 1.98 & 0.79 \\
\hline 2005 & 11 & 1 & $23: 13: 0212.556$ & 40.481 & 2.09 & 0.7 \\
\hline 2005 & 11 & 1 & $23: 52: 3212.558$ & 40.522 & 1.65 & 1.01 \\
\hline 2005 & 11 & 2 & $03: 19: 5412.605$ & 40.515 & 2.75 & 1.29 \\
\hline 2005 & 11 & 2 & $04: 41: 3812.403$ & 40.593 & 2.97 & 0.53 \\
\hline 2005 & 11 & 2 & $07: 49: 4312.605$ & 40.473 & 2.50 & 1.77 \\
\hline 2005 & 11 & 2 & $08: 13: 1112.583$ & 40.489 & 2.84 & 1.97 \\
\hline 2005 & 11 & 2 & $09: 17: 4912.581$ & 40.517 & 2.51 & 0.98 \\
\hline 2005 & 11 & 2 & $10: 11: 2212.516$ & 40.538 & 2.68 & 1.14 \\
\hline 2005 & 11 & 2 & $11: 07: 3712.457$ & 40.559 & 2.31 & 2.06 \\
\hline 2005 & 11 & 2 & $11: 45: 0512.575$ & 40.481 & 2.56 & 0.65 \\
\hline 2005 & 11 & 2 & $12: 13: 2312.578$ & 40.511 & 2.27 & 1.21 \\
\hline 2005 & 11 & 2 & $12: 41: 2112.613$ & 40.456 & 2.92 & 1.41 \\
\hline 2005 & 11 & 2 & $12: 54: 2012.727$ & 40.391 & 2.98 & 1.62 \\
\hline 2005 & 11 & 2 & $13: 11: 3712.599$ & 40.469 & 3.11 & 2.33 \\
\hline 2005 & 11 & 2 & $18: 20: 5512.369$ & 40.595 & 2.48 & 1.29 \\
\hline 2005 & 11 & 2 & $19: 11: 5512.541$ & 40.548 & 2.15 & 1.23 \\
\hline 2005 & 11 & 2 & $19: 49: 0512.321$ & 40.633 & 2.80 & 0.84 \\
\hline 2005 & 11 & 2 & $19: 56: 3912.318$ & 40.632 & 3.06 & 0.58 \\
\hline 2005 & 11 & 2 & $19: 59: 5412.325$ & 40.631 & 3.11 & 0.56 \\
\hline 2005 & 11 & 2 & $20: 10: 1012.568$ & 40.470 & 2.33 & 0.87 \\
\hline 2005 & 11 & 2 & $20: 50: 2511.909$ & 41.018 & 2.44 & 2.37 \\
\hline 2005 & 11 & 2 & $21: 39: 0312.590$ & 40.511 & 2.64 & 1.02 \\
\hline 2005 & 11 & 2 & $22: 01: 1312.588$ & 40.478 & 2.19 & 1.75 \\
\hline 2005 & 11 & 2 & $22: 05: 1512.503$ & 40.557 & 2.47 & 0.68 \\
\hline 2005 & 11 & 2 & $22: 38: 2312.447$ & 40.566 & 2.06 & 0.88 \\
\hline 2005 & 11 & 2 & $23: 27: 3612.615$ & 40.459 & 2.75 & 1.34 \\
\hline 2005 & 11 & 2 & $23: 29: 2712.579$ & 40.495 & 2.62 & 0.98 \\
\hline 2005 & 11 & 2 & $23: 44: 1612.451$ & 40.567 & 1.95 & 1.08 \\
\hline 2005 & 11 & 3 & $00: 31: 3112.562$ & 40.478 & 1.77 & 3.18 \\
\hline 2005 & 11 & 3 & $01: 10: 5012.540$ & 40.496 & 2.15 & 1.15 \\
\hline 2005 & 11 & 3 & $01: 50: 1212.302$ & 40.636 & 2.37 & 0.45 \\
\hline 2005 & 11 & 3 & $01: 51: 5712.545$ & 40.550 & 2.29 & 0.86 \\
\hline 2005 & 11 & 3 & $02: 21: 1912.559$ & 40.555 & 2.26 & 1.03 \\
\hline 2005 & 11 & 3 & $03: 21: 3612.592$ & 40.473 & 2.74 & 1,00 \\
\hline 2005 & 11 & 3 & $03: 25: 0312.571$ & 40.467 & 2.46 & 0.84 \\
\hline 2005 & 11 & 3 & $03: 33: 4812.584$ & 40.477 & 2.59 & 1.79 \\
\hline 2005 & 11 & 3 & $04: 47: 1312.636$ & 40.515 & 1.47 & 0.97 \\
\hline 2005 & 11 & 3 & $05: 12: 1412.607$ & 40.492 & 3.16 & 0.86 \\
\hline 2005 & 11 & 3 & $07: 11: 3712.474$ & 40.559 & 2.29 & 0.6 \\
\hline 2005 & 11 & 3 & $07: 14: 1412.483$ & 40.563 & 2.50 & 0.79 \\
\hline 2005 & 11 & 3 & $10: 28: 1412.725$ & 40.331 & 1.95 & 2.56 \\
\hline 2005 & 11 & 3 & $11: 35: 2612.366$ & 40.609 & 2.47 & 0.59 \\
\hline 2005 & 11 & 3 & $13: 37: 3812.603$ & 40.501 & 2.53 & 0.92 \\
\hline 2005 & 11 & 3 & $14: 53: 0812.447$ & 40.577 & 2.45 & 1.75 \\
\hline 2005 & 11 & 3 & $18: 29: 2212.587$ & 40.478 & 2.45 & 1.18 \\
\hline 2005 & 11 & 3 & $18: 51: 3712.382$ & 40.601 & 2.38 & 0.5 \\
\hline 2005 & 11 & 3 & $19: 40: 3012.585$ & 40.519 & 2.65 & 1.55 \\
\hline 2005 & 11 & 3 & $21: 03: 3211.363$ & 40.932 & 3.66 & 14.28 \\
\hline 2005 & 11 & 3 & $21: 08: 3211.367$ & 40.915 & 2.87 & 6.83 \\
\hline 2005 & 11 & 3 & $21: 22: 2912.547$ & 40.492 & 2.78 & 0.5 \\
\hline 2005 & 11 & 3 & $21: 51: 1112.473$ & 40.560 & 2.71 & 1.13 \\
\hline
\end{tabular}




\begin{tabular}{|c|c|c|c|c|c|c|}
\hline 2005 & 11 & 3 & $23: 25: 4212.568$ & 40.520 & 3.91 & 1.3 \\
\hline 2005 & 11 & 3 & $23: 26: 4011.126$ & 41.002 & 2.50 & 6.45 \\
\hline 2005 & 11 & 3 & $23: 29: 0212.566$ & 40.566 & 2.41 & 4.21 \\
\hline 2005 & 11 & 4 & $00: 06: 1612.593$ & 40.468 & 3.43 & 0.96 \\
\hline 2005 & 11 & 4 & $00: 41: 3912.286$ & 40.641 & 1.73 & 1.11 \\
\hline 2005 & 11 & 4 & $00: 44: 4412.398$ & 40.571 & 1.49 & 1.73 \\
\hline 2005 & 11 & 4 & $00: 51: 5911.370$ & 40.925 & 2.25 & 1.93 \\
\hline 2005 & 11 & 4 & $01: 05: 0912.546$ & 40.500 & 2.17 & 0.75 \\
\hline 2005 & 11 & 4 & $01: 14: 2012.370$ & 40.599 & 1.99 & 0.66 \\
\hline 2005 & 11 & 4 & $01: 15: 3412.543$ & 40.491 & 1.94 & 0.58 \\
\hline 2005 & 11 & 4 & $01: 17: 2712.648$ & 40.552 & 1.90 & 2.01 \\
\hline 2005 & 11 & 4 & $01: 29: 0312.452$ & 40.570 & 1.76 & 1.32 \\
\hline 2005 & 11 & 4 & $01: 36: 1512.574$ & 40.524 & 1.53 & 2.99 \\
\hline 2005 & 11 & 4 & $01: 40: 5312.532$ & 40.558 & 2.29 & 1.9 \\
\hline 2005 & 11 & 4 & $01: 59: 3212.659$ & 40.474 & 1.25 & 3.25 \\
\hline 2005 & 11 & 4 & $02: 54: 1612.522$ & 40.539 & 1.97 & 1.67 \\
\hline 2005 & 11 & 4 & $03: 49: 1512.450$ & 40.563 & 2.15 & 2.02 \\
\hline 2005 & 11 & 4 & $07: 46: 4912.655$ & 40.434 & 2.78 & 3.32 \\
\hline 2005 & 11 & 4 & $11: 01: 1112.366$ & 40.616 & 2.82 & 0.55 \\
\hline 2005 & 11 & 4 & $11: 04: 0412.371$ & 40.616 & 2.97 & 0.98 \\
\hline 2005 & 11 & 4 & $11: 09: 5012.440$ & 40.581 & 2.18 & 1.06 \\
\hline 2005 & 11 & 4 & $11: 17: 2112.472$ & 40.559 & 2.63 & 0.58 \\
\hline 2005 & 11 & 4 & $14: 22: 0112.601$ & 40.475 & 3.10 & 1.11 \\
\hline 2005 & 11 & 4 & $15: 19: 5112.566$ & 40.488 & 2.46 & 1.08 \\
\hline 2005 & 11 & 4 & $15: 34: 1612.352$ & 40.611 & 3.25 & 0.48 \\
\hline 2005 & 11 & 4 & $15: 54: 0712.450$ & 40.606 & 1.88 & 5.05 \\
\hline 2005 & 11 & 4 & $16: 09: 0512.327$ & 40.624 & 2.34 & 0.62 \\
\hline 2005 & 11 & 4 & $16: 38: 2512.344$ & 40.615 & 2.68 & 0.7 \\
\hline 2005 & 11 & 4 & $17: 26: 2712.361$ & 40.621 & 2.39 & 0.74 \\
\hline 2005 & 11 & 4 & $18: 09: 5412.409$ & 40.594 & 2.41 & 1.4 \\
\hline 2005 & 11 & 4 & $18: 57: 0912.349$ & 40.604 & 2.64 & 1.24 \\
\hline 2005 & 11 & 4 & $19: 16: 1112.494$ & 40.546 & 1.78 & 3.44 \\
\hline 2005 & 11 & 4 & $19: 57: 1812.316$ & 40.638 & 2.11 & 0.79 \\
\hline 2005 & 11 & 4 & $20: 27: 2012.453$ & 40.561 & 2.23 & 1.13 \\
\hline 2005 & 11 & 4 & $21: 56: 3412.614$ & 40.528 & 2.01 & 0.79 \\
\hline 2005 & 11 & 4 & $21: 58: 5212.609$ & 40.484 & 3.09 & 1.02 \\
\hline 2005 & 11 & 4 & $22: 04: 1212.588$ & 40.520 & 2.67 & 1.03 \\
\hline 2005 & 11 & 4 & $22: 15: 3712.541$ & 40.544 & 2.33 & 1.44 \\
\hline 2005 & 11 & 4 & $22: 52: 4012.541$ & 40.528 & 2.75 & 0.86 \\
\hline 2005 & 11 & 5 & $00: 03: 4612.357$ & 40.617 & 1.89 & 0.69 \\
\hline 2005 & 11 & 5 & $00: 15: 5612.616$ & 40.541 & 1.93 & 0.6 \\
\hline 2005 & 11 & 5 & $00: 40: 3612.515$ & 40.413 & 2.58 & 1.81 \\
\hline 2005 & 11 & 5 & $00: 47: 3812.438$ & 40.575 & 2.20 & 0.48 \\
\hline 2005 & 11 & 5 & $00: 49: 4612.383$ & 40.596 & 1.86 & 0.62 \\
\hline 2005 & 11 & 5 & $00: 54: 5312.512$ & 40.645 & 2.37 & 1.34 \\
\hline 2005 & 11 & 5 & $00: 57: 4912.577$ & 40.522 & 2.47 & 0.88 \\
\hline 2005 & 11 & 5 & $01: 16: 5312.744$ & 40.350 & 2.29 & 4.61 \\
\hline 2005 & 11 & 5 & $01: 24: 1912.309$ & 40.648 & 2.34 & 1.11 \\
\hline 2005 & 11 & 5 & $01: 35: 0712.577$ & 40.527 & 2.35 & 0.79 \\
\hline 2005 & 11 & 5 & $01: 47: 0112.397$ & 40.610 & 1.67 & 1.08 \\
\hline 2005 & 11 & 5 & $02: 14: 0112.292$ & 40.636 & 1.92 & 1.05 \\
\hline 2005 & 11 & 5 & $02: 24: 0112.562$ & 40.508 & 2.58 & 0.97 \\
\hline 2005 & 11 & 5 & $02: 38: 1812.554$ & 40.533 & 1.71 & 2.97 \\
\hline 2005 & 11 & 5 & $02: 38: 4812.576$ & 40.493 & 2.19 & 8.98 \\
\hline 2005 & 11 & 5 & $02: 48: 5912.664$ & 40.481 & 1.09 & 2.38 \\
\hline 2005 & 11 & 5 & $03: 04: 5912.649$ & 40.532 & 1.97 & 0.63 \\
\hline 2005 & 11 & 5 & $03: 17: 5812.579$ & 40.478 & 2.88 & 1.07 \\
\hline 2005 & 11 & 5 & $05: 05: 5912.347$ & 40.648 & 2.69 & 1.62 \\
\hline 2005 & 11 & 5 & $05: 09: 3012.505$ & 40.537 & 1.98 & 1.67 \\
\hline 2005 & 11 & 5 & $09: 53: 4112.549$ & 40.562 & 2.73 & 2.64 \\
\hline 2005 & 11 & 5 & $10: 29: 0412.590$ & 40.477 & 4.41 & 1.4 \\
\hline 2005 & 11 & 5 & $10: 31: 4012.630$ & 40.455 & 2.99 & 6.2 \\
\hline 2005 & 11 & 5 & $11: 16: 5012.580$ & 40.521 & 2.85 & 0.88 \\
\hline 2005 & 11 & 5 & $12: 16: 5112.545$ & 40.479 & 2.33 & 1.02 \\
\hline 2005 & 11 & 5 & $15: 18: 5512.530$ & 40.531 & 2.43 & 1.34 \\
\hline 2005 & 11 & 5 & $15: 36: 0612.539$ & 40.485 & 2.12 & 1.18 \\
\hline 2005 & 11 & 5 & $15: 57: 2812.590$ & 40.477 & 2.42 & 1.38 \\
\hline 2005 & 11 & 5 & $16: 09: 5212.587$ & 40.471 & 2.55 & 1.56 \\
\hline 2005 & 11 & 5 & $16: 19: 0012.581$ & 40.458 & 2.44 & 0.87 \\
\hline 2005 & 11 & 5 & $16: 48: 2012.946$ & 40.232 & 2.25 & 8.6 \\
\hline 2005 & 11 & 5 & $18: 23: 0412.913$ & 40.266 & 2.86 & 0.73 \\
\hline 2005 & 11 & 5 & $18: 39: 3312.896$ & 40.312 & 2.23 & 0.88 \\
\hline 2005 & 11 & 5 & $18: 48: 2512.574$ & 40.482 & 1.67 & 3.33 \\
\hline 2005 & 11 & 5 & $18: 54: 2612.606$ & 40.493 & 1.97 & 3.79 \\
\hline 2005 & 11 & 5 & $19: 12: 4812.541$ & 40.501 & 2.48 & 0.71 \\
\hline 2005 & 11 & 5 & $20: 08: 5312.658$ & 40.420 & 1.45 & 2.63 \\
\hline
\end{tabular}




\begin{tabular}{|c|c|c|c|c|c|c|}
\hline 2005 & 11 & 5 & $20: 41: 2912.610$ & 40.469 & 2.87 & 1.39 \\
\hline 2005 & 11 & 5 & $22: 06: 4312.600$ & 40.421 & 2.01 & 2.54 \\
\hline 2005 & 11 & 5 & $22: 36: 2112.466$ & 40.567 & 2.58 & 0.43 \\
\hline 2005 & 11 & 5 & $22: 40: 3412.582$ & 40.499 & 2.53 & 0.95 \\
\hline 2005 & 11 & 5 & $22: 48: 2112.622$ & 40.495 & 1.88 & 2.85 \\
\hline 2005 & 11 & 5 & $23: 02: 2012.295$ & 40.643 & 2.87 & 0.86 \\
\hline 2005 & 11 & 5 & $23: 28: 1712.403$ & 40.590 & 2.30 & 0.9 \\
\hline 2005 & 11 & 6 & $00: 27: 5212.550$ & 40.501 & 3.05 & 0.55 \\
\hline 2005 & 11 & 6 & $00: 44: 2512.447$ & 40.584 & 2.53 & 0.42 \\
\hline 2005 & 11 & 6 & $00: 53: 0112.258$ & 40.650 & 2.17 & 1.61 \\
\hline 2005 & 11 & 6 & $01: 15: 5012.604$ & 40.534 & 1.91 & 0.58 \\
\hline 2005 & 11 & 6 & $02: 05: 1312.473$ & 40.566 & 2.65 & 0.46 \\
\hline 2005 & 11 & 6 & $02: 09: 5312.486$ & 40.583 & 2.31 & 3.02 \\
\hline 2005 & 11 & 6 & $02: 22: 1412.450$ & 40.557 & 2.27 & 1.24 \\
\hline 2005 & 11 & 6 & $06: 31: 2612.485$ & 40.550 & 2.44 & 0.78 \\
\hline 2005 & 11 & 6 & $11: 07: 4512.282$ & 40.658 & 2.42 & 1.47 \\
\hline 2005 & 11 & 6 & $14: 32: 2012.341$ & 40.325 & 2.38 & 1.14 \\
\hline 2005 & 11 & 6 & $17: 52: 0112.536$ & 40.531 & 1.63 & 3.51 \\
\hline 2005 & 11 & 6 & $19: 49: 3512.553$ & 40.495 & 2.83 & 0.43 \\
\hline 2005 & 11 & 6 & $19: 54: 4512.550$ & 40.500 & 2.51 & 0.57 \\
\hline 2005 & 11 & 6 & $20: 17: 1912.558$ & 40.524 & 2.21 & 1.44 \\
\hline 2005 & 11 & 6 & $20: 20: 2212.461$ & 40.570 & 2.15 & 0.56 \\
\hline 2005 & 11 & 6 & $20: 34: 4112.548$ & 40.486 & 2.23 & 0.52 \\
\hline 2005 & 11 & 6 & $21: 43: 0312.558$ & 40.456 & 1.93 & 0.62 \\
\hline 2005 & 11 & 6 & $22: 02: 2112.350$ & 40.333 & 2.25 & 1.32 \\
\hline 2005 & 11 & 6 & $22: 10: 3612.381$ & 40.600 & 2.37 & 0.47 \\
\hline 2005 & 11 & 6 & $23: 01: 5712.599$ & 40.471 & 2.29 & 1.26 \\
\hline 2005 & 11 & 6 & $23: 14: 1512.644$ & 40.531 & 1.71 & 0.56 \\
\hline 2005 & 11 & 6 & $23: 47: 0312.871$ & 40.249 & 2.44 & 2.75 \\
\hline 2005 & 11 & 7 & $00: 09: 2812.887$ & 40.297 & 2.97 & 1.14 \\
\hline 2005 & 11 & 7 & $01: 02: 3712.868$ & 40.252 & 3.28 & 0.68 \\
\hline 2005 & 11 & 7 & $01: 07: 4612.870$ & 40.281 & 2.25 & 0.82 \\
\hline 2005 & 11 & 7 & $01: 29: 0012.874$ & 40.249 & 2.94 & 0.66 \\
\hline 2005 & 11 & 7 & $01: 37: 0512.870$ & 40.243 & 2.52 & 0.98 \\
\hline 2005 & 11 & 7 & $01: 57: 1312.605$ & 40.562 & 2.16 & 1.09 \\
\hline 2005 & 11 & 7 & $02: 02: 4513.110$ & 40.935 & 3.02 & 0.94 \\
\hline 2005 & 11 & 7 & $02: 34: 3312.655$ & 40.554 & 2.36 & 1.03 \\
\hline 2005 & 11 & 7 & $09: 45: 3412.491$ & 40.574 & 2.82 & 0.75 \\
\hline 2005 & 11 & 7 & $11: 15: 5213.142$ & 40.961 & 2.93 & 3.08 \\
\hline 2005 & 11 & 7 & $15: 16: 3012.427$ & 40.590 & 2.42 & 0.76 \\
\hline 2005 & 11 & 7 & $18: 16: 2112.579$ & 40.512 & 2.36 & 1.51 \\
\hline 2005 & 11 & 7 & $18: 52: 3212.577$ & 40.454 & 2.13 & 0.65 \\
\hline 2005 & 11 & 7 & $18: 56: 3912.315$ & 40.614 & 2.32 & 0.82 \\
\hline 2005 & 11 & 7 & $20: 51: 2312.610$ & 40.481 & 2.83 & 0.77 \\
\hline 2005 & 11 & 7 & $22: 16: 5112.470$ & 40.559 & 2.19 & 0.68 \\
\hline 2005 & 11 & 7 & $22: 47: 3312.611$ & 40.451 & 2.88 & 1.24 \\
\hline 2005 & 11 & 7 & $23: 34: 5512.623$ & 40.466 & 2.15 & 3.03 \\
\hline 2005 & 11 & 8 & $00: 12: 2012.479$ & 40.558 & 2.31 & 0.57 \\
\hline 2005 & 11 & 8 & $00: 38: 5512.467$ & 40.567 & 3.04 & 0.55 \\
\hline 2005 & 11 & 8 & $00: 45: 2412.466$ & 40.566 & 2.19 & 1.27 \\
\hline 2005 & 11 & 8 & $01: 58: 5512.539$ & 40.525 & 1.84 & 0.7 \\
\hline 2005 & 11 & 8 & $04: 15: 3012.591$ & 40.459 & 2.37 & 0.85 \\
\hline 2005 & 11 & 8 & $09: 36: 3312.544$ & 40.498 & 2.47 & 0.71 \\
\hline 2005 & 11 & 8 & $12: 52: 0912.606$ & 40.457 & 3.26 & 3.79 \\
\hline 2005 & 11 & 8 & $17: 52: 5312.573$ & 40.465 & 2.08 & 0.72 \\
\hline 2005 & 11 & 8 & $18: 46: 5812.402$ & 40.596 & 2.59 & 0.56 \\
\hline 2005 & 11 & 8 & $19: 23: 3312.587$ & 40.488 & 2.13 & 1.77 \\
\hline 2005 & 11 & 8 & $22: 03: 2712.557$ & 40.484 & 2.49 & 0.61 \\
\hline 2005 & 11 & 8 & $22: 55: 1512.584$ & 40.503 & 1.98 & 0.94 \\
\hline 2005 & 11 & 9 & $00: 17: 1612.582$ & 40.477 & 1.95 & 0.61 \\
\hline 2005 & 11 & 9 & $00: 45: 3012.424$ & 40.573 & 2.03 & 1.33 \\
\hline 2005 & 11 & 9 & $01: 15: 2812.765$ & 40.642 & 2.14 & 0.62 \\
\hline 2005 & 11 & 9 & $02: 01: 4512.392$ & 40.597 & 2.13 & 0.57 \\
\hline 2005 & 11 & 9 & $02: 28: 2912.482$ & 40.559 & 2.12 & 0.74 \\
\hline 2005 & 11 & 9 & $04: 06: 3312.627$ & 40.453 & 2.69 & 2.79 \\
\hline 2005 & 11 & 9 & $04: 23: 2512.561$ & 40.494 & 2.48 & 5.3 \\
\hline 2005 & 11 & 9 & $05: 06: 0612.446$ & 40.585 & 2.47 & 1.4 \\
\hline 2005 & 11 & 9 & $08: 26: 2512.473$ & 40.564 & 2.85 & 0.69 \\
\hline 2005 & 11 & 9 & $10: 44: 1512.557$ & 40.495 & 3.07 & 0.73 \\
\hline 2005 & 11 & 9 & $18: 18: 5012.580$ & 40.496 & 2.18 & 1.39 \\
\hline 2005 & 11 & 9 & $18: 22: 4012.584$ & 40.494 & 3.07 & 1.04 \\
\hline 2005 & 11 & 9 & $19: 10: 0712.568$ & 40.539 & 2.34 & 0.7 \\
\hline 2005 & 11 & 9 & $19: 17: 5712.530$ & 40.544 & 2.29 & 3.08 \\
\hline 2005 & 11 & 9 & $19: 56: 3812.600$ & 40.464 & 2.16 & 0.89 \\
\hline 2005 & 11 & 9 & $20: 08: 3412.594$ & 40.473 & 2.26 & 1.47 \\
\hline 2005 & 11 & 9 & $20: 36: 2012.585$ & 40.451 & 1.86 & 0.82 \\
\hline
\end{tabular}




\begin{tabular}{|c|c|c|c|c|c|c|}
\hline 2005 & 11 & 9 & $20: 43: 3112.514$ & 40.541 & 1.97 & 2.39 \\
\hline 2005 & 11 & 9 & $21: 03: 5112.623$ & 40.467 & 1.75 & 1.54 \\
\hline 2005 & 11 & 9 & $21: 37: 4012.599$ & 40.505 & 2.94 & 0.71 \\
\hline 2005 & 11 & 9 & $22: 07: 1912.555$ & 40.493 & 2.88 & 0.45 \\
\hline 2005 & 11 & 9 & $22: 52: 1012.318$ & 40.618 & 2.21 & 0.85 \\
\hline 2005 & 11 & 9 & $22: 54: 1212.321$ & 40.623 & 2.02 & 0.69 \\
\hline 2005 & 11 & 9 & $23: 13: 5112.526$ & 40.526 & 1.88 & 1.14 \\
\hline 2005 & 11 & 9 & $23: 15: 4812.644$ & 40.546 & 2.20 & 0.69 \\
\hline 2005 & 11 & 9 & $23: 29: 4512.591$ & 40.465 & 2.16 & 1.32 \\
\hline 2005 & 11 & 9 & $23: 46: 0812.538$ & 40.533 & 1.81 & 1.02 \\
\hline 2005 & 11 & 10 & $00: 00: 1512.566$ & 40.502 & 2.21 & 1.62 \\
\hline 2005 & 11 & 10 & $00: 52: 2112.548$ & 40.528 & 1.77 & 0.93 \\
\hline 2005 & 11 & 10 & $01: 15: 3212.538$ & 40.595 & 2.52 & 3.24 \\
\hline 2005 & 11 & 10 & $01: 45: 2912.529$ & 40.543 & 2.78 & 1.2 \\
\hline 2005 & 11 & 10 & $06: 49: 4012.511$ & 40.546 & 3.17 & 0.56 \\
\hline 2005 & 11 & 10 & $07: 02: 0912.543$ & 40.496 & 2.50 & 0.75 \\
\hline 2005 & 11 & 10 & $08: 19: 3512.387$ & 40.593 & 3.31 & 1.27 \\
\hline 2005 & 11 & 10 & $11: 35: 3712.579$ & 40.529 & 2.57 & 0.83 \\
\hline 2005 & 11 & 10 & $12: 10: 0812.376$ & 40.629 & 2.55 & 1.12 \\
\hline 2005 & 11 & 10 & $15: 48: 3912.553$ & 40.489 & 2.45 & 0.62 \\
\hline 2005 & 11 & 10 & $19: 50: 3512.605$ & 40.502 & 3.03 & 0.82 \\
\hline 2005 & 11 & 10 & $20: 41: 5512.545$ & 40.531 & 2.07 & 0.76 \\
\hline 2005 & 11 & 10 & $20: 45: 5412.548$ & 40.522 & 2.77 & 1.11 \\
\hline 2005 & 11 & 10 & $21: 59: 4212.472$ & 40.558 & 2.08 & 0.98 \\
\hline 2005 & 11 & 10 & $22: 28: 0612.586$ & 40.505 & 2.23 & 1.22 \\
\hline 2005 & 11 & 10 & $23: 24: 5412.579$ & 40.474 & 2.31 & 0.61 \\
\hline 2005 & 11 & 11 & $05: 22: 3312.615$ & 40.461 & 3.86 & 1.41 \\
\hline 2005 & 11 & 11 & $12: 32: 4212.587$ & 40.478 & 2.81 & 1.28 \\
\hline 2005 & 11 & 11 & $19: 31: 4512.577$ & 40.507 & 2.45 & 0.68 \\
\hline 2005 & 11 & 11 & $20: 27: 5812.601$ & 40.502 & 1.77 & 0.89 \\
\hline 2005 & 11 & 11 & $21: 36: 5212.550$ & 40.495 & 1.96 & 0.65 \\
\hline 2005 & 11 & 11 & $22: 38: 2912.471$ & 40.559 & 2.37 & 0.64 \\
\hline 2005 & 11 & 11 & $23: 03: 5512.553$ & 40.500 & 2.52 & 0.51 \\
\hline 2005 & 11 & 12 & $00: 54: 4812.468$ & 40.568 & 2.46 & 0.63 \\
\hline 2005 & 11 & 12 & $00: 59: 5112.567$ & 40.447 & 1.94 & 0.95 \\
\hline 2005 & 11 & 12 & $10: 27: 0212.592$ & 40.518 & 2.67 & 2.35 \\
\hline 2005 & 11 & 12 & $10: 59: 1312.580$ & 40.500 & 3.64 & 1.05 \\
\hline 2005 & 11 & 12 & $12: 37: 2012.588$ & 40.733 & 3.21 & 1.64 \\
\hline 2005 & 11 & 12 & $18: 06: 1912.482$ & 40.673 & 2.38 & 2.15 \\
\hline 2005 & 11 & 12 & $19: 59: 4112.584$ & 40.471 & 2.41 & 1.11 \\
\hline 2005 & 11 & 12 & $21: 10: 3012.229$ & 40.691 & 2.41 & 0.57 \\
\hline 2005 & 11 & 12 & $21: 13: 2112.589$ & 40.459 & 3.26 & 0.84 \\
\hline 2005 & 11 & 12 & $21: 42: 1212.623$ & 40.525 & 2.03 & 0.84 \\
\hline 2005 & 11 & 12 & $21: 43: 4912.555$ & 40.490 & 1.73 & 2.23 \\
\hline 2005 & 11 & 12 & $21: 51: 2612.639$ & 40.534 & 1.73 & 0.63 \\
\hline 2005 & 11 & 12 & $23: 08: 2612.592$ & 40.505 & 1.99 & 2.43 \\
\hline 2005 & 11 & 13 & $00: 11: 4612.590$ & 40.465 & 2.20 & 1.22 \\
\hline 2005 & 11 & 13 & $00: 25: 0412.548$ & 40.536 & 1.97 & 0.55 \\
\hline 2005 & 11 & 13 & $01: 03: 4312.544$ & 40.550 & 2.66 & 3.64 \\
\hline 2005 & 11 & 13 & $02: 39: 2612.317$ & 40.625 & 2.28 & 0.91 \\
\hline 2005 & 11 & 13 & $09: 03: 2312.585$ & 40.517 & 2.55 & 1.19 \\
\hline 2005 & 11 & 13 & $09: 25: 2113.323$ & 41.088 & 3.99 & 2.88 \\
\hline 2005 & 11 & 13 & $10: 16: 3513.272$ & 41.048 & 3.03 & 3.29 \\
\hline 2005 & 11 & 13 & $12: 41: 5612.551$ & 40.500 & 3.10 & 0.76 \\
\hline 2005 & 11 & 13 & $14: 13: 0512.562$ & 40.477 & 2.53 & 0.68 \\
\hline 2005 & 11 & 13 & $15: 42: 2112.476$ & 40.560 & 2.59 & 0.57 \\
\hline 2005 & 11 & 13 & $18: 21: 4012.489$ & 40.568 & 2.16 & 1.05 \\
\hline 2005 & 11 & 13 & $20: 21: 5812.540$ & 40.545 & 2.13 & 0.55 \\
\hline 2005 & 11 & 13 & $21: 32: 2712.531$ & 40.504 & 1.80 & 1.14 \\
\hline 2005 & 11 & 13 & $23: 35: 1712.594$ & 40.514 & 2.37 & 0.78 \\
\hline 2005 & 11 & 14 & $00: 01: 1912.600$ & 40.502 & 1.77 & 3.22 \\
\hline 2005 & 11 & 14 & $08: 58: 0312.740$ & 40.578 & 2.69 & 0.73 \\
\hline 2005 & 11 & 14 & $09: 36: 1212.736$ & 40.569 & 2.38 & 1.59 \\
\hline 2005 & 11 & 14 & $13: 18: 3112.450$ & 40.577 & 2.76 & 0.73 \\
\hline 2005 & 11 & 14 & $15: 57: 4712.559$ & 40.526 & 2.63 & 0.84 \\
\hline 2005 & 11 & 14 & $18: 09: 1912.576$ & 40.516 & 2.45 & 0.98 \\
\hline 2005 & 11 & 14 & $19: 41: 4713.066$ & 40.904 & 3.05 & 1.56 \\
\hline 2005 & 11 & 14 & $21: 13: 2512.547$ & 40.495 & 2.80 & 0.48 \\
\hline 2005 & 11 & 14 & $22: 16: 4912.625$ & 40.453 & 2.16 & 3.43 \\
\hline 2005 & 11 & 14 & $23: 30: 3412.390$ & 40.598 & 2.87 & 0.45 \\
\hline 2005 & 11 & 14 & $23: 55: 2112.548$ & 40.528 & 2.00 & 1.32 \\
\hline 2005 & 11 & 15 & $00: 26: 1112.301$ & 40.628 & 1.97 & 1.09 \\
\hline 2005 & 11 & 15 & $02: 44: 0612.586$ & 40.464 & 2.28 & 0.9 \\
\hline 2005 & 11 & 15 & $03: 04: 2612.566$ & 40.469 & 2.41 & 1.97 \\
\hline 2005 & 11 & 15 & $08: 13: 1113.095$ & 40.992 & 2.97 & 2.46 \\
\hline 2005 & 11 & 15 & $14: 52: 1913.077$ & 40.917 & 2.48 & 1.27 \\
\hline
\end{tabular}




\begin{tabular}{|c|c|c|c|c|c|c|}
\hline 2005 & 11 & 15 & $16: 04: 1112.400$ & 40.590 & 2.25 & 0.61 \\
\hline 2005 & 11 & 15 & $18: 00: 3512.576$ & 40.511 & 1.91 & 1.21 \\
\hline 2005 & 11 & 15 & $18: 57: 3612.484$ & 40.559 & 1.85 & 0.6 \\
\hline 2005 & 11 & 15 & $20: 34: 0112.509$ & 40.544 & 1.84 & 0.59 \\
\hline 2005 & 11 & 15 & $21: 26: 5713.446$ & 40.847 & 3.22 & 3.5 \\
\hline 2005 & 11 & 15 & $21: 57: 3512.545$ & 40.478 & 2.02 & 0.62 \\
\hline 2005 & 11 & 15 & $22: 17: 2713.424$ & 40.824 & 2.83 & 2.42 \\
\hline 2005 & 11 & 15 & $22: 48: 2712.556$ & 40.478 & 2.41 & 0.85 \\
\hline 2005 & 11 & 15 & $23: 05: 5911.871$ & 41.047 & 2.18 & 2.77 \\
\hline 2005 & 11 & 15 & $23: 13: 4112.593$ & 40.514 & 1.99 & 1.24 \\
\hline 2005 & 11 & 15 & $23: 59: 2512.325$ & 40.517 & 2.00 & 3.08 \\
\hline 2005 & 11 & 16 & $00: 40: 3712.493$ & 40.657 & 2.42 & 0.8 \\
\hline 2005 & 11 & 16 & $01: 33: 3212.679$ & 40.503 & 1.42 & 1.95 \\
\hline 2005 & 11 & 16 & $03: 36: 3913.431$ & 40.842 & 3.19 & 3.57 \\
\hline 2005 & 11 & 16 & $06: 29: 3913.426$ & 40.835 & 3.30 & 2.69 \\
\hline 2005 & 11 & 16 & $07: 43: 1312.468$ & 40.560 & 2.64 & 0.84 \\
\hline 2005 & 11 & 16 & $09: 14: 1012.515$ & 40.558 & 2.27 & 0.81 \\
\hline 2005 & 11 & 16 & $11: 48: 3512.616$ & 40.534 & 2.26 & 0.58 \\
\hline 2005 & 11 & 16 & $13: 18: 1012.594$ & 40.431 & 2.05 & 3.46 \\
\hline 2005 & 11 & 16 & $17: 52: 0612.646$ & 40.530 & 1.42 & 0.63 \\
\hline 2005 & 11 & 16 & $18: 16: 0013.086$ & 40.925 & 3.02 & 1.06 \\
\hline 2005 & 11 & 16 & $18: 30: 2412.434$ & 40.569 & 2.25 & 0.85 \\
\hline 2005 & 11 & 16 & $19: 02: 3112.284$ & 40.664 & 2.26 & 0.89 \\
\hline 2005 & 11 & 16 & $19: 46: 4212.541$ & 40.485 & 2.22 & 0.72 \\
\hline 2005 & 11 & 16 & $20: 18: 4812.312$ & 40.630 & 2.53 & 0.58 \\
\hline 2005 & 11 & 16 & $20: 37: 3512.553$ & 40.532 & 1.67 & 1.99 \\
\hline 2005 & 11 & 16 & $20: 40: 5112.585$ & 40.514 & 1.95 & 1.5 \\
\hline 2005 & 11 & 16 & $21: 28: 0112.630$ & 40.531 & 1.58 & 0.63 \\
\hline 2005 & 11 & 16 & $22: 39: 3412.597$ & 40.501 & 2.69 & 0.76 \\
\hline 2005 & 11 & 16 & $23: 49: 0412.592$ & 40.507 & 2.13 & 0.82 \\
\hline 2005 & 11 & 17 & $00: 13: 2613.428$ & 40.816 & 2.54 & 3.99 \\
\hline 2005 & 11 & 17 & $02: 06: 3512.572$ & 40.466 & 2.28 & 0.71 \\
\hline 2005 & 11 & 17 & $06: 26: 1312.579$ & 40.518 & 2.99 & 0.79 \\
\hline 2005 & 11 & 17 & $07: 28: 3212.544$ & 40.485 & 3.18 & 6.63 \\
\hline 2005 & 11 & 17 & $07: 34: 5912.579$ & 40.463 & 2.83 & 1.88 \\
\hline 2005 & 11 & 17 & $10: 37: 2912.572$ & 40.495 & 2.95 & 1.26 \\
\hline 2005 & 11 & 17 & $14: 05: 0812.573$ & 40.462 & 2.70 & 0.9 \\
\hline 2005 & 11 & 17 & $16: 31: 3812.275$ & 40.644 & 2.76 & 0.49 \\
\hline 2005 & 11 & 18 & $00: 08: 3912.598$ & 40.504 & 1.83 & 1.19 \\
\hline 2005 & 11 & 18 & $01: 13: 4312.514$ & 40.548 & 2.26 & 0.79 \\
\hline 2005 & 11 & 18 & $01: 49: 0312.554$ & 40.481 & 2.43 & 0.68 \\
\hline 2005 & 11 & 18 & $05: 45: 1312.561$ & 40.489 & 3.05 & 0.65 \\
\hline 2005 & 11 & 18 & $12: 01: 4713.408$ & 40.899 & 2.80 & 2.09 \\
\hline 2005 & 11 & 18 & $15: 05: 0112.670$ & 40.370 & 2.40 & 9.61 \\
\hline 2005 & 11 & 18 & $18: 13: 5712.539$ & 40.614 & 2.12 & 1.11 \\
\hline 2005 & 11 & 18 & $18: 26: 4512.330$ & 40.614 & 2.72 & 0.74 \\
\hline 2005 & 11 & 18 & $19: 42: 3412.624$ & 40.530 & 1.83 & 0.92 \\
\hline 2005 & 11 & 18 & $20: 41: 1112.435$ & 40.605 & 1.96 & 1.2 \\
\hline 2005 & 11 & 18 & $23: 27: 2412.653$ & 40.563 & 1.97 & 1.21 \\
\hline 2005 & 11 & 19 & $02: 44: 1112.538$ & 40.483 & 2.31 & 0.59 \\
\hline 2005 & 11 & 19 & $04: 07: 1312.457$ & 40.566 & 2.72 & 0.8 \\
\hline 2005 & 11 & 19 & $05: 08: 5412.556$ & 40.489 & 2.80 & 0.7 \\
\hline 2005 & 11 & 19 & $07: 27: 1712.630$ & 40.473 & 2.35 & 4.45 \\
\hline 2005 & 11 & 19 & $08: 47: 5512.578$ & 40.461 & 2.72 & 0.97 \\
\hline 2005 & 11 & 19 & $15: 23: 2012.302$ & 40.620 & 2.51 & 0.81 \\
\hline 2005 & 11 & 19 & $17: 22: 1513.468$ & 40.863 & 2.92 & 3.25 \\
\hline 2005 & 11 & 19 & $20: 10: 2413.430$ & 40.842 & 2.63 & 2.93 \\
\hline 2005 & 11 & 19 & $22: 17: 3812.603$ & 40.541 & 1.97 & 0.96 \\
\hline 2005 & 11 & 19 & $23: 27: 5112.540$ & 40.535 & 1.62 & 4.76 \\
\hline 2005 & 11 & 19 & $23: 43: 0712.579$ & 40.516 & 1.70 & 1.99 \\
\hline 2005 & 11 & 20 & $00: 12: 1112.619$ & 40.534 & 1.65 & 2.13 \\
\hline 2005 & 11 & 20 & $01: 07: 5812.564$ & 40.536 & 2.17 & 2.03 \\
\hline 2005 & 11 & 20 & $01: 34: 5312.575$ & 40.494 & 2.60 & 1.19 \\
\hline 2005 & 11 & 20 & $01: 52: 1212.302$ & 40.636 & 2.42 & 0.8 \\
\hline 2005 & 11 & 20 & $01: 59: 0613.412$ & 40.845 & 2.65 & 4.14 \\
\hline 2005 & 11 & 20 & $03: 18: 1212.373$ & 40.607 & 2.16 & 1.57 \\
\hline 2005 & 11 & 20 & $03: 49: 1012.510$ & 40.651 & 2.79 & 2.13 \\
\hline 2005 & 11 & 20 & $05: 06: 0012.536$ & 40.645 & 3.11 & 2.74 \\
\hline 2005 & 11 & 20 & $06: 20: 3312.580$ & 40.480 & 2.96 & 1.07 \\
\hline 2005 & 11 & 20 & $11: 15: 4312.524$ & 40.623 & 2.33 & 2.58 \\
\hline 2005 & 11 & 20 & $13: 32: 1912.560$ & 40.480 & 2.41 & 1.44 \\
\hline 2005 & 11 & 20 & $19: 50: 2812.678$ & 40.571 & 1.80 & 1.65 \\
\hline 2005 & 11 & 20 & $20: 59: 3712.640$ & 40.532 & 1.93 & 0.52 \\
\hline 2005 & 11 & 20 & $21: 36: 1512.550$ & 40.479 & 2.30 & 0.5 \\
\hline 2005 & 11 & 20 & $21: 53: 4912.366$ & 40.619 & 1.76 & 0.62 \\
\hline 2005 & 11 & 20 & $22: 11: 1312.586$ & 40.505 & 1.66 & 0.8 \\
\hline
\end{tabular}




\begin{tabular}{|c|c|c|c|c|c|c|}
\hline 2005 & 11 & 20 & $22: 39: 3212.445$ & 40.551 & 2.01 & 1.1 \\
\hline 2005 & 11 & 20 & $23: 06: 2112.656$ & 40.472 & 1.73 & 2.81 \\
\hline 2005 & 11 & 20 & $23: 08: 2112.582$ & 40.521 & 1.90 & 1.79 \\
\hline 2005 & 11 & 20 & $23: 09: 4412.733$ & 40.438 & 1.85 & 2.98 \\
\hline 2005 & 11 & 20 & $23: 32: 4413.433$ & 40.826 & 2.50 & 3.13 \\
\hline 2005 & 11 & 20 & $23: 49: 4312.630$ & 40.536 & 2.01 & 0.63 \\
\hline 2005 & 11 & 20 & $23: 50: 5812.598$ & 40.513 & 1.59 & 0.97 \\
\hline 2005 & 11 & 21 & $01: 00: 4312.745$ & 40.575 & 2.13 & 0.61 \\
\hline 2005 & 11 & 21 & $01: 05: 5812.625$ & 40.535 & 1.70 & 0.82 \\
\hline 2005 & 11 & 21 & $01: 07: 5912.511$ & 40.542 & 1.74 & 2.65 \\
\hline 2005 & 11 & 21 & $03: 19: 3312.272$ & 40.662 & 2.06 & 1.15 \\
\hline 2005 & 11 & 21 & $03: 35: 0612.711$ & 40.534 & 2.80 & 0.78 \\
\hline 2005 & 11 & 21 & $13: 23: 0012.577$ & 40.502 & 2.59 & 2.08 \\
\hline 2005 & 11 & 21 & $16: 16: 1512.560$ & 40.477 & 2.47 & 0.92 \\
\hline 2005 & 11 & 21 & $17: 40: 1312.575$ & 40.451 & 2.83 & 1.04 \\
\hline 2005 & 11 & 21 & $18: 20: 0612.457$ & 40.586 & 1.92 & 1.79 \\
\hline 2005 & 11 & 21 & $18: 35: 5612.581$ & 40.528 & 1.79 & 1.61 \\
\hline 2005 & 11 & 21 & $19: 41: 3312.633$ & 40.541 & 1.79 & 4.08 \\
\hline 2005 & 11 & 21 & $20: 28: 0813.374$ & 40.793 & 2.21 & 1.75 \\
\hline 2005 & 11 & 21 & $20: 44: 3012.582$ & 40.471 & 1.52 & 1.03 \\
\hline 2005 & 11 & 21 & $21: 31: 0012.273$ & 40.658 & 2.58 & 0.78 \\
\hline 2005 & 11 & 21 & $21: 37: 0012.535$ & 40.492 & 2.08 & 0.7 \\
\hline 2005 & 11 & 21 & $22: 01: 1312.445$ & 40.585 & 1.78 & 0.82 \\
\hline 2005 & 11 & 21 & $22: 08: 3712.505$ & 40.599 & 1.62 & 1.34 \\
\hline 2005 & 11 & 21 & $22: 31: 3312.495$ & 40.546 & 2.03 & 0.64 \\
\hline 2005 & 11 & 21 & $22: 44: 1412.579$ & 40.444 & 2.40 & 0.84 \\
\hline 2005 & 11 & 21 & $23: 23: 2012.599$ & 40.511 & 1.68 & 1.58 \\
\hline 2005 & 11 & 21 & $23: 42: 4512.589$ & 40.492 & 2.38 & 0.68 \\
\hline 2005 & 11 & 21 & $23: 50: 2912.635$ & 40.520 & 1.58 & 0.98 \\
\hline 2005 & 11 & 21 & $23: 50: 5312.570$ & 40.529 & 2.00 & 1.59 \\
\hline 2005 & 11 & 22 & $01: 21: 0812.549$ & 40.489 & 1.73 & 1.44 \\
\hline 2005 & 11 & 22 & $01: 38: 0012.539$ & 40.555 & 1.78 & 1.33 \\
\hline 2005 & 11 & 22 & $01: 45: 5312.553$ & 40.500 & 2.48 & 0.76 \\
\hline 2005 & 11 & 22 & $02: 03: 4012.678$ & 40.445 & 1.90 & 4.23 \\
\hline 2005 & 11 & 22 & $02: 16: 5912.604$ & 40.472 & 1.78 & 6.79 \\
\hline 2005 & 11 & 22 & $03: 38: 0312.568$ & 40.517 & 2.30 & 1.3 \\
\hline 2005 & 11 & 22 & $04: 52: 3712.431$ & 40.575 & 2.59 & 1.11 \\
\hline 2005 & 11 & 22 & $06: 41: 2812.547$ & 40.489 & 3.02 & 0.78 \\
\hline 2005 & 11 & 22 & $09: 39: 5312.595$ & 40.458 & 2.36 & 1.33 \\
\hline 2005 & 11 & 22 & $12: 31: 5512.658$ & 40.413 & 2.85 & 1.08 \\
\hline 2005 & 11 & 22 & $13: 46: 5112.554$ & 40.491 & 2.65 & 0.53 \\
\hline 2005 & 11 & 22 & $14: 29: 2012.726$ & 40.575 & 2.89 & 1.12 \\
\hline 2005 & 11 & 22 & $15: 54: 4112.548$ & 40.558 & 2.18 & 0.91 \\
\hline 2005 & 11 & 22 & $15: 56: 5712.608$ & 40.487 & 2.17 & 1.98 \\
\hline 2005 & 11 & 22 & $17: 14: 2512.500$ & 40.535 & 2.10 & 1.8 \\
\hline 2005 & 11 & 22 & $19: 13: 4312.389$ & 40.591 & 1.91 & 1.02 \\
\hline 2005 & 11 & 22 & $19: 45: 1012.572$ & 40.532 & 1.89 & 0.81 \\
\hline 2005 & 11 & 22 & $20: 05: 2112.546$ & 40.487 & 2.41 & 0.44 \\
\hline 2005 & 11 & 22 & $20: 44: 0612.612$ & 40.446 & 2.02 & 1.52 \\
\hline 2005 & 11 & 22 & $20: 55: 0312.888$ & 40.269 & 3.11 & 0.45 \\
\hline 2005 & 11 & 22 & $22: 46: 0013.429$ & 40.825 & 2.20 & 1.2 \\
\hline 2005 & 11 & 22 & $23: 32: 2712.393$ & 40.593 & 1.93 & 0.42 \\
\hline 2005 & 11 & 23 & $00: 04: 5012.570$ & 40.537 & 3.17 & 0.64 \\
\hline 2005 & 11 & 23 & $00: 20: 0812.577$ & 40.513 & 2.19 & 0.73 \\
\hline 2005 & 11 & 23 & $01: 04: 5612.573$ & 40.497 & 1.89 & 1.1 \\
\hline 2005 & 11 & 23 & $02: 31: 2012.546$ & 40.484 & 2.39 & 0.57 \\
\hline 2005 & 11 & 23 & $03: 22: 2713.542$ & 40.051 & 2.91 & 2.66 \\
\hline 2005 & 11 & 23 & $09: 47: 2812.454$ & 40.577 & 2.01 & 1.3 \\
\hline 2005 & 11 & 23 & $12: 43: 0812.606$ & 40.476 & 3.15 & 1.46 \\
\hline 2005 & 11 & 23 & $14: 26: 4912.612$ & 40.518 & 2.60 & 1.77 \\
\hline 2005 & 11 & 23 & $17: 45: 4212.536$ & 40.538 & 2.07 & 0.73 \\
\hline 2005 & 11 & 23 & $20: 42: 2512.512$ & 40.554 & 1.83 & 0.6 \\
\hline 2005 & 11 & 23 & $21: 04: 0012.889$ & 40.270 & 1.92 & 1.16 \\
\hline 2005 & 11 & 23 & $23: 12: 4912.283$ & 40.650 & 2.14 & 1.35 \\
\hline 2005 & 11 & 23 & $23: 31: 4912.470$ & 40.562 & 2.71 & 0.51 \\
\hline 2005 & 11 & 24 & $10: 31: 0212.547$ & 40.496 & 2.50 & 3.27 \\
\hline 2005 & 11 & 24 & $13: 17: 5112.596$ & 40.461 & 2.53 & 4.84 \\
\hline 2005 & 11 & 24 & $14: 05: 3312.356$ & 40.619 & 2.67 & 1.68 \\
\hline 2005 & 11 & 24 & $17: 11: 5812.312$ & 40.629 & 2.40 & 0.82 \\
\hline 2005 & 11 & 24 & $20: 20: 0112.632$ & 40.533 & 1.90 & 0.65 \\
\hline 2005 & 11 & 24 & $21: 39: 1712.549$ & 40.485 & 2.02 & 0.54 \\
\hline 2005 & 11 & 24 & $22: 12: 3912.436$ & 40.587 & 2.10 & 0.51 \\
\hline 2005 & 11 & 24 & $22: 35: 4012.587$ & 40.522 & 1.89 & 0.99 \\
\hline 2005 & 11 & 24 & $22: 48: 4912.598$ & 40.513 & 2.92 & 0.65 \\
\hline 2005 & 11 & 24 & $23: 55: 5012.579$ & 40.517 & 1.73 & 1.46 \\
\hline 2005 & 11 & 24 & $23: 56: 4812.598$ & 40.493 & 1.72 & 2.33 \\
\hline
\end{tabular}




\begin{tabular}{|c|c|c|c|c|c|c|}
\hline 2005 & 11 & 25 & $10: 48: 0512.477$ & 40.556 & 2.44 & 0.91 \\
\hline 2005 & 11 & 25 & $11: 34: 0312.632$ & 40.531 & 2.00 & 2.14 \\
\hline 2005 & 11 & 25 & $13: 15: 0612.581$ & 40.476 & 2.58 & 0.95 \\
\hline 2005 & 11 & 25 & $14: 37: 1513.546$ & 39.921 & 2.79 & 9.13 \\
\hline 2005 & 11 & 25 & $19: 25: 2012.652$ & 40.476 & 2.01 & 2.58 \\
\hline 2005 & 11 & 25 & $19: 26: 5212.543$ & 40.503 & 2.45 & 0.7 \\
\hline 2005 & 11 & 25 & $21: 09: 3812.464$ & 40.545 & 1.74 & 1.35 \\
\hline 2005 & 11 & 25 & $21: 23: 0212.208$ & 40.681 & 1.98 & 0.78 \\
\hline 2005 & 11 & 25 & $22: 17: 3712.428$ & 40.576 & 1.56 & 1.06 \\
\hline 2005 & 11 & 26 & $02: 08: 0612.604$ & 40.526 & 2.16 & 0.75 \\
\hline 2005 & 11 & 26 & $07: 34: 2312.579$ & 40.472 & 2.80 & 2.98 \\
\hline 2005 & 11 & 26 & $16: 56: 3712.857$ & 40.636 & 2.63 & 0.43 \\
\hline 2005 & 11 & 26 & $19: 57: 0012.531$ & 40.545 & 2.19 & 0.66 \\
\hline 2005 & 11 & 26 & $20: 47: 3211.820$ & 41.090 & 3.18 & 3.95 \\
\hline 2005 & 11 & 26 & $21: 32: 0412.581$ & 40.521 & 3.03 & 0.82 \\
\hline 2005 & 11 & 26 & $21: 36: 3611.790$ & 41.163 & 3.11 & 4.52 \\
\hline 2005 & 11 & 26 & $21: 49: 5512.574$ & 40.464 & 2.84 & 0.82 \\
\hline 2005 & 11 & 26 & $22: 19: 4011.790$ & 41.119 & 2.86 & 2.34 \\
\hline 2005 & 11 & 27 & $00: 00: 3011.774$ & 41.146 & 2.95 & 2.29 \\
\hline 2005 & 11 & 27 & $01: 29: 5012.559$ & 40.501 & 2.54 & 0.6 \\
\hline 2005 & 11 & 27 & $09: 31: 2712.485$ & 40.554 & 2.21 & 1.07 \\
\hline 2005 & 11 & 27 & $12: 31: 3712.548$ & 40.492 & 3.04 & 0.83 \\
\hline 2005 & 11 & 27 & $17: 42: 3213.397$ & 40.000 & 3.03 & 1.74 \\
\hline 2005 & 11 & 27 & $20: 34: 2612.588$ & 40.467 & 3.06 & 1.29 \\
\hline 2005 & 11 & 27 & $21: 26: 4912.595$ & 40.470 & 2.66 & 0.93 \\
\hline 2005 & 11 & 27 & $22: 33: 0212.619$ & 40.516 & 1.79 & 0.56 \\
\hline 2005 & 11 & 27 & $23: 39: 2112.343$ & 40.618 & 2.21 & 0.62 \\
\hline 2005 & 11 & 28 & $00: 32: 1112.585$ & 40.471 & 1.78 & 1.44 \\
\hline 2005 & 11 & 28 & $00: 37: 5712.452$ & 40.566 & 1.86 & 0.71 \\
\hline 2005 & 11 & 28 & $00: 55: 0312.669$ & 40.446 & 1.92 & 3.48 \\
\hline 2005 & 11 & 28 & $01: 16: 5012.566$ & 40.478 & 2.75 & 0.84 \\
\hline 2005 & 11 & 28 & $19: 43: 1912.582$ & 40.527 & 2.16 & 0.53 \\
\hline 2005 & 11 & 28 & $22: 07: 2112.424$ & 40.720 & 2.10 & 2.23 \\
\hline 2005 & 11 & 28 & $22: 08: 1712.300$ & 40.628 & 2.21 & 0.6 \\
\hline 2005 & 11 & 28 & $22: 54: 3612.627$ & 40.523 & 1.76 & 0.53 \\
\hline 2005 & 11 & 28 & $23: 47: 5211.801$ & 41.035 & 2.23 & 4.31 \\
\hline 2005 & 11 & 29 & $01: 11: 1013.435$ & 40.857 & 2.17 & 1.58 \\
\hline 2005 & 11 & 29 & $02: 04: 4612.560$ & 40.470 & 2.52 & 0.78 \\
\hline 2005 & 11 & 29 & $02: 21: 4812.544$ & 40.534 & 2.54 & 0.55 \\
\hline 2005 & 11 & 29 & $03: 50: 0112.607$ & 40.533 & 2.55 & 0.5 \\
\hline 2005 & 11 & 29 & $13: 19: 5712.640$ & 40.526 & 2.34 & 0.85 \\
\hline 2005 & 11 & 29 & $16: 40: 0912.550$ & 40.563 & 2.65 & 1.56 \\
\hline 2005 & 11 & 29 & $19: 24: 0912.637$ & 40.526 & 1.93 & 1.04 \\
\hline 2005 & 11 & 29 & $20: 04: 3312.534$ & 40.539 & 1.84 & 1.24 \\
\hline 2005 & 11 & 29 & $20: 45: 5912.557$ & 40.525 & 2.27 & 3.2 \\
\hline 2005 & 11 & 29 & $21: 20: 3012.507$ & 40.536 & 1.70 & 2.28 \\
\hline 2005 & 11 & 29 & $23: 37: 5012.550$ & 40.526 & 1.71 & 3.35 \\
\hline 2005 & 11 & 30 & $00: 22: 5612.574$ & 40.526 & 1.76 & 3.25 \\
\hline 2005 & 11 & 30 & $01: 38: 3412.544$ & 40.491 & 2.56 & 0.57 \\
\hline 2005 & 11 & 30 & $10: 58: 3512.493$ & 40.540 & 2.23 & 0.89 \\
\hline 2005 & 11 & 30 & $18: 36: 5112.539$ & 40.506 & 2.44 & 0.57 \\
\hline 2005 & 11 & 30 & $20: 33: 4412.540$ & 40.484 & 2.33 & 0.5 \\
\hline 2005 & 11 & 30 & $21: 00: 1512.567$ & 40.517 & 2.70 & 0.69 \\
\hline 2005 & 11 & 30 & $21: 51: 3712.622$ & 40.524 & 2.10 & 0.63 \\
\hline 2005 & 11 & 30 & $22: 51: 4512.555$ & 40.520 & 1.91 & 2.06 \\
\hline 2005 & 12 & 1 & $00: 57: 5112.749$ & 40.298 & 1.75 & 7.2 \\
\hline 2005 & 12 & 1 & $01: 10: 4412.682$ & 40.393 & 1.22 & 4.54 \\
\hline 2005 & 12 & 1 & $01: 14: 3312.488$ & 40.565 & 1.77 & 2.13 \\
\hline 2005 & 12 & 1 & $01: 24: 0512.601$ & 40.496 & 1.61 & 3.82 \\
\hline 2005 & 12 & 1 & $01: 27: 2712.636$ & 40.535 & 1.46 & 0.78 \\
\hline 2005 & 12 & 1 & $01: 37: 4512.704$ & 40.271 & 1.56 & 1.7 \\
\hline 2005 & 12 & 1 & $01: 39: 1112.514$ & 40.538 & 1.31 & 3.16 \\
\hline 2005 & 12 & 1 & $02: 33: 0312.661$ & 40.398 & 1.50 & 6.37 \\
\hline 2005 & 12 & 1 & $03: 08: 4412.533$ & 40.605 & 1.79 & 3.19 \\
\hline 2005 & 12 & 1 & $03: 22: 2312.436$ & 40.595 & 1.96 & 1.72 \\
\hline 2005 & 12 & 1 & $03: 34: 5012.541$ & 40.544 & 1.62 & 8.63 \\
\hline 2005 & 12 & 1 & $03: 37: 2712.633$ & 40.560 & 1.99 & 2.29 \\
\hline 2005 & 12 & 1 & $03: 47: 1812.826$ & 40.276 & 3.18 & 0.56 \\
\hline 2005 & 12 & 1 & $05: 07: 5812.589$ & 40.523 & 2.34 & 2.38 \\
\hline 2005 & 12 & 1 & $08: 09: 5012.575$ & 40.504 & 2.66 & 4.74 \\
\hline 2005 & 12 & 1 & $12: 39: 3112.827$ & 40.511 & 2.47 & 6.09 \\
\hline 2005 & 12 & 1 & $13: 30: 3113.623$ & 39.859 & 2.92 & 5.69 \\
\hline 2005 & 12 & 1 & $15: 44: 3212.616$ & 40.543 & 1.77 & 1.24 \\
\hline 2005 & 12 & 1 & $16: 22: 02 \quad 12.493$ & 40.533 & 1.91 & 3.08 \\
\hline 2005 & 12 & 1 & $16: 39: 1512.280$ & 40.644 & 2.40 & 0.88 \\
\hline 2005 & 12 & 1 & $19: 23: 3212.569$ & 40.521 & 2.21 & 1.1 \\
\hline
\end{tabular}




\begin{tabular}{|c|c|c|c|c|c|c|}
\hline 2005 & 12 & 1 & $19: 29: 5312.530$ & 40.546 & 2.08 & 2.81 \\
\hline 2005 & 12 & 1 & $20: 43: 3712.529$ & 40.528 & 1.72 & 2.29 \\
\hline 2005 & 12 & 1 & $21: 21: 3812.613$ & 40.452 & 1.79 & 3.6 \\
\hline 2005 & 12 & 1 & $22: 09: 2212.591$ & 40.503 & 1.76 & 1.17 \\
\hline 2005 & 12 & 1 & $23: 43: 5412.591$ & 40.512 & 1.46 & 2.13 \\
\hline 2005 & 12 & 2 & $00: 02: 3912.550$ & 40.484 & 2.50 & 0.78 \\
\hline 2005 & 12 & 2 & $00: 17: 0412.550$ & 40.498 & 2.98 & 0.42 \\
\hline 2005 & 12 & 2 & $00: 19: 0012.578$ & 40.472 & 1.90 & 2.03 \\
\hline 2005 & 12 & 2 & $01: 03: 1912.598$ & 40.512 & 1.74 & 2.46 \\
\hline 2005 & 12 & 2 & $01: 46: 5012.445$ & 40.578 & 1.90 & 6.82 \\
\hline 2005 & 12 & 2 & $02: 03: 3312.530$ & 40.540 & 1.77 & 4.48 \\
\hline 2005 & 12 & 2 & $02: 07: 0512.622$ & 40.523 & 1.75 & 2.58 \\
\hline 2005 & 12 & 2 & $04: 01: 3212.542$ & 40.525 & 2.25 & 3.2 \\
\hline 2005 & 12 & 2 & $08: 51: 5812.550$ & 40.530 & 2.11 & 0.83 \\
\hline 2005 & 12 & 2 & $13: 57: 5812.627$ & 40.523 & 1.51 & 4.99 \\
\hline 2005 & 12 & 2 & $16: 36: 2312.595$ & 40.520 & 1.76 & 4.11 \\
\hline 2005 & 12 & 2 & $16: 46: 5212.554$ & 40.485 & 2.00 & 6.18 \\
\hline 2005 & 12 & 2 & $18: 22: 3612.626$ & 40.526 & 1.73 & 0.66 \\
\hline 2005 & 12 & 2 & $18: 32: 0512.355$ & 40.597 & 1.83 & 0.75 \\
\hline 2005 & 12 & 2 & $18: 36: 4012.610$ & 40.465 & 1.68 & 2.46 \\
\hline 2005 & 12 & 2 & $18: 42: 3712.548$ & 40.502 & 2.07 & 0.84 \\
\hline 2005 & 12 & 2 & $19: 15: 0312.621$ & 40.544 & 1.50 & 1.77 \\
\hline 2005 & 12 & 2 & $19: 19: 1411.809$ & 41.093 & 3.31 & 8.05 \\
\hline 2005 & 12 & 2 & $19: 50: 1512.829$ & 40.278 & 2.46 & 0.77 \\
\hline 2005 & 12 & 2 & $20: 10: 3312.450$ & 40.566 & 1.88 & 1.28 \\
\hline 2005 & 12 & 2 & $20: 12: 1012.616$ & 40.535 & 1.95 & 0.93 \\
\hline 2005 & 12 & 2 & $21: 22: 5812.659$ & 40.511 & 1.24 & 1.47 \\
\hline 2005 & 12 & 2 & $21: 27: 1712.597$ & 40.514 & 1.56 & 0.88 \\
\hline 2005 & 12 & 2 & $22: 11: 0712.508$ & 40.531 & 1.70 & 4.32 \\
\hline 2005 & 12 & 3 & $00: 03: 2812.573$ & 40.501 & 2.78 & 0.97 \\
\hline 2005 & 12 & 3 & $00: 59: 3512.574$ & 40.502 & 3.07 & 1.22 \\
\hline 2005 & 12 & 3 & $01: 14: 5912.541$ & 40.493 & 2.52 & 0.6 \\
\hline 2005 & 12 & 3 & $02: 52: 5212.512$ & 40.518 & 2.29 & 3.12 \\
\hline 2005 & 12 & 3 & $07: 09: 48 \quad 12.294$ & 40.634 & 3.21 & 0.55 \\
\hline 2005 & 12 & 3 & $15: 55: 4412.534$ & 40.591 & 2.01 & 6.79 \\
\hline 2005 & 12 & 3 & $17: 00: 1712.633$ & 40.525 & 1.67 & 3.05 \\
\hline 2005 & 12 & 3 & $19: 21: 0612.539$ & 40.527 & 1.84 & 3.46 \\
\hline 2005 & 12 & 3 & $19: 39: 0012.591$ & 40.513 & 2.03 & 0.6 \\
\hline 2005 & 12 & 3 & $19: 49: 3212.627$ & 40.467 & 1.82 & 1.56 \\
\hline 2005 & 12 & 3 & $21: 03: 4012.591$ & 40.497 & 2.40 & 1.25 \\
\hline 2005 & 12 & 3 & $22: 31: 5912.623$ & 40.535 & 1.78 & 0.77 \\
\hline 2005 & 12 & 4 & $01: 18: 3712.605$ & 40.535 & 1.71 & 0.62 \\
\hline 2005 & 12 & 4 & $02: 30: 2612.358$ & 40.614 & 2.09 & 0.91 \\
\hline 2005 & 12 & 4 & $02: 32: 4912.350$ & 40.629 & 2.48 & 18.7 \\
\hline 2005 & 12 & 4 & $03: 49: 5112.571$ & 40.477 & 2.42 & 2.61 \\
\hline 2005 & 12 & 4 & $04: 38: 2112.317$ & 40.626 & 2.50 & 1.12 \\
\hline 2005 & 12 & 4 & $06: 24: 5112.348$ & 40.620 & 2.69 & 0.77 \\
\hline 2005 & 12 & 4 & $14: 04: 5912.580$ & 40.527 & 2.59 & 0.92 \\
\hline 2005 & 12 & 4 & $20: 57: 2412.646$ & 40.521 & 1.74 & 0.65 \\
\hline 2005 & 12 & 4 & $21: 41: 4912.488$ & 40.559 & 1.68 & 1.24 \\
\hline 2005 & 12 & 5 & $00: 45: 2212.637$ & 40.476 & 1.65 & 5,00 \\
\hline 2005 & 12 & 5 & $01: 50: 5612.627$ & 40.535 & 2.00 & 0.92 \\
\hline 2005 & 12 & 5 & $08: 23: 3412.345$ & 40.669 & 2.76 & 1.27 \\
\hline 2005 & 12 & 5 & $16: 18: 5312.293$ & 40.624 & 2.95 & 1.61 \\
\hline 2005 & 12 & 5 & $21: 19: 0611.888$ & 40.859 & 2.53 & 2.36 \\
\hline 2005 & 12 & 5 & $22: 36: 5312.541$ & 40.539 & 1.92 & 1.61 \\
\hline 2005 & 12 & 5 & $22: 45: 3412.210$ & 40.681 & 2.67 & 1.3 \\
\hline 2005 & 12 & 5 & $23: 34: 0612.435$ & 40.685 & 2.03 & 1.7 \\
\hline 2005 & 12 & 6 & $00: 29: 2112.583$ & 40.520 & 2.77 & 1.14 \\
\hline 2005 & 12 & 6 & $00: 44: 2312.633$ & 40.531 & 1.98 & 0.57 \\
\hline 2005 & 12 & 6 & $01: 47: 4012.489$ & 40.552 & 1.81 & 2.48 \\
\hline 2005 & 12 & 6 & $03: 23: 0912.316$ & 40.631 & 2.51 & 1.32 \\
\hline 2005 & 12 & 6 & $04: 33: 2912.572$ & 40.464 & 2.44 & 2.86 \\
\hline 2005 & 12 & 6 & $09: 34: 4812.597$ & 40.456 & 2.80 & 1.21 \\
\hline 2005 & 12 & 6 & $11: 36: 5612.402$ & 40.587 & 2.36 & 0.87 \\
\hline 2005 & 12 & 6 & $19: 07: 5412.594$ & 40.498 & 2.55 & 1.2 \\
\hline 2005 & 12 & 6 & $20: 02: 0512.608$ & 40.498 & 1.74 & 2.23 \\
\hline 2005 & 12 & 6 & $21: 22: 5512.374$ & 40.605 & 1.85 & 1.39 \\
\hline 2005 & 12 & 6 & $21: 45: 4212.458$ & 40.558 & 1.71 & 1.13 \\
\hline 2005 & 12 & 6 & $22: 17: 0112.354$ & 40.600 & 1.92 & 1.03 \\
\hline 2005 & 12 & 6 & $22: 44: 1712.614$ & 40.530 & 1.98 & 0.93 \\
\hline 2005 & 12 & 6 & $23: 33: 3012.545$ & 40.504 & 2.42 & 0.54 \\
\hline 2005 & 12 & 7 & $07: 24: 5612.553$ & 40.489 & 2.98 & 0.79 \\
\hline 2005 & 12 & 7 & $09: 11: 0912.538$ & 40.502 & 2.53 & 1.18 \\
\hline 2005 & 12 & 7 & $12: 42: 3112.761$ & 40.432 & 2.89 & 3.79 \\
\hline 2005 & 12 & 7 & $15: 04: 4812.549$ & 40.498 & 2.58 & 0.76 \\
\hline
\end{tabular}




\begin{tabular}{|c|c|c|c|c|c|c|}
\hline 2005 & 12 & 7 & $18: 03: 4512.630$ & 40.533 & 2.17 & 0.67 \\
\hline 2005 & 12 & 7 & $18: 08: 4012.592$ & 40.527 & 2.65 & 0.71 \\
\hline 2005 & 12 & 7 & $18: 13: 3012.631$ & 40.538 & 2.02 & 0.7 \\
\hline 2005 & 12 & 7 & $19: 18: 4512.472$ & 40.563 & 1.56 & 1.07 \\
\hline 2005 & 12 & 7 & $20: 45: 4612.731$ & 40.581 & 1.79 & 0.86 \\
\hline 2005 & 12 & 7 & $21: 35: 4412.638$ & 40.502 & 1.49 & 4.2 \\
\hline 2005 & 12 & 7 & $21: 37: 2712.563$ & 40.536 & 2.42 & 3.17 \\
\hline 2005 & 12 & 7 & $22: 07: 5312.461$ & 40.564 & 1.95 & 3.32 \\
\hline 2005 & 12 & 7 & $22: 43: 4612.566$ & 40.523 & 1.74 & 10.72 \\
\hline 2005 & 12 & 7 & $22: 44: 2712.620$ & 40.534 & 1.55 & 1,00 \\
\hline 2005 & 12 & 7 & $22: 50: 2112.614$ & 40.459 & 1.48 & 2.79 \\
\hline 2005 & 12 & 7 & $22: 51: 1812.466$ & 40.568 & 1.52 & 12.79 \\
\hline 2005 & 12 & 7 & $23: 28: 4012.615$ & 40.539 & 1.93 & 1.44 \\
\hline 2005 & 12 & 8 & $01: 03: 4112.632$ & 40.537 & 2.55 & 0.87 \\
\hline 2005 & 12 & 8 & $01: 23: 5312.589$ & 40.528 & 1.83 & 8.92 \\
\hline 2005 & 12 & 8 & $04: 49: 5512.520$ & 40.528 & 2.68 & 17.53 \\
\hline 2005 & 12 & 8 & $16: 04: 1612.588$ & 40.511 & 2.64 & 1.24 \\
\hline 2005 & 12 & 8 & $19: 31: 0212.732$ & 40.615 & 1.92 & 3.43 \\
\hline 2005 & 12 & 8 & $19: 48: 5812.653$ & 40.521 & 1.52 & 1.25 \\
\hline 2005 & 12 & 8 & $21: 47: 3912.626$ & 40.541 & 1.70 & 0.92 \\
\hline 2005 & 12 & 8 & $23: 52: 3612.549$ & 40.545 & 2.13 & 3.41 \\
\hline 2005 & 12 & 9 & $00: 49: 1012.461$ & 40.567 & 3.18 & 3.22 \\
\hline 2005 & 12 & 9 & $02: 00: 5712.581$ & 40.491 & 2.07 & 1.06 \\
\hline 2005 & 12 & 9 & $03: 16: 1612.620$ & 40.570 & 2.20 & 4.27 \\
\hline 2005 & 12 & 9 & $03: 22: 3412.540$ & 40.482 & 2.37 & 0.68 \\
\hline 2005 & 12 & 9 & $04: 40: 2612.571$ & 40.453 & 2.31 & 0.62 \\
\hline 2005 & 12 & 9 & $10: 01: 1212.613$ & 40.529 & 2.29 & 1.03 \\
\hline 2005 & 12 & 9 & $14: 33: 5112.459$ & 40.568 & 2.82 & 6.75 \\
\hline 2005 & 12 & 9 & $15: 09: 2112.511$ & 40.532 & 2.01 & 1.66 \\
\hline 2005 & 12 & 9 & $19: 26: 0012.750$ & 40.411 & 1.92 & 3.28 \\
\hline 2005 & 12 & 9 & $19: 55: 1212.600$ & 40.471 & 2.10 & 2.05 \\
\hline 2005 & 12 & 9 & $20: 25: 1312.437$ & 40.597 & 2.35 & 2.57 \\
\hline 2005 & 12 & 9 & $21: 34: 5212.611$ & 40.490 & 1.96 & 0.62 \\
\hline 2005 & 12 & 9 & $22: 40: 2612.570$ & 40.543 & 2.76 & 1.05 \\
\hline 2005 & 12 & 9 & $23: 45: 2712.359$ & 40.640 & 2.43 & 0.63 \\
\hline 2005 & 12 & 9 & $23: 59: 3912.603$ & 40.502 & 1.93 & 2.89 \\
\hline 2005 & 12 & 10 & $00: 25: 1812.610$ & 40.528 & 1.90 & 0.53 \\
\hline 2005 & 12 & 10 & $01: 38: 2212.541$ & 40.543 & 2.45 & 1.92 \\
\hline 2005 & 12 & 10 & $04: 55: 2912.525$ & 40.512 & 2.53 & 1.87 \\
\hline 2005 & 12 & 10 & $07: 18: 4212.585$ & 40.507 & 2.77 & 1.36 \\
\hline 2005 & 12 & 10 & $16: 48: 4612.583$ & 40.489 & 3.98 & 0.63 \\
\hline 2005 & 12 & 10 & $16: 50: 5212.592$ & 40.471 & 2.93 & 1.39 \\
\hline 2005 & 12 & 10 & $17: 01: 2712.586$ & 40.479 & 2.22 & 1.3 \\
\hline 2005 & 12 & 10 & $17: 07: 2612.597$ & 40.458 & 2.77 & 1.78 \\
\hline 2005 & 12 & 10 & $18: 24: 5912.574$ & 40.500 & 1.93 & 1.43 \\
\hline 2005 & 12 & 10 & $18: 51: 1412.569$ & 40.544 & 2.24 & 1.02 \\
\hline 2005 & 12 & 10 & $20: 53: 5112.819$ & 40.084 & 2.49 & 12.74 \\
\hline 2005 & 12 & 10 & $20: 56: 4712.642$ & 40.537 & 2.02 & 0.94 \\
\hline 2005 & 12 & 10 & $21: 28: 3112.575$ & 40.518 & 2.13 & 1.02 \\
\hline 2005 & 12 & 11 & $02: 24: 4412.472$ & 40.523 & 2.24 & 1.38 \\
\hline 2005 & 12 & 11 & $08: 29: 0912.651$ & 40.576 & 2.29 & 2.93 \\
\hline 2005 & 12 & 11 & $21: 19: 4312.573$ & 40.457 & 2.09 & 0.59 \\
\hline 2005 & 12 & 11 & $22: 11: 0712.320$ & 40.669 & 2.28 & 1.6 \\
\hline 2005 & 12 & 11 & $22: 32: 0512.619$ & 40.535 & 1.88 & 0.61 \\
\hline 2005 & 12 & 11 & $22: 50: 1612.371$ & 40.319 & 2.60 & 2.22 \\
\hline 2005 & 12 & 11 & $23: 24: 2712.735$ & 40.580 & 2.20 & 0.75 \\
\hline 2005 & 12 & 12 & $01: 34: 4012.594$ & 40.492 & 2.28 & 1.26 \\
\hline 2005 & 12 & 12 & $08: 40: 2412.575$ & 40.490 & 2.89 & 1.28 \\
\hline 2005 & 12 & 12 & $16: 10: 3312.589$ & 40.484 & 3.76 & 1.08 \\
\hline 2005 & 12 & 12 & $19: 09: 2812.549$ & 40.495 & 2.44 & 0.54 \\
\hline 2005 & 12 & 12 & $22: 36: 2312.636$ & 40.527 & 1.70 & 0.6 \\
\hline 2005 & 12 & 12 & $23: 33: 3712.599$ & 40.476 & 3.90 & 0.88 \\
\hline 2005 & 12 & 12 & $23: 36: 1612.611$ & 40.465 & 2.63 & 0.94 \\
\hline 2005 & 12 & 13 & $04: 50: 3812.559$ & 40.482 & 2.51 & 1.77 \\
\hline 2005 & 12 & 13 & $19: 47: 2912.802$ & 40.606 & 2.13 & 1.15 \\
\hline 2005 & 12 & 13 & $20: 28: 1112.626$ & 40.536 & 1.84 & 0.62 \\
\hline 2005 & 12 & 13 & $21: 31: 1312.587$ & 40.474 & 2.10 & 1.91 \\
\hline 2005 & 12 & 13 & $22: 27: 5912.544$ & 40.543 & 2.15 & 0.62 \\
\hline 2005 & 12 & 13 & $22: 47: 5512.525$ & 40.533 & 1.96 & 0.84 \\
\hline 2005 & 12 & 14 & $05: 35: 2612.530$ & 40.536 & 2.80 & 2.2 \\
\hline 2005 & 12 & 14 & $13: 55: 0712.529$ & 40.532 & 2.68 & 2.44 \\
\hline 2005 & 12 & 14 & $16: 12: 1612.540$ & 40.492 & 2.56 & 0.74 \\
\hline 2005 & 12 & 14 & $18: 36: 4612.572$ & 40.500 & 2.55 & 1.13 \\
\hline 2005 & 12 & 14 & $18: 38: 4012.572$ & 40.493 & 2.75 & 1.66 \\
\hline 2005 & 12 & 14 & $20: 36: 2912.583$ & 40.496 & 2.91 & 1.04 \\
\hline 2005 & 12 & 14 & $20: 36: 5412.590$ & 40.489 & 3.40 & 0.54 \\
\hline
\end{tabular}




\begin{tabular}{|c|c|c|c|c|c|c|}
\hline 2005 & 12 & 14 & $21: 23: 1112.572$ & 40.494 & 1.93 & 2.35 \\
\hline 2005 & 12 & 15 & $00: 11: 3512.577$ & 40.498 & 2.65 & 0.56 \\
\hline 2005 & 12 & 15 & $02: 37: 0412.591$ & 40.502 & 1.97 & 1.04 \\
\hline 2005 & 12 & 15 & $09: 37: 1712.583$ & 40.456 & 2.31 & 2.87 \\
\hline 2005 & 12 & 15 & $11: 00: 0512.636$ & 40.533 & 1.99 & 1.36 \\
\hline 2005 & 12 & 15 & $18: 23: 5912.635$ & 40.527 & 2.10 & 0.99 \\
\hline 2005 & 12 & 15 & $19: 23: 4912.559$ & 40.492 & 2.20 & 0.6 \\
\hline 2005 & 12 & 15 & $19: 26: 47 \quad 12.583$ & 40.505 & 2.11 & 1.15 \\
\hline 2005 & 12 & 15 & $20: 49: 0012.566$ & 40.482 & 1.77 & 0.65 \\
\hline 2005 & 12 & 15 & $21: 34: 4812.552$ & 40.496 & 2.55 & 0.62 \\
\hline 2005 & 12 & 15 & $22: 24: 1212.617$ & 40.522 & 1.84 & 3.31 \\
\hline 2005 & 12 & 15 & $23: 52: 1311.363$ & 40.978 & 2.65 & 11.86 \\
\hline 2005 & 12 & 16 & $00: 45: 3512.620$ & 40.538 & 2.34 & 0.96 \\
\hline 2005 & 12 & 16 & $00: 51: 4412.585$ & 40.507 & 1.96 & 1.56 \\
\hline 2005 & 12 & 16 & $00: 53: 0812.552$ & 40.499 & 2.26 & 0.64 \\
\hline 2005 & 12 & 16 & $02: 01: 2312.589$ & 40.509 & 2.09 & 0.61 \\
\hline 2005 & 12 & 16 & $05: 26: 0012.593$ & 40.500 & 2.35 & 1.12 \\
\hline 2005 & 12 & 16 & $08: 03: 3412.601$ & 40.466 & 2.58 & 1.65 \\
\hline 2005 & 12 & 16 & $09: 40: 3412.996$ & 40.658 & 2.90 & 1.16 \\
\hline 2005 & 12 & 16 & $11: 02: 2012.568$ & 40.518 & 2.69 & 1.09 \\
\hline 2005 & 12 & 16 & $17: 47: 3212.620$ & 40.524 & 1.95 & 0.67 \\
\hline 2005 & 12 & 16 & $21: 32: 4112.595$ & 40.501 & 1.94 & 1.49 \\
\hline 2005 & 12 & 16 & $21: 42: 1112.581$ & 40.504 & 2.67 & 0.7 \\
\hline 2005 & 12 & 16 & $21: 47: 1812.664$ & 40.519 & 1.98 & 0.85 \\
\hline 2005 & 12 & 16 & $22: 45: 0812.597$ & 40.533 & 1.86 & 0.69 \\
\hline 2005 & 12 & 17 & $00: 40: 2912.593$ & 40.466 & 4.14 & 0.81 \\
\hline 2005 & 12 & 17 & $00: 44: 0912.579$ & 40.477 & 3.39 & 0.99 \\
\hline 2005 & 12 & 17 & $08: 08: 5112.584$ & 40.502 & 2.83 & 0.86 \\
\hline 2005 & 12 & 17 & $17: 54: 5412.505$ & 40.625 & 2.06 & 2,00 \\
\hline 2005 & 12 & 17 & $18: 09: 2012.620$ & 40.545 & 2.39 & 1.36 \\
\hline 2005 & 12 & 17 & $19: 09: 5912.591$ & 40.484 & 2.21 & 0.79 \\
\hline 2005 & 12 & 17 & $19: 11: 4912.328$ & 40.616 & 2.74 & 1.05 \\
\hline 2005 & 12 & 17 & $20: 17: 2112.611$ & 40.478 & 2.14 & 1.2 \\
\hline 2005 & 12 & 17 & $20: 48: 5912.548$ & 40.500 & 3.03 & 0.58 \\
\hline 2005 & 12 & 17 & $22: 07: 4112.545$ & 40.543 & 1.90 & 1.41 \\
\hline 2005 & 12 & 17 & $22: 12: 0012.532$ & 40.557 & 2.28 & 1.06 \\
\hline 2005 & 12 & 17 & $22: 50: 1412.639$ & 40.526 & 2.04 & 0.95 \\
\hline 2005 & 12 & 18 & $00: 19: 4412.854$ & 40.692 & 1.80 & 1,00 \\
\hline 2005 & 12 & 18 & $00: 21: 2912.582$ & 40.483 & 1.86 & 0.82 \\
\hline 2005 & 12 & 18 & $00: 26: 1312.850$ & 40.269 & 2.08 & 1.21 \\
\hline 2005 & 12 & 18 & $01: 59: 2812.446$ & 40.610 & 2.20 & 2.25 \\
\hline 2005 & 12 & 18 & $06: 08: 4212.532$ & 40.569 & 2.37 & 1.36 \\
\hline 2005 & 12 & 18 & $06: 23: 3912.578$ & 40.499 & 2.58 & 1.66 \\
\hline 2005 & 12 & 18 & $06: 24: 2012.667$ & 40.438 & 2.63 & 3.55 \\
\hline 2005 & 12 & 18 & $11: 24: 1912.328$ & 40.636 & 2.73 & 1.43 \\
\hline 2005 & 12 & 18 & $16: 22: 4912.575$ & 40.478 & 2.86 & 1.26 \\
\hline 2005 & 12 & 18 & $17: 21: 4612.601$ & 40.483 & 2.41 & 0.61 \\
\hline 2005 & 12 & 18 & $17: 52: 3412.576$ & 40.504 & 2.08 & 1.47 \\
\hline 2005 & 12 & 18 & $17: 55: 2812.582$ & 40.496 & 2.08 & 2.02 \\
\hline 2005 & 12 & 18 & $21: 15: 2312.538$ & 40.502 & 2.58 & 0.74 \\
\hline 2005 & 12 & 18 & $22: 50: 0812.555$ & 40.477 & 2.35 & 0.54 \\
\hline 2005 & 12 & 18 & $22: 55: 2012.550$ & 40.498 & 2.10 & 0.54 \\
\hline 2005 & 12 & 18 & $23: 27: 3512.598$ & 40.478 & 4.05 & 0.77 \\
\hline 2005 & 12 & 19 & $01: 41: 2712.565$ & 40.523 & 2.09 & 1.25 \\
\hline 2005 & 12 & 19 & $03: 15: 2512.596$ & 40.505 & 2.31 & 1.04 \\
\hline 2005 & 12 & 19 & $19: 33: 2112.587$ & 40.465 & 2.20 & 2.07 \\
\hline 2005 & 12 & 19 & $20: 22: 1812.500$ & 40.648 & 1.85 & 0.97 \\
\hline 2005 & 12 & 19 & $21: 12: 4912.468$ & 40.573 & 1.73 & 1.41 \\
\hline 2005 & 12 & 19 & $22: 08: 5812.579$ & 40.480 & 1.88 & 1.24 \\
\hline 2005 & 12 & 19 & $23: 24: 0012.579$ & 40.445 & 1.99 & 1.13 \\
\hline 2005 & 12 & 20 & $00: 38: 2012.513$ & 40.547 & 1.62 & 1.36 \\
\hline 2005 & 12 & 20 & $00: 54: 4912.579$ & 40.455 & 2.26 & 0.68 \\
\hline 2005 & 12 & 20 & $05: 11: 0112.600$ & 40.469 & 3.42 & 1.25 \\
\hline 2005 & 12 & 20 & $05: 28: 2712.578$ & 40.499 & 3.07 & 1.18 \\
\hline 2005 & 12 & 21 & $00: 10: 5712.565$ & 40.471 & 1.72 & 2.04 \\
\hline 2005 & 12 & 21 & $19: 00: 0812.625$ & 40.523 & 1.95 & 1.03 \\
\hline 2005 & 12 & 21 & $22: 07: 2212.633$ & 40.534 & 2.46 & 0.67 \\
\hline 2005 & 12 & 22 & $00: 20: 0812.583$ & 40.485 & 2.41 & 0.98 \\
\hline 2005 & 12 & 22 & $01: 47: 3512.448$ & 40.576 & 2.09 & 1.09 \\
\hline 2005 & 12 & 22 & $03: 10: 5012.580$ & 40.483 & 3.10 & 0.88 \\
\hline 2005 & 12 & 22 & $03: 14: 2912.577$ & 40.495 & 4.04 & 0.94 \\
\hline 2005 & 12 & 22 & $15: 43: 2512.627$ & 40.538 & 2.27 & 0.69 \\
\hline 2005 & 12 & 22 & $18: 35: 2912.558$ & 40.495 & 2.35 & 0.8 \\
\hline 2005 & 12 & 22 & $22: 33: 3112.552$ & 40.480 & 2.38 & 0.56 \\
\hline 2005 & 12 & 22 & $23: 00: 0812.546$ & 40.494 & 1.80 & 0.47 \\
\hline 2005 & 12 & 23 & $04: 25: 4212.573$ & 40.540 & 2.89 & 0.68 \\
\hline
\end{tabular}




\begin{tabular}{|c|c|c|c|c|c|c|}
\hline 2005 & 12 & 23 & $10: 59: 1512.543$ & 40.504 & 2.98 & 0.65 \\
\hline 2005 & 12 & 23 & $14: 10: 4512.550$ & 40.492 & 2.52 & 0.53 \\
\hline 2005 & 12 & 23 & $16: 22: 5512.548$ & 40.487 & 2.62 & 0.69 \\
\hline 2005 & 12 & 23 & $21: 19: 1612.603$ & 40.457 & 1.85 & 1.99 \\
\hline 2005 & 12 & 23 & $22: 46: 2612.584$ & 40.483 & 3.17 & 0.84 \\
\hline 2005 & 12 & 23 & $23: 57: 1112.588$ & 40.502 & 1.90 & 0.99 \\
\hline 2005 & 12 & 24 & $02: 32: 3512.587$ & 40.495 & 2.12 & 1.48 \\
\hline 2005 & 12 & 24 & $05: 39: 0212.571$ & 40.514 & 3.20 & 0.88 \\
\hline 2005 & 12 & 24 & $17: 38: 3912.574$ & 40.502 & 2.29 & 2.15 \\
\hline 2005 & 12 & 24 & $19: 01: 1312.575$ & 40.489 & 2.01 & 1.89 \\
\hline 2005 & 12 & 24 & $20: 35: 1812.595$ & 40.505 & 2.46 & 0.92 \\
\hline 2005 & 12 & 24 & $22: 29: 0612.488$ & 40.558 & 1.84 & 1.18 \\
\hline 2005 & 12 & 24 & $23: 00: 4412.594$ & 40.529 & 2.18 & 1.35 \\
\hline 2005 & 12 & 24 & $23: 11: 0912.611$ & 40.473 & 2.14 & 1.11 \\
\hline 2005 & 12 & 25 & $00: 53: 5912.600$ & 40.507 & 2.48 & 0.54 \\
\hline 2005 & 12 & 25 & $02: 54: 4912.451$ & 40.583 & 2.04 & 0.89 \\
\hline 2005 & 12 & 25 & $02: 55: 5912.708$ & 40.459 & 2.30 & 3.51 \\
\hline 2005 & 12 & 25 & $09: 28: 2712.555$ & 40.465 & 2.36 & 3.48 \\
\hline 2005 & 12 & 25 & $17: 23: 0012.595$ & 40.505 & 2.35 & 0.67 \\
\hline 2005 & 12 & 25 & $18: 21: 3212.590$ & 40.465 & 2.27 & 1.46 \\
\hline 2005 & 12 & 25 & $18: 31: 1512.579$ & 40.492 & 2.91 & 0.89 \\
\hline 2005 & 12 & 25 & $19: 51: 3512.543$ & 40.546 & 1.97 & 0.73 \\
\hline 2005 & 12 & 25 & $20: 00: 2912.318$ & 40.631 & 1.79 & 1.3 \\
\hline 2005 & 12 & 25 & $21: 09: 1512.447$ & 40.568 & 1.57 & 0.86 \\
\hline 2005 & 12 & 25 & $21: 12: 2112.554$ & 40.538 & 2.15 & 1.8 \\
\hline 2005 & 12 & 25 & $22: 53: 0012.504$ & 40.524 & 2.07 & 0.84 \\
\hline 2005 & 12 & 25 & $23: 22: 1712.305$ & 40.577 & 1.73 & 0.84 \\
\hline 2005 & 12 & 26 & $01: 43: 3612.614$ & 40.525 & 2.01 & 0.62 \\
\hline 2005 & 12 & 26 & $02: 57: 4312.591$ & 40.462 & 2.69 & 1.26 \\
\hline 2005 & 12 & 26 & $02: 59: 2312.577$ & 40.474 & 2.22 & 1.42 \\
\hline 2005 & 12 & 26 & $11: 33: 1912.585$ & 40.490 & 3.52 & 1.06 \\
\hline 2005 & 12 & 26 & $11: 34: 0812.599$ & 40.495 & 3.62 & 2.97 \\
\hline 2005 & 12 & 26 & $11: 35: 4512.607$ & 40.472 & 3.75 & 1.01 \\
\hline 2005 & 12 & 26 & $18: 38: 1712.553$ & 40.495 & 2.34 & 0.59 \\
\hline 2005 & 12 & 26 & $20: 10: 2612.611$ & 40.483 & 2.21 & 2.18 \\
\hline 2005 & 12 & 26 & $21: 21: 1112.511$ & 40.675 & 2.75 & 9.24 \\
\hline 2005 & 12 & 26 & $23: 00: 5812.628$ & 40.539 & 1.50 & 1.71 \\
\hline 2005 & 12 & 27 & $01: 19: 4112.585$ & 40.499 & 1.83 & 1.68 \\
\hline 2005 & 12 & 27 & $01: 30: 4412.596$ & 40.469 & 2.08 & 1.45 \\
\hline 2005 & 12 & 27 & $01: 41: 3012.605$ & 40.533 & 1.83 & 0.66 \\
\hline 2005 & 12 & 27 & $06: 44: 5712.613$ & 40.474 & 2.38 & 1.3 \\
\hline 2005 & 12 & 27 & $08: 17: 1912.596$ & 40.506 & 2.82 & 1.43 \\
\hline 2005 & 12 & 27 & $10: 16: 1112.563$ & 40.476 & 2.09 & 1.4 \\
\hline 2005 & 12 & 27 & $10: 49: 1212.610$ & 40.524 & 2.38 & 1.05 \\
\hline 2005 & 12 & 27 & $17: 10: 4512.553$ & 40.483 & 2.15 & 0.88 \\
\hline 2005 & 12 & 27 & $17: 43: 1012.543$ & 40.491 & 2.15 & 0.77 \\
\hline 2005 & 12 & 27 & $20: 16: 1012.596$ & 40.468 & 1.70 & 1.61 \\
\hline 2005 & 12 & 27 & $20: 18: 1812.595$ & 40.450 & 1.74 & 1.38 \\
\hline 2005 & 12 & 27 & $22: 10: 1411.275$ & 41.015 & 3.78 & 3.54 \\
\hline 2005 & 12 & 27 & $23: 15: 0112.574$ & 40.492 & 1.80 & 3.92 \\
\hline 2005 & 12 & 27 & $23: 31: 4012.609$ & 40.478 & 1.89 & 2.26 \\
\hline 2005 & 12 & 28 & $00: 36: 5512.595$ & 40.502 & 1.65 & 2.65 \\
\hline 2005 & 12 & 28 & $00: 55: 5512.598$ & 40.515 & 2.08 & 1.52 \\
\hline 2005 & 12 & 28 & $00: 57: 3512.621$ & 40.521 & 1.70 & 2.27 \\
\hline 2005 & 12 & 28 & $02: 54: 4112.567$ & 40.532 & 2.17 & 2.76 \\
\hline 2005 & 12 & 28 & $14: 28: 1212.586$ & 40.467 & 2.83 & 1.77 \\
\hline 2005 & 12 & 28 & $14: 29: 4612.613$ & 40.455 & 2.76 & 3.02 \\
\hline 2005 & 12 & 28 & $18: 08: 0312.551$ & 40.490 & 2.39 & 0.44 \\
\hline 2005 & 12 & 28 & $20: 13: 0512.590$ & 40.488 & 2.15 & 1.73 \\
\hline 2005 & 12 & 28 & $21: 00: 3812.544$ & 40.522 & 1.40 & 3.25 \\
\hline 2005 & 12 & 28 & $21: 01: 3012.505$ & 40.537 & 1.40 & 2.59 \\
\hline 2005 & 12 & 28 & $21: 06: 2412.649$ & 40.495 & 1.73 & 2.19 \\
\hline 2005 & 12 & 28 & $22: 18: 5412.583$ & 40.498 & 2.51 & 1.02 \\
\hline 2005 & 12 & 28 & $22: 19: 3112.595$ & 40.500 & 2.55 & 0.81 \\
\hline 2005 & 12 & 28 & $22: 23: 3412.625$ & 40.465 & 2.62 & 1.62 \\
\hline 2005 & 12 & 28 & $22: 24: 4312.589$ & 40.499 & 2.72 & 1.39 \\
\hline 2005 & 12 & 28 & $22: 27: 1112.577$ & 40.495 & 3.11 & 1.96 \\
\hline 2005 & 12 & 28 & $22: 36: 5112.585$ & 40.485 & 3.20 & 0.96 \\
\hline 2005 & 12 & 28 & $22: 39: 3212.603$ & 40.476 & 2.46 & 2.12 \\
\hline 2005 & 12 & 28 & $22: 40: 0712.675$ & 40.432 & 2.54 & 2.97 \\
\hline 2005 & 12 & 28 & $22: 41: 1412.578$ & 40.486 & 2.56 & 1.84 \\
\hline 2005 & 12 & 28 & $22: 49: 0012.599$ & 40.477 & 2.03 & 1.88 \\
\hline 2005 & 12 & 29 & $00: 47: 3412.567$ & 40.516 & 1.43 & 3.71 \\
\hline 2005 & 12 & 29 & $02: 08: 2112.590$ & 40.506 & 1.82 & 2.51 \\
\hline 2005 & 12 & 29 & $04: 52: 5512.617$ & 40.457 & 2.62 & 2.34 \\
\hline 2005 & 12 & 29 & $05: 44: 5612.586$ & 40.505 & 2.43 & 2.65 \\
\hline
\end{tabular}




\begin{tabular}{|c|c|c|c|c|c|c|}
\hline 2005 & 12 & 29 & $11: 53: 1812.699$ & 40.401 & 2.20 & 5.09 \\
\hline 2005 & 12 & 29 & $21: 58: 0012.577$ & 40.448 & 1.81 & 1.31 \\
\hline 2005 & 12 & 29 & $22: 15: 1312.609$ & 40.469 & 3.83 & 0.87 \\
\hline 2005 & 12 & 29 & $22: 21: 3112.583$ & 40.483 & 2.99 & 1.32 \\
\hline 2005 & 12 & 29 & $22: 41: 3312.595$ & 40.487 & 3.67 & 0.77 \\
\hline 2005 & 12 & 29 & $23: 25: 3512.554$ & 40.496 & 2.37 & 1.26 \\
\hline 2005 & 12 & 29 & $23: 49: 2112.574$ & 40.466 & 1.90 & 0.83 \\
\hline 2005 & 12 & 30 & $00: 48: 2712.584$ & 40.489 & 2.07 & 1.28 \\
\hline 2005 & 12 & 30 & $00: 56: 0812.326$ & 40.595 & 2.08 & 1.03 \\
\hline 2005 & 12 & 30 & $01: 23: 4512.588$ & 40.508 & 2.24 & 0.79 \\
\hline 2005 & 12 & 30 & $02: 22: 2711.880$ & 41.066 & 3.89 & 5,00 \\
\hline 2005 & 12 & 30 & $03: 18: 1712.579$ & 40.474 & 2.24 & 1.9 \\
\hline 2005 & 12 & 30 & $08: 23: 0612.581$ & 40.515 & 3.00 & 1.43 \\
\hline 2005 & 12 & 30 & $09: 41: 1511.845$ & 41.126 & 3.72 & 8.44 \\
\hline 2005 & 12 & 30 & $16: 54: 5312.480$ & 40.545 & 1.73 & 1.31 \\
\hline 2005 & 12 & 30 & $17: 24: 5812.524$ & 40.530 & 1.95 & 2.76 \\
\hline 2005 & 12 & 30 & $18: 24: 2412.548$ & 40.528 & 2.15 & 4.13 \\
\hline 2005 & 12 & 30 & $20: 05: 1012.597$ & 40.511 & 1.79 & 3.14 \\
\hline 2005 & 12 & 30 & $22: 04: 0112.409$ & 40.586 & 2.81 & 0.72 \\
\hline 2005 & 12 & 31 & $00: 42: 3712.570$ & 40.503 & 2.97 & 0.69 \\
\hline 2005 & 12 & 31 & $04: 04: 4612.619$ & 40.522 & 2.21 & 1.54 \\
\hline 2005 & 12 & 31 & $06: 20: 1512.431$ & 40.585 & 3.01 & 0.96 \\
\hline 2005 & 12 & 31 & $06: 53: 5312.597$ & 40.504 & 2.92 & 2.87 \\
\hline 2005 & 12 & 31 & $07: 07: 5412.562$ & 40.474 & 2.59 & 2.67 \\
\hline 2005 & 12 & 31 & $08: 23: 5912.476$ & 40.547 & 1.70 & 1.28 \\
\hline 2005 & 12 & 31 & $18: 56: 1812.782$ & 40.355 & 1.59 & 1.78 \\
\hline 2005 & 12 & 31 & $19: 33: 0512.596$ & 40.477 & 2.03 & 3,00 \\
\hline 2005 & 12 & 31 & $20: 57: 5912.623$ & 40.463 & 1.82 & 2.08 \\
\hline 2006 & 1 & 1 & $00: 08: 5112.588$ & 40.448 & 1.79 & 0.92 \\
\hline 2006 & 1 & 1 & $02: 50: 2812.708$ & 40.425 & 2.14 & 4.12 \\
\hline 2006 & 1 & 1 & $02: 51: 0612.562$ & 40.512 & 2.03 & 1.37 \\
\hline 2006 & 1 & 1 & $08: 00: 5712.569$ & 40.489 & 3.93 & 1.21 \\
\hline 2006 & 1 & 1 & $09: 27: 1112.514$ & 40.513 & 2.52 & 1.3 \\
\hline 2006 & 1 & 1 & $19: 04: 2412.492$ & 40.647 & 2.32 & 1.04 \\
\hline 2006 & 1 & 1 & $20: 26: 2612.648$ & 40.565 & 1.65 & 15.75 \\
\hline 2006 & 1 & 1 & $20: 39: 0012.592$ & 40.429 & 1.48 & 6.04 \\
\hline 2006 & 1 & 1 & $21: 13: 2712.683$ & 40.513 & 1.50 & 1.47 \\
\hline 2006 & 1 & 1 & $21: 35: 4812.589$ & 40.508 & 1.67 & 3.86 \\
\hline 2006 & 1 & 1 & $22: 00: 1012.563$ & 40.532 & 1.48 & 1.21 \\
\hline 2006 & 1 & 2 & $19: 30: 1012.621$ & 40.476 & 1.74 & 3.27 \\
\hline 2006 & 1 & 2 & $19: 46: 0812.547$ & 40.494 & 2.45 & 0.67 \\
\hline 2006 & 1 & 3 & $15: 18: 1912.572$ & 40.466 & 2.56 & 1.19 \\
\hline 2006 & 1 & 3 & $19: 09: 2712.336$ & 40.606 & 2.18 & 1.41 \\
\hline 2006 & 1 & 3 & $19: 44: 1312.569$ & 40.492 & 1.96 & 2.95 \\
\hline 2006 & 1 & 3 & $21: 35: 5712.587$ & 40.464 & 1.75 & 0.96 \\
\hline 2006 & 1 & 4 & $00: 49: 2812.608$ & 40.530 & 1.81 & 1.05 \\
\hline 2006 & 1 & 4 & $14: 30: 0712.587$ & 40.486 & 2.83 & 1.54 \\
\hline 2006 & 1 & 4 & $17: 40: 2212.522$ & 40.574 & 1.96 & 2.83 \\
\hline 2006 & 1 & 4 & $20: 35: 3312.589$ & 40.497 & 1.95 & 1.45 \\
\hline 2006 & 1 & 4 & $20: 53: 4212.581$ & 40.472 & 4.02 & 0.98 \\
\hline 2006 & 1 & 4 & $20: 57: 2412.600$ & 40.518 & 2.70 & 1.35 \\
\hline 2006 & 1 & 4 & $21: 10: 5112.597$ & 40.489 & 2.31 & 1.02 \\
\hline 2006 & 1 & 5 & $00: 19: 1712.559$ & 40.520 & 1.85 & 4.02 \\
\hline 2006 & 1 & 5 & $01: 05: 0112.626$ & 40.535 & 1.98 & 1.1 \\
\hline 2006 & 1 & 5 & $01: 24: 0212.616$ & 40.533 & 1.84 & 1.33 \\
\hline 2006 & 1 & 5 & $03: 23: 4112.608$ & 40.542 & 2.32 & 1.8 \\
\hline 2006 & 1 & 5 & $05: 53: 5112.582$ & 40.489 & 2.61 & 1.82 \\
\hline 2006 & 1 & 5 & $16: 56: 1912.577$ & 40.507 & 2.60 & 1.47 \\
\hline 2006 & 1 & 5 & $17: 07: 1612.599$ & 40.492 & 2.48 & 2.34 \\
\hline 2006 & 1 & 5 & $18: 12: 2912.621$ & 40.502 & 2.17 & 1.68 \\
\hline 2006 & 1 & 5 & $18: 31: 4512.579$ & 40.452 & 1.81 & 1.23 \\
\hline 2006 & 1 & 6 & $06: 01: 2512.566$ & 40.461 & 2.44 & 1.5 \\
\hline 2006 & 1 & 6 & $13: 27: 3112.562$ & 40.461 & 2.94 & 1.02 \\
\hline 2006 & 1 & 6 & $15: 58: 2513.344$ & 40.859 & 3.09 & 2.48 \\
\hline 2006 & 1 & 6 & $17: 14: 0812.535$ & 40.495 & 2.56 & 2.84 \\
\hline 2006 & 1 & 6 & $18: 38: 3812.575$ & 40.514 & 2.54 & 0.75 \\
\hline 2006 & 1 & 6 & $20: 21: 1212.575$ & 40.473 & 2.64 & 1.6 \\
\hline 2006 & 1 & 6 & $20: 21: 4512.575$ & 40.482 & 2.71 & 1.55 \\
\hline 2006 & 1 & 6 & $23: 16: 5112.621$ & 40.536 & 1.87 & 0.66 \\
\hline 2006 & 1 & 7 & $01: 28: 3312.604$ & 40.522 & 1.63 & 1.6 \\
\hline 2006 & 1 & 7 & $08: 24: 2013.359$ & 40.885 & 3.41 & 4.61 \\
\hline 2006 & 1 & 7 & $08: 29: 2513.363$ & 40.893 & 3.32 & 3.83 \\
\hline 2006 & 1 & 7 & $08: 32: 3013.368$ & 40.901 & 3.50 & 3.17 \\
\hline 2006 & 1 & 7 & $09: 55: 2513.367$ & 40.898 & 3.46 & 2.61 \\
\hline 2006 & 1 & 7 & $10: 00: 5813.359$ & 40.894 & 3.13 & 3.18 \\
\hline 2006 & 1 & 7 & $18: 26: 5712.612$ & 40.538 & 1.90 & 1.35 \\
\hline
\end{tabular}




\begin{tabular}{|c|c|c|c|c|c|c|}
\hline 2006 & 1 & 7 & $20: 33: 1912.543$ & 40.486 & 2.68 & 0.67 \\
\hline 2006 & 1 & 7 & $21: 11: 3412.727$ & 40.502 & 1.90 & 11.74 \\
\hline 2006 & 1 & 7 & $23: 41: 0813.324$ & 39.893 & 2.40 & 6.51 \\
\hline 2006 & 1 & 8 & $00: 31: 2812.543$ & 40.498 & 2.18 & 0.58 \\
\hline 2006 & 1 & 8 & $01: 59: 1212.568$ & 40.478 & 1.98 & 1.37 \\
\hline 2006 & 1 & 8 & $19: 40: 0612.580$ & 40.516 & 2.05 & 1.39 \\
\hline 2006 & 1 & 8 & $22: 18: 5212.569$ & 40.459 & 1.78 & 0.81 \\
\hline 2006 & 1 & 8 & $23: 43: 0412.550$ & 40.491 & 2.47 & 0.58 \\
\hline 2006 & 1 & 9 & $08: 48: 4212.562$ & 40.486 & 3.04 & 4.27 \\
\hline 2006 & 1 & 9 & $15: 46: 4612.496$ & 40.550 & 2.27 & 0.86 \\
\hline 2006 & 1 & 9 & $20: 06: 0512.573$ & 40.460 & 1.57 & 1.71 \\
\hline 2006 & 1 & 9 & $21: 51: 0612.614$ & 40.522 & 1.41 & 1.16 \\
\hline 2006 & 1 & 9 & $21: 52: 1312.607$ & 40.526 & 1.40 & 1.27 \\
\hline 2006 & 1 & 9 & $22: 17: 1312.560$ & 40.526 & 2.03 & 0.82 \\
\hline 2006 & 1 & 9 & $23: 54: 5312.337$ & 40.630 & 1.65 & 0.83 \\
\hline 2006 & 1 & 10 & $01: 34: 1712.631$ & 40.531 & 2.70 & 0.67 \\
\hline 2006 & 1 & 10 & $05: 12: 3412.508$ & 40.533 & 2.04 & 2.27 \\
\hline 2006 & 1 & 10 & $05: 28: 4312.529$ & 40.511 & 2.26 & 2.44 \\
\hline 2006 & 1 & 10 & $10: 14: 4412.598$ & 40.510 & 2.57 & 1.65 \\
\hline 2006 & 1 & 10 & $19: 19: 5512.600$ & 40.535 & 1.73 & 0.64 \\
\hline 2006 & 1 & 10 & $23: 53: 2612.574$ & 40.522 & 2.65 & 0.77 \\
\hline 2006 & 1 & 11 & $06: 28: 5212.570$ & 40.451 & 2.51 & 0.81 \\
\hline 2006 & 1 & 11 & $18: 33: 1312.569$ & 40.518 & 1.76 & 0.63 \\
\hline 2006 & 1 & 11 & $18: 54: 0512.599$ & 40.530 & 1.78 & 1.31 \\
\hline 2006 & 1 & 11 & $20: 22: 3212.551$ & 40.508 & 2.23 & 3.76 \\
\hline 2006 & 1 & 11 & $20: 24: 0412.529$ & 40.531 & 1.80 & 2.31 \\
\hline 2006 & 1 & 11 & $20: 31: 3612.619$ & 40.534 & 2.20 & 0.87 \\
\hline 2006 & 1 & 11 & $20: 53: 2212.602$ & 40.540 & 1.59 & 1.98 \\
\hline 2006 & 1 & 11 & $21: 39: 0312.580$ & 40.477 & 2.15 & 1.72 \\
\hline 2006 & 1 & 11 & $21: 40: 2412.614$ & 40.469 & 2.75 & 1.88 \\
\hline 2006 & 1 & 11 & $21: 42: 4012.584$ & 40.476 & 2.17 & 1.38 \\
\hline 2006 & 1 & 11 & 22:06:09 12.541 & 40.538 & 2.03 & 1.73 \\
\hline 2006 & 1 & 12 & $03: 31: 4512.542$ & 40.487 & 2.77 & 0.97 \\
\hline 2006 & 1 & 12 & $09: 21: 4812.510$ & 40.541 & 2.40 & 0.88 \\
\hline 2006 & 1 & 12 & $12: 35: 4812.577$ & 40.465 & 2.21 & 1.1 \\
\hline 2006 & 1 & 12 & $13: 20: 2612.603$ & 40.535 & 2.29 & 2.02 \\
\hline 2006 & 1 & 12 & $19: 31: 3512.548$ & 40.484 & 2.35 & 0.6 \\
\hline 2006 & 1 & 12 & $20: 46: 4912.305$ & 40.615 & 2.10 & 2.94 \\
\hline 2006 & 1 & 12 & $20: 51: 0712.322$ & 40.616 & 1.90 & 0.91 \\
\hline 2006 & 1 & 12 & $23: 57: 1612.551$ & 40.495 & 2.09 & 0.6 \\
\hline 2006 & 1 & 13 & $07: 17: 0312.584$ & 40.489 & 3.23 & 1.06 \\
\hline 2006 & 1 & 13 & $17: 22: 3812.569$ & 40.524 & 2.38 & 1.97 \\
\hline 2006 & 1 & 13 & $17: 50: 4512.445$ & 40.602 & 1.90 & 0.87 \\
\hline 2006 & 1 & 13 & $22: 17: 3812.602$ & 40.533 & 1.88 & 1.64 \\
\hline 2006 & 1 & 13 & $22: 51: 4812.573$ & 40.512 & 2.46 & 1.21 \\
\hline 2006 & 1 & 13 & $23: 19: 4712.570$ & 40.513 & 2.18 & 1.05 \\
\hline 2006 & 1 & 14 & $00: 06: 4212.657$ & 40.474 & 1.90 & 3.14 \\
\hline 2006 & 1 & 14 & $00: 51: 2512.577$ & 40.506 & 1.73 & 1.15 \\
\hline 2006 & 1 & 14 & $03: 30: 3212.558$ & 40.521 & 2.98 & 1.86 \\
\hline 2006 & 1 & 14 & $17: 35: 1212.546$ & 40.548 & 2.12 & 4.11 \\
\hline 2006 & 1 & 14 & $21: 08: 0912.602$ & 40.531 & 1.65 & 0.64 \\
\hline 2006 & 1 & 15 & $01: 02: 3212.573$ & 40.510 & 1.92 & 1.21 \\
\hline 2006 & 1 & 15 & $06: 31: 5312.556$ & 40.486 & 2.47 & 1.33 \\
\hline 2006 & 1 & 15 & $07: 26: 4412.613$ & 40.466 & 2.85 & 1.66 \\
\hline 2006 & 1 & 15 & $07: 41: 1912.567$ & 40.484 & 2.95 & 1.24 \\
\hline 2006 & 1 & 15 & $10: 48: 2412.284$ & 40.618 & 1.92 & 1.04 \\
\hline 2006 & 1 & 15 & $14: 04: 0612.609$ & 40.532 & 2.38 & 1.13 \\
\hline 2006 & 1 & 15 & $22: 13: 0312.561$ & 40.484 & 1.94 & 4.82 \\
\hline 2006 & 1 & 15 & $22: 15: 5613.348$ & 40.875 & 2.14 & 3.67 \\
\hline 2006 & 1 & 16 & $00: 44: 0012.548$ & 40.502 & 1.66 & 1.97 \\
\hline 2006 & 1 & 16 & $00: 46: 2312.572$ & 40.467 & 1.70 & 0.74 \\
\hline 2006 & 1 & 16 & $00: 48: 1612.566$ & 40.463 & 1.65 & 0.97 \\
\hline 2006 & 1 & 16 & $02: 14: 0312.597$ & 40.517 & 3.02 & 0.77 \\
\hline 2006 & 1 & 16 & $19: 33: 3613.159$ & 40.783 & 2.55 & 2.58 \\
\hline 2006 & 1 & 16 & $21: 02: 2012.646$ & 40.544 & 1.92 & 0.72 \\
\hline 2006 & 1 & 16 & $22: 43: 0512.531$ & 40.550 & 2.29 & 0.88 \\
\hline 2006 & 1 & 16 & $23: 11: 3412.628$ & 40.542 & 1.84 & 0.85 \\
\hline 2006 & 1 & 16 & $23: 22: 0812.597$ & 40.521 & 2.63 & 0.85 \\
\hline 2006 & 1 & 17 & $00: 37: 2212.415$ & 40.681 & 2.15 & 1.79 \\
\hline 2006 & 1 & 17 & $00: 49: 2512.444$ & 40.592 & 2.16 & 0.87 \\
\hline 2006 & 1 & 17 & $01: 46: 4313.399$ & 40.942 & 3.50 & 1.43 \\
\hline 2006 & 1 & 17 & $09: 48: 3713.359$ & 40.915 & 3.47 & 1.25 \\
\hline 2006 & 1 & 17 & $16: 16: 1413.395$ & 40.893 & 3.07 & 1.69 \\
\hline 2006 & 1 & 17 & $19: 59: 0211.596$ & 41.013 & 3.75 & 12.28 \\
\hline 2006 & 1 & 17 & $20: 27: 5112.538$ & 40.510 & 2.69 & 0.72 \\
\hline 2006 & 1 & 17 & $22: 31: 2112.557$ & 40.547 & 2.28 & 1.11 \\
\hline
\end{tabular}




\begin{tabular}{|c|c|c|c|c|c|c|}
\hline 2006 & 1 & 18 & $13: 08: 0712.719$ & 40.435 & 2.24 & 4.52 \\
\hline 2006 & 1 & 18 & $16: 48: 3512.432$ & 40.797 & 2.70 & 4.7 \\
\hline 2006 & 1 & 18 & $19: 04: 2012.571$ & 40.508 & 2.02 & 2.4 \\
\hline 2006 & 1 & 18 & $21: 27: 0112.585$ & 40.529 & 2.03 & 0.86 \\
\hline 2006 & 1 & 18 & $21: 28: 4112.423$ & 40.594 & 1.85 & 1.63 \\
\hline 2006 & 1 & 18 & $23: 24: 0012.581$ & 40.518 & 1.86 & 1.58 \\
\hline 2006 & 1 & 19 & $00: 19: 4913.379$ & 40.931 & 3.18 & 2.72 \\
\hline 2006 & 1 & 19 & $01: 16: 4312.533$ & 40.532 & 1.75 & 0.58 \\
\hline 2006 & 1 & 19 & $08: 12: 1612.538$ & 40.502 & 3.03 & 0.63 \\
\hline 2006 & 1 & 19 & $14: 47: 4412.353$ & 40.605 & 2.14 & 1.3 \\
\hline 2006 & 1 & 19 & $19: 58: 5112.557$ & 40.494 & 2.20 & 0.79 \\
\hline 2006 & 1 & 19 & $20: 47: 1212.677$ & 40.582 & 1.51 & 1.21 \\
\hline 2006 & 1 & 19 & $21: 59: 4712.540$ & 40.538 & 1.84 & 0.71 \\
\hline 2006 & 1 & 19 & $22: 07: 2012.648$ & 40.538 & 1.67 & 0.63 \\
\hline 2006 & 1 & 19 & $23: 21: 4412.555$ & 40.520 & 1.56 & 0.65 \\
\hline 2006 & 1 & 20 & $00: 26: 4912.569$ & 40.518 & 1.89 & 0.54 \\
\hline 2006 & 1 & 20 & $00: 33: 5212.564$ & 40.481 & 1.76 & 2.24 \\
\hline 2006 & 1 & 20 & $00: 52: 4912.649$ & 40.525 & 0.83 & 0.75 \\
\hline 2006 & 1 & 20 & $02: 45: 5412.589$ & 40.474 & 2.34 & 1.3 \\
\hline 2006 & 1 & 20 & $08: 34: 3711.543$ & 40.987 & 2.71 & 2.37 \\
\hline 2006 & $\overline{1}$ & 20 & $11: 07: 2612.585$ & 40.481 & 3.02 & 1.39 \\
\hline 2006 & 1 & 20 & $11: 14: 5912.558$ & 40.492 & 2.81 & 0.96 \\
\hline 2006 & 1 & 20 & $11: 20: 2812.565$ & 40.501 & 2.78 & 1.21 \\
\hline 2006 & 1 & 20 & $16: 54: 1212.463$ & 40.640 & 2.26 & 3.21 \\
\hline 2006 & 1 & 20 & $17: 34: 5712.574$ & 40.529 & 2.45 & 0.85 \\
\hline 2006 & 1 & 20 & $18: 58: 1512.579$ & 40.499 & 2.05 & 1.34 \\
\hline 2006 & 1 & 20 & $19: 26: 0812.616$ & 40.474 & 3.81 & 0.83 \\
\hline 2006 & 1 & 20 & $19: 44: 4712.582$ & 40.490 & 3.19 & 1.24 \\
\hline 2006 & 1 & 20 & $20: 42: 3312.460$ & 40.814 & 2.37 & 2.39 \\
\hline 2006 & 1 & 20 & $20: 52: 2112.537$ & 40.551 & 2.42 & 1.16 \\
\hline 2006 & 1 & 20 & $21: 33: 0512.575$ & 40.507 & 2.46 & 0.83 \\
\hline 2006 & 1 & 20 & $21: 39: 3312.586$ & 40.488 & 2.39 & 1.41 \\
\hline 2006 & 1 & 20 & $21: 44: 0512.603$ & 40.480 & 2.48 & 0.86 \\
\hline 2006 & 1 & 20 & $22: 01: 0012.612$ & 40.473 & 2.30 & 1.15 \\
\hline 2006 & $\overline{1}$ & 20 & $22: 03: 1912.578$ & 40.492 & 2.72 & 0.86 \\
\hline 2006 & 1 & 20 & $22: 06: 1512.570$ & 40.480 & 2.57 & 1.02 \\
\hline 2006 & 1 & 21 & $00: 17: 3112.659$ & 40.461 & 2.16 & 1.13 \\
\hline 2006 & 1 & 21 & $02: 08: 2412.623$ & 40.479 & 2.91 & 1.56 \\
\hline 2006 & $\overline{1}$ & 21 & $06: 43: 3812.655$ & 40.476 & 3.61 & 1.37 \\
\hline 2006 & 1 & 21 & $06: 46: 2212.536$ & 40.307 & 3.59 & 1.15 \\
\hline 2006 & 1 & 21 & $09: 42: 1512.582$ & 40.462 & 2.32 & 1.06 \\
\hline 2006 & 1 & 21 & 13:09:59 12.568 & 40.462 & 2.27 & 0.64 \\
\hline 2006 & $\overline{1}$ & 21 & $17: 09: 2112.332$ & 40.596 & 2.51 & 2.88 \\
\hline 2006 & 1 & 21 & $18: 46: 3812.422$ & 40.551 & 2.09 & 1.56 \\
\hline 2006 & 1 & 21 & $19: 09: 2612.565$ & 40.544 & 1.87 & 0.78 \\
\hline 2006 & 1 & 21 & $20: 59: 1412.627$ & 40.532 & 1.81 & 0.96 \\
\hline 2006 & $\overline{1}$ & 21 & $21: 48: 0612.595$ & 40.493 & 2.06 & 0.97 \\
\hline 2006 & 1 & 21 & $22: 23: 2713.380$ & 40.916 & 2.74 & 3.52 \\
\hline 2006 & 1 & 22 & $01: 37: 4312.568$ & 40.504 & 1.98 & 1.04 \\
\hline 2006 & 1 & 22 & $01: 44: 0512.596$ & 40.497 & 2.06 & 0.9 \\
\hline 2006 & 1 & 22 & $04: 10: 1412.655$ & 40.486 & 3.13 & 0.94 \\
\hline 2006 & 1 & 22 & $09: 08: 3912.735$ & 40.212 & 2.54 & 1.08 \\
\hline 2006 & 1 & 22 & $11: 56: 0412.531$ & 40.485 & 2.96 & 1.25 \\
\hline 2006 & 1 & 22 & $13: 01: 3812.461$ & 40.578 & 3.08 & 0.7 \\
\hline 2006 & 1 & 22 & $22: 14: 5112.924$ & 40.389 & 2.27 & 1.04 \\
\hline 2006 & $\overline{1}$ & 22 & $23: 35: 4412.586$ & 40.501 & 2.26 & 0.81 \\
\hline 2006 & 1 & 23 & $00: 55: 5012.569$ & 40.496 & 2.15 & 1.59 \\
\hline 2006 & 1 & 23 & $11: 45: 2612.579$ & 40.499 & 2.42 & 0.94 \\
\hline 2006 & 1 & 23 & $14: 53: 5812.578$ & 40.491 & 3.81 & 0.79 \\
\hline 2006 & 1 & 23 & $14: 58: 0512.602$ & 40.485 & 3.72 & 0.97 \\
\hline 2006 & 1 & 23 & $15: 07: 5812.567$ & 40.496 & 2.47 & 0.89 \\
\hline 2006 & 1 & 23 & $15: 26: 3112.307$ & 40.611 & 2.71 & 1.08 \\
\hline 2006 & 1 & 23 & $16: 33: 1012.572$ & 40.539 & 2.66 & 0.56 \\
\hline 2006 & 1 & 23 & $18: 29: 2212.590$ & 40.511 & 2.68 & 0.99 \\
\hline 2006 & 1 & 23 & $18: 38: 1912.579$ & 40.477 & 2.53 & 1.14 \\
\hline 2006 & 1 & 23 & $21: 14: 0812.565$ & 40.480 & 2.28 & 1.13 \\
\hline 2006 & 1 & 23 & $23: 54: 1012.577$ & 40.511 & 2.22 & 0.88 \\
\hline 2006 & 1 & 24 & $01: 13: 3212.547$ & 40.525 & 1.95 & 0.74 \\
\hline 2006 & 1 & 24 & $14: 28: 2312.470$ & 40.550 & 1.99 & 1.94 \\
\hline 2006 & 1 & 24 & $15: 46: 4612.637$ & 40.533 & 1.57 & 0.94 \\
\hline 2006 & 1 & 24 & $17: 16: 3012.579$ & 40.474 & 2.09 & 1.53 \\
\hline 2006 & 1 & 24 & $17: 20: 2512.604$ & 40.461 & 2.01 & 1.52 \\
\hline 2006 & 1 & 24 & $19: 41: 3712.569$ & 40.500 & 2.27 & 1.34 \\
\hline 2006 & 1 & 24 & $20: 48: 3412.650$ & 40.510 & 1.51 & 0.79 \\
\hline 2006 & 1 & 24 & $22: 16: 3612.595$ & 40.470 & 3.02 & 0.98 \\
\hline 2006 & 1 & 24 & $23: 07: 0512.589$ & 40.505 & 2.14 & 1.09 \\
\hline
\end{tabular}




\begin{tabular}{|c|c|c|c|c|c|c|}
\hline 2006 & 1 & 25 & $01: 21: 0112.587$ & 40.459 & 1.78 & 1.19 \\
\hline 2006 & 1 & 25 & $02: 37: 2112.587$ & 40.470 & 2.53 & 1.01 \\
\hline 2006 & 1 & 25 & $18: 55: 3412.745$ & 40.406 & 1.86 & 0.92 \\
\hline 2006 & 1 & 25 & $19: 19: 4712.550$ & 40.525 & 1.81 & 0.5 \\
\hline 2006 & 1 & 25 & $19: 22: 5112.643$ & 40.522 & 0.86 & 1.01 \\
\hline 2006 & 1 & 25 & $19: 53: 5312.575$ & 40.474 & 1.76 & 0.71 \\
\hline 2006 & 1 & 25 & $20: 23: 1112.428$ & 40.598 & 1.88 & 1.08 \\
\hline 2006 & 1 & 25 & $23: 38: 4512.590$ & 40.469 & 2.38 & 1.04 \\
\hline 2006 & 1 & 25 & $23: 41: 5512.597$ & 40.467 & 2.25 & 1.01 \\
\hline 2006 & 1 & 26 & $00: 16: 5112.616$ & 40.459 & 1.84 & 1.8 \\
\hline 2006 & 1 & 26 & $11: 07: 5112.557$ & 40.490 & 3.34 & 1.14 \\
\hline 2006 & 1 & 26 & $17: 29: 5412.511$ & 40.538 & 2.23 & 0.73 \\
\hline 2006 & 1 & 26 & $17: 41: 5712.585$ & 40.472 & 2.66 & 1.15 \\
\hline 2006 & 1 & 26 & $20: 38: 1912.552$ & 40.491 & 2.13 & 0.59 \\
\hline 2006 & 1 & 26 & $22: 58: 0512.547$ & 40.518 & 1.65 & 3.29 \\
\hline 2006 & 1 & 26 & $23: 29: 4112.570$ & 40.473 & 2.71 & 1.06 \\
\hline 2006 & 1 & 27 & $02: 10: 4612.586$ & 40.456 & 2.17 & 1.47 \\
\hline 2006 & 1 & 27 & $11: 13: 4212.444$ & 40.580 & 2.49 & 1.03 \\
\hline 2006 & 1 & 27 & $17: 33: 1213.391$ & 39.952 & 3.61 & 2.03 \\
\hline 2006 & 1 & 27 & $19: 30: 2112.604$ & 40.518 & 2.32 & 0.97 \\
\hline 2006 & 1 & 27 & $20: 07: 4412.578$ & 40.440 & 1.92 & 0.71 \\
\hline 2006 & 1 & 27 & $20: 20: 0712.545$ & 40.479 & 1.98 & 1.76 \\
\hline 2006 & 1 & 27 & $20: 24: 2012.551$ & 40.489 & 2.37 & 0.63 \\
\hline 2006 & 1 & 27 & $21: 22: 4612.601$ & 40.509 & 1.98 & 0.8 \\
\hline 2006 & 1 & 27 & $21: 33: 1412.650$ & 40.549 & 1.88 & 1.55 \\
\hline 2006 & 1 & 27 & $22: 02: 2812.549$ & 40.493 & 2.67 & 0.97 \\
\hline 2006 & 1 & 28 & $17: 37: 3712.573$ & 40.459 & 1.69 & 1.08 \\
\hline 2006 & 1 & 28 & $23: 56: 2612.668$ & 40.565 & 1.94 & 1.39 \\
\hline 2006 & 1 & 29 & $00: 34: 2612.272$ & 40.651 & 2.20 & 1.92 \\
\hline 2006 & 1 & 29 & $05: 14: 4012.579$ & 40.529 & 2.91 & 0.66 \\
\hline 2006 & 1 & 29 & $06: 51: 0213.360$ & 40.878 & 3.03 & 1.45 \\
\hline 2006 & 1 & 29 & $07: 29: 0612.563$ & 40.489 & 3.01 & 0.42 \\
\hline 2006 & 1 & 29 & $12: 10: 3313.379$ & 40.913 & 3.40 & 1.44 \\
\hline 2006 & 1 & 29 & $21: 52: 0612.473$ & 40.569 & 1.73 & 0.89 \\
\hline 2006 & 1 & 29 & $23: 22: 5112.503$ & 40.559 & 1.89 & 0.86 \\
\hline 2006 & 1 & 30 & $01: 18: 4912.630$ & 40.563 & 2.03 & 1.02 \\
\hline 2006 & 1 & 30 & $13: 14: 0312.252$ & 40.679 & 2.70 & 1.26 \\
\hline 2006 & 1 & 30 & $20: 36: 0612.546$ & 40.498 & 2.12 & 0.49 \\
\hline 2006 & 1 & 30 & $21: 46: 0112.441$ & 40.570 & 1.92 & 0.76 \\
\hline 2006 & 1 & 30 & $23: 13: 5212.606$ & 40.508 & 1.99 & 0.89 \\
\hline 2006 & 1 & 31 & $19: 44: 2612.541$ & 40.489 & 2.51 & 0.58 \\
\hline 2006 & 1 & 31 & $20: 10: 0212.566$ & 40.522 & 2.13 & 0.54 \\
\hline 2006 & 2 & 1 & $07: 49: 3312.533$ & 40.517 & 2.18 & 1.11 \\
\hline 2006 & 2 & 1 & $09: 27: 0712.468$ & 40.549 & 2.07 & 1.27 \\
\hline 2006 & 2 & 1 & $19: 14: 3512.615$ & 40.528 & 1.68 & 0.95 \\
\hline 2006 & 2 & 1 & $20: 07: 1012.501$ & 40.541 & 1.91 & 1.07 \\
\hline 2006 & 2 & 1 & $22: 48: 4412.545$ & 40.490 & 2.34 & 0.6 \\
\hline 2006 & 2 & 1 & $23: 16: 1012.551$ & 40.534 & 1.94 & 0.87 \\
\hline 2006 & 2 & 1 & $23: 51: 1212.558$ & 40.477 & 1.91 & 0.82 \\
\hline 2006 & 2 & 2 & $00: 23: 1312.571$ & 40.461 & 1.90 & 0.82 \\
\hline 2006 & 2 & 2 & $00: 43: 3012.623$ & 40.531 & 1.61 & 1.33 \\
\hline 2006 & 2 & 2 & $02: 07: 4112.599$ & 40.470 & 3.18 & 1.19 \\
\hline 2006 & 2 & 2 & $07: 52: 5412.558$ & 40.481 & 2.27 & 2.49 \\
\hline 2006 & 2 & 2 & $22: 47: 4013.029$ & 40.907 & 2.28 & 1.13 \\
\hline 2006 & 2 & 3 & $01: 27: 3212.527$ & 40.541 & 1.86 & 0.72 \\
\hline 2006 & 2 & 3 & $18: 04: 5012.602$ & 40.479 & 2.22 & 1.76 \\
\hline 2006 & 2 & 4 & $15: 01: 2712.533$ & 40.486 & 2.44 & 1.01 \\
\hline 2006 & 2 & 4 & $17: 31: 0813.389$ & 40.929 & 4.09 & 3.1 \\
\hline 2006 & 2 & 4 & $18: 46: 1112.549$ & 40.549 & 1.87 & 1.68 \\
\hline 2006 & 2 & 4 & $19: 17: 1913.389$ & 40.928 & 2.98 & 1.3 \\
\hline 2006 & 2 & 4 & $19: 32: 3412.546$ & 40.496 & 2.21 & 0.65 \\
\hline 2006 & 2 & 4 & $20: 07: 4813.393$ & 40.932 & 3.67 & 2.81 \\
\hline 2006 & 2 & 5 & $00: 22: 5112.651$ & 40.530 & 1.61 & 0.66 \\
\hline 2006 & 2 & 5 & $15: 51: 1112.570$ & 40.460 & 2.67 & 1.07 \\
\hline 2006 & 2 & 6 & $15: 44: 0512.640$ & 40.539 & 1.90 & 0.71 \\
\hline 2006 & 2 & 6 & $18: 05: 0312.587$ & 40.516 & 1.89 & 0.61 \\
\hline 2006 & 2 & 6 & $19: 59: 4112.428$ & 40.589 & 2.03 & 1.24 \\
\hline 2006 & 2 & 7 & $09: 26: 3212.572$ & 40.451 & 2.21 & 0.69 \\
\hline 2006 & 2 & 7 & $10: 19: 5012.582$ & 40.494 & 2.26 & 0.9 \\
\hline 2006 & 2 & 7 & $21: 43: 1412.595$ & 40.438 & 1.60 & 0.94 \\
\hline 2006 & 2 & 7 & $22: 54: 5512.531$ & 40.485 & 2.49 & 0.91 \\
\hline 2006 & 2 & 8 & $00: 37: 1012.430$ & 40.583 & 2.49 & 0.73 \\
\hline 2006 & 2 & 8 & $08: 35: 0313.235$ & 39.986 & 2.69 & 1.26 \\
\hline 2006 & 2 & 8 & $09: 28: 1912.555$ & 40.480 & 2.27 & 0.78 \\
\hline 2006 & 2 & 8 & $22: 55: 0512.546$ & 40.487 & 2.56 & 0.65 \\
\hline 2006 & 2 & 9 & $21: 10: 2912.510$ & 40.541 & 2.43 & 0.87 \\
\hline
\end{tabular}




\begin{tabular}{|c|c|c|c|c|c|c|}
\hline 2006 & 2 & 9 & $23: 24: 5412.519$ & 40.538 & 1.86 & 0.98 \\
\hline 2006 & 2 & 9 & $23: 40: 0012.645$ & 40.530 & 1.02 & 0.62 \\
\hline 2006 & 2 & 10 & $01: 52: 2211.883$ & 41.067 & 3.09 & 3.38 \\
\hline 2006 & 2 & 10 & 18:09:02 12.529 & 40.547 & 1.96 & 0.71 \\
\hline 2006 & 2 & 10 & $21: 42: 3812.572$ & 40.447 & 1.67 & 0.73 \\
\hline 2006 & 2 & 10 & $22: 20: 0912.557$ & 40.499 & 3.00 & 0.48 \\
\hline 2006 & 2 & 10 & $23: 10: 5112.589$ & 40.523 & 1.81 & 0.75 \\
\hline 2006 & 2 & 11 & $01: 10: 5312.594$ & 40.507 & 2.28 & 0.75 \\
\hline 2006 & 2 & 11 & $01: 17: 0612.862$ & 40.638 & 2.36 & 0.51 \\
\hline 2006 & 2 & 11 & $17: 23: 4912.381$ & 40.608 & 1.25 & 0.92 \\
\hline 2006 & 2 & 11 & $17: 53: 3113.342$ & 39.641 & 2.92 & 2.85 \\
\hline 2006 & 2 & 11 & $21: 52: 5112.395$ & 40.609 & 2.77 & 1.17 \\
\hline 2006 & 2 & 12 & $12: 10: 3512.455$ & 40.579 & 2.57 & 0.82 \\
\hline 2006 & 2 & 12 & $16: 06: 0612.509$ & 40.548 & 2.28 & 0.83 \\
\hline 2006 & 2 & 13 & $00: 58: 1812.578$ & 40.501 & 1.98 & 1.99 \\
\hline 2006 & 2 & 13 & $12: 04: 3313.425$ & 40.948 & 3.86 & 1.47 \\
\hline 2006 & 2 & 13 & $16: 08: 4112.407$ & 40.298 & 2.05 & 2.68 \\
\hline 2006 & 2 & 13 & $16: 14: 0012.404$ & 40.294 & 1.98 & 1.8 \\
\hline 2006 & 2 & 13 & $16: 34: 1613.386$ & 40.927 & 2.83 & 1.49 \\
\hline 2006 & 2 & 13 & $21: 44: 5412.710$ & 40.573 & 1.67 & 0.75 \\
\hline 2006 & 2 & 13 & $23: 59: 3113.410$ & 40.966 & 2.88 & 1.51 \\
\hline 2006 & 2 & 14 & $00: 12: 1113.395$ & 40.931 & 3.66 & 2.08 \\
\hline 2006 & 2 & 14 & $09: 13: 4312.559$ & 40.514 & 2.03 & 1.06 \\
\hline 2006 & 2 & 14 & $12: 31: 4712.622$ & 40.532 & 2.32 & 0.89 \\
\hline 2006 & 2 & 14 & $20: 38: 0212.579$ & 40.522 & 2.05 & 0.65 \\
\hline 2006 & 2 & 14 & $23: 08: 0912.656$ & 40.505 & 1.86 & 0.67 \\
\hline 2006 & 2 & 15 & $02: 53: 2512.378$ & 40.593 & 2.81 & 0.96 \\
\hline 2006 & 2 & 15 & $16: 45: 0112.530$ & 40.549 & 2.32 & 0.87 \\
\hline 2006 & 2 & 15 & $21: 23: 3512.547$ & 40.525 & 1.96 & 0.6 \\
\hline 2006 & 2 & 15 & $23: 47: 4812.527$ & 40.535 & 2.10 & 0.69 \\
\hline 2006 & 2 & 16 & $00: 57: 2412.505$ & 40.544 & 1.55 & 0.94 \\
\hline 2006 & 2 & 16 & $01: 51: 1612.540$ & 40.547 & 2.20 & 0.86 \\
\hline 2006 & 2 & 16 & $02: 10: 5112.652$ & 40.533 & 1.44 & 0.96 \\
\hline 2006 & 2 & 16 & $02: 12: 3212.577$ & 40.511 & 1.79 & 1.3 \\
\hline 2006 & 2 & 16 & $18: 34: 3113.409$ & 40.936 & 2.95 & 1.15 \\
\hline 2006 & 2 & 16 & $19: 55: 0212.648$ & 40.533 & 2.10 & 0.65 \\
\hline 2006 & 2 & 16 & $20: 52: 1312.554$ & 40.522 & 1.97 & 0.6 \\
\hline 2006 & 2 & 16 & $23: 33: 1712.555$ & 40.491 & 2.17 & 1.14 \\
\hline 2006 & 2 & 17 & $09: 51: 1012.580$ & 40.495 & 2.48 & 1.68 \\
\hline 2006 & 2 & 17 & $10: 05: 5412.434$ & 40.623 & 1.73 & 2.62 \\
\hline 2006 & 2 & 17 & $22: 49: 4112.354$ & 40.647 & 1.78 & 4.15 \\
\hline 2006 & 2 & 18 & $00: 50: 5512.562$ & 40.529 & 2.29 & 0.88 \\
\hline 2006 & 2 & 18 & $01: 31: 3212.547$ & 40.525 & 1.56 & 1.39 \\
\hline 2006 & 2 & 18 & $16: 00: 0612.903$ & 39.825 & 2.68 & 2.65 \\
\hline 2006 & 2 & 18 & $17: 31: 0412.621$ & 40.532 & 2.11 & 0.91 \\
\hline 2006 & 2 & 18 & $18: 19: 2612.550$ & 40.538 & 1.80 & 1.03 \\
\hline 2006 & 2 & 18 & $20: 23: 4912.577$ & 40.512 & 1.97 & 0.6 \\
\hline 2006 & 2 & 18 & $22: 23: 5212.861$ & 40.266 & 1.96 & 1.25 \\
\hline 2006 & 2 & 18 & $23: 09: 2012.566$ & 40.465 & 2.13 & 0.59 \\
\hline 2006 & 2 & 18 & $23: 12: 2912.548$ & 40.474 & 1.51 & 0.67 \\
\hline 2006 & 2 & 18 & $23: 37: 2212.570$ & 40.461 & 1.85 & 0.95 \\
\hline 2006 & 2 & 18 & $23: 48: 2812.591$ & 40.466 & 1.78 & 0.51 \\
\hline 2006 & 2 & 19 & $09: 10: 5512.616$ & 40.535 & 2.07 & 1.14 \\
\hline 2006 & 2 & 19 & $18: 02: 2312.339$ & 40.625 & 2.16 & 1.14 \\
\hline 2006 & 2 & 19 & $20: 45: 5812.404$ & 40.589 & 1.73 & 1.03 \\
\hline 2006 & 2 & 19 & $23: 10: 2412.489$ & 40.581 & 1.61 & 1.4 \\
\hline 2006 & 2 & 20 & $02: 39: 2712.396$ & 40.662 & 2.20 & 1.29 \\
\hline 2006 & 2 & 20 & $18: 22: 0612.523$ & 40.622 & 1.94 & 6.33 \\
\hline 2006 & 2 & 21 & $00: 51: 0612.329$ & 40.668 & 2.10 & 1.09 \\
\hline 2006 & 2 & 21 & $00: 54: 2412.323$ & 40.641 & 2.31 & 1.08 \\
\hline 2006 & 2 & 21 & $00: 58: 2312.323$ & 40.651 & 2.27 & 0.94 \\
\hline 2006 & 2 & 21 & $01: 03: 0212.315$ & 40.630 & 2.48 & 0.93 \\
\hline 2006 & 2 & 21 & $01: 05: 4412.317$ & 40.633 & 2.15 & 1.45 \\
\hline 2006 & 2 & 21 & $06: 20: 5612.325$ & 40.617 & 2.62 & 1.23 \\
\hline 2006 & 2 & 21 & $07: 34: 2712.725$ & 40.793 & 2.41 & 2.59 \\
\hline 2006 & 2 & 21 & $19: 43: 0712.576$ & 40.441 & 1.88 & 0.99 \\
\hline 2006 & 2 & 22 & $02: 02: 0213.290$ & 41.054 & 2.89 & 2.34 \\
\hline 2006 & 2 & 22 & $03: 49: 1812.356$ & 40.634 & 3.31 & 0.91 \\
\hline 2006 & 2 & 22 & $04: 01: 5112.575$ & 40.540 & 2.96 & 1.2 \\
\hline 2006 & 2 & 22 & $19: 19: 5412.588$ & 40.483 & 1.33 & 1.71 \\
\hline 2006 & 2 & 22 & $20: 18: 2312.418$ & 40.625 & 1.69 & 1.84 \\
\hline 2006 & 2 & 23 & $01: 58: 0012.588$ & 40.468 & 1.95 & 1.8 \\
\hline 2006 & 2 & 23 & $08: 23: 3112.539$ & 40.493 & 2.31 & 0.56 \\
\hline 2006 & 2 & 23 & $15: 27: 4512.553$ & 40.532 & 2.42 & 0.79 \\
\hline 2006 & 2 & 23 & $21: 21: 0312.576$ & 40.501 & 1.94 & 1.03 \\
\hline 2006 & 2 & 23 & $21: 32: 1712.591$ & 40.497 & 1.80 & 0.49 \\
\hline
\end{tabular}




\begin{tabular}{|c|c|c|c|c|c|c|}
\hline 2006 & 2 & 23 & $22: 22: 1312.326$ & 40.642 & 2.12 & 1.34 \\
\hline 2006 & 2 & 24 & $00: 10: 4012.646$ & 40.516 & 1.36 & 0.76 \\
\hline 2006 & 2 & 24 & $00: 21: 3412.382$ & 40.592 & 1.70 & 0.77 \\
\hline 2006 & 2 & 24 & $02: 40: 5412.654$ & 40.527 & 1.10 & 6.25 \\
\hline 2006 & 2 & 24 & $20: 50: 0212.580$ & 40.515 & 1.82 & 1.33 \\
\hline 2006 & 2 & 24 & $23: 31: 2412.363$ & 40.621 & 2.15 & 1.59 \\
\hline 2006 & 2 & 25 & $07: 23: 2912.523$ & 40.531 & 2.68 & 2,00 \\
\hline 2006 & 2 & 25 & $14: 05: 3412.514$ & 40.518 & 1.63 & 1.24 \\
\hline 2006 & 2 & 25 & $20: 51: 3812.370$ & 40.623 & 1.71 & 0.94 \\
\hline 2006 & 2 & 25 & $23: 55: 3612.523$ & 40.537 & 2.17 & 1.46 \\
\hline 2006 & 2 & 26 & $01: 32: 1612.524$ & 40.441 & 1.38 & 2.63 \\
\hline 2006 & 2 & 26 & $02: 09: 2012.461$ & 40.593 & 2.02 & 0.8 \\
\hline 2006 & 2 & 26 & $03: 47: 0012.342$ & 40.610 & 2.63 & 0.89 \\
\hline 2006 & 2 & 27 & $00: 14: 5112.686$ & 40.557 & 2.03 & 1.15 \\
\hline 2006 & 2 & 27 & $21: 21: 5412.298$ & 40.602 & 1.71 & 1.52 \\
\hline 2006 & 2 & 28 & $20: 39: 0012.566$ & 40.532 & 2.01 & 1.72 \\
\hline 2006 & 2 & 28 & $23: 14: 2612.292$ & 40.615 & 1.72 & 1.01 \\
\hline 2006 & 3 & 1 & $00: 16: 3213.499$ & 39.949 & 2.79 & 2.54 \\
\hline 2006 & 3 & 1 & $00: 19: 5112.490$ & 40.660 & 2.13 & 2.29 \\
\hline 2006 & 3 & 1 & $00: 53: 0513.379$ & 40.940 & 3.06 & 2.83 \\
\hline 2006 & 3 & 1 & $02: 42: 5212.473$ & 40.553 & 2.10 & 0.68 \\
\hline 2006 & 3 & 1 & $08: 47: 5913.498$ & 39.942 & 3.13 & 8.53 \\
\hline 2006 & 3 & 1 & $18: 23: 3312.634$ & 40.548 & 2.25 & 1.07 \\
\hline 2006 & 3 & 1 & $20: 36: 3812.607$ & 40.515 & 3.22 & 0.67 \\
\hline 2006 & 3 & 1 & $21: 36: 2912.551$ & 40.494 & 1.96 & 0.7 \\
\hline 2006 & 3 & 2 & $07: 02: 4512.592$ & 40.449 & 2.01 & 1.33 \\
\hline 2006 & 3 & 2 & $17: 27: 1712.638$ & 40.545 & 2.19 & 0.65 \\
\hline 2006 & 3 & 2 & $18: 04: 3612.644$ & 40.566 & 1.95 & 1.48 \\
\hline 2006 & 3 & 2 & $20: 51: 4712.548$ & 40.489 & 2.19 & 0.52 \\
\hline 2006 & 3 & 2 & $23: 42: 1912.560$ & 40.520 & 1.38 & 3,00 \\
\hline 2006 & 3 & 3 & $20: 13: 2212.620$ & 40.536 & 1.96 & 0.65 \\
\hline 2006 & 3 & 4 & $20: 09: 2512.314$ & 40.618 & 2.17 & 1.15 \\
\hline 2006 & 3 & 4 & $21: 13: 0112.451$ & 40.563 & 1.48 & 1.66 \\
\hline 2006 & 3 & 4 & $22: 58: 5512.534$ & 40.526 & 1.96 & 0.84 \\
\hline 2006 & 3 & 5 & $01: 35: 2112.325$ & 40.630 & 2.01 & 3.47 \\
\hline 2006 & 3 & 5 & $05: 47: 3512.691$ & 40.327 & 2.29 & 3.14 \\
\hline 2006 & 3 & 5 & $11: 51: 1812.567$ & 40.521 & 2.04 & 1.15 \\
\hline 2006 & 3 & 5 & $20: 35: 4712.560$ & 40.519 & 1.84 & 1.01 \\
\hline 2006 & 3 & 5 & $21: 58: 5913.063$ & 40.929 & 2.55 & 1.29 \\
\hline 2006 & 3 & 5 & $23: 09: 5812.552$ & 40.531 & 1.34 & 0.98 \\
\hline 2006 & 3 & 6 & $00: 20: 0112.438$ & 40.580 & 1.71 & 0.83 \\
\hline 2006 & 3 & 6 & $00: 55: 5412.539$ & 40.523 & 1.40 & 0.78 \\
\hline 2006 & 3 & 6 & $01: 10: 4412.570$ & 40.517 & 1.53 & 0.69 \\
\hline 2006 & 3 & 6 & $05: 15: 3512.531$ & 40.509 & 3.02 & 0.71 \\
\hline 2006 & 3 & 6 & $17: 14: 4212.544$ & 40.536 & 1.94 & 0.87 \\
\hline 2006 & 3 & 6 & $20: 25: 0712.587$ & 40.518 & 1.70 & 0.98 \\
\hline 2006 & 3 & 6 & $22: 27: 0412.434$ & 40.594 & 2.30 & 0.66 \\
\hline 2006 & 3 & 6 & $22: 35: 4112.495$ & 40.538 & 1.74 & 0.92 \\
\hline 2006 & 3 & 7 & $00: 22: 1712.313$ & 40.610 & 1.61 & 0.67 \\
\hline 2006 & 3 & 7 & $00: 24: 3912.325$ & 40.624 & 2.46 & 0.85 \\
\hline 2006 & 3 & 7 & $17: 08: 2512.470$ & 40.603 & 2.14 & 3.17 \\
\hline 2006 & 3 & 7 & $17: 54: 5312.351$ & 40.605 & 1.87 & 1.1 \\
\hline 2006 & 3 & 8 & $00: 22: 2112.458$ & 40.565 & 1.52 & 1.35 \\
\hline 2006 & 3 & 8 & $00: 28: 2212.482$ & 40.548 & 1.36 & 0.97 \\
\hline 2006 & 3 & 8 & $01: 55: 4213.417$ & 39.989 & 2.53 & 1.3 \\
\hline 2006 & 3 & 9 & $19: 32: 2312.258$ & 40.685 & 2.31 & 1.67 \\
\hline 2006 & 3 & 9 & $23: 15: 1012.357$ & 40.638 & 2.29 & 0.72 \\
\hline 2006 & 3 & 10 & $16: 36: 4112.540$ & 40.488 & 2.26 & 0.74 \\
\hline 2006 & 3 & 10 & $19: 33: 0112.556$ & 40.534 & 2.09 & 0.82 \\
\hline 2006 & 3 & 10 & $21: 27: 2212.655$ & 40.530 & 1.68 & 0.84 \\
\hline 2006 & 3 & 11 & $06: 01: 1812.589$ & 40.508 & 2.40 & 1.08 \\
\hline 2006 & 3 & 11 & $19: 13: 3712.560$ & 40.489 & 2.23 & 0.78 \\
\hline 2006 & 3 & 11 & $23: 32: 4212.630$ & 40.522 & 1.16 & 1.15 \\
\hline 2006 & 3 & 12 & $02: 29: 5912.578$ & 40.512 & 2.50 & 1.12 \\
\hline 2006 & 3 & 12 & $09: 33: 2312.652$ & 40.473 & 2.44 & 4.08 \\
\hline 2006 & 3 & 12 & $18: 42: 0112.661$ & 40.557 & 2.68 & 0.75 \\
\hline 2006 & 3 & 12 & $22: 03: 4112.745$ & 40.596 & 1.92 & 0.58 \\
\hline 2006 & 3 & 13 & $02: 37: 3612.668$ & 40.559 & 1.99 & 0.89 \\
\hline 2006 & 3 & 15 & $16: 52: 0812.371$ & 40.632 & 2.03 & 1.19 \\
\hline 2006 & 3 & 15 & $18: 52: 5912.331$ & 40.613 & 1.85 & 0.79 \\
\hline 2006 & 3 & 15 & $19: 27: 0013.854$ & 40.523 & 1.98 & 2.47 \\
\hline 2006 & 3 & 17 & $09: 16: 4412.368$ & 40.653 & 2.94 & 0.94 \\
\hline 2006 & 3 & 18 & $01: 23: 2712.352$ & 40.624 & 2.15 & 1.12 \\
\hline 2006 & 3 & 18 & $13: 56: 0512.585$ & 40.575 & 2.67 & 0.83 \\
\hline 2006 & 3 & 18 & $22: 10: 3112.335$ & 40.597 & 2.09 & 2.42 \\
\hline 2006 & 3 & 19 & $07: 33: 1712.557$ & 40.543 & 2.97 & 1.37 \\
\hline
\end{tabular}




\begin{tabular}{|c|c|c|c|c|c|c|}
\hline 2006 & 3 & 19 & $12: 29: 1212.558$ & 40.493 & 2.97 & 0.83 \\
\hline 2006 & 3 & 20 & $02: 03: 3812.529$ & 40.520 & 2.18 & 0.86 \\
\hline 2006 & 3 & 20 & $13: 54: 4112.463$ & 40.562 & 2.30 & 0.95 \\
\hline 2006 & 3 & 20 & $21: 28: 0312.335$ & 40.614 & 2.61 & 0.74 \\
\hline 2006 & 3 & 20 & $22: 15: 5612.344$ & 40.606 & 1.78 & 1.16 \\
\hline 2006 & 3 & 20 & $23: 13: 4212.453$ & 40.560 & 1.53 & 0.78 \\
\hline 2006 & 3 & 20 & $23: 54: 0312.482$ & 40.551 & 1.54 & 0.91 \\
\hline 2006 & 3 & 21 & $00: 31: 3112.578$ & 40.509 & 1.47 & 0.66 \\
\hline 2006 & 3 & 21 & $19: 51: 3012.331$ & 40.609 & 2.16 & 1.1 \\
\hline 2006 & 3 & 22 & $00: 37: 4112.569$ & 40.520 & 1.74 & 0.92 \\
\hline 2006 & 3 & 22 & $01: 04: 4812.522$ & 40.532 & 1.82 & 0.83 \\
\hline 2006 & 3 & 22 & $21: 05: 2312.546$ & 40.489 & 2.07 & 0.9 \\
\hline 2006 & 3 & 23 & $00: 28: 2812.471$ & 40.550 & 1.54 & 0.8 \\
\hline 2006 & 3 & 23 & $00: 37: 0712.598$ & 40.505 & 1.51 & 0.88 \\
\hline 2006 & 3 & 23 & $01: 15: 5712.673$ & 40.354 & 1.68 & 12.11 \\
\hline 2006 & 3 & 25 & $04: 07: 0912.287$ & 40.654 & 2.92 & 1.2 \\
\hline 2006 & 3 & 25 & $21: 56: 3612.352$ & 40.599 & 2.59 & 1.15 \\
\hline 2006 & 4 & 1 & $00: 23: 5312.465$ & 40.563 & 2.42 & 15.37 \\
\hline 2006 & 4 & 4 & $00: 30: 2911.261$ & 40.885 & 3.35 & 11.39 \\
\hline 2006 & 4 & 4 & $22: 26: 1612.325$ & 40.598 & 2.69 & 2.29 \\
\hline 2006 & 4 & 5 & $20: 17: 3912.482$ & 40.479 & 1.76 & 7.84 \\
\hline 2006 & 4 & 5 & $20: 20: 1112.489$ & 40.499 & 1.99 & 3.92 \\
\hline 2006 & 4 & 5 & $21: 13: 0012.401$ & 40.597 & 1.68 & 1.86 \\
\hline 2006 & 4 & 7 & $00: 58: 3412.356$ & 40.620 & 2.19 & 4.03 \\
\hline 2006 & 4 & 10 & $13: 36: 4413.553$ & 38.296 & 5.10 & 12.62 \\
\hline 2006 & 4 & 12 & $01: 17: 0213.017$ & 40.382 & 1.36 & 6.9 \\
\hline 2006 & 4 & 14 & $01: 49: 4512.377$ & 40.604 & 3.27 & 2.46 \\
\hline 2006 & 4 & 14 & $19: 27: 0111.234$ & 40.936 & 2.98 & 14.76 \\
\hline 2006 & 4 & 14 & $23: 21: 2912.394$ & 40.613 & 2.39 & 3.43 \\
\hline 2006 & 4 & 22 & $11: 54: 2111.240$ & 40.935 & 3.20 & 12.4 \\
\hline 2006 & 4 & 22 & $20: 51: 3711.188$ & 40.915 & 3.02 & 13.91 \\
\hline 2006 & 4 & 22 & $21: 42: 4811.274$ & 40.963 & 2.65 & 8.97 \\
\hline 2006 & 4 & 22 & $23: 14: 1511.462$ & 39.804 & 2.24 & 11.93 \\
\hline 2006 & 4 & 22 & $23: 15: 1311.471$ & 39.768 & 2.25 & 5.1 \\
\hline 2006 & 4 & 23 & $21: 04: 5412.546$ & 40.483 & 2.11 & 1.41 \\
\hline 2006 & 4 & 24 & $20: 26: 5113.203$ & 39.890 & 3.25 & 12.81 \\
\hline 2006 & 4 & 25 & $22: 21: 5112.497$ & 40.281 & 2.20 & 4.47 \\
\hline 2006 & 4 & 26 & $21: 58: 2311.244$ & 40.946 & 3.28 & 18.8 \\
\hline 2006 & 4 & 27 & $01: 20: 2511.272$ & 40.920 & 2.58 & 16.46 \\
\hline 2006 & 5 & 1 & $21: 52: 4612.521$ & 40.512 & 1.67 & 10.79 \\
\hline 2006 & 5 & 1 & $22: 42: 5312.326$ & 40.652 & 2.62 & 3.59 \\
\hline 2006 & 5 & 7 & $20: 18: 3412.312$ & 40.589 & 1.95 & 5.38 \\
\hline 2006 & 5 & 18 & $20: 10: 5612.313$ & 40.636 & 2.62 & 1.02 \\
\hline 2006 & 5 & 18 & $21: 56: 0712.330$ & 40.623 & 2.50 & 1.41 \\
\hline 2006 & 5 & 18 & $22: 51: 0711.209$ & 40.846 & 2.49 & 14.31 \\
\hline 2006 & 5 & 26 & $21: 42: 3512.261$ & 40.642 & 2.33 & 1.28 \\
\hline 2006 & 5 & 26 & $23: 09: 5613.028$ & 39.605 & 2.93 & 12.77 \\
\hline 2006 & 5 & 26 & $23: 41: 1212.311$ & 40.623 & 3.75 & 0.94 \\
\hline 2006 & 5 & 27 & $00: 18: 3312.354$ & 40.655 & 1.90 & 3.09 \\
\hline 2006 & 5 & 27 & $00: 23: 2212.327$ & 40.633 & 1.95 & 1.91 \\
\hline 2006 & 5 & 29 & $21: 38: 4612.450$ & 40.582 & 1.82 & 6.31 \\
\hline 2006 & 5 & 30 & $23: 22: 2712.471$ & 40.515 & 1.80 & 9.42 \\
\hline 2006 & 5 & 31 & $23: 11: 0612.389$ & 40.591 & 2.21 & 3.93 \\
\hline 2006 & 6 & 5 & $01: 18: 5912.342$ & 40.636 & 2.31 & 1.9 \\
\hline 2006 & 6 & 10 & $00: 01: 3211.236$ & 40.820 & 3.02 & 13.2 \\
\hline 2006 & 6 & 10 & $21: 33: 0112.334$ & 40.644 & 1.77 & 1.98 \\
\hline 2006 & 6 & 10 & $23: 21: 2512.309$ & 40.627 & 1.93 & 6.64 \\
\hline 2006 & 6 & 11 & $23: 31: 1612.311$ & 40.637 & 2.02 & 1.6 \\
\hline 2006 & 6 & 17 & $12: 39: 0812.340$ & 40.632 & 2.89 & 9.61 \\
\hline 2006 & 6 & 17 & $12: 49: 5012.334$ & 40.637 & 2.97 & 1.88 \\
\hline 2006 & 6 & 17 & $13: 26: 2912.332$ & 40.552 & 3.10 & 15.75 \\
\hline 2006 & 6 & 17 & $13: 38: 1812.340$ & 40.628 & 3.33 & 1.43 \\
\hline 2006 & 6 & 17 & $13: 45: 1612.324$ & 40.625 & 3.54 & 2.25 \\
\hline 2006 & 6 & 17 & $13: 46: 5712.338$ & 40.624 & 3.60 & 1.43 \\
\hline 2006 & 6 & 17 & $13: 55: 0812.342$ & 40.622 & 3.58 & 9.16 \\
\hline 2006 & 6 & 17 & $14: 01: 3012.351$ & 40.610 & 3.68 & 10.52 \\
\hline 2006 & 6 & 17 & $14: 09: 3012.341$ & 40.627 & 4.14 & 15.67 \\
\hline 2006 & 6 & 17 & $14: 17: 2712.310$ & 40.635 & 3.86 & 1.32 \\
\hline 2006 & 6 & 17 & $14: 20: 5912.356$ & 40.620 & 4.16 & 14.31 \\
\hline 2006 & 6 & 17 & $14: 33: 3212.340$ & 40.607 & 4.70 & 2.27 \\
\hline 2006 & 6 & 17 & $14: 45: 1412.346$ & 40.621 & 4.63 & 5.68 \\
\hline 2006 & 6 & 17 & $15: 03: 4512.356$ & 40.622 & 3.59 & 4.82 \\
\hline 2006 & 6 & 17 & $15: 10: 4012.316$ & 40.639 & 3.60 & 2.42 \\
\hline 2006 & 6 & 17 & $15: 20: 2312.381$ & 40.628 & 3.80 & 2.19 \\
\hline 2006 & 6 & 17 & $15: 40: 0512.361$ & 40.599 & 3.88 & 3.76 \\
\hline 2006 & 6 & 17 & $15: 42: 5812.387$ & 40.579 & 4.23 & 6.78 \\
\hline
\end{tabular}




\begin{tabular}{|c|c|c|c|c|c|c|}
\hline 2006 & 6 & 17 & $15: 57: 4612.376$ & 40.589 & 4.07 & 8.15 \\
\hline 2006 & 6 & 17 & $16: 08: 2512.381$ & 40.613 & 3.81 & 7.02 \\
\hline 2006 & 6 & 17 & $16: 20: 4012.364$ & 40.605 & 4.69 & 2.17 \\
\hline 2006 & 6 & 17 & $16: 28: 2812.387$ & 40.611 & 3.81 & 9.38 \\
\hline 2006 & 6 & 17 & $16: 53: 1012.359$ & 40.592 & 4.37 & 2.02 \\
\hline 2006 & 6 & 17 & $17: 17: 4412.365$ & 40.609 & 4.11 & 10.31 \\
\hline 2006 & 6 & 17 & $17: 24: 2012.389$ & 40.588 & 3.37 & 7.01 \\
\hline 2006 & 6 & 17 & $17: 46: 4812.381$ & 40.614 & 3.20 & 7.08 \\
\hline 2006 & 6 & 17 & $18: 36: 1512.381$ & 40.581 & 3.08 & 5.91 \\
\hline 2006 & 6 & 17 & $18: 37: 5112.380$ & 40.608 & 3.31 & 8.06 \\
\hline 2006 & 6 & 17 & $19: 02: 0912.368$ & 40.618 & 3.22 & 1.75 \\
\hline 2006 & 6 & 17 & $19: 11: 1212.353$ & 40.624 & 2.89 & 2.32 \\
\hline 2006 & 6 & 17 & $19: 24: 4212.365$ & 40.628 & 2.68 & 6.87 \\
\hline 2006 & 6 & 17 & $20: 01: 5212.377$ & 40.606 & 2.81 & 6.6 \\
\hline 2006 & 6 & 17 & $20: 06: 4312.378$ & 40.598 & 3.24 & 2.63 \\
\hline 2006 & 6 & 17 & $20: 30: 5612.372$ & 40.623 & 3.04 & 10.05 \\
\hline 2006 & 6 & 17 & $20: 59: 5912.367$ & 40.617 & 2.64 & 1.16 \\
\hline 2006 & 6 & 17 & $21: 15: 3612.380$ & 40.625 & 2.97 & 6.95 \\
\hline 2006 & 6 & 17 & $21: 18: 0012.367$ & 40.621 & 2.75 & 9.17 \\
\hline 2006 & 6 & 17 & $21: 30: 5212.376$ & 40.610 & 3.50 & 5.45 \\
\hline 2006 & 6 & 17 & $21: 42: 2212.377$ & 40.610 & 3.08 & 5.28 \\
\hline 2006 & 6 & 17 & $22: 17: 2512.344$ & 40.621 & 2.55 & 6.1 \\
\hline 2006 & 6 & 17 & $22: 38: 4012.377$ & 40.612 & 2.52 & 1.89 \\
\hline 2006 & 6 & 17 & $23: 23: 4412.363$ & 40.605 & 2.48 & 2.05 \\
\hline 2006 & 6 & 18 & $00: 03: 4012.358$ & 40.603 & 2.43 & 1.89 \\
\hline 2006 & 6 & 18 & $00: 09: 5612.394$ & 40.591 & 2.62 & 7.34 \\
\hline 2006 & 6 & 18 & $00: 46: 1312.355$ & 40.636 & 2.37 & 2.95 \\
\hline 2006 & 6 & 18 & $00: 52: 2312.361$ & 40.623 & 2.46 & 1.43 \\
\hline 2006 & 6 & 18 & $01: 40: 5012.358$ & 40.620 & 2.42 & 3.26 \\
\hline 2006 & 6 & 18 & $02: 26: 5712.395$ & 40.604 & 2.85 & 9.16 \\
\hline 2006 & 6 & 18 & $09: 04: 4312.376$ & 40.623 & 3.72 & 1.55 \\
\hline 2006 & 6 & 18 & $19: 45: 4412.374$ & 40.612 & 2.46 & 5.08 \\
\hline 2006 & 6 & 18 & $22: 48: 5712.395$ & 40.596 & 1.92 & 6.91 \\
\hline 2006 & 6 & 18 & $23: 44: 0112.339$ & 40.616 & 1.99 & 1.37 \\
\hline 2006 & 6 & 19 & $01: 14: 2812.370$ & 40.602 & 2.08 & 1.52 \\
\hline 2006 & 6 & 19 & $01: 37: 3212.354$ & 40.633 & 2.50 & 10.8 \\
\hline 2006 & 6 & 19 & $02: 37: 3012.361$ & 40.615 & 2.59 & 5.76 \\
\hline 2006 & 6 & 19 & $16: 21: 2312.378$ & 40.590 & 3.03 & 10.04 \\
\hline 2006 & 6 & 19 & $19: 16: 1412.371$ & 40.640 & 2.31 & 17.29 \\
\hline 2006 & 6 & 19 & $19: 20: 0512.384$ & 40.629 & 2.76 & 7,00 \\
\hline 2006 & 6 & 19 & $22: 46: 4512.298$ & 40.646 & 2.22 & 2.16 \\
\hline 2006 & 6 & 19 & $22: 56: 3312.383$ & 40.629 & 1.94 & 6.12 \\
\hline 2006 & 6 & 19 & $23: 05: 4912.342$ & 40.646 & 2.88 & 7.26 \\
\hline 2006 & 6 & 19 & $23: 19: 0012.383$ & 40.621 & 2.13 & 5.88 \\
\hline 2006 & 6 & 20 & $04: 51: 3212.359$ & 40.617 & 2.86 & 4.41 \\
\hline 2006 & 6 & 20 & $20: 34: 5212.376$ & 40.611 & 3.04 & 2.17 \\
\hline 2006 & 6 & 20 & $22: 51: 48 \quad 12.344$ & 40.647 & 2.42 & 1.03 \\
\hline 2006 & 6 & 20 & $23: 02: 1912.347$ & 40.639 & 2.31 & 3.16 \\
\hline 2006 & 6 & 20 & $23: 39: 3011.717$ & 41.118 & 3.42 & 7.62 \\
\hline 2006 & 6 & 21 & $16: 05: 3112.541$ & 40.496 & 2.86 & 10.07 \\
\hline 2006 & 6 & 21 & $19: 59: 5012.384$ & 40.604 & 2.65 & 3.82 \\
\hline 2006 & 6 & 21 & $20: 02: 4612.371$ & 40.624 & 2.50 & 5.49 \\
\hline 2006 & 6 & 22 & $15: 49: 2112.392$ & 40.519 & 3.07 & 5.8 \\
\hline 2006 & 6 & 22 & $18: 57: 0312.367$ & 40.621 & 2.60 & 7.56 \\
\hline 2006 & 7 & 14 & $23: 04: 2212.760$ & 40.610 & 2.94 & 2.57 \\
\hline 2006 & 7 & 15 & $01: 20: 4812.750$ & 40.606 & 3.12 & 3.68 \\
\hline 2006 & 7 & 19 & $23: 01: 1212.730$ & 40.670 & 3.23 & 2.52 \\
\hline 2006 & 7 & 24 & $23: 41: 4912.313$ & 40.686 & 2.14 & 1.6 \\
\hline 2006 & 7 & 25 & $20: 00: 2712.359$ & 40.654 & 2.52 & 2.72 \\
\hline 2006 & 7 & 25 & $20: 25: 5412.308$ & 40.630 & 2.13 & 1.07 \\
\hline 2006 & 7 & 25 & $20: 38: 1912.312$ & 40.639 & 2.40 & 1,00 \\
\hline 2006 & 7 & 25 & $20: 41: 2112.305$ & 40.633 & 2.73 & 1.19 \\
\hline 2006 & 7 & 25 & $21: 08: 1012.313$ & 40.626 & 2.72 & 1.04 \\
\hline 2006 & 7 & 25 & $21: 29: 3612.305$ & 40.637 & 2.42 & 2.35 \\
\hline 2006 & 7 & 25 & $21: 48: 1712.339$ & 40.619 & 2.81 & 4.24 \\
\hline 2006 & 7 & 25 & $22: 16: 5312.306$ & 40.655 & 2.62 & 2.55 \\
\hline 2006 & 7 & 25 & $22: 43: 1012.312$ & 40.623 & 2.94 & 1.54 \\
\hline 2006 & 7 & 25 & $22: 51: 2212.324$ & 40.626 & 2.88 & 1.32 \\
\hline 2006 & 7 & 25 & $23: 16: 0612.319$ & 40.628 & 2.53 & 1,00 \\
\hline 2006 & 7 & 26 & $00: 24: 3112.261$ & 40.683 & 2.29 & 1.06 \\
\hline 2006 & 7 & 26 & $00: 30: 3012.321$ & 40.656 & 2.46 & 1.6 \\
\hline 2006 & 7 & 26 & $02: 28: 1712.238$ & 40.683 & 2.92 & 1.33 \\
\hline 2006 & 7 & 26 & $02: 39: 1812.235$ & 40.690 & 2.92 & 1.33 \\
\hline 2006 & 7 & 26 & $04: 52: 4912.270$ & 40.699 & 2.98 & 1.63 \\
\hline 2006 & 7 & 26 & $05: 42: 2312.188$ & 40.698 & 3.29 & 1.23 \\
\hline 2006 & 7 & 26 & $10: 38: 4112.241$ & 40.695 & 2.68 & 0.89 \\
\hline
\end{tabular}




\begin{tabular}{|c|c|c|c|c|c|c|}
\hline 2006 & 7 & 26 & $11: 44: 2712.226$ & 40.723 & 2.70 & 2.14 \\
\hline 2006 & 7 & 27 & $00: 28: 1912.537$ & 40.566 & 1.98 & 2,00 \\
\hline 2006 & 7 & 27 & $21: 02: 1213.371$ & 40.821 & 3.14 & 16.92 \\
\hline 2006 & 7 & 28 & $20: 58: 3712.319$ & 40.612 & 2.11 & 4.02 \\
\hline 2006 & 7 & 29 & $00: 31: 0112.541$ & 40.559 & 2.65 & 1.33 \\
\hline 2006 & 7 & 30 & $22: 53: 4612.774$ & 40.733 & 1.76 & 1.78 \\
\hline 2006 & 8 & 4 & $01: 49: 3011.861$ & 40.907 & 2.30 & 3.65 \\
\hline 2006 & 8 & 7 & $01: 46: 0412.856$ & 40.642 & 2.75 & 3.06 \\
\hline 2006 & 8 & 12 & $19: 42: 5112.473$ & 40.601 & 1.84 & 2.94 \\
\hline 2006 & 8 & 13 & $23: 33: 2112.310$ & 40.625 & 2.00 & 1.55 \\
\hline 2006 & 8 & 17 & $23: 29: 3211.859$ & 41.017 & 2.30 & 3.95 \\
\hline 2006 & 8 & 20 & $23: 09: 3812.307$ & 40.633 & 1.78 & 1.63 \\
\hline 2006 & 8 & 23 & $20: 18: 4513.384$ & 39.986 & 2.46 & 10.17 \\
\hline 2006 & 8 & 23 & $20: 19: 3813.358$ & 39.984 & 2.43 & 3.36 \\
\hline 2006 & 8 & 24 & $00: 51: 2012.484$ & 40.590 & 1.45 & 2.04 \\
\hline 2006 & 8 & 24 & $01: 28: 4813.223$ & 39.964 & 1.97 & 4.24 \\
\hline 2006 & 8 & 27 & $02: 33: 2413.320$ & 39.980 & 2.55 & 4.97 \\
\hline 2006 & 8 & 28 & $00: 38: 5812.242$ & 40.637 & 1.36 & 3.2 \\
\hline 2006 & 8 & 30 & $23: 13: 4012.298$ & 40.642 & 2.00 & 2.55 \\
\hline 2006 & 8 & 31 & $20: 06: 4713.656$ & 39.930 & 2.61 & 5.68 \\
\hline 2006 & 9 & 2 & $20: 44: 5213.566$ & 39.884 & 2.43 & 14.47 \\
\hline 2006 & 9 & 5 & $01: 58: 3412.767$ & 40.616 & 1.99 & 0.8 \\
\hline 2006 & 9 & 5 & $23: 25: 3012.580$ & 40.574 & 1.40 & 1.15 \\
\hline 2006 & 9 & 6 & $20: 24: 2512.877$ & 40.653 & 1.60 & 0.9 \\
\hline 2006 & 9 & 8 & $00: 16: 3512.621$ & 40.563 & 1.76 & 0.95 \\
\hline 2006 & 9 & 8 & $20: 32: 3613.157$ & 41.081 & 1.96 & 2.86 \\
\hline 2006 & 9 & 8 & $22: 11: 1912.582$ & 40.587 & 1.80 & 1.71 \\
\hline 2006 & 9 & 10 & $09: 39: 2712.215$ & 40.690 & 2.85 & 1.21 \\
\hline 2006 & 9 & 10 & $09: 56: 5012.229$ & 40.688 & 2.83 & 1.08 \\
\hline 2006 & 9 & 10 & $10: 25: 0112.262$ & 40.695 & 2.67 & 2.42 \\
\hline 2006 & 9 & 10 & $10: 51: 5412.253$ & 40.708 & 2.79 & 5.34 \\
\hline 2006 & 9 & 10 & $11: 08: 4612.225$ & 40.682 & 2.80 & 2.59 \\
\hline 2006 & 9 & 10 & $11: 14: 0512.265$ & 40.704 & 2.86 & 0.94 \\
\hline 2006 & 9 & 10 & $11: 24: 2412.258$ & 40.702 & 2.86 & 1.5 \\
\hline 2006 & 9 & 10 & $11: 57: 2912.266$ & 40.708 & 2.65 & 2.2 \\
\hline 2006 & 9 & 10 & $12: 01: 0612.241$ & 40.706 & 3.16 & 1.29 \\
\hline 2006 & 9 & 10 & $12: 47: 4412.236$ & 40.703 & 3.11 & 1.31 \\
\hline 2006 & 9 & 10 & $12: 57: 1012.248$ & 40.701 & 3.35 & 1.32 \\
\hline 2006 & 9 & 10 & $13: 09: 4412.211$ & 40.701 & 3.07 & 1.46 \\
\hline 2006 & 9 & 10 & $14: 19: 5912.269$ & 40.703 & 2.69 & 1.71 \\
\hline 2006 & 9 & 10 & $14: 45: 1312.252$ & 40.700 & 2.64 & 3.08 \\
\hline 2006 & 9 & 11 & $01: 05: 3412.255$ & 40.702 & 2.70 & 2.9 \\
\hline 2006 & 9 & 11 & $22: 51: 4012.502$ & 40.578 & 1.52 & 1.77 \\
\hline 2006 & 9 & 13 & $22: 01: 0212.847$ & 41.468 & 1.50 & 2.15 \\
\hline 2006 & 9 & 13 & $23: 29: 5512.517$ & 40.613 & 1.87 & 2.23 \\
\hline 2006 & 9 & 15 & $01: 40: 5513.431$ & 40.783 & 2.26 & 3.51 \\
\hline 2006 & 9 & 18 & $00: 42: 3113.455$ & 39.980 & 1.94 & 9.38 \\
\hline 2006 & 9 & 19 & $01: 30: 3513.385$ & 39.978 & 3.19 & 9.03 \\
\hline 2006 & 9 & 19 & $01: 34: 3313.486$ & 39.970 & 2.31 & 17.38 \\
\hline 2006 & 9 & 19 & $21: 28: 0912.221$ & 40.684 & 2.11 & 0.9 \\
\hline 2006 & 9 & 19 & $23: 58: 4412.501$ & 40.575 & 1.41 & 2.97 \\
\hline 2006 & 9 & 23 & $23: 56: 3113.433$ & 39.987 & 4.36 & 13.2 \\
\hline 2006 & 9 & 25 & $11: 01: 2113.388$ & 39.990 & 4.06 & 4.65 \\
\hline 2006 & 9 & 26 & $23: 51: 0913.431$ & 39.995 & 4.22 & 9.92 \\
\hline 2006 & 10 & 3 & $11: 52: 3713.368$ & 40.841 & 4.19 & 2.01 \\
\hline 2006 & 10 & 10 & $07: 14: 5913.415$ & 39.991 & 2.97 & 14.66 \\
\hline 2006 & 10 & 12 & $10: 47: 4913.353$ & 39.955 & 2.34 & 4.71 \\
\hline 2006 & 10 & 12 & $13: 29: 0813.356$ & 39.982 & 3.46 & 10.29 \\
\hline 2006 & 10 & 12 & $21: 05: 1413.427$ & 39.982 & 3.62 & 13.57 \\
\hline 2006 & 10 & 12 & $22: 03: 1313.539$ & 39.970 & 2.70 & 19.93 \\
\hline 2006 & 10 & 14 & $21: 48: 0113.145$ & 39.861 & 2.55 & 9.23 \\
\hline 2006 & 10 & 20 & $11: 56: 1513.373$ & 39.993 & 2.66 & 6.01 \\
\hline 2006 & 10 & 21 & $12: 30: 5713.332$ & 39.977 & 2.81 & 8.85 \\
\hline 2007 & 3 & 14 & $00: 37: 3112.473$ & 40.557 & 2.19 & 1.29 \\
\hline 2007 & 3 & 14 & $18: 28: 389.841$ & 41.286 & 3.60 & 6.85 \\
\hline 2007 & 3 & 14 & $20: 22: 3612.441$ & 40.547 & 1.80 & 0.78 \\
\hline 2007 & 3 & 14 & $21: 14: 3212.457$ & 40.551 & 1.81 & 0.84 \\
\hline 2007 & 3 & 14 & $23: 11: 1512.379$ & 40.583 & 1.73 & 0.81 \\
\hline 2007 & 3 & 15 & $04: 52: 5012.501$ & 40.537 & 2.08 & 0.99 \\
\hline 2007 & 3 & 15 & $22: 47: 1212.458$ & 40.563 & 1.89 & 0.69 \\
\hline 2007 & 3 & 15 & $23: 09: 3312.454$ & 40.560 & 2.26 & 0.44 \\
\hline 2007 & 3 & 15 & $23: 42: 4112.457$ & 40.554 & 2.10 & 0.45 \\
\hline 2007 & 3 & 16 & $01: 04: 1311.604$ & 40.999 & 2.12 & 1.76 \\
\hline 2007 & 3 & 16 & $02: 04: 3012.470$ & 40.560 & 1.85 & 0.65 \\
\hline 2007 & 3 & 16 & $02: 11: 1812.409$ & 40.571 & 1.89 & 0.51 \\
\hline 2007 & 3 & 16 & $12: 55: 2312.269$ & 40.635 & 2.32 & 0.91 \\
\hline
\end{tabular}




\begin{tabular}{|c|c|c|c|c|c|c|}
\hline 2007 & 3 & 16 & $16: 05: 2411.653$ & 40.958 & 2.16 & 4.52 \\
\hline 2007 & 3 & 16 & $16: 20: 5412.888$ & 39.574 & 3.30 & 5.93 \\
\hline 2007 & 3 & 16 & $18: 19: 1211.632$ & 40.977 & 2.15 & 1.61 \\
\hline 2007 & 3 & 16 & $20: 54: 1412.344$ & 40.593 & 1.90 & 0.63 \\
\hline 2007 & 3 & 16 & $22: 57: 1012.496$ & 40.531 & 1.85 & 0.7 \\
\hline 2007 & 3 & 16 & $23: 27: 2112.447$ & 40.560 & 1.89 & 0.67 \\
\hline 2007 & 3 & 17 & $00: 26: 4412.369$ & 40.590 & 1.83 & 0.87 \\
\hline 2007 & 3 & 17 & $19: 37: 4012.463$ & 40.550 & 2.40 & 0.68 \\
\hline 2007 & 3 & 18 & $00: 06: 2012.543$ & 40.508 & 2.02 & 1.16 \\
\hline 2007 & 3 & 18 & $01: 18: 5812.464$ & 40.554 & 1.94 & 0.63 \\
\hline 2007 & 3 & 18 & $02: 42: 4212.492$ & 40.538 & 2.10 & 1.04 \\
\hline 2007 & 3 & 18 & $05: 33: 0612.441$ & 40.561 & 2.43 & 1.43 \\
\hline 2007 & 3 & 18 & $08: 30: 0112.506$ & 40.547 & 2.26 & 0.79 \\
\hline 2007 & 3 & 18 & $09: 54: 4612.438$ & 40.597 & 2.49 & 0.95 \\
\hline 2007 & 3 & 18 & $17: 29: 3712.457$ & 40.526 & 2.20 & 1.17 \\
\hline 2007 & 3 & 19 & $05: 41: 3612.449$ & 40.560 & 2.35 & 0.71 \\
\hline 2007 & 3 & 19 & $22: 18: 3511.681$ & 39.636 & 2.80 & 0.68 \\
\hline 2007 & 3 & 19 & $23: 18: 4712.508$ & 40.530 & 2.01 & 4.7 \\
\hline 2007 & 3 & 20 & $01: 06: 0412.512$ & 40.541 & 1.95 & 0.65 \\
\hline 2007 & 3 & 20 & $16: 55: 5212.469$ & 40.551 & 2.54 & 0.6 \\
\hline 2007 & 3 & 20 & $22: 17: 4512.602$ & 40.499 & 1.98 & 0.86 \\
\hline 2007 & 3 & 21 & $00: 58: 5112.483$ & 40.528 & 1.99 & 1.7 \\
\hline 2007 & 3 & 21 & $02: 39: 3112.491$ & 40.551 & 2.02 & 0.54 \\
\hline 2007 & 3 & 21 & $03: 58: 4611.561$ & 42.709 & 4.40 & 2.96 \\
\hline 2007 & 3 & 21 & $21: 12: 4412.460$ & 40.575 & 1.93 & 1.51 \\
\hline 2007 & 3 & 21 & $22: 48: 5412.894$ & 40.248 & 2.43 & 3.75 \\
\hline 2007 & 3 & 22 & $21: 17: 3312.486$ & 40.537 & 1.90 & 0.95 \\
\hline 2007 & 3 & 22 & $23: 17: 019.984$ & 41.320 & 2.93 & 10.26 \\
\hline 2007 & 3 & 22 & $23: 23: 579.992$ & 41.362 & 3.24 & 5.39 \\
\hline 2007 & 3 & 23 & $00: 37: 0812.420$ & 40.583 & 1.98 & 0.91 \\
\hline 2007 & 3 & 23 & $00: 56: 4712.480$ & 40.550 & 2.01 & 0.84 \\
\hline 2007 & 3 & 23 & $01: 28: 1712.438$ & 40.560 & 1.89 & 1.8 \\
\hline 2007 & 3 & 23 & $02: 10: 4812.415$ & 40.584 & 2.06 & 0.79 \\
\hline 2007 & 3 & 23 & $02: 58: 2712.750$ & 40.572 & 2.77 & 0.65 \\
\hline 2007 & 3 & 23 & $04: 52: 0413.145$ & 39.742 & 2.87 & 7.87 \\
\hline 2007 & 3 & 23 & $22: 28: 0513.154$ & 39.731 & 2.55 & 5.68 \\
\hline 2007 & 3 & 23 & $22: 56: 2912.312$ & 40.639 & 1.94 & 0.62 \\
\hline 2007 & 3 & 24 & $21: 28: 4312.454$ & 40.562 & 2.04 & 1.17 \\
\hline 2007 & 3 & 24 & $22: 07: 2710.095$ & 41.287 & 3.68 & 5.49 \\
\hline 2007 & 3 & 26 & $12: 47: 1812.419$ & 40.571 & 2.22 & 1.25 \\
\hline 2007 & 3 & 26 & $13: 20: 1312.528$ & 40.527 & 2.24 & 1.15 \\
\hline 2007 & 3 & 27 & $18: 49: 1212.440$ & 40.583 & 2.16 & 1.23 \\
\hline 2007 & 3 & 27 & $23: 08: 2012.338$ & 40.338 & 1.82 & 1.69 \\
\hline 2007 & 3 & 27 & $23: 11: 0512.523$ & 40.529 & 2.01 & 0.64 \\
\hline 2007 & 3 & 27 & $23: 31: 3812.304$ & 40.636 & 1.92 & 0.98 \\
\hline 2007 & 3 & 28 & $01: 44: 3712.485$ & 40.517 & 1.92 & 0.95 \\
\hline 2007 & 3 & 28 & $01: 49: 2911.898$ & 40.990 & 1.95 & 0.46 \\
\hline 2007 & 3 & 28 & $20: 28: 1912.472$ & 40.560 & 2.11 & 1.02 \\
\hline 2007 & 3 & 28 & $23: 12: 5312.542$ & 40.527 & 2.03 & 0.75 \\
\hline 2007 & 3 & 29 & $11: 22: 1412.549$ & 40.542 & 2.28 & 1.5 \\
\hline 2007 & 3 & 29 & $21: 10: 0112.491$ & 40.546 & 2.01 & 0.63 \\
\hline 2007 & 3 & 29 & $22: 44: 3812.461$ & 40.553 & 2.14 & 0.62 \\
\hline 2007 & 3 & 30 & $00: 25: 5212.462$ & 40.544 & 1.83 & 0.94 \\
\hline 2007 & 3 & 30 & $01: 25: 1012.424$ & 40.580 & 2.06 & 0.83 \\
\hline 2007 & 3 & 30 & $09: 00: 5112.472$ & 40.550 & 2.20 & 0.76 \\
\hline 2007 & 3 & 30 & $21: 54: 1812.400$ & 40.584 & 1.85 & 0.69 \\
\hline 2007 & 3 & 31 & $00: 49: 1112.428$ & 40.566 & 1.84 & 1.17 \\
\hline 2007 & 3 & 31 & $23: 11: 1612.352$ & 40.597 & 1.91 & 1.05 \\
\hline 2007 & 4 & 1 & $00: 30: 3011.894$ & 41.527 & 1.97 & 2.42 \\
\hline 2007 & 4 & 1 & $00: 49: 3012.392$ & 40.592 & 1.86 & 0.51 \\
\hline 2007 & 4 & 1 & $02: 47: 0112.463$ & 40.568 & 2.14 & 0.79 \\
\hline 2007 & 4 & 1 & $03: 45: 5111.898$ & 41.543 & 2.40 & 1.87 \\
\hline 2007 & 4 & 1 & $17: 20: 4412.464$ & 40.550 & 2.17 & 1.02 \\
\hline 2007 & 4 & 2 & $16: 35: 2911.897$ & 41.567 & 2.61 & 0.96 \\
\hline 2007 & 4 & 2 & $20: 19: 1311.893$ & 41.556 & 2.44 & 1.42 \\
\hline 2007 & 4 & 2 & $21: 38: 4212.504$ & 40.542 & 1.97 & 1.38 \\
\hline 2007 & 4 & 2 & $22: 15: 0011.884$ & 41.546 & 2.22 & 1.49 \\
\hline 2007 & 4 & 2 & $22: 30: 4412.481$ & 40.547 & 2.07 & 0.82 \\
\hline 2007 & 4 & 2 & $22: 37: 4112.460$ & 40.537 & 1.94 & 0.83 \\
\hline 2007 & 4 & 3 & $01: 34: 1812.429$ & 40.557 & 1.97 & 1.05 \\
\hline 2007 & 4 & 3 & $12: 26: 0312.598$ & 40.500 & 2.52 & 0.64 \\
\hline 2007 & 4 & 3 & $14: 55: 5811.788$ & 41.081 & 3.31 & 0.46 \\
\hline 2007 & 4 & 3 & $15: 08: 4411.814$ & 41.078 & 2.29 & 0.62 \\
\hline 2007 & 4 & 4 & $01: 06: 3512.377$ & 40.591 & 2.03 & 0.66 \\
\hline 2007 & 4 & 4 & $19: 41: 0712.253$ & 40.648 & 2.11 & 0.92 \\
\hline 2007 & 4 & 4 & $22: 17: 1612.501$ & 40.530 & 2.00 & 1.22 \\
\hline
\end{tabular}




\begin{tabular}{|c|c|c|c|c|c|c|}
\hline 2007 & 4 & 4 & $23: 18: 0112.465$ & 40.549 & 2.01 & 0.68 \\
\hline 2007 & 4 & 5 & $00: 10: 5612.362$ & 40.583 & 1.94 & 0.58 \\
\hline 2007 & 4 & 5 & $01: 52: 5912.356$ & 40.577 & 1.94 & 0.84 \\
\hline 2007 & 4 & 5 & $16: 52: 2812.575$ & 40.513 & 2.22 & 1.02 \\
\hline 2007 & 4 & 5 & $21: 30: 4312.382$ & 40.588 & 1.95 & 0.68 \\
\hline 2007 & 4 & 5 & $22: 58: 3612.378$ & 40.590 & 1.97 & 1.12 \\
\hline 2007 & 4 & 5 & $23: 02: 3412.522$ & 40.525 & 1.97 & 0.84 \\
\hline 2007 & 4 & 6 & $01: 39: 2912.511$ & 40.532 & 1.92 & 0.67 \\
\hline 2007 & 4 & 6 & $05: 13: 1812.507$ & 40.530 & 2.17 & 0.8 \\
\hline 2007 & 4 & 6 & $12: 32: 3412.572$ & 40.526 & 2.35 & 0.59 \\
\hline 2007 & 4 & 6 & $14: 20: 5912.454$ & 40.597 & 2.08 & 1.02 \\
\hline 2007 & 4 & 6 & $21: 46: 2611.518$ & 40.911 & 1.81 & 1.48 \\
\hline 2007 & 4 & 6 & $21: 51: 0312.448$ & 40.567 & 1.84 & 0.71 \\
\hline 2007 & 4 & 6 & $23: 11: 0112.443$ & 40.560 & 1.85 & 0.66 \\
\hline 2007 & 4 & 7 & $01: 35: 3412.470$ & 40.547 & 1.85 & 0.9 \\
\hline 2007 & 4 & 7 & $03: 37: 4611.511$ & 40.930 & 2.34 & 0.32 \\
\hline 2007 & 4 & 7 & $16: 29: 5812.429$ & 40.562 & 2.14 & 1.16 \\
\hline 2007 & 4 & 7 & $23: 03: 4012.769$ & 40.608 & 2.02 & 0.89 \\
\hline 2007 & 4 & 7 & $23: 07: 3212.435$ & 40.570 & 2.00 & 0.64 \\
\hline 2007 & 4 & 7 & $23: 46: 4012.418$ & 40.572 & 2.00 & 0.64 \\
\hline 2007 & 4 & 8 & $00: 40: 1312.480$ & 40.547 & 1.73 & 0.61 \\
\hline 2007 & 4 & 8 & $18: 20: 4012.479$ & 40.531 & 1.97 & 0.78 \\
\hline 2007 & 4 & 8 & $22: 25: 3212.332$ & 40.605 & 1.78 & 0.54 \\
\hline 2007 & 4 & 8 & $23: 10: 3112.450$ & 40.545 & 1.80 & 1.7 \\
\hline 2007 & 4 & 9 & $00: 21: 5912.490$ & 40.541 & 1.74 & 3.55 \\
\hline 2007 & 4 & 9 & $00: 22: 3712.427$ & 40.556 & 1.71 & 1.72 \\
\hline 2007 & 4 & 9 & $02: 03: 3312.269$ & 40.394 & 1.72 & 0.89 \\
\hline 2007 & 4 & 9 & $21: 54: 0313.447$ & 39.987 & 2.50 & 6.35 \\
\hline 2007 & 4 & 9 & $22: 49: 1312.389$ & 40.597 & 1.92 & 0.63 \\
\hline 2007 & 4 & 10 & $22: 24: 0312.357$ & 40.602 & 1.89 & 1.12 \\
\hline 2007 & 4 & 10 & $22: 51: 5212.534$ & 40.529 & 1.93 & 0.77 \\
\hline 2007 & 4 & 11 & $00: 01: 5712.390$ & 40.585 & 1.83 & 0.71 \\
\hline 2007 & 4 & 11 & $00: 11: 5412.443$ & 40.567 & 1.89 & 0.61 \\
\hline 2007 & 4 & 11 & $01: 25: 5412.473$ & 40.542 & 2.00 & 0.83 \\
\hline 2007 & 4 & 11 & $09: 12: 0812.565$ & 40.525 & 2.48 & 0.66 \\
\hline 2007 & 4 & 11 & $19: 07: 1310.831$ & 41.218 & 2.84 & 4.25 \\
\hline 2007 & 4 & 12 & $01: 11: 009.962$ & 41.304 & 2.98 & 5.68 \\
\hline 2007 & 4 & 12 & $18: 42: 1712.242$ & 40.663 & 2.13 & 1.32 \\
\hline 2007 & 4 & 12 & $20: 11: 3512.423$ & 40.582 & 2.05 & 0.72 \\
\hline 2007 & 4 & 12 & $22: 04: 0912.435$ & 40.562 & 2.00 & 1.23 \\
\hline 2007 & 4 & 12 & $23: 14: 5012.448$ & 40.569 & 1.97 & 0.57 \\
\hline 2007 & 4 & 13 & $10: 27: 1112.404$ & 40.578 & 2.12 & 0.48 \\
\hline 2007 & 4 & 13 & $18: 53: 5012.409$ & 40.575 & 2.19 & 0.53 \\
\hline 2007 & 4 & 14 & $02: 15: 0512.464$ & 40.554 & 2.02 & 0.72 \\
\hline 2007 & 4 & 14 & $21: 53: 5112.231$ & 40.663 & 1.81 & 0.86 \\
\hline 2007 & 4 & 14 & $22: 19: 2712.555$ & 40.516 & 1.90 & 0.83 \\
\hline 2007 & 4 & 15 & $13: 01: 5011.798$ & 41.108 & 2.15 & 1.69 \\
\hline 2007 & 4 & 15 & $14: 18: 1312.410$ & 40.574 & 2.22 & 0.86 \\
\hline 2007 & 4 & 15 & $20: 02: 3512.479$ & 40.566 & 1.96 & 1.54 \\
\hline 2007 & 4 & 15 & $21: 11: 1212.460$ & 40.560 & 1.99 & 0.81 \\
\hline 2007 & 4 & 16 & $00: 44: 3611.755$ & 41.153 & 2.23 & 0.97 \\
\hline 2007 & 4 & 16 & $01: 09: 5112.411$ & 40.582 & 1.89 & 0.59 \\
\hline 2007 & 4 & 16 & $03: 30: 4612.458$ & 40.555 & 2.18 & 0.62 \\
\hline 2007 & 4 & 16 & $07: 50: 2612.442$ & 40.563 & 2.24 & 0.59 \\
\hline 2007 & 4 & 16 & $13: 36: 0912.447$ & 40.549 & 2.04 & 0.95 \\
\hline 2007 & 4 & 16 & $19: 07: 4612.491$ & 40.532 & 1.92 & 0.73 \\
\hline 2007 & 4 & 16 & $20: 40: 5112.539$ & 40.530 & 1.92 & 0.71 \\
\hline 2007 & 4 & 16 & $23: 43: 0612.435$ & 40.567 & 1.76 & 0.94 \\
\hline 2007 & 4 & 17 & $00: 44: 4212.518$ & 40.535 & 1.84 & 0.78 \\
\hline 2007 & 4 & 17 & $22: 41: 5912.295$ & 40.587 & 1.85 & 2.05 \\
\hline 2007 & 4 & 17 & $22: 51: 4112.360$ & 40.595 & 1.95 & 0.84 \\
\hline 2007 & 4 & 17 & $22: 59: 1910.050$ & 41.355 & 2.96 & 8.08 \\
\hline 2007 & 4 & 17 & $23: 27: 0211.421$ & 41.403 & 2.59 & 1.98 \\
\hline 2007 & 4 & 18 & $00: 17: 0112.501$ & 40.538 & 1.89 & 0.68 \\
\hline 2007 & 4 & 18 & $01: 06: 3512.294$ & 40.598 & 1.80 & 0.49 \\
\hline 2007 & 4 & 18 & $03: 06: 1312.449$ & 40.558 & 2.06 & 1.44 \\
\hline 2007 & 4 & 18 & $03: 46: 4612.390$ & 40.582 & 2.15 & 0.51 \\
\hline 2007 & 4 & 18 & $20: 23: 5712.369$ & 40.587 & 1.84 & 1.09 \\
\hline 2007 & 4 & 18 & $21: 29: 1512.359$ & 40.590 & 1.80 & 0.75 \\
\hline 2007 & 4 & 18 & $22: 31: 0612.563$ & 40.523 & 1.87 & 0.75 \\
\hline 2007 & 4 & 18 & $23: 00: 4811.399$ & 41.412 & 2.15 & 4.78 \\
\hline 2007 & 4 & 18 & $23: 01: 4712.376$ & 40.588 & 1.71 & 0.6 \\
\hline 2007 & 4 & 18 & $23: 31: 1512.505$ & 40.536 & 1.86 & 0.76 \\
\hline 2007 & 4 & 18 & $23: 42: 2712.467$ & 40.551 & 1.92 & 0.62 \\
\hline 2007 & 4 & 19 & $00: 16: 1111.871$ & 41.066 & 1.89 & 0.8 \\
\hline 2007 & 4 & 19 & $00: 38: 0611.878$ & 41.072 & 1.84 & 1.03 \\
\hline
\end{tabular}




\begin{tabular}{|c|c|c|c|c|c|c|}
\hline 2007 & 4 & 19 & $02: 40: 2911.336$ & 41.453 & 2.44 & 1.89 \\
\hline 2007 & 4 & 19 & $19: 29: 5412.386$ & 40.587 & 1.92 & 0.58 \\
\hline 2007 & 4 & 20 & $04: 02: 2212.425$ & 40.585 & 2.07 & 0.87 \\
\hline 2007 & 4 & 20 & $23: 07: 0812.567$ & 40.522 & 1.91 & 0.81 \\
\hline 2007 & 4 & 20 & $23: 15: 2512.529$ & 40.529 & 1.81 & 0.74 \\
\hline 2007 & 4 & 21 & $01: 24: 5412.198$ & 40.685 & 1.79 & 2.07 \\
\hline 2007 & 4 & 21 & $01: 27: 0512.506$ & 40.539 & 1.97 & 0.75 \\
\hline 2007 & 4 & 21 & $02: 20: 5812.463$ & 40.555 & 2.02 & 0.63 \\
\hline 2007 & 4 & 21 & $20: 10: 0812.438$ & 40.554 & 1.90 & 1.34 \\
\hline 2007 & 4 & 21 & $22: 36: 1612.496$ & 40.539 & 1.75 & 1.8 \\
\hline 2007 & 4 & 21 & $22: 39: 2312.443$ & 40.553 & 1.80 & 1.48 \\
\hline 2007 & 4 & 21 & $23: 28: 0812.562$ & 40.525 & 1.81 & 0.73 \\
\hline 2007 & 4 & 21 & $23: 36: 2512.466$ & 40.553 & 1.82 & 0.63 \\
\hline 2007 & 4 & 22 & $00: 15: 5312.345$ & 40.607 & 1.71 & 0.61 \\
\hline 2007 & 4 & 22 & $00: 22: 4012.423$ & 40.575 & 1.81 & 0.61 \\
\hline 2007 & 4 & 22 & $00: 53: 0912.482$ & 40.544 & 1.80 & 0.79 \\
\hline 2007 & 4 & 22 & $01: 11: 5712.392$ & 40.589 & 1.87 & 0.59 \\
\hline 2007 & 4 & 22 & $19: 07: 0112.332$ & 40.609 & 1.88 & 0.63 \\
\hline 2007 & 4 & 22 & $19: 09: 0312.481$ & 40.538 & 1.93 & 1.45 \\
\hline 2007 & 4 & 23 & $20: 17: 3513.204$ & 39.889 & 2.67 & 3.9 \\
\hline 2007 & 4 & 24 & $00: 38: 5712.386$ & 40.583 & 1.96 & 0.7 \\
\hline 2007 & 4 & 24 & $01: 38: 0012.914$ & 40.277 & 2.53 & 2.87 \\
\hline 2007 & 4 & 24 & $18: 53: 0412.444$ & 40.556 & 1.99 & 0.71 \\
\hline 2007 & 4 & 24 & $21: 00: 3212.384$ & 40.588 & 2.22 & 0.62 \\
\hline 2007 & 4 & 24 & $22: 49: 0512.441$ & 40.559 & 1.97 & 1.12 \\
\hline 2007 & 4 & 24 & $23: 38: 5512.478$ & 40.545 & 1.95 & 0.6 \\
\hline 2007 & 4 & 25 & $20: 11: 1612.434$ & 40.561 & 2.06 & 1.06 \\
\hline 2007 & 4 & 25 & $23: 07: 4812.586$ & 40.494 & 2.34 & 0.7 \\
\hline 2007 & 4 & 27 & $18: 00: 4512.370$ & 40.587 & 2.10 & 0.64 \\
\hline 2007 & 4 & 27 & $21: 45: 1312.432$ & 40.563 & 1.88 & 0.93 \\
\hline 2007 & 4 & 28 & $06: 11: 2312.329$ & 40.609 & 2.28 & 0.55 \\
\hline 2007 & 4 & 28 & $19: 11: 3913.298$ & 39.712 & 2.61 & 7.46 \\
\hline 2007 & 4 & 28 & $19: 52: 4212.465$ & 40.555 & 2.01 & 0.76 \\
\hline 2007 & 4 & 28 & $21: 11: 5512.466$ & 40.553 & 1.91 & 0.71 \\
\hline 2007 & 4 & 28 & $21: 25: 4912.418$ & 40.586 & 1.85 & 0.62 \\
\hline 2007 & 4 & 28 & $22: 19: 1812.358$ & 40.596 & 1.83 & 0.94 \\
\hline 2007 & 4 & 29 & $19: 34: 4512.363$ & 40.600 & 1.89 & 0.74 \\
\hline 2007 & 4 & 29 & $20: 56: 2912.464$ & 40.548 & 1.88 & 0.71 \\
\hline 2007 & 4 & 29 & $23: 48: 0012.423$ & 40.566 & 1.85 & 0.97 \\
\hline 2007 & 4 & 30 & $01: 01: 5912.446$ & 40.554 & 1.84 & 1.72 \\
\hline 2007 & 4 & 30 & $07: 44: 4411.711$ & 41.190 & 2.61 & 1.29 \\
\hline 2007 & 4 & 30 & $23: 31: 3712.436$ & 40.563 & 1.88 & 0.6 \\
\hline 2007 & 5 & 1 & $13: 57: 1412.467$ & 40.562 & 2.18 & 0.78 \\
\hline 2007 & 5 & 1 & $22: 15: 3412.439$ & 40.572 & 1.99 & 0.65 \\
\hline 2007 & 5 & 2 & $00: 13: 3613.491$ & 40.789 & 2.46 & 5.41 \\
\hline 2007 & 5 & 2 & $20: 36: 5012.366$ & 40.596 & 1.81 & 0.58 \\
\hline 2007 & 5 & 2 & $23: 31: 4212.375$ & 40.589 & 1.82 & 0.49 \\
\hline 2007 & 5 & 3 & $00: 31: 5312.459$ & 40.542 & 1.70 & 0.66 \\
\hline 2007 & 5 & 3 & $02: 06: 2812.409$ & 40.574 & 1.87 & 1.4 \\
\hline 2007 & 5 & 3 & $17: 52: 3112.370$ & 40.599 & 1.85 & 0.87 \\
\hline 2007 & 5 & 3 & $18: 44: 3912.469$ & 40.545 & 2.02 & 0.58 \\
\hline 2007 & 5 & 3 & $19: 19: 5312.507$ & 40.541 & 1.98 & 0.77 \\
\hline 2007 & 5 & 4 & $00: 29: 0412.398$ & 40.577 & 1.67 & 0.65 \\
\hline 2007 & 5 & 4 & $01: 35: 2112.404$ & 40.593 & 1.77 & 0.57 \\
\hline 2007 & 5 & 4 & $22: 06: 3412.464$ & 40.554 & 1.81 & 0.58 \\
\hline 2007 & 5 & 4 & $22: 24: 2612.439$ & 40.558 & 1.71 & 0.65 \\
\hline 2007 & 5 & 5 & $01: 13: 4212.452$ & 40.559 & 1.80 & 1.1 \\
\hline 2007 & 5 & 5 & $01: 52: 0012.378$ & 40.588 & 1.87 & 0.83 \\
\hline 2007 & 5 & 5 & $05: 11: 4612.493$ & 40.539 & 2.11 & 0.99 \\
\hline 2007 & 5 & 5 & $22: 07: 4911.591$ & 42.685 & 2.71 & 4.04 \\
\hline 2007 & 5 & 6 & $00: 40: 4512.470$ & 40.535 & 1.78 & 1.91 \\
\hline 2007 & 5 & 6 & $00: 52: 4512.469$ & 40.553 & 1.99 & 0.56 \\
\hline 2007 & 5 & 6 & $22: 24: 2210.434$ & 40.003 & 2.75 & 2.07 \\
\hline 2007 & 5 & 6 & $23: 20: 1512.421$ & 40.564 & 1.90 & 2.07 \\
\hline 2007 & 5 & 7 & $00: 12: 0312.411$ & 40.564 & 1.78 & 1.03 \\
\hline 2007 & 5 & 7 & $00: 33: 5313.221$ & 39.826 & 2.39 & 5.52 \\
\hline 2007 & 5 & 7 & $01: 20: 1612.402$ & 40.581 & 1.80 & 1.31 \\
\hline 2007 & 5 & 7 & $19: 37: 1812.528$ & 40.489 & 1.89 & 1.81 \\
\hline 2007 & 5 & 8 & $15: 33: 0311.129$ & 39.693 & 3.17 & 14.29 \\
\hline 2007 & 5 & 8 & $19: 04: 2312.467$ & 40.550 & 1.96 & 0.54 \\
\hline 2007 & 5 & 8 & $23: 05: 0114.059$ & 39.911 & 2.88 & 6.14 \\
\hline 2007 & 5 & 9 & $19: 49: 2712.454$ & 40.557 & 1.87 & 0.68 \\
\hline 2007 & 5 & 10 & $01: 08: 4913.530$ & 40.857 & 2.74 & 19.97 \\
\hline 2007 & 5 & 10 & $19: 20: 3111.645$ & 41.004 & 2.04 & 0.76 \\
\hline 2007 & 5 & 10 & $20: 07: 4713.423$ & 40.862 & 2.34 & 5.58 \\
\hline 2007 & 5 & 11 & $00: 17: 5412.353$ & 40.590 & 1.69 & 0.51 \\
\hline
\end{tabular}




\begin{tabular}{|c|c|c|c|c|c|c|}
\hline 2007 & 5 & 11 & $01: 14: 0212.489$ & 40.535 & 1.83 & 2.16 \\
\hline 2007 & 5 & 11 & $22: 35: 0012.837$ & 40.618 & 2.08 & 0.88 \\
\hline 2007 & 5 & 11 & $22: 56: 2712.493$ & 40.532 & 1.83 & 1.7 \\
\hline 2007 & 5 & 12 & $00: 05: 2613.771$ & 39.778 & 3.75 & 5.41 \\
\hline 2007 & 5 & 12 & $19: 47: 0912.464$ & 40.555 & 2.38 & 0.56 \\
\hline 2007 & 5 & 13 & $16: 37: 0112.447$ & 40.564 & 2.17 & 1.04 \\
\hline 2007 & 5 & 13 & $20: 19: 0512.605$ & 40.494 & 2.10 & 1.03 \\
\hline 2007 & 5 & 13 & $20: 35: 2912.467$ & 40.551 & 2.13 & 0.91 \\
\hline 2007 & 5 & 13 & $22: 04: 5212.424$ & 40.582 & 1.90 & 0.84 \\
\hline 2007 & 5 & 13 & $22: 07: 0111.893$ & 41.359 & 2.30 & 0.66 \\
\hline 2007 & 5 & 14 & $19: 20: 0012.522$ & 40.535 & 1.95 & 0.67 \\
\hline 2007 & 5 & 15 & $00: 20: 4812.450$ & 40.562 & 2.00 & 0.54 \\
\hline 2007 & 5 & 15 & $22: 18: 5612.465$ & 40.550 & 1.88 & 0.61 \\
\hline 2007 & 5 & 15 & $22: 26: 5112.446$ & 40.556 & 1.81 & 0.85 \\
\hline 2007 & 5 & 15 & $22: 58: 5612.455$ & 40.565 & 1.85 & 0.8 \\
\hline 2007 & 5 & 15 & $23: 03: 3112.457$ & 40.559 & 1.93 & 1.12 \\
\hline 2007 & 5 & 16 & $00: 15: 5612.416$ & 40.570 & 1.79 & 1.19 \\
\hline 2007 & 5 & 16 & $21: 09: 4612.285$ & 40.630 & 1.87 & 1.16 \\
\hline 2007 & 5 & 16 & $22: 03: 2111.794$ & 41.098 & 2.23 & 1.04 \\
\hline 2007 & 5 & 16 & $22: 41: 0412.214$ & 40.676 & 1.78 & 0.61 \\
\hline 2007 & 5 & 17 & $00: 14: 3810.310$ & 40.561 & 2.80 & 1.86 \\
\hline 2007 & 5 & 17 & $00: 21: 4010.272$ & 40.575 & 4.22 & 1.54 \\
\hline 2007 & 5 & 17 & $00: 32: 4710.396$ & 40.531 & 3.38 & 5.86 \\
\hline 2007 & 5 & 17 & $01: 41: 3812.274$ & 40.629 & 2.19 & 0.84 \\
\hline 2007 & 5 & 17 & $02: 37: 5210.413$ & 40.602 & 3.66 & 2.98 \\
\hline 2007 & 5 & 17 & $22: 15: 5512.361$ & 40.600 & 1.76 & 0.9 \\
\hline 2007 & 5 & 17 & $22: 29: 2612.515$ & 40.510 & 1.79 & 3.64 \\
\hline 2007 & 5 & 18 & $00: 21: 4712.380$ & 40.599 & 1.66 & 0.77 \\
\hline 2007 & 5 & 18 & $21: 15: 2512.400$ & 40.572 & 2.43 & 1.59 \\
\hline 2007 & 5 & 18 & $23: 23: 3712.470$ & 40.543 & 1.83 & 1.57 \\
\hline 2007 & 5 & 18 & $23: 24: 3812.294$ & 40.597 & 1.91 & 0.5 \\
\hline 2007 & 5 & 18 & $23: 42: 4012.477$ & 40.552 & 1.96 & 0.75 \\
\hline 2007 & 5 & 19 & $06: 01: 5912.330$ & 40.611 & 2.31 & 1.11 \\
\hline 2007 & 5 & 19 & $22: 49: 3410.261$ & 40.563 & 2.97 & 2.13 \\
\hline 2007 & 5 & 19 & $23: 03: 1310.331$ & 40.558 & 2.97 & 9.74 \\
\hline 2007 & 5 & 19 & $23: 52: 3512.326$ & 40.623 & 1.78 & 0.59 \\
\hline 2007 & 5 & 20 & $00: 19: 2412.245$ & 40.653 & 1.94 & 0.58 \\
\hline 2007 & 5 & 20 & $21: 03: 3711.853$ & 39.638 & 2.50 & 4.06 \\
\hline 2007 & 5 & 20 & $21: 27: 0612.555$ & 40.528 & 2.09 & 0.62 \\
\hline 2007 & 5 & 21 & $00: 14: 4012.346$ & 40.627 & 1.91 & 0.71 \\
\hline 2007 & 5 & 21 & $01: 06: 1812.457$ & 40.547 & 1.89 & 1.12 \\
\hline 2007 & 5 & 21 & $21: 19: 5912.487$ & 40.535 & 1.98 & 1.2 \\
\hline 2007 & 5 & 21 & $21: 23: 1712.445$ & 40.562 & 1.88 & 0.65 \\
\hline 2007 & 5 & 21 & $22: 51: 4512.417$ & 40.587 & 1.80 & 0.64 \\
\hline 2007 & 5 & 22 & $00: 25: 1010.269$ & 41.420 & 2.66 & 10.58 \\
\hline 2007 & 5 & 22 & $01: 11: 3812.434$ & 40.568 & 1.77 & 2.27 \\
\hline 2007 & 5 & 22 & $20: 13: 5512.503$ & 40.572 & 1.99 & 1.15 \\
\hline 2007 & 5 & 22 & $20: 23: 0612.389$ & 40.589 & 1.88 & 1.28 \\
\hline 2007 & 5 & 22 & $21: 05: 5312.393$ & 40.554 & 1.81 & 0.91 \\
\hline 2007 & 5 & 22 & $21: 25: 3312.470$ & 40.551 & 1.79 & 0.53 \\
\hline 2007 & 5 & 22 & $22: 49: 0912.376$ & 40.603 & 1.82 & 0.73 \\
\hline 2007 & 5 & 23 & $00: 17: 2312.409$ & 40.570 & 1.72 & 0.78 \\
\hline 2007 & 5 & 23 & $18: 40: 0612.360$ & 40.355 & 1.89 & 0.75 \\
\hline 2007 & 5 & 23 & $18: 50: 3212.359$ & 40.354 & 1.85 & 0.69 \\
\hline 2007 & 5 & 23 & $19: 15: 5812.363$ & 40.340 & 1.68 & 8.21 \\
\hline 2007 & 5 & 23 & $19: 42: 2912.367$ & 40.344 & 1.71 & 8.55 \\
\hline 2007 & 5 & 23 & $20: 47: 2412.369$ & 40.294 & 1.87 & 1.47 \\
\hline 2007 & 5 & 23 & $22: 56: 3812.918$ & 40.246 & 2.06 & 1.87 \\
\hline 2007 & 5 & 24 & $01: 03: 1212.406$ & 40.581 & 1.76 & 0.58 \\
\hline 2007 & 5 & 24 & $18: 57: 0812.493$ & 40.539 & 2.14 & 0.75 \\
\hline 2007 & 5 & 24 & $22: 50: 1812.464$ & 40.556 & 1.77 & 1.21 \\
\hline 2007 & 5 & 24 & $23: 46: 2812.553$ & 40.534 & 1.92 & 0.7 \\
\hline 2007 & 5 & 25 & $01: 39: 3212.393$ & 40.591 & 1.94 & 0.61 \\
\hline 2007 & 5 & 25 & $04: 42: 5212.500$ & 40.550 & 2.17 & 0.87 \\
\hline 2007 & 5 & 25 & $09: 15: 0711.394$ & 39.689 & 3.53 & 18.6 \\
\hline 2007 & 5 & 25 & $09: 17: 0011.401$ & 39.691 & 3.31 & 18.72 \\
\hline 2007 & 5 & 25 & $14: 43: 2012.491$ & 40.530 & 2.15 & 1.09 \\
\hline 2007 & 5 & 25 & $15: 16: 5212.357$ & 40.601 & 2.39 & 0.62 \\
\hline 2007 & 5 & 25 & $21: 23: 1313.388$ & 40.850 & 2.28 & 5.05 \\
\hline 2007 & 5 & 25 & $22: 54: 4412.508$ & 40.540 & 1.87 & 0.62 \\
\hline 2007 & 5 & 25 & $23: 36: 3511.825$ & 41.019 & 1.60 & 1.34 \\
\hline 2007 & 5 & 25 & $23: 38: 5313.379$ & 40.862 & 2.16 & 3.44 \\
\hline 2007 & 5 & 26 & $00: 05: 1512.360$ & 40.607 & 2.09 & 0.62 \\
\hline 2007 & 5 & 26 & $00: 12: 3413.329$ & 40.862 & 2.15 & 3.56 \\
\hline 2007 & 5 & 26 & $00: 38: 4313.379$ & 40.832 & 2.22 & 4.4 \\
\hline 2007 & 5 & 26 & $08: 43: 3912.374$ & 40.595 & 2.21 & 0.72 \\
\hline
\end{tabular}




\begin{tabular}{|c|c|c|c|c|c|c|}
\hline 2007 & 5 & 27 & $08: 07: 1412.453$ & 40.564 & 2.21 & 0.58 \\
\hline 2007 & 5 & 27 & $09: 29: 4212.410$ & 40.589 & 2.22 & 0.7 \\
\hline 2007 & 5 & 28 & $00: 15: 5912.463$ & 40.560 & 1.97 & 0.67 \\
\hline 2007 & 5 & 28 & $00: 35: 4212.470$ & 40.549 & 1.95 & 0.63 \\
\hline 2007 & 5 & 28 & $01: 49: 5612.523$ & 40.534 & 1.91 & 1.17 \\
\hline 2007 & 5 & 28 & $08: 25: 1611.428$ & 39.668 & 3.08 & 19.19 \\
\hline 2007 & 5 & 28 & $11: 32: 2112.438$ & 40.574 & 2.31 & 0.79 \\
\hline 2007 & 5 & 28 & $16: 23: 4513.414$ & 40.866 & 2.43 & 6.99 \\
\hline 2007 & 5 & 28 & $22: 31: 0013.404$ & 40.868 & 2.39 & 5.21 \\
\hline 2007 & 5 & 28 & $22: 57: 4612.420$ & 40.562 & 1.88 & 0.87 \\
\hline 2007 & 5 & 28 & $23: 42: 0611.797$ & 41.099 & 1.86 & 0.74 \\
\hline 2007 & 5 & 29 & $00: 07: 5712.384$ & 40.583 & 1.85 & 1.35 \\
\hline 2007 & 5 & 29 & $01: 05: 2812.222$ & 40.668 & 1.95 & 0.93 \\
\hline 2007 & 5 & 29 & $01: 20: 5712.406$ & 40.589 & 1.87 & 0.63 \\
\hline 2007 & 5 & 29 & $16: 43: 0113.492$ & 40.834 & 3.07 & 5.18 \\
\hline 2007 & 5 & 29 & $22: 16: 2013.428$ & 40.871 & 2.62 & 2.1 \\
\hline 2007 & 5 & 29 & $22: 21: 1213.426$ & 40.889 & 2.62 & 2.07 \\
\hline 2007 & 5 & 29 & $23: 30: 1812.394$ & 40.581 & 1.80 & 1.15 \\
\hline 2007 & 5 & 30 & $00: 55: 1112.454$ & 40.559 & 2.01 & 0.68 \\
\hline 2007 & 5 & 30 & $02: 03: 2612.737$ & 40.615 & 2.12 & 0.74 \\
\hline 2007 & 5 & 30 & $02: 09: 4012.502$ & 40.547 & 1.91 & 1.81 \\
\hline 2007 & 5 & 30 & $23: 53: 5612.466$ & 40.554 & 1.82 & 0.66 \\
\hline 2007 & 5 & 31 & $00: 22: 5013.415$ & 40.864 & 2.46 & 2.28 \\
\hline 2007 & 5 & 31 & $00: 44: 2212.414$ & 40.576 & 1.93 & 0.79 \\
\hline 2007 & 5 & 31 & $19: 29: 5312.465$ & 40.559 & 2.14 & 0.55 \\
\hline 2007 & 5 & 31 & $21: 51: 5912.582$ & 40.480 & 2.02 & 0.97 \\
\hline 2007 & 6 & 1 & $20: 54: 2312.497$ & 40.533 & 1.95 & 6.57 \\
\hline 2007 & 6 & 2 & $22: 20: 5412.575$ & 40.521 & 2.27 & 0.87 \\
\hline 2007 & 6 & 3 & $00: 50: 0412.453$ & 40.550 & 1.84 & 1.25 \\
\hline 2007 & 6 & 3 & $22: 47: 2812.428$ & 40.572 & 2.21 & 0.6 \\
\hline 2007 & 6 & 4 & $10: 04: 2810.297$ & 40.727 & 3.66 & 1.69 \\
\hline 2007 & 6 & 4 & $18: 07: 1112.443$ & 40.565 & 2.14 & 0.91 \\
\hline 2007 & 6 & 7 & $01: 52: 0912.365$ & 40.596 & 2.15 & 0.63 \\
\hline 2007 & 6 & 8 & $00: 42: 4511.855$ & 41.083 & 1.94 & 1.41 \\
\hline 2007 & 6 & 9 & $22: 21: 5812.441$ & 40.568 & 1.96 & 0.68 \\
\hline 2007 & 6 & 10 & $01: 08: 5011.895$ & 41.062 & 1.87 & 4.27 \\
\hline 2007 & 6 & 10 & $18: 46: 2811.515$ & 39.714 & 2.54 & 5.58 \\
\hline 2007 & 6 & 10 & $21: 37: 0711.499$ & 39.702 & 2.55 & 5.74 \\
\hline 2007 & 6 & 13 & $21: 46: 4212.503$ & 40.550 & 2.17 & 0.69 \\
\hline 2007 & 6 & 13 & $22: 06: 3312.445$ & 40.573 & 2.02 & 1.16 \\
\hline 2007 & 6 & 15 & $04: 28: 5713.454$ & 40.092 & 3.42 & 4.74 \\
\hline 2007 & 6 & 15 & $08: 41: 4612.501$ & 40.534 & 2.54 & 1.82 \\
\hline 2007 & 6 & 15 & $22: 03: 3213.349$ & 40.995 & 3.11 & 2.71 \\
\hline 2007 & 6 & 15 & $22: 54: 0912.437$ & 40.566 & 2.08 & 0.59 \\
\hline 2007 & 6 & 16 & $01: 07: 5012.445$ & 40.548 & 1.91 & 1.72 \\
\hline 2007 & 6 & 17 & $00: 04: 2411.951$ & 40.981 & 1.84 & 0.64 \\
\hline 2007 & 6 & 17 & $01: 44: 4612.457$ & 40.548 & 2.10 & 1.77 \\
\hline 2007 & 6 & 17 & $23: 12: 0912.525$ & 40.528 & 1.90 & 0.7 \\
\hline 2007 & 6 & 18 & $01: 23: 2612.454$ & 40.559 & 2.37 & 0.56 \\
\hline 2007 & 6 & 18 & $21: 18: 0212.456$ & 40.560 & 2.30 & 0.55 \\
\hline 2007 & 6 & 19 & $00: 28: 2312.502$ & 40.532 & 2.20 & 0.72 \\
\hline 2007 & 6 & 19 & $02: 17: 5712.085$ & 41.523 & 2.83 & 0.93 \\
\hline 2007 & 6 & 20 & $00: 29: 3512.149$ & 40.895 & 2.17 & 0.85 \\
\hline 2007 & 6 & 21 & $22: 29: 1312.514$ & 40.535 & 2.08 & 0.7 \\
\hline 2007 & 6 & 22 & $20: 56: 4112.434$ & 40.562 & 2.22 & 0.89 \\
\hline 2007 & 6 & 22 & $22: 41: 1212.453$ & 40.550 & 1.93 & 1.29 \\
\hline 2007 & 6 & 22 & $22: 43: 0112.486$ & 40.529 & 1.92 & 0.79 \\
\hline 2007 & 6 & 28 & $02: 46: 1812.582$ & 40.519 & 2.34 & 1.08 \\
\hline 2007 & 7 & 1 & $00: 04: 4012.431$ & 40.577 & 1.89 & 1.02 \\
\hline 2007 & 7 & 1 & $04: 25: 5212.443$ & 40.576 & 2.21 & 0.83 \\
\hline 2007 & 7 & 1 & $22: 08: 0712.387$ & 40.596 & 1.89 & 1.07 \\
\hline 2007 & 7 & 2 & $00: 49: 1112.463$ & 40.550 & 1.87 & 0.97 \\
\hline 2007 & 7 & 2 & $12: 35: 3012.419$ & 40.573 & 2.20 & 0.8 \\
\hline 2007 & 7 & 2 & $21: 40: 5112.274$ & 40.631 & 1.89 & 1.12 \\
\hline 2007 & 7 & 4 & $11: 44: 0412.204$ & 40.678 & 2.06 & 0.88 \\
\hline 2007 & 7 & 4 & $19: 52: 3612.311$ & 40.620 & 2.09 & 0.48 \\
\hline 2007 & 7 & 4 & $22: 20: 4412.434$ & 40.569 & 2.01 & 0.84 \\
\hline 2007 & 7 & 5 & $02: 20: 4812.380$ & 40.594 & 1.98 & 0.54 \\
\hline 2007 & 7 & 5 & $11: 10: 2113.529$ & 39.970 & 2.77 & 8.8 \\
\hline 2007 & 7 & 5 & $16: 47: 5512.485$ & 40.534 & 2.12 & 2.3 \\
\hline 2007 & 7 & 5 & $18: 08: 4912.464$ & 40.542 & 2.08 & 0.56 \\
\hline 2007 & 7 & 5 & $22: 06: 1312.506$ & 40.533 & 2.06 & 0.62 \\
\hline 2007 & 7 & 5 & $23: 10: 5812.450$ & 40.562 & 1.83 & 0.76 \\
\hline 2007 & 7 & 5 & $23: 42: 1012.509$ & 40.535 & 1.97 & 0.87 \\
\hline 2007 & 7 & 6 & $00: 17: 4112.402$ & 40.578 & 1.90 & 0.63 \\
\hline 2007 & 7 & 6 & $01: 41: 2212.493$ & 40.636 & 1.87 & 1.76 \\
\hline
\end{tabular}




\begin{tabular}{|c|c|c|c|c|c|c|}
\hline 2007 & 7 & 6 & $09: 45: 2812.439$ & 40.559 & 2.15 & 0.56 \\
\hline 2007 & 7 & 6 & $11: 47: 4512.510$ & 40.538 & 2.26 & 1.72 \\
\hline 2007 & 7 & 6 & $12: 46: 2211.851$ & 41.054 & 2.17 & 1.22 \\
\hline 2007 & 7 & 6 & $16: 16: 1312.329$ & 40.606 & 2.20 & 0.48 \\
\hline 2007 & 7 & 6 & $20: 54: 4012.438$ & 40.569 & 2.08 & 0.51 \\
\hline 2007 & 7 & 7 & $04: 10: 1612.428$ & 40.570 & 2.16 & 0.87 \\
\hline 2007 & 7 & 7 & $19: 34: 5812.436$ & 40.562 & 2.39 & 0.54 \\
\hline 2007 & 7 & 7 & $20: 13: 4312.475$ & 40.551 & 2.05 & 0.58 \\
\hline 2007 & 7 & 8 & $00: 28: 5912.457$ & 40.544 & 1.90 & 1.24 \\
\hline 2007 & 7 & 8 & $01: 31: 3512.490$ & 40.530 & 1.89 & 1.97 \\
\hline 2007 & 7 & 8 & $18: 31: 3010.302$ & 40.541 & 4.51 & 1.61 \\
\hline 2007 & 7 & 8 & $18: 53: 1310.505$ & 40.381 & 3.34 & 9.46 \\
\hline 2007 & 7 & 8 & $19: 14: 4410.275$ & 40.546 & 4.55 & 6.46 \\
\hline 2007 & 7 & 8 & $20: 07: 3810.271$ & 40.527 & 4.51 & 9.02 \\
\hline 2007 & 7 & 8 & $20: 23: 2610.268$ & 40.559 & 3.54 & 2.93 \\
\hline 2007 & 7 & 8 & $20: 34: 4010.268$ & 40.557 & 3.95 & 1.51 \\
\hline 2007 & 7 & 8 & $20: 40: 5110.304$ & 40.545 & 3.42 & 3.05 \\
\hline 2007 & 7 & 8 & $21: 09: 1710.265$ & 40.587 & 3.99 & 3.06 \\
\hline 2007 & 7 & 8 & $21: 14: 2310.989$ & 40.546 & 2.80 & 12.04 \\
\hline 2007 & 7 & 8 & $21: 22: 3510.343$ & 40.549 & 3.65 & 2.25 \\
\hline 2007 & 7 & 8 & $21: 57: 2310.371$ & 40.434 & 3.28 & 6.63 \\
\hline 2007 & 7 & 9 & $00: 06: 3410.355$ & 40.544 & 3.30 & 2.33 \\
\hline 2007 & 7 & 9 & $03: 35: 4110.302$ & 40.544 & 3.59 & 3.31 \\
\hline 2007 & 7 & 9 & $05: 36: 1810.419$ & 40.489 & 3.29 & 9.04 \\
\hline 2007 & 7 & 9 & $05: 51: 2710.405$ & 40.525 & 3.32 & 8.02 \\
\hline 2007 & 7 & 9 & $14: 58: 1112.416$ & 40.575 & 2.11 & 0.76 \\
\hline 2007 & 7 & 9 & $19: 40: 5712.204$ & 40.677 & 2.15 & 0.93 \\
\hline 2007 & 7 & 9 & $22: 55: 4812.396$ & 40.576 & 1.99 & 0.81 \\
\hline 2007 & 7 & 10 & $20: 31: 3910.276$ & 40.543 & 3.19 & 3.72 \\
\hline 2007 & 7 & 12 & $22: 26: 2812.200$ & 41.006 & 1.84 & 6.08 \\
\hline 2007 & 7 & 13 & $08: 21: 0612.425$ & 40.576 & 2.18 & 3.86 \\
\hline 2007 & 7 & 14 & $19: 19: 5112.412$ & 40.587 & 1.86 & 0.84 \\
\hline 2007 & 7 & 14 & $20: 26: 5812.465$ & 40.557 & 2.19 & 0.8 \\
\hline 2007 & 7 & 14 & $23: 25: 3712.468$ & 40.537 & 1.82 & 1.79 \\
\hline 2007 & 7 & 16 & $00: 27: 5010.353$ & 40.545 & 2.66 & 2.48 \\
\hline 2007 & 7 & 16 & $21: 31: 4512.475$ & 40.545 & 2.09 & 0.88 \\
\hline 2007 & 7 & 17 & $00: 25: 1112.466$ & 40.555 & 2.30 & 0.51 \\
\hline 2007 & 7 & 17 & $06: 38: 4013.365$ & 40.896 & 4.46 & 2.88 \\
\hline 2007 & 7 & 17 & $07: 12: 0513.372$ & 40.895 & 3.50 & 6,00 \\
\hline 2007 & 7 & 17 & $12: 45: 4913.390$ & 40.848 & 3.04 & 5.6 \\
\hline 2007 & 7 & 17 & $16: 47: 3613.378$ & 40.938 & 3.74 & 4.19 \\
\hline 2007 & 7 & 17 & $16: 57: 5813.362$ & 40.904 & 3.74 & 3.14 \\
\hline 2007 & 7 & 17 & $19: 26: 1713.387$ & 40.920 & 2.84 & 8.9 \\
\hline 2007 & 7 & 17 & $21: 52: 1712.474$ & 40.560 & 2.15 & 0.65 \\
\hline 2007 & 7 & 18 & $03: 47: 0113.390$ & 40.904 & 2.98 & 8.02 \\
\hline 2007 & 7 & 18 & $06: 31: 0413.379$ & 40.935 & 3.31 & 8.15 \\
\hline 2007 & 7 & 18 & $12: 09: 4113.389$ & 40.906 & 3.26 & 8.65 \\
\hline 2007 & 7 & 18 & $16: 12: 0911.468$ & 41.048 & 2.36 & 1.55 \\
\hline 2007 & 7 & 18 & $18: 29: 2311.481$ & 41.033 & 2.04 & 1.52 \\
\hline 2007 & 7 & 19 & $13: 59: 1313.360$ & 40.951 & 3.74 & 6.49 \\
\hline 2007 & 7 & 19 & $20: 07: 2813.383$ & 40.910 & 2.73 & 2.93 \\
\hline 2007 & 7 & 19 & $22: 50: 1413.370$ & 40.945 & 3.12 & 5.77 \\
\hline 2007 & 7 & 20 & $00: 39: 4712.472$ & 40.555 & 1.79 & 2.33 \\
\hline 2007 & 7 & 20 & $01: 24: 4912.517$ & 40.510 & 1.95 & 1.51 \\
\hline 2007 & 7 & 20 & $01: 37: 0512.509$ & 40.537 & 1.93 & 0.65 \\
\hline 2007 & 7 & 20 & $01: 50: 2813.303$ & 40.912 & 2.33 & 1.41 \\
\hline 2007 & 7 & 20 & $02: 01: 3913.368$ & 40.940 & 3.07 & 1.21 \\
\hline 2007 & 7 & 20 & $02: 10: 1313.354$ & 40.951 & 2.45 & 6.95 \\
\hline 2007 & 7 & 20 & $03: 15: 0212.483$ & 40.538 & 1.99 & 0.73 \\
\hline 2007 & 7 & 20 & $04: 37: 3913.396$ & 40.882 & 2.74 & 7.74 \\
\hline 2007 & 7 & 20 & $22: 39: 1512.367$ & 40.598 & 1.84 & 0.8 \\
\hline 2007 & 7 & 20 & $23: 08: 5113.369$ & 40.906 & 2.51 & 1.65 \\
\hline 2007 & 7 & 21 & $20: 22: 0813.399$ & 40.842 & 2.39 & 8.4 \\
\hline 2007 & 7 & 21 & $21: 53: 0812.415$ & 40.569 & 1.92 & 0.76 \\
\hline 2007 & 7 & 22 & $00: 16: 0713.393$ & 40.925 & 2.80 & 1.14 \\
\hline 2007 & 7 & 22 & $00: 42: 4012.432$ & 40.564 & 1.79 & 1.93 \\
\hline 2007 & 7 & 22 & $01: 10: 0412.492$ & 40.534 & 1.85 & 0.57 \\
\hline 2007 & 7 & 22 & $01: 18: 5712.456$ & 40.550 & 1.92 & 1.66 \\
\hline 2007 & 7 & 22 & $18: 40: 4612.383$ & 40.589 & 2.04 & 0.77 \\
\hline 2007 & 7 & 23 & $00: 44: 5711.607$ & 42.167 & 2.55 & 9.45 \\
\hline 2007 & 7 & 23 & $00: 58: 1913.037$ & 39.664 & 2.43 & \\
\hline 2007 & 7 & 23 & $20: 58: 1713.377$ & 40.942 & 2.64 & 5.15 \\
\hline 2007 & 7 & 23 & $22: 15: 5012.489$ & 40.538 & 1.93 & 1.64 \\
\hline 2007 & 7 & 23 & $23: 29: 4712.478$ & 40.547 & 1.91 & 0.65 \\
\hline 2007 & 7 & 24 & $20: 07: 5713.377$ & 40.922 & 3.38 & 4.81 \\
\hline 2007 & 7 & 24 & $20: 58: 1713.377$ & 40.942 & 2.35 & 5.15 \\
\hline
\end{tabular}




\begin{tabular}{|c|c|c|c|c|c|c|}
\hline 2007 & 7 & 24 & $22: 15: 5012.482$ & 40.543 & 1.79 & 1.71 \\
\hline 2007 & 7 & 24 & $23: 46: 4111.153$ & 39.999 & 2.24 & 5.5 \\
\hline 2007 & 7 & 25 & $18: 58: 5813.375$ & 40.929 & 2.91 & 2.7 \\
\hline 2007 & 7 & 25 & $21: 27: 0612.445$ & 40.552 & 2.03 & 1.48 \\
\hline 2007 & 7 & 26 & $00: 26: 2512.438$ & 40.570 & 2.20 & 1.04 \\
\hline 2007 & 7 & 26 & $01: 28: 3113.405$ & 40.923 & 2.98 & 2,00 \\
\hline 2007 & 7 & 27 & $02: 20: 4512.877$ & 40.269 & 2.59 & 6.36 \\
\hline 2007 & 7 & 29 & $06: 41: 2713.351$ & 40.920 & 3.52 & 3.05 \\
\hline 2007 & 7 & 30 & $22: 27: 2111.840$ & 41.067 & 2.12 & 1.96 \\
\hline 2007 & 7 & 31 & $04: 18: 2012.441$ & 40.560 & 2.38 & 0.7 \\
\hline 2007 & 7 & 31 & $23: 01: 0112.504$ & 40.537 & 2.14 & 1.12 \\
\hline 2007 & 8 & 2 & $00: 08: 5011.876$ & 40.892 & 1.72 & 1.04 \\
\hline 2007 & 8 & 2 & $00: 18: 1711.868$ & 40.894 & 1.76 & 1.29 \\
\hline 2007 & 8 & 2 & $06: 41: 4112.827$ & 40.606 & 2.50 & 0.62 \\
\hline 2007 & 8 & 2 & $20: 57: 2512.404$ & 40.580 & 1.93 & 0.77 \\
\hline 2007 & 8 & 2 & $22: 25: 5412.439$ & 40.559 & 1.84 & 0.74 \\
\hline 2007 & 8 & 3 & $21: 51: 2111.883$ & 40.941 & 1.81 & 1.18 \\
\hline 2007 & 8 & 4 & $00: 35: 0912.285$ & 40.607 & 1.82 & 2.31 \\
\hline 2007 & 8 & 4 & $01: 53: 4812.423$ & 40.413 & 1.80 & 12.47 \\
\hline 2007 & 8 & 4 & $02: 28: 3312.367$ & 40.603 & 1.97 & 0.81 \\
\hline 2007 & 8 & 4 & $13: 43: 4712.895$ & 40.241 & 2.58 & 1.79 \\
\hline 2007 & 8 & 4 & $23: 42: 1911.777$ & 41.115 & 1.89 & 2.15 \\
\hline 2007 & 8 & 5 & $01: 04: 0211.796$ & 41.117 & 2.01 & 1.08 \\
\hline 2007 & 8 & 5 & $01: 09: 4412.432$ & 40.566 & 1.89 & 0.59 \\
\hline 2007 & 8 & 6 & $00: 25: 3212.511$ & 40.554 & 1.83 & 0.82 \\
\hline 2007 & 8 & 6 & $19: 47: 3012.492$ & 40.531 & 1.92 & 1.58 \\
\hline 2007 & 8 & 6 & $20: 55: 1212.419$ & 40.586 & 1.93 & 0.78 \\
\hline 2007 & 8 & 6 & $21: 46: 2711.880$ & 40.935 & 1.80 & 0.83 \\
\hline 2007 & 8 & 7 & $00: 30: 5711.800$ & 41.118 & 2.24 & 1.77 \\
\hline 2007 & 8 & 7 & $00: 31: 1311.856$ & 41.040 & 2.21 & 12.43 \\
\hline 2007 & 8 & 7 & $05: 17: 2611.796$ & 41.122 & 2.74 & 1.44 \\
\hline 2007 & 8 & 7 & $07: 51: 27 \quad 13.542$ & 40.027 & 3.23 & 8.86 \\
\hline 2007 & 8 & 7 & $14: 36: 2913.489$ & 40.029 & 3.22 & 6.9 \\
\hline 2007 & 8 & 7 & $19: 37: 1712.383$ & 40.581 & 1.90 & 1.23 \\
\hline 2007 & 8 & 8 & $17: 10: 0312.092$ & 40.749 & 2.03 & 1.64 \\
\hline 2007 & 8 & 9 & $22: 47: 2912.505$ & 40.534 & 2.21 & 0.69 \\
\hline 2007 & 8 & 10 & $02: 05: 4212.457$ & 40.548 & 1.98 & 3.69 \\
\hline 2007 & 8 & 10 & $23: 09: 2812.456$ & 40.556 & 1.90 & 0.79 \\
\hline 2007 & 8 & 11 & $01: 19: 1612.514$ & 40.533 & 1.93 & 0.92 \\
\hline 2007 & 8 & 11 & $23: 23: 2310.891$ & 40.874 & 2.31 & 11.37 \\
\hline 2007 & 8 & 11 & $23: 29: 5812.321$ & 40.602 & 1.84 & 1.1 \\
\hline 2007 & 8 & 12 & $01: 17: 4712.171$ & 40.654 & 1.83 & 12.4 \\
\hline 2007 & 8 & 12 & $07: 48: 0212.291$ & 40.620 & 2.35 & 0.94 \\
\hline 2007 & 8 & 12 & $09: 39: 5912.296$ & 40.618 & 2.61 & 1.05 \\
\hline 2007 & 8 & 12 & $09: 40: 5012.310$ & 40.619 & 2.93 & 0.56 \\
\hline 2007 & 8 & 12 & $09: 41: 3112.299$ & 40.620 & 2.85 & 0.58 \\
\hline 2007 & 8 & 12 & $10: 01: 3112.310$ & 40.606 & 2.23 & 0.64 \\
\hline 2007 & 8 & 12 & $10: 02: 3112.299$ & 40.599 & 2.13 & 3.86 \\
\hline 2007 & 8 & 12 & $10: 03: 0012.327$ & 40.598 & 2.13 & 0.8 \\
\hline 2007 & 8 & 12 & $10: 06: 3312.270$ & 40.626 & 2.54 & 0.56 \\
\hline 2007 & 8 & 12 & $10: 08: 4612.595$ & 39.995 & 2.56 & 4.83 \\
\hline 2007 & 8 & 12 & $10: 09: 3512.332$ & 40.539 & 2.31 & 1.29 \\
\hline 2007 & 8 & 12 & $10: 11: 4812.311$ & 40.634 & 2.20 & 2.51 \\
\hline 2007 & 8 & 12 & $10: 13: 5712.297$ & 40.603 & 2.16 & 0.76 \\
\hline 2007 & 8 & 12 & $10: 14: 5912.308$ & 40.644 & 2.16 & 1.58 \\
\hline 2007 & 8 & 12 & $10: 17: 3912.310$ & 40.619 & 2.34 & 1.22 \\
\hline 2007 & 8 & 12 & $10: 17: 5912.316$ & 40.599 & 2.52 & 0.74 \\
\hline 2007 & 8 & 12 & $10: 21: 1812.333$ & 40.583 & 2.22 & 1.34 \\
\hline 2007 & 8 & 12 & $10: 24: 0012.300$ & 40.612 & 2.13 & 1.76 \\
\hline 2007 & 8 & 12 & $10: 25: 2312.322$ & 40.630 & 2.49 & 0.89 \\
\hline 2007 & 8 & 12 & $10: 34: 3012.366$ & 40.612 & 2.64 & 0.71 \\
\hline 2007 & 8 & 12 & $10: 40: 4012.232$ & 40.658 & 2.33 & 2.11 \\
\hline 2007 & 8 & 12 & $10: 44: 1312.329$ & 40.618 & 2.16 & 1.08 \\
\hline 2007 & 8 & 12 & $10: 48: 4512.316$ & 40.609 & 2.47 & 0.52 \\
\hline 2007 & 8 & 12 & $10: 51: 5812.368$ & 40.586 & 3.03 & 0.55 \\
\hline 2007 & 8 & 12 & $10: 58: 2012.317$ & 40.604 & 2.77 & 0.57 \\
\hline 2007 & 8 & 12 & $11: 03: 1512.361$ & 40.593 & 2.26 & 0.59 \\
\hline 2007 & 8 & 12 & $11: 21: 5912.322$ & 40.608 & 2.53 & 0.64 \\
\hline 2007 & 8 & 12 & $11: 36: 3412.336$ & 40.590 & 2.73 & 0.61 \\
\hline 2007 & 8 & 12 & $11: 52: 4512.242$ & 40.671 & 2.48 & 0.7 \\
\hline 2007 & 8 & 12 & $11: 56: 5712.222$ & 40.676 & 2.32 & 1.53 \\
\hline 2007 & 8 & 12 & $12: 09: 0112.227$ & 40.675 & 2.29 & 0.89 \\
\hline 2007 & 8 & 12 & $12: 10: 1012.355$ & 40.609 & 2.15 & 0.94 \\
\hline 2007 & 8 & 12 & $12: 28: 0512.258$ & 40.645 & 2.18 & 1.12 \\
\hline 2007 & 8 & 12 & $12: 40: 1112.346$ & 40.596 & 2.27 & 0.88 \\
\hline 2007 & 8 & 12 & $12: 44: 0512.362$ & 40.603 & 2.64 & 0.61 \\
\hline
\end{tabular}




\begin{tabular}{|c|c|c|c|c|c|c|}
\hline 2007 & 8 & 12 & $13: 25: 3912.255$ & 40.643 & 2.15 & 0.99 \\
\hline 2007 & 8 & 12 & $13: 31: 0812.225$ & 40.673 & 2.28 & 0.72 \\
\hline 2007 & 8 & 12 & $13: 41: 4612.428$ & 40.584 & 2.45 & 0.88 \\
\hline 2007 & 8 & 12 & $13: 50: 4312.351$ & 40.595 & 2.20 & 0.99 \\
\hline 2007 & 8 & 12 & $14: 34: 1312.239$ & 40.642 & 2.31 & 1.44 \\
\hline 2007 & 8 & 12 & $18: 35: 3212.349$ & 40.605 & 2.03 & 0.85 \\
\hline 2007 & 8 & 12 & $19: 10: 4012.423$ & 40.591 & 1.98 & 0.93 \\
\hline 2007 & 8 & 12 & $19: 11: 3712.423$ & 40.590 & 1.99 & 0.81 \\
\hline 2007 & 8 & 12 & $22: 29: 4612.348$ & 40.600 & 1.73 & 0.83 \\
\hline 2007 & 8 & 12 & $23: 06: 0212.306$ & 40.615 & 1.78 & 0.96 \\
\hline 2007 & 8 & 12 & $23: 17: 4712.242$ & 40.662 & 1.92 & 0.74 \\
\hline 2007 & 8 & 12 & $23: 49: 5512.190$ & 40.666 & 1.99 & 0.4 \\
\hline 2007 & 8 & 13 & $00: 28: 0512.334$ & 40.587 & 1.89 & 0.49 \\
\hline 2007 & 8 & 13 & $01: 56: 5412.380$ & 40.600 & 2.09 & 3.6 \\
\hline 2007 & 8 & 13 & $19: 39: 1712.497$ & 40.529 & 1.88 & 0.67 \\
\hline 2007 & 8 & 13 & $22: 51: 4912.528$ & 40.509 & 2.12 & 0.73 \\
\hline 2007 & 8 & 14 & $00: 20: 5912.336$ & 40.606 & 1.76 & 0.7 \\
\hline 2007 & 8 & 14 & $03: 20: 0012.364$ & 40.572 & 2.08 & 0.71 \\
\hline 2007 & 8 & 14 & $09: 36: 2012.297$ & 40.627 & 2.72 & 0.55 \\
\hline 2007 & 8 & 14 & $18: 46: 4012.870$ & 40.635 & 2.87 & 0.7 \\
\hline 2007 & 8 & 15 & $02: 07: 3912.462$ & 40.552 & 1.79 & 5.75 \\
\hline 2007 & 8 & 15 & $03: 09: 4812.559$ & 40.526 & 1.98 & 0.72 \\
\hline 2007 & 8 & 15 & $03: 21: 2712.056$ & 41.280 & 2.39 & 1.58 \\
\hline 2007 & 8 & 15 & $03: 26: 3313.361$ & 41.008 & 2.93 & 9.51 \\
\hline 2007 & 8 & 15 & $07: 50: 5012.440$ & 40.319 & 1.82 & 11.6 \\
\hline 2007 & 8 & 15 & $09: 47: 0411.900$ & 41.048 & 2.46 & 0.95 \\
\hline 2007 & 8 & 15 & $11: 25: 3811.903$ & 41.044 & 2.21 & 1.21 \\
\hline 2007 & 8 & 15 & $11: 25: 3811.901$ & 41.042 & 2.21 & 0.98 \\
\hline 2007 & 8 & 15 & $15: 49: 1312.505$ & 40.543 & 2.30 & 0.66 \\
\hline 2007 & 8 & 15 & $16: 27: 4213.349$ & 40.943 & 2.41 & 6.58 \\
\hline 2007 & 8 & 15 & $19: 01: 1413.371$ & 40.904 & 2.24 & 5.16 \\
\hline 2007 & 8 & 15 & $21: 18: 3513.475$ & 39.993 & 3.04 & 2.06 \\
\hline 2007 & 8 & 15 & $22: 00: 0812.223$ & 40.669 & 2.24 & 0.5 \\
\hline 2007 & 8 & 15 & $22: 12: 2212.245$ & 40.654 & 1.87 & 0.87 \\
\hline 2007 & 8 & 15 & $22: 34: 5913.413$ & 40.922 & 2.43 & 6.71 \\
\hline 2007 & 8 & 15 & $23: 30: 3312.450$ & 40.562 & 1.89 & 0.52 \\
\hline 2007 & 8 & 15 & $23: 58: 0912.440$ & 40.562 & 4.66 & 1.28 \\
\hline 2007 & 8 & 16 & $01: 25: 2812.421$ & 40.566 & 3.29 & 1.29 \\
\hline 2007 & 8 & 16 & $04: 45: 2712.466$ & 40.562 & 2.53 & 0.64 \\
\hline 2007 & 8 & 16 & $19: 46: 3312.446$ & 40.560 & 1.85 & 1.46 \\
\hline 2007 & 8 & 18 & $01: 42: 0012.481$ & 40.536 & 1.92 & 2.04 \\
\hline 2007 & 8 & 19 & $09: 16: 5813.444$ & 40.047 & 3.55 & 6.13 \\
\hline 2007 & 8 & 20 & $00: 18: 3112.454$ & 40.568 & 1.86 & 0.63 \\
\hline 2007 & 8 & 20 & $23: 01: 0312.367$ & 40.590 & 2.29 & 1.31 \\
\hline 2007 & 8 & 21 & $16: 20: 1713.709$ & 40.042 & 3.07 & 4.01 \\
\hline 2007 & 8 & 22 & $00: 46: 3112.502$ & 40.519 & 1.84 & 1.35 \\
\hline 2007 & 8 & 22 & $01: 32: 1012.455$ & 40.559 & 2.23 & 0.55 \\
\hline 2007 & 8 & 22 & $17: 36: 5012.408$ & 40.576 & 2.08 & 0.55 \\
\hline 2007 & 8 & 22 & $21: 17: 1212.426$ & 40.579 & 2.03 & 0.64 \\
\hline 2007 & 8 & 22 & $21: 29: 4612.236$ & 40.661 & 1.89 & 3.35 \\
\hline 2007 & 8 & 23 & $19: 58: 3712.467$ & 40.551 & 1.98 & 0.64 \\
\hline 2007 & 8 & 24 & $22: 37: 4612.525$ & 40.548 & 1.90 & 1.03 \\
\hline 2007 & 8 & 25 & $00: 52: 5612.468$ & 40.547 & 1.83 & 1.17 \\
\hline 2007 & 8 & 26 & $02: 55: 1711.813$ & 41.096 & 2.19 & 1.86 \\
\hline 2007 & 8 & 26 & $02: 56: 2911.809$ & 41.104 & 2.25 & 1.66 \\
\hline 2007 & 8 & 26 & $02: 59: 2411.811$ & 41.106 & 2.01 & 1.75 \\
\hline 2007 & 8 & 26 & $07: 42: 1112.433$ & 40.590 & 2.33 & 0.65 \\
\hline 2007 & 8 & 26 & $16: 26: 1612.434$ & 40.576 & 2.03 & 0.89 \\
\hline 2007 & 8 & 26 & $19: 33: 1112.478$ & 40.538 & 2.01 & 0.75 \\
\hline 2007 & 8 & 26 & $21: 32: 2412.523$ & 40.507 & 1.77 & 1.09 \\
\hline 2007 & 8 & 26 & $21: 51: 3811.265$ & 41.592 & 2.90 & 5.85 \\
\hline 2007 & 8 & 31 & $02: 38: 3611.801$ & 41.119 & 2.49 & 1.48 \\
\hline 2007 & 9 & 1 & $00: 16: 4312.551$ & 40.516 & 1.81 & 1.78 \\
\hline 2007 & 9 & 1 & $19: 44: 3712.443$ & 40.561 & 1.96 & 1.02 \\
\hline 2007 & 9 & 2 & $21: 47: 3412.385$ & 40.574 & 1.79 & 1.29 \\
\hline 2007 & 9 & 2 & $22: 56: 3712.525$ & 40.510 & 1.83 & 1.05 \\
\hline 2007 & 9 & 4 & $03: 17: 2912.403$ & 40.582 & 2.00 & 0.74 \\
\hline 2007 & 9 & 4 & $16: 17: 3812.458$ & 40.566 & 2.13 & 1.5 \\
\hline 2007 & 9 & 4 & $20: 25: 0411.801$ & 41.120 & 1.96 & 2.02 \\
\hline 2007 & 9 & 4 & $20: 46: 0811.789$ & 41.127 & 1.97 & 3.16 \\
\hline 2007 & 9 & 4 & $21: 18: 3211.805$ & 41.116 & 1.97 & 2.76 \\
\hline 2007 & 9 & 4 & $22: 45: 2712.506$ & 40.517 & 1.85 & 1.12 \\
\hline 2007 & 9 & 5 & $01: 14: 0212.537$ & 40.525 & 1.94 & 0.91 \\
\hline 2007 & 9 & 7 & $22: 14: 3012.416$ & 40.586 & 1.95 & 1.24 \\
\hline 2007 & 9 & 9 & $02: 34: 1913.017$ & 39.594 & 2.79 & 7.28 \\
\hline 2007 & 9 & 9 & $21: 26: 1112.460$ & 40.556 & 2.04 & 0.58 \\
\hline
\end{tabular}




\begin{tabular}{|c|c|c|c|c|c|c|}
\hline 2007 & 9 & 10 & $00: 15: 3512.501$ & 40.524 & 1.81 & 7.14 \\
\hline 2007 & 9 & 10 & $10: 32: 4012.467$ & 40.569 & 2.19 & 1.15 \\
\hline 2007 & 9 & 10 & $20: 53: 2512.405$ & 40.579 & 1.88 & 1.82 \\
\hline 2007 & 9 & 10 & $23: 48: 4712.465$ & 40.556 & 1.86 & 0.56 \\
\hline 2007 & 9 & 11 & $01: 17: 4812.421$ & 40.569 & 1.78 & 1.32 \\
\hline 2007 & 9 & 12 & $00: 58: 4412.423$ & 40.575 & 1.99 & 0.61 \\
\hline 2007 & 9 & 12 & $01: 18: 4211.896$ & 40.762 & 1.74 & 2.08 \\
\hline 2007 & 9 & 12 & $02: 18: 2212.445$ & 40.553 & 1.99 & 1.37 \\
\hline 2007 & 9 & 12 & $19: 11: 1712.409$ & 40.561 & 1.94 & 1.41 \\
\hline 2007 & 9 & 13 & $23: 05: 4212.591$ & 40.508 & 1.94 & 0.92 \\
\hline 2007 & 9 & 15 & $17: 00: 5312.453$ & 40.556 & 2.46 & 0.68 \\
\hline 2007 & 9 & 15 & $19: 55: 4812.491$ & 40.531 & 1.87 & 6.24 \\
\hline 2007 & 9 & 16 & $01: 24: 4912.088$ & 41.554 & 2.62 & 5.85 \\
\hline 2007 & 9 & 16 & $06: 55: 5112.457$ & 40.560 & 2.14 & 1.13 \\
\hline 2007 & 9 & 16 & $17: 16: 2912.422$ & 40.556 & 2.03 & 1.46 \\
\hline 2007 & 9 & 17 & $20: 19: 5511.780$ & 41.108 & 2.56 & 1.25 \\
\hline 2007 & 9 & 17 & $20: 34: 0111.736$ & 40.969 & 1.76 & 9.73 \\
\hline 2007 & 9 & 18 & $00: 36: 5512.414$ & 40.577 & 1.75 & 0.61 \\
\hline 2007 & 9 & 20 & $22: 38: 2412.382$ & 40.585 & 1.81 & 0.62 \\
\hline 2007 & 9 & 21 & $00: 52: 5512.462$ & 40.550 & 1.89 & 1.2 \\
\hline 2007 & 9 & 21 & $05: 48: 3512.452$ & 40.572 & 2.09 & 1.35 \\
\hline 2007 & 9 & 22 & $00: 50: 0612.382$ & 40.596 & 1.80 & 0.99 \\
\hline 2007 & 9 & 23 & $13: 38: 4113.365$ & 39.971 & 2.92 & 9.28 \\
\hline 2007 & 9 & 23 & $18: 46: 3812.766$ & 40.559 & 2.79 & 0.66 \\
\hline 2007 & 9 & 23 & $19: 34: 2012.454$ & 40.569 & 1.98 & 1.35 \\
\hline 2007 & 9 & 25 & $00: 30: 5712.228$ & 40.669 & 1.95 & 0.42 \\
\hline 2007 & 9 & 27 & $18: 52: 3512.379$ & 40.587 & 2.22 & 0.54 \\
\hline 2007 & 9 & 28 & $17: 11: 3812.436$ & 40.565 & 2.24 & 0.69 \\
\hline 2007 & 9 & 28 & $20: 45: 2812.995$ & 40.626 & 2.42 & 1.61 \\
\hline 2007 & 9 & 30 & $00: 48: 4712.468$ & 40.551 & 1.95 & 0.82 \\
\hline 2007 & 10 & 1 & $00: 18: 2212.377$ & 40.588 & 1.82 & 0.6 \\
\hline 2007 & 10 & 1 & $01: 25: 3613.380$ & 40.947 & 2.47 & 2.61 \\
\hline 2007 & 10 & 1 & $01: 51: 3213.432$ & 40.859 & 3.08 & 3.16 \\
\hline 2007 & 10 & 1 & $03: 52: 1813.396$ & 40.924 & 3.86 & 13.29 \\
\hline 2007 & 10 & 1 & $03: 55: 0913.382$ & 40.896 & 3.04 & 6.74 \\
\hline 2007 & 10 & 1 & $03: 58: 0313.414$ & 40.837 & 3.08 & 12.27 \\
\hline 2007 & 10 & 1 & $04: 09: 4013.409$ & 40.876 & 3.08 & 5.11 \\
\hline 2007 & 10 & 1 & $16: 19: 4113.364$ & 40.842 & 3.40 & 11.04 \\
\hline 2007 & 10 & 1 & $17: 15: 2313.411$ & 40.898 & 2.63 & 6.8 \\
\hline 2007 & 10 & 1 & $23: 06: 5412.121$ & 40.753 & 1.81 & 0.67 \\
\hline 2007 & 10 & 2 & $00: 25: 589.674$ & 41.420 & 4.26 & 12.78 \\
\hline 2007 & 10 & 2 & $07: 52: 4813.338$ & 40.849 & 3.89 & 10.77 \\
\hline 2007 & 10 & 2 & $09: 00: 2513.385$ & 40.954 & 3.03 & 12.23 \\
\hline 2007 & 10 & 2 & $09: 06: 2813.489$ & 40.855 & 5.15 & 8.52 \\
\hline 2007 & 10 & 2 & $09: 15: 0713.451$ & 40.873 & 3.55 & 2.42 \\
\hline 2007 & 10 & 2 & $10: 09: 0813.448$ & 40.898 & 3.71 & 15.64 \\
\hline 2007 & 10 & 2 & $12: 42: 2213.399$ & 40.854 & 3.64 & 3.77 \\
\hline 2007 & 10 & 2 & $20: 30: 3913.492$ & 40.907 & 3.66 & 6.2 \\
\hline 2007 & 10 & 2 & $21: 12: 5113.406$ & 40.902 & 2.58 & 4,00 \\
\hline 2007 & 10 & 2 & $21: 15: 3313.423$ & 40.859 & 2.46 & 15.57 \\
\hline 2007 & 10 & 3 & $00: 02: 1113.367$ & 40.903 & 2.96 & 3.64 \\
\hline 2007 & 10 & 3 & $02: 30: 4513.413$ & 40.874 & 3.06 & 16.4 \\
\hline 2007 & 10 & 3 & $02: 44: 5413.399$ & 40.870 & 3.51 & 9.59 \\
\hline 2007 & 10 & 3 & $05: 55: 5513.404$ & 40.872 & 3.42 & 4.54 \\
\hline 2007 & 10 & 3 & $16: 57: 4513.105$ & 39.677 & 2.83 & 3.99 \\
\hline 2007 & 10 & 3 & $17: 00: 0913.097$ & 39.686 & 2.72 & 2.97 \\
\hline 2007 & 10 & 3 & $19: 25: 2613.389$ & 40.884 & 3.35 & 3.15 \\
\hline 2007 & 10 & 3 & $20: 13: 2513.419$ & 40.805 & 2.85 & 9.59 \\
\hline 2007 & 10 & 4 & $00: 08: 2112.419$ & 40.571 & 1.95 & 1.3 \\
\hline 2007 & 10 & 4 & $00: 11: 2313.417$ & 40.885 & 2.72 & 2.4 \\
\hline 2007 & 10 & 4 & $00: 58: 4113.412$ & 40.897 & 2.81 & 2.05 \\
\hline 2007 & 10 & 4 & $01: 29: 4213.393$ & 40.855 & 2.46 & 3.06 \\
\hline 2007 & 10 & 4 & $02: 39: 4213.393$ & 40.885 & 2.55 & 2.99 \\
\hline 2007 & 10 & 4 & $08: 49: 3813.357$ & 40.898 & 2.59 & 6.33 \\
\hline 2007 & 10 & 4 & $16: 13: 2613.380$ & 40.879 & 3.46 & 3.46 \\
\hline 2007 & 10 & 4 & $16: 24: 3013.414$ & 40.860 & 2.85 & 2.84 \\
\hline 2007 & 10 & 4 & $18: 25: 3813.374$ & 40.900 & 3.25 & 4.19 \\
\hline 2007 & 10 & 4 & $19: 15: 0413.397$ & 40.843 & 2.41 & 3.18 \\
\hline 2007 & 10 & 4 & $21: 30: 5212.440$ & 40.577 & 2.05 & 0.75 \\
\hline 2007 & 10 & 5 & $08: 44: 0613.343$ & 40.960 & 2.93 & 12.45 \\
\hline 2007 & 10 & 5 & $21: 05: 0012.434$ & 40.566 & 1.86 & 0.55 \\
\hline 2007 & 10 & 5 & $23: 30: 1313.435$ & 40.818 & 2.57 & 8.62 \\
\hline 2007 & 10 & 6 & $01: 28: 1113.428$ & 40.875 & 2.55 & 4.17 \\
\hline 2007 & 10 & 6 & $06: 23: 4812.439$ & 40.576 & 2.33 & 0.83 \\
\hline 2007 & 10 & 6 & $17: 37: 0112.436$ & 40.572 & 1.82 & 1.54 \\
\hline 2007 & 10 & 6 & $22: 03: 1113.424$ & 40.860 & 2.44 & 2.83 \\
\hline
\end{tabular}




\begin{tabular}{|c|c|c|c|c|c|c|}
\hline 2007 & 10 & 6 & $22: 06: 5112.443$ & 40.546 & 1.79 & 1.36 \\
\hline 2007 & 10 & 6 & $22: 16: 0613.422$ & 40.864 & 2.47 & 3.23 \\
\hline 2007 & 10 & 6 & $22: 28: 3613.398$ & 40.872 & 2.40 & 2.79 \\
\hline 2007 & 10 & 6 & $23: 37: 0013.433$ & 40.883 & 2.85 & 2.19 \\
\hline 2007 & 10 & 7 & $00: 19: 4412.853$ & 40.461 & 2.05 & 5.98 \\
\hline 2007 & 10 & 7 & $19: 38: 1612.434$ & 40.562 & 1.94 & 1.34 \\
\hline 2007 & 10 & 7 & $20: 21: 3712.395$ & 40.581 & 2.05 & 0.61 \\
\hline 2007 & 10 & 7 & $23: 28: 0312.424$ & 40.560 & 2.03 & 0.57 \\
\hline 2007 & 10 & 8 & $17: 43: 1812.504$ & 40.529 & 2.07 & 0.88 \\
\hline 2007 & 10 & 8 & $20: 57: 0512.240$ & 40.662 & 1.82 & 0.79 \\
\hline 2007 & 10 & 8 & $22: 56: 4613.419$ & 40.883 & 2.29 & 2.4 \\
\hline 2007 & 10 & 8 & $23: 55: 0612.485$ & 40.529 & 1.83 & 1.1 \\
\hline 2007 & 10 & 9 & $08: 00: 0412.459$ & 40.571 & 2.63 & 0.82 \\
\hline 2007 & 10 & 9 & $18: 04: 0813.414$ & 40.886 & 2.63 & 3.34 \\
\hline 2007 & 10 & 10 & $17: 40: 2612.245$ & 39.063 & 2.57 & 9.28 \\
\hline 2007 & 10 & 10 & $23: 03: 5912.426$ & 40.550 & 1.72 & 0.66 \\
\hline 2007 & 10 & 11 & $04: 54: 1312.392$ & 40.606 & 1.98 & 1.48 \\
\hline 2007 & 10 & 11 & $19: 14: 4912.436$ & 40.732 & 1.89 & 1.16 \\
\hline 2007 & 10 & 11 & $20: 53: 4112.359$ & 40.608 & 1.82 & 0.99 \\
\hline 2007 & 10 & 11 & $23: 00: 2412.407$ & 40.576 & 2.09 & 0.55 \\
\hline 2007 & 10 & 12 & $21: 56: 5312.399$ & 40.564 & 1.91 & 1.32 \\
\hline 2007 & 10 & 12 & $23: 04: 5312.526$ & 40.507 & 1.94 & 2.07 \\
\hline 2007 & 10 & 13 & $19: 21: 4212.371$ & 40.591 & 1.98 & 0.87 \\
\hline 2007 & 10 & 13 & $20: 15: 2312.373$ & 40.589 & 1.95 & 2.21 \\
\hline 2007 & 10 & 14 & $00: 11: 2512.462$ & 40.561 & 2.06 & 0.56 \\
\hline 2007 & 10 & 14 & $03: 10: 5513.388$ & 40.882 & 2.86 & 1.09 \\
\hline 2007 & 10 & 14 & $03: 57: 5513.380$ & 40.879 & 2.85 & 3.93 \\
\hline 2007 & 10 & 15 & $00: 50: 1212.439$ & 40.553 & 1.84 & 2.98 \\
\hline 2007 & 10 & 15 & $05: 03: 2411.799$ & 41.096 & 3.13 & 1.28 \\
\hline 2007 & 10 & 15 & $05: 19: 3911.806$ & 41.087 & 3.27 & 1.24 \\
\hline 2007 & 10 & 15 & $05: 29: 1911.798$ & 41.111 & 2.62 & 1.69 \\
\hline 2007 & 10 & 15 & $05: 30: 5811.793$ & 41.102 & 2.95 & 1.44 \\
\hline 2007 & 10 & 15 & $16: 07: 1311.875$ & 40.937 & 2.20 & 0.68 \\
\hline 2007 & 10 & 16 & $05: 25: 3711.799$ & 41.132 & 2.64 & 2.3 \\
\hline 2007 & 10 & 16 & $22: 45: 5212.349$ & 40.340 & 1.85 & 3.5 \\
\hline 2007 & 10 & 17 & $00: 56: 0911.905$ & 41.527 & 3.03 & 3.33 \\
\hline 2007 & 10 & 17 & $01: 26: 0812.081$ & 40.953 & 1.87 & 10.84 \\
\hline 2007 & 10 & 17 & $23: 15: 5311.792$ & 41.091 & 1.83 & 1.35 \\
\hline 2007 & 10 & 17 & $23: 16: 4611.798$ & 41.098 & 1.86 & 1.14 \\
\hline 2007 & 10 & 18 & $10: 36: 0511.818$ & 41.096 & 2.34 & 1.31 \\
\hline 2007 & 10 & 18 & $17: 13: 0611.902$ & 40.981 & 2.19 & 0.95 \\
\hline 2007 & 10 & 19 & $19: 45: 1312.436$ & 40.562 & 2.06 & 0.57 \\
\hline 2007 & 10 & 19 & $21: 16: 4812.498$ & 40.538 & 2.01 & 0.82 \\
\hline 2007 & 10 & 20 & $01: 37: 2611.791$ & 41.099 & 2.35 & 1.54 \\
\hline 2007 & 10 & 20 & $01: 41: 4913.382$ & 40.878 & 3.03 & 0.95 \\
\hline 2007 & 10 & 20 & $18: 14: 0012.505$ & 40.546 & 1.98 & 0.98 \\
\hline 2007 & 10 & 20 & $23: 14: 0213.378$ & 40.882 & 2.32 & 1.17 \\
\hline 2007 & 10 & 21 & $01: 03: 0912.446$ & 40.547 & 1.96 & 0.96 \\
\hline 2007 & 10 & 21 & $12: 45: 3712.433$ & 40.572 & 2.24 & 1.02 \\
\hline 2007 & 10 & 21 & $20: 44: 1112.497$ & 40.529 & 1.86 & 0.61 \\
\hline 2007 & 10 & 21 & $22: 04: 1713.425$ & 40.894 & 2.42 & 1.9 \\
\hline 2007 & 10 & 22 & $21: 06: 0312.444$ & 40.565 & 1.84 & 0.54 \\
\hline 2007 & 10 & 22 & $23: 32: 0713.445$ & 40.896 & 2.31 & 3.92 \\
\hline 2007 & 10 & 23 & $14: 38: 1012.454$ & 40.572 & 2.09 & 0.74 \\
\hline 2007 & 10 & 23 & $23: 27: 5012.440$ & 40.550 & 1.82 & 0.92 \\
\hline 2007 & 10 & 24 & $00: 39: 1712.378$ & 40.639 & 1.80 & 0.83 \\
\hline 2007 & 10 & 24 & $02: 31: 4912.413$ & 40.584 & 1.81 & 1.22 \\
\hline 2007 & 10 & 24 & $23: 15: 1512.409$ & 40.564 & 1.97 & 0.51 \\
\hline 2007 & 10 & 25 & $22: 14: 4912.376$ & 40.584 & 1.73 & 0.86 \\
\hline 2007 & 10 & 26 & $01: 10: 4612.438$ & 40.481 & 1.69 & 2.97 \\
\hline 2007 & 10 & 26 & $04: 56: 4712.502$ & 40.455 & 2.00 & 1.19 \\
\hline 2007 & 10 & 26 & $06: 48: 0013.913$ & 40.789 & 2.89 & 8.03 \\
\hline 2007 & 10 & 27 & $06: 39: 0812.480$ & 39.953 & 2.23 & 5.93 \\
\hline 2007 & 10 & 27 & $09: 07: 5812.763$ & 40.564 & 2.52 & 0.63 \\
\hline 2007 & 10 & 27 & $10: 23: 0812.473$ & 39.610 & 2.35 & 5.6 \\
\hline 2007 & 10 & 27 & $11: 23: 0412.358$ & 40.891 & 1.99 & 3.0 \\
\hline 2007 & 10 & 27 & $18: 47: 4712.170$ & 39.144 & 2.42 & 10.7 \\
\hline 2007 & 10 & 27 & $20: 08: 2412.232$ & 40.596 & 1.69 & 3.43 \\
\hline 2007 & 10 & 27 & $21: 43: 4611.575$ & 40.466 & 1.91 & 3.96 \\
\hline 2007 & 10 & 27 & $22: 39: 1411.411$ & 40.892 & 1.76 & 1.23 \\
\hline 2007 & 10 & 27 & $22: 39: 1411.297$ & 40.909 & 1.97 & 0.88 \\
\hline 2007 & 10 & 28 & $03: 01: 4510.334$ & 40.638 & 3.34 & 6.05 \\
\hline 2007 & 10 & 28 & $11: 16: 4213.445$ & 40.927 & 3.34 & 5.25 \\
\hline 2007 & 10 & 28 & $19: 45: 5112.264$ & 41.112 & 1.76 & 1.66 \\
\hline 2007 & 10 & 28 & $22: 09: 1113.694$ & 41.716 & 2.55 & 8.75 \\
\hline 2007 & 10 & 29 & $00: 20: 0712.496$ & 40.529 & 1.76 & 0.53 \\
\hline
\end{tabular}




\begin{tabular}{|c|c|c|c|c|c|c|}
\hline 2007 & 10 & 29 & $02: 29: 5212.364$ & 40.598 & 1.89 & 0.58 \\
\hline 2007 & 10 & 29 & $03: 24: 2512.283$ & 40.547 & 1.81 & 3.54 \\
\hline 2007 & 10 & 29 & $08: 10: 3911.158$ & 41.102 & 3.20 & 2.68 \\
\hline 2007 & 10 & 29 & $08: 10: 4011.198$ & 41.051 & 3.16 & 1,00 \\
\hline 2007 & 10 & 29 & $19: 09: 2912.150$ & 40.656 & 1.97 & 6.43 \\
\hline 2007 & 10 & 29 & $19: 40: 4512.389$ & 40.580 & 1.83 & 0.59 \\
\hline 2007 & 10 & 29 & $21: 00: 2412.396$ & 40.535 & 1.67 & 0.88 \\
\hline 2007 & 10 & 29 & $22: 48: 5212.327$ & 40.605 & 1.73 & 0.47 \\
\hline 2007 & 10 & 29 & $23: 17: 3412.488$ & 40.531 & 1.69 & 0.7 \\
\hline 2007 & 10 & 30 & $00: 18: 3712.169$ & 41.459 & 1.97 & 2.57 \\
\hline 2007 & 10 & 30 & $00: 19: 5612.414$ & 40.570 & 1.65 & 0.51 \\
\hline 2007 & 10 & 30 & $01: 43: 4112.334$ & 40.715 & 1.75 & 5.59 \\
\hline 2007 & 10 & 30 & $02: 22: 4813.318$ & 41.157 & 2.96 & 1.77 \\
\hline 2007 & 10 & 30 & $02: 22: 4913.252$ & 41.114 & 2.90 & 1.2 \\
\hline 2007 & 10 & 30 & $02: 26: 1213.221$ & 41.066 & 2.47 & 1.97 \\
\hline 2007 & 10 & 30 & $18: 43: 0313.288$ & 41.088 & 2.31 & 1.89 \\
\hline 2007 & 10 & 30 & $19: 01: 4513.357$ & 40.867 & 2.24 & 2.55 \\
\hline 2007 & 10 & 30 & $19: 01: 4513.348$ & 40.857 & 2.24 & 1.2 \\
\hline 2007 & 10 & 30 & $22: 16: 2312.377$ & 40.592 & 2.37 & 0.37 \\
\hline 2007 & 10 & 31 & $00: 15: 2413.452$ & 40.876 & 2.30 & 2.02 \\
\hline 2007 & 10 & 31 & $00: 15: 2513.422$ & 40.878 & 2.28 & 2.75 \\
\hline 2007 & 10 & 31 & $02: 12: 0013.434$ & 40.919 & 2.42 & 6.57 \\
\hline 2007 & 10 & 31 & $02: 39: 1713.438$ & 40.900 & 2.45 & 9.92 \\
\hline 2007 & 10 & 31 & $13: 21: 0412.435$ & 40.567 & 2.01 & 0.48 \\
\hline 2007 & 10 & 31 & $15: 15: 1610.321$ & 40.042 & 3.25 & 1.35 \\
\hline 2007 & 10 & 31 & $20: 32: 1111.799$ & 41.100 & 1.84 & 0.9 \\
\hline 2007 & 10 & 31 & $20: 32: 1111.804$ & 41.104 & 1.86 & 0.56 \\
\hline 2007 & 10 & 31 & $21: 10: 3012.878$ & 40.632 & 1.99 & 0.62 \\
\hline 2007 & 10 & 31 & $21: 11: 2512.877$ & 40.634 & 2.01 & 0.54 \\
\hline 2007 & 10 & 31 & $22: 49: 2412.413$ & 40.577 & 1.74 & 0.5 \\
\hline 2007 & 10 & 31 & $22: 52: 0712.449$ & 40.549 & 1.73 & 1.04 \\
\hline 2007 & 10 & 31 & $23: 10: 0813.234$ & 41.091 & 2.27 & 2.19 \\
\hline 2007 & 11 & 1 & $00: 51: 5811.752$ & 41.196 & 1.93 & 0.74 \\
\hline 2007 & 11 & 1 & $00: 57: 2511.889$ & 40.915 & 1.68 & 6.49 \\
\hline 2007 & 11 & 1 & $04: 04: 3712.746$ & 40.579 & 2.37 & 0.91 \\
\hline 2007 & 11 & 1 & $19: 26: 3713.319$ & 41.121 & 2.72 & 1,00 \\
\hline 2007 & 11 & 1 & $21: 08: 2112.444$ & 40.560 & 1.84 & 0.49 \\
\hline 2007 & 11 & 1 & $21: 27: 0512.496$ & 40.532 & 1.68 & 1.41 \\
\hline 2007 & 11 & 1 & $21: 31: 2612.400$ & 40.581 & 1.84 & 0.66 \\
\hline 2007 & 11 & 1 & $21: 33: 2812.500$ & 40.538 & 1.73 & 0.84 \\
\hline 2007 & 11 & 2 & $00: 03: 0413.354$ & 41.189 & 2.42 & 4.15 \\
\hline 2007 & 11 & 2 & $00: 22: 0912.225$ & 40.664 & 1.91 & 0.55 \\
\hline 2007 & 11 & 2 & $01: 51: 5513.305$ & 41.108 & 2.52 & 1.05 \\
\hline 2007 & 11 & 2 & $07: 24: 5612.383$ & 40.591 & 2.25 & 0.51 \\
\hline 2007 & 11 & 2 & $22: 55: 0811.179$ & 41.055 & 2.24 & 0.75 \\
\hline 2007 & 11 & 3 & $01: 13: 4912.377$ & 40.586 & 1.84 & 0.49 \\
\hline 2007 & 11 & 3 & $11: 23: 1412.373$ & 40.593 & 2.27 & 0.55 \\
\hline 2007 & 11 & 3 & $20: 47: 0612.916$ & 40.585 & 1.96 & 1.09 \\
\hline 2007 & 11 & 4 & $08: 35: 0013.448$ & 40.868 & 2.62 & 3.36 \\
\hline 2007 & 11 & 4 & $09: 38: 0312.447$ & 40.558 & 2.02 & 0.61 \\
\hline 2007 & 11 & 4 & $20: 47: 1613.512$ & 39.981 & 3.04 & 1.37 \\
\hline 2007 & 11 & 4 & $21: 06: 1713.543$ & 39.988 & 4.53 & 1.32 \\
\hline 2007 & 11 & 4 & $21: 21: 5413.537$ & 39.983 & 3.25 & 1.39 \\
\hline 2007 & 11 & 4 & $21: 36: 2013.473$ & 39.993 & 2.55 & 2.57 \\
\hline 2007 & 11 & 4 & $21: 51: 2113.503$ & 39.936 & 2.52 & 2.09 \\
\hline 2007 & 11 & 4 & $21: 53: 2613.475$ & 39.993 & 2.49 & 3.76 \\
\hline 2007 & 11 & 4 & $21: 56: 4713.508$ & 39.977 & 2.58 & 2.49 \\
\hline 2007 & 11 & 4 & $21: 59: 2813.470$ & 39.999 & 2.51 & 6.87 \\
\hline 2007 & 11 & 4 & $22: 05: 4813.445$ & 39.987 & 2.45 & 3.56 \\
\hline 2007 & 11 & 4 & $22: 07: 2613.396$ & 39.999 & 2.41 & 3.48 \\
\hline 2007 & 11 & 4 & $22: 08: 5313.445$ & 39.995 & 2.44 & 3.5 \\
\hline 2007 & 11 & 4 & $22: 12: 0713.465$ & 39.987 & 2.52 & 3.17 \\
\hline 2007 & 11 & 4 & $22: 18: 1113.536$ & 39.989 & 2.66 & 2.1 \\
\hline 2007 & 11 & 4 & $22: 34: 1513.418$ & 39.994 & 2.43 & 3.01 \\
\hline 2007 & 11 & 4 & $22: 39: 5413.474$ & 39.986 & 2.47 & 2.19 \\
\hline 2007 & 11 & 4 & $22: 43: 0113.523$ & 39.990 & 2.50 & 2.02 \\
\hline 2007 & 11 & 4 & $22: 53: 0013.526$ & 39.973 & 2.52 & 1.99 \\
\hline 2007 & 11 & 4 & $23: 03: 4613.496$ & 39.917 & 2.53 & 4.76 \\
\hline 2007 & 11 & 4 & $23: 06: 0913.508$ & 39.985 & 2.50 & 2.35 \\
\hline 2007 & 11 & 4 & $23: 15: 5113.480$ & 39.952 & 2.46 & 2.7 \\
\hline 2007 & 11 & 4 & $23: 41: 2413.454$ & 39.996 & 2.48 & 4.47 \\
\hline 2007 & 11 & 4 & $23: 52: 0613.492$ & 39.989 & 2.51 & 1.89 \\
\hline 2007 & 11 & 5 & $00: 03: 1613.505$ & 39.999 & 2.44 & 3.63 \\
\hline 2007 & 11 & 5 & $00: 10: 2913.482$ & 39.992 & 2.43 & 1.5 \\
\hline 2007 & 11 & 5 & $00: 19: 0213.521$ & 39.984 & 2.46 & 4.76 \\
\hline 2007 & 11 & 5 & $00: 23: 2313.514$ & 39.974 & 2.49 & 2.67 \\
\hline
\end{tabular}




\begin{tabular}{|c|c|c|c|c|c|c|}
\hline 2007 & 11 & 5 & $00: 28: 0113.496$ & 40.000 & 2.45 & 2.08 \\
\hline 2007 & 11 & 5 & $00: 30: 0413.526$ & 39.977 & 2.56 & 1.53 \\
\hline 2007 & 11 & 5 & $00: 59: 0213.450$ & 39.994 & 2.43 & 4.07 \\
\hline 2007 & 11 & 5 & $01: 04: 0313.400$ & 40.001 & 2.44 & 3.18 \\
\hline 2007 & 11 & 5 & $01: 11: 1013.411$ & 39.986 & 2.50 & 3.93 \\
\hline 2007 & 11 & 5 & $01: 19: 5913.436$ & 39.990 & 2.40 & 2.37 \\
\hline 2007 & 11 & 5 & $01: 22: 2113.501$ & 39.971 & 2.48 & 2.8 \\
\hline 2007 & 11 & 5 & $01: 25: 4513.465$ & 40.006 & 2.43 & 1.9 \\
\hline 2007 & 11 & 5 & $01: 34: 0413.471$ & 39.920 & 2.48 & 6.33 \\
\hline 2007 & 11 & 5 & $02: 04: 4913.453$ & 39.998 & 2.51 & 3.38 \\
\hline 2007 & 11 & 5 & $02: 44: 0813.441$ & 40.953 & 3.49 & 4.81 \\
\hline 2007 & 11 & 5 & $03: 58: 5913.483$ & 39.984 & 2.65 & 3.47 \\
\hline 2007 & 11 & 5 & $04: 03: 2313.424$ & 40.934 & 3.08 & 2.86 \\
\hline 2007 & 11 & 5 & $04: 30: 3413.486$ & 39.990 & 2.82 & 1.41 \\
\hline 2007 & 11 & 5 & $04: 32: 4813.497$ & 40.004 & 2.77 & 2.35 \\
\hline 2007 & 11 & 5 & $06: 12: 0913.522$ & 39.985 & 2.94 & 1.83 \\
\hline 2007 & 11 & 5 & $07: 00: 5713.567$ & 39.970 & 2.87 & 2.11 \\
\hline 2007 & 11 & 5 & $09: 39: 0213.532$ & 39.980 & 2.92 & 1.7 \\
\hline 2007 & 11 & 5 & $12: 15: 5113.519$ & 39.977 & 3.62 & 1.2 \\
\hline 2007 & 11 & 5 & $12: 17: 2413.517$ & 39.977 & 3.93 & 1.51 \\
\hline 2007 & 11 & 5 & $14: 11: 1513.535$ & 39.973 & 2.88 & 1.74 \\
\hline 2007 & 11 & 5 & $14: 20: 0413.478$ & 39.959 & 2.72 & 1.54 \\
\hline 2007 & 11 & 5 & $19: 47: 1013.469$ & 39.991 & 2.47 & 2.71 \\
\hline 2007 & 11 & 5 & $20: 08: 0213.514$ & 39.981 & 2.60 & 1.14 \\
\hline 2007 & 11 & 5 & $20: 10: 1413.510$ & 39.969 & 2.65 & 1.95 \\
\hline 2007 & 11 & 5 & $20: 14: 1613.535$ & 39.986 & 3.45 & 1.24 \\
\hline 2007 & 11 & 5 & $21: 25: 2613.518$ & 39.990 & 2.50 & 2.22 \\
\hline 2007 & 11 & 5 & $21: 39: 4911.820$ & 41.110 & 2.07 & 0.48 \\
\hline 2007 & 11 & 5 & $22: 26: 0113.039$ & 41.174 & 2.36 & 0.97 \\
\hline 2007 & 11 & 5 & $22: 51: 5512.501$ & 40.547 & 1.86 & 0.49 \\
\hline 2007 & 11 & 5 & $23: 23: 0913.431$ & 40.875 & 2.44 & 5.15 \\
\hline 2007 & 11 & 6 & $00: 29: 5113.449$ & 40.896 & 2.42 & 2.45 \\
\hline 2007 & 11 & 6 & $01: 05: 1513.530$ & 39.969 & 2.57 & 1.22 \\
\hline 2007 & 11 & 6 & $05: 21: 3413.456$ & 39.981 & 2.70 & 3.66 \\
\hline 2007 & 11 & 6 & $05: 35: 5013.518$ & 39.964 & 3.05 & 1.5 \\
\hline 2007 & 11 & 6 & $19: 08: 2613.519$ & 39.950 & 2.49 & 1.01 \\
\hline 2007 & 11 & 6 & $20: 09: 4013.490$ & 39.953 & 2.47 & 1.66 \\
\hline 2007 & 11 & 6 & $20: 32: 1813.485$ & 39.989 & 2.42 & 1.93 \\
\hline 2007 & 11 & 6 & $20: 43: 5913.517$ & 39.984 & 2.37 & 2.79 \\
\hline 2007 & 11 & 6 & $21: 58: 1013.517$ & 39.993 & 2.59 & 1.29 \\
\hline 2007 & 11 & 6 & $23: 35: 3413.529$ & 39.979 & 2.43 & 2.23 \\
\hline 2007 & 11 & 6 & $23: 44: 0613.545$ & 39.970 & 4.34 & 1.9 \\
\hline 2007 & 11 & 7 & $00: 18: 1313.529$ & 39.964 & 2.36 & 1.44 \\
\hline 2007 & 11 & 7 & $00: 31: 2812.434$ & 40.569 & 1.91 & 0.72 \\
\hline 2007 & 11 & 7 & $10: 48: 4113.487$ & 39.989 & 2.61 & 3.1 \\
\hline 2007 & 11 & 7 & $11: 09: 0613.507$ & 39.988 & 3.24 & 1.33 \\
\hline 2007 & 11 & 7 & $16: 56: 1713.046$ & 41.164 & 2.83 & 1.06 \\
\hline 2007 & 11 & 7 & $19: 10: 5913.483$ & 39.973 & 2.44 & 1.31 \\
\hline 2007 & 11 & 7 & $22: 57: 2413.427$ & 39.865 & 2.34 & 4.24 \\
\hline 2007 & 11 & 7 & $23: 02: 5213.552$ & 39.990 & 2.37 & 1.21 \\
\hline 2007 & 11 & 8 & $01: 15: 4013.489$ & 39.994 & 2.39 & 2.42 \\
\hline 2007 & 11 & 8 & $02: 03: 0613.484$ & 39.934 & 2.47 & 1.35 \\
\hline 2007 & 11 & 8 & $02: 13: 2213.500$ & 39.954 & 2.41 & 3.85 \\
\hline 2007 & 11 & 8 & $02: 26: 5913.525$ & 39.979 & 2.86 & 2.51 \\
\hline 2007 & 11 & 8 & $03: 21: 4312.449$ & 40.559 & 2.14 & 0.7 \\
\hline 2007 & 11 & 8 & $23: 32: 3413.491$ & 39.968 & 2.32 & 1.7 \\
\hline 2007 & 11 & 9 & $05: 50: 2213.499$ & 39.973 & 2.69 & 2.04 \\
\hline 2007 & 11 & 9 & $11: 00: 1110.184$ & 40.545 & 3.28 & 2.21 \\
\hline 2007 & 11 & 9 & $12: 08: 5110.194$ & 40.614 & 3.78 & 2.16 \\
\hline 2007 & 11 & 9 & $18: 14: 5510.184$ & 40.599 & 2.98 & 2.06 \\
\hline 2007 & 11 & 9 & $21: 16: 5413.491$ & 39.987 & 2.34 & 1.26 \\
\hline 2007 & 11 & 9 & $23: 07: 5113.490$ & 39.973 & 2.46 & 1.47 \\
\hline 2007 & 11 & 10 & $01: 30: 3713.511$ & 39.983 & 2.78 & 1.12 \\
\hline 2007 & 11 & 10 & $13: 22: 5913.481$ & 39.931 & 2.70 & 1,00 \\
\hline 2007 & 11 & 10 & $18: 32: 4113.497$ & 39.927 & 2.61 & 1.36 \\
\hline 2007 & 11 & 10 & $22: 11: 4713.516$ & 39.921 & 2.43 & 3.56 \\
\hline 2007 & 11 & 10 & $23: 37: 0313.518$ & 39.970 & 2.46 & 0.87 \\
\hline 2007 & 11 & 11 & $16: 04: 2412.262$ & 40.668 & 2.89 & 0.45 \\
\hline 2007 & 11 & 11 & $16: 09: 5312.243$ & 40.659 & 2.92 & 0.44 \\
\hline 2007 & 11 & 11 & $16: 35: 4112.263$ & 40.670 & 2.43 & 0.94 \\
\hline 2007 & 11 & 11 & $17: 05: 4512.240$ & 40.659 & 2.34 & 0.6 \\
\hline 2007 & 11 & 11 & $17: 20: 0712.258$ & 40.669 & 2.42 & 0.99 \\
\hline 2007 & 11 & 11 & $17: 35: 5412.241$ & 40.670 & 2.36 & 0.59 \\
\hline 2007 & 11 & 11 & $17: 39: 58 \quad 12.227$ & 40.661 & 2.44 & 0.45 \\
\hline 2007 & 11 & 11 & $17: 49: 5812.240$ & 40.660 & 2.45 & 0.95 \\
\hline 2007 & 11 & 11 & $17: 51: 3412.235$ & 40.681 & 2.52 & 1.26 \\
\hline
\end{tabular}




\begin{tabular}{|c|c|c|c|c|c|c|}
\hline 2007 & 11 & 11 & $18: 11: 4712.238$ & 40.683 & 3.22 & 0.52 \\
\hline 2007 & 11 & 11 & $18: 24: 3412.250$ & 40.679 & 3.07 & 0.57 \\
\hline 2007 & 11 & 11 & $18: 29: 3712.226$ & 40.672 & 2.82 & 0.54 \\
\hline 2007 & 11 & 11 & $18: 34: 0812.214$ & 40.657 & 2.58 & 0.51 \\
\hline 2007 & 11 & 11 & $18: 36: 1512.214$ & 40.678 & 2.69 & 0.49 \\
\hline 2007 & 11 & 11 & $18: 38: 0712.220$ & 40.672 & 2.68 & 0.53 \\
\hline 2007 & 11 & 11 & $18: 54: 0912.205$ & 40.674 & 2.72 & 0.56 \\
\hline 2007 & 11 & 11 & $19: 09: 2312.170$ & 40.682 & 2.46 & 0.62 \\
\hline 2007 & 11 & 11 & $19: 16: 3112.226$ & 40.687 & 2.68 & 0.8 \\
\hline 2007 & 11 & 11 & $19: 22: 0512.209$ & 40.675 & 2.36 & 0.9 \\
\hline 2007 & 11 & 11 & $19: 24: 1812.203$ & 40.688 & 2.45 & 0.77 \\
\hline 2007 & 11 & 11 & $19: 37: 4712.204$ & 40.681 & 2.76 & 0.77 \\
\hline 2007 & 11 & 11 & $19: 40: 1312.221$ & 40.685 & 2.72 & 0.52 \\
\hline 2007 & 11 & 11 & $19: 41: 5112.210$ & 40.670 & 3.01 & 0.37 \\
\hline 2007 & 11 & 11 & $19: 45: 5312.215$ & 40.681 & 2.74 & 0.58 \\
\hline 2007 & 11 & 11 & $19: 48: 0012.203$ & 40.681 & 3.50 & 0.4 \\
\hline 2007 & 11 & 11 & $19: 57: 5712.168$ & 40.680 & 2.75 & 0.47 \\
\hline 2007 & 11 & 11 & $20: 04: 5112.204$ & 40.698 & 2.71 & 0.92 \\
\hline 2007 & 11 & 11 & $20: 13: 5812.205$ & 40.692 & 2.66 & 0.63 \\
\hline 2007 & 11 & 11 & $20: 21: 1012.177$ & 40.672 & 2.94 & 0.5 \\
\hline 2007 & 11 & 11 & $20: 24: 3812.184$ & 40.680 & 2.57 & 0.56 \\
\hline 2007 & 11 & 11 & $20: 26: 1112.190$ & 40.680 & 2.43 & 0.58 \\
\hline 2007 & 11 & 11 & $20: 28: 2012.204$ & 40.691 & 2.49 & 0.57 \\
\hline 2007 & 11 & 11 & $20: 31: 3412.180$ & 40.700 & 2.43 & 1.24 \\
\hline 2007 & 11 & 11 & $20: 32: 2712.222$ & 40.705 & 2.79 & 0.61 \\
\hline 2007 & 11 & 11 & $20: 37: 3412.227$ & 40.690 & 3.69 & 0.47 \\
\hline 2007 & 11 & 11 & $20: 39: 3712.195$ & 40.685 & 2.93 & 0.73 \\
\hline 2007 & 11 & 11 & $20: 41: 2012.191$ & 40.678 & 2.82 & 0.47 \\
\hline 2007 & 11 & 11 & $20: 46: 5812.194$ & 40.687 & 2.60 & 0.77 \\
\hline 2007 & 11 & 11 & $20: 48: 2812.179$ & 40.681 & 2.60 & 0.64 \\
\hline 2007 & 11 & 11 & $20: 50: 3312.178$ & 40.673 & 3.26 & 0.56 \\
\hline 2007 & 11 & 11 & $20: 52: 2912.181$ & 40.694 & 2.67 & 0.55 \\
\hline 2007 & 11 & 11 & $20: 54: 1212.195$ & 40.690 & 2.84 & 0.72 \\
\hline 2007 & 11 & 11 & $20: 56: 1712.253$ & 40.700 & 3.20 & 0.67 \\
\hline 2007 & 11 & 11 & $21: 01: 5412.226$ & 40.685 & 2.52 & 0.87 \\
\hline 2007 & 11 & 11 & $21: 07: 3812.186$ & 40.693 & 2.77 & 0.45 \\
\hline 2007 & 11 & 11 & $21: 10: 3612.200$ & 40.702 & 2.80 & 0.49 \\
\hline 2007 & 11 & 11 & $21: 11: 2212.185$ & 40.689 & 2.81 & 0.47 \\
\hline 2007 & 11 & 11 & $21: 11: 5612.216$ & 40.696 & 2.76 & 0.65 \\
\hline 2007 & 11 & 11 & $21: 14: 2512.170$ & 40.691 & 2.70 & 0.68 \\
\hline 2007 & 11 & 11 & $21: 14: 5112.216$ & 40.695 & 3.05 & 0.42 \\
\hline 2007 & 11 & 11 & $21: 19: 1412.184$ & 40.682 & 2.53 & 0.68 \\
\hline 2007 & 11 & 11 & $21: 19: 4312.191$ & 40.694 & 3.08 & 0.65 \\
\hline 2007 & 11 & 11 & $21: 21: 4012.222$ & 40.691 & 4.56 & 0.4 \\
\hline 2007 & 11 & 11 & $21: 28: 2512.247$ & 40.691 & 2.65 & 0.94 \\
\hline 2007 & 11 & 11 & $21: 34: 4612.167$ & 40.709 & 3.12 & 0.44 \\
\hline 2007 & 11 & 11 & $21: 39: 3212.211$ & 40.695 & 2.96 & 0.57 \\
\hline 2007 & 11 & 11 & $21: 47: 1412.184$ & 40.699 & 2.73 & 0.61 \\
\hline 2007 & 11 & 11 & $21: 49: 3412.181$ & 40.691 & 3.12 & 0.65 \\
\hline 2007 & 11 & 11 & $21: 54: 4312.176$ & 40.686 & 3.05 & 0.74 \\
\hline 2007 & 11 & 11 & $21: 58: 5012.184$ & 40.684 & 3.06 & 0.46 \\
\hline 2007 & 11 & 11 & $22: 02: 4212.179$ & 40.691 & 2.53 & 0.77 \\
\hline 2007 & 11 & 11 & $22: 03: 2112.163$ & 40.686 & 2.75 & 0.98 \\
\hline 2007 & 11 & 11 & $22: 07: 2312.184$ & 40.691 & 2.71 & 0.46 \\
\hline 2007 & 11 & 11 & $22: 10: 0012.182$ & 40.691 & 2.66 & 0.51 \\
\hline 2007 & 11 & 11 & $22: 15: 0612.177$ & 40.703 & 2.68 & 0.49 \\
\hline 2007 & 11 & 11 & $22: 17: 2812.175$ & 40.683 & 2.64 & 0.54 \\
\hline 2007 & 11 & 11 & $22: 21: 5812.169$ & 40.690 & 3.27 & 0.54 \\
\hline 2007 & 11 & 11 & $22: 29: 3012.184$ & 40.701 & 3.44 & 0.46 \\
\hline 2007 & 11 & 11 & $22: 31: 0312.181$ & 40.700 & 2.79 & 0.62 \\
\hline 2007 & 11 & 11 & $22: 32: 5212.177$ & 40.700 & 3.04 & 0.5 \\
\hline 2007 & 11 & 11 & $22: 42: 1212.196$ & 40.693 & 2.68 & 0.6 \\
\hline 2007 & 11 & 11 & $22: 52: 2612.185$ & 40.701 & 3.71 & 0.64 \\
\hline 2007 & 11 & 11 & $23: 05: 2912.173$ & 40.702 & 2.58 & 0.56 \\
\hline 2007 & 11 & 11 & $23: 09: 0412.189$ & 40.696 & 2.97 & 0.61 \\
\hline 2007 & 11 & 11 & $23: 11: 3012.190$ & 40.708 & 3.30 & 0.92 \\
\hline 2007 & 11 & 11 & $23: 12: 3412.193$ & 40.706 & 3.43 & 0.58 \\
\hline 2007 & 11 & 11 & $23: 24: 3612.181$ & 40.704 & 3.20 & 1.24 \\
\hline 2007 & 11 & 11 & $23: 42: 1912.183$ & 40.695 & 3.33 & 0.8 \\
\hline 2007 & 11 & 11 & $23: 44: 3412.165$ & 40.700 & 2.91 & 0.63 \\
\hline 2007 & 11 & 11 & $23: 53: 0512.206$ & 40.703 & 3.83 & 0.64 \\
\hline 2007 & 11 & 12 & $00: 02: 2912.175$ & 40.710 & 2.94 & 0.49 \\
\hline 2007 & 11 & 12 & $00: 19: 3812.171$ & 40.702 & 2.70 & 0.77 \\
\hline 2007 & 11 & 12 & $00: 23: 2712.188$ & 40.708 & 2.72 & 0.74 \\
\hline 2007 & 11 & 12 & $00: 25: 1312.175$ & 40.700 & 2.84 & 0.75 \\
\hline 2007 & 11 & 12 & $00: 27: 2612.152$ & 40.690 & 2.69 & 0.62 \\
\hline
\end{tabular}




\begin{tabular}{|c|c|c|c|c|c|c|}
\hline 2007 & 11 & 12 & $00: 33: 1312.118$ & 40.764 & 2.54 & 0.8 \\
\hline 2007 & 11 & 12 & $00: 35: 0812.183$ & 40.706 & 3.26 & 0.54 \\
\hline 2007 & 11 & 12 & $00: 40: 1812.210$ & 40.679 & 3.68 & 0.39 \\
\hline 2007 & 11 & 12 & $00: 50: 2912.168$ & 40.715 & 3.02 & 0.56 \\
\hline 2007 & 11 & 12 & $00: 52: 3312.157$ & 40.693 & 2.76 & 0.76 \\
\hline 2007 & 11 & 12 & $01: 01: 1012.170$ & 40.702 & 2.44 & 0.62 \\
\hline 2007 & 11 & 12 & $01: 03: 2112.175$ & 40.711 & 2.83 & 0.58 \\
\hline 2007 & 11 & 12 & $01: 08: 2412.164$ & 40.705 & 3.00 & 0.53 \\
\hline 2007 & 11 & 12 & $01: 11: 2012.186$ & 40.704 & 3.23 & 0.68 \\
\hline 2007 & 11 & 12 & $01: 16: 1412.154$ & 40.705 & 2.80 & 0.56 \\
\hline 2007 & 11 & 12 & $01: 18: 2112.162$ & 40.701 & 2.92 & 0.45 \\
\hline 2007 & 11 & 12 & $01: 26: 0012.158$ & 40.690 & 2.94 & 0.51 \\
\hline 2007 & 11 & 12 & $01: 32: 1212.156$ & 40.704 & 3.17 & 0.51 \\
\hline 2007 & 11 & 12 & $01: 36: 5012.155$ & 40.714 & 3.08 & 0.46 \\
\hline 2007 & 11 & 12 & $01: 43: 3312.159$ & 40.703 & 2.98 & 0.65 \\
\hline 2007 & 11 & 12 & $01: 54: 4312.171$ & 40.720 & 3.47 & 0.54 \\
\hline 2007 & 11 & 12 & $01: 59: 0312.180$ & 40.709 & 2.98 & 0.49 \\
\hline 2007 & 11 & 12 & $01: 59: 4112.175$ & 40.712 & 3.53 & 0.57 \\
\hline 2007 & 11 & 12 & $02: 01: 3512.156$ & 40.714 & 2.86 & 0.67 \\
\hline 2007 & 11 & 12 & $02: 02: 1812.170$ & 40.722 & 2.89 & 0.54 \\
\hline 2007 & 11 & 12 & $02: 19: 2512.175$ & 40.704 & 2.93 & 0.55 \\
\hline 2007 & 11 & 12 & $02: 22: 0212.176$ & 40.723 & 2.75 & 0.56 \\
\hline 2007 & 11 & 12 & $02: 36: 1412.155$ & 40.725 & 2.84 & 0.53 \\
\hline 2007 & 11 & 12 & $02: 46: 2912.169$ & 40.721 & 3.57 & 0.44 \\
\hline 2007 & 11 & 12 & $02: 53: 0312.176$ & 40.717 & 3.02 & 0.84 \\
\hline 2007 & 11 & 12 & $03: 06: 1112.175$ & 40.722 & 3.13 & 1.12 \\
\hline 2007 & 11 & 12 & $03: 19: 2512.182$ & 40.696 & 3.16 & 0.92 \\
\hline 2007 & 11 & 12 & $03: 24: 3312.168$ & 40.715 & 3.59 & 0.47 \\
\hline 2007 & 11 & 12 & $03: 30: 1212.164$ & 40.681 & 3.53 & 0.59 \\
\hline 2007 & 11 & 12 & $03: 40: 4212.157$ & 40.691 & 2.94 & 0.77 \\
\hline 2007 & 11 & 12 & $03: 44: 3312.161$ & 40.728 & 2.74 & 0.74 \\
\hline 2007 & 11 & 12 & $03: 50: 2512.163$ & 40.722 & 3.11 & 0.47 \\
\hline 2007 & 11 & 12 & $03: 53: 3612.164$ & 40.722 & 3.47 & 0.48 \\
\hline 2007 & 11 & 12 & $03: 57: 0212.152$ & 40.726 & 2.95 & 0.63 \\
\hline 2007 & 11 & 12 & $03: 58: 2212.147$ & 40.719 & 2.99 & 0.51 \\
\hline 2007 & 11 & 12 & $04: 03: 3312.152$ & 40.727 & 3.07 & 0.48 \\
\hline 2007 & 11 & 12 & $04: 08: 5412.157$ & 40.726 & 3.24 & 0.84 \\
\hline 2007 & 11 & 12 & $04: 09: 5612.128$ & 40.727 & 2.91 & 0.59 \\
\hline 2007 & 11 & 12 & $04: 11: 5112.149$ & 40.718 & 2.94 & 0.6 \\
\hline 2007 & 11 & 12 & $04: 19: 5912.152$ & 40.721 & 3.14 & 0.5 \\
\hline 2007 & 11 & 12 & $04: 25: 0012.170$ & 40.713 & 3.17 & 0.61 \\
\hline 2007 & 11 & 12 & $04: 33: 4112.150$ & 40.723 & 3.12 & 0.43 \\
\hline 2007 & 11 & 12 & $04: 41: 3812.143$ & 40.730 & 2.79 & 0.54 \\
\hline 2007 & 11 & 12 & $04: 46: 2612.136$ & 40.718 & 2.81 & 0.47 \\
\hline 2007 & 11 & 12 & $04: 49: 0412.152$ & 40.730 & 2.97 & 0.43 \\
\hline 2007 & 11 & 12 & $04: 49: 3212.152$ & 40.732 & 2.90 & 0.53 \\
\hline 2007 & 11 & 12 & $05: 04: 4212.149$ & 40.728 & 2.89 & 0.51 \\
\hline 2007 & 11 & 12 & $05: 11: 0612.132$ & 40.715 & 2.74 & 0.66 \\
\hline 2007 & 11 & 12 & $05: 12: 2312.138$ & 40.735 & 2.69 & 0.46 \\
\hline 2007 & 11 & 12 & $05: 17: 4912.161$ & 40.732 & 2.97 & 0.51 \\
\hline 2007 & 11 & 12 & $05: 22: 4512.156$ & 40.725 & 2.72 & 0.47 \\
\hline 2007 & 11 & 12 & $05: 29: 4912.152$ & 40.721 & 2.50 & 0.69 \\
\hline 2007 & 11 & 12 & $05: 32: 5312.180$ & 40.711 & 3.22 & 0.46 \\
\hline 2007 & 11 & 12 & $05: 41: 3412.147$ & 40.732 & 2.98 & 0.69 \\
\hline 2007 & 11 & 12 & $05: 53: 4812.129$ & 40.727 & 2.68 & 0.59 \\
\hline 2007 & 11 & 12 & $05: 57: 1412.139$ & 40.733 & 2.71 & 0.69 \\
\hline 2007 & 11 & 12 & $06: 02: 1612.136$ & 40.728 & 2.69 & 0.45 \\
\hline 2007 & 11 & 12 & $06: 20: 5912.134$ & 40.734 & 2.86 & 0.46 \\
\hline 2007 & 11 & 12 & $06: 26: 0012.171$ & 40.714 & 3.52 & 0.68 \\
\hline 2007 & 11 & 12 & $06: 34: 1112.149$ & 40.727 & 2.52 & 0.74 \\
\hline 2007 & 11 & 12 & $06: 40: 4012.182$ & 40.695 & 2.56 & 0.44 \\
\hline 2007 & 11 & 12 & $06: 43: 0312.128$ & 40.731 & 2.35 & 0.49 \\
\hline 2007 & 11 & 12 & $06: 53: 5412.045$ & 41.065 & 2.08 & 1.25 \\
\hline 2007 & 11 & 12 & $07: 06: 2012.138$ & 40.735 & 2.54 & 0.55 \\
\hline 2007 & 11 & 12 & $07: 20: 3212.161$ & 40.709 & 3.26 & 0.66 \\
\hline 2007 & 11 & 12 & $07: 27: 1212.165$ & 40.711 & 3.39 & 0.5 \\
\hline 2007 & 11 & 12 & $07: 50: 5612.139$ & 40.673 & 2.67 & 0.69 \\
\hline 2007 & 11 & 12 & $07: 53: 3012.129$ & 40.733 & 2.77 & 0.45 \\
\hline 2007 & 11 & 12 & $08: 06: 2712.158$ & 40.719 & 2.72 & 0.54 \\
\hline 2007 & 11 & 12 & $08: 16: 3112.109$ & 40.753 & 2.87 & 0.49 \\
\hline 2007 & 11 & 12 & $08: 32: 0312.129$ & 40.739 & 3.09 & 0.49 \\
\hline 2007 & 11 & 12 & $08: 52: 0912.141$ & 40.751 & 3.08 & 0.57 \\
\hline 2007 & 11 & 12 & $09: 25: 0912.124$ & 40.747 & 3.19 & 0.48 \\
\hline 2007 & 11 & 12 & $09: 34: 3912.121$ & 40.747 & 2.90 & 0.55 \\
\hline 2007 & 11 & 12 & $09: 37: 5012.130$ & 40.728 & 3.17 & 0.48 \\
\hline 2007 & 11 & 12 & $09: 42: 2012.194$ & 40.103 & 3.02 & 6.59 \\
\hline
\end{tabular}




\begin{tabular}{|c|c|c|c|c|c|c|}
\hline 2007 & 11 & 12 & $10: 07: 3912.112$ & 40.743 & 2.68 & 0.67 \\
\hline 2007 & 11 & 12 & $10: 15: 1012.118$ & 40.745 & 2.74 & 0.57 \\
\hline 2007 & 11 & 12 & $10: 20: 0912.117$ & 40.752 & 2.62 & 0.53 \\
\hline 2007 & 11 & 12 & $10: 21: 4112.119$ & 40.739 & 2.73 & 0.41 \\
\hline 2007 & 11 & 12 & $11: 11: 4512.135$ & 40.742 & 3.36 & 0.43 \\
\hline 2007 & 11 & 12 & $11: 45: 1412.178$ & 40.719 & 2.92 & 0.49 \\
\hline 2007 & 11 & 12 & $12: 30: 1812.114$ & 40.737 & 2.40 & 0.72 \\
\hline 2007 & 11 & 12 & $13: 34: 0612.107$ & 40.739 & 2.36 & 0.85 \\
\hline 2007 & 11 & 12 & $13: 57: 1512.083$ & 40.761 & 2.57 & 0.54 \\
\hline 2007 & 11 & 12 & $14: 05: 2912.143$ & 40.740 & 2.99 & 0.39 \\
\hline 2007 & 11 & 12 & $14: 34: 3512.124$ & 40.732 & 3.04 & 0.46 \\
\hline 2007 & 11 & 12 & $15: 33: 0012.082$ & 40.755 & 3.31 & 0.67 \\
\hline 2007 & 11 & 12 & $16: 32: 3912.188$ & 40.712 & 2.57 & 0.51 \\
\hline 2007 & 11 & 12 & $16: 47: 2712.075$ & 40.776 & 2.23 & 0.7 \\
\hline 2007 & 11 & 12 & $17: 24: 1312.095$ & 40.753 & 2.43 & 0.6 \\
\hline 2007 & 11 & 12 & $17: 54: 3512.175$ & 40.717 & 2.75 & 0.61 \\
\hline 2007 & 11 & 12 & $18: 02: 5012.104$ & 40.767 & 3.10 & 0.43 \\
\hline 2007 & 11 & 12 & $18: 29: 0512.182$ & 40.711 & 3.04 & 0.66 \\
\hline 2007 & 11 & 12 & $18: 45: 3612.128$ & 40.742 & 2.64 & 0.4 \\
\hline 2007 & 11 & 12 & $19: 02: 4512.109$ & 40.751 & 2.36 & 0.6 \\
\hline 2007 & 11 & 12 & $19: 23: 5812.114$ & 40.744 & 2.37 & 0.51 \\
\hline 2007 & 11 & 12 & $19: 24: 3312.129$ & 40.748 & 2.58 & 0.55 \\
\hline 2007 & 11 & 12 & $19: 46: 3112.104$ & 40.761 & 2.44 & 0.75 \\
\hline 2007 & 11 & 12 & $20: 12: 4612.078$ & 40.739 & 2.75 & 0.68 \\
\hline 2007 & 11 & 12 & $20: 15: 1712.128$ & 40.752 & 2.83 & 0.49 \\
\hline 2007 & 11 & 12 & $20: 17: 0312.131$ & 40.771 & 3.57 & 1.19 \\
\hline 2007 & 11 & 12 & $20: 59: 0412.102$ & 40.748 & 2.79 & 0.51 \\
\hline 2007 & 11 & 12 & $21: 38: 0812.133$ & 40.736 & 2.66 & 0.39 \\
\hline 2007 & 11 & 12 & $21: 43: 5812.128$ & 40.747 & 2.57 & 0.52 \\
\hline 2007 & 11 & 12 & $21: 50: 0112.122$ & 40.743 & 2.36 & 0.51 \\
\hline 2007 & 11 & 12 & $21: 56: 2412.136$ & 40.736 & 2.60 & 0.46 \\
\hline 2007 & 11 & 12 & $22: 03: 1712.130$ & 40.734 & 3.00 & 0.38 \\
\hline 2007 & 11 & 12 & $22: 07: 0312.136$ & 40.744 & 2.67 & 0.77 \\
\hline 2007 & 11 & 12 & $22: 12: 1412.138$ & 40.757 & 3.00 & 1.04 \\
\hline 2007 & 11 & 12 & $22: 26: 1612.133$ & 40.739 & 2.66 & 0.43 \\
\hline 2007 & 11 & 12 & $22: 26: 5412.123$ & 40.738 & 2.88 & 0.55 \\
\hline 2007 & 11 & 12 & $22: 36: 1712.137$ & 40.754 & 2.84 & 1.27 \\
\hline 2007 & 11 & 12 & $22: 48: 0512.114$ & 40.730 & 2.68 & 0.48 \\
\hline 2007 & 11 & 12 & $22: 50: 2912.130$ & 40.740 & 2.61 & 0.57 \\
\hline 2007 & 11 & 12 & $23: 02: 3812.123$ & 40.737 & 2.84 & 0.44 \\
\hline 2007 & 11 & 12 & $23: 21: 5912.118$ & 40.742 & 2.87 & 0.47 \\
\hline 2007 & 11 & 12 & $23: 24: 4312.125$ & 40.740 & 2.54 & 0.66 \\
\hline 2007 & 11 & 12 & $23: 35: 1612.122$ & 40.738 & 3.15 & 0.48 \\
\hline 2007 & 11 & 12 & $23: 43: 3712.129$ & 40.732 & 3.01 & 0.54 \\
\hline 2007 & 11 & 12 & $23: 46: 5312.123$ & 40.744 & 3.73 & 0.49 \\
\hline 2007 & 11 & 13 & $00: 02: 3012.114$ & 40.747 & 2.30 & 0.45 \\
\hline 2007 & 11 & 13 & $00: 17: 0212.115$ & 40.757 & 2.86 & 0.74 \\
\hline 2007 & 11 & 13 & $00: 26: 1212.123$ & 40.732 & 3.13 & 0.39 \\
\hline 2007 & 11 & 13 & $00: 58: 1012.127$ & 40.733 & 3.11 & 0.41 \\
\hline 2007 & 11 & 13 & $01: 03: 1112.122$ & 40.746 & 2.68 & 0.43 \\
\hline 2007 & 11 & 13 & $01: 09: 0212.105$ & 40.742 & 2.47 & 0.63 \\
\hline 2007 & 11 & 13 & $01: 11: 4512.106$ & 40.752 & 2.40 & 0.47 \\
\hline 2007 & 11 & 13 & $01: 18: 2012.100$ & 40.777 & 3.09 & 0.31 \\
\hline 2007 & 11 & 13 & $01: 28: 3112.107$ & 40.744 & 2.43 & 0.44 \\
\hline 2007 & 11 & 13 & $01: 32: 0112.118$ & 40.745 & 2.73 & 0.42 \\
\hline 2007 & 11 & 13 & $01: 35: 3612.120$ & 40.748 & 2.55 & 0.46 \\
\hline 2007 & 11 & 13 & $01: 39: 2212.120$ & 40.775 & 2.94 & 1.19 \\
\hline 2007 & 11 & 13 & $01: 45: 3812.130$ & 40.773 & 3.53 & 1.21 \\
\hline 2007 & 11 & 13 & $01: 49: 5912.140$ & 40.736 & 2.87 & 0.45 \\
\hline 2007 & 11 & 13 & $01: 53: 4412.111$ & 40.742 & 2.71 & 0.42 \\
\hline 2007 & 11 & 13 & $01: 56: 0612.127$ & 40.743 & 2.82 & 0.56 \\
\hline 2007 & 11 & 13 & $02: 04: 2012.103$ & 40.751 & 2.79 & 0.6 \\
\hline 2007 & 11 & 13 & $02: 08: 2512.120$ & 40.744 & 2.80 & 0.52 \\
\hline 2007 & 11 & 13 & $02: 12: 4212.109$ & 40.755 & 2.82 & 0.46 \\
\hline 2007 & 11 & 13 & $02: 25: 1312.119$ & 40.746 & 2.88 & 0.39 \\
\hline 2007 & 11 & 13 & $02: 33: 4912.115$ & 40.745 & 3.46 & 0.43 \\
\hline 2007 & 11 & 13 & $02: 49: 3812.102$ & 40.761 & 2.53 & 0.54 \\
\hline 2007 & 11 & 13 & $02: 55: 5812.114$ & 40.747 & 2.67 & 0.67 \\
\hline 2007 & 11 & 13 & $03: 14: 2012.116$ & 40.749 & 2.77 & 0.52 \\
\hline 2007 & 11 & 13 & $03: 25: 2812.101$ & 40.761 & 2.49 & 0.4 \\
\hline 2007 & 11 & 13 & $03: 44: 1112.104$ & 40.742 & 2.91 & 0.48 \\
\hline 2007 & 11 & 13 & $03: 45: 4412.111$ & 40.742 & 2.65 & 0.48 \\
\hline 2007 & 11 & 13 & $04: 07: 0312.102$ & 40.763 & 3.20 & 0.48 \\
\hline 2007 & 11 & 13 & $04: 10: 4612.098$ & 40.764 & 2.78 & 0.52 \\
\hline 2007 & 11 & 13 & $04: 18: 1112.112$ & 40.743 & 2.99 & 0.48 \\
\hline 2007 & 11 & 13 & $04: 20: 2312.099$ & 40.755 & 2.71 & 0.55 \\
\hline
\end{tabular}




\begin{tabular}{|c|c|c|c|c|c|c|}
\hline 2007 & 11 & 13 & $04: 24: 2112.098$ & 40.768 & 2.38 & 0.67 \\
\hline 2007 & 11 & 13 & $04: 41: 0112.090$ & 40.760 & 3.13 & 0.55 \\
\hline 2007 & 11 & 13 & $04: 52: 1412.085$ & 40.758 & 2.69 & 0.75 \\
\hline 2007 & 11 & 13 & $04: 55: 4512.094$ & 40.751 & 2.76 & 0.58 \\
\hline 2007 & 11 & 13 & $05: 08: 3812.102$ & 40.760 & 3.12 & 0.43 \\
\hline 2007 & 11 & 13 & $05: 13: 4112.102$ & 40.752 & 2.70 & 0.49 \\
\hline 2007 & 11 & 13 & $05: 20: 0912.095$ & 40.763 & 2.95 & 0.44 \\
\hline 2007 & 11 & 13 & $05: 33: 2512.107$ & 40.741 & 3.58 & 0.44 \\
\hline 2007 & 11 & 13 & $05: 43: 0912.105$ & 40.748 & 3.14 & 0.41 \\
\hline 2007 & 11 & 13 & $05: 50: 1212.093$ & 40.759 & 3.45 & 0.37 \\
\hline 2007 & 11 & 13 & $06: 06: 4312.086$ & 40.748 & 2.91 & 0.53 \\
\hline 2007 & 11 & 13 & $06: 30: 0912.107$ & 40.785 & 3.14 & 1.08 \\
\hline 2007 & 11 & 13 & $06: 36: 1912.097$ & 40.760 & 3.09 & 0.45 \\
\hline 2007 & 11 & 13 & $06: 49: 4512.099$ & 40.746 & 3.09 & 0.38 \\
\hline 2007 & 11 & 13 & $06: 58: 3912.110$ & 40.741 & 2.78 & 0.46 \\
\hline 2007 & 11 & 13 & $07: 09: 1712.082$ & 40.752 & 2.36 & 0.6 \\
\hline 2007 & 11 & 13 & $07: 41: 2912.061$ & 40.762 & 2.64 & 0.49 \\
\hline 2007 & 11 & 13 & $07: 55: 4812.096$ & 40.764 & 2.67 & 0.52 \\
\hline 2007 & 11 & 13 & $08: 04: 5212.088$ & 40.776 & 3.00 & 0.41 \\
\hline 2007 & 11 & 13 & $08: 18: 2412.109$ & 40.734 & 2.90 & 0.43 \\
\hline 2007 & 11 & 13 & $08: 22: 5912.108$ & 40.740 & 2.77 & 0.49 \\
\hline 2007 & 11 & 13 & $08: 41: 2012.082$ & 40.755 & 3.07 & 0.46 \\
\hline 2007 & 11 & 13 & $08: 51: 0312.085$ & 40.750 & 3.12 & 0.52 \\
\hline 2007 & 11 & 13 & $09: 10: 5712.075$ & 40.764 & 2.57 & 0.49 \\
\hline 2007 & 11 & 13 & $09: 28: 4212.066$ & 40.757 & 2.60 & 0.56 \\
\hline 2007 & 11 & 13 & $09: 38: 0612.072$ & 40.770 & 2.59 & 0.39 \\
\hline 2007 & 11 & 13 & $10: 03: 4412.079$ & 40.769 & 2.50 & 0.49 \\
\hline 2007 & 11 & 13 & $10: 06: 0412.101$ & 40.759 & 2.59 & 0.48 \\
\hline 2007 & 11 & 13 & $10: 13: 3512.074$ & 40.767 & 2.39 & 0.56 \\
\hline 2007 & 11 & 13 & $10: 30: 3412.079$ & 40.765 & 2.80 & 0.41 \\
\hline 2007 & 11 & 13 & $10: 34: 1812.100$ & 40.770 & 2.81 & 0.5 \\
\hline 2007 & 11 & 13 & $10: 52: 1712.074$ & 40.763 & 2.86 & 0.46 \\
\hline 2007 & 11 & 13 & $11: 02: 3812.080$ & 40.785 & 2.38 & 0.55 \\
\hline 2007 & 11 & 13 & $11: 12: 1612.075$ & 40.760 & 2.99 & 0.42 \\
\hline 2007 & 11 & 13 & $11: 17: 0812.082$ & 40.772 & 2.65 & 0.35 \\
\hline 2007 & 11 & 13 & $11: 27: 4512.069$ & 40.761 & 2.29 & 0.63 \\
\hline 2007 & 11 & 13 & $11: 52: 4112.049$ & 40.762 & 2.36 & 0.76 \\
\hline 2007 & 11 & 13 & $11: 58: 2212.085$ & 40.764 & 2.35 & 0.85 \\
\hline 2007 & 11 & 13 & $12: 09: 0612.076$ & 40.778 & 2.71 & 0.45 \\
\hline 2007 & 11 & 13 & $12: 21: 5312.068$ & 40.767 & 2.56 & 0.65 \\
\hline 2007 & 11 & 13 & $12: 26: 1312.068$ & 40.771 & 2.49 & 0.63 \\
\hline 2007 & 11 & 13 & $12: 40: 3912.072$ & 40.763 & 2.70 & 0.48 \\
\hline 2007 & 11 & 13 & $13: 19: 2812.093$ & 40.770 & 3.23 & 0.37 \\
\hline 2007 & 11 & 13 & $13: 33: 1412.079$ & 40.770 & 2.89 & 0.43 \\
\hline 2007 & 11 & 13 & $13: 34: 5412.074$ & 40.766 & 2.91 & 0.5 \\
\hline 2007 & 11 & 13 & $14: 16: 5212.121$ & 40.776 & 3.91 & 0.91 \\
\hline 2007 & 11 & 13 & $15: 07: 4712.085$ & 40.763 & 2.62 & 0.41 \\
\hline 2007 & 11 & 13 & $16: 15: 4512.127$ & 40.744 & 2.27 & 0.69 \\
\hline 2007 & 11 & 13 & $16: 47: 2113.480$ & 39.991 & 2.96 & 2.11 \\
\hline 2007 & 11 & 13 & $17: 40: 5612.094$ & 40.761 & 2.90 & 0.37 \\
\hline 2007 & 11 & 13 & $17: 56: 3812.096$ & 40.763 & 2.81 & 0.32 \\
\hline 2007 & 11 & 13 & $18: 09: 1012.081$ & 40.780 & 2.53 & 0.47 \\
\hline 2007 & 11 & 13 & $18: 12: 2412.079$ & 40.760 & 2.32 & 0.51 \\
\hline 2007 & 11 & 13 & $18: 14: 4112.098$ & 40.761 & 2.01 & 0.53 \\
\hline 2007 & 11 & 13 & $18: 42: 4012.121$ & 40.745 & 2.47 & 0.44 \\
\hline 2007 & 11 & 13 & $19: 09: 1112.073$ & 40.786 & 2.42 & 0.47 \\
\hline 2007 & 11 & 13 & $19: 29: 0812.099$ & 40.795 & 2.54 & 1.24 \\
\hline 2007 & 11 & 13 & $19: 34: 4812.080$ & 40.764 & 3.07 & 0.39 \\
\hline 2007 & 11 & 13 & $19: 42: 0612.088$ & 40.766 & 3.12 & 0.42 \\
\hline 2007 & 11 & 13 & $20: 04: 2312.109$ & 40.754 & 2.36 & 1.16 \\
\hline 2007 & 11 & 13 & $20: 06: 0112.042$ & 40.779 & 2.18 & 0.42 \\
\hline 2007 & 11 & 13 & $20: 29: 3012.075$ & 40.773 & 2.42 & 0.44 \\
\hline 2007 & 11 & 13 & $20: 50: 4812.191$ & 40.705 & 2.52 & 0.67 \\
\hline 2007 & 11 & 13 & $21: 29: 4312.109$ & 40.751 & 2.43 & 0.37 \\
\hline 2007 & 11 & 13 & $21: 50: 2812.090$ & 40.751 & 2.26 & 0.48 \\
\hline 2007 & 11 & 13 & $22: 20: 2912.093$ & 40.763 & 2.85 & 0.39 \\
\hline 2007 & 11 & 13 & $22: 26: 5112.123$ & 40.746 & 3.47 & 0.43 \\
\hline 2007 & 11 & 13 & $23: 03: 3612.173$ & 40.706 & 2.63 & 0.64 \\
\hline 2007 & 11 & 13 & $23: 54: 3512.071$ & 40.769 & 2.52 & 0.35 \\
\hline 2007 & 11 & 14 & $00: 05: 5812.060$ & 40.776 & 2.77 & 0.45 \\
\hline 2007 & 11 & 14 & $00: 07: 1112.222$ & 40.679 & 2.64 & 0.53 \\
\hline 2007 & 11 & 14 & $00: 17: 3512.103$ & 40.749 & 2.56 & 0.42 \\
\hline 2007 & 11 & 14 & $00: 36: 1112.084$ & 40.763 & 2.32 & 0.49 \\
\hline 2007 & 11 & 14 & $00: 38: 3012.116$ & 40.746 & 2.24 & 0.46 \\
\hline 2007 & 11 & 14 & $01: 50: 4912.169$ & 40.718 & 3.25 & 0.46 \\
\hline 2007 & 11 & 14 & $02: 07: 3712.188$ & 40.698 & 2.66 & 0.5 \\
\hline
\end{tabular}




\begin{tabular}{|c|c|c|c|c|c|c|}
\hline 2007 & 11 & 14 & $02: 31: 3812.107$ & 40.770 & 2.61 & 0.46 \\
\hline 2007 & 11 & 14 & $04: 09: 3712.107$ & 40.749 & 3.34 & 0.36 \\
\hline 2007 & 11 & 14 & $04: 58: 2512.198$ & 40.681 & 2.95 & 0.59 \\
\hline 2007 & 11 & 14 & $05: 09: 3612.097$ & 40.761 & 2.62 & 0.45 \\
\hline 2007 & 11 & 14 & $05: 43: 2012.117$ & 40.750 & 2.81 & 0.61 \\
\hline 2007 & 11 & 14 & $05: 47: 4412.102$ & 40.746 & 2.55 & 0.63 \\
\hline 2007 & 11 & 14 & $05: 55: 2212.184$ & 40.694 & 2.72 & 0.59 \\
\hline 2007 & 11 & 14 & $06: 32: 3712.096$ & 40.750 & 2.54 & 0.65 \\
\hline 2007 & 11 & 14 & $06: 54: 0712.070$ & 40.780 & 2.77 & 0.44 \\
\hline 2007 & 11 & 14 & $08: 48: 4812.197$ & 40.673 & 2.32 & 0.75 \\
\hline 2007 & 11 & 14 & $08: 56: 3412.070$ & 40.751 & 2.74 & 0.54 \\
\hline 2007 & 11 & 14 & $09: 39: 1312.184$ & 40.687 & 2.46 & 0.56 \\
\hline 2007 & 11 & 14 & $12: 21: 0813.466$ & 40.957 & 3.26 & 9.77 \\
\hline 2007 & 11 & 14 & $12: 37: 4212.111$ & 40.747 & 2.30 & 0.61 \\
\hline 2007 & 11 & 14 & $13: 19: 2613.431$ & 40.938 & 3.48 & 4.85 \\
\hline 2007 & 11 & 14 & $13: 27: 1212.100$ & 40.747 & 2.75 & 0.63 \\
\hline 2007 & 11 & 14 & $13: 46: 2912.076$ & 40.758 & 2.27 & 0.47 \\
\hline 2007 & 11 & 14 & $14: 46: 3913.427$ & 40.925 & 3.20 & 4.13 \\
\hline 2007 & 11 & 14 & $15: 39: 3112.175$ & 40.694 & 3.17 & 0.46 \\
\hline 2007 & 11 & 14 & $17: 48: 4112.207$ & 40.686 & 4.32 & 0.44 \\
\hline 2007 & 11 & 14 & $18: 05: 5713.459$ & 40.926 & 3.30 & 2.51 \\
\hline 2007 & 11 & 14 & $18: 15: 1013.450$ & 40.937 & 3.14 & 2.51 \\
\hline 2007 & 11 & 14 & $18: 19: 0112.068$ & 40.772 & 2.29 & 0.44 \\
\hline 2007 & 11 & 14 & $19: 01: 3513.436$ & 40.932 & 2.73 & 3.81 \\
\hline 2007 & 11 & 14 & $19: 18: 2612.096$ & 40.770 & 2.59 & 0.37 \\
\hline 2007 & 11 & 14 & $19: 30: 1712.093$ & 40.761 & 2.18 & 0.6 \\
\hline 2007 & 11 & 14 & $19: 45: 1312.103$ & 40.752 & 2.23 & 0.38 \\
\hline 2007 & 11 & 14 & $20: 14: 5412.188$ & 40.704 & 3.09 & 0.5 \\
\hline 2007 & 11 & 14 & $20: 33: 2112.119$ & 40.761 & 2.63 & 0.35 \\
\hline 2007 & 11 & 14 & $20: 57: 0113.454$ & 40.904 & 2.43 & 4.23 \\
\hline 2007 & 11 & 14 & $20: 59: 0612.108$ & 40.747 & 2.49 & 0.43 \\
\hline 2007 & 11 & 14 & $21: 02: 1212.094$ & 40.758 & 2.10 & 0.5 \\
\hline 2007 & 11 & 14 & $21: 25: 2012.100$ & 40.735 & 2.03 & 0.67 \\
\hline 2007 & 11 & 14 & $21: 51: 5112.117$ & 40.752 & 2.11 & 0.46 \\
\hline 2007 & 11 & 14 & $21: 53: 4613.439$ & 40.895 & 2.46 & 3.15 \\
\hline 2007 & 11 & 14 & $22: 27: 1412.109$ & 40.748 & 1.99 & 0.46 \\
\hline 2007 & 11 & 14 & $22: 28: 4712.103$ & 40.759 & 2.15 & 0.48 \\
\hline 2007 & 11 & 14 & $22: 42: 2512.110$ & 40.744 & 2.12 & 0.5 \\
\hline 2007 & 11 & 14 & $22: 55: 1412.176$ & 40.706 & 1.95 & 0.44 \\
\hline 2007 & 11 & 14 & $23: 25: 5412.085$ & 40.753 & 1.95 & 0.6 \\
\hline 2007 & 11 & 14 & $23: 30: 4212.089$ & 40.763 & 2.06 & 0.39 \\
\hline 2007 & 11 & 14 & $23: 40: 1412.079$ & 40.777 & 1.88 & 0.55 \\
\hline 2007 & 11 & 15 & $00: 08: 1512.113$ & 40.744 & 1.95 & 0.64 \\
\hline 2007 & 11 & 15 & $00: 09: 5612.119$ & 40.733 & 2.04 & 0.61 \\
\hline 2007 & 11 & 15 & $00: 09: 5612.117$ & 40.732 & 2.00 & 0.56 \\
\hline 2007 & 11 & 15 & $00: 34: 5512.121$ & 40.741 & 1.94 & 0.5 \\
\hline 2007 & 11 & 15 & $00: 52: 4113.426$ & 40.849 & 2.76 & 9.48 \\
\hline 2007 & 11 & 15 & $01: 14: 5812.090$ & 40.755 & 2.87 & 0.35 \\
\hline 2007 & 11 & 15 & $01: 19: 2613.508$ & 39.942 & 2.52 & 1.62 \\
\hline 2007 & 11 & 15 & $01: 33: 0012.117$ & 40.749 & 2.41 & 0.47 \\
\hline 2007 & 11 & 15 & $02: 07: 5913.535$ & 39.957 & 2.57 & 2.22 \\
\hline 2007 & 11 & 15 & $02: 40: 1212.179$ & 40.707 & 2.22 & 0.65 \\
\hline 2007 & 11 & 15 & $04: 47: 2712.164$ & 40.695 & 2.09 & 0.57 \\
\hline 2007 & 11 & 15 & $05: 21: 5312.112$ & 40.744 & 2.99 & 0.43 \\
\hline 2007 & 11 & 15 & $05: 38: 5212.093$ & 40.748 & 2.48 & 0.6 \\
\hline 2007 & 11 & 15 & $06: 24: 4312.088$ & 40.755 & 2.87 & 0.56 \\
\hline 2007 & 11 & 15 & $08: 37: 4012.159$ & 40.708 & 2.61 & 0.49 \\
\hline 2007 & 11 & 15 & $09: 25: 0612.104$ & 40.743 & 2.10 & 0.69 \\
\hline 2007 & 11 & 15 & $09: 51: 5412.113$ & 40.739 & 2.17 & 0.78 \\
\hline 2007 & 11 & 15 & $10: 16: 5612.184$ & 40.688 & 2.15 & 0.94 \\
\hline 2007 & 11 & 15 & $12: 42: 1612.098$ & 40.742 & 2.31 & 1.48 \\
\hline 2007 & 11 & 15 & $14: 28: 2312.108$ & 40.745 & 2.27 & 0.71 \\
\hline 2007 & 11 & 15 & $18: 22: 0112.085$ & 40.773 & 2.97 & 0.4 \\
\hline 2007 & 11 & 15 & $20: 46: 2312.112$ & 40.736 & 2.03 & 0.46 \\
\hline 2007 & 11 & 15 & $21: 06: 5312.096$ & 40.763 & 2.62 & 0.37 \\
\hline 2007 & 11 & 15 & $21: 55: 3812.109$ & 40.763 & 2.20 & 0.49 \\
\hline 2007 & 11 & 15 & $22: 08: 0012.192$ & 40.696 & 2.55 & 0.42 \\
\hline 2007 & 11 & 15 & $23: 30: 2412.146$ & 40.721 & 1.92 & 0.5 \\
\hline 2007 & 11 & 16 & $00: 09: 5612.113$ & 40.741 & 2.00 & 0.51 \\
\hline 2007 & 11 & 16 & $02: 47: 5912.090$ & 40.732 & 2.02 & 0.64 \\
\hline 2007 & 11 & 16 & $03: 24: 1913.543$ & 39.988 & 2.68 & 1.96 \\
\hline 2007 & 11 & 16 & $03: 48: 1012.472$ & 40.566 & 2.21 & 0.93 \\
\hline 2007 & 11 & 16 & $09: 10: 1412.148$ & 40.714 & 2.20 & 0.52 \\
\hline 2007 & 11 & 16 & $15: 42: 1012.120$ & 40.750 & 2.46 & 0.38 \\
\hline 2007 & 11 & 16 & $17: 39: 1112.461$ & 40.561 & 2.44 & 0.54 \\
\hline 2007 & 11 & 16 & $19: 03: 2212.197$ & 40.715 & 2.21 & 1.51 \\
\hline
\end{tabular}




\begin{tabular}{|c|c|c|c|c|c|c|}
\hline 2007 & 11 & 16 & $20: 18: 1713.506$ & 39.994 & 2.52 & 2.06 \\
\hline 2007 & 11 & 16 & $21: 34: 2212.100$ & 40.751 & 2.01 & 0.48 \\
\hline 2007 & 11 & 16 & $22: 16: 5313.438$ & 40.932 & 2.52 & 2.15 \\
\hline 2007 & 11 & 17 & $00: 32: 3112.081$ & 40.754 & 1.93 & 0.55 \\
\hline 2007 & 11 & 17 & $01: 00: 3212.125$ & 40.740 & 1.84 & 1.26 \\
\hline 2007 & 11 & 17 & $02: 02: 5712.105$ & 40.758 & 2.18 & 0.45 \\
\hline 2007 & 11 & 17 & $09: 32: 5312.097$ & 40.737 & 2.27 & 0.45 \\
\hline 2007 & 11 & 17 & $15: 51: 5612.085$ & 40.744 & 2.14 & 1.88 \\
\hline 2007 & 11 & 17 & $16: 25: 5112.148$ & 40.708 & 2.08 & 1.39 \\
\hline 2007 & 11 & 17 & $22: 14: 5613.455$ & 40.936 & 2.38 & 4.88 \\
\hline 2007 & 11 & 18 & $01: 33: 2512.115$ & 40.744 & 3.36 & 0.36 \\
\hline 2007 & 11 & 18 & $02: 46: 3212.086$ & 40.745 & 2.10 & 0.5 \\
\hline 2007 & 11 & 18 & $03: 24: 1013.428$ & 40.913 & 2.53 & 4.61 \\
\hline 2007 & 11 & 18 & $04: 49: 5313.451$ & 40.905 & 3.73 & 4.36 \\
\hline 2007 & 11 & 18 & $07: 38: 0213.458$ & 40.883 & 2.79 & 4.49 \\
\hline 2007 & 11 & 18 & $10: 18: 3312.094$ & 40.742 & 2.64 & 0.58 \\
\hline 2007 & 11 & 18 & $10: 28: 0812.085$ & 40.745 & 2.41 & 0.63 \\
\hline 2007 & 11 & 18 & $20: 28: 2213.544$ & 39.996 & 2.57 & 3.42 \\
\hline 2007 & 11 & 18 & $20: 31: 3913.541$ & 39.991 & 2.59 & 3.17 \\
\hline 2007 & 11 & 18 & $21: 52: 0213.532$ & 39.980 & 3.03 & 1.54 \\
\hline 2007 & 11 & 18 & $22: 52: 0112.100$ & 40.749 & 2.09 & 0.39 \\
\hline 2007 & 11 & 19 & $00: 31: 4311.909$ & 41.039 & 1.89 & 0.34 \\
\hline 2007 & 11 & 19 & $02: 38: 4913.459$ & 40.944 & 3.87 & 6.81 \\
\hline 2007 & 11 & 19 & $03: 33: 4213.334$ & 40.852 & 2.87 & 7.87 \\
\hline 2007 & 11 & 19 & $05: 17: 5712.091$ & 40.735 & 2.31 & 0.87 \\
\hline 2007 & 11 & 19 & $10: 17: 5313.457$ & 40.929 & 3.31 & 4.71 \\
\hline 2007 & 11 & 19 & $22: 50: 5713.425$ & 40.937 & 2.69 & 2.38 \\
\hline 2007 & 11 & 20 & $00: 49: 4812.120$ & 40.751 & 2.13 & 0.45 \\
\hline 2007 & 11 & 20 & $00: 50: 3012.093$ & 40.744 & 2.42 & 0.57 \\
\hline 2007 & 11 & 20 & $01: 08: 0813.524$ & 39.988 & 2.48 & 3.06 \\
\hline 2007 & 11 & 20 & $01: 47: 4913.472$ & 39.995 & 2.47 & 4.1 \\
\hline 2007 & 11 & 20 & $03: 03: 0013.392$ & 40.856 & 2.82 & 1.17 \\
\hline 2007 & 11 & 20 & $05: 40: 4813.456$ & 39.990 & 2.72 & 0.56 \\
\hline 2007 & 11 & 20 & $07: 59: 2712.096$ & 40.733 & 2.49 & 1.44 \\
\hline 2007 & 11 & 21 & $04: 15: 5012.577$ & 40.484 & 2.07 & 1.24 \\
\hline 2007 & 11 & 21 & $08: 12: 2513.452$ & 40.905 & 2.85 & 4.74 \\
\hline 2007 & 11 & 21 & $10: 41: 0811.916$ & 41.072 & 2.09 & 0.49 \\
\hline 2007 & 11 & 21 & $12: 10: 5613.451$ & 40.992 & 3.71 & 7.08 \\
\hline 2007 & 11 & 21 & $17: 37: 3013.551$ & 39.967 & 2.57 & 2.1 \\
\hline 2007 & 11 & 21 & $18: 16: 5613.365$ & 41.047 & 3.10 & 8.05 \\
\hline 2007 & 11 & 21 & $18: 23: 3013.200$ & 41.072 & 2.79 & 14.82 \\
\hline 2007 & 11 & 21 & $23: 22: 5313.535$ & 39.946 & 2.50 & 3.15 \\
\hline 2007 & 11 & 22 & $10: 17: 1013.228$ & 41.093 & 2.86 & 14.52 \\
\hline 2007 & 11 & 22 & $15: 53: 1413.332$ & 41.089 & 2.67 & 9.83 \\
\hline 2007 & 11 & 22 & $16: 16: 4713.280$ & 41.066 & 2.69 & 9.47 \\
\hline 2007 & 11 & 22 & $17: 00: 3011.898$ & 41.035 & 2.02 & 0.32 \\
\hline 2007 & 11 & 22 & $18: 20: 2012.108$ & 40.755 & 2.05 & 0.49 \\
\hline 2007 & 11 & 22 & $18: 55: 2613.431$ & 40.926 & 2.47 & 2.52 \\
\hline 2007 & 11 & 22 & $20: 50: 4312.098$ & 40.755 & 2.01 & 0.49 \\
\hline 2007 & 11 & 22 & $21: 51: 2412.438$ & 40.573 & 1.83 & 0.78 \\
\hline 2007 & 11 & 22 & $23: 05: 4612.244$ & 40.695 & 2.20 & 1.29 \\
\hline 2007 & 11 & 22 & $23: 19: 2912.127$ & 40.759 & 1.99 & 0.57 \\
\hline 2007 & 11 & 23 & $09: 20: 5212.061$ & 40.770 & 2.09 & 0.54 \\
\hline 2007 & 11 & 23 & $18: 37: 5712.494$ & 40.559 & 1.99 & 1.19 \\
\hline 2007 & 11 & 23 & $20: 13: 3113.158$ & 39.635 & 2.47 & 1.23 \\
\hline 2007 & 11 & 23 & $20: 32: 0613.240$ & 41.058 & 2.39 & 12.65 \\
\hline 2007 & 11 & 23 & $22: 30: 5013.525$ & 39.961 & 2.52 & 1.45 \\
\hline 2007 & 11 & 23 & $23: 33: 1213.522$ & 39.976 & 2.53 & 1.83 \\
\hline 2007 & 11 & 24 & $01: 17: 3013.220$ & 41.099 & 2.70 & 11.83 \\
\hline 2007 & 11 & 24 & $07: 51: 5912.430$ & 40.576 & 2.22 & 0.5 \\
\hline 2007 & 11 & 24 & $17: 42: 3312.112$ & 40.743 & 1.99 & 0.51 \\
\hline 2007 & 11 & 24 & $21: 41: 0212.122$ & 40.732 & 1.89 & 0.68 \\
\hline 2007 & 11 & 25 & $06: 26: 5112.127$ & 40.722 & 2.46 & 0.64 \\
\hline 2007 & 11 & 25 & $19: 32: 4412.000$ & 42.364 & 3.28 & 5.3 \\
\hline 2007 & 11 & 25 & $21: 36: 1912.103$ & 40.757 & 1.93 & 0.51 \\
\hline 2007 & 11 & 25 & $22: 13: 3913.505$ & 39.962 & 2.57 & 1.4 \\
\hline 2007 & 11 & 25 & $22: 49: 3312.125$ & 40.747 & 1.86 & 0.53 \\
\hline 2007 & 11 & 26 & $00: 25: 3712.141$ & 40.712 & 1.98 & 0.56 \\
\hline 2007 & 11 & 26 & $02: 14: 0313.422$ & 40.850 & 2.53 & 8.51 \\
\hline 2007 & 11 & 26 & $06: 11: 0713.549$ & 40.013 & 3.32 & 4.06 \\
\hline 2007 & 11 & 26 & $17: 21: 1112.151$ & 40.711 & 2.56 & 0.44 \\
\hline 2007 & 11 & 26 & $19: 29: 2913.530$ & 39.986 & 2.64 & 1.44 \\
\hline 2007 & 11 & 26 & $19: 34: 5012.491$ & 40.537 & 2.09 & 0.72 \\
\hline 2007 & 11 & 26 & $20: 49: 1812.113$ & 40.747 & 2.40 & 0.42 \\
\hline 2007 & 11 & 27 & $20: 06: 2812.077$ & 40.756 & 2.11 & 0.58 \\
\hline 2007 & 11 & 27 & $23: 27: 0513.461$ & 40.910 & 2.39 & 3.93 \\
\hline
\end{tabular}




\begin{tabular}{|c|c|c|c|c|c|c|}
\hline 2007 & 11 & 28 & $01: 38: 5812.809$ & 39.645 & 2.34 & 0.57 \\
\hline 2007 & 11 & 28 & $07: 14: 4313.246$ & 41.086 & 2.92 & 9.18 \\
\hline 2007 & 11 & 28 & $16: 39: 2410.756$ & 41.093 & 2.76 & 4.51 \\
\hline 2007 & 11 & 28 & $17: 48: 4613.449$ & 40.928 & 2.55 & 4.38 \\
\hline 2007 & 11 & 29 & $00: 58: 3011.722$ & 39.590 & 2.28 & 0.46 \\
\hline 2007 & 11 & 29 & $01: 32: 2713.125$ & 39.918 & 2.39 & 0.43 \\
\hline 2007 & 11 & 29 & $02: 24: 5313.264$ & 41.103 & 2.72 & 7.43 \\
\hline 2007 & 11 & 29 & $10: 53: 0512.097$ & 40.750 & 2.24 & 0.57 \\
\hline 2007 & 11 & 29 & $21: 25: 3912.119$ & 40.758 & 2.29 & 0.53 \\
\hline 2007 & 11 & 29 & $22: 50: 5212.118$ & 40.755 & 2.14 & 0.44 \\
\hline 2007 & 11 & 30 & $02: 13: 4010.791$ & 41.091 & 2.77 & 0.83 \\
\hline 2007 & 11 & 30 & $02: 47: 1610.783$ & 41.087 & 2.64 & 4.23 \\
\hline 2007 & 11 & 30 & $17: 09: 3012.860$ & 39.534 & 2.50 & 0.48 \\
\hline 2007 & 12 & 1 & $00: 52: 0312.369$ & 40.609 & 2.04 & 0.37 \\
\hline 2007 & 12 & 1 & $12: 52: 2310.250$ & 40.535 & 3.90 & 1.87 \\
\hline 2007 & 12 & 1 & $13: 45: 3110.250$ & 40.623 & 3.68 & 4.26 \\
\hline 2007 & 12 & 1 & $16: 16: 1910.263$ & 40.596 & 2.88 & 3.73 \\
\hline 2007 & 12 & 1 & $17: 33: 0110.257$ & 40.572 & 2.91 & 2.14 \\
\hline 2007 & 12 & 1 & $17: 56: 1810.270$ & 40.621 & 3.04 & 6.17 \\
\hline 2007 & 12 & 1 & $20: 35: 1613.505$ & 39.905 & 2.48 & 1.64 \\
\hline 2007 & 12 & 2 & $00: 34: 5911.215$ & 39.229 & 2.55 & 1.39 \\
\hline 2007 & 12 & 2 & $01: 48: 4112.095$ & 40.750 & 1.95 & 0.54 \\
\hline 2007 & 12 & 2 & $18: 46: 2812.469$ & 40.567 & 1.93 & 0.79 \\
\hline 2007 & 12 & 2 & $22: 49: 2012.407$ & 40.543 & 1.88 & 0.82 \\
\hline 2007 & 12 & 3 & $00: 47: 4312.152$ & 40.715 & 2.04 & 0.51 \\
\hline 2007 & 12 & 3 & $13: 12: 0410.215$ & 40.592 & 3.70 & 9.54 \\
\hline 2007 & 12 & 3 & $21: 40: 4012.108$ & 40.739 & 1.86 & 0.92 \\
\hline 2007 & 12 & 3 & $22: 01: 3512.461$ & 40.561 & 2.23 & 0.44 \\
\hline 2007 & 12 & 3 & $22: 11: 2012.007$ & 41.259 & 2.02 & 0.76 \\
\hline 2007 & 12 & 4 & $00: 26: 1210.255$ & 40.612 & 2.82 & 4.56 \\
\hline 2007 & 12 & 4 & $01: 14: 1713.328$ & 39.738 & 2.56 & 1.5 \\
\hline 2007 & 12 & 4 & $02: 08: 4913.542$ & 39.972 & 2.64 & 1.27 \\
\hline 2007 & 12 & 4 & $05: 04: 4512.173$ & 40.708 & 2.26 & 1.11 \\
\hline 2007 & 12 & 4 & $18: 10: 0212.434$ & 40.581 & 2.14 & 0.57 \\
\hline 2007 & 12 & 4 & $19: 01: 2413.160$ & 39.644 & 2.50 & 5.48 \\
\hline 2007 & 12 & 4 & $20: 14: 4410.791$ & 41.076 & 2.84 & 0.76 \\
\hline 2007 & 12 & 4 & $20: 16: 2810.792$ & 41.098 & 2.78 & 0.68 \\
\hline 2007 & 12 & 4 & $20: 36: 0710.802$ & 41.115 & 2.60 & 1.05 \\
\hline 2007 & 12 & 4 & $22: 46: 1713.493$ & 39.991 & 2.52 & 2.12 \\
\hline 2007 & 12 & 5 & $00: 57: 0713.521$ & 39.995 & 2.52 & 1.82 \\
\hline 2007 & 12 & 5 & $01: 14: 4713.498$ & 39.936 & 2.50 & 1.8 \\
\hline 2007 & 12 & 5 & $02: 52: 5511.328$ & 39.614 & 2.47 & 0.62 \\
\hline 2007 & 12 & 5 & $10: 08: 0412.540$ & 40.539 & 2.55 & 0.45 \\
\hline 2007 & 12 & 5 & $19: 30: 1413.560$ & 39.959 & 2.52 & 1.57 \\
\hline 2007 & 12 & 5 & $20: 49: 0812.496$ & 40.554 & 1.96 & 0.79 \\
\hline 2007 & 12 & 5 & $22: 33: 5212.498$ & 40.564 & 2.01 & 0.42 \\
\hline 2007 & 12 & 5 & $22: 58: 4512.094$ & 40.748 & 2.15 & 0.62 \\
\hline 2007 & 12 & 6 & $09: 27: 3711.227$ & 39.748 & 2.69 & 0.47 \\
\hline 2007 & 12 & 6 & $17: 36: 4913.507$ & 39.986 & 2.56 & 1.84 \\
\hline 2007 & 12 & 6 & $18: 41: 2112.380$ & 40.602 & 2.07 & 0.68 \\
\hline 2007 & 12 & 6 & $20: 10: 1012.105$ & 40.736 & 2.29 & 0.52 \\
\hline 2007 & 12 & 6 & $22: 06: 3712.505$ & 40.549 & 1.93 & 1.39 \\
\hline 2007 & 12 & 7 & $01: 24: 5514.646$ & 40.017 & 2.95 & 19.77 \\
\hline 2007 & 12 & 7 & $02: 11: 4611.224$ & 39.748 & 2.40 & 0.52 \\
\hline 2007 & 12 & 7 & $16: 30: 2813.489$ & 39.975 & 2.54 & 2.26 \\
\hline 2007 & 12 & 7 & $19: 13: 3512.464$ & 40.552 & 2.01 & 0.6 \\
\hline 2007 & 12 & 7 & $23: 09: 059.525$ & 39.584 & 2.99 & 4.45 \\
\hline 2007 & 12 & 8 & $01: 22: 1611.222$ & 39.742 & 2.35 & 0.41 \\
\hline 2007 & 12 & 8 & $01: 36: 0313.536$ & 39.963 & 2.57 & 2.26 \\
\hline 2007 & 12 & 8 & $12: 49: 3011.838$ & 41.168 & 2.48 & 0.62 \\
\hline 2007 & 12 & 8 & $14: 28: 3811.915$ & 41.481 & 2.56 & 0.41 \\
\hline 2007 & 12 & 8 & $19: 39: 2112.496$ & 40.544 & 1.96 & 1.11 \\
\hline 2007 & 12 & 8 & $19: 55: 4213.543$ & 39.985 & 2.53 & 0.56 \\
\hline 2007 & 12 & 9 & $00: 58: 5812.476$ & 40.545 & 1.84 & 1.3 \\
\hline 2007 & 12 & 9 & $10: 53: 3312.466$ & 39.585 & 2.45 & 0.66 \\
\hline 2007 & 12 & 9 & $11: 49: 3512.444$ & 40.569 & 2.13 & 0.53 \\
\hline 2007 & 12 & 9 & $18: 45: 4012.350$ & 40.336 & 1.63 & 3.73 \\
\hline 2007 & 12 & 9 & $20: 19: 2712.146$ & 40.713 & 2.10 & 0.48 \\
\hline 2007 & 12 & 9 & $23: 44: 0112.146$ & 41.757 & 2.25 & 6.26 \\
\hline 2007 & 12 & 9 & $23: 47: 3711.868$ & 41.076 & 1.80 & 0.39 \\
\hline 2007 & 12 & 10 & $02: 01: 3612.370$ & 40.593 & 1.75 & 0.72 \\
\hline 2007 & 12 & 10 & $11: 24: 1512.339$ & 40.643 & 1.99 & 0.59 \\
\hline 2007 & 12 & 10 & $20: 55: 1113.612$ & 39.913 & 2.47 & 0.78 \\
\hline 2007 & 12 & 10 & $22: 37: 4911.866$ & 41.080 & 1.76 & 0.59 \\
\hline 2007 & 12 & 11 & $00: 35: 3912.394$ & 40.589 & 1.84 & 0.4 \\
\hline 2007 & 12 & 11 & $22: 38: 0210.783$ & 41.100 & 2.47 & 1.17 \\
\hline
\end{tabular}




\begin{tabular}{|c|c|c|c|c|c|c|}
\hline 2007 & 12 & 11 & $22: 58: 5610.790$ & 41.094 & 2.44 & 1.27 \\
\hline 2007 & 12 & 11 & $23: 00: 3710.790$ & 41.091 & 2.44 & 0.92 \\
\hline 2007 & 12 & 13 & $11: 55: 2913.527$ & 39.963 & 2.64 & 1.59 \\
\hline 2007 & 12 & 13 & $19: 13: 4412.453$ & 40.565 & 1.89 & 0.52 \\
\hline 2007 & 12 & 14 & $00: 57: 1612.407$ & 40.600 & 1.87 & 0.48 \\
\hline 2007 & 12 & 14 & $01: 41: 3212.451$ & 40.580 & 1.88 & 0.63 \\
\hline 2007 & 12 & 14 & $08: 45: 3713.136$ & 39.636 & 2.81 & 0.8 \\
\hline 2007 & 12 & 14 & $17: 07: 5712.454$ & 40.562 & 2.17 & 0.54 \\
\hline 2007 & 12 & 14 & $20: 32: 0213.467$ & 39.986 & 2.46 & 2.65 \\
\hline 2007 & 12 & 14 & $23: 02: 4013.513$ & 39.959 & 2.48 & 2.5 \\
\hline 2007 & 12 & 15 & $03: 06: 4812.467$ & 40.211 & 1.81 & 3.14 \\
\hline 2007 & 12 & 15 & $11: 17: 2812.624$ & 39.855 & 2.28 & 10.25 \\
\hline 2007 & 12 & 15 & $15: 00: 5613.147$ & 39.620 & 2.48 & 0.45 \\
\hline 2007 & 12 & 15 & $16: 55: 0712.013$ & 39.908 & 2.21 & 2.38 \\
\hline 2007 & 12 & 16 & $02: 30: 4912.413$ & 40.639 & 1.80 & 0.69 \\
\hline 2007 & 12 & 16 & $03: 20: 0713.102$ & 39.558 & 2.47 & 0.75 \\
\hline 2007 & 12 & 16 & $03: 31: 4512.422$ & 41.002 & 2.14 & 0.61 \\
\hline 2007 & 12 & 16 & $13: 36: 4211.623$ & 40.569 & 1.96 & 0.99 \\
\hline 2007 & 12 & 16 & $16: 45: 3812.162$ & 40.772 & 1.84 & 2.23 \\
\hline 2007 & 12 & 16 & $20: 01: 1913.116$ & 39.593 & 2.50 & 3.24 \\
\hline 2007 & 12 & 16 & $23: 12: 0213.192$ & 39.683 & 2.43 & 0.68 \\
\hline 2007 & 12 & 16 & $23: 59: 3013.143$ & 39.620 & 3.51 & 0.8 \\
\hline 2007 & 12 & 17 & $00: 53: 0212.488$ & 40.554 & 1.80 & 3.27 \\
\hline 2007 & 12 & 17 & $04: 06: 4012.082$ & 40.744 & 1.95 & 3.14 \\
\hline 2007 & 12 & 17 & $14: 10: 5612.416$ & 40.589 & 2.02 & 1.08 \\
\hline 2007 & 12 & 17 & $21: 37: 5912.407$ & 40.596 & 1.80 & 0.6 \\
\hline 2007 & 12 & 17 & $22: 41: 3112.367$ & 40.589 & 1.80 & 0.51 \\
\hline 2007 & 12 & 18 & $00: 43: 5612.446$ & 40.542 & 1.74 & 1.34 \\
\hline 2007 & 12 & 18 & $03: 16: 1012.426$ & 40.582 & 1.89 & 0.46 \\
\hline 2007 & 12 & 18 & $16: 13: 4112.367$ & 40.633 & 1.93 & 0.86 \\
\hline 2007 & 12 & 18 & $23: 43: 0111.916$ & 41.050 & 2.08 & 0.4 \\
\hline 2007 & 12 & 19 & $06: 45: 0012.137$ & 40.708 & 2.09 & 1.98 \\
\hline 2007 & 12 & 19 & $06: 46: 0312.131$ & 40.706 & 2.01 & 2.71 \\
\hline 2007 & 12 & 19 & $15: 08: 0012.403$ & 40.473 & 1.82 & 4.89 \\
\hline 2007 & 12 & 19 & $17: 03: 3711.789$ & 41.055 & 2.60 & 0.51 \\
\hline 2007 & 12 & 19 & $19: 32: 4413.270$ & 40.943 & 2.50 & 18.57 \\
\hline 2007 & 12 & 19 & $19: 49: 3212.134$ & 40.860 & 1.95 & 1.56 \\
\hline 2007 & 12 & 19 & $21: 14: 4012.410$ & 40.027 & 2.02 & 2.61 \\
\hline 2007 & 12 & 19 & $23: 08: 3013.166$ & 39.629 & 3.03 & 0.59 \\
\hline 2007 & 12 & 20 & $02: 31: 2512.513$ & 40.544 & 2.68 & 0.66 \\
\hline 2007 & 12 & 20 & $14: 24: 1112.115$ & 40.742 & 3.35 & 0.42 \\
\hline 2007 & 12 & 20 & $19: 09: 3313.550$ & 39.958 & 2.56 & 2.38 \\
\hline 2007 & 12 & 21 & $00: 34: 0512.426$ & 40.516 & 1.77 & 0.56 \\
\hline 2007 & 12 & 21 & $00: 43: 1412.650$ & 39.709 & 2.19 & 2.41 \\
\hline 2007 & 12 & 21 & $02: 44: 0512.397$ & 40.472 & 1.71 & 1.11 \\
\hline 2007 & 12 & 21 & $10: 08: 0812.197$ & 40.912 & 2.07 & 15.7 \\
\hline 2007 & 12 & 21 & $21: 39: 2512.397$ & 40.600 & 1.77 & 1.46 \\
\hline 2007 & 12 & 23 & $00: 12: 2912.419$ & 40.558 & 1.67 & 1.13 \\
\hline 2007 & 12 & 23 & $01: 10: 2411.968$ & 41.227 & 1.99 & 10.63 \\
\hline 2007 & 12 & 23 & $02: 49: 3512.391$ & 40.562 & 1.75 & 0.96 \\
\hline 2007 & 12 & 23 & $05: 56: 2312.351$ & 40.533 & 1.90 & 0.85 \\
\hline 2007 & 12 & 23 & $09: 40: 1412.470$ & 40.579 & 1.96 & 5.86 \\
\hline 2007 & 12 & 23 & $09: 56: 4412.404$ & 40.567 & 2.00 & 1.74 \\
\hline 2007 & 12 & 23 & $11: 30: 5413.568$ & 39.990 & 2.62 & 2.99 \\
\hline 2007 & 12 & 23 & $16: 53: 1613.544$ & 39.985 & 2.46 & 1.82 \\
\hline 2007 & 12 & 23 & $19: 16: 3912.413$ & 40.596 & 1.72 & 0.65 \\
\hline 2007 & 12 & 23 & $21: 31: 1513.502$ & 39.979 & 2.37 & 3.16 \\
\hline 2007 & 12 & 24 & $03: 35: 2512.403$ & 40.427 & 1.81 & 1.85 \\
\hline 2007 & 12 & 24 & $17: 06: 5612.432$ & 40.542 & 1.94 & 0.88 \\
\hline 2007 & 12 & 24 & $19: 33: 4412.434$ & 39.983 & 1.92 & 1.3 \\
\hline 2007 & 12 & 24 & $20: 19: 2714.125$ & 40.405 & 2.70 & 2.17 \\
\hline 2007 & 12 & 24 & $21: 10: 4312.120$ & 40.908 & 2.11 & 0.75 \\
\hline 2007 & 12 & 24 & $21: 28: 2612.113$ & 40.906 & 2.05 & 0.7 \\
\hline 2007 & 12 & 24 & $22: 02: 2912.122$ & 40.740 & 1.84 & 0.64 \\
\hline 2007 & 12 & 25 & $08: 11: 4412.104$ & 40.723 & 2.17 & 1.03 \\
\hline 2007 & 12 & 25 & $09: 23: 1113.519$ & 39.989 & 2.73 & 1.16 \\
\hline 2007 & 12 & 25 & $11: 55: 5812.411$ & 40.564 & 2.15 & 0.59 \\
\hline 2007 & 12 & 25 & $22: 39: 5112.408$ & 40.548 & 1.72 & 0.6 \\
\hline 2007 & 12 & 26 & $16: 12: 2312.880$ & 39.265 & 2.51 & 2.47 \\
\hline 2007 & 12 & 26 & $22: 54: 47 \quad 12.491$ & 40.528 & 1.95 & 1.55 \\
\hline 2007 & 12 & 27 & $01: 01: 2013.501$ & 39.997 & 2.58 & 1.38 \\
\hline 2007 & 12 & 27 & $02: 47: 3712.505$ & 40.732 & 1.97 & 2.27 \\
\hline 2007 & 12 & 27 & $04: 44: 5013.171$ & 39.681 & 2.66 & 7.33 \\
\hline 2007 & 12 & 27 & $20: 25: 5710.206$ & 40.598 & 2.91 & 2.29 \\
\hline 2007 & 12 & 27 & $22: 16: 4110.265$ & 40.582 & 2.67 & 12.74 \\
\hline 2007 & 12 & 27 & $23: 19: 4112.618$ & 40.947 & 2.06 & 5.56 \\
\hline
\end{tabular}




\begin{tabular}{|c|c|c|c|c|c|c|}
\hline 2007 & 12 & 28 & $16: 41: 3812.522$ & 40.537 & 2.07 & 0.85 \\
\hline 2007 & 12 & 28 & $19: 49: 0414.605$ & 39.910 & 3.03 & 8.22 \\
\hline 2007 & 12 & 28 & $21: 31: 5214.565$ & 39.852 & 2.94 & 17.06 \\
\hline 2007 & 12 & 29 & $14: 35: 4813.511$ & 39.977 & 2.63 & 1.63 \\
\hline 2007 & 12 & 30 & $01: 03: 3013.456$ & 39.995 & 2.51 & 1.05 \\
\hline 2007 & 12 & 30 & $10: 00: 4212.463$ & 40.551 & 2.27 & 0.71 \\
\hline 2007 & 12 & 30 & $11: 58: 2913.502$ & 39.966 & 2.65 & 2.43 \\
\hline 2007 & 12 & 30 & $12: 19: 2113.498$ & 39.989 & 2.69 & 2.94 \\
\hline 2007 & 12 & 30 & $13: 58: 3413.458$ & 39.994 & 2.59 & 1.51 \\
\hline 2007 & 12 & 30 & $19: 52: 2913.540$ & 39.956 & 2.56 & 3.08 \\
\hline 2007 & 12 & 30 & $21: 44: 1813.513$ & 39.978 & 2.54 & 1.77 \\
\hline 2007 & 12 & 30 & $22: 47: 2113.504$ & 39.998 & 2.57 & 1.44 \\
\hline 2007 & 12 & 31 & $06: 30: 0412.412$ & 40.570 & 2.20 & 0.72 \\
\hline 2007 & 12 & 31 & $11: 10: 2912.455$ & 40.566 & 2.29 & 0.51 \\
\hline 2007 & 12 & 31 & $20: 36: 4810.908$ & 39.719 & 2.55 & 1.43 \\
\hline 2008 & 1 & 1 & $02: 58: 3812.858$ & 40.615 & 2.13 & 1.96 \\
\hline 2008 & 1 & 1 & $05: 52: 2412.447$ & 40.568 & 2.08 & 0.46 \\
\hline 2008 & 1 & 1 & $18: 14: 1412.470$ & 40.493 & 1.82 & 0.74 \\
\hline 2008 & 1 & 1 & $22: 42: 1912.859$ & 39.721 & 2.36 & 0.56 \\
\hline 2008 & 1 & 2 & $01: 12: 1611.295$ & 41.646 & 2.70 & 0.82 \\
\hline 2008 & 1 & 2 & $16: 46: 3113.662$ & 39.932 & 2.69 & 7.19 \\
\hline 2008 & 1 & 3 & $12: 21: 0812.420$ & 40.576 & 2.03 & 0.79 \\
\hline 2008 & 1 & 3 & $16: 22: 0612.077$ & 40.749 & 1.87 & 1.37 \\
\hline 2008 & 1 & 3 & $18: 46: 5912.466$ & 40.499 & 1.80 & 1.26 \\
\hline 2008 & 1 & 3 & $19: 16: 2512.363$ & 40.602 & 1.85 & 0.56 \\
\hline 2008 & 1 & 3 & $19: 50: 4612.395$ & 40.587 & 1.87 & 0.61 \\
\hline 2008 & 1 & 3 & $20: 18: 5812.387$ & 40.590 & 1.84 & 0.74 \\
\hline 2008 & 1 & 3 & $20: 30: 2912.884$ & 40.308 & 2.10 & 0.6 \\
\hline 2008 & 1 & 3 & $23: 00: 4312.424$ & 40.584 & 1.88 & 0.81 \\
\hline 2008 & 1 & 4 & $00: 41: 1612.496$ & 40.548 & 1.94 & 1.1 \\
\hline 2008 & 1 & 4 & $13: 14: 5812.216$ & 39.688 & 2.39 & 1.71 \\
\hline 2008 & 1 & 4 & $15: 49: 42 \quad 13.619$ & 39.962 & 2.74 & 6.02 \\
\hline 2008 & 1 & 4 & $18: 51: 4212.392$ & 40.579 & 1.81 & 0.72 \\
\hline 2008 & 1 & 4 & $20: 27: 3111.182$ & 41.064 & 2.39 & 2.12 \\
\hline 2008 & 1 & 4 & $20: 50: 2912.404$ & 40.574 & 1.90 & 0.62 \\
\hline 2008 & 1 & 4 & $21: 17: 3012.408$ & 40.583 & 1.97 & 0.52 \\
\hline 2008 & 1 & 4 & $21: 44: 4112.443$ & 40.563 & 1.98 & 0.53 \\
\hline 2008 & 1 & 4 & $23: 26: 3012.392$ & 40.585 & 1.85 & 0.64 \\
\hline 2008 & 1 & 4 & $23: 38: 2413.593$ & 39.977 & 2.58 & 5.41 \\
\hline 2008 & 1 & 5 & $10: 14: 1312.400$ & 40.594 & 2.14 & 0.4 \\
\hline 2008 & 1 & 6 & $02: 59: 1712.457$ & 40.523 & 1.81 & 1.68 \\
\hline 2008 & 1 & 6 & $06: 16: 5412.447$ & 40.557 & 2.04 & 0.64 \\
\hline 2008 & 1 & 6 & $08: 34: 2012.452$ & 40.550 & 1.91 & 0.72 \\
\hline 2008 & 1 & 6 & $10: 20: 5712.370$ & 40.596 & 1.96 & 0.57 \\
\hline 2008 & 1 & 6 & $10: 46: 1712.450$ & 40.534 & 1.82 & 0.84 \\
\hline 2008 & 1 & 6 & $11: 42: 1012.439$ & 40.573 & 1.92 & 0.55 \\
\hline 2008 & 1 & 6 & $14: 03: 2712.411$ & 40.580 & 2.21 & 0.48 \\
\hline 2008 & 1 & 6 & $21: 58: 0812.379$ & 40.608 & 1.89 & 0.48 \\
\hline 2008 & 1 & 7 & $09: 44: 3813.208$ & 39.532 & 2.71 & 6.1 \\
\hline 2008 & 1 & 7 & $21: 56: 2912.406$ & 40.583 & 1.79 & 0.59 \\
\hline 2008 & 1 & 7 & $23: 27: 0811.308$ & 40.005 & 2.17 & 0.42 \\
\hline 2008 & 1 & 8 & $00: 50: 0612.369$ & 40.598 & 1.82 & 0.47 \\
\hline 2008 & 1 & 8 & $11: 34: 0612.417$ & 40.580 & 1.96 & 0.54 \\
\hline 2008 & 1 & 8 & $21: 24: 3612.383$ & 40.598 & 1.96 & 0.46 \\
\hline 2008 & 1 & 9 & $00: 18: 4012.388$ & 40.594 & 1.94 & 0.72 \\
\hline 2008 & 1 & 9 & $01: 49: 2511.509$ & 39.726 & 2.34 & 0.53 \\
\hline 2008 & 1 & 9 & 03:07:39 12.377 & 40.606 & 2.01 & 0.59 \\
\hline 2008 & 1 & 9 & $03: 17: 4912.364$ & 40.602 & 1.95 & 0.53 \\
\hline 2008 & 1 & 9 & $09: 53: 2012.413$ & 40.578 & 2.04 & 0.67 \\
\hline 2008 & 1 & 9 & $18: 22: 5912.358$ & 40.606 & 1.94 & 1.3 \\
\hline 2008 & 1 & 10 & $02: 42: 2612.463$ & 40.557 & 1.98 & 0.96 \\
\hline 2008 & 1 & 10 & $05: 24: 2312.458$ & 40.548 & 1.96 & 0.66 \\
\hline 2008 & 1 & 10 & $17: 56: 0312.372$ & 40.602 & 2.01 & 0.56 \\
\hline 2008 & 1 & 10 & $21: 46: 4712.455$ & 40.531 & 1.91 & 0.81 \\
\hline 2008 & 1 & 11 & $01: 44: 3212.458$ & 40.566 & 1.98 & 0.49 \\
\hline 2008 & 1 & 11 & $05: 06: 5612.371$ & 40.602 & 1.94 & 0.47 \\
\hline 2008 & 1 & 11 & $18: 49: 1412.494$ & 40.542 & 1.91 & 0.9 \\
\hline 2008 & 1 & 12 & $20: 02: 1112.382$ & 40.589 & 1.83 & 0.54 \\
\hline 2008 & 1 & 12 & $23: 21: 0112.393$ & 40.594 & 1.75 & 1.61 \\
\hline 2008 & 1 & 13 & $00: 15: 0212.407$ & 40.586 & 1.80 & 0.49 \\
\hline 2008 & 1 & 13 & $02: 00: 4112.446$ & 40.570 & 1.83 & 0.5 \\
\hline 2008 & 1 & 13 & $19: 35: 1312.409$ & 40.585 & 1.88 & 0.54 \\
\hline 2008 & 1 & 13 & $23: 25: 4112.000$ & 40.949 & 1.99 & 0.27 \\
\hline 2008 & 1 & 13 & $23: 29: 1012.011$ & 40.941 & 1.90 & 0.41 \\
\hline 2008 & 1 & 14 & $05: 15: 3212.012$ & 40.933 & 1.88 & 3.98 \\
\hline 2008 & 1 & 14 & $19: 16: 5112.011$ & 40.937 & 1.84 & 0.93 \\
\hline
\end{tabular}




\begin{tabular}{|c|c|c|c|c|c|c|}
\hline 2008 & 1 & 14 & $21: 55: 0312.398$ & 40.592 & 1.90 & 0.41 \\
\hline 2008 & 1 & 14 & $21: 59: 5311.234$ & 41.212 & 2.29 & 1.67 \\
\hline 2008 & 1 & 14 & $23: 38: 3811.226$ & 41.216 & 2.29 & 1.31 \\
\hline 2008 & 1 & 15 & $09: 01: 3413.536$ & 39.941 & 2.78 & 2.28 \\
\hline 2008 & 1 & 15 & $12: 15: 4412.446$ & 40.560 & 2.17 & 0.67 \\
\hline 2008 & 1 & 15 & $22: 51: 4713.590$ & 39.920 & 2.74 & 1.46 \\
\hline 2008 & 1 & 16 & $00: 21: 0412.444$ & 40.538 & 2.01 & 0.65 \\
\hline 2008 & 1 & 16 & $01: 33: 5011.832$ & 41.090 & 2.19 & 0.46 \\
\hline 2008 & 1 & 16 & $02: 06: 3812.098$ & 40.743 & 2.12 & 0.5 \\
\hline 2008 & 1 & 16 & $22: 32: 3112.378$ & 40.596 & 1.94 & 0.78 \\
\hline 2008 & 1 & 17 & $00: 23: 0612.475$ & 40.557 & 2.03 & 1.12 \\
\hline 2008 & 1 & 17 & $00: 46: 5912.559$ & 40.535 & 1.98 & 0.98 \\
\hline 2008 & 1 & 17 & $01: 07: 5711.966$ & 39.518 & 2.52 & 0.93 \\
\hline 2008 & 1 & 17 & $15: 57: 4012.112$ & 40.744 & 2.12 & 0.8 \\
\hline 2008 & 1 & 17 & $23: 36: 0512.366$ & 40.610 & 1.94 & 0.52 \\
\hline 2008 & 1 & 18 & $01: 08: 0512.457$ & 40.561 & 1.99 & 0.6 \\
\hline 2008 & 1 & 18 & $03: 15: 4612.014$ & 40.928 & 1.90 & 6.31 \\
\hline 2008 & 1 & 18 & $15: 41: 5112.007$ & 40.912 & 1.94 & 0.82 \\
\hline 2008 & 1 & 18 & $19: 44: 4712.404$ & 40.560 & 1.89 & 1.02 \\
\hline 2008 & 1 & 18 & $22: 42: 2812.429$ & 40.568 & 1.96 & 0.6 \\
\hline 2008 & 1 & 18 & $23: 22: 0112.483$ & 40.532 & 1.86 & 2.21 \\
\hline 2008 & 1 & 19 & $00: 52: 5912.453$ & 40.563 & 1.91 & 0.78 \\
\hline 2008 & 1 & 19 & $01: 32: 4012.431$ & 40.571 & 1.93 & 0.7 \\
\hline 2008 & 1 & 19 & $02: 47: 2311.408$ & 39.661 & 2.46 & 1.4 \\
\hline 2008 & 1 & 19 & $11: 38: 2611.937$ & 41.346 & 2.26 & 0.88 \\
\hline 2008 & 1 & 19 & $22: 49: 2112.192$ & 43.404 & 3.20 & 15.31 \\
\hline 2008 & 1 & 20 & $04: 03: 4010.325$ & 39.528 & 3.23 & 8.94 \\
\hline 2008 & 1 & 20 & $15: 11: 1912.330$ & 40.604 & 1.88 & 0.68 \\
\hline 2008 & 1 & 20 & $17: 45: 5712.369$ & 40.594 & 1.86 & 0.79 \\
\hline 2008 & 1 & 20 & $17: 52: 4213.497$ & 39.994 & 2.49 & 2.15 \\
\hline 2008 & 1 & 20 & $17: 57: 1012.371$ & 40.590 & 2.05 & 0.78 \\
\hline 2008 & 1 & 21 & $01: 32: 4213.614$ & 40.760 & 2.54 & 3.45 \\
\hline 2008 & 1 & 21 & $18: 26: 5313.562$ & 39.951 & 2.60 & 2.47 \\
\hline 2008 & 1 & 21 & $22: 39: 1312.647$ & 40.537 & 1.89 & 2.47 \\
\hline 2008 & 1 & 22 & $03: 05: 3212.391$ & 40.582 & 1.96 & 0.7 \\
\hline 2008 & 1 & 23 & $01: 24: 3412.402$ & 40.582 & 1.90 & 1.24 \\
\hline 2008 & 1 & 23 & $23: 20: 3012.389$ & 40.584 & 1.87 & 0.66 \\
\hline 2008 & 1 & 23 & $23: 33: 2612.359$ & 40.592 & 1.83 & 0.85 \\
\hline 2008 & 1 & 24 & $00: 43: 1312.477$ & 40.555 & 1.84 & 1.29 \\
\hline 2008 & 1 & 24 & $02: 48: 3912.484$ & 40.521 & 1.86 & 1.69 \\
\hline 2008 & 1 & 24 & $08: 02: 3512.387$ & 40.602 & 1.94 & 3.67 \\
\hline 2008 & 1 & 24 & $19: 33: 4712.422$ & 40.581 & 1.94 & 0.98 \\
\hline 2008 & 1 & 24 & $21: 17: 0712.381$ & 40.589 & 1.75 & 0.66 \\
\hline 2008 & 1 & 24 & $22: 41: 2912.415$ & 40.572 & 1.80 & 0.91 \\
\hline 2008 & 1 & 25 & $04: 03: 1712.743$ & 40.591 & 2.75 & 0.89 \\
\hline 2008 & 1 & 25 & $06: 44: 2312.000$ & 40.628 & 2.09 & 1.21 \\
\hline 2008 & 1 & 25 & $16: 41: 0912.448$ & 40.473 & 1.79 & 2.33 \\
\hline 2008 & 1 & 25 & $18: 00: 1512.522$ & 40.544 & 1.82 & 2.64 \\
\hline 2008 & 1 & 25 & $22: 10: 1811.696$ & 41.000 & 1.79 & 0.91 \\
\hline 2008 & 1 & 26 & $01: 06: 2111.696$ & 41.002 & 1.79 & 0.55 \\
\hline 2008 & 1 & 26 & $01: 26: 3912.467$ & 40.549 & 1.87 & 1.98 \\
\hline 2008 & 1 & 26 & $20: 18: 3312.464$ & 40.559 & 2.06 & 0.49 \\
\hline 2008 & 1 & 27 & $08: 28: 4712.452$ & 40.558 & 1.78 & 0.88 \\
\hline 2008 & 1 & 27 & $08: 40: 0312.477$ & 40.565 & 1.90 & 0.58 \\
\hline 2008 & 1 & 27 & $09: 30: 0012.379$ & 40.586 & 1.84 & 0.57 \\
\hline 2008 & 1 & 27 & $11: 32: 4212.399$ & 40.609 & 1.77 & 9.78 \\
\hline 2008 & 1 & 27 & $13: 23: 1012.470$ & 40.556 & 1.81 & 0.8 \\
\hline 2008 & 1 & 27 & $14: 45: 0712.404$ & 40.581 & 1.73 & 1.06 \\
\hline 2008 & 1 & 27 & $18: 35: 2012.390$ & 40.579 & 1.66 & 0.97 \\
\hline 2008 & 1 & 27 & $21: 39: 3912.375$ & 40.596 & 1.64 & 0.6 \\
\hline 2008 & 1 & 27 & $22: 05: 5912.403$ & 40.573 & 1.59 & 0.95 \\
\hline 2008 & 1 & 27 & $22: 56: 2612.416$ & 40.526 & 1.57 & 2.58 \\
\hline 2008 & 1 & 27 & $23: 43: 5512.388$ & 40.580 & 1.55 & 1.05 \\
\hline 2008 & 1 & 27 & $23: 54: 4012.494$ & 40.530 & 1.63 & 0.75 \\
\hline 2008 & 1 & 28 & $00: 50: 5212.378$ & 40.599 & 1.59 & 2.79 \\
\hline 2008 & 1 & 28 & $01: 19: 1812.391$ & 40.582 & 1.49 & 0.79 \\
\hline 2008 & 1 & 28 & $01: 52: 2512.213$ & 40.693 & 1.66 & 0.66 \\
\hline 2008 & 1 & 28 & $03: 47: 1712.406$ & 40.603 & 1.62 & 13.98 \\
\hline 2008 & 1 & 28 & $05: 34: 1912.397$ & 40.577 & 1.70 & 0.75 \\
\hline 2008 & 1 & 28 & $09: 22: 5412.375$ & 40.590 & 1.74 & 1.08 \\
\hline 2008 & 1 & 28 & $11: 09: 1012.424$ & 40.566 & 1.81 & 0.64 \\
\hline 2008 & 1 & 28 & $13: 36: 4412.176$ & 40.713 & 1.84 & 0.65 \\
\hline 2008 & 1 & 28 & $14: 23: 0212.441$ & 40.562 & 1.71 & 0.91 \\
\hline 2008 & 1 & 28 & $16: 54: 0112.379$ & 40.586 & 1.76 & 1.16 \\
\hline 2008 & 1 & 28 & $18: 10: 0112.410$ & 40.573 & 1.67 & 0.78 \\
\hline 2008 & 1 & 28 & $19: 36: 5512.444$ & 40.565 & 1.62 & 1.33 \\
\hline
\end{tabular}




\begin{tabular}{|c|c|c|c|c|c|c|}
\hline 2008 & 1 & 28 & $19: 39: 4512.118$ & 40.757 & 1.80 & 0.84 \\
\hline 2008 & 1 & 28 & $22: 11: 1612.391$ & 40.579 & 1.53 & 1.01 \\
\hline 2008 & 1 & 28 & $22: 25: 2412.612$ & 40.571 & 1.69 & 1.78 \\
\hline 2008 & 1 & 28 & $23: 02: 0312.399$ & 40.579 & 1.51 & 0.98 \\
\hline 2008 & 1 & 28 & $23: 35: 3812.393$ & 40.578 & 1.51 & 1.01 \\
\hline 2008 & 1 & 28 & $23: 40: 3512.434$ & 40.568 & 1.60 & 0.92 \\
\hline 2008 & 1 & 29 & $00: 00: 0912.397$ & 40.588 & 2.33 & 0.56 \\
\hline 2008 & 1 & 29 & $00: 55: 1712.386$ & 40.577 & 1.55 & 1.29 \\
\hline 2008 & 1 & 29 & $01: 06: 3412.387$ & 40.583 & 1.54 & 1.34 \\
\hline 2008 & 1 & 29 & $02: 34: 5212.412$ & 40.576 & 1.56 & 0.76 \\
\hline 2008 & 1 & 29 & $05: 22: 1012.474$ & 40.556 & 1.88 & 0.79 \\
\hline 2008 & 1 & 29 & $08: 48: 0612.500$ & 40.532 & 1.90 & 0.68 \\
\hline 2008 & 1 & 29 & $11: 33: 3212.399$ & 40.578 & 1.73 & 0.9 \\
\hline 2008 & 1 & 29 & $13: 00: 5212.403$ & 40.556 & 1.89 & 0.66 \\
\hline 2008 & 1 & 29 & $14: 17: 2812.123$ & 40.754 & 1.83 & 0.8 \\
\hline 2008 & 1 & 29 & $14: 52: 3412.348$ & 40.606 & 1.72 & 1.64 \\
\hline 2008 & 1 & 29 & $17: 01: 4212.411$ & 40.574 & 1.68 & 0.9 \\
\hline 2008 & 1 & 29 & $23: 32: 0112.392$ & 40.579 & 1.56 & 0.88 \\
\hline 2008 & 1 & 30 & $00: 40: 1812.372$ & 40.595 & 1.58 & 0.81 \\
\hline 2008 & 1 & 30 & $01: 08: 2012.436$ & 40.561 & 1.58 & 1,00 \\
\hline 2008 & 1 & 30 & $01: 15: 0612.419$ & 40.545 & 1.59 & 0.92 \\
\hline 2008 & 1 & 30 & $01: 42: 4912.391$ & 40.582 & 1.58 & 3.68 \\
\hline 2008 & 1 & 30 & $02: 15: 4612.468$ & 40.560 & 1.64 & 2.29 \\
\hline 2008 & 1 & 30 & $02: 18: 1712.415$ & 40.571 & 1.66 & 0.88 \\
\hline 2008 & 1 & 30 & $02: 23: 3612.443$ & 40.564 & 1.64 & 0.76 \\
\hline 2008 & 1 & 30 & $02: 42: 0712.409$ & 40.579 & 1.51 & 0.86 \\
\hline 2008 & 1 & 30 & $02: 59: 1412.448$ & 40.591 & 1.62 & 1.93 \\
\hline 2008 & 1 & 30 & $05: 30: 0812.416$ & 40.573 & 1.74 & 1.02 \\
\hline 2008 & 1 & 30 & $06: 51: 1412.428$ & 40.567 & 1.77 & 0.9 \\
\hline 2008 & 1 & 30 & $07: 01: 5712.423$ & 40.566 & 1.77 & 0.94 \\
\hline 2008 & 1 & 30 & $08: 20: 1112.387$ & 40.587 & 1.91 & 0.99 \\
\hline 2008 & 1 & 30 & $14: 22: 28 \quad 12.362$ & 40.602 & 1.67 & 0.83 \\
\hline 2008 & 1 & 30 & $14: 51: 4012.411$ & 40.568 & 1.57 & 0.87 \\
\hline 2008 & 1 & 30 & $16: 21: 2812.395$ & 40.579 & 1.53 & 1.03 \\
\hline 2008 & 1 & 30 & $17: 21: 1312.442$ & 40.578 & 1.66 & 0.77 \\
\hline 2008 & 1 & 30 & $17: 28: 1812.384$ & 40.586 & 1.47 & 0.83 \\
\hline 2008 & 1 & 30 & $20: 04: 2312.343$ & 40.634 & 1.63 & 4.97 \\
\hline 2008 & 1 & 30 & $20: 25: 4612.421$ & 40.532 & 1.65 & 1.15 \\
\hline 2008 & 1 & 30 & $21: 32: 4112.404$ & 40.577 & 1.60 & 0.73 \\
\hline 2008 & 1 & 30 & $21: 35: 0012.411$ & 40.571 & 1.57 & 0.82 \\
\hline 2008 & 1 & 30 & $21: 41: 5512.585$ & 40.531 & 1.86 & 0.55 \\
\hline 2008 & 1 & 31 & $02: 46: 2812.361$ & 40.603 & 1.65 & 0.71 \\
\hline 2008 & 1 & 31 & $04: 09: 2412.365$ & 40.600 & 1.66 & 0.6 \\
\hline 2008 & 1 & 31 & $05: 47: 4112.113$ & 40.762 & 1.86 & 0.81 \\
\hline 2008 & 1 & 31 & $06: 14: 3712.425$ & 40.566 & 1.72 & 0.81 \\
\hline 2008 & 1 & 31 & $07: 29: 2212.406$ & 40.572 & 1.75 & 0.97 \\
\hline 2008 & 1 & 31 & $09: 17: 0712.461$ & 40.555 & 1.90 & 0.82 \\
\hline 2008 & 1 & 31 & $17: 49: 1112.449$ & 40.563 & 1.85 & 0.98 \\
\hline 2008 & 1 & 31 & $17: 55: 0212.434$ & 40.567 & 1.73 & 0.92 \\
\hline 2008 & 1 & 31 & $20: 23: 4012.441$ & 40.562 & 1.81 & 0.91 \\
\hline 2008 & 1 & 31 & $23: 19: 0512.467$ & 40.571 & 1.71 & 1,00 \\
\hline 2008 & 2 & 1 & $02: 26: 5212.365$ & 40.595 & 1.74 & 0.74 \\
\hline 2008 & 2 & 1 & $03: 32: 4912.407$ & 40.574 & 1.72 & 0.99 \\
\hline 2008 & 2 & 1 & $09: 48: 3712.489$ & 40.549 & 1.76 & 0.77 \\
\hline 2008 & 2 & 1 & $10: 56: 1512.438$ & 40.560 & 1.82 & 0.58 \\
\hline 2008 & 2 & 1 & $11: 09: 3212.493$ & 40.558 & 1.84 & 1.52 \\
\hline 2008 & 2 & 1 & $11: 43: 0612.383$ & 40.588 & 1.76 & 1.01 \\
\hline 2008 & 2 & 1 & $13: 20: 3412.363$ & 40.603 & 1.90 & 0.56 \\
\hline 2008 & 2 & 1 & $16: 05: 2712.400$ & 40.573 & 1.78 & 0.98 \\
\hline 2008 & 2 & 1 & $17: 02: 2712.365$ & 40.601 & 1.85 & 1.25 \\
\hline 2008 & 2 & 1 & $17: 02: 2812.391$ & 40.590 & 1.83 & 1.74 \\
\hline 2008 & 2 & 1 & $19: 44: 0412.472$ & 40.554 & 1.76 & 1.18 \\
\hline 2008 & 2 & 1 & $20: 03: 5912.389$ & 40.585 & 1.62 & 0.86 \\
\hline 2008 & 2 & 1 & $22: 30: 5512.378$ & 40.590 & 1.63 & 0.52 \\
\hline 2008 & 2 & 1 & $22: 35: 0611.761$ & 41.126 & 1.92 & 0.48 \\
\hline 2008 & 2 & 1 & $22: 53: 0212.473$ & 40.553 & 1.63 & 0.8 \\
\hline 2008 & 2 & 1 & $23: 41: 3812.368$ & 40.589 & 1.54 & 0.42 \\
\hline 2008 & 2 & 2 & $00: 45: 0510.823$ & 39.948 & 2.48 & 6.45 \\
\hline 2008 & 2 & 2 & $01: 08: 4312.405$ & 40.581 & 1.52 & 1.15 \\
\hline 2008 & 2 & 2 & $01: 30: 0712.465$ & 40.572 & 1.72 & 1.96 \\
\hline 2008 & 2 & 2 & $01: 36: 4812.466$ & 40.564 & 1.71 & 0.94 \\
\hline 2008 & 2 & 2 & $02: 10: 2112.408$ & 40.648 & 1.65 & 1.5 \\
\hline 2008 & 2 & 2 & $02: 42: 3512.500$ & 40.545 & 1.66 & 1.7 \\
\hline 2008 & 2 & 2 & $02: 49: 1712.367$ & 40.596 & 1.57 & 3.54 \\
\hline 2008 & 2 & 2 & $02: 59: 5912.295$ & 40.644 & 1.72 & 1.6 \\
\hline 2008 & 2 & 2 & $03: 22: 0312.393$ & 40.583 & 1.67 & 1.11 \\
\hline
\end{tabular}




\begin{tabular}{|c|c|c|c|c|c|c|}
\hline 2008 & 2 & 2 & $03: 43: 0012.407$ & 40.604 & 1.56 & 2.67 \\
\hline 2008 & 2 & 2 & $03: 47: 3212.293$ & 40.640 & 1.76 & 5.17 \\
\hline 2008 & 2 & 2 & $03: 58: 2412.456$ & 40.517 & 1.73 & 1.18 \\
\hline 2008 & 2 & 2 & $04: 06: 0010.813$ & 39.913 & 3.13 & 5,00 \\
\hline 2008 & 2 & 2 & $04: 34: 5912.401$ & 40.564 & 1.76 & 0.91 \\
\hline 2008 & 2 & 2 & $05: 39: 3712.366$ & 40.595 & 1.79 & 1.25 \\
\hline 2008 & 2 & 2 & $07: 29: 0512.476$ & 40.570 & 1.81 & 1.55 \\
\hline 2008 & 2 & 2 & $09: 57: 1512.395$ & 40.587 & 1.76 & 0.92 \\
\hline 2008 & 2 & 2 & $11: 27: 2112.364$ & 40.601 & 1.79 & 0.59 \\
\hline 2008 & 2 & 2 & $11: 40: 0612.362$ & 40.596 & 1.75 & 0.64 \\
\hline 2008 & 2 & 2 & $12: 57: 3912.386$ & 40.580 & 1.76 & 0.96 \\
\hline 2008 & 2 & 2 & $13: 00: 0812.431$ & 40.577 & 1.77 & 13.32 \\
\hline 2008 & 2 & 2 & $13: 11: 0612.406$ & 40.541 & 1.80 & 1.44 \\
\hline 2008 & 2 & 2 & $14: 13: 0812.438$ & 40.569 & 1.70 & 0.84 \\
\hline 2008 & 2 & 2 & $15: 51: 2812.430$ & 40.567 & 1.63 & 0.88 \\
\hline 2008 & 2 & 2 & $16: 25: 4112.357$ & 40.534 & 1.64 & 2.44 \\
\hline 2008 & 2 & 2 & $17: 11: 0612.390$ & 40.620 & 1.60 & 2.68 \\
\hline 2008 & 2 & 2 & $17: 47: 2712.363$ & 40.604 & 1.75 & 0.81 \\
\hline 2008 & 2 & 2 & $19: 13: 1312.384$ & 40.588 & 1.70 & 3.78 \\
\hline 2008 & 2 & 2 & $19: 19: 1712.367$ & 40.585 & 1.65 & 8.89 \\
\hline 2008 & 2 & 2 & $19: 28: 4312.384$ & 40.580 & 1.73 & 9,00 \\
\hline 2008 & 2 & 2 & $20: 10: 3912.386$ & 40.603 & 1.61 & 1.74 \\
\hline 2008 & 2 & 2 & $20: 34: 0912.395$ & 40.576 & 1.46 & 0.98 \\
\hline 2008 & 2 & 2 & $20: 48: 5612.372$ & 40.598 & 1.61 & 0.59 \\
\hline 2008 & 2 & 2 & $21: 14: 0312.420$ & 40.526 & 1.64 & 2.77 \\
\hline 2008 & 2 & 2 & $21: 20: 5112.370$ & 40.603 & 1.65 & 0.68 \\
\hline 2008 & 2 & 2 & $21: 48: 5012.367$ & 40.591 & 1.60 & 0.71 \\
\hline 2008 & 2 & 2 & $21: 52: 5212.427$ & 40.536 & 1.58 & 1.53 \\
\hline 2008 & 2 & 2 & $22: 12: 0212.468$ & 40.567 & 1.62 & 3.32 \\
\hline 2008 & 2 & 2 & $23: 21: 3612.343$ & 40.599 & 1.61 & 0.69 \\
\hline 2008 & 2 & 2 & $23: 41: 3812.369$ & 40.597 & 1.65 & 0.53 \\
\hline 2008 & 2 & 3 & $00: 06: 3812.393$ & 40.586 & 1.48 & 4.21 \\
\hline 2008 & 2 & 3 & $01: 27: 4412.404$ & 40.574 & 1.59 & 0.89 \\
\hline 2008 & 2 & 3 & $02: 13: 4212.541$ & 40.543 & 1.82 & 0.37 \\
\hline 2008 & 2 & 3 & $02: 16: 2612.370$ & 40.588 & 1.62 & 8.02 \\
\hline 2008 & 2 & 3 & $03: 11: 2212.383$ & 40.587 & 1.67 & 3.1 \\
\hline 2008 & 2 & 3 & $03: 42: 5412.503$ & 40.538 & 1.90 & 0.51 \\
\hline 2008 & 2 & 3 & $03: 50: 2312.472$ & 40.552 & 1.76 & 6.23 \\
\hline 2008 & 2 & 3 & $03: 53: 2812.392$ & 40.581 & 1.66 & 0.82 \\
\hline 2008 & 2 & 3 & $05: 35: 5212.422$ & 40.568 & 1.77 & 1.01 \\
\hline 2008 & 2 & 3 & $08: 08: 3912.386$ & 40.583 & 2.28 & 1.02 \\
\hline 2008 & 2 & 3 & $08: 41: 4112.392$ & 40.580 & 1.89 & 0.96 \\
\hline 2008 & 2 & 3 & $09: 26: 3912.395$ & 40.579 & 1.86 & 0.75 \\
\hline 2008 & 2 & 3 & $10: 11: 0912.435$ & 40.518 & 1.83 & 3.69 \\
\hline 2008 & 2 & 3 & $10: 52: 4712.366$ & 40.608 & 1.84 & 1.4 \\
\hline 2008 & 2 & 3 & $11: 31: 1012.370$ & 40.590 & 1.95 & 0.76 \\
\hline 2008 & 2 & 3 & $11: 54: 5712.385$ & 40.575 & 1.80 & 1.02 \\
\hline 2008 & 2 & 3 & $12: 17: 3112.398$ & 40.578 & 1.74 & 0.8 \\
\hline 2008 & 2 & 3 & $13: 32: 1712.429$ & 40.560 & 1.81 & 1.54 \\
\hline 2008 & 2 & 3 & $14: 28: 5112.361$ & 40.606 & 1.81 & 0.63 \\
\hline 2008 & 2 & 3 & $15: 24: 4212.388$ & 40.580 & 1.70 & 0.97 \\
\hline 2008 & 2 & 3 & $16: 53: 2512.501$ & 40.544 & 1.74 & 0.9 \\
\hline 2008 & 2 & 3 & $17: 18: 2612.483$ & 40.549 & 1.72 & 0.85 \\
\hline 2008 & 2 & 3 & $17: 29: 0912.372$ & 40.592 & 1.74 & 5.18 \\
\hline 2008 & 2 & 3 & $17: 46: 3512.385$ & 40.582 & 1.67 & 0.9 \\
\hline 2008 & 2 & 3 & $19: 06: 5912.441$ & 40.559 & 1.66 & 0.9 \\
\hline 2008 & 2 & 3 & $20: 20: 1212.471$ & 40.554 & 1.76 & 1.48 \\
\hline 2008 & 2 & 3 & $20: 25: 3612.379$ & 40.585 & 1.65 & 1.18 \\
\hline 2008 & 2 & 3 & $20: 49: 1912.428$ & 40.594 & 1.67 & 1.92 \\
\hline 2008 & 2 & 3 & $20: 54: 1512.317$ & 40.620 & 1.73 & 0.64 \\
\hline 2008 & 2 & 3 & $23: 28: 1512.375$ & 40.529 & 1.67 & 1.82 \\
\hline 2008 & 2 & 3 & $23: 45: 4212.123$ & 40.755 & 1.86 & 0.58 \\
\hline 2008 & 2 & 3 & $23: 45: 4212.116$ & 40.748 & 1.86 & 0.47 \\
\hline 2008 & 2 & 4 & $00: 20: 3612.376$ & 40.614 & 1.68 & 0.85 \\
\hline 2008 & 2 & 4 & $00: 23: 3012.466$ & 40.555 & 1.71 & 0.8 \\
\hline 2008 & 2 & 4 & $01: 35: 5912.460$ & 40.523 & 1.79 & 1.45 \\
\hline 2008 & 2 & 4 & $01: 38: 0912.366$ & 40.571 & 1.77 & 2.77 \\
\hline 2008 & 2 & 4 & $01: 41: 1412.475$ & 40.551 & 1.81 & 0.89 \\
\hline 2008 & 2 & 4 & $01: 49: 3712.408$ & 40.578 & 1.65 & 1.15 \\
\hline 2008 & 2 & 4 & $02: 14: 0312.367$ & 40.587 & 1.73 & 1.07 \\
\hline 2008 & 2 & 4 & $03: 01: 5412.409$ & 40.573 & 1.73 & 0.97 \\
\hline 2008 & 2 & 4 & $03: 20: 3912.377$ & 40.589 & 1.82 & 2.88 \\
\hline 2008 & 2 & 4 & $04: 02: 4112.452$ & 40.560 & 2.01 & 0.46 \\
\hline 2008 & 2 & 4 & $04: 21: 0412.450$ & 40.559 & 1.84 & 0.97 \\
\hline 2008 & 2 & 4 & $05: 01: 2712.379$ & 40.593 & 1.83 & 1.06 \\
\hline 2008 & 2 & 4 & $05: 29: 3412.424$ & 40.577 & 1.86 & 0.48 \\
\hline
\end{tabular}




\begin{tabular}{|c|c|c|c|c|c|c|}
\hline 2008 & 2 & 4 & $09: 00: 2512.405$ & 40.577 & 1.86 & 0.59 \\
\hline 2008 & 2 & 4 & $10: 36: 3312.402$ & 40.575 & 1.78 & 0.96 \\
\hline 2008 & 2 & 4 & $15: 05: 3012.420$ & 40.568 & 1.68 & 0.98 \\
\hline 2008 & 2 & 4 & $16: 19: 3412.409$ & 40.576 & 1.71 & 0.68 \\
\hline 2008 & 2 & 4 & $17: 37: 0912.390$ & 40.580 & 1.84 & 1.01 \\
\hline 2008 & 2 & 4 & $18: 21: 3812.332$ & 40.553 & 2.08 & 0.61 \\
\hline 2008 & 2 & 4 & $18: 31: 0712.412$ & 40.572 & 1.78 & 0.93 \\
\hline 2008 & 2 & 4 & $18: 48: 0312.371$ & 40.597 & 1.77 & 0.5 \\
\hline 2008 & 2 & 4 & $19: 01: 3912.406$ & 40.575 & 1.71 & 1.02 \\
\hline 2008 & 2 & 4 & $19: 06: 2212.400$ & 40.580 & 1.78 & 0.88 \\
\hline 2008 & 2 & 4 & $19: 07: 2812.405$ & 40.533 & 1.73 & 2.85 \\
\hline 2008 & 2 & 4 & $19: 15: 0412.393$ & 40.582 & 1.69 & 0.81 \\
\hline 2008 & 2 & 4 & $19: 19: 1412.457$ & 40.560 & 1.71 & 2.51 \\
\hline 2008 & 2 & 4 & $19: 35: 3212.448$ & 40.546 & 1.73 & 0.83 \\
\hline 2008 & 2 & 4 & $22: 34: 2311.866$ & 41.020 & 1.95 & 0.5 \\
\hline 2008 & 2 & 4 & $22: 55: 1512.496$ & 40.541 & 1.76 & 0.86 \\
\hline 2008 & 2 & 4 & $23: 29: 5112.567$ & 40.412 & 1.78 & 1.03 \\
\hline 2008 & 2 & 5 & $00: 14: 1912.375$ & 40.595 & 1.70 & 1.02 \\
\hline 2008 & 2 & 5 & $00: 16: 3212.392$ & 40.584 & 1.78 & 0.84 \\
\hline 2008 & 2 & 5 & $00: 22: 1812.401$ & 40.579 & 1.68 & 0.91 \\
\hline 2008 & 2 & 5 & $01: 47: 2813.488$ & 40.852 & 2.55 & 1.63 \\
\hline 2008 & 2 & 5 & $02: 23: 2113.445$ & 40.805 & 2.72 & 1.92 \\
\hline 2008 & 2 & 5 & $02: 28: 0713.484$ & 40.844 & 3.04 & 1.61 \\
\hline 2008 & 2 & 5 & $04: 22: 0913.486$ & 40.846 & 3.20 & 1.65 \\
\hline 2008 & 2 & 5 & $08: 41: 1413.481$ & 40.856 & 3.63 & 4.65 \\
\hline 2008 & 2 & 5 & $10: 33: 5413.470$ & 40.844 & 3.10 & 1.8 \\
\hline 2008 & 2 & 5 & $13: 46: 0712.312$ & 40.607 & 2.05 & 0.65 \\
\hline 2008 & 2 & 5 & $14: 01: 0113.484$ & 40.819 & 2.81 & 2.16 \\
\hline 2008 & 2 & 5 & $19: 39: 0113.481$ & 40.848 & 2.83 & 1.83 \\
\hline 2008 & 2 & 5 & $21: 28: 0513.442$ & 40.816 & 2.48 & 1.73 \\
\hline 2008 & 2 & 5 & $22: 29: 4813.460$ & 40.865 & 2.46 & 6.2 \\
\hline 2008 & 2 & 6 & $00: 14: 5513.477$ & 40.838 & 2.63 & 1.84 \\
\hline 2008 & 2 & 6 & $13: 26: 0613.474$ & 40.835 & 2.79 & 3.17 \\
\hline 2008 & 2 & 6 & $21: 36: 5512.398$ & 40.616 & 1.95 & 0.79 \\
\hline 2008 & 2 & 6 & $22: 09: 1913.472$ & 40.839 & 2.53 & 3.61 \\
\hline 2008 & 2 & 6 & $22: 40: 5313.603$ & 40.708 & 2.54 & 1.57 \\
\hline 2008 & 2 & 7 & $00: 06: 2812.502$ & 40.543 & 1.92 & 1.4 \\
\hline 2008 & 2 & 7 & $04: 28: 1513.478$ & 40.835 & 2.61 & 2.12 \\
\hline 2008 & 2 & 7 & $06: 39: 2811.919$ & 41.761 & 2.64 & 2.28 \\
\hline 2008 & 2 & 7 & $13: 00: 3313.472$ & 40.830 & 2.69 & 2.09 \\
\hline 2008 & 2 & 8 & $01: 19: 2512.450$ & 40.573 & 2.04 & 0.46 \\
\hline 2008 & 2 & 8 & $17: 29: 3612.445$ & 40.573 & 1.94 & 1.6 \\
\hline 2008 & 2 & 8 & $21: 00: 4113.421$ & 39.979 & 2.53 & 1.6 \\
\hline 2008 & 2 & 8 & $21: 38: 4811.635$ & 39.665 & 2.37 & 0.62 \\
\hline 2008 & 2 & 8 & $23: 38: 3013.475$ & 40.851 & 2.55 & 3.52 \\
\hline 2008 & 2 & 9 & $00: 31: 4812.312$ & 40.609 & 1.88 & 0.59 \\
\hline 2008 & 2 & 9 & $00: 33: 5312.931$ & 41.158 & 2.35 & 0.73 \\
\hline 2008 & 2 & 9 & $04: 13: 2213.152$ & 39.646 & 2.83 & 0.99 \\
\hline 2008 & 2 & 9 & $08: 13: 5012.939$ & 41.168 & 2.60 & 1.31 \\
\hline 2008 & 2 & 9 & $12: 32: 0613.480$ & 40.849 & 2.64 & 1.47 \\
\hline 2008 & 2 & 9 & $15: 58: 5412.440$ & 40.541 & 1.94 & 2.15 \\
\hline 2008 & 2 & 9 & $17: 47: 3112.376$ & 40.591 & 1.89 & 0.69 \\
\hline 2008 & 2 & 9 & $19: 38: 2513.181$ & 39.770 & 2.62 & 1.81 \\
\hline 2008 & 2 & 9 & $22: 42: 0111.720$ & 39.687 & 2.27 & 1.01 \\
\hline 2008 & 2 & 10 & $01: 17: 1012.957$ & 41.177 & 2.82 & 3.97 \\
\hline 2008 & 2 & 10 & $02: 09: 2313.176$ & 39.764 & 2.50 & 2.17 \\
\hline 2008 & 2 & 10 & $05: 23: 449.396$ & 40.077 & 3.59 & 9.17 \\
\hline 2008 & 2 & 10 & $09: 42: 4312.923$ & 41.182 & 2.47 & 1.04 \\
\hline 2008 & 2 & 10 & $10: 12: 4412.927$ & 41.166 & 2.50 & 1.38 \\
\hline 2008 & 2 & 10 & $16: 18: 3712.397$ & 40.573 & 1.84 & 0.82 \\
\hline 2008 & 2 & 10 & $17: 41: 1610.488$ & 40.835 & 2.60 & 3.57 \\
\hline 2008 & 2 & 10 & $19: 10: 0912.361$ & 40.600 & 1.81 & 0.54 \\
\hline 2008 & 2 & 10 & $19: 14: 0710.525$ & 40.849 & 2.78 & 0.66 \\
\hline 2008 & 2 & 10 & $21: 40: 3212.442$ & 40.563 & 1.80 & 0.77 \\
\hline 2008 & 2 & 11 & $00: 11: 3212.931$ & 41.167 & 2.33 & 1.04 \\
\hline 2008 & 2 & 11 & $00: 33: 2612.934$ & 41.174 & 2.27 & 5.54 \\
\hline 2008 & 2 & 11 & $00: 51: 2212.930$ & 41.164 & 2.25 & 1.39 \\
\hline 2008 & 2 & 11 & $02: 48: 0412.944$ & 41.143 & 2.24 & 6.06 \\
\hline 2008 & 2 & 12 & $14: 45: 1912.444$ & 40.561 & 2.14 & 0.71 \\
\hline 2008 & 2 & 12 & $21: 13: 0912.463$ & 40.570 & 1.90 & 0.88 \\
\hline 2008 & 2 & 12 & $23: 02: 3512.438$ & 40.571 & 1.88 & 0.67 \\
\hline 2008 & 2 & 13 & $01: 33: 1412.390$ & 40.586 & 1.79 & 0.71 \\
\hline 2008 & 2 & 13 & $01: 35: 3012.379$ & 40.581 & 1.82 & 2.03 \\
\hline 2008 & 2 & 13 & $11: 08: 5012.330$ & 40.603 & 2.22 & 0.57 \\
\hline 2008 & 2 & 13 & $17: 46: 3812.375$ & 40.587 & 1.88 & 0.58 \\
\hline 2008 & 2 & 13 & $18: 38: 1913.606$ & 40.764 & 2.44 & 1.68 \\
\hline
\end{tabular}




\begin{tabular}{|c|c|c|c|c|c|c|}
\hline 2008 & 2 & 13 & $22: 30: 5212.454$ & 40.568 & 2.11 & 0.49 \\
\hline 2008 & 2 & 13 & $23: 34: 3612.411$ & 40.577 & 1.79 & 0.61 \\
\hline 2008 & 2 & 13 & $23: 41: 4312.503$ & 40.553 & 2.13 & 0.67 \\
\hline 2008 & 2 & 14 & $18: 03: 3112.083$ & 40.735 & 1.88 & 0.88 \\
\hline 2008 & 2 & 14 & $23: 21: 0412.390$ & 40.579 & 1.85 & 0.83 \\
\hline 2008 & 2 & 14 & $23: 44: 0413.197$ & 39.796 & 2.41 & 4.93 \\
\hline 2008 & 2 & 15 & $00: 46: 4612.456$ & 40.573 & 2.28 & 0.69 \\
\hline 2008 & 2 & 15 & $03: 09: 0212.324$ & 40.578 & 1.93 & 1.56 \\
\hline 2008 & 2 & 15 & $11: 11: 3212.464$ & 40.557 & 2.06 & 0.58 \\
\hline 2008 & 2 & 15 & $17: 43: 2012.448$ & 40.556 & 2.23 & 0.64 \\
\hline 2008 & 2 & 15 & $21: 07: 1613.182$ & 39.764 & 2.55 & 2.33 \\
\hline 2008 & 2 & 16 & $03: 56: 0412.410$ & 40.576 & 1.93 & 0.53 \\
\hline 2008 & 2 & 16 & $09: 27: 0513.775$ & 39.911 & 3.19 & 1.84 \\
\hline 2008 & 2 & 16 & $17: 44: 0412.432$ & 40.569 & 1.84 & 1.2 \\
\hline 2008 & 2 & 16 & $20: 27: 1312.448$ & 40.561 & 2.06 & 2.01 \\
\hline 2008 & 2 & 16 & $23: 48: 4612.364$ & 40.599 & 1.96 & 0.6 \\
\hline 2008 & 2 & 17 & $17: 19: 5012.912$ & 39.802 & 2.70 & 0.47 \\
\hline 2008 & 2 & 17 & $23: 55: 3212.455$ & 40.116 & 1.95 & 1.96 \\
\hline 2008 & 2 & 18 & $00: 48: 3612.482$ & 40.555 & 1.90 & 1.92 \\
\hline 2008 & 2 & 18 & $08: 54: 5712.588$ & 40.519 & 2.30 & 1.46 \\
\hline 2008 & 2 & 18 & $13: 23: 1013.995$ & 40.672 & 3.25 & 6.22 \\
\hline 2008 & 2 & 18 & $18: 58: 5813.175$ & 39.746 & 2.75 & 1.24 \\
\hline 2008 & 2 & 19 & $01: 10: 3412.384$ & 40.569 & 1.78 & 0.9 \\
\hline 2008 & 2 & 19 & $02: 07: 2512.408$ & 40.582 & 2.02 & 0.64 \\
\hline 2008 & 2 & 19 & $02: 09: 3910.542$ & 40.788 & 3.10 & 0.7 \\
\hline 2008 & 2 & 19 & $03: 28: 1113.826$ & 40.548 & 3.19 & 2.57 \\
\hline 2008 & 2 & 19 & $11: 38: 1313.517$ & 39.974 & 2.62 & 1.58 \\
\hline 2008 & 2 & 19 & $13: 46: 5712.377$ & 40.601 & 2.08 & 0.6 \\
\hline 2008 & 2 & 20 & $23: 58: 4910.527$ & 40.809 & 2.94 & 0.96 \\
\hline 2008 & 2 & 21 & $01: 09: 4712.883$ & 41.136 & 2.50 & 2.83 \\
\hline 2008 & 2 & 21 & $01: 47: 3212.923$ & 41.154 & 2.59 & 0.63 \\
\hline 2008 & 2 & 21 & $08: 25: 0312.925$ & 41.180 & 2.60 & 1.16 \\
\hline 2008 & 2 & 21 & $20: 03: 5111.459$ & 41.110 & 2.09 & 1.4 \\
\hline 2008 & 2 & 21 & $22: 12: 3610.747$ & 39.645 & 2.57 & 1.55 \\
\hline 2008 & 2 & 22 & $00: 54: 489.986$ & 39.921 & 3.30 & 0.88 \\
\hline 2008 & 2 & 23 & $03: 05: 3210.577$ & 40.769 & 2.76 & 7.45 \\
\hline 2008 & 2 & 24 & $16: 59: 2812.913$ & 39.803 & 3.16 & 0.41 \\
\hline 2008 & 2 & 25 & $01: 01: 5212.380$ & 40.587 & 2.02 & 0.6 \\
\hline 2008 & 2 & 25 & $12: 55: 1912.412$ & 40.571 & 2.21 & 0.66 \\
\hline 2008 & 2 & 25 & $18: 08: 1810.848$ & 41.201 & 2.77 & 4.61 \\
\hline 2008 & 2 & 25 & $22: 32: 0312.500$ & 40.037 & 2.24 & 3.4 \\
\hline 2008 & 2 & 26 & $16: 10: 0912.949$ & 41.162 & 2.43 & 6.47 \\
\hline 2008 & 2 & 27 & $15: 02: 3212.921$ & 41.620 & 2.62 & 3.89 \\
\hline 2008 & 2 & 27 & $22: 37: 399.930$ & 39.882 & 2.84 & 1.48 \\
\hline 2008 & 2 & 27 & $22: 43: 1612.479$ & 40.578 & 1.86 & 1.21 \\
\hline 2008 & 2 & 28 & $00: 10: 3611.532$ & 41.070 & 1.88 & 10.19 \\
\hline 2008 & 2 & 28 & $02: 05: 2813.492$ & 40.009 & 2.50 & 4.62 \\
\hline 2008 & 2 & 28 & $13: 35: 3512.955$ & 41.166 & 2.45 & 1.47 \\
\hline 2008 & 2 & 28 & $16: 20: 1712.427$ & 40.580 & 2.03 & 0.69 \\
\hline 2008 & 2 & 28 & $17: 04: 1413.741$ & 39.947 & 2.72 & 5.85 \\
\hline 2008 & 2 & 28 & $20: 04: 3412.958$ & 41.161 & 2.31 & 1.68 \\
\hline 2008 & 2 & 28 & $20: 54: 1712.372$ & 40.578 & 1.75 & 1.91 \\
\hline 2008 & 2 & 28 & $23: 41: 3312.388$ & 40.581 & 1.84 & 0.74 \\
\hline 2008 & 2 & 29 & $00: 11: 0613.399$ & 40.806 & 2.40 & 8.76 \\
\hline 2008 & 2 & 29 & $01: 28: 2912.385$ & 40.587 & 1.91 & 0.83 \\
\hline 2008 & 2 & 29 & $01: 56: 0111.917$ & 41.018 & 1.85 & 0.56 \\
\hline 2008 & 2 & 29 & $02: 29: 0412.419$ & 40.576 & 1.94 & 0.55 \\
\hline 2008 & 2 & 29 & $02: 29: 4712.765$ & 39.601 & 2.42 & 0.89 \\
\hline 2008 & 2 & 29 & $18: 59: 5713.666$ & 40.720 & 2.85 & 4.96 \\
\hline 2008 & 2 & 29 & $19: 46: 3112.366$ & 40.572 & 2.08 & 0.8 \\
\hline 2008 & 3 & 1 & $17: 17: 4112.464$ & 40.570 & 2.20 & 0.58 \\
\hline 2008 & 3 & 10 & $23: 32: 0210.753$ & 39.866 & 2.67 & 2.86 \\
\hline 2008 & 3 & 11 & $01: 01: 0313.363$ & 39.814 & 2.58 & 1.01 \\
\hline 2008 & 3 & 11 & $09: 08: 3113.345$ & 39.983 & 2.64 & 1.07 \\
\hline 2008 & 3 & 11 & $10: 11: 0613.349$ & 39.796 & 2.73 & 1,00 \\
\hline 2008 & 3 & 11 & $21: 11: 2512.386$ & 40.596 & 1.92 & 0.73 \\
\hline 2008 & 3 & 11 & $23: 50: 2713.548$ & 39.994 & 2.64 & 3.76 \\
\hline 2008 & 3 & 12 & $00: 36: 5912.386$ & 40.573 & 1.84 & 1.25 \\
\hline 2008 & 3 & 12 & $05: 47: 3212.405$ & 40.579 & 2.12 & 0.56 \\
\hline 2008 & 3 & 12 & $23: 04: 3713.570$ & 39.847 & 2.61 & 12.56 \\
\hline 2008 & 3 & 13 & $00: 36: 4212.494$ & 40.547 & 1.89 & 0.64 \\
\hline 2008 & 3 & 13 & $02: 23: 3412.447$ & 40.562 & & 1.22 \\
\hline 2008 & 3 & 13 & $20: 34: 5812.437$ & 40.565 & 2.01 & 0.54 \\
\hline 2008 & 3 & 14 & $02: 17: 2912.478$ & 40.536 & 1.96 & 0.98 \\
\hline 2008 & 3 & 14 & $04: 03: 5412.459$ & 40.548 & 2.14 & 0.73 \\
\hline 2008 & 3 & 14 & $14: 13: 5713.250$ & 41.043 & 3.24 & 5.1 \\
\hline
\end{tabular}




\begin{tabular}{|c|c|c|c|c|c|c|}
\hline 2008 & 3 & 15 & $00: 29: 2112.402$ & 40.577 & 1.89 & 0.63 \\
\hline 2008 & 3 & 15 & $05: 14: 5013.298$ & 41.062 & 3.09 & 1.53 \\
\hline 2008 & 3 & 15 & $15: 08: 5012.848$ & 40.638 & 2.57 & 1.4 \\
\hline 2008 & 3 & 16 & $01: 30: 1412.413$ & 40.581 & 2.12 & 0.43 \\
\hline 2008 & 3 & 16 & $04: 31: 5613.288$ & 41.068 & 3.07 & 3.74 \\
\hline 2008 & 3 & 16 & $09: 02: 4911.858$ & 42.010 & 2.78 & 5.85 \\
\hline 2008 & 3 & 16 & $20: 45: 1012.465$ & 40.552 & 1.99 & 0.86 \\
\hline 2008 & 3 & 16 & $21: 36: 0212.458$ & 40.566 & 1.94 & 1.08 \\
\hline 2008 & 3 & 17 & $00: 03: 5312.955$ & 39.519 & 2.50 & 1.38 \\
\hline 2008 & 3 & 17 & $00: 11: 4210.335$ & 40.978 & 2.69 & 2.21 \\
\hline 2008 & 3 & 17 & $01: 55: 4413.384$ & 41.087 & 2.73 & 11.64 \\
\hline 2008 & 3 & 17 & $09: 54: 0411.822$ & 39.131 & 2.78 & 3.35 \\
\hline 2008 & 3 & 17 & $15: 53: 2113.256$ & 41.057 & 2.57 & 19.36 \\
\hline 2008 & 3 & 17 & $20: 19: 0812.442$ & 40.568 & 2.02 & 0.66 \\
\hline 2008 & 3 & 17 & $22: 22: 4712.367$ & 40.595 & 1.90 & 0.68 \\
\hline 2008 & 3 & 17 & $22: 55: 2011.292$ & 41.450 & 2.35 & 2.02 \\
\hline 2008 & 3 & 17 & $23: 23: 1711.281$ & 41.460 & 2.32 & 2.71 \\
\hline 2008 & 3 & 18 & $00: 15: 0411.857$ & 42.000 & 2.40 & 1.72 \\
\hline 2008 & 3 & 18 & $00: 31: 0812.431$ & 40.593 & 1.88 & 0.53 \\
\hline 2008 & 3 & 18 & $01: 04: 0012.458$ & 40.567 & 1.96 & 0.65 \\
\hline 2008 & 3 & 18 & $01: 21: 3112.395$ & 40.626 & 1.91 & 0.89 \\
\hline 2008 & 3 & 18 & $02: 39: 0813.635$ & 40.699 & 2.64 & 1.38 \\
\hline 2008 & 3 & 18 & $03: 14: 2311.814$ & 39.514 & 2.46 & 0.9 \\
\hline 2008 & 3 & 18 & $07: 22: 2914.600$ & 40.242 & 3.27 & 7.11 \\
\hline 2008 & 3 & 18 & $13: 03: 5513.673$ & 40.712 & 2.76 & 1.9 \\
\hline 2008 & 3 & 18 & $13: 21: 3613.503$ & 40.000 & 2.75 & 0.95 \\
\hline 2008 & 3 & 18 & $23: 15: 5610.359$ & 40.998 & 2.79 & 2.54 \\
\hline 2008 & 3 & 18 & $23: 17: 3210.306$ & 41.015 & 2.74 & 2.07 \\
\hline 2008 & 3 & 18 & $23: 31: 0710.395$ & 41.005 & 2.85 & 2.23 \\
\hline 2008 & 3 & 20 & $00: 10: 3012.396$ & 40.582 & 1.96 & 0.37 \\
\hline 2008 & 3 & 20 & $02: 10: 5813.252$ & 41.073 & 2.46 & 10.15 \\
\hline 2008 & 3 & 20 & $09: 37: 4412.484$ & 40.545 & 2.06 & 0.48 \\
\hline 2008 & 3 & 20 & $20: 40: 3313.149$ & 39.941 & 2.47 & 0.41 \\
\hline 2008 & 3 & 20 & $22: 05: 1813.670$ & 40.047 & 2.56 & 5.69 \\
\hline 2008 & 3 & 20 & $22: 58: 3612.351$ & 40.582 & 3.70 & 0.63 \\
\hline 2008 & 3 & 20 & $23: 12: 0612.449$ & 40.562 & 3.24 & 0.51 \\
\hline 2008 & 3 & 21 & $02: 31: 3313.633$ & 39.914 & 2.67 & 1,00 \\
\hline 2008 & 3 & 21 & $04: 46: 0413.479$ & 40.001 & 2.66 & 1.42 \\
\hline 2008 & 3 & 21 & $09: 45: 2213.143$ & 39.932 & 2.62 & 0.58 \\
\hline 2008 & 3 & 21 & $21: 07: 1612.380$ & 40.610 & 2.65 & 0.81 \\
\hline 2008 & 3 & 21 & $22: 21: 5512.353$ & 40.606 & 1.95 & 0.45 \\
\hline 2008 & 3 & 21 & $23: 00: 5112.358$ & 40.597 & 1.81 & 6.2 \\
\hline 2008 & 3 & 21 & $23: 26: 0512.367$ & 40.601 & 1.85 & 1.02 \\
\hline 2008 & 3 & 22 & $00: 24: 1712.353$ & 40.597 & 1.77 & 1.09 \\
\hline 2008 & 3 & 22 & $00: 28: 5612.379$ & 40.595 & 1.80 & 11.47 \\
\hline 2008 & 3 & 22 & $23: 10: 1712.415$ & 40.566 & 1.79 & 1.27 \\
\hline 2008 & 3 & 23 & $00: 27: 5312.487$ & 40.541 & 1.80 & 1.86 \\
\hline 2008 & 3 & 23 & $03: 27: 2313.126$ & 39.907 & 2.46 & 13.07 \\
\hline 2008 & 3 & 23 & $12: 18: 4311.739$ & 41.966 & 2.76 & 12.34 \\
\hline 2008 & 3 & 23 & $16: 03: 5912.804$ & 39.596 & 2.61 & 0.54 \\
\hline 2008 & 3 & 23 & $21: 01: 4512.426$ & 40.563 & 1.91 & 1.26 \\
\hline 2008 & 3 & 23 & $23: 13: 0712.467$ & 40.557 & 1.83 & 1.42 \\
\hline 2008 & 3 & 24 & $02: 24: 4112.501$ & 40.535 & 1.92 & 0.61 \\
\hline 2008 & 3 & 24 & $02: 26: 3612.497$ & 40.536 & 1.99 & 0.67 \\
\hline 2008 & 3 & 24 & $17: 48: 2512.523$ & 40.539 & 2.24 & 0.52 \\
\hline 2008 & 3 & 24 & $22: 08: 3212.439$ & 40.571 & 1.96 & 0.44 \\
\hline 2008 & 3 & 25 & $02: 17: 4912.362$ & 40.604 & 1.98 & 0.46 \\
\hline 2008 & 3 & 25 & $10: 01: 2512.485$ & 40.535 & 2.04 & 0.66 \\
\hline 2008 & 3 & 25 & $15: 05: 2312.379$ & 40.590 & 1.95 & 0.63 \\
\hline 2008 & 3 & 25 & $23: 42: 0713.515$ & 39.949 & 2.58 & 0.73 \\
\hline 2008 & 3 & 26 & $00: 06: 5213.513$ & 39.947 & 2.47 & 1.1 \\
\hline 2008 & 3 & 26 & $18: 41: 3613.017$ & 39.610 & 2.53 & 10.04 \\
\hline 2008 & 3 & 27 & $01: 42: 1512.322$ & 39.713 & 2.21 & 0.77 \\
\hline 2008 & 3 & 27 & $01: 55: 0210.351$ & 41.031 & 2.89 & 5.3 \\
\hline 2008 & 3 & 27 & $03: 01: 5712.454$ & 40.578 & 1.96 & 0.84 \\
\hline 2008 & 3 & 28 & $00: 53: 1612.493$ & 40.557 & 2.00 & 0.57 \\
\hline 2008 & 3 & 29 & $00: 50: 0713.921$ & 40.561 & 2.99 & 1.41 \\
\hline 2008 & 3 & 29 & $01: 12: 3912.792$ & 39.465 & 2.51 & 0.53 \\
\hline 2008 & 3 & 29 & $02: 20: 1612.577$ & 40.486 & 1.97 & 1.48 \\
\hline 2008 & 3 & 29 & $02: 29: 2212.434$ & 40.553 & 1.95 & 0.64 \\
\hline 2008 & 3 & 29 & $10: 56: 3811.884$ & 41.410 & 2.30 & 0.44 \\
\hline 2008 & 3 & 29 & $17: 01: 1912.325$ & 40.611 & 1.93 & 0.74 \\
\hline 2008 & 3 & 29 & $23: 17: 2413.664$ & 40.739 & 2.53 & 1.48 \\
\hline 2008 & 3 & 30 & $21: 54: 4212.489$ & 40.539 & 1.92 & 0.51 \\
\hline 2008 & 3 & 31 & $02: 15: 0412.449$ & 40.555 & 1.91 & 0.76 \\
\hline 2008 & 3 & 31 & $03: 38: 4512.256$ & 40.646 & 2.24 & 0.83 \\
\hline
\end{tabular}




\begin{tabular}{|c|c|c|c|c|c|c|}
\hline 2008 & 3 & 31 & $10: 40: 4412.222$ & 40.666 & 2.15 & 0.73 \\
\hline 2008 & 3 & 31 & $12: 49: 3712.199$ & 40.682 & 2.32 & 0.65 \\
\hline 2008 & 3 & 31 & $13: 41: 4412.194$ & 40.684 & 2.34 & 0.67 \\
\hline 2008 & 3 & 31 & $15: 11: 1212.199$ & 40.691 & 2.22 & 0.83 \\
\hline 2008 & 3 & 31 & $15: 11: 5212.183$ & 40.687 & 2.10 & 0.84 \\
\hline 2008 & 3 & 31 & $15: 14: 4512.190$ & 40.686 & 2.10 & 1.07 \\
\hline 2008 & 3 & 31 & $15: 18: 5812.181$ & 40.687 & 2.08 & 0.82 \\
\hline 2008 & 3 & 31 & $15: 33: 4712.195$ & 40.686 & 2.13 & 1.09 \\
\hline 2008 & 3 & 31 & $15: 37: 2612.191$ & 40.684 & 2.17 & 1.22 \\
\hline 2008 & 3 & 31 & $15: 47: 0612.192$ & 40.690 & 2.24 & 0.79 \\
\hline 2008 & 3 & 31 & $16: 22: 2912.192$ & 40.689 & 2.05 & 0.98 \\
\hline 2008 & 3 & 31 & $16: 22: 4312.200$ & 40.681 & 2.31 & 0.82 \\
\hline 2008 & 3 & 31 & $16: 23: 1312.181$ & 40.689 & 2.44 & 0.59 \\
\hline 2008 & 3 & 31 & $16: 40: 1312.193$ & 40.686 & 2.36 & 0.47 \\
\hline 2008 & 3 & 31 & $16: 43: 5912.202$ & 40.656 & 2.52 & 0.42 \\
\hline 2008 & 3 & 31 & $18: 54: 1812.190$ & 40.697 & 2.35 & 0.43 \\
\hline 2008 & 3 & 31 & $19: 08: 3612.186$ & 40.693 & 2.29 & 0.47 \\
\hline 2008 & 3 & 31 & $19: 15: 1612.177$ & 40.696 & 2.30 & 0.5 \\
\hline 2008 & 3 & 31 & $19: 17: 0912.179$ & 40.697 & 2.24 & 0.53 \\
\hline 2008 & 3 & 31 & $19: 18: 1212.188$ & 40.697 & 2.77 & 0.41 \\
\hline 2008 & 3 & 31 & $19: 22: 1112.183$ & 40.695 & 2.44 & 0.45 \\
\hline 2008 & 3 & 31 & $19: 27: 0812.182$ & 40.697 & 2.48 & 0.53 \\
\hline 2008 & 3 & 31 & $19: 32: 1412.170$ & 40.690 & 2.32 & 0.51 \\
\hline 2008 & 3 & 31 & $19: 41: 5612.179$ & 40.699 & 2.52 & 0.68 \\
\hline 2008 & 3 & 31 & $19: 43: 1012.190$ & 40.703 & 2.42 & 0.61 \\
\hline 2008 & 3 & 31 & $19: 46: 0212.178$ & 40.697 & 2.77 & 0.47 \\
\hline 2008 & 3 & 31 & $19: 53: 5612.186$ & 40.696 & 2.47 & 0.56 \\
\hline 2008 & 3 & 31 & $20: 01: 3012.188$ & 40.701 & 3.01 & 0.42 \\
\hline 2008 & 3 & 31 & $20: 03: 0912.183$ & 40.699 & 2.26 & 0.46 \\
\hline 2008 & 3 & 31 & $20: 08: 4412.179$ & 40.700 & 2.44 & 0.4 \\
\hline 2008 & 3 & 31 & $20: 09: 4212.187$ & 40.704 & 2.49 & 0.44 \\
\hline 2008 & 3 & 31 & $20: 10: 4712.182$ & 40.700 & 2.75 & 0.4 \\
\hline 2008 & 3 & 31 & $20: 15: 0512.178$ & 40.699 & 2.83 & 0.36 \\
\hline 2008 & 3 & 31 & $20: 17: 3412.179$ & 40.702 & 2.71 & 0.39 \\
\hline 2008 & 3 & 31 & $20: 19: 0812.182$ & 40.704 & 2.51 & 0.44 \\
\hline 2008 & 3 & 31 & $20: 20: 0912.182$ & 40.701 & 2.28 & 0.37 \\
\hline 2008 & 3 & 31 & $20: 21: 1512.184$ & 40.701 & 2.55 & 0.43 \\
\hline 2008 & 3 & 31 & $20: 31: 5212.182$ & 40.702 & 2.32 & 0.43 \\
\hline 2008 & 3 & 31 & $20: 34: 1912.182$ & 40.703 & 2.60 & 0.4 \\
\hline 2008 & 3 & 31 & $20: 39: 2612.185$ & 40.704 & 2.39 & 0.52 \\
\hline 2008 & 3 & 31 & $20: 41: 0012.178$ & 40.702 & 3.00 & 0.38 \\
\hline 2008 & 3 & 31 & $20: 42: 1212.182$ & 40.705 & 2.37 & 0.47 \\
\hline 2008 & 3 & 31 & $20: 43: 0812.172$ & 40.701 & 2.54 & 0.51 \\
\hline 2008 & 3 & 31 & $20: 44: 1812.183$ & 40.702 & 2.28 & 0.38 \\
\hline 2008 & 3 & 31 & $20: 46: 1912.181$ & 40.702 & 2.37 & 0.47 \\
\hline 2008 & 3 & 31 & $20: 47: 3912.182$ & 40.701 & 2.51 & 0.37 \\
\hline 2008 & 3 & 31 & $20: 49: 0712.184$ & 40.703 & 2.47 & 0.45 \\
\hline 2008 & 3 & 31 & $20: 49: 4212.178$ & 40.705 & 2.98 & 0.39 \\
\hline 2008 & 3 & 31 & $20: 51: 1712.182$ & 40.699 & 2.52 & 0.46 \\
\hline 2008 & 3 & 31 & $20: 54: 4912.179$ & 40.701 & 2.90 & 0.4 \\
\hline 2008 & 3 & 31 & $20: 56: 0612.178$ & 40.704 & 3.21 & 0.36 \\
\hline 2008 & 3 & 31 & $20: 58: 0412.176$ & 40.698 & 2.40 & 0.47 \\
\hline 2008 & 3 & 31 & $21: 03: 0712.180$ & 40.711 & 3.30 & 0.32 \\
\hline 2008 & 3 & 31 & $21: 10: 4112.219$ & 40.680 & 2.72 & 0.66 \\
\hline 2008 & 3 & 31 & $21: 15: 3212.179$ & 40.709 & 2.40 & 0.53 \\
\hline 2008 & 3 & 31 & $21: 16: 5312.178$ & 40.702 & 2.43 & 0.42 \\
\hline 2008 & 3 & 31 & $21: 19: 0912.186$ & 40.701 & 2.61 & 0.59 \\
\hline 2008 & 3 & 31 & $21: 19: 5812.178$ & 40.706 & 2.40 & 0.52 \\
\hline 2008 & 3 & 31 & $21: 23: 5012.182$ & 40.704 & 2.81 & 0.59 \\
\hline 2008 & 3 & 31 & $21: 31: 3412.170$ & 40.702 & 3.03 & 0.38 \\
\hline 2008 & 3 & 31 & $21: 34: 0912.181$ & 40.706 & 2.67 & 0.38 \\
\hline 2008 & 3 & 31 & $21: 34: 2712.182$ & 40.705 & 2.81 & 0.54 \\
\hline 2008 & 3 & 31 & $21: 37: 3512.162$ & 40.699 & 2.43 & 0.67 \\
\hline 2008 & 3 & 31 & $21: 39: 0512.174$ & 40.701 & 2.97 & 0.42 \\
\hline 2008 & 3 & 31 & $21: 40: 0512.182$ & 40.706 & 2.55 & 0.42 \\
\hline 2008 & 3 & 31 & $21: 44: 0312.174$ & 40.705 & 2.73 & 0.42 \\
\hline 2008 & 3 & 31 & $21: 46: 4012.194$ & 40.704 & 2.54 & 2.67 \\
\hline 2008 & 3 & 31 & $21: 49: 5812.176$ & 40.708 & 3.03 & 0.39 \\
\hline 2008 & 3 & 31 & $21: 53: 0112.189$ & 40.694 & 2.38 & 0.61 \\
\hline 2008 & 3 & 31 & $21: 54: 5112.160$ & 40.700 & 2.29 & 0.78 \\
\hline 2008 & 3 & 31 & $21: 55: 0312.172$ & 40.699 & 2.93 & 0.38 \\
\hline 2008 & 3 & 31 & $21: 57: 3812.173$ & 40.703 & 2.68 & 0.4 \\
\hline 2008 & 3 & 31 & $21: 59: 0612.175$ & 40.705 & 2.58 & 0.39 \\
\hline 2008 & 3 & 31 & $22: 00: 1312.158$ & 40.698 & 2.38 & 0.81 \\
\hline 2008 & 3 & 31 & $22: 02: 0312.170$ & 40.705 & 2.82 & 0.43 \\
\hline 2008 & 3 & 31 & $22: 02: 3312.177$ & 40.703 & 2.69 & 0.42 \\
\hline
\end{tabular}




\begin{tabular}{|c|c|c|c|c|c|c|}
\hline 2008 & 3 & 31 & $22: 05: 4112.174$ & 40.707 & 2.83 & 0.41 \\
\hline 2008 & 3 & 31 & $22: 07: 3912.179$ & 40.708 & 2.48 & 0.45 \\
\hline 2008 & 3 & 31 & $22: 09: 4012.174$ & 40.705 & 2.78 & 0.45 \\
\hline 2008 & 3 & 31 & $22: 15: 2212.174$ & 40.708 & 3.05 & 0.35 \\
\hline 2008 & 3 & 31 & $22: 16: 5112.171$ & 40.704 & 2.92 & 0.41 \\
\hline 2008 & 3 & 31 & $22: 20: 5612.169$ & 40.699 & 2.56 & 0.42 \\
\hline 2008 & 3 & 31 & $22: 23: 4712.179$ & 40.714 & 2.63 & 0.75 \\
\hline 2008 & 3 & 31 & $22: 24: 1512.177$ & 40.707 & 2.73 & 0.53 \\
\hline 2008 & 3 & 31 & $22: 27: 3012.175$ & 40.706 & 2.58 & 0.47 \\
\hline 2008 & 3 & 31 & $22: 28: 4012.182$ & 40.703 & 2.30 & 0.76 \\
\hline 2008 & 3 & 31 & $22: 28: 5112.176$ & 40.705 & 2.53 & 0.5 \\
\hline 2008 & 3 & 31 & $22: 33: 3712.176$ & 40.710 & 2.60 & 0.45 \\
\hline 2008 & 3 & 31 & $22: 35: 2912.170$ & 40.712 & 2.40 & 0.5 \\
\hline 2008 & 3 & 31 & $22: 36: 4012.178$ & 40.710 & 2.45 & 0.5 \\
\hline 2008 & 3 & 31 & $22: 38: 1912.170$ & 40.709 & 2.80 & 0.42 \\
\hline 2008 & 3 & 31 & $22: 46: 2512.194$ & 40.708 & 2.73 & 0.48 \\
\hline 2008 & 3 & 31 & $22: 47: 0212.177$ & 40.710 & 2.61 & 0.5 \\
\hline 2008 & 3 & 31 & $22: 50: 3212.169$ & 40.709 & 2.94 & 0.41 \\
\hline 2008 & 3 & 31 & $22: 52: 4312.167$ & 40.707 & 2.91 & 0.39 \\
\hline 2008 & 3 & 31 & $22: 53: 3412.175$ & 40.708 & 2.46 & 0.42 \\
\hline 2008 & 3 & 31 & $22: 54: 4012.175$ & 40.705 & 2.50 & 0.39 \\
\hline 2008 & 3 & 31 & $22: 54: 5912.171$ & 40.708 & 2.46 & 0.56 \\
\hline 2008 & 3 & 31 & $22: 55: 1612.174$ & 40.714 & 3.00 & 0.37 \\
\hline 2008 & 3 & 31 & $22: 56: 3312.188$ & 40.703 & 2.40 & 0.5 \\
\hline 2008 & 3 & 31 & $22: 56: 5612.170$ & 40.709 & 2.61 & 0.46 \\
\hline 2008 & 3 & 31 & $22: 57: 3012.175$ & 40.707 & 2.33 & 0.54 \\
\hline 2008 & 3 & 31 & $22: 57: 5512.165$ & 40.707 & 2.49 & 0.5 \\
\hline 2008 & 3 & 31 & $22: 59: 2212.185$ & 40.716 & 2.38 & 0.77 \\
\hline 2008 & 3 & 31 & $22: 59: 3912.171$ & 40.707 & 3.25 & 0.39 \\
\hline 2008 & 3 & 31 & $23: 00: 2212.176$ & 40.708 & 2.55 & 0.45 \\
\hline 2008 & 3 & 31 & $23: 04: 5212.167$ & 40.711 & 2.49 & 0.38 \\
\hline 2008 & 3 & 31 & $23: 05: 4912.177$ & 40.707 & 2.59 & 0.54 \\
\hline 2008 & 3 & 31 & $23: 07: 0412.176$ & 40.700 & 2.38 & 0.75 \\
\hline 2008 & 3 & 31 & $23: 08: 1612.171$ & 40.708 & 3.21 & 0.35 \\
\hline 2008 & 3 & 31 & $23: 12: 0512.171$ & 40.708 & 2.84 & 0.35 \\
\hline 2008 & 3 & 31 & $23: 16: 4312.167$ & 40.712 & 2.59 & 0.42 \\
\hline 2008 & 3 & 31 & $23: 17: 1912.177$ & 40.709 & 2.75 & 0.4 \\
\hline 2008 & 3 & 31 & $23: 17: 4312.171$ & 40.710 & 3.32 & 0.41 \\
\hline 2008 & 3 & 31 & $23: 27: 1012.162$ & 40.710 & 2.83 & 0.4 \\
\hline 2008 & 3 & 31 & $23: 36: 5212.180$ & 40.719 & 2.98 & 0.53 \\
\hline 2008 & 3 & 31 & $23: 38: 1412.171$ & 40.718 & 3.25 & 0.34 \\
\hline 2008 & 3 & 31 & $23: 41: 0512.169$ & 40.712 & 2.73 & 0.44 \\
\hline 2008 & 3 & 31 & $23: 44: 3512.165$ & 40.712 & 3.01 & 0.4 \\
\hline 2008 & 3 & 31 & $23: 45: 1212.173$ & 40.703 & 2.78 & 0.55 \\
\hline 2008 & 3 & 31 & $23: 53: 2512.161$ & 40.709 & 2.62 & 0.5 \\
\hline 2008 & 3 & 31 & $23: 55: 3312.172$ & 40.711 & 3.54 & 0.37 \\
\hline 2008 & 3 & 31 & $23: 57: 3612.165$ & 40.711 & 3.08 & 0.36 \\
\hline 2008 & 4 & 1 & $00: 02: 4812.167$ & 40.701 & 2.74 & 0.71 \\
\hline 2008 & 4 & 1 & $00: 11: 1612.169$ & 40.714 & 3.46 & 0.35 \\
\hline 2008 & 4 & 1 & $00: 18: 0612.164$ & 40.703 & 2.80 & 0.42 \\
\hline 2008 & 4 & 1 & $00: 25: 0012.170$ & 40.714 & 2.67 & 0.36 \\
\hline 2008 & 4 & 1 & $00: 27: 1912.165$ & 40.712 & 2.71 & 0.41 \\
\hline 2008 & 4 & 1 & $00: 32: 1212.164$ & 40.714 & 2.29 & 0.5 \\
\hline 2008 & 4 & 1 & $00: 33: 0312.164$ & 40.713 & 2.95 & 0.4 \\
\hline 2008 & 4 & 1 & $00: 34: 4312.161$ & 40.708 & 2.62 & 0.43 \\
\hline 2008 & 4 & 1 & $00: 36: 0512.164$ & 40.717 & 2.87 & 0.35 \\
\hline 2008 & 4 & 1 & $00: 38: 1212.165$ & 40.713 & 3.42 & 0.33 \\
\hline 2008 & 4 & 1 & $00: 44: 4412.174$ & 40.713 & 2.55 & 0.43 \\
\hline 2008 & 4 & 1 & $00: 46: 1612.165$ & 40.717 & 2.48 & 0.38 \\
\hline 2008 & 4 & 1 & $01: 08: 3712.166$ & 40.707 & 2.59 & 0.5 \\
\hline 2008 & 4 & 1 & $01: 09: 0112.161$ & 40.711 & 2.64 & 0.64 \\
\hline 2008 & 4 & 1 & $01: 13: 1012.159$ & 40.714 & 2.46 & 0.55 \\
\hline 2008 & 4 & 1 & $01: 13: 5412.159$ & 40.714 & 2.62 & 0.41 \\
\hline 2008 & 4 & 1 & $01: 15: 4612.161$ & 40.716 & 3.05 & 0.33 \\
\hline 2008 & 4 & 1 & $01: 18: 1112.152$ & 40.704 & 2.57 & 0.82 \\
\hline 2008 & 4 & 1 & $01: 22: 2812.160$ & 40.710 & 2.55 & 0.59 \\
\hline 2008 & 4 & 1 & $01: 27: 0212.167$ & 40.716 & 3.52 & 0.43 \\
\hline 2008 & 4 & 1 & $01: 33: 5812.162$ & 40.718 & 2.73 & 0.42 \\
\hline 2008 & 4 & 1 & $01: 38: 3312.165$ & 40.719 & 3.00 & 0.33 \\
\hline 2008 & 4 & 1 & $01: 41: 5112.166$ & 40.717 & 2.59 & 0.4 \\
\hline 2008 & 4 & 1 & $01: 43: 0212.164$ & 40.717 & 2.78 & 0.39 \\
\hline 2008 & 4 & 1 & $01: 44: 2512.168$ & 40.713 & 2.55 & 0.38 \\
\hline 2008 & 4 & 1 & $01: 47: 4112.166$ & 40.716 & 3.34 & 0.35 \\
\hline 2008 & 4 & 1 & $02: 19: 3012.138$ & 40.711 & 2.52 & 0.72 \\
\hline 2008 & 4 & 1 & $02: 42: 1512.137$ & 40.711 & 2.42 & 0.84 \\
\hline 2008 & 4 & 1 & $02: 46: 2212.150$ & 40.721 & 2.67 & 0.46 \\
\hline
\end{tabular}




\begin{tabular}{|c|c|c|c|c|c|c|}
\hline 2008 & 4 & 1 & $03: 01: 4712.141$ & 40.716 & 2.68 & 0.81 \\
\hline 2008 & 4 & 1 & $03: 34: 5312.150$ & 40.722 & 2.45 & 0.74 \\
\hline 2008 & 4 & 1 & $03: 36: 3612.130$ & 40.707 & 2.36 & 0.85 \\
\hline 2008 & 4 & 1 & $03: 50: 3912.156$ & 40.718 & 2.65 & 0.67 \\
\hline 2008 & 4 & 1 & $03: 54: 1812.163$ & 40.713 & 2.75 & 0.66 \\
\hline 2008 & 4 & 1 & $04: 01: 1112.157$ & 40.719 & 2.75 & 0.59 \\
\hline 2008 & 4 & 1 & $04: 33: 1612.138$ & 40.720 & 2.48 & 0.74 \\
\hline 2008 & 4 & 1 & $04: 34: 1912.148$ & 40.715 & 2.41 & 0.81 \\
\hline 2008 & 4 & 1 & $04: 42: 3712.174$ & 40.723 & 2.57 & 1.86 \\
\hline 2008 & 4 & 1 & $08: 56: 1212.162$ & 40.718 & 2.47 & 0.63 \\
\hline 2008 & 4 & 1 & $09: 20: 1212.154$ & 40.720 & 2.28 & 0.64 \\
\hline 2008 & 4 & 1 & $09: 48: 2812.167$ & 40.716 & 2.62 & 0.4 \\
\hline 2008 & 4 & 1 & $11: 42: 0612.150$ & 40.724 & 2.65 & 0.63 \\
\hline 2008 & 4 & 1 & $11: 52: 3212.156$ & 40.717 & 2.67 & 0.46 \\
\hline 2008 & 4 & 1 & $11: 54: 1112.159$ & 40.715 & 2.54 & 0.69 \\
\hline 2008 & 4 & 1 & $12: 12: 4912.168$ & 40.717 & 2.74 & 0.48 \\
\hline 2008 & 4 & 1 & $12: 43: 2512.143$ & 40.716 & 2.47 & 0.61 \\
\hline 2008 & 4 & 1 & $13: 46: 1412.137$ & 40.718 & 2.53 & 0.72 \\
\hline 2008 & 4 & 1 & $14: 45: 4512.148$ & 40.719 & 2.30 & 0.62 \\
\hline 2008 & 4 & 1 & $15: 11: 0812.138$ & 40.721 & 2.36 & 0.66 \\
\hline 2008 & 4 & 1 & $17: 39: 3512.182$ & 40.682 & 3.18 & 0.38 \\
\hline 2008 & 4 & 1 & $17: 48: 4412.156$ & 40.710 & 2.53 & 0.44 \\
\hline 2008 & 4 & 1 & $18: 08: 0412.109$ & 40.751 & 2.78 & 0.46 \\
\hline 2008 & 4 & 1 & $18: 13: 3112.119$ & 40.726 & 2.38 & 0.58 \\
\hline 2008 & 4 & 1 & $18: 45: 2212.149$ & 40.721 & 2.53 & 0.41 \\
\hline 2008 & 4 & 1 & $20: 19: 0212.145$ & 40.718 & 2.23 & 0.7 \\
\hline 2008 & 4 & 1 & $20: 41: 1112.165$ & 40.717 & 2.71 & 0.35 \\
\hline 2008 & 4 & 1 & $21: 57: 5912.153$ & 40.723 & 2.29 & 0.36 \\
\hline 2008 & 4 & 1 & $22: 37: 1712.142$ & 40.722 & 1.94 & 0.56 \\
\hline 2008 & 4 & 1 & $23: 26: 1812.161$ & 40.718 & 2.15 & 0.43 \\
\hline 2008 & 4 & 1 & $23: 31: 3612.170$ & 40.718 & 2.50 & 0.4 \\
\hline 2008 & 4 & 2 & $00: 14: 5912.150$ & 40.722 & 2.38 & 0.37 \\
\hline 2008 & 4 & 2 & $00: 45: 0312.154$ & 40.712 & 2.23 & 0.36 \\
\hline 2008 & 4 & 2 & $01: 25: 5712.162$ & 40.719 & 2.43 & 0.51 \\
\hline 2008 & 4 & 2 & $04: 21: 0312.113$ & 40.754 & 2.97 & 0.47 \\
\hline 2008 & 4 & 2 & $04: 29: 4312.146$ & 40.718 & 2.60 & 0.74 \\
\hline 2008 & 4 & 2 & $06: 07: 1212.138$ & 40.703 & 2.23 & 0.91 \\
\hline 2008 & 4 & 2 & $08: 23: 4412.157$ & 40.712 & 2.23 & 0.81 \\
\hline 2008 & 4 & 2 & $11: 15: 1512.142$ & 40.715 & 2.24 & 0.69 \\
\hline 2008 & 4 & 2 & $11: 20: 1312.146$ & 40.713 & 2.29 & 0.7 \\
\hline 2008 & 4 & 2 & $12: 42: 48 \quad 12.122$ & 40.715 & 2.23 & 0.73 \\
\hline 2008 & 4 & 2 & $13: 39: 3212.136$ & 40.726 & 2.32 & 0.86 \\
\hline 2008 & 4 & 2 & $14: 14: 5112.128$ & 40.719 & 2.23 & 0.85 \\
\hline 2008 & 4 & 2 & $14: 32: 4412.147$ & 40.710 & 2.27 & 0.56 \\
\hline 2008 & 4 & 2 & $14: 36: 1112.168$ & 40.702 & 2.28 & 0.69 \\
\hline 2008 & 4 & 2 & $15: 11: 4512.141$ & 40.728 & 2.26 & 0.82 \\
\hline 2008 & 4 & 2 & $16: 16: 0212.139$ & 40.724 & 2.43 & 0.5 \\
\hline 2008 & 4 & 2 & $17: 35: 2812.162$ & 40.711 & 2.34 & 0.48 \\
\hline 2008 & 4 & 2 & $18: 58: 4812.167$ & 40.704 & 2.17 & 0.61 \\
\hline 2008 & 4 & 2 & $19: 39: 3412.114$ & 40.752 & 2.30 & 0.39 \\
\hline 2008 & 4 & 2 & $20: 14: 1812.157$ & 40.718 & 2.29 & 0.42 \\
\hline 2008 & 4 & 2 & $20: 25: 2512.160$ & 40.718 & 2.59 & 0.43 \\
\hline 2008 & 4 & 2 & $21: 20: 1112.158$ & 40.716 & 2.08 & 0.44 \\
\hline 2008 & 4 & 2 & $22: 14: 3812.153$ & 40.730 & 2.18 & 0.38 \\
\hline 2008 & 4 & 2 & $23: 02: 5512.162$ & 40.723 & 2.11 & 0.4 \\
\hline 2008 & 4 & 3 & $00: 43: 1712.155$ & 40.714 & 2.03 & 0.45 \\
\hline 2008 & 4 & 3 & $01: 01: 2712.154$ & 40.714 & 2.03 & 0.6 \\
\hline 2008 & 4 & 3 & $01: 49: 2412.178$ & 40.702 & 1.95 & 0.92 \\
\hline 2008 & 4 & 3 & $09: 52: 0712.165$ & 40.700 & 2.13 & 0.7 \\
\hline 2008 & 4 & 3 & $11: 38: 4012.170$ & 40.704 & 2.11 & 0.66 \\
\hline 2008 & 4 & 3 & $13: 33: 4212.111$ & 40.747 & 2.60 & 0.43 \\
\hline 2008 & 4 & 3 & $15: 12: 3412.119$ & 40.662 & 2.19 & 3.02 \\
\hline 2008 & 4 & 3 & $16: 59: 2012.165$ & 40.701 & 2.17 & 0.54 \\
\hline 2008 & 4 & 3 & $17: 45: 3812.176$ & 40.674 & 2.18 & 0.62 \\
\hline 2008 & 4 & 3 & $21: 14: 3812.186$ & 40.703 & 2.14 & 0.71 \\
\hline 2008 & 4 & 3 & $22: 41: 2812.154$ & 40.717 & 2.01 & 0.62 \\
\hline 2008 & 4 & 3 & $22: 49: 5012.167$ & 40.705 & 1.92 & 0.59 \\
\hline 2008 & 4 & 4 & $00: 01: 1612.164$ & 40.725 & 2.69 & 0.49 \\
\hline 2008 & 4 & 4 & $00: 23: 3112.161$ & 40.718 & 2.06 & 0.63 \\
\hline 2008 & 4 & 4 & $01: 19: 4312.181$ & 40.700 & 2.01 & 0.43 \\
\hline 2008 & 4 & 4 & $01: 30: 5612.177$ & 40.707 & 2.02 & 0.59 \\
\hline 2008 & 4 & 4 & $03: 29: 2112.144$ & 40.705 & 2.04 & 1.14 \\
\hline 2008 & 4 & 4 & $13: 52: 1713.486$ & 40.872 & 2.85 & 0.78 \\
\hline 2008 & 4 & 4 & $15: 13: 5312.153$ & 40.709 & 2.15 & 2.08 \\
\hline 2008 & 4 & 4 & $17: 02: 2913.485$ & 40.849 & 2.71 & 0.77 \\
\hline 2008 & 4 & 4 & $19: 06: 1611.931$ & 41.252 & 2.17 & 1.51 \\
\hline
\end{tabular}




\begin{tabular}{|c|c|c|c|c|c|c|}
\hline 2008 & 4 & 4 & $21: 09: 4511.228$ & 40.862 & 2.21 & 0.46 \\
\hline 2008 & 4 & 4 & $21: 28: 4813.517$ & 40.889 & 2.54 & 4.27 \\
\hline 2008 & 4 & 4 & $22: 53: 0013.491$ & 40.858 & 2.53 & 1.3 \\
\hline 2008 & 4 & 4 & $22: 59: 0713.459$ & 40.852 & 2.50 & 2.41 \\
\hline 2008 & 4 & 4 & $23: 08: 5311.875$ & 41.024 & 1.91 & 0.4 \\
\hline 2008 & 4 & 5 & $01: 29: 3613.474$ & 40.861 & 2.82 & 4.27 \\
\hline 2008 & 4 & 5 & $02: 51: 4012.162$ & 40.716 & 2.34 & 0.42 \\
\hline 2008 & 4 & 5 & $05: 12: 4812.102$ & 40.754 & 2.20 & 0.72 \\
\hline 2008 & 4 & 5 & $09: 59: 2312.446$ & 40.569 & 2.34 & 0.49 \\
\hline 2008 & 4 & 5 & $10: 23: 5311.938$ & 41.264 & 2.55 & 0.32 \\
\hline 2008 & 4 & 5 & $16: 45: 2113.477$ & 40.861 & 2.62 & 1.86 \\
\hline 2008 & 4 & 5 & $19: 23: 3612.424$ & 40.577 & 2.00 & 0.56 \\
\hline 2008 & 4 & 5 & $23: 09: 2311.917$ & 41.252 & 2.04 & 0.89 \\
\hline 2008 & 4 & 7 & $10: 32: 5713.405$ & 39.982 & 2.97 & 0.99 \\
\hline 2008 & 4 & 8 & $21: 27: 1811.976$ & 41.350 & 2.24 & 0.51 \\
\hline 2008 & 4 & 9 & $00: 10: 1512.455$ & 40.547 & 2.30 & 0.59 \\
\hline 2008 & 4 & 12 & $07: 51: 2211.635$ & 41.026 & 2.50 & 0.33 \\
\hline 2008 & 4 & 12 & $22: 59: 2013.454$ & 39.963 & 2.67 & 1.08 \\
\hline 2008 & 4 & 13 & $02: 01: 229.966$ & 40.296 & 3.10 & 11.03 \\
\hline 2008 & 4 & 13 & $18: 46: 0613.397$ & 40.955 & 2.54 & 7.13 \\
\hline 2008 & 4 & 13 & $21: 03: 0012.261$ & 40.643 & 1.97 & 0.66 \\
\hline 2008 & 4 & 13 & $21: 08: 5613.413$ & 39.967 & 2.63 & 0.65 \\
\hline 2008 & 4 & 13 & $21: 52: 2313.402$ & 39.968 & 2.58 & 0.69 \\
\hline 2008 & 4 & 13 & $22: 29: 5413.410$ & 40.916 & 2.93 & 1.29 \\
\hline 2008 & 4 & 14 & $00: 04: 5912.389$ & 40.585 & 1.96 & 0.41 \\
\hline 2008 & 4 & 14 & $00: 27: 5712.427$ & 40.569 & 1.86 & 1.02 \\
\hline 2008 & 4 & 14 & $01: 44: 0813.443$ & 40.925 & 2.84 & 13.43 \\
\hline 2008 & 4 & 14 & $02: 32: 5613.421$ & 40.882 & 2.72 & 1.12 \\
\hline 2008 & 4 & 14 & $03: 39: 4913.425$ & 40.912 & 3.35 & 2.47 \\
\hline 2008 & 4 & 14 & $05: 16: 5413.421$ & 40.903 & 2.79 & 2.17 \\
\hline 2008 & 4 & 14 & $11: 37: 0413.453$ & 40.967 & 2.77 & 3.81 \\
\hline 2008 & 4 & 14 & $14: 47: 3513.381$ & 40.988 & 2.77 & 5.53 \\
\hline 2008 & 4 & 15 & $09: 37: 1612.082$ & 40.769 & 2.23 & 0.7 \\
\hline 2008 & 4 & 15 & $18: 14: 4712.473$ & 40.546 & 2.09 & 0.73 \\
\hline 2008 & 4 & 15 & $20: 47: 4912.417$ & 40.582 & 1.98 & 0.48 \\
\hline 2008 & 4 & 15 & $21: 00: 2412.440$ & 40.560 & 1.92 & 1.04 \\
\hline 2008 & 4 & 16 & $02: 22: 5712.386$ & 40.588 & 2.07 & 0.76 \\
\hline 2008 & 4 & 16 & $05: 15: 3313.456$ & 40.870 & 2.79 & 1.39 \\
\hline 2008 & 4 & 16 & $09: 41: 2812.667$ & 40.496 & 2.24 & 1.08 \\
\hline 2008 & 4 & 16 & $17: 53: 4112.942$ & 40.323 & 3.30 & 0.32 \\
\hline 2008 & 4 & 16 & $23: 29: 0712.453$ & 40.566 & 2.29 & 0.42 \\
\hline 2008 & 4 & 17 & $06: 49: 2112.403$ & 40.594 & 2.24 & 0.51 \\
\hline 2008 & 4 & 17 & $20: 43: 0113.566$ & 40.722 & 2.52 & 2.74 \\
\hline 2008 & 4 & 18 & $00: 48: 1113.665$ & 39.913 & 2.66 & 1.48 \\
\hline 2008 & 4 & 18 & $09: 53: 0213.435$ & 40.827 & 2.64 & 4.19 \\
\hline 2008 & 4 & 18 & $18: 10: 4312.433$ & 40.574 & 2.10 & 0.49 \\
\hline 2008 & 4 & 18 & $22: 36: 4613.452$ & 40.835 & 2.57 & 4.02 \\
\hline 2008 & 4 & 19 & $00: 43: 3712.277$ & 40.643 & 1.94 & 0.48 \\
\hline 2008 & 4 & 19 & $10: 27: 4412.127$ & 39.683 & 2.59 & 0.54 \\
\hline 2008 & 4 & 19 & $17: 40: 4413.417$ & 40.834 & 2.74 & 6.06 \\
\hline 2008 & 4 & 19 & $17: 44: 0813.463$ & 40.876 & 2.63 & 1.59 \\
\hline 2008 & 4 & 19 & $22: 30: 4512.388$ & 40.581 & 1.95 & 0.86 \\
\hline 2008 & 4 & 20 & $03: 04: 1212.379$ & 40.597 & 2.08 & 0.48 \\
\hline 2008 & 4 & 20 & $16: 43: 5013.414$ & 39.958 & 2.98 & 0.57 \\
\hline 2008 & 4 & 20 & $22: 18: 5212.134$ & 39.688 & 2.29 & 0.53 \\
\hline 2008 & 4 & 21 & $01: 31: 2311.180$ & 39.951 & 2.36 & 1.56 \\
\hline 2008 & 4 & 21 & $09: 09: 0212.450$ & 40.557 & 2.12 & 0.71 \\
\hline 2008 & 4 & 21 & $09: 32: 2312.522$ & 40.527 & 2.09 & 0.98 \\
\hline 2008 & 4 & 21 & $12: 25: 3312.376$ & 40.593 & 2.44 & 0.44 \\
\hline 2008 & 4 & 21 & $21: 47: 2612.751$ & 40.556 & 2.10 & 1.1 \\
\hline 2008 & 4 & 22 & $00: 45: 4213.747$ & 40.498 & 2.64 & 1.71 \\
\hline 2008 & 4 & 22 & $00: 45: 4313.890$ & 40.536 & 2.71 & 1.72 \\
\hline 2008 & 4 & 22 & $10: 04: 0613.420$ & 40.980 & 2.69 & 5.89 \\
\hline 2008 & 4 & 22 & $18: 00: 1310.349$ & 40.960 & 3.04 & 2.59 \\
\hline 2008 & 4 & 23 & $00: 51: 5112.439$ & 40.559 & 1.94 & 0.59 \\
\hline 2008 & 4 & 23 & $01: 40: 3910.409$ & 40.960 & 2.83 & 6.77 \\
\hline 2008 & 4 & 23 & $03: 53: 0212.402$ & 40.576 & 2.07 & 0.96 \\
\hline 2008 & 4 & 23 & $09: 05: 1911.911$ & 41.027 & 2.13 & 0.94 \\
\hline 2008 & 4 & 23 & $11: 51: 2611.783$ & 39.093 & 2.93 & 6.95 \\
\hline 2008 & 4 & 23 & $21: 55: 2912.450$ & 40.567 & 1.89 & 1.38 \\
\hline 2008 & 4 & 23 & $22: 58: 1712.449$ & 40.551 & 1.90 & 0.62 \\
\hline 2008 & 4 & 24 & $10: 30: 0511.599$ & 42.910 & 3.55 & 9.57 \\
\hline 2008 & 4 & 24 & $22: 34: 3912.392$ & 40.593 & 1.91 & 0.6 \\
\hline 2008 & 4 & 25 & $00: 06: 1213.485$ & 40.851 & 2.52 & 1.26 \\
\hline 2008 & 4 & 25 & $01: 19: 3011.340$ & 39.811 & 2.41 & 0.57 \\
\hline 2008 & 4 & 25 & $10: 23: 2713.228$ & 39.798 & 2.91 & 0.42 \\
\hline
\end{tabular}




\begin{tabular}{|c|c|c|c|c|c|c|}
\hline 2008 & 4 & 25 & $19: 04: 4212.117$ & 40.746 & 2.49 & 0.36 \\
\hline 2008 & 4 & 25 & $21: 50: 2112.256$ & 39.628 & 2.35 & 0.4 \\
\hline 2008 & 4 & 26 & $10: 02: 0610.591$ & 39.876 & 3.44 & 4.13 \\
\hline 2008 & 4 & 26 & $15: 52: 0113.399$ & 39.987 & 2.63 & 0.93 \\
\hline 2008 & 4 & 26 & $16: 07: 2413.403$ & 39.992 & 2.68 & 0.5 \\
\hline 2008 & 4 & 26 & $20: 32: 3712.505$ & 40.544 & 1.99 & 0.46 \\
\hline 2008 & 4 & 27 & $05: 36: 0312.853$ & 39.477 & 2.60 & 0.59 \\
\hline 2008 & 4 & 27 & $13: 52: 0412.905$ & 39.800 & 2.50 & 0.52 \\
\hline 2008 & 4 & 27 & $16: 51: 5212.406$ & 39.662 & 2.39 & 0.63 \\
\hline 2008 & 4 & 27 & $20: 43: 5813.501$ & 39.996 & 2.54 & 1.15 \\
\hline 2008 & 4 & 27 & $21: 50: 4612.866$ & 40.634 & 2.15 & 0.68 \\
\hline 2008 & 4 & 27 & $22: 12: 1112.489$ & 40.542 & 1.83 & 0.81 \\
\hline 2008 & 4 & 27 & $22: 32: 4712.491$ & 40.535 & 1.90 & 1.09 \\
\hline 2008 & 4 & 27 & $23: 29: 3911.542$ & 41.111 & 1.99 & 0.98 \\
\hline 2008 & 4 & 28 & $00: 29: 2411.982$ & 40.932 & 1.84 & 1.56 \\
\hline 2008 & 4 & 28 & $18: 24: 2812.445$ & 40.562 & 2.22 & 0.56 \\
\hline 2008 & 4 & 28 & $20: 48: 5412.990$ & 39.793 & 2.71 & 0.82 \\
\hline 2008 & 4 & 28 & $21: 09: 3112.366$ & 40.603 & 2.11 & 0.55 \\
\hline 2008 & 4 & 28 & $22: 18: 1513.471$ & 40.828 & 2.49 & 2.14 \\
\hline 2008 & 4 & 29 & $00: 06: 5811.712$ & 42.918 & 5.00 & 2.29 \\
\hline 2008 & 4 & 29 & $01: 07: 3011.706$ & 42.913 & 4.96 & 2.75 \\
\hline 2008 & 4 & 29 & $01: 47: 4811.740$ & 42.866 & 4.31 & 2.37 \\
\hline 2008 & 4 & 29 & $09: 40: 3411.693$ & 42.902 & 3.45 & 3.07 \\
\hline 2008 & 4 & 29 & $13: 16: 0811.727$ & 42.918 & 3.13 & 6.13 \\
\hline 2008 & 4 & 29 & $17: 04: 5211.735$ & 42.836 & 4.24 & 5,00 \\
\hline 2008 & 4 & 29 & $20: 17: 2811.729$ & 42.943 & 3.30 & 3.29 \\
\hline 2008 & 4 & 29 & $22: 41: 2811.717$ & 42.921 & 4.42 & 1,00 \\
\hline 2008 & 4 & 29 & $22: 56: 0911.660$ & 42.947 & 3.35 & 5.66 \\
\hline 2008 & 4 & 30 & $00: 52: 2811.640$ & 42.815 & 2.99 & 6.25 \\
\hline 2008 & 4 & 30 & $08: 33: 0511.720$ & 42.930 & 4.11 & 5,00 \\
\hline 2008 & 4 & 30 & $10: 31: 5311.776$ & 42.853 & 4.08 & 2.32 \\
\hline 2008 & 4 & 30 & $22: 01: 2912.599$ & 40.508 & 2.01 & 0.74 \\
\hline 2008 & 5 & 1 & $02: 24: 5113.234$ & 39.812 & 2.77 & 0.98 \\
\hline 2008 & 5 & 3 & $22: 47: 2213.483$ & 40.799 & 2.93 & 1.12 \\
\hline 2008 & 5 & 3 & $23: 43: 5613.168$ & 39.722 & 2.56 & 1.3 \\
\hline 2008 & 5 & 4 & $01: 36: 1912.506$ & 40.543 & 2.01 & 0.76 \\
\hline 2008 & 5 & 4 & $07: 55: 5212.949$ & 39.493 & 2.78 & 0.65 \\
\hline 2008 & 5 & 4 & $21: 06: 2711.622$ & 43.041 & 3.67 & 6.67 \\
\hline 2008 & 5 & 4 & $23: 10: 4715.250$ & 39.694 & 3.26 & 8.24 \\
\hline 2008 & 5 & 5 & $01: 28: 2512.389$ & 40.582 & 1.99 & 0.41 \\
\hline 2008 & 5 & 5 & $01: 45: 4712.321$ & 40.606 & 2.01 & 0.56 \\
\hline 2008 & 5 & 5 & $18: 28: 2511.577$ & 41.045 & 2.08 & 0.85 \\
\hline 2008 & 5 & 5 & $22: 29: 3511.733$ & 41.184 & 2.00 & 1.8 \\
\hline 2008 & 5 & 5 & $22: 53: 3912.428$ & 40.577 & 1.90 & 3.35 \\
\hline 2008 & 5 & 6 & $01: 49: 2712.507$ & 40.532 & 2.04 & 1.02 \\
\hline 2008 & 5 & 6 & $09: 50: 0713.514$ & 40.868 & 2.83 & 2.03 \\
\hline 2008 & 5 & 6 & $22: 29: 4112.385$ & 40.595 & 1.96 & 0.65 \\
\hline 2008 & 5 & 7 & $12: 59: 3113.399$ & 40.884 & 2.69 & 4.39 \\
\hline 2008 & 5 & 7 & $13: 21: 4513.109$ & 39.772 & 2.68 & 3.51 \\
\hline 2008 & 5 & 7 & $22: 50: 2012.388$ & 40.576 & 1.87 & 1.32 \\
\hline 2008 & 5 & 8 & $22: 31: 4213.665$ & 39.909 & 2.75 & 1.53 \\
\hline 2008 & 5 & 9 & $10: 31: 5812.544$ & 40.525 & 2.09 & 0.6 \\
\hline 2008 & 5 & 9 & $13: 51: 0212.427$ & 40.578 & 2.26 & 0.84 \\
\hline 2008 & 5 & 9 & $16: 12: 3313.341$ & 39.971 & 2.72 & 0.75 \\
\hline 2008 & 5 & 9 & $23: 23: 0112.561$ & 40.511 & 2.25 & 1.1 \\
\hline 2008 & 5 & 9 & $23: 37: 1313.316$ & 39.985 & 2.57 & 0.51 \\
\hline 2008 & 5 & 10 & $00: 25: 3612.316$ & 40.403 & 1.86 & 0.92 \\
\hline 2008 & 5 & 10 & $01: 04: 5011.447$ & 41.108 & 2.20 & 0.61 \\
\hline 2008 & 5 & 10 & $02: 41: 2112.363$ & 40.602 & 2.17 & 0.57 \\
\hline 2008 & 5 & 10 & $04: 43: 4411.431$ & 41.110 & 2.38 & 1.28 \\
\hline 2008 & 5 & 10 & $09: 48: 1812.329$ & 40.606 & 2.20 & 0.57 \\
\hline 2008 & 5 & 10 & $23: 21: 0311.870$ & 39.491 & 2.50 & 1.04 \\
\hline 2008 & 5 & 10 & $23: 23: 1812.602$ & 40.496 & 2.11 & 0.61 \\
\hline 2008 & 5 & 10 & $23: 28: 0313.632$ & 39.922 & 2.63 & 2.57 \\
\hline 2008 & 5 & 11 & $01: 40: 1711.634$ & 43.094 & 3.16 & 6.43 \\
\hline 2008 & 5 & 11 & $22: 25: 2711.435$ & 41.111 & 2.35 & 0.79 \\
\hline 2008 & 5 & 11 & $22: 45: 209.290$ & 40.091 & 3.17 & 2.88 \\
\hline 2008 & 5 & 12 & $00: 25: 1711.872$ & 39.492 & 2.93 & 0.52 \\
\hline 2008 & 5 & 12 & $06: 22: 5211.731$ & 39.630 & 2.71 & 0.56 \\
\hline 2008 & 5 & 12 & $15: 32: 4212.388$ & 40.583 & 2.13 & 0.57 \\
\hline 2008 & 5 & 12 & $18: 41: 5113.023$ & 40.742 & 2.38 & 1.17 \\
\hline 2008 & 5 & 12 & $22: 36: 2711.608$ & 40.858 & 1.79 & 10.17 \\
\hline 2008 & 5 & 12 & $22: 54: 0511.443$ & 41.108 & 2.15 & 0.94 \\
\hline 2008 & 5 & 13 & $00: 06: 0312.114$ & 40.765 & 1.82 & 0.71 \\
\hline 2008 & 5 & 13 & $02: 25: 3213.528$ & 39.964 & 2.67 & 1.67 \\
\hline 2008 & 5 & 13 & $04: 54: 5813.755$ & 40.637 & 2.79 & 1.82 \\
\hline
\end{tabular}




\begin{tabular}{|c|c|c|c|c|c|c|}
\hline 2008 & 5 & 13 & $17: 34: 4512.471$ & 40.542 & 2.15 & 0.97 \\
\hline 2008 & 5 & 13 & $22: 07: 1412.489$ & 40.543 & 1.93 & 1.06 \\
\hline 2008 & 5 & 13 & $22: 47: 4510.503$ & 39.965 & 2.67 & 2.36 \\
\hline 2008 & 5 & 13 & $22: 52: 1413.693$ & 39.841 & 2.62 & 1.14 \\
\hline 2008 & 5 & 14 & $05: 29: 0312.375$ & 40.593 & 2.22 & 0.62 \\
\hline 2008 & 5 & 14 & $11: 17: 4312.107$ & 40.917 & 2.36 & 0.81 \\
\hline 2008 & 5 & 14 & $19: 04: 3412.468$ & 40.552 & 2.22 & 0.49 \\
\hline 2008 & 5 & 15 & $01: 00: 3812.581$ & 40.503 & 1.88 & 1.1 \\
\hline 2008 & 5 & 16 & $01: 06: 2511.575$ & 40.995 & 2.11 & 0.84 \\
\hline 2008 & 5 & 16 & $03: 25: 4312.465$ & 40.555 & 2.28 & 0.38 \\
\hline 2008 & 5 & 16 & $04: 26: 0213.233$ & 39.798 & 3.09 & 0.39 \\
\hline 2008 & 5 & 17 & $00: 58: 3212.383$ & 40.595 & 1.84 & 1.16 \\
\hline 2008 & 5 & 17 & $16: 03: 5110.401$ & 40.020 & 2.96 & 2.46 \\
\hline 2008 & 5 & 17 & $22: 40: 059.271$ & 40.091 & 3.18 & 1.73 \\
\hline 2008 & 5 & 17 & $22: 57: 3313.492$ & 39.944 & 2.56 & 0.97 \\
\hline 2008 & 5 & 18 & $00: 08: 0512.495$ & 40.542 & 1.96 & 0.75 \\
\hline 2008 & 5 & 18 & $16: 49: 2913.450$ & 39.990 & 2.68 & 0.82 \\
\hline 2008 & 5 & 18 & $22: 11: 5513.237$ & 39.793 & 2.58 & 0.51 \\
\hline 2008 & 5 & 18 & $22: 15: 1712.558$ & 40.549 & 1.97 & 2.46 \\
\hline 2008 & 5 & 19 & $01: 41: 569.290$ & 40.083 & 3.35 & 2.01 \\
\hline 2008 & 5 & 19 & $01: 49: 2312.397$ & 40.579 & 1.93 & 0.78 \\
\hline 2008 & 5 & 19 & $08: 43: 3512.399$ & 40.575 & 2.12 & 0.58 \\
\hline 2008 & 5 & 19 & $18: 56: 2012.363$ & 40.592 & 2.08 & 0.97 \\
\hline 2008 & 5 & 19 & $19: 23: 3511.776$ & 41.019 & 1.87 & 4.69 \\
\hline 2008 & 5 & 19 & $23: 26: 309.260$ & 40.058 & 3.21 & 2.67 \\
\hline 2008 & 5 & 19 & $23: 46: 1512.495$ & 40.533 & 1.93 & 0.7 \\
\hline 2008 & 5 & 20 & $00: 03: 3712.342$ & 40.628 & 1.99 & 0.51 \\
\hline 2008 & 5 & 20 & $00: 03: 3712.339$ & 40.627 & 1.99 & 0.4 \\
\hline 2008 & 5 & 20 & $00: 59: 3213.244$ & 39.774 & 2.49 & 4.93 \\
\hline 2008 & 5 & 20 & $01: 44: 3712.470$ & 40.552 & 2.12 & 0.53 \\
\hline 2008 & 5 & 20 & $01: 44: 3712.467$ & 40.552 & 2.13 & 0.41 \\
\hline 2008 & 5 & 20 & $02: 06: 019.380$ & 40.162 & 4.19 & 1.75 \\
\hline 2008 & 5 & 20 & $02: 59: 359.316$ & 40.100 & 3.86 & 1.53 \\
\hline 2008 & 5 & 20 & $07: 01: 199.287$ & 40.114 & 3.65 & 1.87 \\
\hline 2008 & 5 & 20 & $09: 30: 189.217$ & 40.052 & 3.42 & 5.7 \\
\hline 2008 & 5 & 20 & $11: 20: 2613.866$ & 40.559 & 2.87 & 3.05 \\
\hline 2008 & 5 & 20 & $13: 25: 309.306$ & 40.113 & 3.53 & 2.04 \\
\hline 2008 & 5 & 20 & $19: 35: 3712.593$ & 40.506 & 2.53 & 0.87 \\
\hline 2008 & 5 & 21 & $00: 41: 009.202$ & 40.022 & 3.15 & 4.72 \\
\hline 2008 & 5 & 21 & $00: 46: 409.232$ & 40.063 & 3.13 & 2.44 \\
\hline 2008 & 5 & 21 & $02: 00: 489.196$ & 40.009 & 3.41 & 1.33 \\
\hline 2008 & 5 & 21 & $03: 58: 4912.382$ & 40.581 & 2.08 & 1.65 \\
\hline 2008 & 5 & 21 & $16: 03: 2513.512$ & 39.875 & 2.66 & 2.22 \\
\hline 2008 & 5 & 21 & $22: 53: 1112.407$ & 40.574 & 1.91 & 0.55 \\
\hline 2008 & 5 & 22 & $00: 16: 0614.013$ & 40.466 & 2.80 & 2.23 \\
\hline 2008 & 5 & 22 & $00: 23: 0714.007$ & 40.469 & 3.13 & 1.27 \\
\hline 2008 & 5 & 22 & $01: 28: 589.221$ & 40.046 & 3.32 & 3.43 \\
\hline 2008 & 5 & 22 & $07: 33: 149.229$ & 40.035 & 3.51 & 1.45 \\
\hline 2008 & 5 & 22 & $15: 18: 0812.497$ & 40.556 & 2.44 & 0.54 \\
\hline 2008 & 5 & 22 & $19: 14: 559.244$ & 40.065 & 3.49 & 1.53 \\
\hline 2008 & 5 & 23 & $00: 22: 5712.409$ & 40.583 & 1.92 & 0.44 \\
\hline 2008 & 5 & 23 & $00: 26: 549.325$ & 40.098 & 3.11 & 2.62 \\
\hline 2008 & 5 & 23 & $01: 07: 299.277$ & 40.097 & 3.22 & 2.35 \\
\hline 2008 & 5 & 23 & $11: 58: 0412.457$ & 40.517 & 2.16 & 1,00 \\
\hline 2008 & 5 & 23 & $13: 15: 3811.613$ & 40.909 & 2.10 & 0.98 \\
\hline 2008 & 5 & 23 & $14: 34: 019.282$ & 40.115 & 3.49 & 2.16 \\
\hline 2008 & 5 & 23 & $21: 27: 499.281$ & 40.060 & 3.29 & 9,00 \\
\hline 2008 & 5 & 23 & $22: 12: 2412.425$ & 40.588 & 1.99 & 0.52 \\
\hline 2008 & 5 & 24 & $16: 40: 4812.402$ & 40.557 & 2.09 & 2.18 \\
\hline 2008 & 5 & 24 & $20: 43: 5113.277$ & 39.812 & 2.76 & 0.55 \\
\hline 2008 & 5 & 24 & $21: 10: 4011.732$ & 41.384 & 2.37 & 0.74 \\
\hline 2008 & 5 & 24 & $22: 17: 2212.949$ & 39.575 & 2.64 & 0.43 \\
\hline 2008 & 5 & 24 & $23: 48: 269.265$ & 40.086 & 3.18 & 2.26 \\
\hline 2008 & 5 & 25 & $10: 45: 0312.455$ & 40.535 & 2.17 & 0.83 \\
\hline 2008 & 5 & 25 & $20: 12: 469.180$ & 40.008 & 3.32 & 8.21 \\
\hline 2008 & 5 & 25 & $21: 19: 3911.559$ & 42.050 & 2.72 & 5.99 \\
\hline 2008 & 5 & 25 & $21: 43: 4913.552$ & 39.899 & 2.68 & 1.17 \\
\hline 2008 & 5 & 25 & $22: 10: 5212.409$ & 40.582 & 1.96 & 0.44 \\
\hline 2008 & 5 & 26 & $03: 07: 1913.166$ & 39.704 & 2.76 & 1.27 \\
\hline 2008 & 5 & 26 & $10: 53: 2213.550$ & 39.900 & 2.81 & 0.65 \\
\hline 2008 & 5 & 26 & $17: 15: 2712.884$ & 40.319 & 2.67 & 0.51 \\
\hline 2008 & 5 & 26 & 20:09:17 12.454 & 40.562 & 2.01 & 0.57 \\
\hline 2008 & 5 & 26 & $21: 45: 0510.301$ & 40.992 & 2.94 & 0.64 \\
\hline 2008 & 5 & 26 & $23: 32: 329.229$ & 40.043 & 3.67 & 3.16 \\
\hline 2008 & 5 & 26 & $23: 43: 209.211$ & 40.024 & 3.35 & 2.68 \\
\hline 2008 & 5 & 26 & $23: 48: 009.211$ & 40.036 & 3.44 & 2.69 \\
\hline
\end{tabular}




\begin{tabular}{|c|c|c|c|c|c|c|}
\hline 2008 & 5 & 27 & $00: 24: 079.216$ & 40.037 & 3.29 & 2.66 \\
\hline 2008 & 5 & 27 & $04: 41: 049.174$ & 40.009 & 3.84 & 7.86 \\
\hline 2008 & 5 & 27 & $12: 30: 3312.876$ & 40.265 & 2.71 & 0.37 \\
\hline 2008 & 5 & 27 & $20: 58: 1013.635$ & 39.908 & 3.12 & 0.86 \\
\hline 2008 & 5 & 27 & $21: 01: 4913.465$ & 40.006 & 3.53 & 1.43 \\
\hline 2008 & 5 & 27 & $22: 30: 2612.433$ & 40.572 & 1.94 & 0.46 \\
\hline 2008 & 5 & 27 & $22: 51: 4213.552$ & 39.901 & 2.66 & 0.76 \\
\hline 2008 & 5 & 28 & $00: 04: 2613.555$ & 39.899 & 2.60 & 2.54 \\
\hline 2008 & 5 & 28 & $00: 20: 0912.460$ & 40.558 & 2.39 & 0.36 \\
\hline 2008 & 5 & 28 & $02: 08: 0311.543$ & 41.122 & 2.24 & 1.41 \\
\hline 2008 & 5 & 28 & $06: 34: 489.226$ & 39.977 & 4.29 & 6.56 \\
\hline 2008 & 5 & 28 & $09: 47: 1912.582$ & 40.521 & 2.20 & 1.14 \\
\hline 2008 & 5 & 28 & $21: 05: 2011.954$ & 41.292 & 2.32 & 0.45 \\
\hline 2008 & 5 & 29 & $00: 07: 5512.322$ & 40.608 & 1.95 & 0.46 \\
\hline 2008 & 5 & 29 & $07: 07: 5912.518$ & 40.544 & 2.19 & 1.05 \\
\hline 2008 & 5 & 29 & $08: 38: 2414.587$ & 39.813 & 3.14 & 5.28 \\
\hline 2008 & 5 & 29 & $09: 02: 3413.335$ & 41.044 & 3.26 & 4.78 \\
\hline 2008 & 5 & 29 & $18: 11: 0711.195$ & 40.108 & 2.63 & 0.69 \\
\hline 2008 & 5 & 29 & 19:08:08 12.964 & 39.556 & 2.61 & 1.75 \\
\hline 2008 & 5 & 29 & $20: 51: 4612.437$ & 40.571 & 2.05 & 0.98 \\
\hline 2008 & 5 & 30 & $19: 21: 5913.549$ & 39.900 & 2.68 & 1.25 \\
\hline 2008 & 5 & 31 & $03: 06: 5113.370$ & 41.133 & 3.62 & 4.01 \\
\hline 2008 & 6 & 1 & $00: 28: 4413.282$ & 41.027 & 2.32 & 5.84 \\
\hline 2008 & 6 & 1 & $01: 27: 1512.524$ & 40.540 & 2.00 & 0.5 \\
\hline 2008 & 6 & 1 & $01: 44: 5812.497$ & 40.539 & 1.96 & 0.76 \\
\hline 2008 & 6 & 1 & $02: 29: 3812.386$ & 40.581 & 2.19 & 0.83 \\
\hline 2008 & 6 & 1 & $11: 12: 3812.887$ & 40.627 & 2.39 & 5.06 \\
\hline 2008 & 6 & 1 & $11: 16: 2313.304$ & 41.049 & 3.03 & 8.58 \\
\hline 2008 & 6 & 1 & $14: 15: 4112.585$ & 40.466 & 2.24 & 1.83 \\
\hline 2008 & 6 & 1 & $15: 52: 0212.572$ & 40.535 & 2.42 & 1.31 \\
\hline 2008 & 6 & 1 & $16: 30: 4912.582$ & 40.527 & 2.44 & 0.85 \\
\hline 2008 & 6 & 2 & $02: 10: 0412.394$ & 40.592 & 2.01 & 0.79 \\
\hline 2008 & 6 & 2 & $04: 56: 0312.836$ & 40.627 & 2.86 & 0.57 \\
\hline 2008 & 6 & 2 & $18: 37: 4311.954$ & 41.395 & 2.10 & 1.86 \\
\hline 2008 & 6 & 2 & $18: 54: 1512.409$ & 40.588 & 1.90 & 0.7 \\
\hline 2008 & 6 & 2 & $20: 35: 1712.604$ & 40.507 & 1.98 & 0.8 \\
\hline 2008 & 6 & 2 & $21: 35: 3312.358$ & 40.586 & 2.04 & 0.48 \\
\hline 2008 & 6 & 3 & $00: 43: 4813.325$ & 41.054 & 2.91 & 4.3 \\
\hline 2008 & 6 & 3 & $03: 20: 0213.316$ & 41.052 & 2.96 & 9.56 \\
\hline 2008 & 6 & 3 & $03: 41: 4513.370$ & 41.071 & 2.67 & 11.13 \\
\hline 2008 & 6 & 3 & $21: 22: 2812.316$ & 40.608 & 1.91 & 0.74 \\
\hline 2008 & 6 & 4 & $00: 46: 0912.428$ & 40.581 & 1.90 & 0.58 \\
\hline 2008 & 6 & 4 & $01: 11: 1413.302$ & 41.049 & 2.80 & 6.98 \\
\hline 2008 & 6 & 4 & $04: 42: 0513.321$ & 41.067 & 3.28 & 7.39 \\
\hline 2008 & 6 & 4 & $09: 31: 2613.323$ & 41.044 & 2.78 & 6.63 \\
\hline 2008 & 6 & 4 & $18: 57: 0812.870$ & 40.637 & 2.30 & 0.62 \\
\hline 2008 & 6 & 4 & $22: 10: 1612.954$ & 39.718 & 2.51 & 0.5 \\
\hline 2008 & 6 & 4 & $22: 56: 2713.491$ & 39.999 & 2.63 & 2.8 \\
\hline 2008 & 6 & 4 & $23: 37: 5812.871$ & 40.632 & 2.24 & 0.51 \\
\hline 2008 & 6 & 5 & $03: 27: 1112.418$ & 40.577 & 2.24 & 0.95 \\
\hline 2008 & 6 & 5 & $11: 05: 3112.455$ & 40.566 & 2.01 & 0.69 \\
\hline 2008 & 6 & 5 & $23: 41: 0512.316$ & 40.614 & 1.90 & 0.82 \\
\hline 2008 & 6 & 6 & $16: 44: 0412.398$ & 40.544 & 2.00 & 1.72 \\
\hline 2008 & 6 & 6 & $19: 53: 1412.497$ & 40.539 & 1.89 & 0.66 \\
\hline 2008 & 6 & 6 & $22: 01: 5213.565$ & 39.823 & 2.61 & 5.82 \\
\hline 2008 & 6 & 7 & $00: 10: 3112.382$ & 40.592 & 1.96 & 0.61 \\
\hline 2008 & 6 & 7 & $23: 15: 2612.478$ & 40.547 & 1.93 & 0.96 \\
\hline 2008 & 6 & 8 & $01: 19: 0912.324$ & 40.604 & 1.99 & 0.36 \\
\hline 2008 & 6 & 8 & $10: 22: 0111.745$ & 39.044 & 2.91 & 1.25 \\
\hline 2008 & 6 & 8 & $22: 48: 2712.414$ & 40.577 & 1.96 & 0.6 \\
\hline 2008 & 6 & 9 & $03: 30: 2612.468$ & 40.555 & 2.08 & 1.26 \\
\hline 2008 & 6 & 9 & $14: 56: 4412.490$ & 40.547 & 2.22 & 0.58 \\
\hline 2008 & 6 & 9 & $18: 58: 1512.112$ & 40.751 & 2.36 & 0.42 \\
\hline 2008 & 6 & 9 & $22: 14: 4312.430$ & 40.571 & 1.92 & 0.43 \\
\hline 2008 & 6 & 10 & $21: 02: 2112.932$ & 39.557 & 2.58 & 0.58 \\
\hline 2008 & 6 & 10 & $22: 33: 3812.391$ & 40.532 & 1.89 & 1.05 \\
\hline 2008 & 6 & 10 & $22: 51: 4613.673$ & 39.938 & 2.65 & 4.73 \\
\hline 2008 & 6 & 11 & $00: 58: 5611.557$ & 41.045 & 1.97 & 1.06 \\
\hline 2008 & 6 & 11 & $08: 18: 3812.432$ & 40.558 & 2.21 & 0.77 \\
\hline 2008 & 6 & 11 & $08: 27: 4011.802$ & 43.000 & 3.47 & 4.09 \\
\hline 2008 & 6 & 11 & $21: 41: 4112.452$ & 40.553 & 2.03 & 0.54 \\
\hline 2008 & 6 & 12 & $00: 20: 5012.445$ & 40.570 & 2.04 & 0.79 \\
\hline 2008 & 6 & 12 & $08: 14: 1811.546$ & 41.060 & 2.62 & 0.62 \\
\hline 2008 & 6 & 13 & $00: 21: 4412.307$ & 40.613 & 1.91 & 0.77 \\
\hline 2008 & 6 & 13 & $20: 39: 4712.520$ & 40.539 & 2.05 & 1.34 \\
\hline 2008 & 6 & 14 & $04: 47: 5913.555$ & 39.941 & 2.83 & 3.15 \\
\hline
\end{tabular}




\begin{tabular}{|c|c|c|c|c|c|c|}
\hline 2008 & 6 & 14 & $21: 58: 1310.351$ & 40.965 & 2.96 & 2.26 \\
\hline 2008 & 6 & 14 & $23: 46: 1712.461$ & 40.547 & 1.95 & 0.95 \\
\hline 2008 & 6 & 15 & $21: 54: 4010.521$ & 40.017 & 3.06 & 3.33 \\
\hline 2008 & 6 & 18 & $00: 34: 3812.450$ & 40.559 & 2.01 & 0.41 \\
\hline 2008 & 6 & 18 & $02: 26: 1312.466$ & 40.538 & 2.04 & 1.11 \\
\hline 2008 & 6 & 18 & $20: 54: 5512.394$ & 40.570 & 2.04 & 0.48 \\
\hline 2008 & 6 & 18 & $22: 29: 2112.352$ & 40.599 & 1.99 & 0.51 \\
\hline 2008 & 6 & 19 & $23: 08: 2611.866$ & 40.986 & 1.98 & 0.9 \\
\hline 2008 & 6 & 19 & $23: 46: 1911.863$ & 41.002 & 1.87 & 1.59 \\
\hline 2008 & 6 & 20 & $19: 35: 4213.620$ & 39.916 & 2.71 & 4,00 \\
\hline 2008 & 6 & 21 & $01: 16: 0112.885$ & 40.278 & 2.44 & 0.32 \\
\hline 2008 & 6 & 21 & $23: 15: 4912.435$ & 40.562 & 2.01 & 0.43 \\
\hline 2008 & 6 & 22 & $00: 26: 2012.391$ & 40.584 & 1.92 & 0.52 \\
\hline 2008 & 6 & 22 & $00: 27: 3613.629$ & 39.873 & 2.68 & 11.18 \\
\hline 2008 & 6 & 22 & $01: 20: 2012.409$ & 40.580 & 1.94 & 0.54 \\
\hline 2008 & 6 & 22 & $22: 38: 1112.406$ & 40.581 & 1.94 & 0.47 \\
\hline 2008 & 6 & 22 & $22: 42: 5712.397$ & 40.579 & 1.86 & 0.55 \\
\hline 2008 & 6 & 23 & $07: 49: 1712.447$ & 40.557 & 2.18 & 0.46 \\
\hline 2008 & 6 & 23 & $16: 38: 3212.590$ & 40.515 & 2.27 & 0.48 \\
\hline 2008 & 6 & 24 & $01: 15: 3612.377$ & 40.589 & 1.97 & 0.46 \\
\hline 2008 & 6 & 24 & $06: 40: 3912.402$ & 40.550 & 2.13 & 0.64 \\
\hline 2008 & 6 & 24 & $21: 44: 1712.387$ & 40.611 & 2.00 & 0.4 \\
\hline 2008 & 6 & 25 & $01: 06: 0612.328$ & 40.602 & 2.01 & 0.5 \\
\hline 2008 & 6 & 26 & $00: 29: 0812.396$ & 40.595 & 1.88 & 0.56 \\
\hline 2008 & 6 & 26 & $18: 27: 1913.505$ & 39.988 & 2.72 & 1.76 \\
\hline 2008 & 6 & 26 & $21: 49: 4812.933$ & 39.795 & 2.57 & 1.58 \\
\hline 2008 & 6 & 27 & $18: 50: 4211.362$ & 39.802 & 2.54 & 1.34 \\
\hline 2008 & 6 & 27 & $20: 02: 4512.504$ & 40.542 & 1.97 & 0.56 \\
\hline 2008 & 6 & 27 & $20: 45: 4712.473$ & 40.549 & 1.91 & 0.67 \\
\hline 2008 & 6 & 28 & $00: 32: 5912.493$ & 40.539 & 1.92 & 1.13 \\
\hline 2008 & 6 & 28 & $19: 13: 4812.456$ & 40.557 & 2.13 & 0.84 \\
\hline 2008 & 6 & 28 & $20: 48: 0312.903$ & 39.543 & 2.55 & 0.75 \\
\hline 2008 & 6 & 29 & $09: 56: 3112.925$ & 39.537 & 2.65 & 0.91 \\
\hline 2008 & 6 & 29 & $13: 07: 4413.261$ & 40.030 & 2.82 & 2.09 \\
\hline 2008 & 6 & 29 & $21: 39: 0412.472$ & 40.549 & 2.00 & 0.48 \\
\hline 2008 & 6 & 29 & $23: 44: 0012.394$ & 40.600 & 1.89 & 0.61 \\
\hline 2008 & 6 & 30 & $00: 25: 4712.319$ & 40.600 & 1.89 & 0.49 \\
\hline 2008 & 6 & 30 & $19: 19: 0812.384$ & 40.592 & 2.09 & 0.53 \\
\hline 2008 & 6 & 30 & $21: 49: 3811.836$ & 41.240 & 2.10 & 0.63 \\
\hline 2008 & 6 & 30 & $22: 50: 0812.385$ & 40.586 & 1.95 & 0.36 \\
\hline 2008 & 6 & 30 & $23: 56: 3612.397$ & 40.583 & 1.97 & 0.47 \\
\hline 2008 & 7 & 1 & $02: 39: 1712.414$ & 40.576 & 2.11 & 0.76 \\
\hline 2008 & 7 & 1 & $11: 24: 3811.961$ & 40.870 & 2.85 & 0.29 \\
\hline 2008 & 7 & 1 & $21: 36: 3612.490$ & 40.542 & 2.11 & 0.39 \\
\hline 2008 & 7 & 1 & $22: 13: 3612.019$ & 40.966 & 1.77 & 1.06 \\
\hline 2008 & 7 & 1 & $23: 11: 2712.468$ & 40.556 & 2.08 & 0.54 \\
\hline 2008 & 7 & 3 & $00: 18: 3912.382$ & 40.592 & 1.95 & 0.67 \\
\hline 2008 & 7 & 3 & $06: 28: 1012.852$ & 40.628 & 2.86 & 0.55 \\
\hline 2008 & 7 & 3 & $10: 05: 0312.515$ & 39.553 & 2.79 & 0.65 \\
\hline 2008 & 7 & 3 & $16: 59: 1612.375$ & 40.594 & 2.37 & 0.41 \\
\hline 2008 & 7 & 3 & $19: 02: 0612.333$ & 40.604 & 1.99 & 0.62 \\
\hline 2008 & 7 & 3 & $19: 37: 3512.452$ & 40.574 & 1.97 & 0.54 \\
\hline 2008 & 7 & 3 & $22: 48: 1712.424$ & 40.570 & 1.83 & 0.35 \\
\hline 2008 & 7 & 4 & $00: 44: 0312.504$ & 40.538 & 1.90 & 0.49 \\
\hline 2008 & 7 & 4 & $22: 14: 0612.616$ & 39.659 & 2.37 & 0.95 \\
\hline 2008 & 7 & 5 & $00: 42: 3913.118$ & 41.204 & 2.45 & 1.12 \\
\hline 2008 & 7 & 5 & $00: 44: 2711.839$ & 40.951 & 1.92 & 0.66 \\
\hline 2008 & 7 & 6 & $01: 17: 5012.399$ & 40.610 & 1.92 & 3.42 \\
\hline 2008 & 7 & 6 & $09: 31: 3312.520$ & 40.534 & 2.13 & 0.67 \\
\hline 2008 & 7 & 7 & $00: 35: 3312.458$ & 40.546 & 1.96 & 0.87 \\
\hline 2008 & 7 & 7 & $01: 17: 3112.426$ & 40.573 & 1.93 & 0.49 \\
\hline 2008 & 7 & 7 & $02: 28: 3612.395$ & 40.585 & 1.91 & 0.64 \\
\hline 2008 & 7 & 7 & $09: 16: 4812.399$ & 40.588 & 2.27 & 0.67 \\
\hline 2008 & 7 & 8 & $05: 56: 3112.451$ & 40.555 & 2.29 & 0.6 \\
\hline 2008 & 7 & 8 & $19: 55: 0012.417$ & 40.578 & 2.62 & 0.52 \\
\hline 2008 & 7 & 9 & $06: 06: 1012.432$ & 40.566 & 2.20 & 0.68 \\
\hline 2008 & 7 & 9 & $08: 24: 2812.298$ & 40.622 & 2.04 & 1.54 \\
\hline 2008 & 7 & 9 & $08: 41: 4112.267$ & 40.652 & 1.87 & 0.69 \\
\hline 2008 & 7 & 9 & $08: 53: 1912.288$ & 40.636 & 1.93 & 0.65 \\
\hline 2008 & 7 & 9 & $09: 27: 5112.281$ & 40.624 & 1.93 & 0.58 \\
\hline 2008 & 7 & 9 & $09: 30: 0912.294$ & 40.622 & 1.91 & 0.53 \\
\hline 2008 & 7 & 9 & $10: 04: 4512.316$ & 40.611 & 1.96 & 0.53 \\
\hline 2008 & 7 & 9 & $10: 14: 0612.308$ & 40.622 & 2.10 & 0.36 \\
\hline 2008 & 7 & 9 & $10: 15: 3312.292$ & 40.610 & 2.00 & 0.56 \\
\hline 2008 & 7 & 9 & $10: 42: 1312.272$ & 40.642 & 2.02 & 0.83 \\
\hline 2008 & 7 & 9 & $10: 48: 4812.282$ & 40.634 & 1.91 & 0.71 \\
\hline
\end{tabular}




\begin{tabular}{|c|c|c|c|c|c|c|}
\hline 2008 & 7 & 9 & $11: 44: 2912.269$ & 40.639 & 1.99 & 0.65 \\
\hline 2008 & 7 & 9 & $12: 07: 2712.264$ & 40.647 & 2.05 & 0.7 \\
\hline 2008 & 7 & 9 & $14: 03: 0212.274$ & 40.650 & 2.02 & 0.93 \\
\hline 2008 & 7 & 9 & $14: 07: 5512.254$ & 40.646 & 2.01 & 0.68 \\
\hline 2008 & 7 & 9 & $14: 10: 5312.252$ & 40.651 & 2.13 & 0.62 \\
\hline 2008 & 7 & 9 & $14: 25: 4112.255$ & 40.649 & 2.07 & 0.71 \\
\hline 2008 & 7 & 9 & $14: 26: 2212.258$ & 40.650 & 2.00 & 0.65 \\
\hline 2008 & 7 & 9 & $15: 01: 1812.251$ & 40.653 & 1.99 & 0.75 \\
\hline 2008 & 7 & 9 & $15: 13: 0612.230$ & 40.682 & 2.25 & 0.49 \\
\hline 2008 & 7 & 9 & $16: 02: 1812.213$ & 40.669 & 2.02 & 0.64 \\
\hline 2008 & 7 & 9 & $16: 12: 4812.235$ & 40.680 & 2.00 & 0.45 \\
\hline 2008 & 7 & 9 & $16: 14: 4912.213$ & 40.660 & 1.98 & 0.65 \\
\hline 2008 & 7 & 9 & $16: 19: 1612.218$ & 40.672 & 1.93 & 1.49 \\
\hline 2008 & 7 & 9 & $16: 31: 2612.207$ & 40.671 & 2.33 & 0.65 \\
\hline 2008 & 7 & 9 & $16: 44: 3912.458$ & 40.561 & 2.50 & 0.82 \\
\hline 2008 & 7 & 9 & $16: 48: 5012.233$ & 40.684 & 1.95 & 0.61 \\
\hline 2008 & 7 & 9 & $16: 54: 2312.216$ & 40.662 & 1.99 & 0.95 \\
\hline 2008 & 7 & 9 & $16: 59: 4212.249$ & 40.679 & 2.34 & 0.57 \\
\hline 2008 & 7 & 9 & $17: 03: 5012.203$ & 40.673 & 2.09 & 0.51 \\
\hline 2008 & 7 & 9 & $17: 06: 5712.191$ & 40.642 & 1.97 & 0.83 \\
\hline 2008 & 7 & 9 & $17: 22: 0812.211$ & 40.670 & 2.00 & 0.82 \\
\hline 2008 & 7 & 9 & $17: 25: 1012.187$ & 40.684 & 1.94 & 1.51 \\
\hline 2008 & 7 & 9 & $17: 27: 3912.220$ & 40.667 & 2.01 & 0.73 \\
\hline 2008 & 7 & 9 & $17: 30: 5112.214$ & 40.674 & 2.44 & 0.42 \\
\hline 2008 & 7 & 9 & $17: 32: 5712.227$ & 40.674 & 2.00 & 0.74 \\
\hline 2008 & 7 & 9 & $17: 42: 3612.227$ & 40.667 & 2.17 & 0.45 \\
\hline 2008 & 7 & 9 & $17: 45: 5112.235$ & 40.675 & 2.08 & 0.69 \\
\hline 2008 & 7 & 9 & $17: 48: 0412.252$ & 40.674 & 2.24 & 0.58 \\
\hline 2008 & 7 & 9 & $17: 49: 3712.242$ & 40.667 & 2.13 & 0.81 \\
\hline 2008 & 7 & 9 & $17: 51: 1912.198$ & 40.668 & 2.31 & 0.48 \\
\hline 2008 & 7 & 9 & $17: 52: 0812.219$ & 40.672 & 2.23 & 0.64 \\
\hline 2008 & 7 & 9 & $17: 53: 5512.230$ & 40.662 & 2.22 & 0.57 \\
\hline 2008 & 7 & 9 & $17: 53: 5512.238$ & 40.666 & 2.22 & 0.52 \\
\hline 2008 & 7 & 9 & $17: 56: 3512.208$ & 40.674 & 2.06 & 0.57 \\
\hline 2008 & 7 & 9 & $17: 58: 2612.233$ & 40.662 & 2.02 & 0.7 \\
\hline 2008 & 7 & 9 & $17: 59: 3512.239$ & 40.665 & 2.00 & 0.88 \\
\hline 2008 & 7 & 9 & $18: 05: 0312.226$ & 40.667 & 2.11 & 0.47 \\
\hline 2008 & 7 & 9 & $18: 06: 0112.249$ & 40.655 & 2.00 & 0.51 \\
\hline 2008 & 7 & 9 & $18: 11: 4612.216$ & 40.679 & 2.41 & 0.49 \\
\hline 2008 & 7 & 9 & $18: 12: 5912.236$ & 40.683 & 2.30 & 0.58 \\
\hline 2008 & 7 & 9 & $18: 16: 1612.226$ & 40.674 & 2.66 & 0.45 \\
\hline 2008 & 7 & 9 & $18: 20: 2112.260$ & 40.655 & 2.04 & 0.61 \\
\hline 2008 & 7 & 9 & $18: 23: 0312.256$ & 40.702 & 2.14 & 0.58 \\
\hline 2008 & 7 & 9 & $18: 30: 2212.223$ & 40.683 & 2.08 & 0.54 \\
\hline 2008 & 7 & 9 & $18: 41: 5312.235$ & 40.673 & 2.28 & 0.43 \\
\hline 2008 & 7 & 9 & $18: 42: 25 \quad 12.239$ & 40.676 & 2.76 & 0.49 \\
\hline 2008 & 7 & 9 & $18: 44: 1912.226$ & 40.667 & 2.48 & 0.39 \\
\hline 2008 & 7 & 9 & $18: 51: 1112.207$ & 40.671 & 2.23 & 0.49 \\
\hline 2008 & 7 & 9 & $18: 56: 45 \quad 12.242$ & 40.688 & 1.98 & 0.74 \\
\hline 2008 & 7 & 9 & $18: 57: 2912.231$ & 40.675 & 1.95 & 0.85 \\
\hline 2008 & 7 & 9 & $19: 00: 4112.226$ & 40.668 & 2.61 & 0.37 \\
\hline 2008 & 7 & 9 & $19: 06: 4612.216$ & 40.674 & 2.74 & 0.5 \\
\hline 2008 & 7 & 9 & $19: 09: 0712.230$ & 40.668 & 2.24 & 0.52 \\
\hline 2008 & 7 & 9 & $19: 15: 5512.225$ & 40.676 & 2.98 & 0.39 \\
\hline 2008 & 7 & 9 & $19: 20: 5312.182$ & 40.677 & 2.37 & 0.53 \\
\hline 2008 & 7 & 9 & $19: 28: 1212.234$ & 40.663 & 2.03 & 1.47 \\
\hline 2008 & 7 & 9 & $19: 31: 2112.222$ & 40.664 & 1.98 & 0.48 \\
\hline 2008 & 7 & 9 & $19: 34: 2912.228$ & 40.676 & 2.32 & 0.39 \\
\hline 2008 & 7 & 9 & $19: 47: 5312.196$ & 40.678 & 2.64 & 0.48 \\
\hline 2008 & 7 & 9 & $20: 00: 0412.222$ & 40.672 & 3.18 & 0.31 \\
\hline 2008 & 7 & 9 & $20: 09: 4612.225$ & 40.671 & 2.28 & 0.37 \\
\hline 2008 & 7 & 9 & $20: 13: 1412.207$ & 40.674 & 2.80 & 0.42 \\
\hline 2008 & 7 & 9 & $20: 14: 1012.221$ & 40.664 & 2.62 & 0.47 \\
\hline 2008 & 7 & 9 & $20: 20: 1112.230$ & 40.667 & 2.84 & 0.41 \\
\hline 2008 & 7 & 9 & $20: 22: 1812.223$ & 40.671 & 3.14 & 0.36 \\
\hline 2008 & 7 & 9 & $20: 47: 3312.216$ & 40.677 & 2.90 & 0.43 \\
\hline 2008 & 7 & 9 & $20: 59: 3512.226$ & 40.673 & 2.25 & 0.52 \\
\hline 2008 & 7 & 9 & $21: 00: 1712.199$ & 40.681 & 2.27 & 0.43 \\
\hline 2008 & 7 & 9 & $21: 03: 1812.231$ & 40.676 & 2.04 & 0.5 \\
\hline 2008 & 7 & 9 & $21: 18: 1712.199$ & 40.680 & 2.14 & 0.56 \\
\hline 2008 & 7 & 9 & $21: 19: 0912.186$ & 40.697 & 2.41 & 0.6 \\
\hline 2008 & 7 & 9 & $21: 26: 1912.264$ & 40.641 & 1.77 & 0.58 \\
\hline 2008 & 7 & 9 & $21: 27: 2212.185$ & 40.695 & 1.92 & 0.78 \\
\hline 2008 & 7 & 9 & $21: 28: 5012.209$ & 40.682 & 1.91 & 0.51 \\
\hline 2008 & 7 & 9 & $21: 32: 4112.234$ & 40.669 & 2.87 & 0.46 \\
\hline 2008 & 7 & 9 & $21: 35: 4812.218$ & 40.676 & 2.01 & 0.57 \\
\hline
\end{tabular}




\begin{tabular}{|c|c|c|c|c|c|c|}
\hline 2008 & 7 & 9 & $21: 36: 5112.227$ & 40.676 & 2.06 & 0.44 \\
\hline 2008 & 7 & 9 & $21: 48: 5312.176$ & 40.699 & 2.14 & 0.49 \\
\hline 2008 & 7 & 9 & $21: 53: 5912.178$ & 40.691 & 2.00 & 0.38 \\
\hline 2008 & 7 & 9 & $22: 04: 5912.192$ & 40.685 & 2.04 & 0.47 \\
\hline 2008 & 7 & 9 & $22: 08: 5012.200$ & 40.686 & 1.89 & 0.61 \\
\hline 2008 & 7 & 9 & $22: 22: 0312.204$ & 40.677 & 2.21 & 0.49 \\
\hline 2008 & 7 & 9 & $22: 25: 3612.219$ & 40.681 & 2.09 & 0.42 \\
\hline 2008 & 7 & 9 & $22: 39: 3212.231$ & 40.679 & 2.19 & 0.41 \\
\hline 2008 & 7 & 9 & $22: 39: 5112.188$ & 40.686 & 2.70 & 0.45 \\
\hline 2008 & 7 & 9 & $22: 45: 2312.185$ & 40.688 & 2.05 & 0.36 \\
\hline 2008 & 7 & 9 & $22: 46: 5312.194$ & 40.682 & 2.12 & 0.4 \\
\hline 2008 & 7 & 9 & $22: 47: 2212.198$ & 40.688 & 2.40 & 0.45 \\
\hline 2008 & 7 & 9 & $22: 49: 5812.204$ & 40.688 & 2.04 & 0.48 \\
\hline 2008 & 7 & 9 & $22: 51: 0912.196$ & 40.691 & 2.43 & 0.4 \\
\hline 2008 & 7 & 9 & $23: 07: 3512.198$ & 40.683 & 2.98 & 0.53 \\
\hline 2008 & 7 & 9 & $23: 11: 2312.166$ & 40.697 & 2.07 & 0.38 \\
\hline 2008 & 7 & 9 & $23: 12: 5912.181$ & 40.686 & 2.04 & 0.56 \\
\hline 2008 & 7 & 9 & $23: 14: 5212.199$ & 40.696 & 2.17 & 0.49 \\
\hline 2008 & 7 & 9 & $23: 16: 5512.196$ & 40.686 & 2.25 & 0.4 \\
\hline 2008 & 7 & 9 & $23: 23: 3712.197$ & 40.688 & 1.95 & 0.36 \\
\hline 2008 & 7 & 9 & $23: 25: 3612.220$ & 40.672 & 2.25 & 0.42 \\
\hline 2008 & 7 & 9 & $23: 29: 2112.197$ & 40.690 & 2.32 & 0.53 \\
\hline 2008 & 7 & 9 & $23: 33: 5312.169$ & 40.693 & 2.12 & 0.54 \\
\hline 2008 & 7 & 9 & $23: 37: 0412.200$ & 40.684 & 1.86 & 0.44 \\
\hline 2008 & 7 & 9 & $23: 40: 1612.217$ & 40.674 & 2.82 & 0.35 \\
\hline 2008 & 7 & 9 & $23: 45: 3112.192$ & 40.691 & 1.83 & 0.48 \\
\hline 2008 & 7 & 9 & $23: 46: 1712.192$ & 40.686 & 1.81 & 0.45 \\
\hline 2008 & 7 & 9 & $23: 50: 5212.174$ & 40.681 & 1.94 & 0.66 \\
\hline 2008 & 7 & 9 & $23: 58: 0412.174$ & 40.693 & 2.05 & 0.43 \\
\hline 2008 & 7 & 9 & $23: 59: 4712.184$ & 40.690 & 2.11 & 0.54 \\
\hline 2008 & 7 & 10 & $00: 05: 0112.206$ & 40.683 & 2.55 & 0.39 \\
\hline 2008 & 7 & 10 & $00: 07: 2512.207$ & 40.665 & 2.06 & 0.68 \\
\hline 2008 & 7 & 10 & $00: 11: 1612.232$ & 40.679 & 1.93 & 0.45 \\
\hline 2008 & 7 & 10 & $00: 12: 0812.170$ & 40.697 & 2.39 & 0.4 \\
\hline 2008 & 7 & 10 & $00: 14: 4612.180$ & 40.696 & 2.23 & 0.42 \\
\hline 2008 & 7 & 10 & $00: 19: 0312.219$ & 40.674 & 1.80 & 0.61 \\
\hline 2008 & 7 & 10 & $00: 20: 2312.161$ & 40.695 & 2.00 & 0.64 \\
\hline 2008 & 7 & 10 & $00: 22: 5712.174$ & 40.700 & 2.15 & 0.44 \\
\hline 2008 & 7 & 10 & $00: 23: 5612.176$ & 40.697 & 2.35 & 0.31 \\
\hline 2008 & 7 & 10 & $00: 37: 0112.197$ & 40.688 & 2.18 & 0.39 \\
\hline 2008 & 7 & 10 & $00: 37: 2812.192$ & 40.691 & 2.38 & 0.54 \\
\hline 2008 & 7 & 10 & $00: 41: 2412.165$ & 40.695 & 2.10 & 0.41 \\
\hline 2008 & 7 & 10 & $00: 43: 5812.167$ & 40.696 & 2.39 & 0.4 \\
\hline 2008 & 7 & 10 & $00: 44: 5912.183$ & 40.693 & 2.20 & 0.52 \\
\hline 2008 & 7 & 10 & $00: 47: 0512.197$ & 40.686 & 2.57 & 0.45 \\
\hline 2008 & 7 & 10 & $00: 53: 4112.171$ & 40.683 & 1.72 & 0.69 \\
\hline 2008 & 7 & 10 & $01: 04: 0412.241$ & 40.676 & 2.44 & 0.43 \\
\hline 2008 & 7 & 10 & $01: 06: 1212.224$ & 40.677 & 2.06 & 0.43 \\
\hline 2008 & 7 & 10 & $01: 12: 4512.181$ & 40.685 & 2.08 & 0.49 \\
\hline 2008 & 7 & 10 & $01: 16: 1112.174$ & 40.697 & 2.21 & 0.37 \\
\hline 2008 & 7 & 10 & $01: 21: 0412.177$ & 40.701 & 2.25 & 0.4 \\
\hline 2008 & 7 & 10 & $01: 22: 2412.182$ & 40.701 & 2.26 & 0.38 \\
\hline 2008 & 7 & 10 & $01: 29: 4212.184$ & 40.688 & 2.41 & 0.46 \\
\hline 2008 & 7 & 10 & $01: 35: 3612.178$ & 40.704 & 2.71 & 0.36 \\
\hline 2008 & 7 & 10 & $01: 40: 2012.169$ & 40.700 & 2.75 & 0.36 \\
\hline 2008 & 7 & 10 & $01: 42: 2712.161$ & 40.699 & 2.09 & 0.64 \\
\hline 2008 & 7 & 10 & $01: 52: 3012.200$ & 40.694 & 2.61 & 0.37 \\
\hline 2008 & 7 & 10 & $02: 03: 3212.196$ & 40.691 & 2.16 & 0.62 \\
\hline 2008 & 7 & 10 & $02: 05: 1012.177$ & 40.687 & 2.13 & 0.4 \\
\hline 2008 & 7 & 10 & $02: 06: 4312.173$ & 40.696 & 2.10 & 0.4 \\
\hline 2008 & 7 & 10 & $02: 14: 1212.190$ & 40.691 & 2.19 & 0.42 \\
\hline 2008 & 7 & 10 & $02: 17: 1512.231$ & 40.679 & 2.23 & 0.42 \\
\hline 2008 & 7 & 10 & $02: 18: 3712.189$ & 40.688 & 2.42 & 0.56 \\
\hline 2008 & 7 & 10 & $02: 21: 5612.175$ & 40.706 & 2.63 & 0.46 \\
\hline 2008 & 7 & 10 & $02: 31: 5012.176$ & 40.698 & 2.22 & 0.52 \\
\hline 2008 & 7 & 10 & $02: 33: 1612.181$ & 40.699 & 2.13 & 0.6 \\
\hline 2008 & 7 & 10 & $02: 35: 1412.167$ & 40.693 & 1.87 & 1,00 \\
\hline 2008 & 7 & 10 & $02: 49: 2412.187$ & 40.687 & 2.48 & 0.4 \\
\hline 2008 & 7 & 10 & $03: 02: 2412.155$ & 40.701 & 2.41 & 0.45 \\
\hline 2008 & 7 & 10 & $03: 04: 5112.176$ & 40.706 & 2.25 & 0.63 \\
\hline 2008 & 7 & 10 & $03: 14: 4412.193$ & 40.698 & 2.77 & 0.37 \\
\hline 2008 & 7 & 10 & $03: 20: 5512.175$ & 40.705 & 3.19 & 0.4 \\
\hline 2008 & 7 & 10 & $03: 45: 3412.167$ & 40.698 & 2.16 & 0.76 \\
\hline 2008 & 7 & 10 & $04: 11: 3612.175$ & 40.698 & 2.49 & 0.75 \\
\hline 2008 & 7 & 10 & $04: 28: 3812.172$ & 40.695 & 2.42 & 0.75 \\
\hline 2008 & 7 & 10 & $04: 42: 3312.177$ & 40.692 & 2.70 & 0.5 \\
\hline
\end{tabular}




\begin{tabular}{|c|c|c|c|c|c|c|}
\hline 2008 & 7 & 10 & $05: 01: 3312.167$ & 40.697 & 2.23 & 0.86 \\
\hline 2008 & 7 & 10 & $05: 06: 5412.181$ & 40.708 & 2.62 & 0.61 \\
\hline 2008 & 7 & 10 & $05: 40: 3812.181$ & 40.704 & 2.85 & 0.43 \\
\hline 2008 & 7 & 10 & $05: 43: 2412.175$ & 40.697 & 2.64 & 0.52 \\
\hline 2008 & 7 & 10 & $06: 18: 5012.184$ & 40.692 & 2.49 & 0.48 \\
\hline 2008 & 7 & 10 & $06: 37: 2712.180$ & 40.699 & 2.79 & 0.38 \\
\hline 2008 & 7 & 10 & $07: 04: 2512.184$ & 40.693 & 2.35 & 0.56 \\
\hline 2008 & 7 & 10 & $07: 21: 2212.155$ & 40.698 & 2.61 & 0.47 \\
\hline 2008 & 7 & 10 & $07: 49: 4812.172$ & 40.703 & 2.34 & 0.66 \\
\hline 2008 & 7 & 10 & $08: 34: 4712.176$ & 40.698 & 2.53 & 0.5 \\
\hline 2008 & 7 & 10 & $08: 37: 1812.181$ & 40.702 & 3.32 & 0.42 \\
\hline 2008 & 7 & 10 & $08: 57: 2512.164$ & 40.696 & 2.45 & 0.4 \\
\hline 2008 & 7 & 10 & $09: 12: 0612.175$ & 40.699 & 2.73 & 0.5 \\
\hline 2008 & 7 & 10 & $09: 24: 1412.163$ & 40.696 & 2.58 & 0.54 \\
\hline 2008 & 7 & 10 & $10: 02: 5212.160$ & 40.706 & 2.93 & 0.45 \\
\hline 2008 & 7 & 10 & $10: 09: 4712.153$ & 40.701 & 2.74 & 0.49 \\
\hline 2008 & 7 & 10 & $10: 32: 4812.162$ & 40.707 & 3.11 & 0.44 \\
\hline 2008 & 7 & 10 & $10: 52: 0212.160$ & 40.709 & 2.69 & 0.55 \\
\hline 2008 & 7 & 10 & $10: 59: 5312.174$ & 40.699 & 3.03 & 0.43 \\
\hline 2008 & 7 & 10 & $11: 10: 4312.160$ & 40.709 & 3.50 & 0.43 \\
\hline 2008 & 7 & 10 & $11: 28: 3612.161$ & 40.705 & 2.71 & 0.37 \\
\hline 2008 & 7 & 10 & $11: 31: 0712.165$ & 40.705 & 3.48 & 0.34 \\
\hline 2008 & 7 & 10 & $12: 13: 4312.149$ & 40.710 & 2.83 & 0.54 \\
\hline 2008 & 7 & 10 & $12: 58: 0212.212$ & 40.681 & 2.88 & 0.69 \\
\hline 2008 & 7 & 10 & $13: 17: 2912.146$ & 40.708 & 2.52 & 0.51 \\
\hline 2008 & 7 & 10 & $14: 21: 4812.126$ & 40.714 & 2.20 & 0.65 \\
\hline 2008 & 7 & 10 & $14: 38: 4712.143$ & 40.705 & 2.39 & 1.37 \\
\hline 2008 & 7 & 10 & $14: 45: 1012.213$ & 40.677 & 2.29 & 0.52 \\
\hline 2008 & 7 & 10 & $16: 23: 5712.178$ & 40.699 & 2.24 & 0.45 \\
\hline 2008 & 7 & 10 & $16: 24: 2912.176$ & 40.695 & 2.31 & 0.53 \\
\hline 2008 & 7 & 10 & $16: 43: 5712.175$ & 40.714 & 2.79 & 0.42 \\
\hline 2008 & 7 & 10 & $17: 21: 3312.174$ & 40.700 & 2.06 & 0.49 \\
\hline 2008 & 7 & 10 & $17: 32: 0712.174$ & 40.700 & 2.80 & 0.41 \\
\hline 2008 & 7 & 10 & $18: 04: 0712.174$ & 40.694 & 2.15 & 0.52 \\
\hline 2008 & 7 & 10 & $18: 10: 4112.155$ & 40.708 & 2.25 & 0.33 \\
\hline 2008 & 7 & 10 & $20: 03: 3412.500$ & 40.517 & 1.86 & 0.79 \\
\hline 2008 & 7 & 10 & $20: 26: 0512.180$ & 40.716 & 1.99 & 0.56 \\
\hline 2008 & 7 & 10 & $20: 30: 3912.220$ & 40.676 & 1.99 & 0.62 \\
\hline 2008 & 7 & 10 & $20: 57: 0812.185$ & 40.698 & 1.95 & 0.61 \\
\hline 2008 & 7 & 10 & $21: 38: 2912.163$ & 40.703 & 1.74 & 0.67 \\
\hline 2008 & 7 & 10 & $22: 00: 0412.169$ & 40.695 & 1.83 & 0.43 \\
\hline 2008 & 7 & 10 & $23: 18: 1512.223$ & 40.668 & 1.98 & 0.43 \\
\hline 2008 & 7 & 10 & $23: 44: 1812.211$ & 40.671 & 1.79 & 0.52 \\
\hline 2008 & 7 & 11 & $01: 51: 3612.199$ & 40.688 & 2.06 & 0.51 \\
\hline 2008 & 7 & 11 & $03: 32: 3412.238$ & 40.682 & 2.16 & 0.58 \\
\hline 2008 & 7 & 11 & $04: 07: 5012.134$ & 40.704 & 2.24 & 0.62 \\
\hline 2008 & 7 & 11 & $05: 18: 4212.174$ & 40.710 & 2.82 & 0.45 \\
\hline 2008 & 7 & 11 & $05: 57: 2112.428$ & 40.562 & 2.21 & 0.8 \\
\hline 2008 & 7 & 12 & $00: 20: 3313.510$ & 39.995 & 2.45 & 1.46 \\
\hline 2008 & 7 & 12 & $01: 24: 4611.892$ & 41.370 & 2.06 & 0.68 \\
\hline 2008 & 7 & 12 & $01: 44: 3612.154$ & 40.704 & 1.91 & 0.62 \\
\hline 2008 & 7 & 13 & $00: 59: 1812.464$ & 40.559 & 2.01 & 0.66 \\
\hline 2008 & 7 & 13 & $01: 06: 5512.186$ & 40.695 & 1.82 & 0.85 \\
\hline 2008 & 7 & 13 & $01: 35: 2312.189$ & 40.693 & 1.78 & 0.77 \\
\hline 2008 & 7 & 13 & $09: 36: 3012.138$ & 40.701 & 1.88 & 1.19 \\
\hline 2008 & 7 & 13 & $12: 34: 1612.400$ & 40.601 & 1.89 & 0.77 \\
\hline 2008 & 7 & 13 & $14: 08: 1412.126$ & 40.702 & 2.22 & 0.61 \\
\hline 2008 & 7 & 13 & $19: 14: 4312.884$ & 39.575 & 2.53 & 0.77 \\
\hline 2008 & 7 & 13 & $22: 02: 1412.152$ & 40.714 & 2.02 & 0.47 \\
\hline 2008 & 7 & 13 & $22: 06: 4512.511$ & 40.547 & 1.86 & 0.73 \\
\hline 2008 & 7 & 13 & $22: 10: 3812.157$ & 40.716 & 2.30 & 0.53 \\
\hline 2008 & 7 & 13 & $23: 22: 5912.168$ & 40.709 & 1.72 & 0.6 \\
\hline 2008 & 7 & 14 & $03: 41: 4512.460$ & 40.548 & 1.98 & 0.57 \\
\hline 2008 & 7 & 14 & $12: 05: 4213.446$ & 39.976 & 2.65 & 15.9 \\
\hline 2008 & 7 & 14 & $22: 22: 5511.977$ & 39.657 & 2.41 & 0.67 \\
\hline 2008 & 7 & 15 & $00: 46: 4212.246$ & 40.665 & 1.84 & 0.46 \\
\hline 2008 & 7 & 15 & $21: 06: 5113.134$ & 39.676 & 2.51 & 1.39 \\
\hline 2008 & 7 & 15 & $21: 55: 2812.399$ & 40.585 & 1.99 & 0.51 \\
\hline 2008 & 7 & 16 & $02: 15: 5712.155$ & 40.711 & 1.94 & 0.65 \\
\hline 2008 & 7 & 18 & $15: 12: 5512.458$ & 40.557 & 2.07 & 0.56 \\
\hline 2008 & 7 & 19 & $00: 13: 1812.511$ & 40.536 & 2.04 & 0.36 \\
\hline 2008 & 7 & 19 & $00: 29: 5912.369$ & 40.600 & 1.88 & 0.48 \\
\hline 2008 & 7 & 19 & $17: 57: 4112.561$ & 39.550 & 2.44 & 0.82 \\
\hline 2008 & 7 & 20 & $23: 33: 1312.445$ & 40.554 & 1.83 & 0.82 \\
\hline 2008 & 7 & 21 & $15: 43: 1912.501$ & 40.548 & 2.02 & 0.59 \\
\hline 2008 & 7 & 21 & $18: 27: 0310.911$ & 41.252 & 2.53 & 5.73 \\
\hline
\end{tabular}




\begin{tabular}{|c|c|c|c|c|c|c|}
\hline 2008 & 7 & 21 & $20: 17: 3912.399$ & 40.582 & 1.88 & 0.58 \\
\hline 2008 & 7 & 21 & $20: 54: 3313.437$ & 40.020 & 2.47 & 2.18 \\
\hline 2008 & 7 & 21 & $22: 55: 4812.400$ & 40.572 & 1.81 & 0.78 \\
\hline 2008 & 7 & 22 & $03: 20: 1312.461$ & 40.562 & 1.92 & 0.89 \\
\hline 2008 & 7 & 22 & $20: 54: 3212.253$ & 41.671 & 2.29 & 0.7 \\
\hline 2008 & 7 & 22 & $22: 59: 1412.235$ & 41.651 & 2.26 & 1.43 \\
\hline 2008 & 7 & 22 & $23: 44: 2412.476$ & 40.548 & 1.78 & 0.61 \\
\hline 2008 & 7 & 23 & $00: 09: 3712.259$ & 41.672 & 2.26 & 0.64 \\
\hline 2008 & 7 & 23 & $01: 40: 4812.214$ & 41.583 & 2.26 & 3,00 \\
\hline 2008 & 7 & 23 & $17: 00: 4512.381$ & 40.604 & 2.07 & 1.01 \\
\hline 2008 & 7 & 26 & $23: 41: 4413.429$ & 40.018 & 2.50 & 4.03 \\
\hline 2008 & 7 & 27 & $20: 34: 0512.948$ & 40.197 & 2.43 & 1.08 \\
\hline 2008 & 7 & 27 & $21: 12: 3212.401$ & 40.584 & 1.85 & 0.49 \\
\hline 2008 & 7 & 27 & $23: 19: 5411.357$ & 39.902 & 2.31 & 0.37 \\
\hline 2008 & 7 & 28 & $06: 17: 4412.417$ & 40.573 & 2.15 & 0.62 \\
\hline 2008 & 7 & 28 & $08: 37: 5712.394$ & 40.583 & 2.22 & 0.4 \\
\hline 2008 & 7 & 28 & $15: 36: 2713.490$ & 40.008 & 2.72 & 4.51 \\
\hline 2008 & 7 & 29 & $02: 24: 1912.386$ & 40.627 & 1.88 & 0.98 \\
\hline 2008 & 7 & 29 & $15: 24: 409.773$ & 39.997 & 3.60 & 7.68 \\
\hline 2008 & 7 & 29 & $21: 54: 5512.306$ & 40.605 & 1.90 & 0.57 \\
\hline 2008 & 7 & 30 & $22: 04: 2912.541$ & 40.526 & 1.89 & 0.65 \\
\hline 2008 & 7 & 30 & $23: 37: 5812.388$ & 40.583 & 1.83 & 0.74 \\
\hline 2008 & 7 & 31 & $00: 13: 0910.867$ & 41.217 & 2.61 & 1.45 \\
\hline 2008 & 7 & 31 & $04: 24: 2812.202$ & 41.562 & 2.37 & 2.1 \\
\hline 2008 & 7 & 31 & $11: 10: 5512.002$ & 40.931 & 1.95 & 2.06 \\
\hline 2008 & 7 & 31 & $22: 54: 5212.421$ & 40.569 & 2.00 & 0.52 \\
\hline 2008 & 8 & 1 & $00: 31: 2612.492$ & 40.533 & 1.84 & 0.45 \\
\hline 2008 & 8 & 1 & $00: 41: 1512.371$ & 40.595 & 1.83 & 0.82 \\
\hline 2008 & 8 & 1 & $12: 55: 2813.898$ & 40.569 & 3.95 & 1.23 \\
\hline 2008 & 8 & 1 & $13: 10: 4613.797$ & 40.551 & 3.41 & 2.75 \\
\hline 2008 & 8 & 1 & $16: 35: 0211.873$ & 41.079 & 2.65 & 0.52 \\
\hline 2008 & 8 & 2 & $10: 02: 0813.414$ & 40.007 & 2.64 & 1.94 \\
\hline 2008 & 8 & 2 & $10: 49: 2812.463$ & 40.558 & 2.09 & 0.73 \\
\hline 2008 & 8 & 2 & $20: 04: 2113.493$ & 39.973 & 2.55 & 2.64 \\
\hline 2008 & 8 & 2 & $20: 39: 5911.630$ & 39.681 & 2.36 & 0.85 \\
\hline 2008 & 8 & 2 & $21: 30: 3213.449$ & 39.978 & 2.46 & 1.53 \\
\hline 2008 & 8 & 2 & $23: 44: 2612.371$ & 40.590 & 1.79 & 0.5 \\
\hline 2008 & 8 & 3 & $23: 35: 3712.366$ & 40.599 & 1.83 & 0.47 \\
\hline 2008 & 8 & 3 & $23: 41: 5813.453$ & 39.966 & 2.40 & 1.69 \\
\hline 2008 & 8 & 4 & $00: 17: 3412.420$ & 40.574 & 1.76 & 0.35 \\
\hline 2008 & 8 & 4 & $01: 45: 4112.808$ & 39.704 & 2.28 & 0.9 \\
\hline 2008 & 8 & 4 & $02: 41: 2012.371$ & 40.597 & 1.88 & 0.36 \\
\hline 2008 & 8 & 4 & $02: 46: 2212.598$ & 40.501 & 1.88 & 0.5 \\
\hline 2008 & 8 & 4 & $03: 13: 5312.419$ & 40.569 & 1.94 & 0.44 \\
\hline 2008 & 8 & 5 & $02: 18: 1212.942$ & 40.183 & 2.19 & 0.58 \\
\hline 2008 & 8 & 5 & $17: 42: 2912.172$ & 40.701 & 2.74 & 0.46 \\
\hline 2008 & 8 & 6 & $00: 04: 5012.309$ & 40.610 & 1.83 & 0.42 \\
\hline 2008 & 8 & 6 & $20: 20: 4412.355$ & 40.602 & 1.76 & 0.51 \\
\hline 2008 & 8 & 6 & $21: 37: 1412.385$ & 40.221 & 1.73 & 1.36 \\
\hline 2008 & 8 & 6 & $23: 41: 0112.466$ & 40.555 & 1.91 & 0.45 \\
\hline 2008 & 8 & 7 & $01: 15: 1412.347$ & 40.602 & 1.72 & 0.48 \\
\hline 2008 & 8 & 7 & $06: 26: 2512.493$ & 40.539 & 2.17 & 0.38 \\
\hline 2008 & 8 & 8 & $00: 21: 4012.461$ & 40.556 & 1.76 & 0.45 \\
\hline 2008 & 8 & 8 & $19: 38: 5612.313$ & 40.611 & 2.04 & 0.4 \\
\hline 2008 & 8 & 9 & $00: 56: 4612.418$ & 40.560 & 1.71 & 0.61 \\
\hline 2008 & 8 & 9 & $01: 14: 2212.406$ & 40.576 & 1.77 & 0.42 \\
\hline 2008 & 8 & 9 & $04: 58: 5312.426$ & 40.565 & 1.93 & 0.56 \\
\hline 2008 & 8 & 9 & $20: 48: 5812.454$ & 40.557 & 1.92 & 0.4 \\
\hline 2008 & 8 & 9 & $21: 53: 2912.491$ & 40.551 & 1.90 & 0.49 \\
\hline 2008 & 8 & 10 & $00: 27: 1212.310$ & 40.611 & 1.72 & 0.46 \\
\hline 2008 & 8 & 11 & $00: 23: 4212.407$ & 40.578 & 1.82 & 0.4 \\
\hline 2008 & 8 & 11 & $01: 00: 3612.440$ & 40.564 & 1.83 & 0.37 \\
\hline 2008 & 8 & 12 & $01: 19: 0612.445$ & 40.562 & 1.77 & 0.43 \\
\hline 2008 & 8 & 12 & $02: 54: 3312.314$ & 40.618 & 1.76 & 0.47 \\
\hline 2008 & 8 & 12 & $15: 09: 0312.449$ & 40.560 & 2.17 & 0.65 \\
\hline 2008 & 8 & 13 & $00: 26: 2912.449$ & 40.557 & 1.78 & 0.42 \\
\hline 2008 & 8 & 13 & $17: 29: 0412.368$ & 40.595 & 1.96 & 0.53 \\
\hline 2008 & 8 & 13 & $20: 31: 3712.411$ & 40.579 & 1.89 & 0.37 \\
\hline 2008 & 8 & 14 & $00: 06: 2412.488$ & 40.538 & 1.81 & 0.62 \\
\hline 2008 & 8 & 14 & $01: 44: 1512.356$ & 40.576 & 1.80 & 0.56 \\
\hline 2008 & 8 & 14 & $02: 40: 1912.436$ & 40.561 & 2.01 & 0.84 \\
\hline 2008 & 8 & 14 & $07: 37: 3212.415$ & 40.584 & 2.35 & 0.55 \\
\hline 2008 & 8 & 14 & $09: 12: 3412.364$ & 40.592 & 1.95 & 0.73 \\
\hline 2008 & 8 & 15 & $00: 09: 1912.446$ & 40.555 & 1.70 & 0.73 \\
\hline 2008 & 8 & 15 & $02: 42: 5012.399$ & 40.563 & 1.84 & 0.7 \\
\hline 2008 & 8 & 15 & $02: 51: 0311.934$ & 41.521 & 2.38 & 1.05 \\
\hline
\end{tabular}




\begin{tabular}{|c|c|c|c|c|c|c|}
\hline 2008 & 8 & 15 & $04: 28: 2812.501$ & 40.531 & 2.00 & 0.81 \\
\hline 2008 & 8 & 15 & $10: 28: 1111.973$ & 41.259 & 2.35 & 0.76 \\
\hline 2008 & 8 & 15 & $13: 45: 5212.463$ & 40.564 & 2.06 & 0.66 \\
\hline 2008 & 8 & 15 & $19: 02: 4413.135$ & 39.690 & 2.47 & 1.55 \\
\hline 2008 & 8 & 16 & $01: 53: 0712.438$ & 40.566 & 1.78 & 0.44 \\
\hline 2008 & 8 & 16 & $20: 49: 2612.531$ & 39.549 & 2.34 & 0.96 \\
\hline 2008 & 8 & 17 & $08: 39: 4012.025$ & 39.717 & 2.34 & 1.56 \\
\hline 2008 & 8 & 17 & $19: 12: 4115.107$ & 39.345 & 3.16 & 18.43 \\
\hline 2008 & 8 & 17 & $19: 39: 4913.612$ & 39.873 & 2.54 & 11.87 \\
\hline 2008 & 8 & 18 & $05: 21: 1312.368$ & 40.591 & 1.91 & 0.54 \\
\hline 2008 & 8 & 18 & $17: 41: 1612.521$ & 40.475 & 2.12 & 0.63 \\
\hline 2008 & 8 & 18 & $18: 40: 5512.375$ & 40.564 & 1.82 & 0.64 \\
\hline 2008 & 8 & 18 & $20: 40: 1712.378$ & 40.579 & 2.26 & 0.53 \\
\hline 2008 & 8 & 18 & $22: 46: 4712.400$ & 40.588 & 1.77 & 0.46 \\
\hline 2008 & 8 & 19 & $00: 08: 5912.493$ & 40.539 & 1.75 & 0.4 \\
\hline 2008 & 8 & 19 & $00: 10: 3512.382$ & 40.577 & 1.70 & 0.47 \\
\hline 2008 & 8 & 19 & $01: 14: 4712.383$ & 40.602 & 1.74 & 0.59 \\
\hline 2008 & 8 & 19 & $01: 35: 4212.300$ & 40.611 & 1.76 & 0.53 \\
\hline 2008 & 8 & 19 & $22: 05: 2613.305$ & 40.066 & 2.51 & 0.57 \\
\hline 2008 & 8 & 20 & $02: 43: 2312.392$ & 40.585 & 1.91 & 0.51 \\
\hline 2008 & 8 & 21 & $20: 12: 4212.577$ & 40.518 & 1.94 & 0.61 \\
\hline 2008 & 8 & 21 & $22: 44: 5112.500$ & 40.543 & 1.85 & 0.43 \\
\hline 2008 & 8 & 22 & $22: 04: 4111.961$ & 39.781 & 2.30 & 0.29 \\
\hline 2008 & 8 & 23 & $02: 35: 3312.434$ & 40.574 & 1.96 & 0.41 \\
\hline 2008 & 8 & 23 & $03: 44: 1212.320$ & 40.606 & 1.95 & 0.52 \\
\hline 2008 & 8 & 24 & $02: 17: 0512.377$ & 40.593 & 1.79 & 0.6 \\
\hline 2008 & 8 & 24 & $02: 30: 4812.371$ & 40.593 & 1.75 & 0.7 \\
\hline 2008 & 8 & 24 & $11: 48: 4912.367$ & 40.590 & 1.92 & 0.59 \\
\hline 2008 & 8 & 24 & $21: 48: 1112.354$ & 40.588 & 1.73 & 0.32 \\
\hline 2008 & 8 & 24 & $21: 49: 4312.384$ & 40.602 & 1.82 & 0.44 \\
\hline 2008 & 8 & 24 & $22: 27: 3412.452$ & 40.571 & 1.75 & 0.48 \\
\hline 2008 & 8 & 25 & $00: 48: 5212.376$ & 40.098 & 1.77 & 5.01 \\
\hline 2008 & 8 & 25 & $00: 49: 2212.357$ & 40.565 & 1.76 & 0.74 \\
\hline 2008 & 8 & 25 & $00: 53: 4912.349$ & 40.569 & 1.77 & 0.61 \\
\hline 2008 & 8 & 25 & $00: 59: 3412.353$ & 40.585 & 1.69 & 0.67 \\
\hline 2008 & 8 & 25 & $01: 01: 5912.351$ & 40.599 & 1.70 & 0.69 \\
\hline 2008 & 8 & 25 & $02: 24: 0412.360$ & 40.586 & 2.20 & 0.62 \\
\hline 2008 & 8 & 25 & $02: 26: 1612.361$ & 40.591 & 2.10 & 0.6 \\
\hline 2008 & 8 & 25 & $02: 43: 2512.740$ & 40.555 & 2.29 & 0.61 \\
\hline 2008 & 8 & 25 & $04: 04: 5612.870$ & 39.481 & 2.60 & 0.48 \\
\hline 2008 & 8 & 25 & $19: 01: 3112.580$ & 39.623 & 2.38 & 0.65 \\
\hline 2008 & 8 & 26 & $22: 08: 2512.431$ & 40.569 & 1.85 & 0.41 \\
\hline 2008 & 8 & 26 & $22: 55: 5212.312$ & 40.612 & 1.74 & 0.49 \\
\hline 2008 & 8 & 27 & $01: 38: 3412.406$ & 40.578 & 1.79 & 0.46 \\
\hline 2008 & 8 & 28 & $00: 04: 5712.418$ & 40.576 & 1.72 & 0.54 \\
\hline 2008 & 8 & 28 & $20: 45: 5412.402$ & 40.579 & 1.80 & 0.41 \\
\hline 2008 & 8 & 29 & $02: 23: 5112.383$ & 40.581 & 1.77 & 0.47 \\
\hline 2008 & 8 & 29 & $14: 54: 0312.369$ & 40.604 & 2.10 & 0.5 \\
\hline 2008 & 8 & 29 & $21: 50: 2713.532$ & 39.995 & 2.39 & 1.64 \\
\hline 2008 & 8 & 29 & $23: 35: 3512.471$ & 40.536 & 1.71 & 0.61 \\
\hline 2008 & 8 & 30 & $00: 07: 0812.324$ & 40.618 & 1.86 & 0.47 \\
\hline 2008 & 8 & 30 & $03: 16: 1712.490$ & 40.538 & 1.86 & 0.98 \\
\hline 2008 & 8 & 30 & $08: 35: 0712.599$ & 40.507 & 2.04 & 0.96 \\
\hline 2008 & 8 & 30 & $20: 03: 2312.370$ & 40.582 & 1.83 & 1.22 \\
\hline 2008 & 8 & 31 & $00: 22: 3612.366$ & 40.591 & 1.73 & 0.73 \\
\hline 2008 & 8 & 31 & $02: 02: 0412.397$ & 40.584 & 1.83 & 0.64 \\
\hline 2008 & 8 & 31 & $07: 38: 5812.374$ & 40.601 & 1.93 & 0.71 \\
\hline 2008 & 8 & 31 & $08: 38: 3512.448$ & 40.581 & 2.02 & 0.65 \\
\hline 2008 & 8 & 31 & $08: 47: 5712.397$ & 40.583 & 2.02 & 0.53 \\
\hline 2008 & 8 & 31 & $16: 22: 4313.875$ & 40.561 & 4.10 & 2.73 \\
\hline 2008 & 8 & 31 & $18: 30: 5912.025$ & 40.948 & 1.90 & 0.46 \\
\hline 2008 & 8 & 31 & $20: 12: 5513.040$ & 39.520 & 2.52 & 1.47 \\
\hline 2008 & 9 & 1 & $02: 09: 3312.094$ & 41.488 & 2.46 & 0.54 \\
\hline 2008 & 9 & 1 & $06: 23: 1812.062$ & 41.524 & 2.37 & 5.2 \\
\hline 2008 & 9 & 1 & $07: 00: 4412.417$ & 40.573 & 2.06 & 1.08 \\
\hline 2008 & 9 & 2 & $11: 05: 3312.466$ & 40.556 & 2.34 & 0.45 \\
\hline 2008 & 9 & 2 & $18: 20: 0912.489$ & 40.531 & 2.09 & 0.8 \\
\hline 2008 & 9 & 3 & $18: 14: 1212.087$ & 41.670 & 2.52 & 0.74 \\
\hline 2008 & 9 & 3 & $18: 51: 5012.450$ & 40.556 & 1.95 & 0.73 \\
\hline 2008 & 9 & 3 & $19: 11: 1312.104$ & 41.647 & 2.43 & 0.44 \\
\hline 2008 & 9 & 3 & $19: 20: 1812.066$ & 41.662 & 2.34 & 0.91 \\
\hline 2008 & 9 & 3 & $19: 35: 3712.095$ & 41.657 & 2.35 & 1.46 \\
\hline 2008 & 9 & 3 & $19: 48: 0412.062$ & 41.613 & 2.24 & 1.56 \\
\hline 2008 & 9 & 3 & $20: 01: 1712.039$ & 41.614 & 2.24 & 1.96 \\
\hline 2008 & 9 & 3 & $20: 13: 5012.127$ & 41.676 & 2.27 & 1.51 \\
\hline 2008 & 9 & 3 & $20: 37: 3712.087$ & 41.628 & 2.26 & 1.69 \\
\hline
\end{tabular}




\begin{tabular}{|c|c|c|c|c|c|c|}
\hline 2008 & 9 & 3 & $20: 38: 3712.099$ & 41.618 & 2.22 & 0.58 \\
\hline 2008 & 9 & 3 & $20: 47: 5712.083$ & 41.633 & 2.23 & 0.97 \\
\hline 2008 & 9 & 3 & $20: 52: 1212.090$ & 41.615 & 2.28 & 0.62 \\
\hline 2008 & 9 & 3 & $21: 03: 0112.130$ & 41.612 & 2.21 & 1.9 \\
\hline 2008 & 9 & 3 & $21: 07: 5612.099$ & 41.663 & 2.24 & 1.57 \\
\hline 2008 & 9 & 3 & $21: 22: 2612.080$ & 41.620 & 2.34 & 0.72 \\
\hline 2008 & 9 & 3 & $22: 14: 0112.080$ & 41.664 & 2.28 & 0.59 \\
\hline 2008 & 9 & 3 & $22: 15: 4712.061$ & 41.559 & 2.18 & 2.02 \\
\hline 2008 & 9 & 3 & $22: 16: 2512.095$ & 41.646 & 2.23 & 2.65 \\
\hline 2008 & 9 & 3 & $22: 24: 1512.015$ & 41.607 & 2.36 & 1.37 \\
\hline 2008 & 9 & 3 & $22: 27: 0812.101$ & 41.630 & 2.17 & 1.19 \\
\hline 2008 & 9 & 3 & $22: 39: 1112.080$ & 41.614 & 2.23 & 0.93 \\
\hline 2008 & 9 & 3 & $22: 58: 3212.101$ & 41.636 & 2.84 & 0.99 \\
\hline 2008 & 9 & 3 & $23: 22: 2012.098$ & 41.656 & 2.40 & 0.69 \\
\hline 2008 & 9 & 3 & $23: 33: 1412.097$ & 41.670 & 2.61 & 0.88 \\
\hline 2008 & 9 & 3 & $23: 35: 2512.096$ & 41.673 & 2.33 & 0.92 \\
\hline 2008 & 9 & 4 & $00: 16: 2711.981$ & 41.695 & 2.28 & 4.48 \\
\hline 2008 & 9 & 4 & $00: 36: 3312.092$ & 41.630 & 2.32 & 0.62 \\
\hline 2008 & 9 & 4 & $00: 57: 4012.023$ & 41.679 & 2.32 & 2.38 \\
\hline 2008 & 9 & 4 & $01: 17: 5212.098$ & 41.628 & 2.29 & 1.77 \\
\hline 2008 & 9 & 4 & $01: 18: 5912.097$ & 41.646 & 2.30 & 0.54 \\
\hline 2008 & 9 & 4 & $01: 28: 2912.122$ & 41.705 & 2.50 & 1.27 \\
\hline 2008 & 9 & 4 & $01: 32: 0612.088$ & 41.635 & 2.28 & 0.73 \\
\hline 2008 & 9 & 4 & $01: 39: 4312.101$ & 41.639 & 2.28 & 1.32 \\
\hline 2008 & 9 & 4 & $01: 51: 2312.098$ & 41.622 & 2.28 & 0.67 \\
\hline 2008 & 9 & 4 & $02: 33: 4212.090$ & 41.644 & 2.33 & 0.74 \\
\hline 2008 & 9 & 4 & $02: 55: 5612.058$ & 41.668 & 2.30 & 1.51 \\
\hline 2008 & 9 & 4 & $07: 11: 3912.081$ & 41.666 & 3.03 & 0.45 \\
\hline 2008 & 9 & 4 & $10: 38: 4112.380$ & 40.587 & 2.34 & 0.72 \\
\hline 2008 & 9 & 4 & $15: 34: 5712.089$ & 41.656 & 2.47 & 0.55 \\
\hline 2008 & 9 & 4 & $15: 40: 4712.097$ & 41.610 & 2.44 & 1.45 \\
\hline 2008 & 9 & 4 & $16: 24: 5712.097$ & 41.604 & 2.33 & 2.26 \\
\hline 2008 & 9 & 4 & $17: 44: 4712.115$ & 41.625 & 2.35 & 2.34 \\
\hline 2008 & 9 & 4 & $17: 54: 5712.072$ & 41.622 & 2.42 & 4.35 \\
\hline 2008 & 9 & 4 & $19: 49: 4212.080$ & 41.661 & 2.33 & 0.79 \\
\hline 2008 & 9 & 4 & $19: 52: 4712.082$ & 41.652 & 2.31 & 1.22 \\
\hline 2008 & 9 & 4 & $19: 56: 3712.092$ & 41.642 & 2.37 & 0.67 \\
\hline 2008 & 9 & 4 & $20: 01: 1412.071$ & 41.656 & 2.28 & 0.94 \\
\hline 2008 & 9 & 5 & $00: 26: 5512.083$ & 41.649 & 2.37 & 1.45 \\
\hline 2008 & 9 & 5 & $00: 31: 1312.099$ & 41.605 & 2.43 & 1.26 \\
\hline 2008 & 9 & 5 & $00: 33: 5012.076$ & 41.663 & 2.42 & 1.22 \\
\hline 2008 & 9 & 5 & $00: 35: 5012.104$ & 41.645 & 2.42 & 1.34 \\
\hline 2008 & 9 & 5 & $00: 47: 4812.092$ & 41.672 & 2.53 & 0.54 \\
\hline 2008 & 9 & 5 & $00: 52: 4712.102$ & 41.680 & 2.31 & 0.44 \\
\hline 2008 & 9 & 5 & $02: 09: 0612.085$ & 41.624 & 2.29 & 0.68 \\
\hline 2008 & 9 & 5 & $03: 57: 5512.080$ & 41.667 & 2.47 & 1.57 \\
\hline 2008 & 9 & 5 & $05: 53: 4512.083$ & 41.638 & 2.45 & 0.72 \\
\hline 2008 & 9 & 5 & $06: 57: 5212.108$ & 41.651 & 2.41 & 0.59 \\
\hline 2008 & 9 & 5 & $10: 09: 3712.090$ & 41.629 & 2.58 & 2.02 \\
\hline 2008 & 9 & 5 & $10: 28: 0111.473$ & 41.887 & 2.81 & 2.65 \\
\hline 2008 & 9 & 5 & $10: 37: 4812.071$ & 41.604 & 2.38 & 1.37 \\
\hline 2008 & 9 & 5 & $10: 43: 4212.075$ & 41.632 & 2.48 & 1.19 \\
\hline 2008 & 9 & 5 & $10: 49: 5712.069$ & 41.668 & 2.47 & 0.93 \\
\hline 2008 & 9 & 5 & $11: 05: 3712.082$ & 41.631 & 2.41 & 0.99 \\
\hline 2008 & 9 & 5 & $12: 13: 2912.074$ & 41.643 & 2.51 & 0.44 \\
\hline 2008 & 9 & 5 & $12: 16: 2112.089$ & 41.670 & 2.66 & 0.35 \\
\hline 2008 & 9 & 5 & $14: 12: 2512.102$ & 41.622 & 2.67 & 1.19 \\
\hline 2008 & 9 & 5 & $14: 41: 2512.110$ & 41.643 & 2.55 & 0.55 \\
\hline 2008 & 9 & 5 & $15: 07: 2312.116$ & 41.630 & 2.49 & 1.95 \\
\hline 2008 & 9 & 5 & $16: 22: 3911.986$ & 41.659 & 2.42 & 0.72 \\
\hline 2008 & 9 & 5 & $17: 59: 3712.078$ & 41.677 & 2.52 & 3.18 \\
\hline 2008 & 9 & 5 & $20: 56: 1914.885$ & 40.070 & 4.06 & 0.65 \\
\hline 2008 & 9 & 5 & $23: 46: 4413.679$ & 39.953 & 2.52 & 1.55 \\
\hline 2008 & 9 & 5 & $23: 49: 2212.330$ & 40.603 & 1.69 & 0.9 \\
\hline 2008 & 9 & 5 & $23: 51: 5112.322$ & 40.609 & 1.74 & 0.33 \\
\hline 2008 & 9 & 6 & $00: 32: 0012.374$ & 40.588 & 1.69 & 0.42 \\
\hline 2008 & 9 & 6 & $02: 25: 5014.747$ & 39.922 & 3.04 & 0.37 \\
\hline 2008 & 9 & 6 & $13: 59: 4114.759$ & 39.974 & 3.07 & 0.33 \\
\hline 2008 & 9 & 7 & $01: 34: 0211.411$ & 39.826 & 2.32 & 0.26 \\
\hline 2008 & 9 & 7 & $04: 10: 3611.878$ & 41.375 & 2.29 & 0.37 \\
\hline 2008 & 9 & 7 & $05: 12: 2312.087$ & 41.649 & 2.51 & 0.36 \\
\hline 2008 & 9 & 7 & $09: 07: 5612.077$ & 41.586 & 2.40 & 0.53 \\
\hline 2008 & 9 & 7 & $12: 02: 0111.781$ & 39.135 & 2.69 & 0.4 \\
\hline 2008 & 9 & 7 & $15: 10: 3712.441$ & 40.570 & 2.45 & 0.37 \\
\hline 2008 & 9 & 7 & $20: 14: 5711.950$ & 41.604 & 2.47 & 0.43 \\
\hline 2008 & 9 & 7 & $20: 23: 1712.093$ & 41.634 & 2.53 & 0.47 \\
\hline
\end{tabular}




\begin{tabular}{|c|c|c|c|c|c|c|}
\hline 2008 & 9 & 7 & $21: 00: 3712.088$ & 41.639 & 2.33 & 0.65 \\
\hline 2008 & 9 & 7 & $21: 10: 4512.057$ & 41.654 & 2.21 & 0.42 \\
\hline 2008 & 9 & 7 & $22: 04: 2212.090$ & 41.629 & 2.14 & 0.53 \\
\hline 2008 & 9 & 7 & $22: 53: 1312.045$ & 41.646 & 2.19 & 0.37 \\
\hline 2008 & 9 & 7 & $23: 17: 1212.091$ & 41.635 & 2.25 & 0.62 \\
\hline 2008 & 9 & 7 & $23: 17: 5712.042$ & 41.626 & 2.18 & 0.56 \\
\hline 2008 & 9 & 8 & $00: 19: 0612.412$ & 40.563 & 1.73 & 1.19 \\
\hline 2008 & 9 & 9 & $11: 01: 2212.070$ & 41.636 & 2.40 & 1.48 \\
\hline 2008 & 9 & 9 & $16: 41: 0312.431$ & 40.573 & 1.94 & 0.51 \\
\hline 2008 & 9 & 10 & $00: 39: 3713.509$ & 40.641 & 2.38 & 1.48 \\
\hline 2008 & 9 & 10 & $00: 50: 1512.085$ & 41.656 & 2.21 & 0.68 \\
\hline 2008 & 9 & 10 & $07: 14: 2613.472$ & 39.934 & 2.50 & 1.19 \\
\hline 2008 & 9 & 10 & $08: 05: 5612.442$ & 40.553 & 1.90 & 0.62 \\
\hline 2008 & 9 & 10 & $15: 20: 1312.081$ & 40.758 & 1.91 & 5.17 \\
\hline 2008 & 9 & 10 & $16: 35: 3112.477$ & 40.558 & 1.82 & 0.65 \\
\hline 2008 & 9 & 10 & $19: 09: 3612.394$ & 40.591 & 2.11 & 0.46 \\
\hline 2008 & 9 & 10 & $22: 26: 0312.400$ & 40.579 & 1.77 & 0.52 \\
\hline 2008 & 9 & 11 & $01: 34: 2012.353$ & 40.607 & 2.63 & 0.35 \\
\hline 2008 & 9 & 11 & $05: 02: 2512.371$ & 40.595 & 1.91 & 0.74 \\
\hline 2008 & 9 & 11 & $06: 40: 3212.424$ & 40.558 & 1.81 & 0.89 \\
\hline 2008 & 9 & 11 & $08: 56: 1212.925$ & 39.551 & 2.55 & 0.44 \\
\hline 2008 & 9 & 11 & $20: 35: 5512.433$ & 40.569 & 1.82 & 0.35 \\
\hline 2008 & 9 & 11 & $23: 15: 3013.487$ & 39.930 & 2.60 & 1.19 \\
\hline 2008 & 9 & 11 & $23: 43: 1012.115$ & 40.760 & 1.79 & 0.55 \\
\hline 2008 & 9 & 12 & $00: 15: 4513.505$ & 39.941 & 2.39 & 1.95 \\
\hline 2008 & 9 & 12 & $03: 21: 0113.524$ & 39.952 & 3.51 & 0.72 \\
\hline 2008 & 9 & 12 & $04: 12: 1713.547$ & 39.930 & 2.80 & 3.18 \\
\hline 2008 & 9 & 12 & $04: 58: 2413.479$ & 39.927 & 2.76 & 0.65 \\
\hline 2008 & 9 & 12 & $07: 47: 2813.454$ & 39.935 & 2.51 & 1.55 \\
\hline 2008 & 9 & 12 & $17: 48: 4412.397$ & 40.553 & 1.82 & 0.74 \\
\hline 2008 & 9 & 12 & $18: 58: 0713.524$ & 39.932 & 2.50 & 1.2 \\
\hline 2008 & 9 & 12 & $21: 06: 0913.483$ & 39.931 & 2.57 & 0.8 \\
\hline 2008 & 9 & 12 & $21: 08: 4613.492$ & 39.934 & 2.51 & 0.73 \\
\hline 2008 & 9 & 12 & $21: 18: 4412.375$ & 40.592 & 1.86 & 0.47 \\
\hline 2008 & 9 & 12 & $21: 27: 0313.520$ & 39.935 & 2.67 & 1.21 \\
\hline 2008 & 9 & 12 & $22: 36: 5012.409$ & 40.578 & 1.79 & 0.44 \\
\hline 2008 & 9 & 12 & $22: 41: 4413.681$ & 39.971 & 2.53 & 3.35 \\
\hline 2008 & 9 & 13 & $01: 27: 3913.280$ & 39.870 & 2.31 & 2.48 \\
\hline 2008 & 9 & 13 & $17: 23: 1312.290$ & 40.605 & 1.79 & 1.65 \\
\hline 2008 & 9 & 13 & $22: 27: 2413.468$ & 39.903 & 2.40 & 4.03 \\
\hline 2008 & 9 & 13 & $22: 48: 2812.328$ & 40.611 & 2.06 & 0.45 \\
\hline 2008 & 9 & 13 & $23: 25: 4712.459$ & 40.552 & 1.90 & 0.45 \\
\hline 2008 & 9 & 14 & $00: 01: 5413.512$ & 39.941 & 2.63 & 1.71 \\
\hline 2008 & 9 & 14 & $17: 10: 5112.443$ & 40.565 & 2.04 & 0.39 \\
\hline 2008 & 9 & 14 & $20: 16: 2312.344$ & 40.606 & 1.91 & 0.65 \\
\hline 2008 & 9 & 14 & $20: 38: 1312.323$ & 40.595 & 1.92 & 0.48 \\
\hline 2008 & 9 & 14 & $21: 44: 5413.511$ & 39.920 & 2.60 & 0.86 \\
\hline 2008 & 9 & 15 & $00: 44: 0512.333$ & 40.595 & 2.07 & 0.29 \\
\hline 2008 & 9 & 15 & $16: 08: 4512.395$ & 40.601 & 2.18 & 0.39 \\
\hline 2008 & 9 & 15 & $19: 50: 4212.401$ & 40.596 & 2.06 & 0.36 \\
\hline 2008 & 9 & 15 & $21: 00: 0411.448$ & 40.985 & 2.36 & 0.8 \\
\hline 2008 & 9 & 15 & $21: 03: 0111.451$ & 40.993 & 2.23 & 0.9 \\
\hline 2008 & 9 & 15 & $21: 19: 4612.334$ & 40.590 & 1.99 & 0.33 \\
\hline 2008 & 9 & 15 & $21: 22: 5012.392$ & 40.588 & 1.85 & 0.42 \\
\hline 2008 & 9 & 15 & $21: 45: 2012.339$ & 40.590 & 1.91 & 0.37 \\
\hline 2008 & 9 & 15 & $23: 05: 4712.394$ & 40.602 & 1.92 & 0.33 \\
\hline 2008 & 9 & 16 & $00: 06: 0212.337$ & 40.588 & 2.18 & 0.26 \\
\hline 2008 & 9 & 16 & $01: 22: 1412.375$ & 40.599 & 1.93 & 0.37 \\
\hline 2008 & 9 & 16 & $01: 29: 4812.387$ & 40.599 & 2.26 & 0.36 \\
\hline 2008 & 9 & 16 & $02: 01: 5912.417$ & 40.571 & 1.91 & 0.53 \\
\hline 2008 & 9 & 16 & $11: 26: 2913.452$ & 39.933 & 2.64 & 1.54 \\
\hline 2008 & 9 & 16 & $20: 22: 0412.300$ & 40.597 & 1.79 & 0.62 \\
\hline 2008 & 9 & 16 & $23: 16: 0113.222$ & 39.799 & 2.33 & 0.84 \\
\hline 2008 & 9 & 17 & $01: 25: 3013.513$ & 39.912 & 2.52 & 1.27 \\
\hline 2008 & 9 & 17 & $08: 36: 1813.419$ & 40.916 & 2.51 & 5.03 \\
\hline 2008 & 9 & 17 & $18: 04: 4311.922$ & 41.198 & 2.42 & 1.25 \\
\hline 2008 & 9 & 17 & $21: 12: 2712.066$ & 41.624 & 2.28 & 1.04 \\
\hline 2008 & 9 & 17 & $22: 08: 1212.460$ & 40.552 & 1.77 & 0.41 \\
\hline 2008 & 9 & 18 & $01: 13: 3012.255$ & 40.638 & 1.76 & 0.72 \\
\hline 2008 & 9 & 18 & $03: 05: 1012.408$ & 40.585 & 1.97 & 0.76 \\
\hline 2008 & 9 & 18 & $13: 49: 5011.925$ & 39.520 & 2.97 & 0.59 \\
\hline 2008 & 9 & 18 & $16: 57: 5412.398$ & 40.574 & 1.86 & 0.53 \\
\hline 2008 & 9 & 18 & $18: 15: 4511.907$ & 39.525 & 2.33 & 0.84 \\
\hline 2008 & 9 & 18 & $18: 52: 4712.450$ & 40.559 & 1.89 & 0.42 \\
\hline 2008 & 9 & 18 & $21: 29: 2112.486$ & 40.521 & 1.76 & 0.85 \\
\hline 2008 & 9 & 18 & $22: 31: 2713.614$ & 40.727 & 2.42 & 2.46 \\
\hline
\end{tabular}




\begin{tabular}{|c|c|c|c|c|c|c|}
\hline 2008 & 9 & 18 & $23: 12: 2312.133$ & 39.721 & 2.07 & 0.67 \\
\hline 2008 & 9 & 19 & $06: 15: 2212.464$ & 40.528 & 2.00 & 1.08 \\
\hline 2008 & 9 & 19 & $11: 50: 3212.385$ & 40.571 & 1.98 & 1.85 \\
\hline 2008 & 9 & 19 & $13: 21: 4412.450$ & 40.552 & 1.97 & 0.53 \\
\hline 2008 & 9 & 19 & $16: 23: 2112.299$ & 40.617 & 1.85 & 1.34 \\
\hline 2008 & 9 & 19 & $16: 55: 0812.312$ & 40.609 & 1.85 & 0.61 \\
\hline 2008 & 9 & 19 & $20: 14: 3911.875$ & 41.547 & 2.18 & 2.06 \\
\hline 2008 & 9 & 19 & $21: 54: 3212.361$ & 40.594 & 1.82 & 0.42 \\
\hline 2008 & 9 & 19 & $23: 10: 3112.402$ & 40.584 & 1.74 & 0.55 \\
\hline 2008 & 9 & 20 & $00: 56: 4312.417$ & 40.564 & 1.74 & 0.66 \\
\hline 2008 & 9 & 20 & $16: 15: 3912.382$ & 40.586 & 1.84 & 0.6 \\
\hline 2008 & 9 & 20 & $19: 51: 0212.267$ & 40.626 & 1.75 & 0.71 \\
\hline 2008 & 9 & 20 & $19: 55: 3113.508$ & 39.953 & 2.40 & 2.44 \\
\hline 2008 & 9 & 21 & $00: 05: 3111.946$ & 39.526 & 2.27 & 0.53 \\
\hline 2008 & 9 & 21 & $05: 26: 4312.414$ & 40.595 & 1.90 & 0.76 \\
\hline 2008 & 9 & 21 & $13: 33: 0412.371$ & 40.615 & 1.98 & 0.82 \\
\hline 2008 & 9 & 21 & $18: 54: 5312.488$ & 40.534 & 1.78 & 0.48 \\
\hline 2008 & 9 & 21 & $23: 35: 1013.433$ & 39.979 & 2.37 & 1.92 \\
\hline 2008 & 9 & 22 & $03: 48: 3113.515$ & 39.944 & 3.19 & 1.74 \\
\hline 2008 & 9 & 22 & $05: 49: 5211.888$ & 41.531 & 2.53 & 1.14 \\
\hline 2008 & 9 & 22 & $07: 43: 3413.445$ & 39.942 & 2.55 & 2.06 \\
\hline 2008 & 9 & 22 & $16: 17: 4212.497$ & 40.538 & 2.09 & 0.43 \\
\hline 2008 & 9 & 24 & $02: 10: 4812.407$ & 40.585 & 1.89 & 0.53 \\
\hline 2008 & 9 & 24 & $22: 59: 1212.447$ & 40.567 & 1.92 & 0.42 \\
\hline 2008 & 9 & 24 & $23: 23: 5912.410$ & 40.579 & 1.78 & 0.38 \\
\hline 2008 & 9 & 25 & $01: 13: 3812.434$ & 40.569 & 1.80 & 0.49 \\
\hline 2008 & 9 & 25 & $10: 54: 3912.402$ & 40.590 & 1.84 & 0.68 \\
\hline 2008 & 9 & 25 & $19: 58: 4212.327$ & 40.615 & 1.91 & 0.49 \\
\hline 2008 & 9 & 25 & $22: 21: 0812.978$ & 40.342 & 2.09 & 0.55 \\
\hline 2008 & 9 & 26 & $17: 32: 3812.447$ & 40.558 & 1.89 & 0.79 \\
\hline 2008 & 9 & 26 & $18: 43: 0212.471$ & 40.539 & 1.93 & 0.65 \\
\hline 2008 & 9 & 26 & $20: 54: 1811.285$ & 41.661 & 3.98 & 0.77 \\
\hline 2008 & 9 & 27 & $20: 58: 1713.541$ & 40.649 & 2.40 & 3.17 \\
\hline 2008 & 9 & 27 & $20: 58: 1813.569$ & 40.687 & 2.42 & 0.84 \\
\hline 2008 & 9 & 27 & $21: 43: 2312.386$ & 40.596 & 1.68 & 0.5 \\
\hline 2008 & 9 & 27 & $23: 08: 0812.426$ & 40.570 & 1.79 & 2.44 \\
\hline 2008 & 9 & 28 & $09: 01: 3413.509$ & 39.929 & 2.64 & 2.1 \\
\hline 2008 & 9 & 28 & $18: 54: 1113.552$ & 39.971 & 2.97 & 2.24 \\
\hline 2008 & 9 & 28 & $23: 23: 1412.584$ & 40.511 & 2.06 & 0.5 \\
\hline 2008 & 9 & 29 & $04: 11: 5513.502$ & 39.927 & 2.70 & 0.97 \\
\hline 2008 & 9 & 29 & $20: 48: 4512.416$ & 40.557 & 1.78 & 0.59 \\
\hline 2008 & 9 & 30 & $01: 29: 5412.438$ & 40.554 & 1.74 & 0.55 \\
\hline 2008 & 9 & 30 & $22: 28: 4012.387$ & 40.581 & 1.73 & 0.54 \\
\hline 2008 & 10 & 1 & $00: 54: 0212.369$ & 40.599 & 1.79 & 0.72 \\
\hline 2008 & 10 & 1 & $13: 34: 1112.333$ & 40.613 & 2.08 & 0.67 \\
\hline 2008 & 10 & 1 & $16: 42: 2512.327$ & 40.617 & 2.03 & 0.55 \\
\hline 2008 & 10 & 1 & $16: 49: 3612.332$ & 40.611 & 1.85 & 0.45 \\
\hline 2008 & 10 & 1 & $18: 19: 0612.334$ & 40.624 & 2.16 & 0.47 \\
\hline 2008 & 10 & 1 & $22: 18: 5512.373$ & 40.591 & 1.96 & 0.46 \\
\hline 2008 & 10 & 1 & $22: 35: 4812.449$ & 40.566 & 1.77 & 0.56 \\
\hline 2008 & 10 & 1 & $23: 55: 4911.826$ & 39.939 & 2.08 & 0.49 \\
\hline 2008 & 10 & 2 & $00: 56: 2013.396$ & 40.042 & 2.29 & 2.2 \\
\hline 2008 & 10 & 2 & $02: 39: 3112.383$ & 40.601 & 1.86 & 0.6 \\
\hline 2008 & 10 & 2 & $04: 25: 1612.452$ & 40.552 & 2.27 & 0.62 \\
\hline 2008 & 10 & 2 & $07: 01: 4212.355$ & 40.609 & 2.12 & 0.85 \\
\hline 2008 & 10 & 2 & $10: 49: 4612.747$ & 40.606 & 2.05 & 0.65 \\
\hline 2008 & 10 & 2 & $17: 27: 2311.250$ & 39.598 & 2.50 & 0.76 \\
\hline 2008 & 10 & 2 & $22: 51: 4712.352$ & 40.602 & 2.01 & 0.4 \\
\hline 2008 & 10 & 2 & $23: 14: 5112.739$ & 39.622 & 2.33 & 0.7 \\
\hline 2008 & 10 & 3 & $00: 56: 0412.361$ & 40.593 & 2.47 & 0.37 \\
\hline 2008 & 10 & 3 & $01: 20: 3112.753$ & 40.557 & 1.94 & 0.52 \\
\hline 2008 & 10 & 3 & $06: 14: 3712.361$ & 40.614 & 2.38 & 1.37 \\
\hline 2008 & 10 & 4 & $00: 42: 3912.399$ & 40.578 & 1.81 & 0.63 \\
\hline 2008 & 10 & 4 & $03: 19: 1312.880$ & 40.285 & 2.37 & 0.49 \\
\hline 2008 & 10 & 4 & $16: 05: 1112.459$ & 40.562 & 1.98 & 0.49 \\
\hline 2008 & 10 & 4 & $23: 10: 4912.436$ & 40.569 & 1.73 & 0.57 \\
\hline 2008 & 10 & 5 & $00: 39: 4812.415$ & 40.568 & 1.82 & 0.68 \\
\hline 2008 & 10 & 5 & $06: 14: 0413.642$ & 39.928 & 2.75 & 0.9 \\
\hline 2008 & 10 & 5 & $20: 37: 3412.304$ & 40.636 & 1.94 & 1.68 \\
\hline 2008 & 10 & 5 & $23: 15: 4110.965$ & 39.742 & 3.28 & 0.54 \\
\hline 2008 & 10 & 6 & $01: 33: 179.674$ & 39.854 & 2.95 & 16.01 \\
\hline 2008 & 10 & 6 & $21: 14: 0212.376$ & 40.593 & 1.84 & 0.76 \\
\hline 2008 & 10 & 7 & $19: 36: 5713.795$ & 40.515 & 2.72 & 12.52 \\
\hline 2008 & 10 & 9 & $02: 06: 5513.468$ & 39.869 & 2.61 & 1.51 \\
\hline 2008 & 10 & 9 & $16: 46: 5213.860$ & 40.548 & 3.01 & 1.89 \\
\hline 2008 & 10 & 9 & $20: 02: 2113.843$ & 40.530 & 2.77 & 1.45 \\
\hline
\end{tabular}




\begin{tabular}{|c|c|c|c|c|c|c|}
\hline 2008 & 10 & 9 & $21: 04: 4912.382$ & 40.591 & 2.37 & 0.42 \\
\hline 2008 & 10 & 9 & $23: 04: 3012.311$ & 40.614 & 2.23 & 0.42 \\
\hline 2008 & 10 & 10 & $01: 04: 3312.663$ & 39.577 & 2.52 & 0.92 \\
\hline 2008 & 10 & 10 & $01: 11: 0713.890$ & 40.539 & 2.78 & 1.35 \\
\hline 2008 & 10 & 10 & $23: 06: 3713.714$ & 39.970 & 2.69 & 0.64 \\
\hline 2008 & 10 & 12 & $19: 45: 0714.848$ & 40.044 & 3.21 & 19,00 \\
\hline 2008 & 10 & 12 & $23: 15: 5412.444$ & 40.560 & 1.93 & 0.73 \\
\hline 2008 & 10 & 13 & $18: 32: 3513.914$ & 40.557 & 2.93 & 2.75 \\
\hline 2008 & 10 & 13 & $18: 57: 1312.463$ & 40.558 & 2.13 & 0.57 \\
\hline 2008 & 10 & 13 & $20: 21: 1212.968$ & 39.545 & 2.64 & 1.93 \\
\hline 2008 & 10 & 13 & $22: 12: 3912.418$ & 40.577 & 2.17 & 0.45 \\
\hline 2008 & 10 & 13 & $23: 28: 3013.897$ & 40.539 & 2.71 & 1.08 \\
\hline 2008 & 10 & 14 & $01: 43: 0413.669$ & 39.865 & 2.73 & 0.8 \\
\hline 2008 & 10 & 14 & $21: 29: 2512.354$ & 40.497 & 1.93 & 1.3 \\
\hline 2008 & 10 & 15 & $12: 12: 5712.514$ & 40.536 & 2.24 & 0.41 \\
\hline 2008 & 10 & 15 & $21: 56: 0510.889$ & 40.791 & 2.49 & 1.46 \\
\hline 2008 & 10 & 15 & $23: 10: 4912.388$ & 40.588 & 1.98 & 0.44 \\
\hline 2008 & 10 & 15 & $23: 56: 0912.121$ & 41.719 & 2.29 & 1.8 \\
\hline 2008 & 10 & 16 & $00: 03: 2512.440$ & 40.569 & 2.38 & 0.29 \\
\hline 2008 & 10 & 16 & $00: 52: 1212.398$ & 40.585 & 1.99 & 0.48 \\
\hline 2008 & 10 & 16 & $08: 07: 4112.371$ & 40.601 & 2.13 & 0.62 \\
\hline 2008 & 10 & 16 & $23: 14: 5712.383$ & 40.584 & 1.66 & 0.57 \\
\hline 2008 & 10 & 17 & $00: 16: 4212.379$ & 40.593 & 1.69 & 0.5 \\
\hline 2008 & 10 & 17 & $01: 10: 4112.309$ & 40.611 & 1.86 & 0.43 \\
\hline 2008 & 10 & 17 & $05: 13: 5112.306$ & 40.612 & 2.01 & 0.84 \\
\hline 2008 & 10 & 17 & $06: 35: 4312.310$ & 40.607 & 2.06 & 0.57 \\
\hline 2008 & 10 & 17 & $06: 44: 0112.323$ & 40.629 & 2.23 & 1.39 \\
\hline 2008 & 10 & 17 & $06: 48: 5012.313$ & 40.607 & 2.36 & 0.72 \\
\hline 2008 & 10 & 17 & $06: 51: 0312.322$ & 40.590 & 2.29 & 0.57 \\
\hline 2008 & 10 & 17 & $06: 56: 0512.309$ & 40.607 & 2.40 & 0.46 \\
\hline 2008 & 10 & 17 & $06: 56: 3612.313$ & 40.606 & 2.42 & 0.54 \\
\hline 2008 & 10 & 17 & $07: 04: 5412.313$ & 40.612 & 2.40 & 0.82 \\
\hline 2008 & 10 & 17 & $07: 06: 3812.309$ & 40.610 & 2.48 & 0.73 \\
\hline 2008 & 10 & 17 & $07: 09: 1812.317$ & 40.612 & 2.58 & 0.74 \\
\hline 2008 & 10 & 17 & $07: 10: 2912.320$ & 40.594 & 2.71 & 0.46 \\
\hline 2008 & 10 & 17 & $07: 15: 4012.326$ & 40.602 & 2.76 & 0.58 \\
\hline 2008 & 10 & 17 & $07: 16: 0712.323$ & 40.607 & 2.81 & 0.76 \\
\hline 2008 & 10 & 17 & $07: 22: 0112.344$ & 40.605 & 2.76 & 0.82 \\
\hline 2008 & 10 & 17 & $07: 22: 4612.330$ & 40.616 & 3.33 & 0.47 \\
\hline 2008 & 10 & 17 & $07: 23: 1512.321$ & 40.604 & 3.30 & 0.72 \\
\hline 2008 & 10 & 17 & $07: 28: 2112.327$ & 40.609 & 3.27 & 0.35 \\
\hline 2008 & 10 & 17 & $07: 35: 5712.319$ & 40.602 & 2.74 & 0.55 \\
\hline 2008 & 10 & 17 & $07: 36: 5812.340$ & 40.609 & 2.89 & 0.8 \\
\hline 2008 & 10 & 17 & $07: 37: 4312.320$ & 40.634 & 2.65 & 1.3 \\
\hline 2008 & 10 & 17 & $07: 38: 1012.327$ & 40.597 & 2.69 & 0.52 \\
\hline 2008 & 10 & 17 & $07: 38: 5712.328$ & 40.609 & 2.92 & 0.37 \\
\hline 2008 & 10 & 17 & $07: 42: 1412.339$ & 40.605 & 3.26 & 0.5 \\
\hline 2008 & 10 & 17 & $07: 55: 0212.354$ & 40.595 & 3.48 & 0.6 \\
\hline 2008 & 10 & 17 & $07: 56: 1412.339$ & 40.609 & 3.78 & 0.59 \\
\hline 2008 & 10 & 17 & $07: 58: 1412.347$ & 40.599 & 3.39 & 0.38 \\
\hline 2008 & 10 & 17 & $08: 03: 1112.328$ & 40.615 & 2.99 & 0.74 \\
\hline 2008 & 10 & 17 & $08: 04: 1112.333$ & 40.610 & 3.30 & 0.4 \\
\hline 2008 & 10 & 17 & $08: 09: 5312.328$ & 40.616 & 3.14 & 0.58 \\
\hline 2008 & 10 & 17 & $08: 19: 0112.339$ & 40.599 & 3.01 & 0.41 \\
\hline 2008 & 10 & 17 & $08: 27: 4412.370$ & 40.601 & 3.02 & 0.62 \\
\hline 2008 & 10 & 17 & $08: 30: 4712.363$ & 40.578 & 2.76 & 0.61 \\
\hline 2008 & 10 & 17 & $08: 37: 1812.316$ & 40.616 & 3.52 & 0.34 \\
\hline 2008 & 10 & 17 & $08: 42: 0512.355$ & 40.592 & 3.71 & 0.44 \\
\hline 2008 & 10 & 17 & $08: 47: 1112.382$ & 40.576 & 3.47 & 0.5 \\
\hline 2008 & 10 & 17 & $08: 55: 3212.370$ & 40.599 & 3.46 & 0.72 \\
\hline 2008 & 10 & 17 & $08: 57: 3512.346$ & 40.600 & 4.27 & 0.51 \\
\hline 2008 & 10 & 17 & $09: 00: 3112.361$ & 40.597 & 3.81 & 0.43 \\
\hline 2008 & 10 & 17 & $09: 03: 0512.382$ & 40.604 & 3.25 & 0.75 \\
\hline 2008 & 10 & 17 & $09: 05: 0912.359$ & 40.592 & 3.70 & 0.49 \\
\hline 2008 & 10 & 17 & $09: 06: 1112.365$ & 40.585 & 3.23 & 1.88 \\
\hline 2008 & 10 & 17 & $09: 07: 3412.363$ & 40.590 & 3.23 & 0.69 \\
\hline 2008 & 10 & 17 & $09: 09: 2412.358$ & 40.592 & 3.80 & 0.33 \\
\hline 2008 & 10 & 17 & $09: 13: 2312.381$ & 40.603 & 3.74 & 0.54 \\
\hline 2008 & 10 & 17 & $09: 20: 5912.355$ & 40.589 & 3.99 & 0.51 \\
\hline 2008 & 10 & 17 & $09: 24: 0512.360$ & 40.585 & 3.67 & 0.4 \\
\hline 2008 & 10 & 17 & $09: 28: 4812.360$ & 40.569 & 3.48 & 0.62 \\
\hline 2008 & 10 & 17 & $09: 30: 2812.400$ & 40.588 & 3.10 & 1.27 \\
\hline 2008 & 10 & 17 & $09: 30: 4512.368$ & 40.595 & 3.59 & 0.7 \\
\hline 2008 & 10 & 17 & $09: 33: 1612.358$ & 40.587 & 3.29 & 0.52 \\
\hline 2008 & 10 & 17 & $09: 40: 3912.354$ & 40.594 & 4.63 & 0.35 \\
\hline 2008 & 10 & 17 & $09: 43: 2412.378$ & 40.592 & 3.81 & 0.48 \\
\hline
\end{tabular}




\begin{tabular}{|c|c|c|c|c|c|c|}
\hline 2008 & 10 & 17 & $09: 48: 5812.370$ & 40.585 & 4.04 & 0.36 \\
\hline 2008 & 10 & 17 & $09: 51: 5912.371$ & 40.587 & 3.17 & 0.47 \\
\hline 2008 & 10 & 17 & $10: 04: 2512.373$ & 40.577 & 3.12 & 0.83 \\
\hline 2008 & 10 & 17 & $10: 06: 3912.374$ & 40.580 & 3.32 & 0.37 \\
\hline 2008 & 10 & 17 & $10: 08: 1212.367$ & 40.572 & 3.39 & 1.29 \\
\hline 2008 & 10 & 17 & $10: 08: 1212.368$ & 40.587 & 3.44 & 0.39 \\
\hline 2008 & 10 & 17 & $10: 13: 1612.383$ & 40.577 & 3.46 & 0.66 \\
\hline 2008 & 10 & 17 & $10: 15: 5112.382$ & 40.577 & 3.28 & 0.52 \\
\hline 2008 & 10 & 17 & $10: 16: 3912.384$ & 40.573 & 3.46 & 0.52 \\
\hline 2008 & 10 & 17 & $10: 24: 0812.375$ & 40.578 & 3.73 & 0.38 \\
\hline 2008 & 10 & 17 & $10: 26: 0812.351$ & 40.586 & 3.93 & 0.47 \\
\hline 2008 & 10 & 17 & $10: 30: 4212.377$ & 40.576 & 3.40 & 0.71 \\
\hline 2008 & 10 & 17 & $10: 47: 3912.382$ & 40.573 & 2.68 & 0.92 \\
\hline 2008 & 10 & 17 & $10: 57: 1412.361$ & 40.592 & 4.66 & 0.48 \\
\hline 2008 & 10 & 17 & $11: 01: 1512.389$ & 40.573 & 3.86 & 0.37 \\
\hline 2008 & 10 & 17 & $11: 02: 3612.386$ & 40.564 & 3.48 & 0.5 \\
\hline 2008 & 10 & 17 & $11: 04: 4712.379$ & 40.566 & 3.66 & 0.37 \\
\hline 2008 & 10 & 17 & $11: 08: 0512.393$ & 40.587 & 3.63 & 0.47 \\
\hline 2008 & 10 & 17 & $11: 15: 1612.393$ & 40.573 & 3.48 & 0.48 \\
\hline 2008 & 10 & 17 & $11: 18: 1812.400$ & 40.585 & 3.80 & 0.41 \\
\hline 2008 & 10 & 17 & $11: 24: 3812.389$ & 40.578 & 3.27 & 1.16 \\
\hline 2008 & 10 & 17 & $11: 28: 3612.396$ & 40.580 & 3.02 & 0.93 \\
\hline 2008 & 10 & 17 & $11: 30: 3412.394$ & 40.575 & 2.82 & 0.67 \\
\hline 2008 & 10 & 17 & $11: 38: 1912.389$ & 40.574 & 3.53 & 0.49 \\
\hline 2008 & 10 & 17 & $11: 40: 1412.402$ & 40.568 & 3.00 & 0.51 \\
\hline 2008 & 10 & 17 & $11: 47: 3912.392$ & 40.572 & 2.99 & 0.64 \\
\hline 2008 & 10 & 17 & $11: 51: 1312.401$ & 40.579 & 3.76 & 0.3 \\
\hline 2008 & 10 & 17 & $12: 02: 0512.395$ & 40.578 & 3.74 & 0.39 \\
\hline 2008 & 10 & 17 & $12: 08: 4812.406$ & 40.561 & 3.22 & 0.48 \\
\hline 2008 & 10 & 17 & $12: 10: 3412.393$ & 40.576 & 3.07 & 0.65 \\
\hline 2008 & 10 & 17 & $12: 12: 0412.383$ & 40.576 & 3.02 & 0.77 \\
\hline 2008 & 10 & 17 & $12: 18: 3612.397$ & 40.574 & 3.52 & 0.42 \\
\hline 2008 & 10 & 17 & $12: 20: 0612.378$ & 40.574 & 3.01 & 0.39 \\
\hline 2008 & 10 & 17 & $12: 24: 4312.375$ & 40.580 & 3.22 & 0.72 \\
\hline 2008 & 10 & 17 & $12: 26: 2312.410$ & 40.569 & 3.39 & 0.5 \\
\hline 2008 & 10 & 17 & $12: 32: 2812.379$ & 40.587 & 2.96 & 0.59 \\
\hline 2008 & 10 & 17 & $12: 39: 0912.401$ & 40.557 & 3.45 & 0.38 \\
\hline 2008 & 10 & 17 & $12: 41: 3412.386$ & 40.569 & 3.46 & 0.43 \\
\hline 2008 & 10 & 17 & $12: 45: 5112.386$ & 40.584 & 3.28 & 0.5 \\
\hline 2008 & 10 & 17 & $12: 47: 4812.437$ & 40.560 & 2.73 & 1.22 \\
\hline 2008 & 10 & 17 & $12: 52: 2512.405$ & 40.578 & 3.59 & 0.61 \\
\hline 2008 & 10 & 17 & $13: 06: 0812.416$ & 40.568 & 4.01 & 0.35 \\
\hline 2008 & 10 & 17 & $13: 21: 0612.420$ & 40.584 & 3.10 & 0.87 \\
\hline 2008 & 10 & 17 & $13: 25: 3612.407$ & 40.568 & 3.15 & 0.41 \\
\hline 2008 & 10 & 17 & $13: 30: 3712.376$ & 40.581 & 2.74 & 0.52 \\
\hline 2008 & 10 & 17 & $13: 32: 2312.410$ & 40.559 & 2.92 & 0.79 \\
\hline 2008 & 10 & 17 & $13: 32: 2312.404$ & 40.572 & 3.69 & 0.37 \\
\hline 2008 & 10 & 17 & $13: 34: 4212.408$ & 40.564 & 3.28 & 0.37 \\
\hline 2008 & 10 & 17 & $13: 37: 5612.392$ & 40.564 & 2.85 & 0.44 \\
\hline 2008 & 10 & 17 & $13: 39: 4112.428$ & 40.574 & 3.32 & 0.47 \\
\hline 2008 & 10 & 17 & $13: 47: 4112.412$ & 40.577 & 2.79 & 0.58 \\
\hline 2008 & 10 & 17 & $13: 53: 2812.413$ & 40.560 & 2.94 & 0.8 \\
\hline 2008 & 10 & 17 & $13: 53: 5912.375$ & 40.590 & 2.86 & 0.38 \\
\hline 2008 & 10 & 17 & $13: 55: 5112.415$ & 40.561 & 3.33 & 0.64 \\
\hline 2008 & 10 & 17 & $13: 58: 4912.401$ & 40.586 & 3.34 & 0.33 \\
\hline 2008 & 10 & 17 & $14: 01: 1512.392$ & 40.583 & 2.81 & 0.41 \\
\hline 2008 & 10 & 17 & $14: 05: 0112.400$ & 40.574 & 3.17 & 0.62 \\
\hline 2008 & 10 & 17 & $14: 08: 3112.415$ & 40.554 & 2.89 & 0.75 \\
\hline 2008 & 10 & 17 & $14: 10: 1512.370$ & 40.588 & 2.80 & 0.37 \\
\hline 2008 & 10 & 17 & $14: 17: 5812.417$ & 40.573 & 2.61 & 0.8 \\
\hline 2008 & 10 & 17 & $14: 22: 3612.394$ & 40.553 & 3.01 & 0.48 \\
\hline 2008 & 10 & 17 & $14: 27: 0412.404$ & 40.551 & 2.43 & 0.58 \\
\hline 2008 & 10 & 17 & $14: 29: 4112.392$ & 40.579 & 2.92 & 0.38 \\
\hline 2008 & 10 & 17 & $14: 31: 2812.402$ & 40.566 & 3.63 & 0.56 \\
\hline 2008 & 10 & 17 & $14: 36: 3912.404$ & 40.575 & 2.84 & 0.56 \\
\hline 2008 & 10 & 17 & $14: 39: 0212.426$ & 40.578 & 2.67 & 0.45 \\
\hline 2008 & 10 & 17 & $14: 44: 3012.426$ & 40.580 & 2.67 & 0.53 \\
\hline 2008 & 10 & 17 & $14: 47: 1312.403$ & 40.570 & 3.59 & 0.33 \\
\hline 2008 & 10 & 17 & $14: 49: 3112.388$ & 40.570 & 2.58 & 0.48 \\
\hline 2008 & 10 & 17 & $15: 01: 4412.412$ & 40.567 & 3.31 & 0.44 \\
\hline 2008 & 10 & 17 & $15: 03: 3712.375$ & 40.580 & 2.66 & 0.56 \\
\hline 2008 & 10 & 17 & $15: 21: 1612.405$ & 40.564 & 3.36 & 0.46 \\
\hline 2008 & 10 & 17 & $15: 24: 3612.407$ & 40.579 & 2.63 & 0.46 \\
\hline 2008 & 10 & 17 & $15: 27: 1612.426$ & 40.566 & 2.99 & 0.55 \\
\hline 2008 & 10 & 17 & $15: 38: 0112.426$ & 40.551 & 2.41 & 0.88 \\
\hline 2008 & 10 & 17 & $15: 39: 1012.423$ & 40.581 & 2.95 & 0.43 \\
\hline
\end{tabular}




\begin{tabular}{|c|c|c|c|c|c|c|}
\hline 2008 & 10 & 17 & $15: 39: 4112.396$ & 40.565 & 3.24 & 0.46 \\
\hline 2008 & 10 & 17 & $15: 43: 0412.406$ & 40.563 & 2.47 & 1.24 \\
\hline 2008 & 10 & 17 & $15: 45: 2012.405$ & 40.567 & 4.34 & 0.27 \\
\hline 2008 & 10 & 17 & $15: 50: 5612.378$ & 40.566 & 2.63 & 0.61 \\
\hline 2008 & 10 & 17 & $15: 53: 0512.401$ & 40.585 & 2.50 & 0.97 \\
\hline 2008 & 10 & 17 & $15: 55: 1212.418$ & 40.582 & 2.38 & 0.59 \\
\hline 2008 & 10 & 17 & $15: 57: 5412.379$ & 40.575 & 2.46 & 0.58 \\
\hline 2008 & 10 & 17 & $16: 02: 4812.410$ & 40.567 & 3.52 & 0.46 \\
\hline 2008 & 10 & 17 & $16: 04: 1312.432$ & 40.572 & 2.87 & 0.55 \\
\hline 2008 & 10 & 17 & $16: 06: 5112.408$ & 40.564 & 2.30 & 0.85 \\
\hline 2008 & 10 & 17 & $16: 12: 3512.378$ & 40.583 & 2.74 & 0.76 \\
\hline 2008 & 10 & 17 & $16: 20: 4112.384$ & 40.584 & 2.71 & 0.39 \\
\hline 2008 & 10 & 17 & $16: 26: 3812.380$ & 40.574 & 2.44 & 0.4 \\
\hline 2008 & 10 & 17 & $16: 27: 5512.388$ & 40.579 & 2.84 & 0.41 \\
\hline 2008 & 10 & 17 & $16: 29: 1112.382$ & 40.569 & 3.37 & 0.5 \\
\hline 2008 & 10 & 17 & $16: 32: 4412.415$ & 40.569 & 3.25 & 0.35 \\
\hline 2008 & 10 & 17 & $16: 37: 1512.411$ & 40.568 & 2.45 & 0.79 \\
\hline 2008 & 10 & 17 & $16: 40: 1812.400$ & 40.563 & 2.72 & 0.41 \\
\hline 2008 & 10 & 17 & $16: 41: 1912.404$ & 40.561 & 2.52 & 0.53 \\
\hline 2008 & 10 & 17 & $16: 44: 1512.399$ & 40.575 & 2.52 & 0.51 \\
\hline 2008 & 10 & 17 & $16: 46: 0112.390$ & 40.578 & 3.05 & 0.4 \\
\hline 2008 & 10 & 17 & $16: 47: 3912.406$ & 40.571 & 3.35 & 0.33 \\
\hline 2008 & 10 & 17 & $16: 53: 3212.416$ & 40.564 & 2.16 & 0.94 \\
\hline 2008 & 10 & 17 & $16: 54: 3712.350$ & 40.574 & 2.30 & 1.85 \\
\hline 2008 & 10 & 17 & $16: 56: 5512.405$ & 40.575 & 2.18 & 0.55 \\
\hline 2008 & 10 & 17 & $16: 59: 3612.387$ & 40.573 & 2.60 & 0.37 \\
\hline 2008 & 10 & 17 & $17: 04: 1312.352$ & 40.599 & 2.57 & 0.78 \\
\hline 2008 & 10 & 17 & $17: 07: 5112.418$ & 40.547 & 2.50 & 0.55 \\
\hline 2008 & 10 & 17 & $17: 12: 4812.364$ & 40.594 & 2.59 & 0.4 \\
\hline 2008 & 10 & 17 & $17: 14: 5812.368$ & 40.596 & 2.40 & 0.57 \\
\hline 2008 & 10 & 17 & $17: 17: 1912.407$ & 40.570 & 2.35 & 0.56 \\
\hline 2008 & 10 & 17 & $17: 21: 1112.353$ & 40.595 & 2.86 & 0.41 \\
\hline 2008 & 10 & 17 & $17: 24: 1612.409$ & 40.565 & 3.20 & 0.37 \\
\hline 2008 & 10 & 17 & $17: 27: 1712.374$ & 40.572 & 3.01 & 0.44 \\
\hline 2008 & 10 & 17 & $17: 30: 4712.336$ & 40.620 & 2.43 & 0.79 \\
\hline 2008 & 10 & 17 & $17: 36: 1812.372$ & 40.579 & 2.33 & 0.54 \\
\hline 2008 & 10 & 17 & $17: 39: 4812.402$ & 40.567 & 3.38 & 0.39 \\
\hline 2008 & 10 & 17 & $17: 43: 2312.380$ & 40.573 & 2.33 & 0.46 \\
\hline 2008 & 10 & 17 & $17: 45: 5612.416$ & 40.566 & 2.37 & 0.79 \\
\hline 2008 & 10 & 17 & $17: 50: 2012.409$ & 40.560 & 2.41 & 0.67 \\
\hline 2008 & 10 & 17 & $17: 53: 2312.406$ & 40.563 & 2.60 & 0.63 \\
\hline 2008 & 10 & 17 & $17: 53: 5112.402$ & 40.571 & 3.19 & 0.37 \\
\hline 2008 & 10 & 17 & $18: 02: 2012.401$ & 40.571 & 2.70 & 0.5 \\
\hline 2008 & 10 & 17 & $18: 07: 3312.401$ & 40.543 & 2.74 & 0.61 \\
\hline 2008 & 10 & 17 & $18: 11: 4912.384$ & 40.582 & 2.59 & 0.38 \\
\hline 2008 & 10 & 17 & $18: 13: 2712.379$ & 40.586 & 2.84 & 0.43 \\
\hline 2008 & 10 & 17 & $18: 14: 5912.400$ & 40.586 & 2.61 & 0.46 \\
\hline 2008 & 10 & 17 & $18: 24: 1912.379$ & 40.584 & 2.49 & 0.39 \\
\hline 2008 & 10 & 17 & $18: 28: 3712.366$ & 40.588 & 2.69 & 0.42 \\
\hline 2008 & 10 & 17 & $18: 31: 2212.381$ & 40.577 & 2.96 & 0.39 \\
\hline 2008 & 10 & 17 & $18: 37: 4812.393$ & 40.580 & 2.35 & 0.62 \\
\hline 2008 & 10 & 17 & $18: 39: 2112.376$ & 40.596 & 2.45 & 0.46 \\
\hline 2008 & 10 & 17 & $18: 39: 4312.355$ & 40.593 & 3.17 & 0.46 \\
\hline 2008 & 10 & 17 & $18: 41: 0812.382$ & 40.575 & 2.61 & 0.51 \\
\hline 2008 & 10 & 17 & $18: 44: 2712.387$ & 40.573 & 2.62 & 0.61 \\
\hline 2008 & 10 & 17 & $18: 48: 4012.364$ & 40.592 & 2.63 & 0.42 \\
\hline 2008 & 10 & 17 & $18: 53: 2512.388$ & 40.565 & 2.54 & 0.36 \\
\hline 2008 & 10 & 17 & $18: 54: 5712.402$ & 40.568 & 3.20 & 0.35 \\
\hline 2008 & 10 & 17 & $19: 01: 5212.389$ & 40.574 & 2.62 & 0.43 \\
\hline 2008 & 10 & 17 & $19: 02: 3112.373$ & 40.585 & 2.80 & 0.71 \\
\hline 2008 & 10 & 17 & $19: 04: 0412.403$ & 40.559 & 2.39 & 0.5 \\
\hline 2008 & 10 & 17 & $19: 13: 3412.350$ & 40.603 & 2.65 & 0.3 \\
\hline 2008 & 10 & 17 & $19: 16: 3012.412$ & 40.565 & 2.42 & 0.66 \\
\hline 2008 & 10 & 17 & $19: 22: 0712.369$ & 40.605 & 2.55 & 0.42 \\
\hline 2008 & 10 & 17 & $19: 25: 5612.370$ & 40.588 & 2.81 & 0.5 \\
\hline 2008 & 10 & 17 & $19: 32: 2412.454$ & 40.614 & 2.69 & 1.45 \\
\hline 2008 & 10 & 17 & $19: 34: 2812.376$ & 40.581 & 2.64 & 0.94 \\
\hline 2008 & 10 & 17 & $19: 35: 1412.361$ & 40.600 & 3.07 & 0.42 \\
\hline 2008 & 10 & 17 & $19: 42: 4612.402$ & 40.571 & 2.56 & 0.36 \\
\hline 2008 & 10 & 17 & $19: 52: 1712.320$ & 40.620 & 2.39 & 0.48 \\
\hline 2008 & 10 & 17 & $19: 55: 4812.404$ & 40.568 & 2.13 & 0.78 \\
\hline 2008 & 10 & 17 & $19: 57: 5012.351$ & 40.597 & 2.60 & 0.34 \\
\hline 2008 & 10 & 17 & $20: 01: 4812.402$ & 40.570 & 2.49 & 0.57 \\
\hline 2008 & 10 & 17 & $20: 04: 2412.395$ & 40.571 & 2.67 & 0.42 \\
\hline 2008 & 10 & 17 & $20: 09: 4612.405$ & 40.565 & 2.92 & 0.52 \\
\hline 2008 & 10 & 17 & $20: 10: 4112.387$ & 40.568 & 2.52 & 0.45 \\
\hline
\end{tabular}




\begin{tabular}{|c|c|c|c|c|c|c|}
\hline 2008 & 10 & 17 & $20: 16: 0412.389$ & 40.583 & 2.69 & 0.48 \\
\hline 2008 & 10 & 17 & $20: 18: 2212.373$ & 40.582 & 2.64 & 0.57 \\
\hline 2008 & 10 & 17 & $20: 20: 2312.405$ & 40.560 & 2.31 & 1.93 \\
\hline 2008 & 10 & 17 & $20: 23: 5112.358$ & 40.589 & 2.73 & 0.44 \\
\hline 2008 & 10 & 17 & $20: 24: 2412.359$ & 40.592 & 4.15 & 0.31 \\
\hline 2008 & 10 & 17 & $20: 28: 0112.425$ & 40.579 & 3.02 & 0.66 \\
\hline 2008 & 10 & 17 & $20: 33: 0712.430$ & 40.561 & 2.33 & 0.4 \\
\hline 2008 & 10 & 17 & $20: 33: 5512.403$ & 40.578 & 2.42 & 0.54 \\
\hline 2008 & 10 & 17 & $20: 43: 1012.381$ & 40.574 & 2.66 & 0.33 \\
\hline 2008 & 10 & 17 & $20: 44: 2412.373$ & 40.581 & 2.30 & 0.49 \\
\hline 2008 & 10 & 17 & $20: 47: 1912.387$ & 40.569 & 2.02 & 0.72 \\
\hline 2008 & 10 & 17 & $20: 49: 5612.361$ & 40.588 & 2.77 & 0.32 \\
\hline 2008 & 10 & 17 & $20: 51: 1412.391$ & 40.573 & 2.96 & 0.4 \\
\hline 2008 & 10 & 17 & $20: 55: 4212.378$ & 40.591 & 2.71 & 0.33 \\
\hline 2008 & 10 & 17 & 21:03:12 12.392 & 40.588 & 2.42 & 0.39 \\
\hline 2008 & 10 & 17 & $21: 04: 3112.350$ & 40.594 & 2.64 & 0.57 \\
\hline 2008 & 10 & 17 & $21: 07: 1312.388$ & 40.585 & 2.28 & 0.52 \\
\hline 2008 & 10 & 17 & $21: 08: 2912.424$ & 40.559 & 2.59 & 0.43 \\
\hline 2008 & 10 & 17 & $21: 16: 3712.335$ & 40.609 & 2.31 & 0.42 \\
\hline 2008 & 10 & 17 & $21: 17: 1512.346$ & 40.592 & 2.20 & 0.64 \\
\hline 2008 & 10 & 17 & $21: 22: 4112.415$ & 40.569 & 2.19 & 0.47 \\
\hline 2008 & 10 & 17 & $21: 28: 1412.356$ & 40.596 & 2.48 & 0.35 \\
\hline 2008 & 10 & 17 & $21: 30: 4012.415$ & 40.565 & 2.66 & 0.31 \\
\hline 2008 & 10 & 17 & $21: 32: 2812.352$ & 40.609 & 2.54 & 0.4 \\
\hline 2008 & 10 & 17 & $21: 33: 5712.426$ & 40.574 & 2.83 & 0.38 \\
\hline 2008 & 10 & 17 & $21: 40: 1512.374$ & 40.582 & 2.55 & 0.35 \\
\hline 2008 & 10 & 17 & $21: 42: 0612.382$ & 40.580 & 2.40 & 0.4 \\
\hline 2008 & 10 & 17 & $21: 46: 3212.389$ & 40.557 & 2.09 & 0.67 \\
\hline 2008 & 10 & 17 & $21: 52: 1512.354$ & 40.586 & 2.38 & 0.31 \\
\hline 2008 & 10 & 17 & $21: 56: 1412.413$ & 40.569 & 3.44 & 0.38 \\
\hline 2008 & 10 & 17 & $21: 58: 1512.415$ & 40.576 & 2.97 & 0.37 \\
\hline 2008 & 10 & 17 & $22: 01: 4912.398$ & 40.563 & 2.07 & 0.85 \\
\hline 2008 & 10 & 17 & $22: 18: 0612.384$ & 40.585 & 2.60 & 0.52 \\
\hline 2008 & 10 & 17 & $22: 24: 4512.358$ & 40.590 & 2.60 & 0.47 \\
\hline 2008 & 10 & 17 & $22: 31: 2812.432$ & 40.554 & 2.00 & 0.64 \\
\hline 2008 & 10 & 17 & $22: 33: 4312.358$ & 40.591 & 2.08 & 0.45 \\
\hline 2008 & 10 & 17 & $22: 37: 3112.380$ & 40.581 & 2.46 & 0.51 \\
\hline 2008 & 10 & 17 & $22: 44: 4512.375$ & 40.583 & 2.04 & 0.78 \\
\hline 2008 & 10 & 17 & $22: 46: 4412.366$ & 40.592 & 2.59 & 0.51 \\
\hline 2008 & 10 & 17 & $22: 51: 3412.397$ & 40.571 & 2.12 & 0.66 \\
\hline 2008 & 10 & 17 & $22: 53: 1512.368$ & 40.617 & 2.02 & 0.49 \\
\hline 2008 & 10 & 17 & $22: 58: 3512.342$ & 40.594 & 2.16 & 0.77 \\
\hline 2008 & 10 & 17 & $23: 04: 5812.355$ & 40.596 & 1.94 & 0.54 \\
\hline 2008 & 10 & 17 & $23: 07: 0812.363$ & 40.590 & 2.03 & 0.7 \\
\hline 2008 & 10 & 17 & $23: 10: 0312.342$ & 40.616 & 2.12 & 0.42 \\
\hline 2008 & 10 & 17 & $23: 12: 2512.407$ & 40.574 & 2.09 & 0.46 \\
\hline 2008 & 10 & 17 & $23: 14: 5312.358$ & 40.604 & 2.21 & 0.4 \\
\hline 2008 & 10 & 17 & $23: 18: 4312.373$ & 40.585 & 2.75 & 0.38 \\
\hline 2008 & 10 & 17 & $23: 20: 5512.377$ & 40.580 & 2.87 & 0.32 \\
\hline 2008 & 10 & 17 & $23: 25: 0712.386$ & 40.582 & 2.80 & 0.43 \\
\hline 2008 & 10 & 17 & $23: 29: 3812.358$ & 40.603 & 3.23 & 0.34 \\
\hline 2008 & 10 & 17 & $23: 37: 0612.393$ & 40.571 & 2.76 & 0.45 \\
\hline 2008 & 10 & 17 & $23: 41: 1312.407$ & 40.566 & 2.68 & 0.35 \\
\hline 2008 & 10 & 17 & $23: 48: 2812.386$ & 40.574 & 2.37 & 0.4 \\
\hline 2008 & 10 & 17 & $23: 51: 3712.453$ & 40.563 & 2.47 & 0.4 \\
\hline 2008 & 10 & 17 & $23: 54: 2512.411$ & 40.576 & 2.00 & 1.12 \\
\hline 2008 & 10 & 17 & $23: 58: 4112.411$ & 40.579 & 2.01 & 0.74 \\
\hline 2008 & 10 & 18 & $00: 05: 4512.389$ & 40.577 & 2.34 & 0.45 \\
\hline 2008 & 10 & 18 & $00: 08: 4712.388$ & 40.578 & 2.42 & 0.39 \\
\hline 2008 & 10 & 18 & $00: 10: 5412.356$ & 40.600 & 2.25 & 0.36 \\
\hline 2008 & 10 & 18 & $00: 14: 1512.418$ & 40.569 & 2.50 & 0.5 \\
\hline 2008 & 10 & 18 & $00: 15: 4412.356$ & 40.604 & 2.18 & 0.61 \\
\hline 2008 & 10 & 18 & $00: 17: 1012.378$ & 40.578 & 2.20 & 0.45 \\
\hline 2008 & 10 & 18 & $00: 20: 4512.354$ & 40.608 & 2.50 & 0.42 \\
\hline 2008 & 10 & 18 & $00: 23: 4712.372$ & 40.586 & 2.46 & 0.36 \\
\hline 2008 & 10 & 18 & $00: 25: 3712.382$ & 40.574 & 2.34 & 0.51 \\
\hline 2008 & 10 & 18 & $00: 30: 4212.351$ & 40.592 & 2.28 & 0.36 \\
\hline 2008 & 10 & 18 & $00: 31: 5112.405$ & 40.569 & 2.41 & 0.43 \\
\hline 2008 & 10 & 18 & $00: 35: 2512.369$ & 40.591 & 2.17 & 0.43 \\
\hline 2008 & 10 & 18 & $00: 42: 4212.358$ & 40.579 & 2.12 & 0.41 \\
\hline 2008 & 10 & 18 & $00: 45: 1512.386$ & 40.584 & 2.29 & 0.52 \\
\hline 2008 & 10 & 18 & $00: 58: 4712.384$ & 40.583 & 1.99 & 0.4 \\
\hline 2008 & 10 & 18 & $00: 59: 4912.371$ & 40.586 & 2.08 & 0.37 \\
\hline 2008 & 10 & 18 & $01: 01: 2912.352$ & 40.592 & 2.15 & 0.41 \\
\hline 2008 & 10 & 18 & $01: 03: 3012.349$ & 40.595 & 2.24 & 0.4 \\
\hline 2008 & 10 & 18 & $01: 06: 3212.389$ & 40.571 & 2.58 & 0.3 \\
\hline
\end{tabular}




\begin{tabular}{|c|c|c|c|c|c|c|}
\hline 2008 & 10 & 18 & $01: 11: 3312.346$ & 40.593 & 2.42 & 0.3 \\
\hline 2008 & 10 & 18 & $01: 13: 5112.357$ & 40.587 & 2.52 & 0.36 \\
\hline 2008 & 10 & 18 & $01: 17: 4312.348$ & 40.588 & 2.02 & 0.66 \\
\hline 2008 & 10 & 18 & $01: 20: 4312.371$ & 40.583 & 1.98 & 0.59 \\
\hline 2008 & 10 & 18 & $01: 28: 0212.392$ & 40.581 & 1.94 & 0.63 \\
\hline 2008 & 10 & 18 & $01: 38: 5812.357$ & 40.592 & 2.18 & 0.4 \\
\hline 2008 & 10 & 18 & $01: 47: 0912.350$ & 40.595 & 1.97 & 0.46 \\
\hline 2008 & 10 & 18 & $01: 47: 4812.352$ & 40.591 & 2.27 & 0.51 \\
\hline 2008 & 10 & 18 & $01: 49: 0112.387$ & 40.572 & 2.03 & 0.38 \\
\hline 2008 & 10 & 18 & $01: 54: 0212.379$ & 40.579 & 2.64 & 0.33 \\
\hline 2008 & 10 & 18 & $01: 55: 3212.369$ & 40.587 & 2.14 & 0.44 \\
\hline 2008 & 10 & 18 & $01: 56: 2712.355$ & 40.600 & 2.23 & 0.64 \\
\hline 2008 & 10 & 18 & $01: 57: 3312.388$ & 40.583 & 2.47 & 0.46 \\
\hline 2008 & 10 & 18 & $01: 58: 4312.593$ & 40.513 & 2.81 & 0.61 \\
\hline 2008 & 10 & 18 & $02: 05: 4012.351$ & 40.613 & 2.43 & 0.5 \\
\hline 2008 & 10 & 18 & $02: 07: 1412.380$ & 40.575 & 2.67 & 0.46 \\
\hline 2008 & 10 & 18 & $02: 11: 0312.399$ & 40.570 & 2.09 & 0.71 \\
\hline 2008 & 10 & 18 & $02: 12: 5912.393$ & 40.511 & 1.87 & 0.77 \\
\hline 2008 & 10 & 18 & $02: 18: 1012.357$ & 40.598 & 2.53 & 0.33 \\
\hline 2008 & 10 & 18 & $02: 20: 4412.385$ & 40.569 & 2.04 & 0.88 \\
\hline 2008 & 10 & 18 & $02: 22: 3812.362$ & 40.584 & 2.01 & 0.46 \\
\hline 2008 & 10 & 18 & $02: 30: 5612.438$ & 40.560 & 2.44 & 0.71 \\
\hline 2008 & 10 & 18 & $02: 32: 2712.340$ & 40.619 & 2.19 & 0.49 \\
\hline 2008 & 10 & 18 & $02: 36: 2612.347$ & 40.585 & 2.68 & 0.36 \\
\hline 2008 & 10 & 18 & $02: 42: 2512.389$ & 40.570 & 2.05 & 0.51 \\
\hline 2008 & 10 & 18 & $02: 44: 2712.370$ & 40.586 & 2.51 & 0.64 \\
\hline 2008 & 10 & 18 & $02: 54: 4612.369$ & 40.594 & 2.49 & 0.4 \\
\hline 2008 & 10 & 18 & $02: 57: 5212.393$ & 40.576 & 2.15 & 0.39 \\
\hline 2008 & 10 & 18 & $03: 03: 5612.367$ & 40.594 & 2.11 & 0.67 \\
\hline 2008 & 10 & 18 & $03: 05: 0612.356$ & 40.592 & 2.38 & 0.35 \\
\hline 2008 & 10 & 18 & $03: 09: 3612.374$ & 40.583 & 2.32 & 0.41 \\
\hline 2008 & 10 & 18 & $03: 16: 1012.371$ & 40.598 & 2.16 & 0.53 \\
\hline 2008 & 10 & 18 & $03: 28: 4912.381$ & 40.578 & 2.63 & 0.38 \\
\hline 2008 & 10 & 18 & $03: 30: 1812.330$ & 40.607 & 2.31 & 0.46 \\
\hline 2008 & 10 & 18 & $03: 32: 2012.372$ & 40.587 & 2.90 & 0.32 \\
\hline 2008 & 10 & 18 & $03: 48: 5712.388$ & 40.580 & 2.54 & 0.41 \\
\hline 2008 & 10 & 18 & $03: 50: 2312.352$ & 40.611 & 2.46 & 0.51 \\
\hline 2008 & 10 & 18 & $03: 52: 4612.306$ & 40.598 & 2.25 & 0.58 \\
\hline 2008 & 10 & 18 & $03: 59: 3112.387$ & 40.579 & 2.37 & 0.7 \\
\hline 2008 & 10 & 18 & $04: 02: 3812.387$ & 40.583 & 3.18 & 0.32 \\
\hline 2008 & 10 & 18 & $04: 08: 1612.417$ & 40.574 & 2.40 & 0.36 \\
\hline 2008 & 10 & 18 & $04: 16: 2112.407$ & 40.576 & 2.10 & 0.94 \\
\hline 2008 & 10 & 18 & $04: 21: 2912.407$ & 40.576 & 2.08 & 0.56 \\
\hline 2008 & 10 & 18 & $04: 23: 4412.342$ & 40.590 & 2.67 & 0.4 \\
\hline 2008 & 10 & 18 & $04: 26: 2812.354$ & 40.583 & 2.45 & 0.62 \\
\hline 2008 & 10 & 18 & $04: 29: 5412.375$ & 40.581 & 2.34 & 0.46 \\
\hline 2008 & 10 & 18 & $04: 43: 5112.370$ & 40.590 & 2.25 & 0.7 \\
\hline 2008 & 10 & 18 & $04: 47: 4712.349$ & 40.605 & 2.21 & 0.55 \\
\hline 2008 & 10 & 18 & $04: 54: 0012.348$ & 40.594 & 2.50 & 0.53 \\
\hline 2008 & 10 & 18 & $04: 57: 5212.370$ & 40.589 & 2.18 & 0.52 \\
\hline 2008 & 10 & 18 & $04: 58: 3312.387$ & 40.576 & 2.47 & 0.42 \\
\hline 2008 & 10 & 18 & $05: 05: 3412.431$ & 40.571 & 2.64 & 0.41 \\
\hline 2008 & 10 & 18 & $05: 09: 3012.359$ & 40.603 & 2.27 & 1.39 \\
\hline 2008 & 10 & 18 & $05: 13: 0812.349$ & 40.590 & 2.04 & 0.51 \\
\hline 2008 & 10 & 18 & $05: 14: 3112.410$ & 40.560 & 2.19 & 0.65 \\
\hline 2008 & 10 & 18 & $05: 18: 3712.370$ & 40.588 & 2.89 & 0.41 \\
\hline 2008 & 10 & 18 & $05: 21: 3112.382$ & 40.579 & 2.17 & 0.54 \\
\hline 2008 & 10 & 18 & $05: 34: 0412.367$ & 40.567 & 2.33 & 0.57 \\
\hline 2008 & 10 & 18 & $05: 43: 0012.378$ & 40.573 & 2.07 & 0.44 \\
\hline 2008 & 10 & 18 & $05: 58: 1212.382$ & 40.572 & 2.32 & 0.39 \\
\hline 2008 & 10 & 18 & $06: 09: 2712.396$ & 40.560 & 2.37 & 0.68 \\
\hline 2008 & 10 & 18 & $06: 14: 3012.406$ & 40.563 & 2.16 & 0.49 \\
\hline 2008 & 10 & 18 & $06: 17: 2612.401$ & 40.569 & 2.27 & 0.54 \\
\hline 2008 & 10 & 18 & $06: 19: 1812.372$ & 40.583 & 2.21 & 0.43 \\
\hline 2008 & 10 & 18 & $06: 29: 0312.410$ & 40.560 & 2.81 & 0.34 \\
\hline 2008 & 10 & 18 & $06: 29: 5812.374$ & 40.584 & 2.60 & 0.43 \\
\hline 2008 & 10 & 18 & $06: 48: 0212.397$ & 40.569 & 2.49 & 0.42 \\
\hline 2008 & 10 & 18 & $06: 49: 0012.391$ & 40.543 & 2.22 & 2.37 \\
\hline 2008 & 10 & 18 & $06: 53: 1912.383$ & 40.577 & 2.38 & 0.41 \\
\hline 2008 & 10 & 18 & $06: 53: 5712.340$ & 40.593 & 2.34 & 0.45 \\
\hline 2008 & 10 & 18 & $07: 01: 2012.349$ & 40.592 & 2.26 & 0.5 \\
\hline 2008 & 10 & 18 & $07: 07: 3512.391$ & 40.563 & 2.17 & 0.66 \\
\hline 2008 & 10 & 18 & $07: 13: 2412.391$ & 40.579 & 3.09 & 0.38 \\
\hline 2008 & 10 & 18 & $07: 23: 5112.355$ & 40.594 & 2.16 & 0.48 \\
\hline 2008 & 10 & 18 & $07: 36: 3512.392$ & 40.561 & 2.36 & 1.04 \\
\hline 2008 & 10 & 18 & $07: 40: 3212.354$ & 40.596 & 2.33 & 0.35 \\
\hline
\end{tabular}




\begin{tabular}{|c|c|c|c|c|c|c|}
\hline 2008 & 10 & 18 & $07: 51: 0612.358$ & 40.605 & 2.21 & 0.47 \\
\hline 2008 & 10 & 18 & $07: 56: 0812.339$ & 40.600 & 2.41 & 0.39 \\
\hline 2008 & 10 & 18 & $08: 03: 4412.328$ & 40.593 & 2.08 & 0.47 \\
\hline 2008 & 10 & 18 & $08: 08: 5312.369$ & 40.587 & 2.10 & 0.92 \\
\hline 2008 & 10 & 18 & $08: 10: 2112.372$ & 40.581 & 2.11 & 0.82 \\
\hline 2008 & 10 & 18 & $08: 17: 3512.387$ & 40.599 & 2.15 & 0.77 \\
\hline 2008 & 10 & 18 & $08: 27: 0812.344$ & 40.591 & 2.45 & 0.74 \\
\hline 2008 & 10 & 18 & $08: 30: 3812.390$ & 40.567 & 2.29 & 0.75 \\
\hline 2008 & 10 & 18 & $08: 34: 0812.362$ & 40.582 & 2.08 & 0.58 \\
\hline 2008 & 10 & 18 & $08: 44: 0912.380$ & 40.560 & 2.29 & 1.18 \\
\hline 2008 & 10 & 18 & $08: 51: 0912.372$ & 40.581 & 1.97 & 0.63 \\
\hline 2008 & 10 & 18 & $08: 53: 1712.377$ & 40.581 & 2.26 & 0.97 \\
\hline 2008 & 10 & 18 & $08: 57: 2312.361$ & 40.583 & 2.23 & 0.41 \\
\hline 2008 & 10 & 18 & $09: 02: 3712.360$ & 40.586 & 2.07 & 0.57 \\
\hline 2008 & 10 & 18 & $09: 03: 1912.354$ & 40.599 & 2.38 & 0.67 \\
\hline 2008 & 10 & 18 & $09: 04: 5912.378$ & 40.581 & 1.98 & 0.91 \\
\hline 2008 & 10 & 18 & $09: 15: 2312.381$ & 40.586 & 1.95 & 0.69 \\
\hline 2008 & 10 & 18 & $09: 18: 0612.370$ & 40.560 & 2.05 & 0.47 \\
\hline 2008 & 10 & 18 & $09: 24: 1712.354$ & 40.599 & 2.68 & 0.33 \\
\hline 2008 & 10 & 18 & $09: 26: 2212.358$ & 40.593 & 2.14 & 0.42 \\
\hline 2008 & 10 & 18 & $09: 30: 2712.357$ & 40.646 & 2.09 & 0.97 \\
\hline 2008 & 10 & 18 & $09: 32: 5612.405$ & 40.576 & 1.99 & 0.53 \\
\hline 2008 & 10 & 18 & $09: 36: 3012.348$ & 40.606 & 2.60 & 0.37 \\
\hline 2008 & 10 & 18 & $09: 38: 1912.370$ & 40.584 & 2.67 & 0.35 \\
\hline 2008 & 10 & 18 & $09: 40: 4312.371$ & 40.557 & 2.17 & 0.67 \\
\hline 2008 & 10 & 18 & $09: 41: 3212.345$ & 40.604 & 2.55 & 0.54 \\
\hline 2008 & 10 & 18 & $09: 47: 2812.361$ & 40.587 & 2.32 & 0.45 \\
\hline 2008 & 10 & 18 & $09: 53: 2212.346$ & 40.594 & 2.38 & 0.41 \\
\hline 2008 & 10 & 18 & $10: 01: 1312.349$ & 40.590 & 2.51 & 0.36 \\
\hline 2008 & 10 & 18 & $10: 02: 2012.354$ & 40.595 & 2.63 & 0.4 \\
\hline 2008 & 10 & 18 & $10: 06: 4512.382$ & 40.583 & 2.71 & 0.68 \\
\hline 2008 & 10 & 18 & $10: 15: 4812.383$ & 40.584 & 2.13 & 0.62 \\
\hline 2008 & 10 & 18 & $10: 23: 4612.342$ & 40.599 & 2.07 & 0.54 \\
\hline 2008 & 10 & 18 & $10: 40: 5612.372$ & 40.590 & 2.42 & 0.4 \\
\hline 2008 & 10 & 18 & $10: 49: 4012.381$ & 40.573 & 2.01 & 0.96 \\
\hline 2008 & 10 & 18 & $10: 57: 5512.382$ & 40.585 & 2.13 & 0.5 \\
\hline 2008 & 10 & 18 & $11: 03: 1412.349$ & 40.591 & 2.23 & 0.4 \\
\hline 2008 & 10 & 18 & $11: 04: 3312.350$ & 40.595 & 2.41 & 0.46 \\
\hline 2008 & 10 & 18 & $11: 07: 1712.389$ & 40.577 & 2.84 & 0.62 \\
\hline 2008 & 10 & 18 & $11: 13: 1012.358$ & 40.591 & 2.24 & 0.48 \\
\hline 2008 & 10 & 18 & $11: 29: 4312.389$ & 40.569 & 2.37 & 0.71 \\
\hline 2008 & 10 & 18 & $11: 31: 1212.393$ & 40.574 & 2.60 & 0.38 \\
\hline 2008 & 10 & 18 & $11: 41: 0912.386$ & 40.575 & 2.34 & 0.41 \\
\hline 2008 & 10 & 18 & $11: 59: 3512.341$ & 40.593 & 2.78 & 0.45 \\
\hline 2008 & 10 & 18 & $12: 03: 0412.343$ & 40.595 & 2.64 & 0.5 \\
\hline 2008 & 10 & 18 & $12: 10: 1812.399$ & 40.580 & 2.28 & 0.78 \\
\hline 2008 & 10 & 18 & $12: 33: 5712.403$ & 40.576 & 2.19 & 0.56 \\
\hline 2008 & 10 & 18 & $12: 36: 2312.406$ & 40.560 & 2.00 & 0.44 \\
\hline 2008 & 10 & 18 & $12: 43: 1812.396$ & 40.574 & 2.59 & 0.6 \\
\hline 2008 & 10 & 18 & $12: 45: 3112.385$ & 40.576 & 2.71 & 0.86 \\
\hline 2008 & 10 & 18 & $12: 56: 2312.358$ & 40.592 & 2.65 & 0.53 \\
\hline 2008 & 10 & 18 & $12: 57: 0912.363$ & 40.589 & 4.12 & 0.5 \\
\hline 2008 & 10 & 18 & $13: 05: 2912.348$ & 40.592 & 2.70 & 0.46 \\
\hline 2008 & 10 & 18 & $13: 11: 5612.415$ & 40.562 & 2.32 & 0.81 \\
\hline 2008 & 10 & 18 & $13: 16: 3112.412$ & 40.564 & 2.14 & 0.58 \\
\hline 2008 & 10 & 18 & $13: 19: 2712.385$ & 40.588 & 2.53 & 0.52 \\
\hline 2008 & 10 & 18 & $13: 30: 2412.361$ & 40.597 & 2.83 & 0.43 \\
\hline 2008 & 10 & 18 & $13: 38: 5312.342$ & 40.592 & 2.15 & 0.58 \\
\hline 2008 & 10 & 18 & $14: 03: 2112.326$ & 40.603 & 2.03 & 0.51 \\
\hline 2008 & 10 & 18 & $14: 19: 1912.378$ & 40.590 & 2.40 & 0.46 \\
\hline 2008 & 10 & 18 & $14: 22: 3612.344$ & 40.592 & 2.47 & 0.42 \\
\hline 2008 & 10 & 18 & $14: 27: 1212.421$ & 40.570 & 2.28 & 0.37 \\
\hline 2008 & 10 & 18 & $15: 04: 4212.397$ & 40.573 & 2.07 & 0.52 \\
\hline 2008 & 10 & 18 & $15: 13: 5512.406$ & 40.577 & 2.07 & 0.87 \\
\hline 2008 & 10 & 18 & $15: 20: 3412.365$ & 40.582 & 2.31 & 0.46 \\
\hline 2008 & 10 & 18 & $15: 25: 1312.386$ & 40.574 & 2.01 & 0.5 \\
\hline 2008 & 10 & 18 & $15: 27: 4512.389$ & 40.576 & 2.48 & 0.43 \\
\hline 2008 & 10 & 18 & $15: 43: 4812.347$ & 40.591 & 2.65 & 0.43 \\
\hline 2008 & 10 & 18 & $15: 54: 5612.342$ & 40.602 & 2.63 & 0.34 \\
\hline 2008 & 10 & 18 & $16: 05: 1612.368$ & 40.585 & 2.29 & 0.54 \\
\hline 2008 & 10 & 18 & $16: 21: 5712.382$ & 40.602 & 2.14 & 0.85 \\
\hline 2008 & 10 & 18 & $16: 24: 5412.397$ & 40.573 & 2.20 & 0.39 \\
\hline 2008 & 10 & 18 & $16: 26: 0612.401$ & 40.562 & 2.90 & 0.39 \\
\hline 2008 & 10 & 18 & $16: 30: 5712.403$ & 40.543 & 2.33 & 0.48 \\
\hline 2008 & 10 & 18 & $16: 34: 4912.363$ & 40.589 & 1.94 & 0.54 \\
\hline 2008 & 10 & 18 & $16: 42: 2512.395$ & 40.581 & 2.53 & 0.36 \\
\hline
\end{tabular}




\begin{tabular}{|c|c|c|c|c|c|c|}
\hline 2008 & 10 & 18 & $16: 46: 3212.396$ & 40.569 & 1.92 & 1.28 \\
\hline 2008 & 10 & 18 & $16: 51: 1812.350$ & 40.589 & 2.07 & 0.49 \\
\hline 2008 & 10 & 18 & $16: 56: 0812.459$ & 40.562 & 2.04 & 0.45 \\
\hline 2008 & 10 & 18 & $17: 09: 3612.353$ & 40.607 & 2.07 & 0.53 \\
\hline 2008 & 10 & 18 & $17: 13: 3212.350$ & 40.592 & 1.92 & 0.55 \\
\hline 2008 & 10 & 18 & $17: 18: 3812.366$ & 40.615 & 2.34 & 0.6 \\
\hline 2008 & 10 & 18 & $17: 22: 2412.389$ & 40.579 & 1.91 & 0.99 \\
\hline 2008 & 10 & 18 & $17: 23: 0712.383$ & 40.586 & 2.33 & 0.39 \\
\hline 2008 & 10 & 18 & $17: 30: 3812.364$ & 40.585 & 2.00 & 0.47 \\
\hline 2008 & 10 & 18 & $17: 32: 1012.371$ & 40.574 & 1.88 & 0.53 \\
\hline 2008 & 10 & 18 & $17: 53: 5212.358$ & 40.601 & 2.02 & 0.56 \\
\hline 2008 & 10 & 18 & $17: 57: 1412.370$ & 40.585 & 1.96 & 0.66 \\
\hline 2008 & 10 & 18 & $17: 59: 3212.389$ & 40.577 & 2.06 & 0.57 \\
\hline 2008 & 10 & 18 & $18: 05: 4512.358$ & 40.589 & 2.29 & 0.36 \\
\hline 2008 & 10 & 18 & $18: 09: 3712.403$ & 40.562 & 2.19 & 0.58 \\
\hline 2008 & 10 & 18 & $18: 14: 3512.376$ & 40.587 & 2.79 & 0.39 \\
\hline 2008 & 10 & 18 & $18: 17: 0812.407$ & 40.575 & 2.07 & 0.47 \\
\hline 2008 & 10 & 18 & $18: 19: 0712.367$ & 40.593 & 2.24 & 0.42 \\
\hline 2008 & 10 & 18 & $18: 24: 5512.358$ & 40.593 & 2.12 & 0.55 \\
\hline 2008 & 10 & 18 & $18: 29: 2412.362$ & 40.593 & 2.53 & 0.41 \\
\hline 2008 & 10 & 18 & $18: 33: 2812.354$ & 40.579 & 2.44 & 0.35 \\
\hline 2008 & 10 & 18 & $18: 47: 2312.355$ & 40.587 & 2.41 & 0.49 \\
\hline 2008 & 10 & 18 & $18: 54: 5212.424$ & 40.564 & 2.03 & 0.49 \\
\hline 2008 & 10 & 18 & $19: 11: 4812.334$ & 40.594 & 1.90 & 0.62 \\
\hline 2008 & 10 & 18 & $19: 12: 3912.372$ & 40.575 & 2.10 & 0.43 \\
\hline 2008 & 10 & 18 & $19: 16: 3112.371$ & 40.583 & 2.01 & 0.5 \\
\hline 2008 & 10 & 18 & $19: 18: 5412.351$ & 40.595 & 2.29 & 0.51 \\
\hline 2008 & 10 & 18 & $19: 24: 2912.354$ & 40.597 & 2.20 & 0.33 \\
\hline 2008 & 10 & 18 & $19: 27: 1112.352$ & 40.601 & 2.20 & 0.42 \\
\hline 2008 & 10 & 18 & $19: 27: 5712.355$ & 40.603 & 2.23 & 0.59 \\
\hline 2008 & 10 & 18 & $19: 31: 2512.348$ & 40.594 & 2.10 & 0.37 \\
\hline 2008 & 10 & 18 & $19: 34: 4312.360$ & 40.584 & 1.95 & 0.42 \\
\hline 2008 & 10 & 18 & $19: 36: 4312.340$ & 40.593 & 2.31 & 0.41 \\
\hline 2008 & 10 & 18 & $19: 49: 5812.365$ & 40.588 & 2.00 & 0.4 \\
\hline 2008 & 10 & 18 & $19: 50: 4812.347$ & 40.595 & 1.92 & 0.76 \\
\hline 2008 & 10 & 18 & $19: 54: 4612.432$ & 40.568 & 1.97 & 0.41 \\
\hline 2008 & 10 & 18 & $20: 06: 0812.398$ & 40.569 & 1.96 & 0.64 \\
\hline 2008 & 10 & 18 & $20: 07: 3512.387$ & 40.574 & 2.11 & 0.65 \\
\hline 2008 & 10 & 18 & $20: 10: 3412.380$ & 40.580 & 2.26 & 0.47 \\
\hline 2008 & 10 & 18 & $20: 25: 4712.376$ & 40.583 & 2.57 & 0.43 \\
\hline 2008 & 10 & 18 & $20: 27: 0312.343$ & 40.601 & 1.97 & 0.71 \\
\hline 2008 & 10 & 18 & $20: 36: 2212.368$ & 40.595 & 1.90 & 0.88 \\
\hline 2008 & 10 & 18 & $20: 41: 0412.356$ & 40.592 & 2.24 & 0.44 \\
\hline 2008 & 10 & 18 & $20: 46: 1012.339$ & 40.591 & 1.92 & 0.49 \\
\hline 2008 & 10 & 18 & $20: 56: 2112.405$ & 40.566 & 1.86 & 0.46 \\
\hline 2008 & 10 & 18 & $20: 58: 3612.389$ & 40.549 & 1.91 & 2.15 \\
\hline 2008 & 10 & 18 & $21: 01: 2812.377$ & 40.578 & 1.85 & 0.38 \\
\hline 2008 & 10 & 18 & $21: 01: 5612.371$ & 40.586 & 1.93 & 0.67 \\
\hline 2008 & 10 & 18 & $21: 09: 0912.353$ & 40.589 & 2.24 & 0.44 \\
\hline 2008 & 10 & 18 & $21: 11: 4312.345$ & 40.596 & 1.89 & 0.45 \\
\hline 2008 & 10 & 18 & $21: 15: 2012.360$ & 40.617 & 2.08 & 0.38 \\
\hline 2008 & 10 & 18 & $21: 17: 0012.360$ & 40.590 & 2.29 & 0.44 \\
\hline 2008 & 10 & 18 & $21: 17: 4912.378$ & 40.584 & 2.34 & 0.41 \\
\hline 2008 & 10 & 18 & $21: 21: 0212.353$ & 40.588 & 2.82 & 0.36 \\
\hline 2008 & 10 & 18 & $21: 25: 2512.352$ & 40.593 & 2.20 & 0.29 \\
\hline 2008 & 10 & 18 & $21: 30: 3412.354$ & 40.584 & 1.96 & 0.59 \\
\hline 2008 & 10 & 18 & $21: 34: 1612.357$ & 40.602 & 2.25 & 0.91 \\
\hline 2008 & 10 & 18 & $21: 46: 1312.364$ & 40.587 & 2.03 & 0.55 \\
\hline 2008 & 10 & 18 & $21: 50: 0612.366$ & 40.590 & 2.13 & 0.42 \\
\hline 2008 & 10 & 18 & $21: 58: 0212.351$ & 40.592 & 1.93 & 0.47 \\
\hline 2008 & 10 & 18 & $22: 02: 0512.408$ & 40.574 & 1.92 & 0.57 \\
\hline 2008 & 10 & 18 & $22: 08: 5212.361$ & 40.574 & 1.98 & 1.43 \\
\hline 2008 & 10 & 18 & $22: 09: 4112.368$ & 40.589 & 2.50 & 0.38 \\
\hline 2008 & 10 & 18 & $22: 10: 3312.344$ & 40.593 & 2.45 & 0.48 \\
\hline 2008 & 10 & 18 & $22: 16: 2112.356$ & 40.593 & 2.45 & 0.35 \\
\hline 2008 & 10 & 18 & $22: 20: 5612.347$ & 40.599 & 2.05 & 0.4 \\
\hline 2008 & 10 & 18 & $22: 33: 3212.406$ & 40.580 & 2.17 & 1.35 \\
\hline 2008 & 10 & 18 & $22: 36: 2812.368$ & 40.590 & 2.07 & 0.35 \\
\hline 2008 & 10 & 18 & $22: 43: 5312.396$ & 40.581 & 2.02 & 0.99 \\
\hline 2008 & 10 & 18 & $22: 49: 2212.363$ & 40.595 & 2.76 & 0.6 \\
\hline 2008 & 10 & 18 & $22: 52: 2812.360$ & 40.611 & 2.00 & 0.53 \\
\hline 2008 & 10 & 18 & $22: 53: 2312.356$ & 40.603 & 2.15 & 0.35 \\
\hline 2008 & 10 & 18 & $23: 01: 0212.375$ & 40.588 & 1.98 & 0.68 \\
\hline 2008 & 10 & 18 & $23: 07: 0112.356$ & 40.595 & 2.92 & 0.31 \\
\hline 2008 & 10 & 18 & $23: 11: 2612.391$ & 40.583 & 1.98 & 0.52 \\
\hline 2008 & 10 & 18 & $23: 15: 3212.352$ & 40.592 & 1.88 & 0.5 \\
\hline
\end{tabular}




\begin{tabular}{|c|c|c|c|c|c|c|}
\hline 2008 & 10 & 18 & $23: 18: 2512.341$ & 40.594 & 2.19 & 0.37 \\
\hline 2008 & 10 & 18 & $23: 30: 1212.385$ & 40.564 & 2.05 & 1.32 \\
\hline 2008 & 10 & 18 & $23: 36: 4612.453$ & 40.565 & 2.08 & 0.97 \\
\hline 2008 & 10 & 18 & $23: 37: 3412.382$ & 40.578 & 2.41 & 0.38 \\
\hline 2008 & 10 & 18 & $23: 46: 0212.336$ & 40.593 & 1.76 & 0.6 \\
\hline 2008 & 10 & 18 & $23: 51: 0912.344$ & 40.598 & 1.82 & 0.53 \\
\hline 2008 & 10 & 18 & $23: 51: 4512.376$ & 40.571 & 1.83 & 0.74 \\
\hline 2008 & 10 & 18 & $23: 53: 5212.393$ & 40.575 & 2.28 & 0.63 \\
\hline 2008 & 10 & 18 & $23: 55: 0412.396$ & 40.560 & 1.83 & 0.63 \\
\hline 2008 & 10 & 19 & $00: 02: 3612.344$ & 40.597 & 2.32 & 0.36 \\
\hline 2008 & 10 & 19 & $00: 08: 2712.333$ & 40.609 & 1.88 & 0.41 \\
\hline 2008 & 10 & 19 & $00: 09: 3312.340$ & 40.598 & 1.93 & 0.36 \\
\hline 2008 & 10 & 19 & $00: 11: 2712.370$ & 40.590 & 1.87 & 0.51 \\
\hline 2008 & 10 & 19 & $00: 13: 5312.424$ & 40.568 & 1.92 & 0.54 \\
\hline 2008 & 10 & 19 & $00: 16: 3812.347$ & 40.588 & 1.87 & 0.45 \\
\hline 2008 & 10 & 19 & $00: 19: 4312.387$ & 40.576 & 1.76 & 1.34 \\
\hline 2008 & 10 & 19 & $00: 21: 0712.389$ & 40.570 & 1.81 & 1.29 \\
\hline 2008 & 10 & 19 & $00: 27: 2512.386$ & 40.586 & 2.68 & 0.3 \\
\hline 2008 & 10 & 19 & $00: 34: 2512.389$ & 40.581 & 2.15 & 0.33 \\
\hline 2008 & 10 & 19 & $00: 36: 2612.411$ & 40.565 & 3.19 & 0.37 \\
\hline 2008 & 10 & 19 & $00: 40: 2012.419$ & 40.573 & 2.29 & 0.28 \\
\hline 2008 & 10 & 19 & $00: 42: 2312.405$ & 40.564 & 2.07 & 0.65 \\
\hline 2008 & 10 & 19 & $00: 45: 4612.374$ & 40.587 & 2.70 & 6.26 \\
\hline 2008 & 10 & 19 & $00: 45: 4612.198$ & 41.760 & 3.27 & 0.37 \\
\hline 2008 & 10 & 19 & $00: 54: 5612.344$ & 40.601 & 1.96 & 0.46 \\
\hline 2008 & 10 & 19 & $00: 56: 1812.403$ & 40.553 & 1.91 & 0.45 \\
\hline 2008 & 10 & 19 & $01: 01: 1212.345$ & 40.590 & 1.84 & 0.43 \\
\hline 2008 & 10 & 19 & $01: 01: 4512.351$ & 40.600 & 2.01 & 0.45 \\
\hline 2008 & 10 & 19 & $01: 05: 3812.419$ & 40.561 & 1.95 & 0.8 \\
\hline 2008 & 10 & 19 & $01: 08: 1912.351$ & 40.599 & 1.84 & 0.71 \\
\hline 2008 & 10 & 19 & $01: 12: 3612.346$ & 40.602 & 1.80 & 1.15 \\
\hline 2008 & 10 & 19 & $01: 28: 2612.381$ & 40.568 & 1.98 & 0.48 \\
\hline 2008 & 10 & 19 & $01: 33: 5012.350$ & 40.592 & 1.95 & 0.58 \\
\hline 2008 & 10 & 19 & $01: 34: 2812.370$ & 40.590 & 1.97 & 0.76 \\
\hline 2008 & 10 & 19 & $01: 35: 3112.361$ & 40.590 & 2.40 & 0.53 \\
\hline 2008 & 10 & 19 & $01: 38: 2012.361$ & 40.590 & 2.01 & 0.46 \\
\hline 2008 & 10 & 19 & $01: 47: 3812.351$ & 40.583 & 1.91 & 0.66 \\
\hline 2008 & 10 & 19 & $01: 48: 3112.361$ & 40.595 & 2.26 & 0.68 \\
\hline 2008 & 10 & 19 & $01: 53: 0312.434$ & 40.574 & 2.14 & 1.01 \\
\hline 2008 & 10 & 19 & $02: 00: 0312.430$ & 40.564 & 2.85 & 0.34 \\
\hline 2008 & 10 & 19 & $02: 05: 0512.390$ & 40.586 & 2.12 & 0.81 \\
\hline 2008 & 10 & 19 & $02: 06: 5512.431$ & 40.568 & 2.12 & 0.63 \\
\hline 2008 & 10 & 19 & $02: 07: 5112.354$ & 40.598 & 2.59 & 0.49 \\
\hline 2008 & 10 & 19 & $02: 15: 5912.424$ & 40.564 & 2.51 & 0.41 \\
\hline 2008 & 10 & 19 & $02: 34: 1612.349$ & 40.590 & 2.07 & 0.52 \\
\hline 2008 & 10 & 19 & $02: 35: 2012.378$ & 40.571 & 2.08 & 0.45 \\
\hline 2008 & 10 & 19 & $02: 41: 5612.403$ & 40.568 & 1.95 & 0.87 \\
\hline 2008 & 10 & 19 & $02: 52: 3812.344$ & 40.599 & 2.02 & 0.62 \\
\hline 2008 & 10 & 19 & $03: 00: 3712.365$ & 40.582 & 2.13 & 0.56 \\
\hline 2008 & 10 & 19 & $03: 12: 5412.370$ & 40.579 & 2.18 & 0.57 \\
\hline 2008 & 10 & 19 & $03: 44: 2412.339$ & 40.595 & 2.18 & 0.4 \\
\hline 2008 & 10 & 19 & $03: 53: 1012.389$ & 40.574 & 2.64 & 0.54 \\
\hline 2008 & 10 & 19 & $04: 04: 1012.352$ & 40.613 & 2.50 & 0.51 \\
\hline 2008 & 10 & 19 & $04: 29: 1412.368$ & 40.588 & 2.16 & 0.61 \\
\hline 2008 & 10 & 19 & $04: 36: 2812.365$ & 40.589 & 2.17 & 0.86 \\
\hline 2008 & 10 & 19 & $04: 50: 0912.375$ & 40.585 & 2.29 & 0.38 \\
\hline 2008 & 10 & 19 & $04: 53: 1612.352$ & 40.592 & 2.24 & 0.58 \\
\hline 2008 & 10 & 19 & $05: 30: 2612.386$ & 40.582 & 3.46 & 0.47 \\
\hline 2008 & 10 & 19 & $05: 31: 3712.385$ & 40.577 & 2.96 & 0.6 \\
\hline 2008 & 10 & 19 & $05: 59: 3612.378$ & 40.592 & 2.61 & 0.62 \\
\hline 2008 & 10 & 19 & $06: 07: 3312.400$ & 40.572 & 2.43 & 0.57 \\
\hline 2008 & 10 & 19 & $06: 12: 2612.363$ & 40.579 & 2.40 & 0.52 \\
\hline 2008 & 10 & 19 & $06: 23: 5712.353$ & 40.591 & 2.71 & 0.44 \\
\hline 2008 & 10 & 19 & $06: 44: 3812.390$ & 40.581 & 2.59 & 0.59 \\
\hline 2008 & 10 & 19 & $07: 11: 0312.350$ & 40.587 & 2.43 & 0.67 \\
\hline 2008 & 10 & 19 & $07: 13: 2812.341$ & 40.603 & 2.47 & 0.5 \\
\hline 2008 & 10 & 19 & $07: 19: 1912.345$ & 40.595 & 2.38 & 0.52 \\
\hline 2008 & 10 & 19 & $07: 54: 1912.369$ & 40.584 & 2.15 & 1.14 \\
\hline 2008 & 10 & 19 & $08: 05: 1412.350$ & 40.603 & 2.48 & 0.56 \\
\hline 2008 & 10 & 19 & $08: 13: 5012.370$ & 40.584 & 2.12 & 0.45 \\
\hline 2008 & 10 & 19 & $08: 15: 3212.370$ & 40.585 & 2.28 & 0.49 \\
\hline 2008 & 10 & 19 & $08: 21: 0012.355$ & 40.607 & 2.34 & 0.59 \\
\hline 2008 & 10 & 19 & $08: 23: 4112.358$ & 40.591 & 2.49 & 0.38 \\
\hline 2008 & 10 & 19 & $08: 28: 1412.357$ & 40.598 & 2.37 & 0.46 \\
\hline 2008 & 10 & 19 & $08: 53: 1712.352$ & 40.585 & 2.57 & 0.46 \\
\hline 2008 & 10 & 19 & $09: 23: 2612.361$ & 40.595 & 2.20 & 0.46 \\
\hline
\end{tabular}




\begin{tabular}{|c|c|c|c|c|c|c|}
\hline 2008 & 10 & 19 & $09: 25: 0212.395$ & 40.569 & 2.32 & 0.41 \\
\hline 2008 & 10 & 19 & $09: 34: 5412.373$ & 40.587 & 2.02 & 0.58 \\
\hline 2008 & 10 & 19 & $09: 46: 0712.375$ & 40.583 & 2.31 & 0.67 \\
\hline 2008 & 10 & 19 & $09: 51: 5412.357$ & 40.588 & 2.29 & 0.46 \\
\hline 2008 & 10 & 19 & $10: 27: 0112.378$ & 40.575 & 3.32 & 0.46 \\
\hline 2008 & 10 & 19 & $10: 46: 1912.386$ & 40.560 & 2.18 & 0.59 \\
\hline 2008 & 10 & 19 & $10: 53: 5812.369$ & 40.590 & 2.70 & 0.49 \\
\hline 2008 & 10 & 19 & $10: 56: 4212.355$ & 40.598 & 2.38 & 0.49 \\
\hline 2008 & 10 & 19 & $11: 04: 1912.402$ & 40.568 & 2.22 & 0.46 \\
\hline 2008 & 10 & 19 & $11: 06: 5512.374$ & 40.576 & 1.98 & 1.2 \\
\hline 2008 & 10 & 19 & $11: 08: 1412.356$ & 40.599 & 1.99 & 0.94 \\
\hline 2008 & 10 & 19 & $11: 10: 5812.363$ & 40.582 & 2.38 & 0.5 \\
\hline 2008 & 10 & 19 & $11: 12: 1412.356$ & 40.594 & 2.41 & 0.62 \\
\hline 2008 & 10 & 19 & $11: 16: 1112.352$ & 40.594 & 2.58 & 1.04 \\
\hline 2008 & 10 & 19 & $11: 20: 0512.356$ & 40.601 & 2.72 & 0.47 \\
\hline 2008 & 10 & 19 & $11: 25: 5912.375$ & 40.573 & 2.14 & 0.59 \\
\hline 2008 & 10 & 19 & $11: 31: 3412.356$ & 40.609 & 2.16 & 0.45 \\
\hline 2008 & 10 & 19 & $11: 47: 5112.331$ & 40.599 & 2.04 & 0.43 \\
\hline 2008 & 10 & 19 & $11: 56: 4612.367$ & 40.581 & 2.17 & 0.6 \\
\hline 2008 & 10 & 19 & $12: 05: 0412.374$ & 40.571 & 2.17 & 0.56 \\
\hline 2008 & 10 & 19 & $12: 44: 5912.387$ & 40.565 & 2.42 & 1,00 \\
\hline 2008 & 10 & 19 & $13: 31: 4812.395$ & 40.581 & 2.36 & 0.41 \\
\hline 2008 & 10 & 19 & $13: 32: 4312.390$ & 40.579 & 2.58 & 0.37 \\
\hline 2008 & 10 & 19 & $13: 35: 1712.391$ & 40.580 & 2.51 & 0.49 \\
\hline 2008 & 10 & 19 & $13: 49: 2012.354$ & 40.592 & 2.24 & 0.39 \\
\hline 2008 & 10 & 19 & $14: 03: 0812.358$ & 40.588 & 2.23 & 0.5 \\
\hline 2008 & 10 & 19 & $14: 24: 0112.355$ & 40.590 & 2.10 & 0.55 \\
\hline 2008 & 10 & 19 & $14: 29: 0612.371$ & 40.587 & 2.65 & 0.42 \\
\hline 2008 & 10 & 19 & $15: 26: 0812.354$ & 40.588 & 2.20 & 0.54 \\
\hline 2008 & 10 & 19 & $15: 32: 2612.359$ & 40.592 & 2.50 & 0.45 \\
\hline 2008 & 10 & 19 & $15: 39: 3712.380$ & 40.577 & 2.21 & 0.78 \\
\hline 2008 & 10 & 19 & $15: 47: 0112.353$ & 40.590 & 2.02 & 0.48 \\
\hline 2008 & 10 & 19 & $15: 51: 0612.367$ & 40.585 & 2.29 & 0.63 \\
\hline 2008 & 10 & 19 & $16: 15: 3412.384$ & 40.579 & 1.85 & 0.58 \\
\hline 2008 & 10 & 19 & $16: 24: 5412.347$ & 40.592 & 1.98 & 0.46 \\
\hline 2008 & 10 & 19 & $16: 35: 5312.343$ & 40.593 & 1.90 & 0.51 \\
\hline 2008 & 10 & 19 & $16: 43: 1712.357$ & 40.583 & 1.86 & 2.52 \\
\hline 2008 & 10 & 19 & $16: 49: 1112.406$ & 40.575 & 2.31 & 0.47 \\
\hline 2008 & 10 & 19 & $17: 12: 5012.357$ & 40.621 & 2.11 & 0.47 \\
\hline 2008 & 10 & 19 & $17: 21: 1212.340$ & 40.602 & 2.17 & 0.62 \\
\hline 2008 & 10 & 19 & $17: 30: 3612.366$ & 40.574 & 1.93 & 0.8 \\
\hline 2008 & 10 & 19 & $17: 35: 5612.356$ & 40.610 & 2.07 & 0.75 \\
\hline 2008 & 10 & 19 & $17: 51: 0012.360$ & 40.589 & 1.97 & 0.84 \\
\hline 2008 & 10 & 19 & $18: 01: 5312.352$ & 40.594 & 2.18 & 0.41 \\
\hline 2008 & 10 & 19 & $18: 04: 2712.345$ & 40.587 & 2.36 & 0.49 \\
\hline 2008 & 10 & 19 & $18: 09: 4112.433$ & 40.569 & 2.26 & 0.39 \\
\hline 2008 & 10 & 19 & $18: 17: 4912.346$ & 40.597 & 1.88 & 1.12 \\
\hline 2008 & 10 & 19 & $18: 30: 2812.354$ & 40.602 & 1.91 & 0.57 \\
\hline 2008 & 10 & 19 & $18: 32: 1012.359$ & 40.607 & 1.85 & 0.57 \\
\hline 2008 & 10 & 19 & $18: 39: 3912.342$ & 40.595 & 2.44 & 0.31 \\
\hline 2008 & 10 & 19 & $18: 44: 1812.342$ & 40.597 & 2.10 & 0.35 \\
\hline 2008 & 10 & 19 & $18: 47: 0412.360$ & 40.595 & 2.13 & 0.45 \\
\hline 2008 & 10 & 19 & $18: 59: 3112.366$ & 40.588 & 1.98 & 0.73 \\
\hline 2008 & 10 & 19 & $19: 03: 5412.337$ & 40.598 & 2.06 & 0.7 \\
\hline 2008 & 10 & 19 & $19: 07: 5312.361$ & 40.585 & 2.19 & 0.4 \\
\hline 2008 & 10 & 19 & $19: 32: 3612.344$ & 40.599 & 2.42 & 0.44 \\
\hline 2008 & 10 & 19 & $19: 36: 4412.391$ & 40.566 & 2.03 & 0.86 \\
\hline 2008 & 10 & 19 & $19: 41: 1112.354$ & 40.597 & 2.00 & 0.48 \\
\hline 2008 & 10 & 19 & $19: 43: 5612.356$ & 40.583 & 1.85 & 0.53 \\
\hline 2008 & 10 & 19 & $19: 46: 2412.381$ & 40.581 & 1.92 & 0.57 \\
\hline 2008 & 10 & 19 & $19: 47: 52 \quad 12.336$ & 40.596 & 1.84 & 0.69 \\
\hline 2008 & 10 & 19 & $19: 53: 4012.345$ & 40.597 & 1.90 & 0.52 \\
\hline 2008 & 10 & 19 & $19: 57: 3512.361$ & 40.589 & 2.35 & 0.39 \\
\hline 2008 & 10 & 19 & $20: 26: 3412.337$ & 40.594 & 1.93 & 0.31 \\
\hline 2008 & 10 & 19 & $20: 30: 5712.370$ & 40.587 & 2.02 & 0.4 \\
\hline 2008 & 10 & 19 & $20: 33: 2412.409$ & 40.568 & 2.01 & 0.41 \\
\hline 2008 & 10 & 19 & $20: 44: 2412.383$ & 40.578 & 1.92 & 0.43 \\
\hline 2008 & 10 & 19 & $21: 08: 1512.431$ & 40.580 & 2.66 & 0.33 \\
\hline 2008 & 10 & 19 & $21: 15: 5912.426$ & 40.550 & 1.83 & 0.47 \\
\hline 2008 & 10 & 19 & $21: 19: 4612.359$ & 40.590 & 1.90 & 0.38 \\
\hline 2008 & 10 & 19 & $21: 31: 4012.354$ & 40.591 & 1.92 & 0.38 \\
\hline 2008 & 10 & 19 & $21: 37: 1212.377$ & 40.581 & 2.03 & 0.43 \\
\hline 2008 & 10 & 19 & $22: 00: 0512.435$ & 40.567 & 1.91 & 0.34 \\
\hline 2008 & 10 & 19 & $22: 20: 1112.343$ & 40.602 & 2.07 & 0.37 \\
\hline 2008 & 10 & 19 & $22: 22: 4412.336$ & 40.584 & 2.02 & 0.49 \\
\hline 2008 & 10 & 19 & $22: 26: 2712.378$ & 40.583 & 1.92 & 0.49 \\
\hline
\end{tabular}




\begin{tabular}{|c|c|c|c|c|c|c|}
\hline 2008 & 10 & 19 & $22: 29: 5212.389$ & 40.568 & 1.88 & 0.48 \\
\hline 2008 & 10 & 19 & $22: 32: 3312.412$ & 40.568 & 2.05 & 0.34 \\
\hline 2008 & 10 & 19 & $22: 43: 3012.385$ & 40.585 & 1.95 & 0.54 \\
\hline 2008 & 10 & 19 & $22: 49: 0612.355$ & 40.588 & 1.97 & 0.31 \\
\hline 2008 & 10 & 19 & $23: 27: 3212.343$ & 40.593 & 1.82 & 0.4 \\
\hline 2008 & 10 & 19 & $23: 29: 0212.375$ & 40.578 & 2.20 & 0.34 \\
\hline 2008 & 10 & 19 & $23: 32: 2012.354$ & 40.593 & 1.98 & 0.41 \\
\hline 2008 & 10 & 19 & $23: 45: 2412.356$ & 40.583 & 1.82 & 0.44 \\
\hline 2008 & 10 & 19 & $23: 47: 5912.331$ & 40.605 & 2.01 & 0.35 \\
\hline 2008 & 10 & 19 & $23: 51: 1212.340$ & 40.593 & 2.10 & 0.39 \\
\hline 2008 & 10 & 19 & $23: 53: 5112.461$ & 40.559 & 2.42 & 0.38 \\
\hline 2008 & 10 & 20 & $00: 20: 5012.394$ & 40.579 & 1.81 & 0.45 \\
\hline 2008 & 10 & 20 & $00: 27: 5712.660$ & 39.510 & 2.28 & 0.52 \\
\hline 2008 & 10 & 20 & $00: 33: 3112.392$ & 40.569 & 2.02 & 0.43 \\
\hline 2008 & 10 & 20 & $00: 36: 4612.390$ & 40.571 & 2.26 & 0.35 \\
\hline 2008 & 10 & 20 & $00: 43: 0612.399$ & 40.569 & 2.20 & 0.33 \\
\hline 2008 & 10 & 20 & $00: 44: 5412.360$ & 40.585 & 1.91 & 0.44 \\
\hline 2008 & 10 & 20 & $00: 49: 2012.362$ & 40.583 & 2.08 & 0.39 \\
\hline 2008 & 10 & 20 & $00: 52: 3312.358$ & 40.587 & 2.81 & 0.47 \\
\hline 2008 & 10 & 20 & $01: 06: 0512.396$ & 40.576 & 2.67 & 0.36 \\
\hline 2008 & 10 & 20 & $01: 17: 5012.366$ & 40.585 & 1.81 & 0.54 \\
\hline 2008 & 10 & 20 & $01: 38: 5612.358$ & 40.591 & 3.07 & 0.38 \\
\hline 2008 & 10 & 20 & $01: 43: 5012.384$ & 40.574 & 2.47 & 0.4 \\
\hline 2008 & 10 & 20 & $02: 02: 0012.373$ & 40.572 & 2.00 & 0.49 \\
\hline 2008 & 10 & 20 & $02: 11: 3912.389$ & 40.574 & 2.01 & 0.68 \\
\hline 2008 & 10 & 20 & $02: 36: 4512.431$ & 40.578 & 2.27 & 0.43 \\
\hline 2008 & 10 & 20 & $02: 40: 4812.388$ & 40.575 & 2.18 & 0.37 \\
\hline 2008 & 10 & 20 & $03: 14: 5312.355$ & 40.577 & 2.09 & 0.76 \\
\hline 2008 & 10 & 20 & $03: 58: 4112.361$ & 40.602 & 2.06 & 0.48 \\
\hline 2008 & 10 & 20 & $04: 11: 1012.361$ & 40.611 & 2.14 & 0.83 \\
\hline 2008 & 10 & 20 & $04: 28: 4112.355$ & 40.606 & 2.42 & 0.44 \\
\hline 2008 & 10 & 20 & $04: 31: 2412.370$ & 40.618 & 2.28 & 0.94 \\
\hline 2008 & 10 & 20 & $04: 41: 4612.397$ & 40.581 & 2.24 & 0.4 \\
\hline 2008 & 10 & 20 & $04: 55: 0312.353$ & 40.609 & 2.51 & 0.38 \\
\hline 2008 & 10 & 20 & $05: 05: 2712.404$ & 40.583 & 2.37 & 0.52 \\
\hline 2008 & 10 & 20 & $05: 25: 5112.367$ & 40.598 & 3.19 & 0.43 \\
\hline 2008 & 10 & 20 & $05: 39: 0812.368$ & 40.586 & 2.99 & 0.36 \\
\hline 2008 & 10 & 20 & $05: 55: 3412.396$ & 40.576 & 2.76 & 0.35 \\
\hline 2008 & 10 & 20 & $06: 22: 4212.380$ & 40.583 & 2.42 & 0.62 \\
\hline 2008 & 10 & 20 & $06: 58: 07 \quad 12.362$ & 40.593 & 2.42 & 0.42 \\
\hline 2008 & 10 & 20 & $07: 05: 2212.358$ & 40.594 & 2.42 & 0.45 \\
\hline 2008 & 10 & 20 & $08: 07: 2012.389$ & 40.575 & 2.19 & 0.66 \\
\hline 2008 & 10 & 20 & $08: 34: 1012.378$ & 40.590 & 2.18 & 0.45 \\
\hline 2008 & 10 & 20 & $09: 59: 2012.339$ & 40.593 & 2.27 & 0.44 \\
\hline 2008 & 10 & 20 & $10: 04: 3712.367$ & 40.574 & 2.12 & 0.79 \\
\hline 2008 & 10 & 20 & $10: 20: 3112.381$ & 40.576 & 1.95 & 0.78 \\
\hline 2008 & 10 & 20 & $12: 10: 1512.361$ & 40.593 & 2.34 & 0.41 \\
\hline 2008 & 10 & 20 & $12: 19: 0912.341$ & 40.599 & 2.13 & 0.49 \\
\hline 2008 & 10 & 20 & $15: 42: 4112.345$ & 40.581 & 2.45 & 0.58 \\
\hline 2008 & 10 & 20 & $15: 56: 1512.337$ & 40.592 & 2.30 & 0.49 \\
\hline 2008 & 10 & 20 & $16: 02: 5312.357$ & 40.588 & 2.51 & 0.41 \\
\hline 2008 & 10 & 20 & $16: 33: 0912.373$ & 40.585 & 2.45 & 0.43 \\
\hline 2008 & 10 & 20 & $17: 42: 07 \quad 12.367$ & 40.583 & 2.20 & 0.42 \\
\hline 2008 & 10 & 20 & $17: 50: 4912.347$ & 40.596 & 2.38 & 0.36 \\
\hline 2008 & 10 & 20 & $18: 51: 0012.415$ & 40.570 & 1.96 & 0.39 \\
\hline 2008 & 10 & 20 & $18: 53: 1112.393$ & 40.570 & 2.36 & 0.42 \\
\hline 2008 & 10 & 20 & $19: 29: 0712.359$ & 40.585 & 2.11 & 0.34 \\
\hline 2008 & 10 & 20 & $19: 32: 0412.370$ & 40.586 & 2.41 & 0.43 \\
\hline 2008 & 10 & 20 & $19: 53: 5312.391$ & 40.574 & 2.06 & 0.34 \\
\hline 2008 & 10 & 20 & $20: 00: 0812.394$ & 40.569 & 2.13 & 0.43 \\
\hline 2008 & 10 & 20 & $20: 11: 5212.371$ & 40.586 & 2.38 & 0.41 \\
\hline 2008 & 10 & 20 & $20: 56: 3412.365$ & 40.590 & 1.86 & 0.51 \\
\hline 2008 & 10 & 20 & $21: 29: 0712.334$ & 40.622 & 2.19 & 0.41 \\
\hline 2008 & 10 & 20 & $22: 10: 2812.422$ & 41.887 & 2.42 & 1.26 \\
\hline 2008 & 10 & 20 & $22: 48: 4012.362$ & 40.586 & 2.07 & 0.41 \\
\hline 2008 & 10 & 20 & $23: 02: 2412.354$ & 40.583 & 2.28 & 0.36 \\
\hline 2008 & 10 & 20 & $23: 10: 5412.359$ & 40.617 & 2.17 & 0.7 \\
\hline 2008 & 10 & 20 & $23: 28: 0012.372$ & 40.585 & 2.09 & 0.37 \\
\hline 2008 & 10 & 20 & $23: 41: 0112.342$ & 40.597 & 1.81 & 0.36 \\
\hline 2008 & 10 & 21 & $00: 06: 2512.367$ & 40.585 & 1.97 & 0.4 \\
\hline 2008 & 10 & 21 & $00: 14: 5612.385$ & 40.585 & 2.05 & 0.49 \\
\hline 2008 & 10 & 21 & $00: 35: 3712.361$ & 40.595 & 2.41 & 0.42 \\
\hline 2008 & 10 & 21 & $00: 48: 1812.362$ & 40.595 & 1.93 & 0.37 \\
\hline 2008 & 10 & 21 & $01: 04: 0112.405$ & 40.570 & 1.85 & 0.65 \\
\hline 2008 & 10 & 21 & $01: 22: 0312.408$ & 40.562 & 1.97 & 0.5 \\
\hline 2008 & 10 & 21 & $01: 40: 5312.387$ & 40.572 & 2.04 & 0.35 \\
\hline
\end{tabular}




\begin{tabular}{|c|c|c|c|c|c|c|}
\hline 2008 & 10 & 21 & $02: 19: 5412.418$ & 40.550 & 1.98 & 0.47 \\
\hline 2008 & 10 & 21 & $02: 28: 5412.345$ & 40.613 & 2.34 & 0.42 \\
\hline 2008 & 10 & 21 & $02: 48: 5112.333$ & 40.601 & 1.95 & 0.57 \\
\hline 2008 & 10 & 21 & $03: 24: 3612.359$ & 40.591 & 2.25 & 0.78 \\
\hline 2008 & 10 & 21 & $03: 35: 0112.363$ & 40.588 & 2.05 & 0.87 \\
\hline 2008 & 10 & 21 & $03: 38: 5712.370$ & 40.590 & 2.19 & 0.62 \\
\hline 2008 & 10 & 21 & $04: 42: 2612.385$ & 40.579 & 2.38 & 0.53 \\
\hline 2008 & 10 & 21 & $05: 56: 0512.743$ & 40.600 & 2.25 & 1.01 \\
\hline 2008 & 10 & 21 & $07: 53: 5312.372$ & 40.585 & 2.26 & 0.53 \\
\hline 2008 & 10 & 21 & $10: 25: 2612.365$ & 40.601 & 2.28 & 0.37 \\
\hline 2008 & 10 & 21 & $10: 38: 3512.359$ & 40.590 & 2.44 & 0.43 \\
\hline 2008 & 10 & 21 & $10: 47: 3312.350$ & 40.592 & 2.57 & 0.52 \\
\hline 2008 & 10 & 21 & $13: 23: 2312.386$ & 40.568 & 2.31 & 0.58 \\
\hline 2008 & 10 & 21 & $13: 27: 5612.351$ & 40.599 & 2.27 & 0.58 \\
\hline 2008 & 10 & 21 & $14: 04: 3912.346$ & 40.609 & 2.50 & 1.18 \\
\hline 2008 & 10 & 21 & $15: 46: 5812.425$ & 40.555 & 3.54 & 0.44 \\
\hline 2008 & 10 & 21 & $16: 06: 2312.429$ & 40.566 & 2.05 & 0.6 \\
\hline 2008 & 10 & 21 & $16: 32: 3112.361$ & 40.593 & 1.94 & 0.8 \\
\hline 2008 & 10 & 21 & $16: 54: 2512.390$ & 40.581 & 2.00 & 0.56 \\
\hline 2008 & 10 & 21 & $17: 05: 3312.343$ & 40.595 & 1.99 & 0.38 \\
\hline 2008 & 10 & 21 & $17: 17: 4712.424$ & 40.564 & 2.12 & 0.34 \\
\hline 2008 & 10 & 21 & $18: 17: 5012.342$ & 40.591 & 1.93 & 0.6 \\
\hline 2008 & 10 & 21 & $18: 28: 5212.357$ & 40.592 & 1.88 & 0.41 \\
\hline 2008 & 10 & 21 & $18: 31: 2212.358$ & 40.587 & 2.05 & 0.61 \\
\hline 2008 & 10 & 21 & $19: 11: 5612.365$ & 40.581 & 2.18 & 0.41 \\
\hline 2008 & 10 & 21 & $19: 21: 3212.349$ & 40.595 & 1.95 & 0.4 \\
\hline 2008 & 10 & 21 & $19: 27: 4112.368$ & 40.597 & 2.07 & 0.52 \\
\hline 2008 & 10 & 21 & $19: 36: 5912.373$ & 40.588 & 2.07 & 0.38 \\
\hline 2008 & 10 & 21 & $19: 48: 0812.415$ & 40.556 & 2.06 & 0.42 \\
\hline 2008 & 10 & 21 & $20: 20: 3112.378$ & 40.573 & 1.98 & 0.44 \\
\hline 2008 & 10 & 21 & $21: 13: 5212.353$ & 40.588 & 1.88 & 0.35 \\
\hline 2008 & 10 & 21 & $21: 24: 5012.384$ & 40.580 & 2.62 & 0.41 \\
\hline 2008 & 10 & 21 & $21: 59: 4812.496$ & 40.552 & 2.40 & 0.33 \\
\hline 2008 & 10 & 21 & $22: 07: 2412.382$ & 40.575 & 2.02 & 0.47 \\
\hline 2008 & 10 & 21 & $22: 25: 1212.387$ & 40.563 & 1.83 & 0.62 \\
\hline 2008 & 10 & 21 & $22: 36: 3912.424$ & 40.580 & 1.87 & 0.43 \\
\hline 2008 & 10 & 21 & $22: 55: 4012.347$ & 40.597 & 2.07 & 0.38 \\
\hline 2008 & 10 & 21 & $23: 32: 2812.409$ & 40.570 & 2.24 & 0.34 \\
\hline 2008 & 10 & 21 & $23: 55: 1612.369$ & 40.592 & 1.98 & 0.35 \\
\hline 2008 & 10 & 22 & $00: 15: 0412.413$ & 40.568 & 2.81 & 0.35 \\
\hline 2008 & 10 & 22 & $00: 33: 5612.354$ & 40.584 & 1.98 & 0.45 \\
\hline 2008 & 10 & 22 & $00: 55: 2512.357$ & 40.602 & 1.89 & 0.39 \\
\hline 2008 & 10 & 22 & $01: 06: 4912.376$ & 40.584 & 2.56 & 0.35 \\
\hline 2008 & 10 & 22 & $01: 35: 1512.336$ & 40.606 & 1.78 & 1.37 \\
\hline 2008 & 10 & 22 & $01: 41: 2612.345$ & 40.593 & 2.56 & 0.59 \\
\hline 2008 & 10 & 22 & $02: 21: 3112.355$ & 40.592 & 2.44 & 0.42 \\
\hline 2008 & 10 & 22 & $02: 44: 4312.355$ & 40.590 & 2.04 & 0.46 \\
\hline 2008 & 10 & 22 & $03: 47: 0412.350$ & 40.589 & 3.05 & 0.39 \\
\hline 2008 & 10 & 22 & $04: 07: 5212.378$ & 40.578 & 2.23 & 0.5 \\
\hline 2008 & 10 & 22 & $04: 32: 0812.384$ & 40.597 & 2.26 & 0.96 \\
\hline 2008 & 10 & 22 & $05: 02: 1212.363$ & 40.335 & 1.99 & 1.12 \\
\hline 2008 & 10 & 22 & $07: 04: 5612.312$ & 40.593 & 2.20 & 0.68 \\
\hline 2008 & 10 & 22 & $08: 14: 1712.345$ & 40.588 & 2.15 & 0.41 \\
\hline 2008 & 10 & 22 & $08: 50: 2012.412$ & 40.573 & 2.08 & 0.68 \\
\hline 2008 & 10 & 22 & $10: 50: 1812.381$ & 40.590 & 2.02 & 0.59 \\
\hline 2008 & 10 & 22 & $15: 51: 5012.399$ & 40.573 & 2.08 & 0.47 \\
\hline 2008 & 10 & 22 & $16: 27: 4112.361$ & 40.603 & 2.24 & 0.47 \\
\hline 2008 & 10 & 22 & $18: 23: 2512.368$ & 40.338 & 1.81 & 1.17 \\
\hline 2008 & 10 & 22 & $18: 30: 1512.383$ & 40.571 & 1.97 & 0.58 \\
\hline 2008 & 10 & 22 & $19: 02: 5712.382$ & 40.586 & 2.52 & 0.47 \\
\hline 2008 & 10 & 22 & $19: 07: 5012.369$ & 40.588 & 2.32 & 0.42 \\
\hline 2008 & 10 & 22 & $19: 22: 5212.372$ & 40.581 & 1.98 & 0.45 \\
\hline 2008 & 10 & 22 & $19: 34: 4112.354$ & 40.581 & 2.15 & 0.63 \\
\hline 2008 & 10 & 22 & $19: 39: 3412.360$ & 40.582 & 2.02 & 0.46 \\
\hline 2008 & 10 & 22 & $20: 02: 1812.384$ & 40.578 & 2.14 & 0.57 \\
\hline 2008 & 10 & 22 & $20: 12: 1912.340$ & 40.587 & 2.07 & 0.49 \\
\hline 2008 & 10 & 22 & $20: 12: 4312.346$ & 40.584 & 2.51 & 0.73 \\
\hline 2008 & 10 & 22 & $20: 24: 0912.450$ & 40.570 & 1.82 & 0.44 \\
\hline 2008 & 10 & 22 & $20: 35: 1512.361$ & 40.582 & 2.38 & 0.36 \\
\hline 2008 & 10 & 22 & $20: 38: 3912.356$ & 40.579 & 2.18 & 0.33 \\
\hline 2008 & 10 & 22 & $20: 44: 4712.361$ & 40.580 & 1.91 & 0.6 \\
\hline 2008 & 10 & 22 & $21: 00: 2612.389$ & 40.583 & 1.98 & 0.38 \\
\hline 2008 & 10 & 22 & $22: 12: 5612.379$ & 40.585 & 1.86 & 0.44 \\
\hline 2008 & 10 & 22 & $23: 04: 2712.390$ & 40.586 & 2.02 & 0.36 \\
\hline 2008 & 10 & 22 & $23: 44: 1712.419$ & 40.574 & 1.77 & 0.45 \\
\hline 2008 & 10 & 23 & $01: 58: 2612.392$ & 40.584 & 1.76 & 0.5 \\
\hline
\end{tabular}




\begin{tabular}{|c|c|c|c|c|c|c|}
\hline 2008 & 10 & 23 & $02: 53: 2112.419$ & 40.583 & 2.34 & 0.34 \\
\hline 2008 & 10 & 23 & $05: 11: 3412.421$ & 40.575 & 2.62 & 0.42 \\
\hline 2008 & 10 & 23 & $10: 54: 4112.292$ & 40.615 & 2.06 & 0.87 \\
\hline 2008 & 10 & 23 & $14: 18: 4812.407$ & 40.583 & 2.42 & 0.69 \\
\hline 2008 & 10 & 23 & $14: 34: 5012.400$ & 40.564 & 2.13 & 0.54 \\
\hline 2008 & 10 & 23 & $15: 52: 5412.401$ & 40.577 & 2.01 & 0.62 \\
\hline 2008 & 10 & 23 & $19: 52: 0012.382$ & 40.585 & 1.90 & 0.47 \\
\hline 2008 & 10 & 23 & $20: 00: 5112.386$ & 40.574 & 1.98 & 0.39 \\
\hline 2008 & 10 & 23 & $22: 12: 1312.436$ & 40.569 & 1.78 & 0.52 \\
\hline 2008 & 10 & 23 & $22: 25: 4312.334$ & 40.601 & 1.84 & 0.47 \\
\hline 2008 & 10 & 23 & $22: 26: 2712.361$ & 40.337 & 1.73 & 3.04 \\
\hline 2008 & 10 & 23 & $22: 59: 4012.433$ & 40.563 & 1.74 & 0.55 \\
\hline 2008 & 10 & 24 & $00: 45: 5212.401$ & 40.562 & 1.74 & 0.7 \\
\hline 2008 & 10 & 24 & $00: 55: 1912.411$ & 40.561 & 1.71 & 0.57 \\
\hline 2008 & 10 & 24 & $01: 41: 1512.397$ & 40.588 & 1.83 & 0.6 \\
\hline 2008 & 10 & 24 & $01: 54: 3612.419$ & 40.578 & 1.86 & 0.41 \\
\hline 2008 & 10 & 24 & $03: 40: 4712.397$ & 40.578 & 2.07 & 0.56 \\
\hline 2008 & 10 & 24 & $04: 36: 0812.372$ & 40.602 & 2.14 & 0.66 \\
\hline 2008 & 10 & 24 & $15: 20: 4012.398$ & 40.574 & 2.01 & 0.72 \\
\hline 2008 & 10 & 24 & $18: 15: 4712.436$ & 40.572 & 1.91 & 0.43 \\
\hline 2008 & 10 & 24 & $19: 45: 4312.460$ & 40.560 & 2.07 & 0.43 \\
\hline 2008 & 10 & 24 & $22: 05: 4412.400$ & 40.564 & 1.81 & 0.51 \\
\hline 2008 & 10 & 24 & $23: 36: 5112.422$ & 40.570 & 1.75 & 0.47 \\
\hline 2008 & 10 & 25 & $07: 00: 5712.387$ & 40.574 & 2.22 & 0.99 \\
\hline 2008 & 10 & 25 & $07: 46: 5412.451$ & 40.567 & 2.42 & 0.54 \\
\hline 2008 & 10 & 25 & $08: 50: 2412.339$ & 40.588 & 2.28 & 0.68 \\
\hline 2008 & 10 & 25 & $16: 17: 5312.357$ & 40.589 & 2.00 & 0.51 \\
\hline 2008 & 10 & 25 & $17: 28: 2212.365$ & 40.342 & 1.89 & 0.8 \\
\hline 2008 & 10 & 25 & $17: 58: 0612.367$ & 40.593 & 3.85 & 0.53 \\
\hline 2008 & 10 & 25 & $23: 01: 4512.376$ & 40.585 & 1.72 & 1.28 \\
\hline 2008 & 10 & 26 & $00: 22: 3812.363$ & 40.338 & 1.85 & 0.59 \\
\hline 2008 & 10 & 26 & $00: 54: 3112.400$ & 40.571 & 1.82 & 0.53 \\
\hline 2008 & 10 & 26 & $17: 00: 2012.391$ & 40.567 & 2.16 & 0.47 \\
\hline 2008 & 10 & 26 & $20: 11: 4312.393$ & 40.576 & 1.81 & 0.46 \\
\hline 2008 & 10 & 26 & $20: 29: 4912.381$ & 40.566 & 1.75 & 0.62 \\
\hline 2008 & 10 & 26 & $20: 52: 2612.365$ & 40.575 & 1.83 & 0.56 \\
\hline 2008 & 10 & 26 & $23: 38: 2412.363$ & 40.589 & 1.71 & 0.66 \\
\hline 2008 & 10 & 27 & $00: 09: 3812.470$ & 40.553 & 1.81 & 0.48 \\
\hline 2008 & 10 & 27 & $04: 45: 4712.377$ & 40.581 & 2.10 & 0.66 \\
\hline 2008 & 10 & 27 & $05: 51: 3512.361$ & 40.338 & 2.04 & 1.58 \\
\hline 2008 & 10 & 27 & $08: 28: 5812.419$ & 40.575 & 2.20 & 0.37 \\
\hline 2008 & 10 & 27 & $12: 45: 3712.394$ & 40.581 & 2.45 & 0.54 \\
\hline 2008 & 10 & 27 & $16: 59: 1012.466$ & 40.548 & 2.03 & 0.56 \\
\hline 2008 & 10 & 27 & $19: 17: 1212.341$ & 40.596 & 1.81 & 0.54 \\
\hline 2008 & 10 & 27 & $21: 24: 1612.357$ & 40.595 & 2.32 & 0.31 \\
\hline 2008 & 10 & 27 & $22: 39: 5712.385$ & 40.571 & 1.70 & 0.8 \\
\hline 2008 & 10 & 27 & $22: 41: 1313.687$ & 39.925 & 2.41 & 0.55 \\
\hline 2008 & 10 & 27 & $22: 49: 4112.358$ & 40.593 & 1.75 & 0.35 \\
\hline 2008 & 10 & 27 & $23: 51: 1112.371$ & 40.600 & 2.24 & 0.34 \\
\hline 2008 & 10 & 28 & $01: 44: 2312.386$ & 40.560 & 1.72 & 0.56 \\
\hline 2008 & 10 & 28 & $02: 01: 2612.385$ & 40.584 & 1.90 & 0.53 \\
\hline 2008 & 10 & 28 & $03: 02: 2212.355$ & 40.499 & 2.03 & 0.38 \\
\hline 2008 & 10 & 28 & $09: 46: 1312.352$ & 40.609 & 2.49 & 0.54 \\
\hline 2008 & 10 & 29 & $01: 11: 2712.427$ & 40.559 & 1.97 & 0.5 \\
\hline 2008 & 10 & 29 & $02: 19: 4312.425$ & 40.560 & 1.84 & 0.79 \\
\hline 2008 & 10 & 29 & $07: 22: 3212.351$ & 40.608 & 2.57 & 0.36 \\
\hline 2008 & 10 & 29 & $18: 13: 3312.361$ & 40.613 & 1.89 & 0.55 \\
\hline 2008 & 10 & 29 & $19: 14: 4212.475$ & 40.554 & 1.86 & 0.76 \\
\hline 2008 & 10 & 30 & $00: 28: 0910.610$ & 39.916 & 2.51 & 5.55 \\
\hline 2008 & 10 & 30 & $00: 33: 5313.686$ & 39.918 & 2.45 & 0.59 \\
\hline 2008 & 10 & 30 & $01: 21: 4314.383$ & 40.239 & 2.66 & 6.72 \\
\hline 2008 & 10 & 30 & $03: 25: 0013.832$ & 40.550 & 2.67 & 1.41 \\
\hline 2008 & 10 & 30 & $18: 04: 0713.687$ & 39.925 & 2.54 & 5.52 \\
\hline 2008 & 10 & 30 & $19: 01: 0113.886$ & 40.529 & 2.60 & 1.97 \\
\hline 2008 & 10 & 30 & $21: 54: 0311.688$ & 39.622 & 2.27 & 0.57 \\
\hline 2008 & 10 & 30 & $23: 39: 2312.169$ & 40.695 & 1.68 & 1.69 \\
\hline 2008 & 10 & 31 & $00: 26: 3612.792$ & 40.335 & 1.91 & 0.29 \\
\hline 2008 & 10 & 31 & $01: 43: 5313.561$ & 39.904 & 2.46 & 2,00 \\
\hline 2008 & 10 & 31 & $06: 39: 2411.865$ & 41.573 & 2.51 & 0.84 \\
\hline 2008 & 10 & 31 & $16: 03: 0711.899$ & 41.301 & 2.13 & 0.5 \\
\hline 2008 & 10 & 31 & $16: 50: 2313.678$ & 39.933 & 2.56 & 1.31 \\
\hline 2008 & 10 & 31 & $23: 25: 2013.677$ & 39.938 & 2.55 & 0.81 \\
\hline 2008 & 10 & 31 & $23: 43: 0212.726$ & 39.493 & 2.34 & 1.36 \\
\hline 2008 & 11 & 1 & $00: 42: 4613.632$ & 39.941 & 3.07 & 0.56 \\
\hline 2008 & 11 & 1 & $02: 26: 2312.407$ & 40.570 & 2.54 & 0.5 \\
\hline 2008 & 11 & 1 & $03: 58: 5812.446$ & 40.535 & 2.11 & 1.4 \\
\hline
\end{tabular}




\begin{tabular}{|c|c|c|c|c|c|c|}
\hline 2008 & 11 & 1 & $08: 11: 0212.397$ & 40.487 & 2.31 & 2.25 \\
\hline 2008 & 11 & 1 & $10: 06: 4212.498$ & 39.542 & 2.59 & 2.41 \\
\hline 2008 & 11 & 1 & $13: 52: 5513.691$ & 39.922 & 2.72 & 0.76 \\
\hline 2008 & 11 & 1 & $14: 32: 2412.356$ & 40.604 & 2.45 & 0.77 \\
\hline 2008 & 11 & 1 & $15: 43: 1411.560$ & 40.987 & 2.26 & 1.49 \\
\hline 2008 & 11 & 1 & $17: 13: 0010.882$ & 39.724 & 2.64 & 1.45 \\
\hline 2008 & 11 & 1 & $19: 57: 4711.892$ & 40.915 & 1.93 & 2.09 \\
\hline 2008 & 11 & 1 & $21: 14: 0811.212$ & 39.712 & 2.47 & 1.52 \\
\hline 2008 & 11 & 1 & $21: 56: 5412.498$ & 39.598 & 2.33 & 0.87 \\
\hline 2008 & 11 & 1 & $22: 42: 2212.463$ & 40.547 & 1.83 & 0.6 \\
\hline 2008 & 11 & 1 & $23: 34: 0612.347$ & 40.601 & 1.80 & 1.02 \\
\hline 2008 & 11 & 2 & $00: 39: 1113.557$ & 39.904 & 2.62 & 0.55 \\
\hline 2008 & 11 & 2 & $03: 01: 1512.487$ & 40.569 & 2.05 & 1.13 \\
\hline 2008 & 11 & 2 & $09: 33: 4612.328$ & 40.599 & 2.15 & 1,00 \\
\hline 2008 & 11 & 2 & $19: 10: 1010.620$ & 39.859 & 2.81 & 6.29 \\
\hline 2008 & 11 & 2 & $19: 16: 2212.421$ & 40.578 & 1.91 & 0.9 \\
\hline 2008 & 11 & 2 & $21: 50: 5113.686$ & 39.919 & 2.94 & 5.97 \\
\hline 2008 & 11 & 3 & $00: 03: 2313.684$ & 39.916 & 2.70 & 0.67 \\
\hline 2008 & 11 & 3 & $00: 13: 3013.691$ & 39.918 & 2.68 & 4.77 \\
\hline 2008 & 11 & 3 & $00: 33: 0513.675$ & 39.939 & 2.68 & 0.75 \\
\hline 2008 & 11 & 3 & $00: 34: 4212.445$ & 40.570 & 2.22 & 0.51 \\
\hline 2008 & 11 & 3 & $00: 42: 4913.896$ & 40.560 & 2.71 & 1.39 \\
\hline 2008 & 11 & 3 & $01: 03: 1813.520$ & 39.988 & 2.73 & 0.44 \\
\hline 2008 & 11 & 3 & $01: 07: 1413.892$ & 40.578 & 2.75 & 2.79 \\
\hline 2008 & 11 & 3 & $01: 46: 3613.901$ & 40.570 & 2.73 & 1.7 \\
\hline 2008 & 11 & 3 & $02: 18: 1213.883$ & 40.520 & 2.75 & 1.64 \\
\hline 2008 & 11 & 3 & $07: 30: 3813.556$ & 39.906 & 2.81 & 4.36 \\
\hline 2008 & 11 & 3 & $11: 10: 0613.835$ & 40.530 & 3.01 & 0.69 \\
\hline 2008 & 11 & 3 & $11: 17: 1013.843$ & 40.552 & 3.08 & 2.75 \\
\hline 2008 & 11 & 3 & $12: 25: 0613.859$ & 40.535 & 3.44 & 1.17 \\
\hline 2008 & 11 & 3 & $12: 28: 3713.899$ & 40.604 & 3.32 & 2.62 \\
\hline 2008 & 11 & 3 & $15: 32: 1313.811$ & 40.510 & 2.77 & 2.17 \\
\hline 2008 & 11 & 3 & $16: 46: 3113.857$ & 40.512 & 2.89 & 1.71 \\
\hline 2008 & 11 & 3 & $18: 21: 4613.829$ & 40.552 & 3.06 & 1.34 \\
\hline 2008 & 11 & 3 & $18: 30: 3413.897$ & 40.521 & 2.77 & 1.58 \\
\hline 2008 & 11 & 3 & $18: 36: 3213.833$ & 40.520 & 2.77 & 0.94 \\
\hline 2008 & 11 & 3 & $19: 36: 0913.852$ & 40.530 & 2.86 & 6.46 \\
\hline 2008 & 11 & 3 & $20: 15: 1912.484$ & 40.544 & 2.06 & 0.44 \\
\hline 2008 & 11 & 3 & $20: 42: 2913.821$ & 40.573 & 3.02 & 4.96 \\
\hline 2008 & 11 & 3 & $22: 21: 1013.878$ & 40.541 & 2.77 & 1.54 \\
\hline 2008 & 11 & 3 & $22: 29: 4912.368$ & 40.611 & 2.61 & 0.35 \\
\hline 2008 & 11 & 3 & $23: 52: 1813.870$ & 40.522 & 3.24 & 1.29 \\
\hline 2008 & 11 & 4 & $00: 06: 1113.849$ & 40.559 & 2.58 & 0.95 \\
\hline 2008 & 11 & 4 & $01: 18: 1113.823$ & 40.517 & 2.62 & 1.84 \\
\hline 2008 & 11 & 4 & $02: 54: 4913.844$ & 40.553 & 2.77 & 1.54 \\
\hline 2008 & 11 & 4 & $03: 03: 2313.816$ & 40.535 & 2.78 & 1.33 \\
\hline 2008 & 11 & 4 & $06: 02: 2713.858$ & 40.566 & 3.22 & 2.6 \\
\hline 2008 & 11 & 4 & $06: 22: 4813.823$ & 40.505 & 2.98 & 4.2 \\
\hline 2008 & 11 & 4 & $13: 13: 1013.678$ & 39.937 & 2.77 & 1.26 \\
\hline 2008 & 11 & 4 & $15: 06: 4513.555$ & 39.920 & 2.70 & 1.35 \\
\hline 2008 & 11 & 4 & $17: 13: 0413.844$ & 40.562 & 3.14 & 1.41 \\
\hline 2008 & 11 & 4 & $18: 23: 3213.886$ & 40.536 & 2.62 & 2.33 \\
\hline 2008 & 11 & 4 & $19: 33: 5013.679$ & 39.927 & 2.58 & 4.04 \\
\hline 2008 & 11 & 4 & $20: 48: 5112.872$ & 40.624 & 2.07 & 1.1 \\
\hline 2008 & 11 & 4 & $21: 33: 1413.673$ & 39.986 & 2.61 & 5.73 \\
\hline 2008 & 11 & 4 & $23: 45: 5513.805$ & 40.012 & 2.60 & 3.87 \\
\hline 2008 & 11 & 5 & $00: 53: 4013.542$ & 39.919 & 2.53 & 1.73 \\
\hline 2008 & 11 & 5 & $01: 05: 2013.549$ & 39.886 & 2.56 & 0.71 \\
\hline 2008 & 11 & 5 & $01: 12: 5412.448$ & 40.566 & 1.96 & 0.57 \\
\hline 2008 & 11 & 5 & $05: 51: 3313.916$ & 40.550 & 2.89 & 4.2 \\
\hline 2008 & 11 & 5 & $11: 58: 2912.597$ & 40.493 & 2.46 & 0.68 \\
\hline 2008 & 11 & 5 & $21: 25: 4312.433$ & 40.547 & 1.75 & 1.76 \\
\hline 2008 & 11 & 5 & $23: 46: 4613.685$ & 39.929 & 2.57 & 0.65 \\
\hline 2008 & 11 & 6 & $00: 15: 1012.095$ & 40.775 & 1.76 & 1.37 \\
\hline 2008 & 11 & 6 & $00: 45: 1313.484$ & 40.866 & 2.61 & 1.25 \\
\hline 2008 & 11 & 7 & $11: 41: 5312.394$ & 40.576 & 2.35 & 0.46 \\
\hline 2008 & 11 & 7 & $20: 39: 1311.915$ & 41.218 & 2.20 & 0.38 \\
\hline 2008 & 11 & 7 & $23: 22: 4312.382$ & 40.583 & 1.99 & 0.57 \\
\hline 2008 & 11 & 7 & $23: 31: 3012.817$ & 39.592 & 2.36 & 2.88 \\
\hline 2008 & 11 & 7 & $23: 34: 3312.385$ & 40.583 & 1.96 & 0.51 \\
\hline 2008 & 11 & 8 & $00: 00: 2712.327$ & 40.541 & 1.89 & 1.06 \\
\hline 2008 & 11 & 8 & $18: 38: 2312.374$ & 40.567 & 1.92 & 1.84 \\
\hline 2008 & 11 & 8 & $18: 40: 0411.292$ & 41.542 & 2.62 & 0.77 \\
\hline 2008 & 11 & 8 & $19: 15: 3412.507$ & 40.540 & 1.97 & 0.62 \\
\hline 2008 & 11 & 8 & $19: 35: 4411.318$ & 41.595 & 2.92 & 0.78 \\
\hline 2008 & 11 & 8 & $21: 24: 0312.457$ & 40.556 & 1.85 & 0.63 \\
\hline
\end{tabular}




\begin{tabular}{|c|c|c|c|c|c|c|}
\hline 2008 & 11 & 8 & $23: 25: 3612.514$ & 40.536 & 1.83 & 0.65 \\
\hline 2008 & 11 & 9 & $00: 04: 3311.369$ & 39.712 & 2.52 & 0.71 \\
\hline 2008 & 11 & 9 & $00: 31: 4712.313$ & 40.608 & 1.92 & 0.46 \\
\hline 2008 & 11 & 9 & $01: 53: 0513.695$ & 39.920 & 2.60 & 0.66 \\
\hline 2008 & 11 & 9 & $22: 34: 5112.385$ & 40.568 & 1.94 & 0.57 \\
\hline 2008 & 11 & 9 & $22: 36: 1612.393$ & 40.560 & 1.91 & 0.51 \\
\hline 2008 & 11 & 10 & $02: 17: 5214.643$ & 40.047 & 3.11 & 7.91 \\
\hline 2008 & 11 & 10 & $17: 42: 3112.366$ & 40.576 & 1.77 & 0.77 \\
\hline 2008 & 11 & 10 & $23: 52: 2013.516$ & 39.982 & 2.42 & 0.76 \\
\hline 2008 & 11 & 12 & $00: 03: 4013.442$ & 40.008 & 2.35 & 1.57 \\
\hline 2008 & 11 & 12 & $18: 41: 0812.597$ & 40.506 & 2.03 & 0.73 \\
\hline 2008 & 11 & 12 & $18: 57: 2912.371$ & 40.585 & 2.09 & 0.57 \\
\hline 2008 & 11 & 13 & $19: 57: 1013.663$ & 39.934 & 2.52 & 0.9 \\
\hline 2008 & 11 & 14 & $00: 52: 4813.672$ & 39.908 & 2.46 & 0.72 \\
\hline 2008 & 11 & 14 & $20: 12: 1312.436$ & 40.567 & 1.86 & 0.46 \\
\hline 2008 & 11 & 14 & $23: 48: 5912.352$ & 40.565 & 1.75 & 0.63 \\
\hline 2008 & 11 & 15 & $04: 14: 0912.387$ & 40.269 & 1.94 & 2.02 \\
\hline 2008 & 11 & 15 & $10: 18: 3812.461$ & 40.556 & 2.24 & 0.69 \\
\hline 2008 & 11 & 15 & $10: 42: 1212.379$ & 40.310 & 1.96 & 0.81 \\
\hline 2008 & 11 & 15 & $14: 12: 3312.376$ & 40.319 & 2.18 & 0.75 \\
\hline 2008 & 11 & 15 & $20: 11: 0012.364$ & 40.585 & 1.96 & 0.43 \\
\hline 2008 & 11 & 15 & $21: 14: 3811.883$ & 41.174 & 2.02 & 0.45 \\
\hline 2008 & 11 & 16 & $08: 37: 1412.372$ & 40.337 & 2.05 & 0.52 \\
\hline 2008 & 11 & 16 & $17: 16: 4412.374$ & 40.336 & 2.72 & 8.49 \\
\hline 2008 & 11 & 16 & $17: 50: 4512.424$ & 40.571 & 3.12 & 0.58 \\
\hline 2008 & 11 & 16 & $20: 15: 5113.054$ & 39.576 & 2.60 & 1.45 \\
\hline 2008 & 11 & 16 & $23: 11: 3713.428$ & 40.882 & 2.33 & 1.37 \\
\hline 2008 & 11 & 17 & $00: 42: 5713.696$ & 39.917 & 2.61 & 5.24 \\
\hline 2008 & 11 & 17 & $03: 08: 4612.441$ & 40.570 & 2.50 & 0.42 \\
\hline 2008 & 11 & 17 & $15: 56: 2412.403$ & 40.577 & 2.00 & 0.81 \\
\hline 2008 & 11 & 18 & $20: 10: 2212.921$ & 39.682 & 2.46 & 2.18 \\
\hline 2008 & 11 & 18 & $23: 15: 2813.795$ & 39.978 & 2.63 & 1.3 \\
\hline 2008 & 11 & 19 & $18: 36: 0613.278$ & 39.926 & 2.38 & 0.44 \\
\hline 2008 & 11 & 20 & $02: 33: 2712.520$ & 40.535 & 2.09 & 0.57 \\
\hline 2008 & 11 & 21 & $17: 52: 2111.913$ & 39.465 & 2.39 & 0.97 \\
\hline 2008 & 11 & 21 & $23: 08: 4013.026$ & 40.683 & 2.10 & 0.6 \\
\hline 2008 & 11 & 21 & $23: 31: 5613.703$ & 39.923 & 2.50 & 2.35 \\
\hline 2008 & 11 & 22 & $21: 17: 5811.929$ & 41.213 & 1.89 & 0.53 \\
\hline 2008 & 11 & 23 & $01: 17: 5911.885$ & 41.569 & 2.19 & 1.3 \\
\hline 2008 & 11 & 23 & $01: 44: 4611.876$ & 41.560 & 2.14 & 0.87 \\
\hline 2008 & 11 & 23 & $08: 13: 1412.589$ & 40.497 & 2.38 & 0.86 \\
\hline 2008 & 11 & 23 & $20: 08: 5011.919$ & 41.509 & 2.16 & 0.83 \\
\hline 2008 & 11 & 23 & $22: 00: 2113.893$ & 40.009 & 2.55 & 1.2 \\
\hline 2008 & 11 & 24 & $18: 34: 1113.657$ & 39.895 & 2.51 & 5.46 \\
\hline 2008 & 11 & 24 & $21: 53: 0111.790$ & 42.073 & 2.41 & 4.29 \\
\hline 2008 & 11 & 25 & $21: 17: 0311.872$ & 41.132 & 1.85 & 0.56 \\
\hline 2008 & 11 & 25 & $22: 36: 2411.915$ & 41.539 & 3.23 & 0.5 \\
\hline 2008 & 11 & 26 & $00: 10: 1711.881$ & 41.166 & 2.08 & 0.33 \\
\hline 2008 & 11 & 26 & $00: 43: 1911.887$ & 41.147 & 1.83 & 0.39 \\
\hline 2008 & 11 & 26 & $01: 22: 1512.360$ & 40.616 & 1.73 & 0.49 \\
\hline 2008 & 11 & 26 & $10: 20: 1311.884$ & 41.157 & 2.26 & 0.43 \\
\hline 2008 & 11 & 26 & $10: 34: 3711.868$ & 41.145 & 2.19 & 0.67 \\
\hline 2008 & 11 & 26 & $23: 47: 0312.354$ & 40.497 & 1.86 & 0.42 \\
\hline 2008 & 11 & 27 & $20: 15: 4012.377$ & 40.486 & 1.90 & 0.67 \\
\hline 2008 & 11 & 27 & $21: 21: 5613.150$ & 39.646 & 2.82 & 0.56 \\
\hline 2008 & 11 & 27 & $21: 52: 3912.998$ & 40.753 & 2.01 & 0.59 \\
\hline 2008 & 11 & 28 & $08: 07: 5912.367$ & 40.594 & 2.25 & 0.65 \\
\hline 2008 & 11 & 28 & $20: 57: 5612.989$ & 39.679 & 2.35 & 1.14 \\
\hline 2008 & 11 & 28 & $21: 39: 5812.939$ & 39.645 & 2.32 & 4.89 \\
\hline 2008 & 11 & 28 & $23: 45: 3713.679$ & 39.920 & 2.48 & 1.3 \\
\hline 2008 & 11 & 29 & $17: 39: 2012.135$ & 40.896 & 2.03 & 0.34 \\
\hline 2008 & 11 & 29 & $19: 05: 5712.117$ & 40.908 & 1.89 & 0.7 \\
\hline 2008 & 11 & 29 & $20: 43: 4312.703$ & 39.582 & 2.29 & 0.41 \\
\hline 2008 & 11 & 29 & $21: 30: 4413.667$ & 39.929 & 2.50 & 0.86 \\
\hline 2008 & 11 & 29 & $22: 12: 4312.068$ & 39.628 & 2.24 & 0.85 \\
\hline 2008 & 11 & 29 & $23: 38: 0913.744$ & 40.424 & 2.49 & 1.49 \\
\hline 2008 & 11 & 30 & $01: 30: 1513.135$ & 41.004 & 2.10 & 1.49 \\
\hline 2008 & 11 & 30 & $03: 08: 5912.358$ & 40.603 & 2.04 & 0.69 \\
\hline 2008 & 11 & 30 & $20: 49: 4713.803$ & 40.469 & 2.49 & 2.37 \\
\hline 2008 & 11 & 30 & $21: 46: 5813.405$ & 40.895 & 2.32 & 1.31 \\
\hline 2008 & 11 & 30 & $22: 39: 3013.410$ & 40.905 & 2.24 & 0.73 \\
\hline 2008 & 12 & 20 & $05: 42: 1312.420$ & 39.710 & 2.57 & 0.58 \\
\hline 2008 & 12 & 20 & $17: 34: 4212.418$ & 40.574 & 2.13 & 0.54 \\
\hline 2008 & 12 & 21 & $00: 28: 3012.475$ & 40.549 & 1.88 & 0.65 \\
\hline 2008 & 12 & 22 & $01: 45: 4510.399$ & 40.542 & 3.05 & 3.15 \\
\hline 2008 & 12 & 22 & $02: 01: 5711.902$ & 41.318 & 2.22 & 0.61 \\
\hline
\end{tabular}




\begin{tabular}{|c|c|c|c|c|c|c|}
\hline 2008 & 12 & 22 & $04: 37: 0810.346$ & 40.552 & 3.96 & 1.74 \\
\hline 2008 & 12 & 22 & $18: 58: 2310.376$ & 40.542 & 2.89 & 1.41 \\
\hline 2008 & 12 & 23 & $11: 07: 1210.682$ & 41.390 & 2.93 & 2.47 \\
\hline 2008 & 12 & 24 & $14: 24: 5512.363$ & 40.620 & 2.38 & 0.55 \\
\hline 2008 & 12 & 24 & $18: 41: 2712.352$ & 40.493 & 2.11 & 0.47 \\
\hline 2008 & 12 & 24 & $20: 26: 3212.863$ & 40.629 & 2.17 & 0.36 \\
\hline 2008 & 12 & 25 & $20: 04: 3213.714$ & 39.908 & 2.56 & 1.3 \\
\hline 2008 & 12 & 25 & $22: 21: 5913.467$ & 39.934 & 2.45 & 0.58 \\
\hline 2008 & 12 & 26 & $00: 13: 5412.434$ & 40.579 & 1.89 & 0.6 \\
\hline 2008 & 12 & 26 & $05: 41: 2013.250$ & 41.069 & 2.83 & 2.06 \\
\hline 2008 & 12 & 26 & $11: 26: 2013.263$ & 41.065 & 2.73 & 0.88 \\
\hline 2008 & 12 & 26 & $17: 12: 0012.439$ & 40.570 & 2.03 & 0.41 \\
\hline 2008 & 12 & 26 & $23: 14: 0811.955$ & 41.515 & 2.12 & 1.16 \\
\hline 2008 & 12 & 27 & $02: 19: 2511.288$ & 39.704 & 2.68 & 0.56 \\
\hline 2009 & 1 & 1 & $02: 59: 4211.971$ & 41.251 & 2.08 & 1.01 \\
\hline 2009 & 1 & 1 & $03: 13: 1712.463$ & 40.563 & 2.21 & 0.56 \\
\hline 2009 & 1 & 2 & $23: 01: 5912.410$ & 40.566 & 1.84 & 0.88 \\
\hline 2009 & 1 & 3 & $15: 16: 5413.472$ & 39.932 & 2.69 & 0.69 \\
\hline 2009 & 1 & 3 & $23: 14: 2713.710$ & 39.939 & 3.65 & 0.5 \\
\hline 2009 & 1 & 4 & $15: 16: 1711.963$ & 41.279 & 2.43 & 0.39 \\
\hline 2009 & 1 & 4 & $22: 02: 4313.473$ & 39.933 & 2.51 & 0.79 \\
\hline 2009 & 1 & 5 & $03: 38: 5213.467$ & 39.944 & 2.68 & 0.97 \\
\hline 2009 & 1 & 8 & $00: 12: 0612.321$ & 40.599 & 1.72 & 0.74 \\
\hline 2009 & 1 & 9 & $00: 05: 0312.505$ & 40.538 & 1.78 & 0.81 \\
\hline 2009 & 1 & 9 & $01: 30: 5914.861$ & 39.517 & 2.97 & 10.74 \\
\hline 2009 & 1 & 10 & $02: 08: 4811.956$ & 41.275 & 2.09 & 0.46 \\
\hline 2009 & 1 & 10 & $20: 09: 0310.363$ & 40.986 & 2.78 & 6.12 \\
\hline 2009 & 1 & 11 & $04: 06: 3713.510$ & 39.927 & 2.93 & 0.49 \\
\hline 2009 & 1 & 11 & $21: 08: 2412.430$ & 40.570 & 1.92 & 0.63 \\
\hline 2009 & 1 & 11 & $22: 15: 2813.651$ & 39.918 & 2.66 & 1.61 \\
\hline 2009 & 1 & 12 & $23: 55: 5912.134$ & 40.898 & 1.80 & 0.56 \\
\hline 2009 & 1 & 13 & $15: 11: 2013.509$ & 39.927 & 2.66 & 0.6 \\
\hline 2009 & 1 & 13 & $20: 07: 1112.431$ & 40.566 & 1.93 & 0.5 \\
\hline 2009 & 1 & 14 & $09: 20: 5013.649$ & 39.921 & 2.84 & 1.45 \\
\hline 2009 & 1 & 14 & $22: 03: 3612.851$ & 40.153 & 2.71 & 0.34 \\
\hline 2009 & 1 & 16 & $03: 28: 3912.851$ & 40.153 & 3.33 & 0.35 \\
\hline 2009 & 1 & 17 & $20: 11: 3912.448$ & 40.561 & 2.05 & 0.57 \\
\hline 2009 & 1 & 17 & $23: 24: 2712.247$ & 39.640 & 2.50 & 0.88 \\
\hline 2009 & 1 & 18 & $01: 00: 5012.208$ & 39.639 & 2.38 & 0.83 \\
\hline 2009 & 1 & 19 & $23: 52: 1813.428$ & 40.925 & 2.48 & 0.83 \\
\hline 2009 & 1 & 21 & $23: 43: 4412.344$ & 40.587 & 2.04 & 0.62 \\
\hline 2009 & 1 & 22 & $02: 04: 0112.256$ & 39.641 & 2.58 & 0.43 \\
\hline 2009 & 1 & 23 & $01: 34: 3811.928$ & 41.276 & 2.05 & 0.55 \\
\hline 2009 & 1 & 23 & $08: 25: 0013.678$ & 39.910 & 3.01 & 1,00 \\
\hline 2009 & 1 & 23 & $23: 50: 1212.511$ & 40.539 & 1.92 & 0.45 \\
\hline 2009 & 1 & 24 & $00: 09: 2712.347$ & 40.594 & 1.75 & 0.9 \\
\hline 2009 & 1 & 24 & $19: 28: 4612.463$ & 40.556 & 2.33 & 0.64 \\
\hline 2009 & 1 & 24 & $22: 16: 5011.912$ & 41.479 & 2.14 & 0.55 \\
\hline 2009 & 1 & 25 & $07: 49: 5612.752$ & 40.628 & 2.29 & 0.92 \\
\hline 2009 & 1 & 25 & $21: 51: 0912.327$ & 40.605 & 2.01 & 0.44 \\
\hline 2009 & 1 & 26 & $02: 01: 5212.460$ & 40.557 & 2.33 & 0.46 \\
\hline 2009 & 1 & 26 & $10: 03: 5811.886$ & 41.072 & 2.29 & 0.47 \\
\hline 2009 & 1 & 26 & $10: 27: 3311.900$ & 41.075 & 2.31 & 0.41 \\
\hline 2009 & 1 & 26 & $13: 32: 4712.366$ & 40.586 & 2.16 & 0.73 \\
\hline 2009 & 1 & 27 & $22: 44: 5412.487$ & 40.541 & 1.96 & 0.56 \\
\hline 2009 & 1 & 28 & $00: 37: 2013.520$ & 39.938 & 2.48 & 0.69 \\
\hline 2009 & 1 & 29 & $03: 52: 0212.454$ & 40.558 & 1.98 & 1.46 \\
\hline 2009 & 1 & 29 & $07: 04: 4513.139$ & 39.927 & 2.72 & 0.47 \\
\hline 2009 & 1 & 29 & $22: 26: 4713.124$ & 39.671 & 2.92 & 0.71 \\
\hline 2009 & 1 & 30 & $01: 36: 3212.484$ & 40.534 & 1.89 & 0.9 \\
\hline 2009 & 1 & 30 & $01: 45: 2712.448$ & 40.558 & 1.87 & 0.53 \\
\hline 2009 & 1 & 30 & $21: 09: 2412.400$ & 40.601 & 1.86 & 0.67 \\
\hline 2009 & 1 & 30 & $21: 56: 3212.326$ & 40.609 & 1.79 & 0.76 \\
\hline 2009 & 1 & 30 & $23: 28: 5912.355$ & 40.596 & 1.96 & 0.6 \\
\hline 2009 & 1 & 30 & $23: 45: 2812.341$ & 40.591 & 2.41 & 0.32 \\
\hline 2009 & 1 & 31 & $00: 43: 5912.376$ & 40.584 & 1.86 & 0.7 \\
\hline 2009 & 1 & 31 & $02: 40: 0012.770$ & 39.470 & 2.92 & 0.89 \\
\hline 2009 & 1 & 31 & $17: 48: 1312.855$ & 39.522 & 2.42 & 0.91 \\
\hline 2009 & 1 & 31 & $22: 14: 5611.890$ & 41.581 & 2.20 & 2.09 \\
\hline 2009 & 2 & 1 & $02: 38: 4212.042$ & 41.609 & 2.35 & 4.87 \\
\hline 2009 & 2 & 1 & $17: 16: 3712.472$ & 40.554 & 2.06 & 0.58 \\
\hline 2009 & 2 & 2 & $01: 19: 2213.688$ & 39.904 & 2.52 & 0.78 \\
\hline 2009 & 2 & 2 & $15: 14: 5511.296$ & 39.595 & 2.87 & 0.58 \\
\hline 2009 & 2 & 3 & $23: 43: 1312.464$ & 40.559 & 1.97 & 0.71 \\
\hline 2009 & 2 & 5 & $05: 44: 1013.293$ & 41.068 & 2.82 & 4.77 \\
\hline 2009 & 2 & 6 & $00: 39: 0111.305$ & 41.600 & 2.48 & 0.8 \\
\hline
\end{tabular}




\begin{tabular}{|c|c|c|c|c|c|c|}
\hline 2009 & 2 & 6 & $14: 22: 5712.180$ & 39.695 & 2.56 & 0.66 \\
\hline 2009 & 2 & 6 & $21: 33: 0211.338$ & 41.630 & 3.08 & 1.09 \\
\hline 2009 & 2 & 6 & $23: 04: 0711.870$ & 41.015 & 1.91 & 0.71 \\
\hline 2009 & 2 & 7 & $02: 13: 1311.309$ & 41.633 & 2.65 & 1.57 \\
\hline 2009 & 2 & 7 & $10: 35: 2712.465$ & 40.560 & 2.50 & 0.54 \\
\hline 2009 & 2 & 7 & $17: 25: 1511.290$ & 41.595 & 2.74 & 3.74 \\
\hline 2009 & 2 & 7 & $18: 35: 5911.338$ & 41.604 & 3.89 & 1.05 \\
\hline 2009 & 2 & 7 & $18: 41: 4511.355$ & 41.612 & 3.26 & 1.34 \\
\hline 2009 & 2 & 7 & $20: 08: 1811.325$ & 41.657 & 2.67 & 1.72 \\
\hline 2009 & 2 & 8 & $06: 43: 2912.932$ & 39.565 & 2.77 & 3.62 \\
\hline 2009 & 2 & 8 & $15: 14: 4511.246$ & 41.561 & 2.80 & 1.77 \\
\hline 2009 & 2 & 8 & $19: 28: 2311.343$ & 41.592 & 2.94 & 1.34 \\
\hline 2009 & 2 & 8 & $19: 34: 0211.346$ & 41.625 & 2.57 & 0.67 \\
\hline 2009 & 2 & 9 & $01: 38: 1911.303$ & 41.598 & 2.88 & 1.1 \\
\hline 2009 & 2 & 9 & $22: 24: 2313.428$ & 39.998 & 2.48 & 0.84 \\
\hline 2009 & 2 & 10 & $20: 31: 5511.947$ & 41.206 & 2.86 & 6.28 \\
\hline 2009 & 2 & 11 & $01: 16: 3211.956$ & 41.178 & 2.44 & 6.44 \\
\hline 2009 & 2 & 11 & $16: 17: 4311.947$ & 41.188 & 2.42 & 0.68 \\
\hline 2009 & 2 & 11 & $16: 33: 4311.944$ & 41.197 & 2.17 & 2.46 \\
\hline 2009 & 2 & 11 & $17: 11: 3212.312$ & 40.616 & 2.15 & 0.64 \\
\hline 2009 & 2 & 11 & $17: 13: 4812.289$ & 40.606 & 2.07 & 0.58 \\
\hline 2009 & 2 & 11 & $17: 17: 0512.321$ & 40.614 & 2.15 & 0.76 \\
\hline 2009 & 2 & 11 & $17: 20: 0312.313$ & 40.611 & 2.18 & 0.65 \\
\hline 2009 & 2 & 11 & $17: 27: 5012.306$ & 40.614 & 2.26 & 0.56 \\
\hline 2009 & 2 & 11 & $17: 29: 4412.303$ & 40.615 & 2.30 & 0.58 \\
\hline 2009 & 2 & 11 & $17: 32: 1712.314$ & 40.615 & 2.55 & 0.55 \\
\hline 2009 & 2 & 11 & $17: 37: 2011.949$ & 41.188 & 2.30 & 0.56 \\
\hline 2009 & 2 & 11 & $17: 44: 3512.318$ & 40.611 & 2.27 & 0.74 \\
\hline 2009 & 2 & 11 & $17: 53: 2112.332$ & 40.614 & 2.62 & 0.72 \\
\hline 2009 & 2 & 11 & $17: 57: 3312.319$ & 40.608 & 2.71 & 0.73 \\
\hline 2009 & 2 & 11 & $17: 58: 1512.322$ & 40.603 & 3.17 & 0.7 \\
\hline 2009 & 2 & 11 & $17: 59: 5512.341$ & 40.615 & 2.96 & 1.23 \\
\hline 2009 & 2 & 11 & $18: 24: 2412.374$ & 40.636 & 2.97 & 1.71 \\
\hline 2009 & 2 & 11 & $18: 29: 2612.326$ & 40.608 & 2.94 & 0.62 \\
\hline 2009 & 2 & 11 & $18: 31: 2412.331$ & 40.624 & 2.84 & 0.73 \\
\hline 2009 & 2 & 11 & $18: 34: 2312.344$ & 40.609 & 3.00 & 0.4 \\
\hline 2009 & 2 & 11 & $18: 36: 4212.313$ & 40.618 & 3.04 & 0.71 \\
\hline 2009 & 2 & 11 & $18: 41: 1312.325$ & 40.625 & 3.06 & 0.52 \\
\hline 2009 & 2 & 11 & $18: 48: 2612.318$ & 40.616 & 3.22 & 0.52 \\
\hline 2009 & 2 & 11 & $18: 49: 4512.318$ & 40.618 & 3.08 & 0.34 \\
\hline 2009 & 2 & 11 & $18: 51: 2612.315$ & 40.618 & 2.86 & 0.81 \\
\hline 2009 & 2 & 11 & $18: 52: 5412.297$ & 40.621 & 2.99 & 0.64 \\
\hline 2009 & 2 & 11 & $18: 54: 2412.314$ & 40.617 & 3.28 & 0.3 \\
\hline 2009 & 2 & 11 & $18: 57: 4712.317$ & 40.618 & 3.41 & 0.51 \\
\hline 2009 & 2 & 11 & $19: 00: 5612.315$ & 40.608 & 3.09 & 0.61 \\
\hline 2009 & 2 & 11 & $19: 08: 4712.325$ & 40.622 & 3.23 & 0.35 \\
\hline 2009 & 2 & 11 & $19: 14: 0412.307$ & 40.616 & 2.96 & 0.36 \\
\hline 2009 & 2 & 11 & $19: 15: 0212.296$ & 40.620 & 2.67 & 0.52 \\
\hline 2009 & 2 & 11 & $19: 25: 3512.325$ & 40.537 & 2.49 & 2.68 \\
\hline 2009 & 2 & 11 & $19: 28: 3512.337$ & 40.620 & 2.94 & 0.59 \\
\hline 2009 & 2 & 11 & $19: 33: 3612.432$ & 40.623 & 2.71 & 2.5 \\
\hline 2009 & 2 & 11 & $19: 35: 1412.343$ & 40.603 & 2.80 & 0.35 \\
\hline 2009 & 2 & 11 & $19: 41: 0912.371$ & 40.625 & 2.55 & 1,00 \\
\hline 2009 & 2 & 11 & $19: 52: 3712.368$ & 40.584 & 2.77 & 0.39 \\
\hline 2009 & 2 & 11 & $19: 59: 5812.291$ & 40.625 & 2.85 & 0.53 \\
\hline 2009 & 2 & 11 & $20: 08: 0812.366$ & 40.615 & 2.67 & 0.91 \\
\hline 2009 & 2 & 11 & $20: 09: 2712.332$ & 40.628 & 3.09 & 0.53 \\
\hline 2009 & 2 & 11 & $20: 13: 0912.342$ & 40.598 & 2.75 & 0.75 \\
\hline 2009 & 2 & 11 & $20: 17: 4212.370$ & 40.599 & 3.11 & 0.71 \\
\hline 2009 & 2 & 11 & $20: 19: 4812.298$ & 40.625 & 2.71 & 0.4 \\
\hline 2009 & 2 & 11 & $20: 22: 4812.358$ & 40.600 & 3.59 & 0.49 \\
\hline 2009 & 2 & 11 & $20: 29: 1112.293$ & 40.622 & 2.67 & 0.69 \\
\hline 2009 & 2 & 11 & $20: 29: 4712.320$ & 40.617 & 3.15 & 0.52 \\
\hline 2009 & 2 & 11 & $20: 38: 4012.377$ & 40.595 & 2.82 & 1.31 \\
\hline 2009 & 2 & 11 & $20: 40: 3612.410$ & 40.580 & 2.87 & 0.74 \\
\hline 2009 & 2 & 11 & $20: 43: 3012.388$ & 40.602 & 2.97 & 0.72 \\
\hline 2009 & 2 & 11 & $20: 49: 3512.354$ & 40.590 & 3.61 & 0.35 \\
\hline 2009 & 2 & 11 & $21: 05: 0112.358$ & 40.596 & 3.19 & 0.42 \\
\hline 2009 & 2 & 11 & $21: 14: 3212.366$ & 40.597 & 3.40 & 0.63 \\
\hline 2009 & 2 & 11 & $21: 20: 5212.366$ & 40.587 & 3.39 & 0.42 \\
\hline 2009 & 2 & 11 & $21: 34: 4612.381$ & 40.573 & 2.75 & 0.51 \\
\hline 2009 & 2 & 11 & $21: 37: 2012.343$ & 40.598 & 2.58 & 0.42 \\
\hline 2009 & 2 & 11 & $21: 39: 5412.366$ & 40.593 & 2.98 & 0.41 \\
\hline 2009 & 2 & 11 & $21: 40: 3712.383$ & 40.605 & 3.53 & 0.93 \\
\hline 2009 & 2 & 11 & $21: 47: 5112.367$ & 40.594 & 2.84 & 0.62 \\
\hline 2009 & 2 & 11 & $21: 50: 2212.361$ & 40.605 & 3.01 & 0.43 \\
\hline
\end{tabular}




\begin{tabular}{|c|c|c|c|c|c|c|}
\hline 2009 & 2 & 11 & $21: 53: 3212.379$ & 40.596 & 3.63 & 1.13 \\
\hline 2009 & 2 & 11 & $21: 55: 5212.349$ & 40.520 & 2.84 & 0.49 \\
\hline 2009 & 2 & 11 & $22: 17: 3612.360$ & 40.600 & 3.67 & 0.35 \\
\hline 2009 & 2 & 11 & $22: 20: 0912.363$ & 40.595 & 3.26 & 0.46 \\
\hline 2009 & 2 & 11 & $22: 27: 5012.394$ & 40.581 & 2.58 & 0.59 \\
\hline 2009 & 2 & 11 & $22: 31: 2112.380$ & 40.611 & 2.94 & 0.49 \\
\hline 2009 & 2 & 11 & $22: 34: 4412.369$ & 40.592 & 2.63 & 0.5 \\
\hline 2009 & 2 & 11 & $22: 36: 2412.351$ & 40.589 & 2.72 & 1.52 \\
\hline 2009 & 2 & 11 & $22: 42: 4012.409$ & 40.567 & 2.56 & 1.05 \\
\hline 2009 & 2 & 11 & $22: 45: 5212.370$ & 40.590 & 3.80 & 0.36 \\
\hline 2009 & 2 & 11 & $22: 50: 2512.383$ & 40.581 & 2.80 & 0.6 \\
\hline 2009 & 2 & 11 & $22: 57: 4012.370$ & 40.586 & 2.85 & 0.49 \\
\hline 2009 & 2 & 11 & $22: 59: 4612.352$ & 40.610 & 2.82 & 0.36 \\
\hline 2009 & 2 & 11 & $23: 04: 1812.364$ & 40.582 & 2.45 & 0.74 \\
\hline 2009 & 2 & 11 & $23: 08: 4012.371$ & 40.605 & 2.85 & 0.43 \\
\hline 2009 & 2 & 11 & $23: 11: 5512.384$ & 40.575 & 2.79 & 0.34 \\
\hline 2009 & 2 & 11 & $23: 15: 0112.372$ & 40.593 & 2.65 & 0.5 \\
\hline 2009 & 2 & 11 & $23: 19: 4512.364$ & 40.599 & 3.70 & 0.35 \\
\hline 2009 & 2 & 11 & $23: 28: 1212.379$ & 40.570 & 2.87 & 0.52 \\
\hline 2009 & 2 & 11 & $23: 38: 3612.381$ & 40.588 & 3.32 & 0.41 \\
\hline 2009 & 2 & 11 & $23: 42: 1912.356$ & 40.604 & 2.39 & 0.68 \\
\hline 2009 & 2 & 11 & $23: 49: 1912.364$ & 40.599 & 4.00 & 0.36 \\
\hline 2009 & 2 & 11 & $23: 58: 5312.333$ & 40.608 & 2.21 & 0.41 \\
\hline 2009 & 2 & 12 & $00: 03: 2412.313$ & 40.609 & 2.22 & 0.57 \\
\hline 2009 & 2 & 12 & $00: 04: 2012.322$ & 40.438 & 2.12 & 1.37 \\
\hline 2009 & 2 & 12 & $00: 08: 0212.383$ & 40.592 & 2.08 & 0.41 \\
\hline 2009 & 2 & 12 & $00: 10: 0712.345$ & 40.599 & 2.64 & 0.45 \\
\hline 2009 & 2 & 12 & $00: 14: 0412.359$ & 40.614 & 2.18 & 0.46 \\
\hline 2009 & 2 & 12 & $00: 20: 3412.357$ & 40.615 & 2.01 & 0.53 \\
\hline 2009 & 2 & 12 & $00: 21: 5812.345$ & 40.611 & 2.11 & 0.96 \\
\hline 2009 & 2 & 12 & $00: 22: 3112.339$ & 40.629 & 2.22 & 0.95 \\
\hline 2009 & 2 & 12 & $00: 24: 1212.348$ & 40.621 & 2.26 & 1.05 \\
\hline 2009 & 2 & 12 & $00: 30: 1912.363$ & 40.594 & 2.10 & 0.84 \\
\hline 2009 & 2 & 12 & $00: 32: 3812.338$ & 40.601 & 2.04 & 0.39 \\
\hline 2009 & 2 & 12 & $00: 33: 1212.329$ & 40.584 & 2.46 & 1.45 \\
\hline 2009 & 2 & 12 & $00: 37: 1112.318$ & 40.608 & 2.02 & 0.47 \\
\hline 2009 & 2 & 12 & $00: 41: 4112.339$ & 40.597 & 2.09 & 0.75 \\
\hline 2009 & 2 & 12 & $00: 43: 3612.357$ & 40.611 & 2.23 & 0.82 \\
\hline 2009 & 2 & 12 & $00: 48: 2812.320$ & 40.621 & 1.94 & 0.69 \\
\hline 2009 & 2 & 12 & $00: 52: 3512.352$ & 40.580 & 2.67 & 0.5 \\
\hline 2009 & 2 & 12 & $01: 01: 2612.365$ & 40.591 & 3.50 & 0.31 \\
\hline 2009 & 2 & 12 & $01: 05: 4812.367$ & 40.581 & 2.36 & 0.58 \\
\hline 2009 & 2 & 12 & $01: 14: 0812.328$ & 40.606 & 2.08 & 0.46 \\
\hline 2009 & 2 & 12 & $01: 15: 3912.331$ & 40.603 & 2.07 & 0.88 \\
\hline 2009 & 2 & 12 & $01: 17: 3212.335$ & 40.603 & 2.21 & 0.54 \\
\hline 2009 & 2 & 12 & $01: 22: 2712.365$ & 40.621 & 1.98 & 0.84 \\
\hline 2009 & 2 & 12 & $01: 23: 2112.329$ & 40.631 & 2.26 & 0.63 \\
\hline 2009 & 2 & 12 & $01: 25: 2512.358$ & 40.595 & 2.21 & 0.79 \\
\hline 2009 & 2 & 12 & $01: 33: 2012.381$ & 40.610 & 2.29 & 1.23 \\
\hline 2009 & 2 & 12 & $01: 43: 2312.384$ & 40.554 & 1.98 & 1.17 \\
\hline 2009 & 2 & 12 & $01: 47: 3312.357$ & 40.591 & 2.22 & 0.69 \\
\hline 2009 & 2 & 12 & $01: 53: 1212.340$ & 40.603 & 2.01 & 0.91 \\
\hline 2009 & 2 & 12 & $01: 55: 3812.346$ & 40.601 & 2.04 & 0.5 \\
\hline 2009 & 2 & 12 & $02: 00: 1012.348$ & 40.585 & 2.16 & 0.77 \\
\hline 2009 & 2 & 12 & $02: 01: 2112.323$ & 40.609 & 2.25 & 0.65 \\
\hline 2009 & 2 & 12 & $02: 05: 1012.352$ & 40.609 & 2.07 & 0.49 \\
\hline 2009 & 2 & 12 & $02: 07: 5812.351$ & 40.614 & 2.50 & 0.41 \\
\hline 2009 & 2 & 12 & $02: 10: 2812.355$ & 40.601 & 2.16 & 0.7 \\
\hline 2009 & 2 & 12 & $02: 19: 0012.348$ & 40.605 & 2.09 & 0.64 \\
\hline 2009 & 2 & 12 & $02: 28: 4312.316$ & 40.617 & 2.01 & 0.56 \\
\hline 2009 & 2 & 12 & $02: 30: 3112.323$ & 40.604 & 2.00 & 0.64 \\
\hline 2009 & 2 & 12 & $02: 41: 0312.369$ & 40.596 & 2.28 & 0.57 \\
\hline 2009 & 2 & 12 & $02: 43: 2012.346$ & 40.585 & 2.13 & 0.66 \\
\hline 2009 & 2 & 12 & $02: 49: 4012.357$ & 40.615 & 2.24 & 0.69 \\
\hline 2009 & 2 & 12 & $02: 51: 0312.376$ & 40.584 & 2.49 & 0.45 \\
\hline 2009 & 2 & 12 & $02: 53: 2612.351$ & 40.601 & 2.44 & 0.52 \\
\hline 2009 & 2 & 12 & $03: 00: 1012.375$ & 40.585 & 2.42 & 0.55 \\
\hline 2009 & 2 & 12 & $03: 33: 1612.312$ & 40.612 & 2.60 & 0.45 \\
\hline 2009 & 2 & 12 & $04: 09: 1612.390$ & 40.614 & 2.10 & 1.39 \\
\hline 2009 & 2 & 12 & $04: 29: 4512.373$ & 40.623 & 2.18 & 1.15 \\
\hline 2009 & 2 & 12 & $05: 10: 1412.320$ & 40.614 & 2.09 & 1.3 \\
\hline 2009 & 2 & 12 & $05: 24: 4612.319$ & 40.607 & 2.19 & 0.62 \\
\hline 2009 & 2 & 12 & $05: 46: 3912.312$ & 40.605 & 2.06 & 0.86 \\
\hline 2009 & 2 & 12 & $05: 57: 2612.361$ & 40.613 & 2.84 & 0.77 \\
\hline 2009 & 2 & 12 & $06: 02: 5312.338$ & 40.604 & 2.30 & 0.85 \\
\hline 2009 & 2 & 12 & $07: 05: 3412.355$ & 40.587 & 2.09 & 1.01 \\
\hline
\end{tabular}




\begin{tabular}{|c|c|c|c|c|c|c|}
\hline 2009 & 2 & 12 & $08: 35: 3912.325$ & 40.603 & 2.15 & 0.63 \\
\hline 2009 & 2 & 12 & $08: 56: 3211.949$ & 41.181 & 2.29 & 0.64 \\
\hline 2009 & 2 & 12 & $09: 10: 2512.343$ & 40.520 & 2.19 & 0.73 \\
\hline 2009 & 2 & 12 & $11: 02: 0012.361$ & 40.598 & 2.28 & 0.53 \\
\hline 2009 & 2 & 12 & $12: 50: 5312.371$ & 40.595 & 2.10 & 0.65 \\
\hline 2009 & 2 & 12 & $13: 01: 0012.352$ & 40.600 & 2.22 & 0.64 \\
\hline 2009 & 2 & 12 & $13: 02: 5612.372$ & 40.587 & 2.21 & 1.1 \\
\hline 2009 & 2 & 12 & $13: 05: 5112.328$ & 40.607 & 2.07 & 0.6 \\
\hline 2009 & 2 & 12 & $13: 11: 4612.362$ & 40.587 & 2.11 & 0.54 \\
\hline 2009 & 2 & 12 & $13: 16: 0812.381$ & 40.596 & 2.21 & 1.06 \\
\hline 2009 & 2 & 12 & $14: 00: 3912.368$ & 40.627 & 2.20 & 1.26 \\
\hline 2009 & 2 & 12 & $15: 12: 0312.311$ & 40.606 & 1.96 & 0.88 \\
\hline 2009 & 2 & 12 & $15: 32: 0112.349$ & 40.612 & 2.14 & 0.82 \\
\hline 2009 & 2 & 12 & $16: 25: 5812.330$ & 40.608 & 2.25 & 0.67 \\
\hline 2009 & 2 & 12 & $16: 33: 4412.357$ & 40.603 & 2.33 & 0.55 \\
\hline 2009 & 2 & 12 & $16: 54: 4312.358$ & 40.570 & 2.08 & 1.97 \\
\hline 2009 & 2 & 12 & $18: 12: 5012.347$ & 40.606 & 2.43 & 0.75 \\
\hline 2009 & 2 & 12 & $18: 20: 4912.358$ & 40.585 & 2.07 & 0.79 \\
\hline 2009 & 2 & 12 & $18: 39: 4612.364$ & 40.604 & 1.93 & 0.66 \\
\hline 2009 & 2 & 12 & $18: 45: 3112.311$ & 40.612 & 1.95 & 0.82 \\
\hline 2009 & 2 & 12 & $18: 54: 4012.317$ & 40.608 & 2.15 & 0.48 \\
\hline 2009 & 2 & 12 & $19: 04: 4512.325$ & 40.606 & 2.41 & 0.4 \\
\hline 2009 & 2 & 12 & $19: 20: 2112.318$ & 40.611 & 1.92 & 0.67 \\
\hline 2009 & 2 & 12 & $20: 03: 3912.329$ & 40.599 & 2.06 & 0.57 \\
\hline 2009 & 2 & 12 & $20: 09: 5012.356$ & 40.599 & 2.11 & 0.57 \\
\hline 2009 & 2 & 12 & $20: 25: 2412.311$ & 40.612 & 2.11 & 0.42 \\
\hline 2009 & 2 & 12 & $21: 05: 1012.328$ & 40.612 & 2.62 & 0.36 \\
\hline 2009 & 2 & 12 & $21: 13: 0012.370$ & 40.621 & 2.09 & 1.29 \\
\hline 2009 & 2 & 12 & $22: 02: 0112.330$ & 40.607 & 1.98 & 0.44 \\
\hline 2009 & 2 & 12 & $22: 54: 1111.956$ & 41.191 & 2.25 & 0.35 \\
\hline 2009 & 2 & 12 & $23: 09: 3112.366$ & 40.598 & 1.97 & 0.49 \\
\hline 2009 & 2 & 12 & $23: 11: 1912.355$ & 40.596 & 1.96 & 0.62 \\
\hline 2009 & 2 & 12 & $23: 28: 0912.316$ & 40.607 & 1.99 & 0.62 \\
\hline 2009 & 2 & 12 & $23: 50: 2212.367$ & 40.589 & 2.03 & 0.64 \\
\hline 2009 & 2 & 13 & $00: 20: 5012.326$ & 40.610 & 1.96 & 0.58 \\
\hline 2009 & 2 & 13 & $00: 25: 1112.328$ & 40.610 & 1.89 & 0.63 \\
\hline 2009 & 2 & 13 & $00: 26: 0712.330$ & 40.610 & 1.87 & 0.59 \\
\hline 2009 & 2 & 13 & $00: 53: 5511.925$ & 41.213 & 1.98 & 0.58 \\
\hline 2009 & 2 & 13 & $01: 04: 1012.314$ & 40.608 & 2.00 & 0.57 \\
\hline 2009 & 2 & 13 & $01: 04: 4112.366$ & 40.590 & 2.06 & 0.78 \\
\hline 2009 & 2 & 13 & $01: 18: 3512.374$ & 40.592 & 2.02 & 0.66 \\
\hline 2009 & 2 & 13 & $01: 34: 5212.367$ & 40.576 & 2.02 & 0.83 \\
\hline 2009 & 2 & 13 & $01: 54: 4012.323$ & 40.611 & 1.96 & 0.67 \\
\hline 2009 & 2 & 13 & $01: 58: 2012.328$ & 40.618 & 2.04 & 0.39 \\
\hline 2009 & 2 & 13 & $02: 23: 2012.353$ & 40.603 & 2.08 & 0.73 \\
\hline 2009 & 2 & 13 & $02: 31: 5512.350$ & 40.594 & 2.34 & 0.34 \\
\hline 2009 & 2 & 13 & $03: 10: 1612.317$ & 40.610 & 2.28 & 0.71 \\
\hline 2009 & 2 & 13 & $03: 32: 1012.301$ & 40.626 & 2.02 & 0.85 \\
\hline 2009 & 2 & 13 & $04: 32: 1712.309$ & 40.612 & 2.14 & 1.23 \\
\hline 2009 & 2 & 13 & $04: 48: 0712.339$ & 40.605 & 2.27 & 0.58 \\
\hline 2009 & 2 & 13 & $04: 57: 0412.331$ & 40.597 & 2.32 & 0.58 \\
\hline 2009 & 2 & 13 & $06: 06: 5012.358$ & 40.599 & 2.67 & 0.4 \\
\hline 2009 & 2 & 13 & $07: 49: 0612.330$ & 40.603 & 2.33 & 0.58 \\
\hline 2009 & 2 & 13 & $07: 50: 5112.327$ & 40.610 & 2.20 & 0.75 \\
\hline 2009 & 2 & 13 & $10: 15: 5912.372$ & 40.602 & 2.20 & 0.54 \\
\hline 2009 & 2 & 13 & $17: 13: 2012.353$ & 40.595 & 2.25 & 0.57 \\
\hline 2009 & 2 & 13 & $17: 40: 3112.345$ & 40.619 & 2.21 & 0.82 \\
\hline 2009 & 2 & 13 & $18: 09: 3212.360$ & 40.585 & 2.27 & 0.4 \\
\hline 2009 & 2 & 13 & $20: 15: 5012.312$ & 40.605 & 2.00 & 0.51 \\
\hline 2009 & 2 & 13 & $21: 23: 3712.370$ & 40.577 & 2.11 & 0.8 \\
\hline 2009 & 2 & 13 & $21: 35: 5411.937$ & 41.200 & 2.07 & 1.02 \\
\hline 2009 & 2 & 13 & $23: 16: 3111.951$ & 41.186 & 2.03 & 0.5 \\
\hline 2009 & 2 & 13 & $23: 35: 4711.940$ & 41.189 & 1.98 & 6.57 \\
\hline 2009 & 2 & 14 & $03: 17: 5912.372$ & 40.607 & 2.60 & 0.5 \\
\hline 2009 & 2 & 14 & $16: 43: 3312.354$ & 40.614 & 2.10 & 0.7 \\
\hline 2009 & 2 & 14 & $18: 53: 0311.299$ & 39.600 & 2.56 & 0.88 \\
\hline 2009 & 2 & 14 & $21: 24: 0212.316$ & 40.630 & 2.03 & 2.75 \\
\hline 2009 & 2 & 14 & $22: 24: 0812.349$ & 40.611 & 2.15 & 0.4 \\
\hline 2009 & 2 & 14 & $23: 49: 3712.349$ & 40.591 & 2.44 & 0.49 \\
\hline 2009 & 2 & 15 & $01: 35: 0712.395$ & 40.285 & 1.93 & 0.84 \\
\hline 2009 & 2 & 15 & $10: 28: 4013.047$ & 39.625 & 2.71 & 5.99 \\
\hline 2009 & 2 & 15 & $15: 09: 4011.948$ & 41.188 & 2.40 & 0.32 \\
\hline 2009 & 2 & 15 & $16: 40: 4011.959$ & 41.180 & 2.12 & 0.87 \\
\hline 2009 & 2 & 15 & $20: 06: 1612.444$ & 40.555 & 2.15 & 0.51 \\
\hline 2009 & 2 & 15 & $20: 10: 2313.135$ & 39.947 & 3.08 & 0.34 \\
\hline 2009 & 2 & 15 & $21: 39: 5611.954$ & 41.167 & 2.05 & 0.61 \\
\hline
\end{tabular}




\begin{tabular}{|c|c|c|c|c|c|c|}
\hline 2009 & 2 & 15 & $23: 21: 5611.931$ & 41.191 & 2.01 & 0.65 \\
\hline 2009 & 2 & 15 & $23: 46: 0511.950$ & 41.206 & 1.95 & 0.77 \\
\hline 2009 & 2 & 16 & $00: 58: 3311.940$ & 41.194 & 2.00 & 0.71 \\
\hline 2009 & 2 & 16 & $01: 06: 0511.936$ & 41.202 & 1.95 & 0.6 \\
\hline 2009 & 2 & 16 & $16: 28: 2312.350$ & 40.607 & 2.63 & 0.51 \\
\hline 2009 & 2 & 16 & $17: 30: 3913.048$ & 39.601 & 3.12 & 0.78 \\
\hline 2009 & 2 & 16 & $17: 30: 4512.351$ & 40.608 & 2.40 & 0.42 \\
\hline 2009 & 2 & 17 & $00: 08: 4413.669$ & 40.002 & 2.58 & 1.39 \\
\hline 2009 & 2 & 17 & $01: 39: 5812.374$ & 40.598 & 1.88 & 0.94 \\
\hline 2009 & 2 & 18 & $14: 20: 2411.950$ & 41.186 & 2.39 & 0.61 \\
\hline 2009 & 2 & 18 & $19: 56: 5112.018$ & 40.931 & 1.78 & 0.84 \\
\hline 2009 & 2 & 19 & $00: 39: 1212.114$ & 40.910 & 1.99 & 0.56 \\
\hline 2009 & 2 & 19 & $00: 59: 5612.137$ & 40.906 & 1.87 & 0.53 \\
\hline 2009 & 2 & 19 & $01: 18: 2212.130$ & 40.909 & 1.85 & 0.61 \\
\hline 2009 & 2 & 19 & $01: 38: 1312.137$ & 40.907 & 1.85 & 0.52 \\
\hline 2009 & 2 & 19 & $02: 33: 1713.080$ & 39.550 & 2.93 & 0.4 \\
\hline 2009 & 2 & 19 & $02: 50: 1211.953$ & 41.200 & 2.70 & 0.32 \\
\hline 2009 & 2 & 19 & $04: 00: 3311.950$ & 41.198 & 2.98 & 0.31 \\
\hline 2009 & 2 & 20 & $02: 57: 2212.366$ & 40.603 & 2.73 & 0.37 \\
\hline 2009 & 2 & 20 & $03: 00: 0412.341$ & 40.613 & 2.56 & 0.45 \\
\hline 2009 & 2 & 22 & $01: 24: 2113.627$ & 39.838 & 2.62 & 0.96 \\
\hline 2009 & 2 & 22 & $02: 10: 4813.624$ & 39.855 & 2.71 & 1.01 \\
\hline 2009 & 2 & 22 & $02: 30: 1211.951$ & 41.601 & 2.33 & 1.76 \\
\hline 2009 & 2 & 22 & $02: 34: 2012.374$ & 40.348 & 2.09 & 0.59 \\
\hline 2009 & 2 & 22 & $04: 57: 3012.374$ & 40.350 & 2.44 & 0.4 \\
\hline 2009 & 2 & 22 & $07: 12: 4212.376$ & 40.345 & 2.24 & 0.64 \\
\hline 2009 & 2 & 22 & $11: 58: 4713.619$ & 39.868 & 3.02 & 1.06 \\
\hline 2009 & 2 & 22 & $13: 48: 5013.052$ & 39.567 & 2.73 & 0.89 \\
\hline 2009 & 2 & 23 & $02: 08: 3513.065$ & 39.574 & 2.55 & 1.21 \\
\hline 2009 & 2 & 24 & $01: 59: 2313.644$ & 39.813 & 2.69 & 1.81 \\
\hline 2009 & 2 & 24 & $19: 38: 0613.042$ & 39.617 & 2.54 & 1.63 \\
\hline 2009 & 2 & 25 & $00: 07: 2112.062$ & 41.275 & 1.96 & 0.88 \\
\hline 2009 & 2 & 25 & $00: 11: 2712.477$ & 40.550 & 2.01 & 0.55 \\
\hline 2009 & 2 & 25 & $03: 43: 0313.258$ & 39.940 & 2.56 & 0.74 \\
\hline 2009 & 2 & 25 & $11: 28: 1511.951$ & 41.193 & 2.26 & 0.66 \\
\hline 2009 & 2 & 25 & $23: 17: 4812.350$ & 40.595 & 1.84 & 0.5 \\
\hline 2009 & 2 & 27 & $00: 36: 3411.926$ & 41.216 & 3.14 & 0.32 \\
\hline 2009 & 2 & 27 & $01: 24: 0711.930$ & 41.206 & 2.16 & 0.38 \\
\hline 2009 & 2 & 27 & $01: 54: 1311.931$ & 41.218 & 2.56 & 0.45 \\
\hline 2009 & 2 & 27 & $02: 32: 2411.917$ & 41.207 & 2.20 & 0.67 \\
\hline 2009 & 2 & 27 & $02: 33: 3811.909$ & 41.249 & 2.22 & 0.66 \\
\hline 2009 & 2 & 27 & $04: 43: 0211.922$ & 41.208 & 3.15 & 0.4 \\
\hline 2009 & 2 & 27 & $06: 00: 2011.932$ & 41.208 & 2.72 & 0.56 \\
\hline 2009 & 2 & 27 & $08: 32: 1411.895$ & 41.211 & 2.48 & 1.73 \\
\hline 2009 & 2 & 27 & $15: 26: 1011.904$ & 41.227 & 2.31 & 1.03 \\
\hline 2009 & 2 & 27 & $16: 08: 4911.934$ & 41.207 & 2.33 & 0.78 \\
\hline 2009 & 2 & 27 & $16: 15: 2811.943$ & 41.207 & 2.33 & 0.48 \\
\hline 2009 & 2 & 27 & $16: 36: 2111.940$ & 41.207 & 2.36 & 0.54 \\
\hline 2009 & 2 & 27 & $16: 39: 2211.916$ & 41.209 & 2.52 & 0.75 \\
\hline 2009 & 2 & 27 & $16: 56: 3311.921$ & 41.207 & 2.31 & 0.83 \\
\hline 2009 & 2 & 27 & $16: 57: 5311.969$ & 41.174 & 2.20 & 0.54 \\
\hline 2009 & 2 & 27 & $17: 10: 0511.946$ & 41.216 & 2.39 & 0.32 \\
\hline 2009 & 2 & 27 & $17: 22: 2611.922$ & 41.208 & 2.21 & 0.82 \\
\hline 2009 & 2 & 27 & $18: 02: 4211.932$ & 41.209 & 2.60 & 0.46 \\
\hline 2009 & 2 & 27 & $18: 52: 5811.931$ & 41.207 & 2.24 & 0.81 \\
\hline 2009 & 2 & 27 & $22: 38: 2911.935$ & 41.212 & 2.35 & 0.33 \\
\hline 2009 & 2 & 27 & $22: 49: 3011.945$ & 41.205 & 2.10 & 0.36 \\
\hline 2009 & 2 & 28 & $12: 22: 5911.917$ & 41.218 & 2.32 & 3.41 \\
\hline 2009 & 2 & 28 & $13: 53: 2113.014$ & 39.685 & 2.65 & 3.29 \\
\hline 2009 & 2 & 28 & $22: 37: 2512.367$ & 40.584 & 1.83 & 1.05 \\
\hline 2009 & 3 & 1 & $00: 29: 0511.948$ & 41.207 & 2.01 & 0.42 \\
\hline 2009 & 3 & 1 & $12: 25: 0911.913$ & 41.216 & 2.36 & 0.59 \\
\hline 2009 & 3 & 1 & $18: 23: 4912.460$ & 40.559 & 2.71 & 0.42 \\
\hline 2009 & 3 & 2 & $18: 01: 2511.928$ & 41.211 & 2.46 & 0.35 \\
\hline 2009 & 3 & 2 & $19: 00: 3313.207$ & 39.742 & 2.76 & 0.75 \\
\hline 2009 & 3 & 2 & $19: 07: 4411.937$ & 41.206 & 2.54 & 0.36 \\
\hline 2009 & 3 & 2 & $19: 09: 5311.935$ & 41.207 & 2.45 & 0.5 \\
\hline 2009 & 3 & 2 & $20: 17: 0911.930$ & 41.210 & 2.29 & 0.35 \\
\hline 2009 & 3 & 2 & $20: 20: 2011.930$ & 41.208 & 2.24 & 0.37 \\
\hline 2009 & 3 & 2 & $20: 30: 0811.928$ & 41.213 & 2.67 & 0.38 \\
\hline 2009 & 3 & 2 & $20: 50: 1911.924$ & 41.211 & 2.13 & 0.35 \\
\hline 2009 & 3 & 2 & $21: 19: 4011.934$ & 41.215 & 2.23 & 1.25 \\
\hline 2009 & 3 & 2 & $21: 55: 1111.933$ & 41.213 & 3.66 & 0.38 \\
\hline 2009 & 3 & 2 & $22: 00: 5711.926$ & 41.216 & 2.44 & 0.32 \\
\hline 2009 & 3 & 2 & $22: 07: 4211.931$ & 41.214 & 2.26 & 0.38 \\
\hline 2009 & 3 & 2 & $22: 25: 1311.928$ & 41.220 & 2.88 & 0.42 \\
\hline
\end{tabular}




\begin{tabular}{|c|c|c|c|c|c|c|}
\hline 2009 & 3 & 2 & $22: 26: 5911.943$ & 41.210 & 2.11 & 0.58 \\
\hline 2009 & 3 & 2 & $22: 33: 0111.924$ & 41.214 & 2.99 & 0.41 \\
\hline 2009 & 3 & 2 & $22: 40: 5811.920$ & 41.204 & 2.20 & 0.47 \\
\hline 2009 & 3 & 2 & $22: 50: 3411.941$ & 41.203 & 2.07 & 0.57 \\
\hline 2009 & 3 & 2 & $22: 53: 0711.938$ & 41.205 & 1.98 & 0.72 \\
\hline 2009 & 3 & 2 & $23: 05: 5311.940$ & 41.213 & 2.17 & 0.6 \\
\hline 2009 & 3 & 2 & $23: 11: 3811.934$ & 41.203 & 2.43 & 0.55 \\
\hline 2009 & 3 & 2 & $23: 55: 0611.937$ & 41.210 & 3.40 & 0.35 \\
\hline 2009 & 3 & 3 & $00: 25: 3711.940$ & 41.205 & 2.08 & 0.36 \\
\hline 2009 & 3 & 3 & $00: 36: 4111.918$ & 41.213 & 2.06 & 0.41 \\
\hline 2009 & 3 & 3 & $00: 42: 3411.919$ & 41.217 & 1.99 & 0.35 \\
\hline 2009 & 3 & 3 & $01: 10: 0811.935$ & 41.208 & 2.54 & 0.3 \\
\hline 2009 & 3 & 3 & $01: 33: 1411.918$ & 41.217 & 2.07 & 0.32 \\
\hline 2009 & 3 & 3 & $03: 14: 4511.955$ & 41.188 & 2.25 & 0.53 \\
\hline 2009 & 3 & 3 & $04: 07: 1911.944$ & 41.191 & 2.27 & 1.36 \\
\hline 2009 & 3 & 3 & $14: 12: 0211.920$ & 41.217 & 3.50 & 0.31 \\
\hline 2009 & 3 & 3 & $14: 28: 2911.921$ & 41.217 & 3.67 & 0.37 \\
\hline 2009 & 3 & 3 & $14: 39: 1911.933$ & 41.209 & 2.47 & 0.44 \\
\hline 2009 & 3 & 3 & $14: 49: 1111.961$ & 41.208 & 2.45 & 0.61 \\
\hline 2009 & 3 & 3 & $14: 53: 2511.972$ & 41.212 & 3.54 & 1.03 \\
\hline 2009 & 3 & 3 & $17: 35: 4313.068$ & 39.590 & 2.67 & 1.63 \\
\hline 2009 & 3 & 3 & $20: 56: 3011.953$ & 41.187 & 1.85 & 0.66 \\
\hline 2009 & 3 & 3 & $23: 06: 4911.926$ & 41.210 & 1.89 & 0.66 \\
\hline 2009 & 3 & 4 & $01: 29: 5311.625$ & 39.678 & 3.05 & 0.27 \\
\hline 2009 & 3 & 4 & $22: 59: 3711.938$ & 41.200 & 1.97 & 0.41 \\
\hline 2009 & 3 & 6 & $21: 04: 1911.929$ & 41.207 & 2.08 & 0.41 \\
\hline 2009 & 3 & 6 & $22: 46: 2911.919$ & 41.211 & 2.03 & 0.47 \\
\hline 2009 & 3 & 6 & $23: 17: 4311.935$ & 41.207 & 2.24 & 0.35 \\
\hline 2009 & 3 & 6 & $23: 29: 3411.928$ & 41.211 & 2.14 & 0.4 \\
\hline 2009 & 3 & 6 & $23: 40: 3311.928$ & 41.206 & 2.16 & 0.4 \\
\hline 2009 & 3 & 7 & $00: 13: 5411.927$ & 41.206 & 2.06 & 0.64 \\
\hline 2009 & 3 & 7 & $00: 46: 2711.929$ & 41.213 & 2.33 & 0.34 \\
\hline 2009 & 3 & 7 & $01: 39: 2213.072$ & 39.583 & 2.88 & 1.56 \\
\hline 2009 & 3 & 8 & $13: 59: 2611.934$ & 41.208 & 2.43 & 0.39 \\
\hline 2009 & 3 & 11 & $00: 28: 479.794$ & 39.892 & 3.55 & 0.96 \\
\hline 2009 & 3 & 11 & $23: 44: 1412.514$ & 40.519 & 2.23 & 1.17 \\
\hline 2009 & 3 & 12 & $01: 28: 2212.434$ & 40.561 & 2.22 & 0.54 \\
\hline 2009 & 3 & 12 & $19: 28: 5413.050$ & 39.606 & 2.74 & 0.78 \\
\hline 2009 & 3 & 13 & $10: 57: 1013.792$ & 39.728 & 2.95 & 2.19 \\
\hline 2009 & 3 & 13 & $20: 35: 1413.063$ & 39.559 & 3.09 & 2.53 \\
\hline 2009 & 3 & 14 & $00: 11: 2413.457$ & 40.791 & 2.61 & 1.78 \\
\hline 2009 & 3 & 17 & $21: 43: 0112.367$ & 40.576 & 2.11 & 0.43 \\
\hline 2009 & 3 & 18 & $21: 01: 1513.132$ & 39.954 & 2.44 & 0.52 \\
\hline 2009 & 3 & 19 & $03: 36: 3711.911$ & 41.056 & 2.10 & 0.41 \\
\hline 2009 & 3 & 20 & $15: 05: 2212.778$ & 39.474 & 2.75 & 2.26 \\
\hline 2009 & 3 & 23 & $00: 37: 5213.431$ & 40.872 & 2.55 & 5.89 \\
\hline 2009 & 3 & 25 & $18: 46: 0511.320$ & 39.642 & 2.75 & 0.93 \\
\hline 2009 & 3 & 26 & $00: 17: 0912.845$ & 39.551 & 2.55 & 0.88 \\
\hline 2009 & 3 & 27 & $02: 10: 0711.763$ & 41.118 & 2.01 & 0.84 \\
\hline 2009 & 3 & 28 & $23: 14: 2711.431$ & 40.987 & 2.21 & 0.66 \\
\hline 2009 & 3 & 28 & $23: 21: 4412.130$ & 40.900 & 1.85 & 0.48 \\
\hline 2009 & 3 & 29 & $18: 06: 3513.628$ & 40.756 & 2.82 & 6.51 \\
\hline 2009 & 3 & 29 & $23: 20: 5211.956$ & 41.271 & 2.14 & 0.27 \\
\hline 2009 & 3 & 30 & $23: 37: 1711.976$ & 41.255 & 1.96 & 0.69 \\
\hline 2009 & 3 & 31 & $01: 04: 5712.476$ & 40.551 & 2.09 & 0.6 \\
\hline 2009 & 3 & 31 & $17: 54: 3312.261$ & 39.599 & 2.43 & 0.55 \\
\hline 2009 & 4 & 1 & $02: 30: 2911.956$ & 41.275 & 2.07 & 0.63 \\
\hline 2009 & 4 & 2 & $22: 56: 3013.400$ & 39.981 & 2.61 & 1.16 \\
\hline 2009 & 4 & 3 & $15: 32: 2211.957$ & 41.274 & 2.21 & 0.57 \\
\hline 2009 & 4 & 4 & $00: 19: 5913.350$ & 40.922 & 2.41 & 3.99 \\
\hline 2009 & 4 & 4 & $08: 27: 5911.971$ & 41.286 & 2.37 & 0.45 \\
\hline 2009 & 4 & 5 & $01: 25: 1313.497$ & 39.962 & 2.60 & 1.59 \\
\hline 2009 & 4 & 5 & $05: 49: 1511.947$ & 41.289 & 2.36 & 0.62 \\
\hline 2009 & 4 & 5 & $09: 32: 4511.942$ & 41.278 & 2.16 & 0.76 \\
\hline 2009 & 4 & 5 & $21: 21: 2711.947$ & 41.274 & 1.98 & 0.49 \\
\hline 2009 & 4 & 6 & $03: 43: 1911.945$ & 41.275 & 2.29 & 0.44 \\
\hline 2009 & 4 & 6 & $21: 23: 2913.563$ & 39.951 & 2.58 & 5.06 \\
\hline 2009 & 4 & 6 & $23: 31: 0611.294$ & 39.669 & 2.35 & 1.07 \\
\hline 2009 & 4 & 11 & $07: 21: 2312.413$ & 40.572 & 2.21 & 0.69 \\
\hline 2009 & 4 & 11 & $22: 56: 3112.607$ & 40.425 & 1.97 & 1.23 \\
\hline 2009 & 4 & 12 & $00: 43: 2111.369$ & 39.794 & 2.53 & 0.39 \\
\hline 2009 & 4 & 12 & $02: 57: 2912.370$ & 40.346 & 2.12 & 1.37 \\
\hline 2009 & 4 & 13 & $19: 09: 4911.868$ & 41.005 & 2.32 & 0.36 \\
\hline 2009 & 4 & 14 & $01: 43: 2611.869$ & 40.992 & 1.91 & 1.88 \\
\hline 2009 & 4 & 16 & $14: 42: 5711.853$ & 42.137 & 3.08 & 1.02 \\
\hline 2009 & 4 & 16 & $21: 32: 0114.490$ & 39.958 & 3.22 & 5.75 \\
\hline
\end{tabular}




\begin{tabular}{|c|c|c|c|c|c|c|}
\hline 2009 & 4 & 16 & $22: 49: 2713.052$ & 39.602 & 2.62 & 0.66 \\
\hline 2009 & 4 & 17 & $20: 44: 3513.613$ & 39.934 & 2.69 & 1.27 \\
\hline 2009 & 4 & 17 & $23: 21: 2713.512$ & 40.012 & 2.69 & 2.26 \\
\hline 2009 & 4 & 18 & $20: 41: 2213.215$ & 39.775 & 2.96 & 0.76 \\
\hline 2009 & 4 & 19 & $05: 54: 2613.051$ & 39.618 & 2.76 & 0.71 \\
\hline 2009 & 4 & 19 & $19: 14: 1213.052$ & 39.603 & 2.87 & 0.66 \\
\hline 2009 & 4 & 20 & $23: 22: 1013.471$ & 40.008 & 2.56 & 3.48 \\
\hline 2009 & 4 & 21 & $20: 37: 5412.869$ & 40.263 & 2.32 & 0.55 \\
\hline 2009 & 4 & 24 & $00: 40: 4613.563$ & 39.891 & 2.65 & 0.96 \\
\hline 2009 & 4 & 26 & 19:39: 1611.067 & 40.905 & 2.85 & 0.66 \\
\hline 2009 & 4 & 26 & $22: 04: 0211.105$ & 40.865 & 2.35 & 0.79 \\
\hline 2009 & 4 & 27 & $00: 05: 1711.072$ & 40.909 & 2.49 & 0.49 \\
\hline 2009 & 4 & 27 & $14: 22: 0511.074$ & 40.906 & 2.95 & 0.5 \\
\hline 2009 & 4 & 27 & $20: 29: 2611.058$ & 40.913 & 2.62 & 0.65 \\
\hline 2009 & 4 & 30 & $18: 39: 0612.281$ & 40.624 & 2.05 & 2.66 \\
\hline 2009 & 4 & 30 & $21: 08: 5912.597$ & 40.501 & 2.07 & 0.83 \\
\hline 2009 & 5 & 2 & $21: 54: 2113.736$ & 39.964 & 2.72 & 3.93 \\
\hline 2009 & 5 & 3 & $06: 27: 1211.445$ & 39.928 & 2.86 & 0.72 \\
\hline 2009 & 5 & 4 & $01: 44: 0711.441$ & 39.933 & 2.46 & 0.72 \\
\hline 2009 & 5 & 4 & $17: 39: 5511.704$ & 42.017 & 3.04 & 13.8 \\
\hline 2009 & 5 & 4 & $19: 39: 2111.653$ & 39.726 & 3.02 & 0.43 \\
\hline 2009 & 5 & 5 & $18: 07: 0812.956$ & 39.650 & 2.52 & 3.25 \\
\hline 2009 & 5 & 6 & $17: 41: 2711.734$ & 42.135 & 2.66 & 1.16 \\
\hline 2009 & 5 & 8 & $09: 31: 1612.466$ & 40.550 & 2.34 & 0.78 \\
\hline 2009 & 5 & 8 & $09: 38: 5011.659$ & 42.304 & 2.92 & 2.22 \\
\hline 2009 & 5 & 8 & $21: 50: 1812.471$ & 40.537 & 2.39 & 0.54 \\
\hline 2009 & 5 & 10 & $19: 11: 1412.287$ & 40.625 & 2.01 & 0.76 \\
\hline 2009 & 5 & 11 & $00: 07: 5511.720$ & 42.132 & 2.54 & 1.14 \\
\hline 2009 & 5 & 11 & $02: 57: 5311.241$ & 40.895 & 2.43 & 0.7 \\
\hline 2009 & 5 & 11 & $19: 25: 4213.055$ & 39.563 & 2.59 & 1.09 \\
\hline 2009 & 5 & 12 & $23: 17: 2711.905$ & 41.369 & 2.04 & 0.71 \\
\hline 2009 & 5 & 13 & $15: 34: 2011.723$ & 41.751 & 2.49 & 1.91 \\
\hline 2009 & 5 & 13 & $19: 11: 2513.386$ & 40.003 & 2.60 & 1.73 \\
\hline 2009 & 5 & 14 & $19: 56: 1214.235$ & 40.269 & 2.89 & 2.35 \\
\hline 2009 & 5 & 14 & $22: 48: 0113.467$ & 39.993 & 2.90 & 0.99 \\
\hline 2009 & 5 & 15 & $20: 49: 4214.371$ & 40.120 & 3.24 & 3,00 \\
\hline 2009 & 5 & 15 & $23: 43: 5913.104$ & 39.623 & 2.53 & 0.64 \\
\hline 2009 & 5 & 16 & $03: 53: 1913.461$ & 39.990 & 2.84 & 1.42 \\
\hline 2009 & 5 & 16 & $08: 28: 3612.447$ & 40.560 & 2.40 & 0.7 \\
\hline 2009 & 5 & 16 & $20: 28: 0411.949$ & 42.240 & 3.09 & 1.02 \\
\hline 2009 & 5 & 16 & $22: 06: 2813.463$ & 39.989 & 2.70 & 1.4 \\
\hline 2009 & 5 & 17 & $00: 08: 5011.751$ & 42.090 & 2.90 & 1.51 \\
\hline 2009 & 5 & 17 & $04: 26: 4813.646$ & 40.684 & 2.89 & 2.19 \\
\hline 2009 & 5 & 17 & $09: 29: 3211.772$ & 42.038 & 2.78 & 2.51 \\
\hline 2009 & 5 & 17 & $09: 30: 1311.741$ & 42.053 & 3.22 & 0.86 \\
\hline 2009 & 5 & 17 & $10: 01: 5111.768$ & 42.046 & 2.97 & 1.04 \\
\hline 2009 & 5 & 17 & $10: 21: 1711.765$ & 42.056 & 2.78 & 0.89 \\
\hline 2009 & 5 & 17 & $10: 44: 3211.762$ & 42.053 & 2.94 & 1.3 \\
\hline 2009 & 5 & 17 & $11: 01: 3211.754$ & 42.072 & 2.76 & 0.8 \\
\hline 2009 & 5 & 17 & $11: 14: 1411.762$ & 42.048 & 2.74 & 0.93 \\
\hline 2009 & 5 & 18 & $03: 43: 5611.765$ & 42.044 & 2.90 & 6.49 \\
\hline 2009 & 5 & 19 & $22: 07: 2214.652$ & 39.862 & 2.98 & 12.46 \\
\hline 2009 & 5 & 20 & $20: 30: 3212.491$ & 40.552 & 1.97 & 0.67 \\
\hline 2009 & 5 & 22 & $04: 10: 2312.364$ & 40.606 & 2.33 & 0.58 \\
\hline 2009 & 5 & 23 & $23: 44: 1412.291$ & 40.627 & 1.95 & 0.62 \\
\hline 2009 & 5 & 26 & $18: 09: 3415.918$ & 39.234 & 3.70 & 9.15 \\
\hline 2009 & 5 & 27 & $20: 13: 3213.606$ & 39.919 & 2.67 & 0.77 \\
\hline 2009 & 5 & 28 & $18: 18: 0013.658$ & 39.967 & 2.74 & 0.9 \\
\hline 2009 & 5 & 30 & $09: 44: 1113.849$ & 41.501 & 3.20 & 2.94 \\
\hline 2009 & 5 & 30 & $13: 00: 5712.420$ & 40.578 & 2.32 & 1.13 \\
\hline 2009 & 5 & 30 & $14: 12: 4812.470$ & 40.553 & 2.34 & 0.65 \\
\hline 2009 & 5 & 31 & $01: 33: 2012.381$ & 40.584 & 2.08 & 0.79 \\
\hline 2009 & 6 & 1 & $02: 29: 1611.685$ & 39.709 & 2.72 & 0.5 \\
\hline 2009 & 6 & 1 & $13: 24: 2413.787$ & 41.456 & 3.52 & 1.85 \\
\hline 2009 & 6 & 1 & $20: 51: 1014.119$ & 39.871 & 2.91 & 1.94 \\
\hline 2009 & 6 & 2 & $17: 46: 4413.085$ & 39.631 & 2.89 & 1.58 \\
\hline 2009 & 6 & 2 & $18: 49: 0812.300$ & 40.619 & 2.05 & 0.59 \\
\hline 2009 & 6 & 3 & $00: 41: 0711.841$ & 42.280 & 2.63 & 1.02 \\
\hline 2009 & 6 & 3 & $00: 59: 0012.397$ & 40.578 & 1.96 & 0.6 \\
\hline 2009 & 6 & 3 & $21: 41: 5713.097$ & 39.650 & 2.77 & 0.94 \\
\hline 2009 & 6 & 4 & $21: 36: 0212.347$ & 40.331 & 1.90 & 0.58 \\
\hline 2009 & 6 & 5 & $00: 12: 5112.470$ & 40.548 & 1.95 & 0.82 \\
\hline 2009 & 6 & 5 & $01: 21: 4712.287$ & 40.621 & 1.82 & 1.08 \\
\hline 2009 & 6 & 5 & $07: 36: 3711.593$ & 42.039 & 3.00 & 0.75 \\
\hline 2009 & 6 & 6 & $18: 49: 5212.353$ & 40.582 & 2.03 & 0.54 \\
\hline 2009 & 6 & 7 & $00: 29: 0712.251$ & 40.968 & 1.86 & 0.95 \\
\hline
\end{tabular}




\begin{tabular}{|c|c|c|c|c|c|c|}
\hline 2009 & 6 & 7 & $17: 07: 5412.292$ & 40.603 & 2.14 & 0.73 \\
\hline 2009 & 6 & 8 & $16: 10: 3110.568$ & 39.770 & 2.94 & 7.63 \\
\hline 2009 & 6 & 9 & $13: 38: 4611.437$ & 39.943 & 2.48 & 2.64 \\
\hline 2009 & 6 & 10 & $18: 23: 1512.416$ & 40.576 & 2.12 & 0.54 \\
\hline 2009 & 6 & 11 & $01: 39: 3211.893$ & 41.424 & 2.19 & 0.45 \\
\hline 2009 & 6 & 11 & $13: 06: 5411.934$ & 41.205 & 2.13 & 0.57 \\
\hline 2009 & 6 & 11 & $20: 48: 3911.443$ & 39.893 & 2.39 & 3.26 \\
\hline 2009 & 6 & 12 & $20: 52: 2513.523$ & 39.990 & 2.75 & 2.19 \\
\hline 2009 & 6 & 13 & $01: 50: 0513.046$ & 39.598 & 2.54 & 1.25 \\
\hline 2009 & 6 & 13 & $02: 38: 3813.219$ & 39.781 & 2.68 & 1.43 \\
\hline 2009 & 6 & 15 & $01: 29: 1613.210$ & 39.786 & 2.59 & 1.01 \\
\hline 2009 & 6 & 16 & $18: 30: 4512.281$ & 40.630 & 1.99 & 1.31 \\
\hline 2009 & 6 & 16 & $20: 44: 2012.460$ & 40.541 & 2.70 & 0.67 \\
\hline 2009 & 6 & 17 & $18: 25: 1412.432$ & 40.565 & 2.11 & 0.49 \\
\hline 2009 & 6 & 18 & $00: 28: 3711.645$ & 41.008 & 1.90 & 2.05 \\
\hline 2009 & 6 & 18 & $01: 19: 4713.433$ & 39.991 & 2.66 & 2.04 \\
\hline 2009 & 6 & 18 & $10: 08: 0211.599$ & 40.952 & 2.00 & 5,00 \\
\hline 2009 & 6 & 18 & $10: 44: 2911.649$ & 41.023 & 2.12 & 0.55 \\
\hline 2009 & 6 & 18 & $21: 18: 1712.483$ & 40.556 & 2.13 & 2.41 \\
\hline 2009 & 6 & 19 & $10: 45: 1711.656$ & 41.016 & 1.96 & 2.97 \\
\hline 2009 & 6 & 19 & $11: 07: 0011.623$ & 40.975 & 2.10 & 0.56 \\
\hline 2009 & 6 & 20 & $14: 31: 1812.606$ & 40.489 & 2.79 & 0.56 \\
\hline 2009 & 6 & 22 & $00: 36: 2112.436$ & 40.566 & 1.95 & 0.74 \\
\hline 2009 & 6 & 23 & $17: 07: 1712.328$ & 40.343 & 2.13 & 0.49 \\
\hline 2009 & 6 & 23 & $17: 14: 4912.329$ & 40.343 & 2.41 & 0.36 \\
\hline 2009 & 6 & 23 & $22: 18: 1312.611$ & 39.578 & 2.47 & 0.9 \\
\hline 2009 & 6 & 24 & $17: 24: 0711.851$ & 41.093 & 2.13 & 1.45 \\
\hline 2009 & 6 & 24 & $23: 00: 4212.500$ & 40.538 & 2.10 & 0.76 \\
\hline 2009 & 6 & 25 & $20: 11: 4612.444$ & 40.566 & 2.35 & 0.54 \\
\hline 2009 & 6 & 25 & $20: 29: 0012.461$ & 40.559 & 2.25 & 0.64 \\
\hline 2009 & 6 & 25 & $21: 16: 5313.489$ & 40.823 & 2.75 & 2.07 \\
\hline 2009 & 6 & 26 & $00: 29: 2413.139$ & 39.690 & 2.63 & 1.38 \\
\hline 2009 & 6 & 26 & $01: 19: 0011.610$ & 41.022 & 1.81 & 0.78 \\
\hline 2009 & 6 & 26 & $08: 23: 5112.986$ & 40.687 & 2.66 & 0.57 \\
\hline 2009 & 6 & 26 & $19: 42: 0011.604$ & 41.041 & 2.33 & 0.56 \\
\hline 2009 & 6 & 26 & $21: 16: 2511.593$ & 41.003 & 1.93 & 2.08 \\
\hline 2009 & 6 & 26 & $21: 23: 4011.623$ & 41.043 & 2.00 & 3.33 \\
\hline 2009 & 6 & 26 & $21: 45: 0911.615$ & 41.040 & 1.91 & 0.61 \\
\hline 2009 & 6 & 26 & $21: 48: 2311.609$ & 41.044 & 2.06 & 0.89 \\
\hline 2009 & 6 & 26 & $22: 03: 4811.609$ & 41.034 & 1.87 & 0.85 \\
\hline 2009 & 6 & 26 & $22: 06: 3311.618$ & 41.030 & 1.85 & 0.7 \\
\hline 2009 & 6 & 26 & $23: 07: 3611.609$ & 41.031 & 1.85 & 1.17 \\
\hline 2009 & 6 & 27 & $00: 40: 1711.619$ & 41.046 & 1.87 & 1.07 \\
\hline 2009 & 6 & 27 & $01: 10: 5611.620$ & 41.023 & 1.99 & 0.66 \\
\hline 2009 & 6 & 27 & $07: 50: 0311.596$ & 41.018 & 2.09 & 2.4 \\
\hline 2009 & 6 & 27 & $09: 53: 4811.615$ & 41.045 & 2.21 & 0.9 \\
\hline 2009 & 6 & 27 & $12: 13: 0211.598$ & 41.022 & 2.15 & 2.35 \\
\hline 2009 & 6 & 27 & $23: 31: 0511.600$ & 41.038 & 1.87 & 0.56 \\
\hline 2009 & 6 & 28 & $00: 11: 5311.611$ & 41.041 & 1.96 & 0.95 \\
\hline 2009 & 6 & 28 & $00: 46: 5611.600$ & 41.037 & 1.85 & 0.73 \\
\hline 2009 & 6 & 28 & $01: 22: 4911.602$ & 41.039 & 1.96 & 0.73 \\
\hline 2009 & 6 & 28 & $01: 51: 3511.602$ & 41.037 & 1.96 & 1.01 \\
\hline 2009 & 6 & 28 & $02: 49: 4711.613$ & 41.037 & 2.33 & 0.65 \\
\hline 2009 & 6 & 28 & $08: 05: 0512.281$ & 40.629 & 2.07 & 1.1 \\
\hline 2009 & 6 & 28 & $08: 32: 1612.278$ & 40.545 & 2.02 & 2.14 \\
\hline 2009 & 6 & 28 & $08: 46: 3212.279$ & 40.624 & 2.09 & 1.75 \\
\hline 2009 & 6 & 28 & $08: 56: 0612.279$ & 40.624 & 2.23 & 2.11 \\
\hline 2009 & 6 & 28 & $09: 01: 1712.524$ & 40.465 & 2.21 & 11.15 \\
\hline 2009 & 6 & 28 & $09: 14: 4912.291$ & 40.618 & 2.17 & 0.94 \\
\hline 2009 & 6 & 28 & $09: 16: 1512.258$ & 40.663 & 2.06 & 2.07 \\
\hline 2009 & 6 & 28 & $09: 17: 5012.296$ & 40.619 & 2.22 & 1.14 \\
\hline 2009 & 6 & 28 & $09: 41: 4512.302$ & 40.625 & 2.39 & 0.5 \\
\hline 2009 & 6 & 28 & $09: 48: 5412.345$ & 40.613 & 2.36 & 2.28 \\
\hline 2009 & 6 & 28 & $09: 57: 5112.305$ & 40.617 & 2.62 & 0.49 \\
\hline 2009 & 6 & 28 & $10: 16: 3212.301$ & 40.618 & 2.94 & 0.46 \\
\hline 2009 & 6 & 28 & $10: 17: 1512.307$ & 40.637 & 2.98 & 0.96 \\
\hline 2009 & 6 & 28 & $10: 18: 5012.296$ & 40.623 & 2.53 & 0.45 \\
\hline 2009 & 6 & 28 & $10: 20: 0512.303$ & 40.620 & 3.51 & 0.38 \\
\hline 2009 & 6 & 28 & $12: 10: 2012.216$ & 40.681 & 2.18 & 0.74 \\
\hline 2009 & 6 & 28 & $12: 20: 4812.334$ & 40.620 & 2.39 & 1.26 \\
\hline 2009 & 6 & 28 & $12: 43: 0412.310$ & 40.627 & 2.41 & 0.79 \\
\hline 2009 & 6 & 28 & $13: 07: 1312.253$ & 40.644 & 2.36 & 1.03 \\
\hline 2009 & 6 & 28 & $13: 09: 48 \quad 12.262$ & 40.661 & 2.35 & 2.13 \\
\hline 2009 & 6 & 28 & $13: 21: 3012.210$ & 40.674 & 2.37 & 0.96 \\
\hline 2009 & 6 & 28 & $13: 22: 4112.228$ & 40.672 & 2.42 & 1.16 \\
\hline 2009 & 6 & 28 & $13: 28: 5612.231$ & 40.673 & 2.26 & 2.06 \\
\hline
\end{tabular}




\begin{tabular}{|c|c|c|c|c|c|c|}
\hline 2009 & 6 & 28 & $14: 14: 5012.246$ & 40.643 & 2.16 & 1.98 \\
\hline 2009 & 6 & 28 & $14: 38: 0512.251$ & 40.644 & 2.42 & 0.79 \\
\hline 2009 & 6 & 28 & $16: 14: 0212.234$ & 40.671 & 2.26 & 0.76 \\
\hline 2009 & 6 & 28 & $16: 52: 1312.206$ & 40.683 & 2.31 & 0.95 \\
\hline 2009 & 6 & 28 & $17: 16: 3512.228$ & 40.684 & 2.27 & 1.92 \\
\hline 2009 & 6 & 28 & $17: 37: 3612.219$ & 40.677 & 2.36 & 1.92 \\
\hline 2009 & 6 & 28 & $20: 07: 3311.616$ & 41.023 & 2.05 & 0.73 \\
\hline 2009 & 6 & 29 & $01: 00: 3411.600$ & 41.035 & 1.96 & 1.26 \\
\hline 2009 & 6 & 29 & $06: 30: 1311.592$ & 40.913 & 2.03 & 3.77 \\
\hline 2009 & 6 & 29 & $07: 29: 0911.600$ & 41.036 & 2.22 & 0.82 \\
\hline 2009 & 6 & 29 & $11: 29: 5211.607$ & 41.046 & 2.38 & 1.45 \\
\hline 2009 & 6 & 29 & $22: 06: 0811.914$ & 41.160 & 2.20 & 1.04 \\
\hline 2009 & 6 & 29 & $23: 30: 0211.911$ & 41.172 & 2.11 & 0.55 \\
\hline 2009 & 6 & 30 & $11: 09: 2712.468$ & 40.555 & 2.52 & 0.57 \\
\hline 2009 & 6 & 30 & $22: 18: 0311.904$ & 41.173 & 2.01 & 0.96 \\
\hline 2009 & 6 & 30 & $22: 55: 0612.245$ & 40.651 & 2.06 & 1.27 \\
\hline 2009 & 7 & 2 & $01: 26: 0412.354$ & 40.590 & 2.06 & 4.61 \\
\hline 2009 & 7 & 4 & $23: 51: 3112.411$ & 40.565 & 2.02 & 2.07 \\
\hline 2009 & 7 & 5 & $16: 47: 2013.369$ & 39.975 & 2.83 & 1.39 \\
\hline 2009 & 7 & 5 & $22: 05: 1112.057$ & 39.641 & 2.42 & 1.13 \\
\hline 2009 & 7 & 6 & $00: 21: 5013.048$ & 39.533 & 2.68 & 0.56 \\
\hline 2009 & 7 & 7 & $11: 00: 1911.987$ & 40.797 & 2.14 & 3.24 \\
\hline 2009 & 7 & 7 & $17: 07: 3013.234$ & 39.823 & 2.82 & 0.54 \\
\hline 2009 & 7 & 7 & $22: 30: 3013.230$ & 39.739 & 2.62 & 1.03 \\
\hline 2009 & 7 & 9 & $16: 00: 3911.947$ & 41.189 & 2.24 & 0.42 \\
\hline 2009 & 7 & 9 & $16: 38: 0411.950$ & 41.174 & 2.11 & 0.95 \\
\hline 2009 & 7 & 10 & $00: 21: 1812.328$ & 40.606 & 2.01 & 0.77 \\
\hline 2009 & 7 & 10 & $01: 48: 3212.358$ & 40.486 & 1.98 & 1.42 \\
\hline 2009 & 7 & 10 & $06: 21: 2711.894$ & 41.076 & 2.07 & 0.83 \\
\hline 2009 & 7 & 10 & $06: 23: 5011.897$ & 41.067 & 2.22 & 0.53 \\
\hline 2009 & 7 & 10 & $23: 07: 5212.443$ & 40.563 & 2.07 & 0.68 \\
\hline 2009 & 7 & 11 & $22: 12: 0612.809$ & 39.572 & 2.56 & 2.42 \\
\hline 2009 & 7 & 11 & $22: 39: 5813.555$ & 40.687 & 2.61 & 7.46 \\
\hline 2009 & 7 & 12 & $04: 29: 1911.591$ & 40.992 & 2.26 & 0.75 \\
\hline 2009 & 7 & 12 & $07: 59: 1012.261$ & 40.653 & 2.18 & 1.78 \\
\hline 2009 & 7 & 12 & $16: 17: 5912.459$ & 40.563 & 2.39 & 0.57 \\
\hline 2009 & 7 & 12 & $21: 19: 5811.617$ & 40.980 & 2.10 & 0.95 \\
\hline 2009 & 7 & 12 & $22: 01: 3511.595$ & 40.996 & 2.83 & 0.42 \\
\hline 2009 & 7 & 12 & $22: 36: 0311.591$ & 40.994 & 2.16 & 0.56 \\
\hline 2009 & 7 & 12 & $23: 41: 0711.591$ & 40.995 & 2.06 & 0.85 \\
\hline 2009 & 7 & 12 & $23: 52: 4111.586$ & 40.990 & 1.97 & 0.89 \\
\hline 2009 & 7 & 12 & $23: 54: 4111.593$ & 40.989 & 1.97 & 0.83 \\
\hline 2009 & 7 & 13 & $00: 10: 4211.591$ & 40.989 & 2.00 & 0.85 \\
\hline 2009 & 7 & 13 & $00: 15: 4911.606$ & 40.992 & 1.95 & 0.74 \\
\hline 2009 & 7 & 13 & $03: 54: 5511.598$ & 41.009 & 2.14 & 1.01 \\
\hline 2009 & 7 & 13 & $10: 21: 2511.342$ & 40.718 & 2.31 & 7.79 \\
\hline 2009 & 7 & 13 & $17: 27: 2812.461$ & 39.671 & 2.57 & 0.81 \\
\hline 2009 & 7 & 13 & $23: 23: 5012.492$ & 40.538 & 2.03 & 1.21 \\
\hline 2009 & 7 & 14 & $01: 47: 2313.137$ & 39.648 & 2.62 & 1.14 \\
\hline 2009 & 7 & 14 & $09: 00: 0912.248$ & 40.650 & 2.19 & 1.23 \\
\hline 2009 & 7 & 14 & $21: 16: 2811.976$ & 41.253 & 2.06 & 0.88 \\
\hline 2009 & 7 & 15 & $19: 34: 4312.175$ & 40.704 & 2.03 & 0.95 \\
\hline 2009 & 7 & 16 & $01: 04: 3511.896$ & 41.068 & 1.97 & 0.69 \\
\hline 2009 & 7 & 16 & $18: 43: 2411.947$ & 41.266 & 2.31 & 0.57 \\
\hline 2009 & 7 & 16 & $20: 09: 1111.952$ & 41.269 & 2.30 & 0.92 \\
\hline 2009 & 7 & 16 & $20: 45: 2412.903$ & 39.498 & 2.79 & 8.9 \\
\hline 2009 & 7 & 17 & $01: 50: 1212.471$ & 40.553 & 2.27 & 0.7 \\
\hline 2009 & 7 & 17 & $02: 21: 1711.953$ & 41.188 & 2.27 & 0.57 \\
\hline 2009 & 7 & 17 & $12: 41: 2211.966$ & 41.169 & 2.13 & 1.52 \\
\hline 2009 & 7 & 18 & $19: 57: 3112.996$ & 40.746 & 2.39 & 6.27 \\
\hline 2009 & 7 & 18 & $21: 50: 4914.598$ & 39.873 & 3.13 & 6.39 \\
\hline 2009 & 7 & 18 & $23: 09: 0511.869$ & 40.952 & 1.83 & 3.23 \\
\hline 2009 & 7 & 19 & $11: 34: 2312.463$ & 40.549 & 2.26 & 0.55 \\
\hline 2009 & 7 & 19 & $23: 49: 3511.330$ & 40.029 & 2.36 & 4.97 \\
\hline 2009 & 7 & 20 & $03: 09: 1212.187$ & 39.739 & 2.60 & 1.23 \\
\hline 2009 & 7 & 21 & $00: 50: 1512.614$ & 40.454 & 2.11 & 1.55 \\
\hline 2009 & 7 & 21 & $14: 56: 1812.712$ & 40.573 & 2.44 & 3.34 \\
\hline 2009 & 7 & 21 & $17: 25: 2812.307$ & 40.612 & 2.15 & 1.2 \\
\hline 2009 & 7 & 21 & $18: 46: 2014.500$ & 40.445 & 3.13 & $10 . e$ \\
\hline 2009 & 7 & 21 & $20: 59: 3113.552$ & 39.885 & 2.80 & 1.45 \\
\hline 2009 & 7 & 22 & $12: 22: 0913.377$ & 40.021 & 2.97 & 6.88 \\
\hline 2009 & 7 & 22 & $20: 35: 4112.454$ & 40.564 & 2.08 & 0.61 \\
\hline 2009 & 7 & 23 & $04: 50: 4211.868$ & 41.245 & 2.31 & 2.06 \\
\hline 2009 & 7 & 23 & $05: 59: 0311.375$ & 40.922 & 2.40 & 2.32 \\
\hline 2009 & 7 & 23 & $06: 01: 2211.377$ & 40.922 & 2.90 & 0.45 \\
\hline 2009 & 7 & 23 & $22: 03: 1713.673$ & 39.941 & 2.79 & 0.99 \\
\hline
\end{tabular}




\begin{tabular}{|c|c|c|c|c|c|c|}
\hline 2009 & 7 & 23 & $22: 54: 4812.435$ & 40.577 & 2.20 & 0.43 \\
\hline 2009 & 7 & 24 & $00: 33: 1710.995$ & 39.673 & 2.63 & 2.32 \\
\hline 2009 & 7 & 25 & $02: 09: 1311.011$ & 39.682 & 3.16 & 5.09 \\
\hline 2009 & 7 & 25 & $11: 27: 5312.425$ & 40.572 & 2.26 & 0.67 \\
\hline 2009 & 7 & 26 & $06: 13: 2013.547$ & 39.825 & 2.94 & 7.15 \\
\hline 2009 & 7 & 26 & $19: 59: 0613.538$ & 39.899 & 2.82 & 0.67 \\
\hline 2009 & 7 & 27 & $07: 04: 4413.462$ & 39.958 & 3.08 & 1.6 \\
\hline 2009 & 7 & 27 & $09: 26: 1213.533$ & 39.891 & 3.01 & 0.97 \\
\hline 2009 & 7 & 28 & $00: 05: 0713.061$ & 39.578 & 2.82 & 1.92 \\
\hline 2009 & 7 & 28 & $01: 43: 4812.122$ & 40.754 & 2.14 & 1.2 \\
\hline 2009 & 7 & 28 & $10: 47: 3013.278$ & 39.825 & 2.78 & 0.7 \\
\hline 2009 & 7 & 29 & $06: 58: 1313.476$ & 40.013 & 3.00 & 1.84 \\
\hline 2009 & 7 & 29 & $11: 37: 2713.179$ & 39.816 & 2.76 & 0.97 \\
\hline 2009 & 7 & 29 & $19: 31: 0712.908$ & 39.617 & 2.64 & 3.39 \\
\hline 2009 & 7 & 29 & $23: 27: 3913.587$ & 39.799 & 2.83 & 2.12 \\
\hline 2009 & 7 & 30 & $06: 41: 4311.539$ & 42.193 & 3.31 & 1.01 \\
\hline 2009 & 7 & 30 & $22: 35: 1013.550$ & 39.899 & 2.73 & 0.67 \\
\hline 2009 & 7 & 30 & $23: 53: 2213.557$ & 39.853 & 2.81 & 7,00 \\
\hline 2009 & 8 & 1 & $19: 25: 1913.297$ & 39.836 & 3.01 & 2.75 \\
\hline 2009 & 8 & 1 & $22: 27: 0714.283$ & 40.268 & 3.09 & 0.4 \\
\hline 2009 & 8 & 2 & $22: 26: 5012.871$ & 40.631 & 2.78 & 0.49 \\
\hline 2009 & 8 & 3 & $00: 45: 3712.061$ & 41.654 & 2.30 & 0.84 \\
\hline 2009 & 8 & 3 & $00: 46: 1311.984$ & 41.560 & 2.24 & 5.99 \\
\hline 2009 & 8 & 3 & $01: 57: 5512.808$ & 39.638 & 2.62 & 0.32 \\
\hline 2009 & 8 & 3 & $09: 12: 3312.061$ & 41.615 & 2.78 & 0.87 \\
\hline 2009 & 8 & 3 & $20: 37: 2013.709$ & 39.904 & 2.93 & 0.51 \\
\hline 2009 & 8 & 3 & $21: 13: 4812.960$ & 39.584 & 2.74 & 0.34 \\
\hline 2009 & 8 & 5 & $23: 13: 0812.434$ & 40.570 & 2.26 & 0.61 \\
\hline 2009 & 8 & 13 & $20: 49: 0111.696$ & 39.596 & 2.54 & 3.54 \\
\hline 2009 & 8 & 14 & $22: 50: 5114.757$ & 39.873 & 3.14 & 2.13 \\
\hline 2009 & 8 & 15 & $01: 41: 3512.096$ & 41.273 & 1.94 & 0.75 \\
\hline 2009 & 8 & 20 & $13: 29: 3412.740$ & 40.641 & 2.62 & 0.94 \\
\hline 2009 & 8 & 21 & $00: 25: 2412.591$ & 40.506 & 2.08 & 1.23 \\
\hline 2009 & 8 & 22 & $01: 46: 1611.937$ & 39.587 & 2.49 & 1.22 \\
\hline 2009 & 8 & 25 & $01: 30: 4111.538$ & 41.211 & 2.13 & 0.97 \\
\hline 2009 & 8 & 25 & $10: 35: 5811.558$ & 41.208 & 2.53 & 0.76 \\
\hline 2009 & 8 & 25 & $20: 02: 2211.561$ & 41.194 & 2.64 & 0.87 \\
\hline 2009 & 8 & 26 & $06: 24: 0011.553$ & 41.206 & 3.63 & 0.74 \\
\hline 2009 & 8 & 27 & $02: 45: 2012.016$ & 39.691 & 2.44 & 0.97 \\
\hline 2009 & 8 & 28 & $22: 30: 1210.254$ & 40.569 & 2.71 & 1.78 \\
\hline 2009 & 8 & 29 & $03: 09: 3012.501$ & 40.534 & 2.34 & 1.55 \\
\hline 2009 & 8 & 30 & $00: 02: 119.611$ & 39.395 & 3.77 & 1.45 \\
\hline 2009 & 9 & 1 & $11: 15: 1712.463$ & 40.553 & 2.55 & 1.92 \\
\hline 2009 & 9 & 1 & $14: 12: 2312.008$ & 40.930 & 2.37 & 1.84 \\
\hline 2009 & 9 & 5 & $09: 27: 0611.461$ & 40.721 & 2.18 & 2.06 \\
\hline 2009 & 9 & 5 & $14: 51: 0411.898$ & 41.286 & 2.16 & 1.14 \\
\hline 2009 & 9 & 8 & $00: 10: 5413.505$ & 39.596 & 2.69 & 2.12 \\
\hline 2009 & 9 & 8 & $04: 42: 4912.590$ & 40.502 & 2.36 & 0.32 \\
\hline 2009 & 9 & 9 & $04: 08: 5412.436$ & 40.573 & 2.29 & 3.23 \\
\hline 2009 & 9 & 10 & $06: 23: 3512.840$ & 40.642 & 2.41 & 4.97 \\
\hline 2009 & 9 & 10 & $07: 17: 3912.472$ & 40.534 & 2.15 & 1.92 \\
\hline 2009 & 9 & 10 & $12: 12: 3911.909$ & 41.057 & 2.09 & 0.99 \\
\hline 2009 & 9 & 10 & $15: 16: 1911.956$ & 41.455 & 2.35 & 1.84 \\
\hline 2009 & 9 & 10 & $17: 57: 3811.954$ & 41.447 & 2.45 & 0.97 \\
\hline 2009 & 9 & 11 & $20: 51: 1013.521$ & 39.623 & 2.69 & 1.6 \\
\hline 2009 & 9 & 12 & $03: 23: 4312.304$ & 39.337 & 2.52 & 0.67 \\
\hline 2009 & 9 & 13 & $03: 22: 5812.453$ & 40.540 & 2.04 & 0.67 \\
\hline 2009 & 9 & 13 & $20: 39: 0612.558$ & 40.440 & 2.04 & 0.61 \\
\hline 2009 & 9 & 14 & $11: 46: 1012.754$ & 39.536 & 2.71 & 0.42 \\
\hline 2009 & 9 & 14 & $22: 09: 2110.222$ & 41.038 & 2.87 & 0.49 \\
\hline 2009 & 9 & 14 & $22: 15: 2910.212$ & 41.033 & 2.86 & 0.34 \\
\hline 2009 & 9 & 14 & $23: 22: 5712.026$ & 41.223 & 1.88 & 1.55 \\
\hline 2009 & 9 & 15 & $22: 22: 1912.463$ & 40.547 & 1.93 & 1.52 \\
\hline 2009 & 9 & 16 & $05: 58: 4412.398$ & 40.560 & 2.22 & 1.42 \\
\hline 2009 & 9 & 17 & $23: 20: 1112.461$ & 40.554 & 2.04 & 1.45 \\
\hline 2009 & 9 & 18 & $10: 38: 2611.686$ & 39.923 & 2.39 & 1.39 \\
\hline 2009 & 9 & 18 & $16: 37: 1012.466$ & 40.550 & 2.24 & 1.6 \\
\hline 2009 & 9 & 18 & $23: 18: 3411.482$ & 40.981 & 2.16 & 1.78 \\
\hline 2009 & 9 & 19 & $00: 45: 1711.320$ & 41.996 & 2.68 & 1.84 \\
\hline 2009 & 9 & 19 & $17: 56: 079.530$ & 40.101 & 4.66 & 1.92 \\
\hline 2009 & 9 & 19 & $20: 21: 349.509$ & 40.096 & 3.25 & 0.32 \\
\hline 2009 & 9 & 19 & $23: 28: 219.518$ & 40.074 & 3.01 & 0.34 \\
\hline 2009 & 9 & 20 & $00: 51: 159.552$ & 40.145 & 2.81 & 1.78 \\
\hline 2009 & 9 & 20 & $16: 29: 0711.318$ & 41.990 & 2.65 & 0.45 \\
\hline 2009 & 9 & 20 & $18: 11: 0011.295$ & 42.002 & 2.85 & 0.42 \\
\hline 2009 & 9 & 22 & $01: 10: 3811.955$ & 41.285 & 1.89 & 0.4 \\
\hline
\end{tabular}




$\begin{array}{lllllll}2009 & 9 & 24 & 07: 56: 4612.877 & 39.766 & 2.91 & 0.43 \\ 2009 & 9 & 25 & 01: 47: 1113.630 & 39.961 & 2.69 & 0.49 \\ 2009 & 9 & 25 & 18: 27: 4013.579 & 39.830 & 2.73 & 1.01 \\ 2009 & 9 & 25 & 22: 49: 2211.622 & 39.769 & 2.80 & 1.14 \\ 2009 & 9 & 27 & 05: 29: 0411.918 & 41.703 & 2.54 & 1.92 \\ 2009 & 9 & 27 & 23: 15: 0511.627 & 41.018 & 1.79 & 1.42 \\ 2009 & 9 & 28 & 00: 51: 2411.948 & 41.189 & 1.90 & 1.23 \\ 2009 & 10 & 2 & 10: 17: 1111.789 & 42.271 & 3.04 & 1.42 \\ 2009 & 10 & 2 & 15: 51: 5313.669 & 39.909 & 3.00 & 1.22 \\ 2009 & 10 & 2 & 16: 35: 2913.672 & 39.902 & 3.44 & 1.78 \\ 2009 & 10 & 2 & 20: 29: 4413.559 & 39.804 & 2.65 & 1.45 \\ 2009 & 10 & 3 & 01: 00: 3313.672 & 39.899 & 2.74 & 1.01 \\ 2009 & 10 & 3 & 01: 37: 3312.440 & 40.568 & 2.14 & 1.22 \\ 2009 & 10 & 3 & 05: 58: 1011.791 & 42.238 & 3.11 & 1.42 \\ 2009 & 10 & 5 & 21: 47: 4912.398 & 40.581 & 2.04 & 1.23 \\ 2009 & 10 & 5 & 23: 47: 3815.300 & 39.345 & 3.22 & 1.92 \\ 2009 & 10 & 6 & 22: 42: 1912.433 & 40.574 & 1.85 & 1.55 \\ 2009 & 10 & 7 & 00: 46: 3312.421 & 40.558 & 1.64 & 1.84\end{array}$

